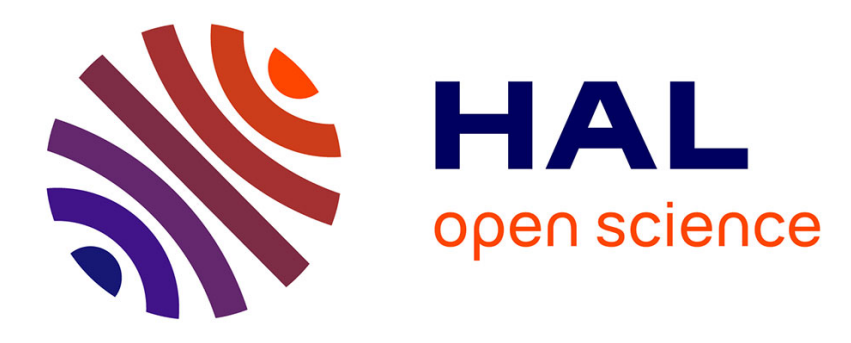

\title{
Re-entry radiation aerothermodynamics in the vacuum ultraviolet
}

Umar Sheikh

\section{To cite this version:}

Umar Sheikh. Re-entry radiation aerothermodynamics in the vacuum ultraviolet. Engineering Sciences [physics]. Ecole Centrale Paris, 2014. English. NNT : 2014ECAP0036 • tel-01088680

\section{HAL Id: tel-01088680 \\ https://theses.hal.science/tel-01088680}

Submitted on 28 Nov 2014

HAL is a multi-disciplinary open access archive for the deposit and dissemination of scientific research documents, whether they are published or not. The documents may come from teaching and research institutions in France or abroad, or from public or private research centers.
L'archive ouverte pluridisciplinaire HAL, est destinée au dépôt et à la diffusion de documents scientifiques de niveau recherche, publiés ou non, émanant des établissements d'enseignement et de recherche français ou étrangers, des laboratoires publics ou privés. 


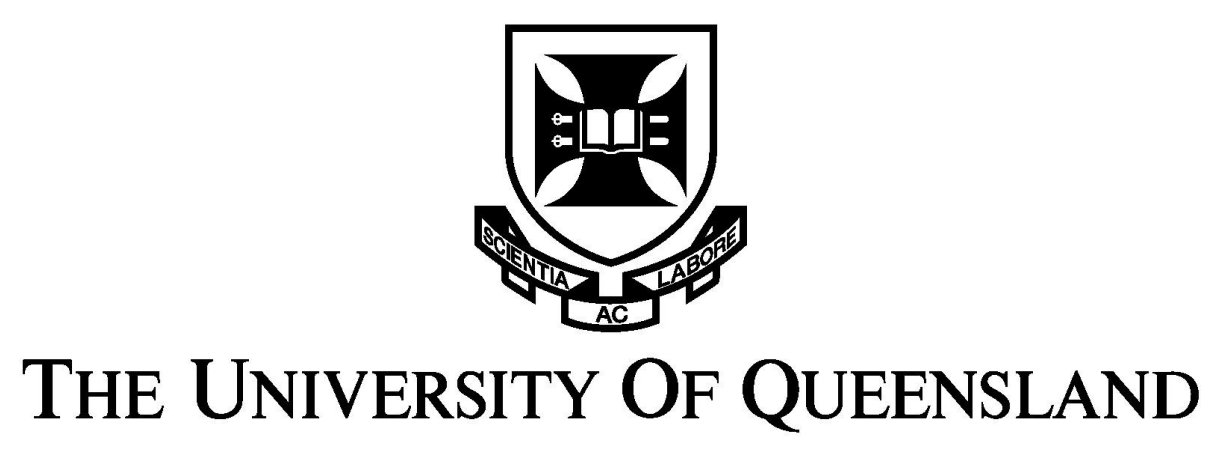

A U S T R A L I A

\section{Re-Entry Radiation Aerothermodynamics in the Vacuum Ultraviolet}

Umar A Sheikh

Bachelor of Aerospace Engineering (Honours IA)

A thesis submitted for the degree for the Doctor of Philosophy at

The University of Queensland in 2014

School of Mechanical and Mining Engineering

in Cotutelle with Ecole Centrale Paris

Laboratoire d'Énergétique Moléculaire et Macroscopique,

Combustion (EM2C), CNRS UPR288 


\begin{abstract}
$\underline{\text { Abstract }}$
A major design challenge for re-entry capsules lies in the modelling of convective and radiative heat transfer to the surface of the vehicle. At certain points on superorbital re-entry trajectories, up to $40 \%$ of the total radiative heat flux is contributed by the vacuum ultra-violet (VUV) spectral range and it is in this spectral range that the largest uncertainties lie. The high level of uncertainty in the VUV is a result of a lack of published experimental data due to difficulties encountered in measuring radiation in the VUV, such as strong absorption by most optical materials and air. Additional complexities of the VUV spectral range include its strongly self-absorbing nature and spectral line broadening.

The primary goal of this study was to obtain calibrated spectral measurements in the VUV that enable the investigation of physical processes occurring in the shock layer that influence the incident radiative heat flux. In particular, the issues to be investigated were the variation in spectral radiance observed across a shock layer compared to the spectral radiance measured through the surface, the effects of self-absorption on spectral line intensity and the broadening of spectral lines in the VUV as a function of depth of radiating flow field. The measurements made across and through the surface of a model provide the first set of calibrated experimental results for the validation of computational codes used to predict incident radiative heat flux. Measurements made with a varying depth of radiating flow field provide a unique set of experimental data for the validation of radiation transport models and broadening coefficients. This study also used computational simulations to investigate the accuracy of a flow field solver coupled with two reaction rate schemes and compared the spectra produced using Specair with experimentally measured values.
\end{abstract}

To achieve these goals, an optical system was designed to measure the VUV radiative emission produced around a blunt two-dimensional model in a spatially resolved manner across the shock layer. Spatial resolution allowed for spectral measurements to be made in both the equilibrium and non-equilibrium parts of the shock layer. A second optical system was designed to obtain measurements of VUV radiation incident on the surface of the model. This system incorporated a window in the surface with a mirror housed within the model to deflect the radiation out of the test section and into the detection system. To effectively vary the depth of the radiating flow field, the length of a two-dimensional model was varied, changing the depth of the shock layer being observed.

The X2 expansion tube was used to create the high enthalpy flows required to produce radiating shock layers. Two flow conditions were created for this study that represented flight equivalent velocities of $10.0 \mathrm{~km} / \mathrm{s}$ and $12.2 \mathrm{~km} / \mathrm{s}$. The spectroscopy system utilized for this study consisted of an evacuated McPherson NOVA 225 spectrometer coupled to 
an Andor iStar VUV enhanced intensified charge coupled device. An evacuated light tube sealed with a magnesium fluoride window was required to extend the evacuated light path to the model and avoid any absorption by molecular oxygen. An in-situ calibration of the VUV spectroscopy system was conducted using a deuterium lamp located in the position of the radiating shock layer.

The integrated incident spectral radiance measured through the surface of the model between $115 \mathrm{~nm}$ and $180 \mathrm{~nm}$ was $0.744 \mathrm{~W} / \mathrm{cm}^{2} \mathrm{sr}$ for the $10.0 \mathrm{~km} / \mathrm{s}$ condition and $12.3 \mathrm{~W} / \mathrm{cm}^{2} \mathrm{sr}$ for the faster $12.2 \mathrm{~km} / \mathrm{s}$ condition. These values were $25 \%$ and $31 \%$ respectively of the integrated spectral radiance measured in the equilibrium region when viewing across the shock layer. The repeatability of the experimental results obtained by the VUV emission spectroscopy system was measured to be better than $15 \%$ for spectral line intensity and $20 \%$ for integrated intensity between $115 \mathrm{~nm}$ and $180 \mathrm{~nm}$. All spectral lines measured were found to be self-absorbing to varying degrees for the $10.0 \mathrm{~km} / \mathrm{s}$ condition and strongly self-absorbing for the $12.2 \mathrm{~km} / \mathrm{s}$ condition. Spectral line broadening of the strongly selfabsorbing lines was also observed.

Computational simulations of the flow field around each model were made using the inhouse solver Eilmer 3 utilising the Park finite reaction rate scheme. Using the computed flow field as inputs into Specair, spectra were generated for both conditions and models. Simulations of the self-absorption experiments predicted self-absorption of all spectral lines investigated and showed good agreement with the measured values for most spectral lines. The computed broadened spectral line width was found to be an under prediction of the measured spectral line width.

An extension to this study was the creation of a VUV emission spectroscopy system for use on the plasma torch at Ecole Centrale Paris (ECP). The plasma torch produces air at thermochemical equilibrium and can therefore be used to validate Einstein coefficients used in the prediction of spectral line intensities without the complexities of the nonequilibrium regions observed in the expansion tube. The flow condition used for this study had a peak temperature of $6,600 \mathrm{~K}$ and the torch was operated at atmospheric pressure.

A nitrogen flush through the optical path was employed to reduce absorption by molecular oxygen. To maintain the structural integrity of the viewing window at the edge of the plasma, a water cooled copper housing assembly was designed. Simulations conducted using the COMSOL Multiphysics program predicted the thermal stresses on the window would not exceed the apparent elastic limit. Experiments were conducted to investigate variance in transmission of the viewing window due to surface degradation and absorption by molecular oxygen between the edge of the plasma and the viewing window. Through 
the observation of the $174 \mathrm{~nm}$ nitrogen doublet and the $777 \mathrm{~nm}$ oxygen triplet, it was concluded that surface degradation of the viewing window was occurring when the window was closer than $5 \mathrm{~mm}$ from the plasma edge. It was also found that there was molecular oxygen absorbing a fraction of the signal at this distance.

A further goal of the plasma torch VUV spectroscopy system was to establish a system capable of varying the depth of the radiating flow field to investigate self-absorption and spectral line broadening. A water cooled copper pipe was used as a fence and traversed through the plasma during operation. Spectral measurements of the nitrogen doublet at $174 \mathrm{~nm}$ and the oxygen triplet at $777 \mathrm{~nm}$ were made at $1 \mathrm{~mm}$ spacings. Self-absorption of the $174 \mathrm{~nm}$ nitrogen doublet was confirmed and no spectral line broadening was observed.

Calibration of the ECP VUV spectroscopy system was carried out using an argon miniarc placed at the location of the plasma. The in-situ calibration was carried out after the experiment to account for any damage that occurred to the window during testing and any absorption through the optical path by molecular oxygen. Due to the observed degradation of the window surface during the test time and unquantified level of absorption between the viewing window and the plasma, a large uncertainty was estimated for the calibration resulting in an inability to validate the Einstein coefficients of the nitrogen doublet at $174 \mathrm{~nm}$.

Through this study two VUV spectroscopy systems were created on two separate facilities and a benchmark dataset was obtained viewing shock layers across and through the surface of a model. The levels of self-absorption and spectral line broadening were also quantified for varying depths of radiating flow fields providing a unique set of results for validation of radiation transport solvers. Computational simulations of the flow field conducted with Eilmer 3, in conjunction with the Park reaction rate scheme, were used to create spectra with Specair and the results were compared against experimentally measured values. 


\section{Declaration by author}

This thesis is composed of my original work, and contains no material previously published or written by another person except where due reference has been made in the text. I have clearly stated the contribution by others to jointly-authored works that I have included in my thesis.

I have clearly stated the contribution of others to my thesis as a whole, including statistical assistance, survey design, data analysis, significant technical procedures, professional editorial advice, and any other original research work used or reported in my thesis. The content of my thesis is the result of work I have carried out since the commencement of my research higher degree candidature and does not include a substantial part of work that has been submitted to qualify for the award of any other degree or diploma in any university or other tertiary institution. I have clearly stated which parts of my thesis, if any, have been submitted to qualify for another award.

I acknowledge that an electronic copy of my thesis must be lodged with the University Library and, subject to the General Award Rules of The University of Queensland, immediately made available for research and study in accordance with the Copyright Act 1968.

I acknowledge that copyright of all material contained in my thesis resides with the copyright holder(s) of that material. Where appropriate I have obtained copyright permission from the copyright holder to reproduce material in this thesis. 


\section{Publications during candidature}

U. A. Sheikh. Vacuum ultraviolet measurements on the surface of a blunt body for reentry flows. In 11th International Workshop on Shock Tube Technology, University of Queensland, Brisbane, Australia., 2011

U. A. Sheikh, R. G. Morgan, and T. J. McIntyre. Self-absorption of vacuum ultraviolet radiation in superorbital flows. In 5th International Workshop on Radiation of High Temperature Gases in Atmospheric Entry, Barcelona, Spain, 2012

U. A Sheikh, R. G. Morgan, F. Zander, T. N. Eichmann, and T. J. McIntrye. Vacuum ultraviolet emission spectroscopy system for superorbital reentries. In 18th AIAA International Space Planes and Hypersonic Systems and Technologies Conference, Tours, France., 2012

U. A Sheikh, C. Jacobs, C. O. Laux, R. G. Morgan, and T. J McIntyre. Measurements of high enthalpy radiating flows in the vacuum ultraviolet. In 29th International Symposium on Shock Waves, Madison, WI, USA., 2013

U. A. Sheikh, C. Jacobs, R. G. Morgan, C. O. Laux, and T. J. McIntyre. Re-entry radiative heating studies in the vacuum ultraviolet. In Aerospace Thematic Workshop., Aussois, France, 2013

U. A Sheikh, C. O. Laux, R. G. Morgan, and T. J McIntyre. Through surface and across surface vacuum ultraviolet spectral measurements in an expansion tube. In 44th AIAA Thermophysics Conference, San Diego, California, USA., 2013

U. A Sheikh, R. G Morgan, and T.J McIntyre. Vacuum ultraviolet spectral measurements for superorbital earth entry in the x2 expansion tube. AIAA (Submitted), 2014

C Jacobs, U.A Sheikh, M MacDonald, and C.O Laux. Vacuum ultraviolet radiation studies in a plasma torch facility from 170 - $200 \mathrm{~nm}$. In 44th AIAA Thermophysics Conference, San Diego, California, USA., 2013. AIAA

P. Leyland, T. McIntyre, U. Sheikh, T. Eichmann, F. Zander, R. Morgan, S. Loehle, T. Hermann, F. deFillippis, E. Trifoni, and G. Cillo. Vuv radiation measurements for radiation-abalation coupling. In 5th International Workshop on Radiation of High Temperature Gases in Atmospheric Entry, Barcelona, Spain 
R. G. Morgan, T. J. McIntyre, and U. A. Sheikh. Simulation of radiating flows in impulsive facilities. In 49th AIAA Aerospace Sciences Meeting including the New Horizons Forum and Aerospace Exposition, Orlando, Florida, 2011

R. G. Morgan, U. A Sheikh, , and D. E. Gildfind. Standing shock formation in a nonreflected shock tube. In The 28th International Symposium on Shock Waves, Manchester, UK., 2011

H Porat, U. A Sheikh, R. G Morgan, and T. J McIntyre. Vacuum ultraviolet emission spectroscopy at titan and mars atmospheric entry conditions. In 44th AIAA Thermophysics Conference, San Diego, California, USA., 2013

F. Zander, Morgan R. G., Sheikh U. A., Buttsworth D. G., and Teakle P. R. Hot wall reentry testing in hypersonic impulse facilities. AIAA, 51:476-484, 2012

F. Zander, Morgan R. G., Sheikh U. A., Buttsworth D. G., and Teakle P. R. Hot wall testing methodology for impulse facilities. In 18th AIAA International Space Planes and Hypersonic Systems and Technologies Conference, Tours, France, 2012

F Zander, P. A Jacobs, U. A Sheikh, and R. G Morgan. High speed imaging of spherical shock standoff in hypervelocity flows. In 18th Australasian Fluid Mechanics Conference. Australasian Fluid Mechanics Society, 2012

F Zander, P. A Jacobs, U. A Sheikh, D. Buttsworth, and R. G Morgan. High speed imaging of $\mathrm{x} 2$ expansion tube flows. In 30th International Congress on High-Speed Imaging and Photonics, 2012

P Leyland, T McIntyre, R Morgan, P Jacobs, F Zander, U Sheikh, T Eichmann, E Fahy, O Joshi, G Duffa, et al. Radiation-ablation coupling for capsule reentry heating via simulation and expansion tube investigations. In 5th European Conference for Aeronautics and Space Sciences (EUCASS), 2013

\section{Publications included in this thesis}

No publications included. 


\section{Contributions by others to the thesis}

Christophe Laux and Alex Louzet contributed significantly to the Résumé.

Carolyn Jacobs computed the temperature profile presented in Figure 6.2.

Carolyn Jacobs computed the simulated spectrum used in Figure 6.23 and Figure 6.24.

Statement of parts of the thesis submitted to qualify for the award of another degree

None. 


\section{Acknowledgements}

First and foremost, a massive thankyou to my supervisors Richard, Tim and Christophe. This project and the amazing opportunities I've been privileged to experience would not have been possible without the work you guys have put in.

Richard I don't know how many people get a supervisor who can come up with an answer to the most technical question using first order principles and the back of an envelope in two minutes and be within $2 \%$ of the value. I guess the fishing trips and barbeques make up for the error. Thanks mate!

Tim It's very hard to slip a typo or an average sentence by you. You are always the first to read everything and amazingly thorough. Not to mention your huge wealth of knowledge on optics and all things physics related. I've greatly appreciated the way you've explained these things to me when it all got pretty hairy. Thank you Tim.

Christophe I believe your willingness to include me in an array of projects and bring me along to the various meetings and conferences will go a long way towards shaping my career. These opportunities have also given me a lot of insight into how research is carried out on a global scale and its applications. Your passion for spectroscopy and knowledge of radiative heating has been instrumental in my understanding of the field and the analysis carried out in this project. Merci ami.

To everyone at UQ, I have adored my years at the Centre for Hypersonics. The team atmosphere that exists is amazing. I am gen $\mathrm{Y}$ and love instant gratification, and working with you guys I have definitely been able to get this. So huge thanks to Fabs, Hadas, Elise, Dyl, Brades, Troy, Grump, Doc, James, Jorge, Christoher, Brad, Han and everyone at the CfH. Especially those who've had the 'pleasure' of operating the tunnel with the VUV gear installed, thanks for your patience. To Brian and Frans, thank you for all your hard work in putting the together the high vacuum bits, building perfects model off a crude drawing and altering pieces as fast as humanly possible. And of course, the hardest working man for the CfH who doesn't actually work for the CfH, PJ. You're a legend.

I would also like to acknowledge those who have been involved in my extra-curricular activities in Brisbane. In particular embodiment of menacement that is Quest and my fellow cohort of execs. There was never a time when I couldn't call you guys to escape the $\mathrm{PhD}$ world. I feel very lucky to have crossed paths with you guys upon my move up to Brisbane. 
Heading to ECP and living in Paris for a year was an amazing adventure and it would not have been possible without all the work you put in Caz. I cannot thank you enough. And working with you and Rowdy on the plasma torch was great fun. I would also like to thank everyone else at ECP for all the laughs and good times over the year.

To my Parisian partner in crime, Coco, thank you so much for the ten million things you helped me out with. I often describe starting out in Paris as being a baby again, which would pretty much make you a parent. A very corrupting and irresponsible parent, which I greatly appreciated! And to the Baz, Fanny and gang from UJ2CP, it was great to have such a good group of people to share Paris life with. You made it feel like home. To everyone I've met on my travels, old friends and new, thanks for having me on your couches, showing me your home towns and coming on adventures with me.

And last but not least, my friends and family back home in Melbourne. You guys are always there for me to provide me with everything I could possibly ask for. I always count down the days until I'm back home and can slip back into my old life as if I never left. This means a great deal to me. Thank you so much.

This work was supported by the Australian Post-graduate Award, Eiffel Doctorale Program, Ablative Thermal Protective Systems grant from the Australian Research Council, Radiation-Shapes-Thermal Protection Investigations for High Speed Earth Re-entry (RASTAS SPEAR) grant from the European Union Seventh Framework Program and Ablation Radiation Coupling grant from the European Space Agency. 


\section{Keywords}

Hypersonics, radiation, radiative heat transfer, vacuum ultraviolet, expansion tubes, plasma, spectroscopy, radiation modelling.

Australian and New Zealand Standard Research Classifications (ANZSRC)

$\begin{array}{ccc}020503 & \text { Nonlinear Optics and Spectroscopy } & 45 \% \\ 090107 & \text { Hypersonic Propulsion and Hypersonic Aerodynamics } & 40 \% \\ 091501 & \text { Computational Fluid Dynamics } & 15 \%\end{array}$

Field of Research (FoR) Classification

FoR code: 0901, Aerospace Engineering 100\% 


\section{THESE}

présentée par

\section{Umar A Sheikh}

pour l'obtention du

GRADE de DOCTEUR

Formation doctorale: Énergétique

Laboratoire d'accueil : $\quad$ Laboratoire d'Énergétique Moléculaire et Macroscopique, Combustion (EM2C) du CNRS et de l'ECP

Laboratoire partenaire: The Centre for Hypersonics School of Mechanical and Mining Engineering The University of Queensland, Australia

\section{Aérothermique rayonnement de rentrée avec un accent sur le rayonnement ultraviolet $\mathrm{du}$ vide}

Soutenue le 07 Mars 2014 devant le jury composé de:

Prof. Prof Christophe Laux Directeur de thèse Ecole Centrale Paris

Prof Richard Morgan

A. Prof Tim McIntyre

Dr Vincent Wheatley

Prof Olivier Chazot

Dr. Viviana Lago

Prof Penelope Sanderson
Directeur de thèse The University of Queensland

Directeur de thèse The University of Queensland

Président

The University of Queensland

Rapporteur

von Karman Institute

Rapporteur

CNRS

Convenor
The University of Queensland
Ecole Centrale des Arts et Manufactures

Grand Établissement sous tutelle

du Ministère de l'Éducation Nationale

Grande Voie des Vignes

92295 Châtenay-Malabry Cedex

Tél : 33 (1) 41131000

Télex : 634991 F EC PARIS
Laboratoire d'Énergétique

Moléculaire et Macroscopique,

Combustion (E.M2.C.)

UPR 288, CNRS et Ecole Centrale Paris

Tél : 33 (1) 41131031

Fax : 33 (1) 47028035

$2014-\mathrm{xx}$ 


\section{Résumé}

L'un des défis majeurs pour la conception des capsules de rentrée concerne la modélisation des transferts convectifs et radiatifs à la surface du véhicule. A certains points des trajectoires de rentrée super-orbitale, jusqu'à $40 \%$ du flux radiatif total émane du domaine spectral VUV (vacuum ultraviolet), or c'est dans ce domaine que les incertitudes sont les plus fortes. Ce haut niveau d'incertitudes est dû en particulier à un manque de données expérimentales fiables. Le rayonnement VUV est en effet difficile à mesurer en raison de la forte absorption de l'air et des optiques utilisées pour sa mesure. Des difficultés d'analyse supplémentaires sont causées par le fort degré d'auto-absorption et par l'élargissement spectral des raies dans le VUV.

L'objectif central de cette étude était d'obtenir des spectres d'émission expérimentaux calibrés dans le VUV afin d'étudier les processus physico-chimiques dans la couche de choc qui contrôlent le flux radiatif. Plus précisément, les objectifs étaient de comparer les spectres observés parallèlement et perpendiculairement à la couche de choc, d'étudier les effets sur l'intensité des raies spectrales émises dans le VUV de l'auto-absorption et de l'élargissement spectral en fonction de la profondeur de champ radiatif (épaisseur optique). Les mesures effectuées perpendiculairement et parallèlement à la surface d'une maquette placée dans l'écoulement représentent un premier jeu de données expérimentales calibrées dans le VUV qui seront utiles pour valider les codes de calcul destinés à prédire le flux radiatif incident. Les mesures obtenues pour différentes profondeurs de champ radiatif représentent quant à elles un ensemble de données expérimentales uniques pour la validation des modèles de transport radiatif et des coefficients d'élargissement des raies. Cette étude s'appuie également sur des simulations numériques afin d'évaluer les prédictions d'un solveur d'écoulement couplé à deux schémas cinétiques à travers la comparaison des spectres mesurés avec les spectres simulés par le code radiatif Specair.

Pour atteindre ces objectifs, un banc optique a été conçu et mis en place pour mesurer l'intensité du rayonnement VUV produit autour d'une maquette bidimensionnelle émoussée, avec une résolution spatiale suffisante pour résoudre le profil d'émission dans la couche de choc. La résolution spatiale a été choisie de façon à pouvoir effectuer des mesures du rayonnement dans les zones d'équilibre et hors équilibre de la couche de choc. Un deuxième système a été conçu pour obtenir des mesures du rayonnement VUV incident sur la surface de la maquette. Ce système est constitué d'un hublot placé sur la surface de la maquette et d'un miroir logé à l'intérieur de la maquette pour transmettre le rayonnement vers le système de détection. La profondeur du champ radiatif peut être variée en modifiant la longueur de la maquette, ce qui change l'épaisseur de la couche de choc observée.

Le tunnel à détente X2 a été utilisé pour créer les écoulements à haute enthalpie nécessaires 
pour produire les couches de choc émissives. Deux conditions d'écoulement ont été générées pour cette étude de façon à reproduire des vitesses équivalentes de vol de $10 \mathrm{~km} / \mathrm{s}$ et $12.2 \mathrm{~km} / \mathrm{s}$. Le système spectroscopique utilisé pour ces études comprend un spectromètre McPherson NOVA 225 sous vide couplé à une caméra ICCD Andor iStar de réponse renforcée dans le VUV. Un tube optique scellé par une fenêtre en fluorine a été installé pour prolonger le trajet optique sous vide jusqu'à la maquette de façon à éliminer l'absorption par l'oxygène moléculaire. Le système spectroscopique a été calibré in situ avec une lampe à deutérium placée à l'endroit de la couche de choc rayonnante.

L'intensité spectrale incidente sur la surface de la maquette, intégrée entre $115 \mathrm{~nm}$ et $180 \mathrm{~nm}$, est de $0.744 \mathrm{~W} / \mathrm{cm}^{2}$ sr pour une vitesse d'écoulement de $10 \mathrm{~km} / \mathrm{s}$ et $12,3 \mathrm{~W} / \mathrm{cm}^{2} \mathrm{sr}$ à $12.2 \mathrm{~km} / \mathrm{s}$. Ces valeurs représentent respectivement $25 \%$ et $31 \%$ de l'intensité spectrale totale mesurée dans la région d'équilibre lors de l'observation à travers la couche de choc. La reproductibilité des résultats expérimentaux obtenus par spectroscopie d'émission VUV a été déterminée à environ $15 \%$ pour l'intensité des raies et $20 \%$ pour l'intensité totale intégrée entre $115 \mathrm{~nm}$ et $180 \mathrm{~nm}$. Toutes les raies spectrales mesurées se sont révélées auto-absorbantes à des degrés variables pour les conditions de vol à $10.0 \mathrm{~km} / \mathrm{s}$, et très fortement auto-absorbantes à $12.2 \mathrm{~km} / \mathrm{s}$. Un élargissement spectral des raies fortement auto-absorbantes a aussi été observé.

Des simulations numériques de l'écoulement autour de chaque maquette ont été réalisées avec le solveur Eilmer 3 en utilisant les schémas cinétiques à taux finis de Gupta et de Park. Les spectres d'émission ont ensuite été générés avec le code de rayonnement Specair aux conditions de pression et de températures calculées pour les deux simulations. Ces travaux démontrent que le schéma cinétique de Park offre une meilleure prédiction de l'intensité totale du rayonnement VUV à $10.0 \mathrm{~km} / \mathrm{s}$ et celui de Gupta pour le cas à $12.2 \mathrm{~km} / \mathrm{s}$. Les simulations des expériences d'auto-absorption prédisent que toutes les raies étudiées sont auto-absorbées et un bon accord a été obtenu entre les mesures et les simulations pour la plupart des raies observées. En revanche, l'élargissement spectral calculé sous-estime l'élargissement mesuré.

Les travaux ont été étendus à la création d'un système de spectroscopie d'émission VUV pour la torche plasma inductive de l'Ecole Centrale Paris (ECP). La torche plasma génère de l'air à l'équilibre thermochimique et peut donc être utilisée pour valider les modèles radiatifs, en particulier les coefficients d'Einstein utilisés pour la prédiction de l'intensité des raies spectrales indépendamment de la complexité liées aux phénomènes hors équilibre observés dans le tube à détente. L'écoulement utilisé pour ces investigations est à la pression atmosphérique avec une température maximale de 6,600 K.

Une purge d'azote a été appliquée sur le trajet optique afin de réduire l'absorption de 
l'oxygène moléculaire. Pour maintenir l'intégrité structurelle du hublot d'observation en contact avec le plasma, un système refroidi par eau a été conçu. Des simulations menées avec le logiciel COMSOL Multiphysics ont permis de prédire les contraintes thermiques sur le hublot et de démontrer que celles-ci ne dépassent pas la limite élastique apparente du matériau. Une étude expérimentale a été menée pour déterminer la dégradation de la transmission du hublot liée à la proximité du plasma ainsi que l'absorption de l'oxygène moléculaire entre le bord du plasma et le hublot. La mesure de l'intensité du doublet d'azote atomique à $174 \mathrm{~nm}$ et du triplet d'oxygène atomique à $777 \mathrm{~nm}$ a permis de conclure que la dégradation de surface du hublot se produit lorsque celui-ci est situé à moins de $5 \mathrm{~mm}$ du bord du plasma. A cette distance, il a été montré qu'une fraction du signal est absorbée par les molécules d'oxygène.

Un deuxième objectif était de développer un système capable d'étudier les spectres émis par la torche sur des profondeurs de champ radiatif variables afin de caractériser l'autoabsorption et l'élargissement des raies spectrales. Un tube de cuivre refroidi à l'eau, installé sur une translation micrométrique, a été placé dans le plasma de façon à bloquer le rayonnement au-delà d'une distance ajustable. Les émissions du doublet d'azote atomique à $174 \mathrm{~nm}$ et du triplet oxygène atomique à $777 \mathrm{~nm}$ ont été mesurées sur des profondeurs optiques variables avec un pas de $1 \mathrm{~mm}$. L'auto-absorption du doublet à $174 \mathrm{~nm}$ a été confirmée.

La calibration du système spectroscopique VUV de l'ECP a été réalisée au moyen d'un mini-arc d'argon placé en lieu et place du plasma. Cette calibration in situ a été menée à la fin des mesures avec le plasma, de façon à prendre en compte l'effet d'une dégradation potentielle du hublot pendant les tests ainsi que l'absorption par l'oxygène moléculaire sur le trajet optique. En raison de la dégradation observée de la surface du hublot sur la durée du test et d'un niveau indéterminé d'absorption entre le hublot et le plasma, il existe une forte incertitude sur les spectres calibrés. Des pistes d'amélioration du système ont été proposées pour permettre de valider dans le futur le modèle radiatif du doublet d'azote à $174 \mathrm{~nm}$.

Grace aux travaux de cette thèse, deux systèmes spectroscopiques VUV ont été conçus et mis en place sur un tube de détente et une torche à plasma. Un ensemble de données expérimentales de référence sur l'intensité spectrale émise parallèlement et perpendiculairement à la couche de choc ont été obtenues. Le degré d'auto-absorption et l'élargissement des raies spectrales ont été quantifiés pour différentes profondeurs de champ radiatif, fournissant ainsi un ensemble de données de réference pour la validation des codes de rayonnement. Les simulations numériques de l'écoulement, réalisées avec Eilmer3 en utilisant les schémas cinétiques de Gupta et de Park, ont permis de générer des spectres d'émission à l'aide du logiciel Specair, et les résultats ont été comparés aux mesures expérimentales. 


\section{Contents}

Abstract ii

Publications $\quad$ vi

\begin{tabular}{|ll}
\hline Acknowledgements & ix
\end{tabular}

Résumé xiii

Table of Contents $\quad$ xviii

$\begin{array}{ll}\text { List of Figures } & \text { Xxv }\end{array}$

$\begin{array}{ll}\text { List of Tables } & \text { xxvii }\end{array}$

$\begin{array}{ll}\text { Nomenclature } & \text { xxviii }\end{array}$

$\begin{array}{lll}1 & \text { Introduction } & 1\end{array}$

1.1 Research Objectives . . . . . . . . . . . . . . . . . . . . . . . . . . . 3

2 Radiative Heat Flux $\quad 5$

2.1 Black Body Radiation and Self-Absorption . . . . . . . . . . . . . . . . . . . . 9

2.2 Flow Field Radiation Coupling Effects . . . . . . . . . . . . . . . . . . . . . . . . . . . . 10

2.3 Measurement of Emission Spectra . . . . . . . . . . . . . . . . . . . . . . 11

2.3 .1 Design of Optical Systems . . . . . . . . . . . . . . . . . . . . 12

$2.3 .2 \quad$ Spectral Line Broadening Processes . . . . . . . . . . . . . . . . . . 14

$2.3 .3 \quad$ Absorption Cross-Sections . . . . . . . . . . . . . . . . . . . . 15

\begin{tabular}{lll}
\hline 3 & Literature Review & 17
\end{tabular}

$3.1 \quad$ Flight Experiments $\ldots \ldots \ldots \ldots$

3.2 Planetary Exploration and Non-Equilibrium Radiation . . . . . . . . . . . 20

3.3 Re-Entry Observation Missions _ . . . . . . . . . . . . . . . . . . 20

3.4 Ground Testing Facilities . . . . . . . . . . . . . . . . . . . . . . . . . . . . 21

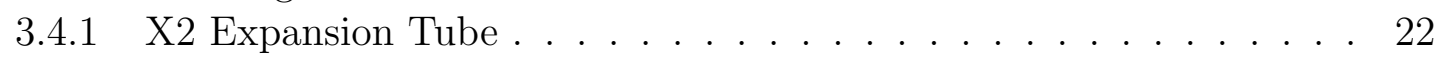

3.4 .2 Plasma Torch at Ecole Centrale Paris . . . . . . . . . . . . . . . . . 24

3.4 .3 Ground Testing Radiation Data . . . . . . . . . . . . . . . . . . . 27

3.5 Radiation Modelling Programs . . . . . . . . . . . . . . . . . . . 30 


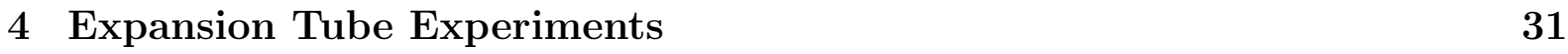

4.1 Model Design . . . . . . . . . . . . . . . . . . . . . . 31

$4.1 .1 \quad$ Measuring VUV Radiation Through the Surface . . . . . . . . . . . 31

$4.1 .2 \quad$ Measuring VUV Radiation Across the Surface . . . . . . . . . . . . . 35

4.1 .3 Model Shape . . . . . . . . . . . . . . . . . . . . . . 38

4.1 .4 Self-Absorption Experiments . . . . . . . . . . . . . . . . . . . . . . . . . . . . . . . . . 4

4.2 VUV Emission Spectroscopy System … . . . . . . . . . . . . . 42

4.2 .1 Optical Configuration . . . . . . . . . . . . . . . . 45

4.2 .2 High Vacuum Optical Chamber Design . . . . . . . . . . . . . . . . 46

$4.2 .3 \quad$ Alignment of Optical Components . . . . . . . . . . . . . . . . . . . 48

4.2 .4 Vacuum Pressure Requirements . . . . . . . . . . . . . . . . . . . 50

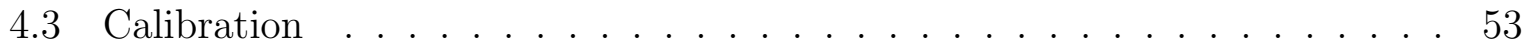

4.3 .1 Validity of Calibration Data . . . . . . . . . . . . . 56

4.3 .2 Calculation of the Calibration Factor . . . . . . . . . . . . . . . . . 60

4.4 Operating Conditions . . . . . . . . . . . . . . . . . . 62

4.4 .1 Experimental and Computational Uncertainties . . . . . . . . . 66

4.5 Shot Validity Analysis Tool . . . . . . . . . . . . . . . . . . . . 67

4.5 .1 Time Resolved Radiative Emission Measurement . . . . . . . . . . 68

$4.5 .2 \quad$ High Speed Imaging System . . . . . . . . . . . . . . . . . . . . . . 69

4.5.3 High Temporal Resolution Shock Speed Measurement System ............................. 70

4.5 .4 Sample Results . . . . . . . . . . . . . . . . . . . 72

4.6 Repeatability . . . . . . . . . . . . . . . . . . . . . . . . 74

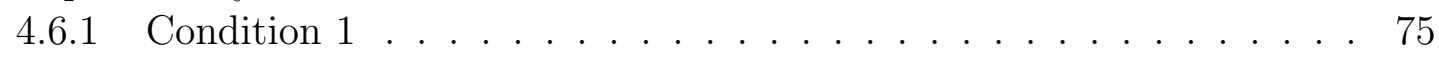

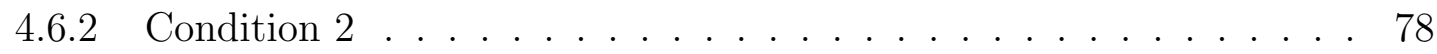

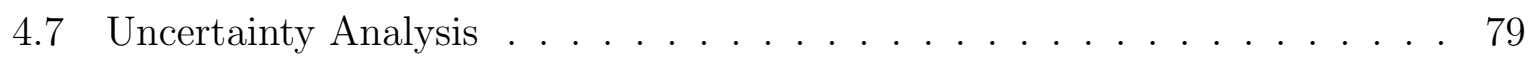

4.8 Expansion Tube Experimental Datasets . . . . . . . . . . . . . . . . . . . . 80

4.8 .1 Sample Image Processing. . . . . . . . . . . . . . . . . . . . . . 80

4.8 .2 Through Surface and Across Surface Spectral Comparison . . . . . 83

4.8.3 Self-Absorption Experiments . . . . . . . . . . . . . . . . . 86

$\begin{array}{lll}5 & \text { Computational Reconstruction of Expansion Tube Experiments } & 89\end{array}$

5.1 Across and Through Surface Model Results . . . . . . . . . . . . . . . . . 90

5.2 Self-Absorption Experiments . . . . . . . . . . . . . . . . . . . . 97

5.3 Conclusions . . . . . . . . . . . . . . . . . . . . . . . 107

$\begin{array}{llr}6 & \text { Plasma Torch Experiments } & 108\end{array}$

6.1 Operating Conditions . . . . . . . . . . . . . . . . . . . . 108

6.2 Optical System Design . . . . . . . . . . . . . . . . . . . . . . 112

6.2.1 Proof of Concept Testing - Removal of Molecular Oxygen Absorption Through a Nitrogen Flush . . . . . . . . . . . . . . . 112

6.2 .2 General Set-up for VUV Diagnostics of Plasma Emission . . . . . . 116

6.2 .3 Window Housing Design . . . . . . . . . . . . . . . . 118

6.2 .4 Calibration . . . . . . . . . . . . . . . . . . . . . 122

6.3 Spectral Measurements Between $170 \mathrm{~nm}$ and $200 \mathrm{~nm}$. . . . . . . . . . . . . 126

6.3 .1 Comparisons With Simulations and Previous Studies . . . . . . . . 128 
6.4 Self-Absorption Experiment . . . . . . . . . . . . . . . . . . . 131

6.4 .1 Measurements . . . . . . . . . . . . . . . . . . . . 132

6.4 .2 Comparison With Simulations . . . . . . . . . . . . . . . . . 134

\begin{tabular}{llr}
\hline & Conclusions & 136
\end{tabular}

7.1 Future Work . . . . . . . . . . . . . . . . . . . . . . . . . . . . . . . . . . . 139

\begin{tabular}{ll}
\hline A Optical Aberrations & 154
\end{tabular}

\begin{tabular}{ll}
\hline B ICCD & 156
\end{tabular}

\begin{tabular}{ll}
\hline C Repeatability Testing Shots & 159
\end{tabular}

\begin{tabular}{ll}
\hline D Calibrated Expansion Tube Datasets & 169
\end{tabular}

D.1 Through Surface and Across Surface Experiments . . . . . . . . . . . . . . 169

D.2 $\quad$ Self-Absorption Experiments . . . . . . . . . . . . . . . . . . 178

\begin{tabular}{ll}
\hline E Computational Simulation Code & 190
\end{tabular}

E.1 $\quad$ Square Model . . . . . . . . . . . . . . . . . . . . . . . . . . . . . . . . . . 190

E.2 $\quad$ Cylindrical Model . . . . . . . . . . . . . . . . . . . . . . . . . . . . . . . . 192 


\section{List of Figures}

1.1 Variation of convective and radiative heat fluxes with increasing velocity.

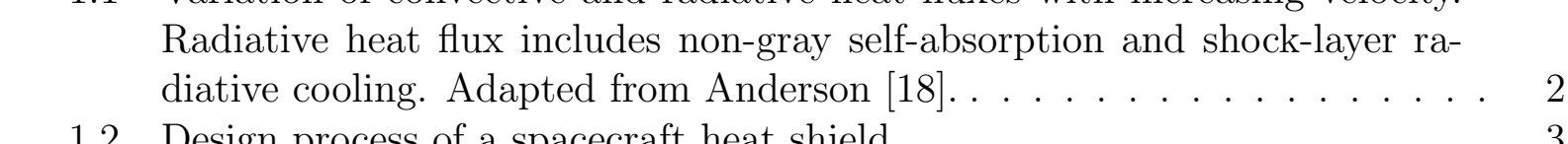

1.2 Design process of a spacecraft heat shield. . . . . . . . . . . . . 3

2.1 Thermochemical energy storage mechanisms; electronic, vibrational and rotational. Adapted from [19] . . . . . . . . . . . . . . 6

2.2 Possible spectral lines for hydrogen and iron . . . . . . . . . . . . . . . . . 7

2.3 Effects of shock heating in air at varying velocities and altitudes. Adapted from Anderson [18] $\ldots \ldots \ldots \ldots$. . . . . . . . . . . . . . . . . . . . . 8

2.4 Depiction of the varying regions possible within a shock layer. Adapted from [18] . . . . . . . . . . . . . . . . . . . . . . . . . . . . . . . 9

2.5 Planck's law limiting radiative emission and causing self-absorption to occur. Simulation conducted using Specair for $\mathrm{CO}(4+)$ at a temperature of $6,600 \mathrm{~K}$, pressure of $2,000 \mathrm{~Pa}$ and $1.0 \mathrm{~cm}$ depth of radiating flow field. . . . 10

2.6 Broadening effects on an atomic nitrogen triplet calculated using Specair. . 14

2.7 Absorption cross-sections for molecular oxygen [20], $\mathrm{O}_{2}$ Schumann-Runge

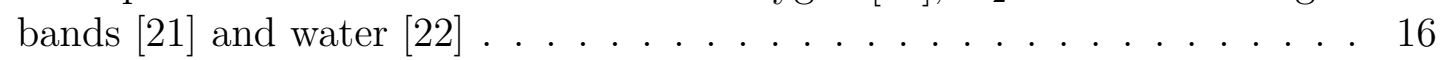

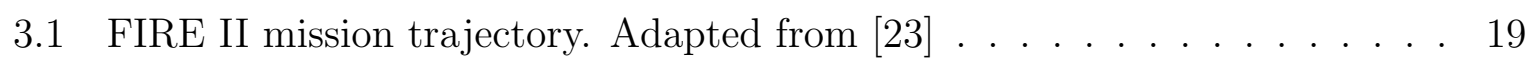

3.2 Stagnation temperature and test time ranges of various ground testing facilities. Adapted from Bertin [24]. . . . . . . . . . . . . . . 22

3.3 Schematic of the X2 expansion tube configuration used for this study. ST13 and AT1-6 PCBs are the shock tube and acceleration tube pressure trans-

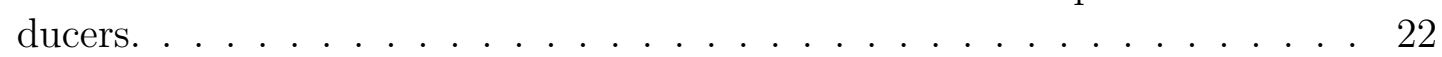

3.4 Schematic of the plasma torch $[25] \ldots \ldots \ldots \ldots \ldots$

3.5 Intensity of the magnetic field without a discharge (a) and with a discharge

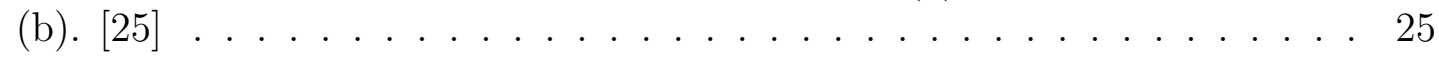

3.6 Representative temperature profiles for a high swirl and low swirl condition. 27

3.7 Schematic arrangement of the Palumbo arc jet experiment [26] $\ldots . . . . .29$

$4.1 \quad$ Proof of concept model with perspex front end and open mirror housing. . 32

4.2 Mylar diaphragm fragments impacting on windows. The window during the steady test time can be seen in the top left image. Top right image shows the arrival of a large piece of Mylar diaphragm and its impact on the window. . . . . . . . . . . . . . . . . 33 
4.3 Top down view of high vacuum path to model with mirror housing assembly

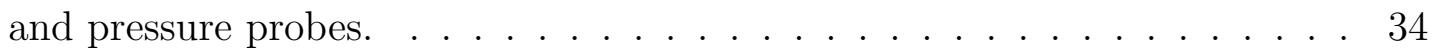

$4.4 \quad$ Additional bracing installed to ensure model position is maintained. . . . . 35

4.5 Top down view of initial fence and window position with no gap to the blunt model. . . . . . . . . . . . . . . . . 35

$4.6 \quad$ Perturbations created by the fence and stagnation region between the fence and the model. . . . . . . . . . . . . . . . . 36

4.7 Top down view of second fence and window position with a $10 \mathrm{~mm}$ gap to the blunt model. . . . . . . . . . . . . . . . . . 37

4.8 Steady flow for over $130 \mu$ s achieved with a $10 \mathrm{~mm}$ gap. Counter in bottom

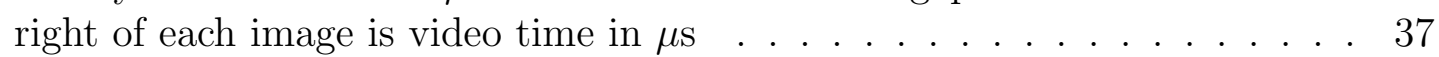

4.9 Left - Side on view of idealised two-dimensional shock stand off. Right Three dimensional view of idealised region used for mass flow balance. . . . 38

4.10 Shock stand off correction for varying aspect ratio square bodies in supersonic and hypersonic flow. An aspect ratio of 3.6 produces a 0.76 correction factor between two-dimensional and three-dimensional shock stand offs. . . 40

4.11 Top down view of vaporised aluminium impacting the surface. Flow from left to right. . . . . . . . . . . . . . . . . . . . 40

4.12 Self-absorption experiments concept, top down view (left). Area imaged spatially onto spectrometer when looking across the cylinder (right). . . . . . 41

4.13 Shock stand off correction for varying aspect ratio cylinders in supersonic and hypersonic flow [27]. . . . . . . . . . . . . . . . . . . . 42

4.14 Depiction of a Czerny-Turner configuration. . . . . . . . . . . . . . . 43

4.15 Left - Normal incidence concave grating Seya-Namioka monochromator. Right - Turning concave grating design. . . . . . . . . . . . . . . . . . . 43

4.16 Imaging optics to observe radiation across and through the surface of the models. Image drawn to scale. . . . . . . . . . . . . . . . . . . . 45

4.17 Extension of spectrometer table and rotation of spectrometer for enhanced compatibility with test section. . . . . . . . . . . . . . 47

4.18 Deformation of the optical chamber with an atmospheric pressure force. . . 48

4.19 (Left) - Alignment tool mounted onto the edges of the flat model.(Right) Installation of railings in the optical chamber and the mounting poles used in the alignment process. . . . . . . . . . . . . . . . . . . . . . . 49

4.20 A chain block was used to move the test section into firing position. This was required to account for movement of the test section due to vacuum forces. . . . . . . . . . . . . . . . . . . . 49

4.21 Blurred spectral focus at spatial focal point and sharp spectral focus at slit

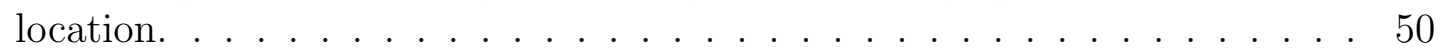

4.22 Transmission percentage of VUV radiation through $1.0 \mathrm{~m}$ of air at varying

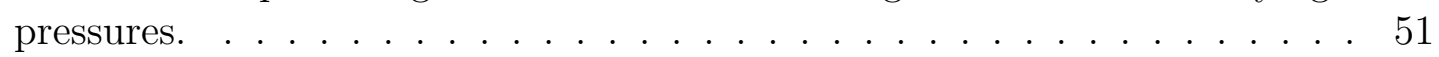

4.23 Transmission percentage of VUV radiation through $1.0 \mathrm{~m}$ of water vapour at varying pressures. . . . . . . . . . . . . . 51

4.24 Results of a $10^{-4}$ Torr vacuum pressure on the transmission of the VUV radiation through an optical path of $401.3 \mathrm{~cm}$ with a molecular oxygen mole fraction of 0.21 and a water vapour mole fraction of $0.5 . \quad$. . . . . . 52 
4.25 Array of vacuum pumping options available and their ranges. Source [28] . 53

4.26 Calibration lamp mounted in place of the model. . . . . . . . . . . . . . . 54

4.27 Calibration lamp internal dimensions . . . . . . . . . . . . . . . 55

4.28 Linear response of the camera with variation in exposure time of the ICCD 55

4.29 Left - Superimposed calibration images depicting ICCD array ranges tested.

Right - Results of integrating each image indicate there negligible variation in pixel sensitivity across the ICCD. The slight discrepancy at the longer wavelength cut off is due to the circular shape of the intensifier clipping

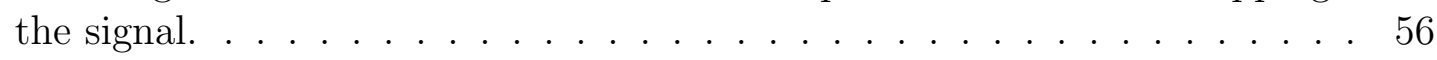

4.30 Scaled absorption cross-sections of water vapour and molecular oxygen su-

perimposed on the measured and known spectral datasets. . . . . . . . . . 57

4.31 Spectra of the deuterium lamp during start up . . . . . . . . . . . . . 58

4.32 Spatial variation in the location of peak radiation output due to a twisting angle between the grating and the camera . . . . . . . . . . . . . . 59

4.33 Results of a 0.375 degree rotation of the recorded image on the position of the peak spectral radiation output. . . . . . . . . . . . . . . 59

4.34 Spectral averaging or smoothing of measured data to achieve a spectral bandwidth matching that of the given calibration dataset. . . . . . . . . . 60

4.35 Measured and published transmissions of magnesium fluoride windows. Publish transmission from Crystran [29]. . . . . . . . . . . . . . . . . 61

4.36 Final system sensitivity measured for both optical systems. The across system sensitivity here is calculated for a $10.0 \mathrm{~mm}$ aperture. . . . . . . . . 61

4.37 Pitot rake arrangement with conical glancing pressure probes for conditions testing. [30] $\ldots \ldots \ldots \ldots \ldots \ldots$. . . . . . . . . . . . . . . . . . 64

4.38 Experimental pitot traces for condition 1 and 2. Estimated test time for condition 1 is $26.55 \mathrm{~ms}$ to $26.70 \mathrm{~ms}$ and estimated test time for condition 2 is $26.55 \mathrm{~ms}$ to $26.65 \mathrm{~ms}$. . . . . . . . . . . . . . . . . . . . . . . 65

4.39 Measured core flow size and variation in pitot pressure at distances from the nozzle exit for condition 1 . . . . . . . . . . . . . . . 66

4.40 Measured static pressure values at the downstream end of the acceleration tube for condition 1 (Left) and condition 2 (Right). . . . . . . . . . . . 67

4.41 Schematic of a photomultiplier tube. [31] $\ldots \ldots \ldots$. . . . . . . . . 68

4.42 Optical system used with VNIR and UV spectroscopy system now used with a PMT. Adapted from [32] . . . . . . . . . . . . . . . . . . . . . 69

4.43 Spectral response of the Shimadzu HPV-1 high speed camera [33] . . . . . 70

4.44 Top down view of installed photodiode mount assembly (left). Schematic of photodiode mounts assembly (right). . . . . . . . . . . . . . . 71

4.45 schematic of the amplifier and terminator circuit designed for each photodiode. Adapted from Zander [34] . . . . . . . . . . . . . . . . . . . 71

4.46 Signal traces from the photodiode array. Shock speed calculations between the three pairs of photodiodes produces $11.1 \mathrm{~km} / \mathrm{s}, 7.3 \mathrm{~km} / \mathrm{s}$ and $20.0 \mathrm{~km} / \mathrm{s}$. A curve of best fit predicts $10.1 \mathrm{~km} / \mathrm{s} . \ldots \ldots \ldots . \ldots 73$

4.47 Sample shot validity analysis plot. . . . . . . . . . . . . . . . . 73

4.48 Chemical species responsible for each spectral line observed. Subscript D denotes a double peak. . . . . . . . . . . . . . . . . 75 
4.49 Results from repeatability study of condition 1 across the VUV range. . . . 75

4.50 Results from repeatability study of condition 1 for the lower half of the VUV range. . . . . . . . . . . . . . . . 76

4.51 Results from the integration analysis of condition 1 . . . . . . . . . 77

4.52 Integrated values of the Planck curve between $120 \mathrm{~nm}$ and $180 \mathrm{~nm}$ at various temperatures, normalised at 10,000 K. . . . . . . . . . . . 78

4.53 Results from repeatability study of condition $2 . \quad \ldots . . . . . .79$

4.54 Raw image acquired on the ICCD across at flat $90 \mathrm{~mm}$ model at condition 2.21

4.55 Calibrated image across a $90 \mathrm{~mm}$ model at condition $2.2 . . . .82$

4.56 Comparison of across surface and through surface spectral measurements at condition 1. . . . . . . . . . . . . . . . . 83

4.57 Comparison of across surface and through surface spectral measurements

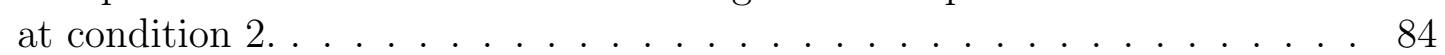

4.58 Ratios of the selected peak heights when observing through the surface compared to across the surface. Molecular oxygen absorption spectrum superimposed to investigate boundary layer oxygen absorption. . . . . . . . . 85

4.59 (Left) - Ratios of spectral line peaks for condition 1. (Right) - Ratios of spectral line peaks for condition 2. Nitrogen lines selected are $120.0 \mathrm{~nm}$, $124.3 \mathrm{~nm}, 131.1 \mathrm{~nm}, 132.0 \mathrm{~nm}, 141.2 \mathrm{~nm}, 149.3 \mathrm{~nm}, 174.3 \mathrm{~nm}$ and $174.5 \mathrm{~nm}$. Oxygen line at $130.5 \mathrm{~nm} . \ldots \ldots \ldots$. . . . . . . . . . 87

4.60 Comparison of VUV emission measured across a $90 \mathrm{~mm}$ square and cylindrical model for condition 1. . . . . . . . . . . . . . . 87

4.61 Comparison of VUV emission measured across a $90 \mathrm{~mm}$ square and cylindrical model for condition 1. . . . . . . . . . . . . . . . 88

4.62 (Left) - Minimal peak broadening observed for condition 1 at the $141 \mathrm{~nm}$ line. (Right) - Strong peak broadening observed for the faster test condition on a self-absorbing line. . . . . . . . . . . . . . . . . 88

5.1 Structured grids used in the simulation of both models. . . . . . . . . . . . 90

5.2 Time resolved images of shock arrival and flow establishment over the model. 91

5.3 Computed stagnation streamline temperatures. $\ldots \ldots \ldots$. . . . . . . . . 92

5.4 Computed mass fractions at the stagnation streamline. . . . . . . . . . . . 92

5.5 Computed and experimentally measured across surface spectra for condi-

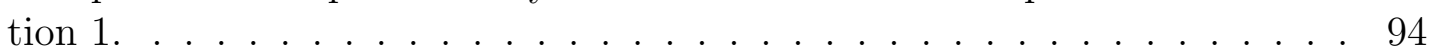

5.6 Computed and experimentally measured through surface spectra for condition 1. . . . . . . . . . . . . . . . . . . . 94

5.7 Computed and experimentally measured across surface spectra for condi-

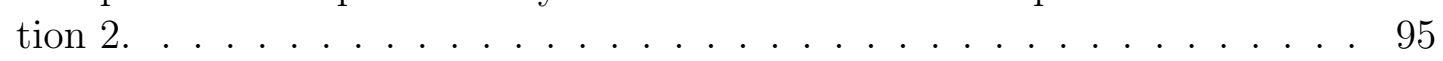

5.8 Computed and experimentally measured through surface spectra for condition 2. . . . . . . . . . . . . . . . . 95

5.9 Computed stagnation streamline temperatures at both conditions. . . . . . 97

5.10 Computed stagnation streamline mass fractions using the Park reaction scheme at both conditions. . . . . . . . . . . . . . . . . 98

5.11 Computed and spectra across a $90 \mathrm{~mm}$ cylindrical model at condition 1. . . 99

5.12 Computed and measured spectra across a $45 \mathrm{~mm}$ cylindrical model at condition 1. . . . . . . . . . . . . . . . . . . . . . . . . . . . . 
5.13 Computed and measured spectra across a $20 \mathrm{~mm}$ cylindrical model at condition 1. . . . . . . . . . . . . . . . . 100

5.14 Computed and measured spectra across a $90 \mathrm{~mm}$ cylindrical model at condition 2. . . . . . . . . . . . . . . . . 101

5.15 Computed and measured spectra across a $45 \mathrm{~mm}$ cylindrical model at con-

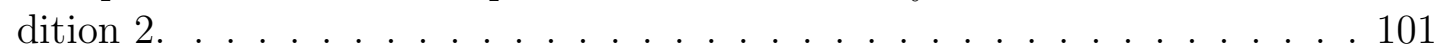

5.16 Computed and measured spectra across a $20 \mathrm{~mm}$ cylindrical model at condition 2. . . . . . . . . . . . . . . . . . . . 102

5.17 Experimentally measured and computed spectral line peak height ratios for condition 1.

5.18 Experimentally measured and computed spectral line peak height ratios for condition 2 .

5.19 Spectral line intensities for condition 1 as a function of depth of radiating flow field, normalised at $90 \mathrm{~mm}$ depth of radiating flow field. (Top) Computed results. (Bottom) - Experimental measurements. . . . . . . . . 104

5.20 Spectral line intensities for condition 2 as a function of depth of radiating flow field, normalised at $90 \mathrm{~mm}$ depth of radiating flow field. (Top) Computed results. (Bottom) - Experimental measurements. . . . . . . . . 105

5.21 Experimentally measured and computed spectral line widths for the $141.2 \mathrm{~nm}$ peak at condition 1. . . . . . . . . . . . . . . 106

5.22 Experimentally measured and computed spectral line widths for the $149.3 \mathrm{~nm}$ peak at condition 2 .

6.1 Optical system used to measure the atomic oxygen triplet at $777 \mathrm{~nm}[8]$. . . 110

6.2 Measured temperature profile with upper and lower uncertainty limits. . . 111

6.3 Influence of molecular oxygen contaminants on transmitted signal through an optical path of $3 \mathrm{~m} . ~ \ldots \ldots \ldots \ldots . \ldots 113$

6.4 Initial nitrogen flush testing configuration with the mini-arc placed at the optical entrance of the spectrometer whilst nitrogen is flushed through it. . 114

6.5 Increase in transmission through the optical path during 145 minutes of flushing the spectrometer with nitrogen. . . . . . . . . . . . . . . . . 114

6.6 Increase in radiation transmission between $170 \mathrm{~nm}$ and $180 \mathrm{~nm}$ during 180 minutes of flushing the spectrometer with nitrogen. . . . . . . . . . . . . 115

6.7 Increase in transmission through the optical path of the nitrogen and carbon lines between $173 \mathrm{~nm}$ and $177 \mathrm{~nm}$ after 0,3 and 20 hours of flushing the spectrometer with nitrogen. . . . . . . . . . . . . . . . . 116

6.8 Optical configuration and nitrogen flow path for plasma torch experiments. 117

6.9 Final set up of the spectrometer, optical chamber, water cooled window

housing and the plasma torch. . . . . . . . . . . . . 118

6.10 Final design of the water cooled window housing assembly. . . . . . . . . . . 119

6.11 Results of the COMSOL Multiphysics simulation showing the steady state temperature of the window within the water cooled copper housing. . . . . 120

6.12 Plasma emission spectra obtained in the VUV with the water cooled window housing at $10 \mathrm{~mm}, 7.5 \mathrm{~mm}, 6 \mathrm{~mm}, 5 \mathrm{~mm}$ and $4 \mathrm{~mm}$ from the edge of the nozzle. . . . . . . . . . . . . . . . . . . 120 
6.13 Plasma emission spectra obtained in the VUV with the window housing at $5 \mathrm{~mm}, 4 \mathrm{~mm}, 3 \mathrm{~mm}, 2 \mathrm{~mm}$ and $1 \mathrm{~mm}$ from the edge of the nozzle. . . . . . 121

6.14 Integrated and normalised values of spectral measurements in the VUV and NIR . . . . . . . . . . . . . . . . . . . 122

6.15 Schematic of the in-situ calibration approach with a shroud to contain the nitrogen flush used to remove molecular oxygen between the viewing window and the mini-arc. . . . . . . . . . . . . . . . . . 123

6.16 Spectral response of the system using in-situ calibration between $171 \mathrm{~nm}$ and $179 \mathrm{~nm}$ with interpolation across contaminant spectral lines. . . . . . . 123

6.17 Spectral data obtained using the mini-arc with nitrogen and carbon contamination lines. . . . . . . . . . . . . . . . . 124

6.18 Spectral response of the system using in-situ calibration between $170 \mathrm{~nm}$ and $200 \mathrm{~nm}$ with interpolation across contaminant spectral lines. . . . . . . 124

6.19 Uncalibrated, spatially resolved spectral image of plasma torch emission between $171 \mathrm{~nm}$ and $179 \mathrm{~nm}$. . . . . . . . . . . . . . . . . 126

6.20 Uncalibrated spectrum of plasma emission from $170 \mathrm{~nm}$ to $200 \mathrm{~nm}$. . . . . . 127

6.21 Calibrated and spatially averaged spectrum of plasma emission from $170 \mathrm{~nm}$

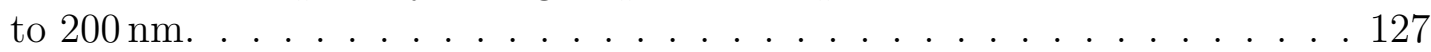

6.22 Calibrated and spatially averaged spectra at torch start up and after 52 minutes of operation. . . . . . . . . . . . . . 128

6.23 Comparison of the measured spectrum and the spectrum computed by

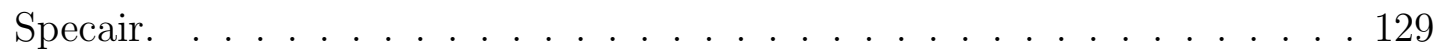

6.24 Comparison of measured spectra calibrated using an in-situ approach and a non-in-situ calibration and the spectrum computed by Specair. . . . . . . 129

6.25 Radiation absorption of a slab of air at ambient temperature and atmospheric pressure with a molecular oxygen mole fraction of 0.21 at over various path lengths. The level of absorption predicted by the comparison of Specair simulation and the measured values using the two calibration approaches at a distance of $5 \mathrm{~mm}$ from the plasma edge are also presented. 130

6.26 Concept of the traversable water cooled fence across the plasma to limit

the depth of radiating flow field observed. . . . . . . . . . . . . . 131

6.27 Water cooled fence during operation. . . . . . . . . . . . . . . . . 132

6.28 Spectral line peak intensities at various depths of radiating flow fields normalised using the measured intensities at a $50 \mathrm{~mm}$ depth of radiating flow field. Spectral lines compared are the $174.3 \mathrm{~nm}$ and $174.5 \mathrm{~nm}$ atomic nitrogen lines and the $777.2 \mathrm{~nm}$ atomic oxygen line. . . . . . . . . . . . . . . 133

6.29 Normalised spectral lines of the $174.3 \mathrm{~nm}$ and $174.5 \mathrm{~nm}$ nitrogen doublet at varying depths of radiating flow field. . . . . . . . . . . . . . . 133

6.30 Measured and computed normalised spectral lines of the $174.3 \mathrm{~nm}$ and $174.5 \mathrm{~nm}$ nitrogen doublet at varying depths of radiating flow field. . . . . 134

6.31 (Left) - Spectral line profile for various instrument broadening functions for the $174.27 \mathrm{~nm}$ atomic nitrogen spectral line. (Right) - spectral line profile for various instrument functions for the $174.53 \mathrm{~nm}$ atomic nitrogen spectral line. Both simulations conducted at atmospheric pressure at $6,700 \mathrm{~K}$ with a $4.5 \mathrm{~cm}$ depth of radiating flow field. . . . . . . . . . . . . . . 135 
A.1 Effects of A. spherical aberration, B. comatic, C. astigmatism, D. curvature of field, E. distortion and F. chromatic aberration . . . . . . . . . . . 155

B.1 Cross section of a Princeton Instruments Generation II Image Intensifier and CCD array. [35] . . . . . . . . . . . . . . . 157

C.1 x2s1908 - Repeatability test shot at condition 1. . . . . . . . . . . . . . . 160

C.2 x2s1917 - Repeatability test shot at condition 1. . . . . . . . . . . . . . . . 161

C.3 x2s1918 - Repeatability test shot at condition 1. . . . . . . . . . . . . . . . 162

C.4 x2s1919 - Repeatability test shot at condition 1. . . . . . . . . . . . . . . 163

C.5 x2s1907 - Repeatability test shot at condition 2. . . . . . . . . . . . . . . 164

C.6 x2s1920 - Repeatability test shot at condition 2. . . . . . . . . . . . . . 165

C.7 x2s1922 - Repeatability test shot at condition 2. . . . . . . . . . . . . . . 166

C.8 x2s1923 - Repeatability test shot at condition 2. . . . . . . . . . . . . . . . 167

C.9 x2s1924 - Repeatability test shot at condition 2. . . . . . . . . . . . . . . . 168

D.1 x2s1938 - Primary shot data for across the surface at condition 1. . . . . . 170

D.2 x2s1936 - Repeat shot data for across the surface at condition 1. . . . . . . 171

D.3 x2s1954 - Primary shot data for through surface at condition 1. . . . . . . 172

D.4 x2s1953 - Repeat shot data for through surface at condition 1. . . . . . . . 173

D.5 x2s1940 - Primary shot data for across the surface at condition 2. . . . . . 174

D.6 x2s1939 - Repeat shot data for across the surface at condition 2. . . . . . . 175

D.7 x2s1957 - Primary shot data for through surface at condition 2. . . . . . . 176

D.8 x2s1958 - Repeat shot data for through surface at condition 2. . . . . . . . 177

D.9 x2s1926 - Primary shot data for across the surface of a $90 \mathrm{~mm}$ model at

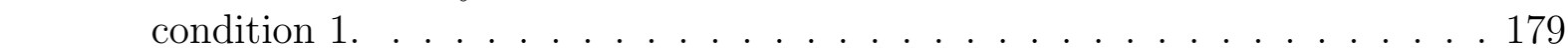

D.10 x2s1927 - Repeat shot data for across the surface of a $90 \mathrm{~mm}$ model at

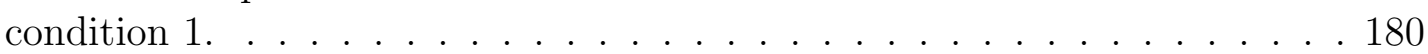

D.11 x2s1932 - Primary shot data for across the surface of a $45 \mathrm{~mm}$ model at

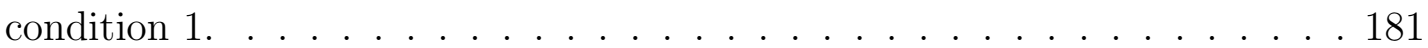

D.12 x2s1933 - Repeat shot data for across the surface of a $45 \mathrm{~mm}$ model at condition 1. . . . . . . . . . . . . . . . . . . 182

D.13 x2s1946 - Primary shot data for across the surface of a $20 \mathrm{~mm}$ model at

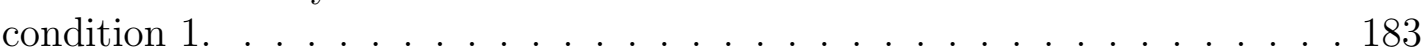

D.14 x2s1945 - Repeat shot data for across the surface of a $20 \mathrm{~mm}$ model at condition 1. . . . . . . . . . . . . . . . . . . . 184

D.15 x2s1931 - Primary shot data for across the surface of a $90 \mathrm{~mm}$ model at

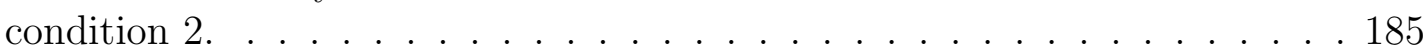

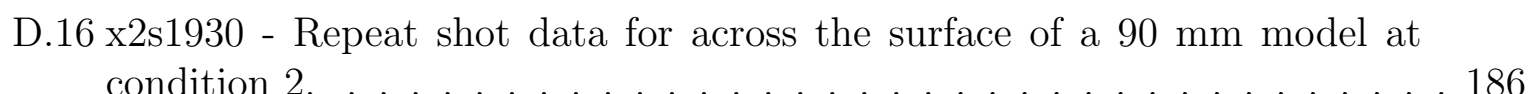

D.17 x2s1942 - Primary shot data for across the surface of a $45 \mathrm{~mm}$ model at condition 2. . . . . . . . . . . . . . . . . . 187

D.18 x2s1943 - Primary shot data for across the surface of a $20 \mathrm{~mm}$ model at condition 2. . . . . . . . . . . . . . . . . . . . . 188

D.19 x2s1944 - Repeat shot data for across the surface of a $20 \mathrm{~mm}$ model at condition 2. . . . . . . . . . . . . . . . . . . . . . . . . . . 189 


\section{List of Tables}

2.1 Effect of varying aperture on circle of confusion for a $500 \mathrm{~mm}$ focal mirror system with a magnification of 1.5 and a depth of field of $100 \mathrm{~mm}$. . . . . 13

3.1 Re-entry flight observation missions of the 2000s. . . . . . . . . . . . 21

3.2 Emission spectroscopy capabilities of various hypersonic facilities around. . 28

4.1 Comparison of the three spectroscopy systems considered. . . . . . . . . . 44

4.2 Details of optical systems produced. . . . . . . . . . . . . . 46

$4.3 \quad$ Fill pressures for condition 1. Uncertainties taken from Gildfind [30] . . . . 62

4.4 Free stream test conditions calculated using Pitot and available measured

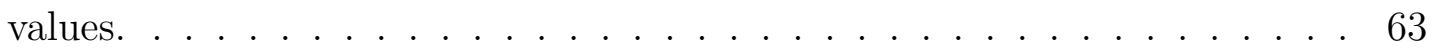

4.5 Total integrated counts and normalised integrals for condition 1 when observing across the surface of the model. . . . . . . . . . . . . . 76

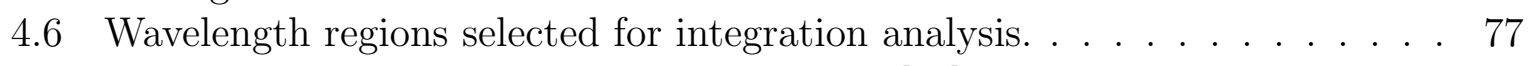

4.7 Relative uncertainty of the calibration lamp [36]. . . . . . . . . . . . . 80

4.8 Nine atomic lines selected for analysis of boundary layer absorption . . . . 84

4.9 Total VUV radiative heat flux measurements across the surface in the equilibrium region and incident on the surface having passed through the boundary layer for condition $1.6 \ldots \ldots \ldots$. . . . . . . . . . . 86

4.10 Total VUV radiation heat flux measurements across the surface in the equilibrium region and incident on the surface having passed through the boundary layer for condition $2 . \ldots \ldots \ldots$. . . . . . . . . . . 86

$5.1 \quad$ Steady region properties calculated using Eilmer 3 for both conditions with the flat model. . . . . . . . . . . . . . . . . . 93

5.2 Integrated values for the VUV spectrum and ratios of observed across surface measurements to the through surface measurements. . . . . . . . . . . 96

$5.3 \quad$ Steady region properties calculated using Eilmer 3 for both conditions with the cylindrical model. . . . . . . . . . . . . . . . . 99

6.1 Operational parameters for tuned plasma torch condition. . . . . . . . . . 109

6.2 Mole fractions for three representative plasma torch locations . . . . . . . . . 112

6.3 Contributing factors in the total uncertainty of the in-situ mini-arc cali-

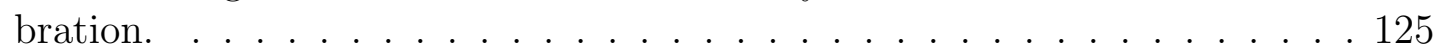

C.1 Shots conducted for repeatability study. . . . . . . . . . . . . . . . 159

D.1 Shots conducted for across and through surface comparison experiments. . 169 
D.2 Shots conducted for self-absorption experiments with a $19 \mathrm{~mm}$ radius of curvature cylinder.

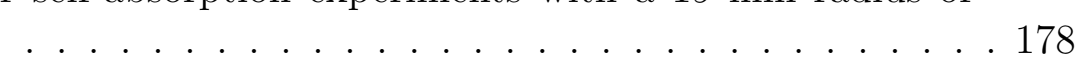




\section{Nomenclature}

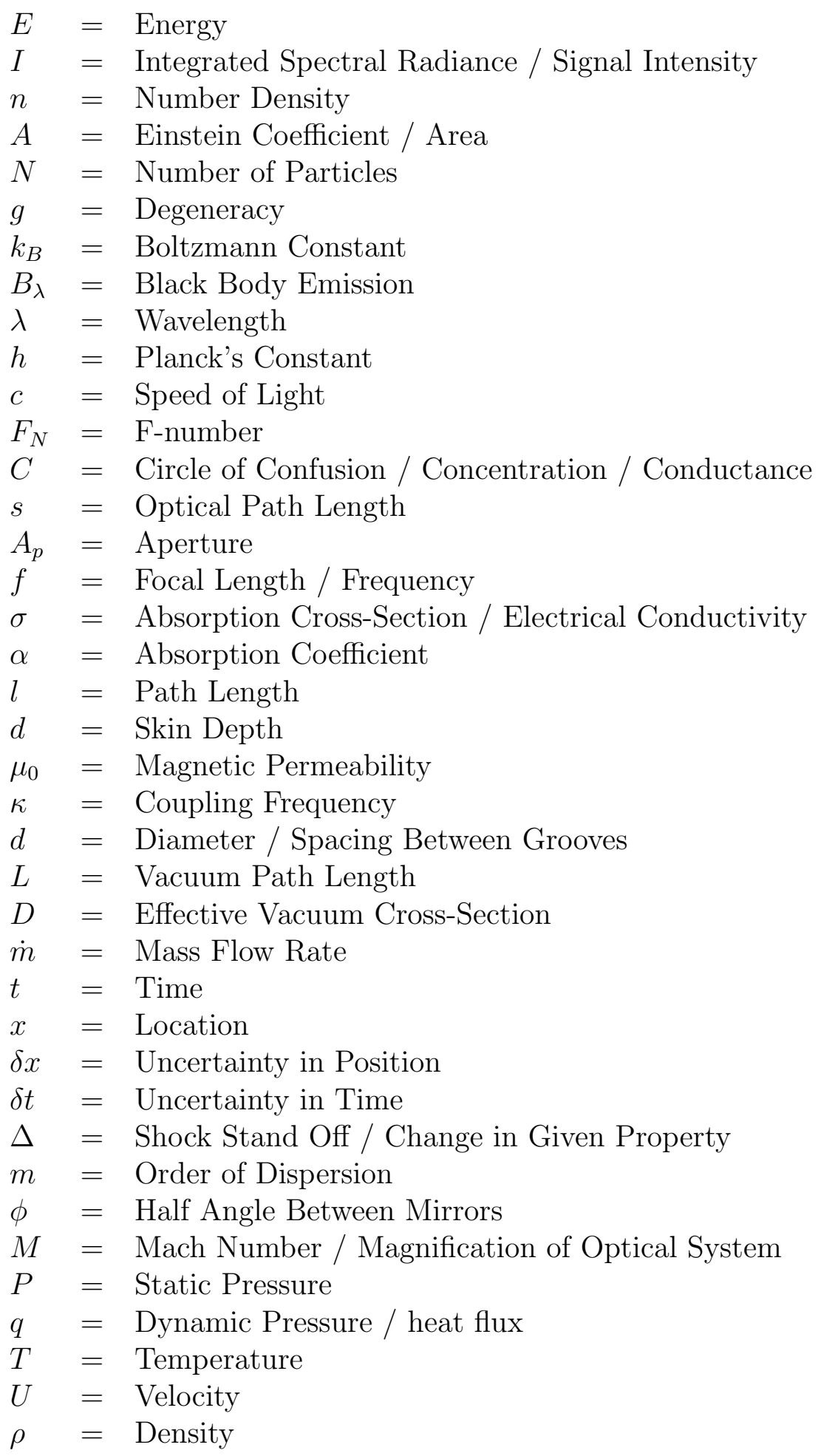


Subscripts

$$
\begin{aligned}
& u=\text { Upper State } \\
& l=\text { Lower State } \\
& o=\text { Object } \\
& i=\text { Image / Initial } \\
& r=\text { Radiative }
\end{aligned}
$$

Superscripts

$$
\circ=\text { Degrees }
$$

Acronyms

$$
\begin{aligned}
& A R=\text { Aspect Ratio } \\
& C A D=\text { Computer Aided Design } \\
& C C D=\text { Charge Coupled Device } \\
& C F D=\text { Computation Fluid Dynamics } \\
& I C C D=\text { Intensified CCD } \\
& I R=\text { Infrared } \\
& N I=\text { National Instruments } \\
& P M T=\text { Photo Multiplier Tube } \\
& T P S=\text { Thermal Protection Systems } \\
& U V=\text { Ultraviolet } \\
& V U V=\text { Vacuum Ultraviolet }
\end{aligned}
$$





\section{Chapter 1}

\section{Introduction}

The atmospheric entry component of a mission lasts only minutes but is one of the most critical stages of a mission due to the high heat loads encountered by the spacecraft. As a spacecraft enters the atmosphere at super-orbital velocity, a strong shock wave is formed directly in front of it. The shock wave processes the atmospheric gas into a dense, hot shock layer with peak temperatures in excess of $10,000 \mathrm{~K}$. The sudden increase in gas temperature as it is shock processed is due to high kinetic energy of the spacecraft being transferred to the air. Higher amounts of energy within the shock layer result in higher temperatures and therefore a larger heat flux to the spacecraft.

There are two main forms of heat transfer that occur in a shock layer; convective and radiative. Convective heat flux is the transfer of thermal energy as hot gas passes over the surface of a vehicle. Convective heating is significant even at relatively low temperatures and as such it can be readily studied using basic sources such as flames or electrical heaters. This has led to a large knowledge base of the process along with analytical models and empirical methods to calculate the resulting convective heat flux. In hypersonic flows the calculation of convective heat flux is more complex due to the potential of boundary layer transition, catalytic surface recombination and the introduction of particles from an ablating surface. Radiative heat flux is the outcome of transitions from higher quantum energy states to lower ones resulting in the emission of photons that impact the surface, transferring their energy. It is a phenomenon only observable in high temperature gases and does not scale simply, as shown in Figure 1.1. Consequently it is much more difficult to study and this has resulted in a limited understanding of the phenomenon.

Spacecraft commonly employ a thermal protection system (TPS) to overcome the the high heat loads experienced during super-orbital entry. The purpose of the TPS is to prevent the high heat loads from compromising the integrity of the load bearing structure of the vehicle. To design an efficient TPS that can withstand high shock layer temperatures, a 


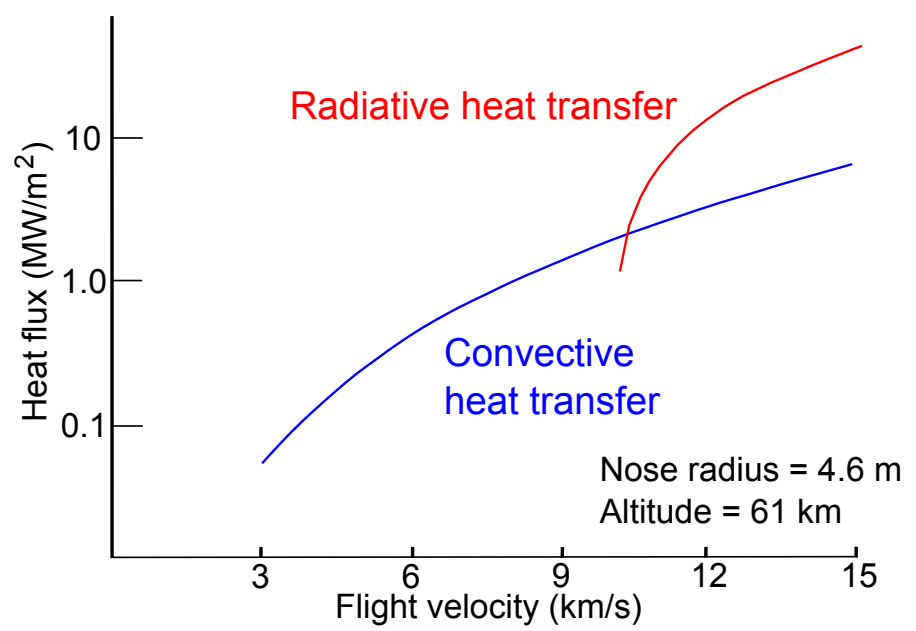

Figure 1.1: Variation of convective and radiative heat fluxes with increasing velocity. Radiative heat flux includes non-gray self-absorption and shock-layer radiative cooling. Adapted from Anderson [18].

designer must be able to accurately calculate the heat flux and heat transfer mechanism to the TPS at all points on the spacecraft's trajectory. The current approach used to calculate the heat flux is the application of computational models. Computational models used in this field are constructed with theoretical models and tailored using available flight and ground testing data. The theoretical models used require accurate knowledge of the probability of each quantum transition occurring, known as the Einstein coefficients, and the number density in each quantum level. Einstein coefficients are generally found experimentally and hence have an associated uncertainty. The chemical composition influencing the number density can vary depending on the finite-rate reaction scheme used in its calculation.

Due to the high cost of space flights, there have been very few flights ever conducted for the purpose of re-entry heating studies. As well, only a limited amount of experimental data exists due to the highly specialised nature of the facilities required to recreate the flow environment ahead of a re-entry spacecraft. This fact is highlighted in the high velocity region of a trajectory where facilities require extreme amounts of energy, and complex systems to channel that energy, in order to produce flight conditions. The lack of available data to test computational models results in significant uncertainties in the total heat flux calculated. These uncertainties result in large safety margins in the design of heat shields leading to costly excess weight. 


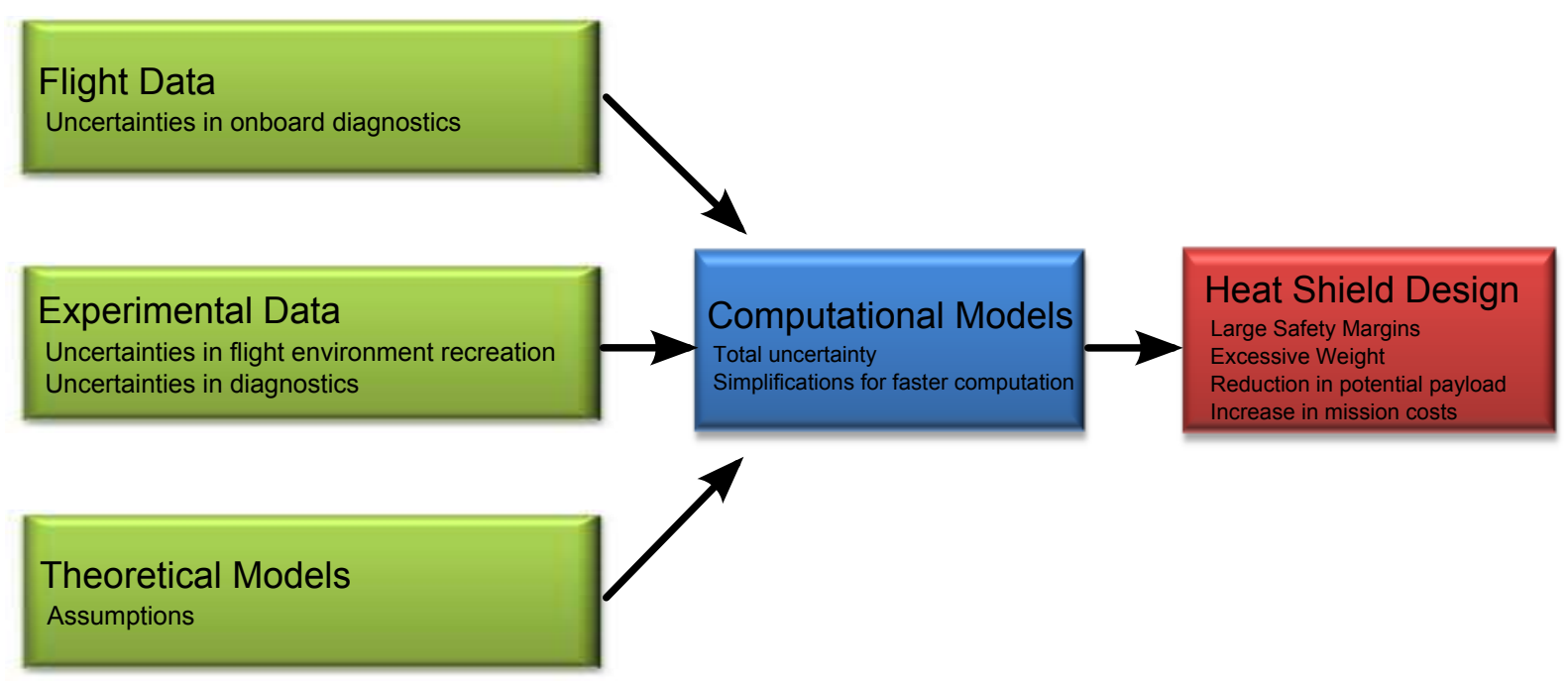

Figure 1.2: Design process of a spacecraft heat shield.

\subsection{Research Objectives}

The primary aim of this research was to use an expansion tube to create an aerothermodynamic environment representative of a real flight shock layer and investigate the physical processes that influence radiative heating. In particular, the vacuum ultraviolet (VUV) part of the radiative spectrum was to be investigated as it contributes $30-50 \%$ of the total radiative heat flux during peak heating for a lunar return trajectory [37]. The VUV spectral range is also responsible for the largest uncertainties when computing the heat flux to the surface of a vehicle [38]. Specifically, this was to be done through the creation of a system capable of measuring radiation across the shock layer in a spatially resolved manner and comparing this with measurements of VUV radiation incident on the surface of a model. Such a system provides insights into the radiative transfer mechanisms, flow field coupling phenomena and provide a dataset for the validation of radiation modelling codes.

Spectral data obtained on the expansion tube was to be simulated using Eilmer 3, the in-house computational fluid dynamics package at The Centre for Hypersonics, and the radiation modelling code Specair. These simulations were to be conducted using the Park [39] finite-rate reaction scheme and comparisons made with measured values. Specair was selected as the radiation modelling program as it uses Einstein coefficients from the NIST database, the primary source for most radiation modelling programs, ensuring this was a valuable comparison. 
To complement the research goals for the expansion tube, another system capable of measurements in the VUV was to be created for use on the plasma torch at Ecole Centrale Paris. As the flow in the plasma torch is in thermochemical equilibrium, it is possible to validate the Einstein coefficients without the non-equilibrium complexities observed in the expansion tubes.

A further aim of this research was to study the length scales of self-absorption in the VUV along with the impact of self-absorption and broadening on the total spectral radiance measured. This entailed investigating which spectral lines in the VUV spectral range are self-absorbed and the effects of varying levels of self-absorption on the measured spectrum. To experimentally study this phenomenon in the expansion tube, the length of a two dimensional model was to be varied consequently scaling the depth of the radiating flow field observed. For the plasma torch experiments, the observed depth of the radiating flow field was to be altered through the use of a traversing fence. It was intended that the data obtained in this study would be used to test the radiative transport properties of Specair and validate the broadening coefficients used. These results also provide a unique dataset for the validation of all radiation transport solvers. 


\section{Chapter 2}

\section{Radiative Heat Flux}

There are four mechanisms with which a molecule stores energy: translational, electronic, vibrational and rotational. Translational energy of a molecule is simply its the kinetic energy stored in its momentum. Rotational energy is measured though the rotational velocity of atoms about the centre of mass of the molecule. Vibrational energy is the thermochemical energy stored in the vibrations of atoms within a molecule and electronic energy is determined by the electrostatic interaction of the electron with other electrons and the nucleus.

Each electron configuration, and consequently electronic state, is dependent on the molecule's internuclear separation, as shown in Figure 2.1. With increasing energy, the electrons are more loosely bound in the excited states and consequently the molecule's potential energy becomes shallower and broader. For each electronic energy state, there are many bound vibrational energy states and for each vibrational energy state, there are many bound rotational energy states. Combining the four mechanisms, the total thermochemical energy of a molecule can be represented by Equation 2.1.

$$
E_{\text {total }}=E_{\text {translational }}+E_{\text {electronic }}+E_{\text {vibtraional }}+E_{\text {rotational }}
$$




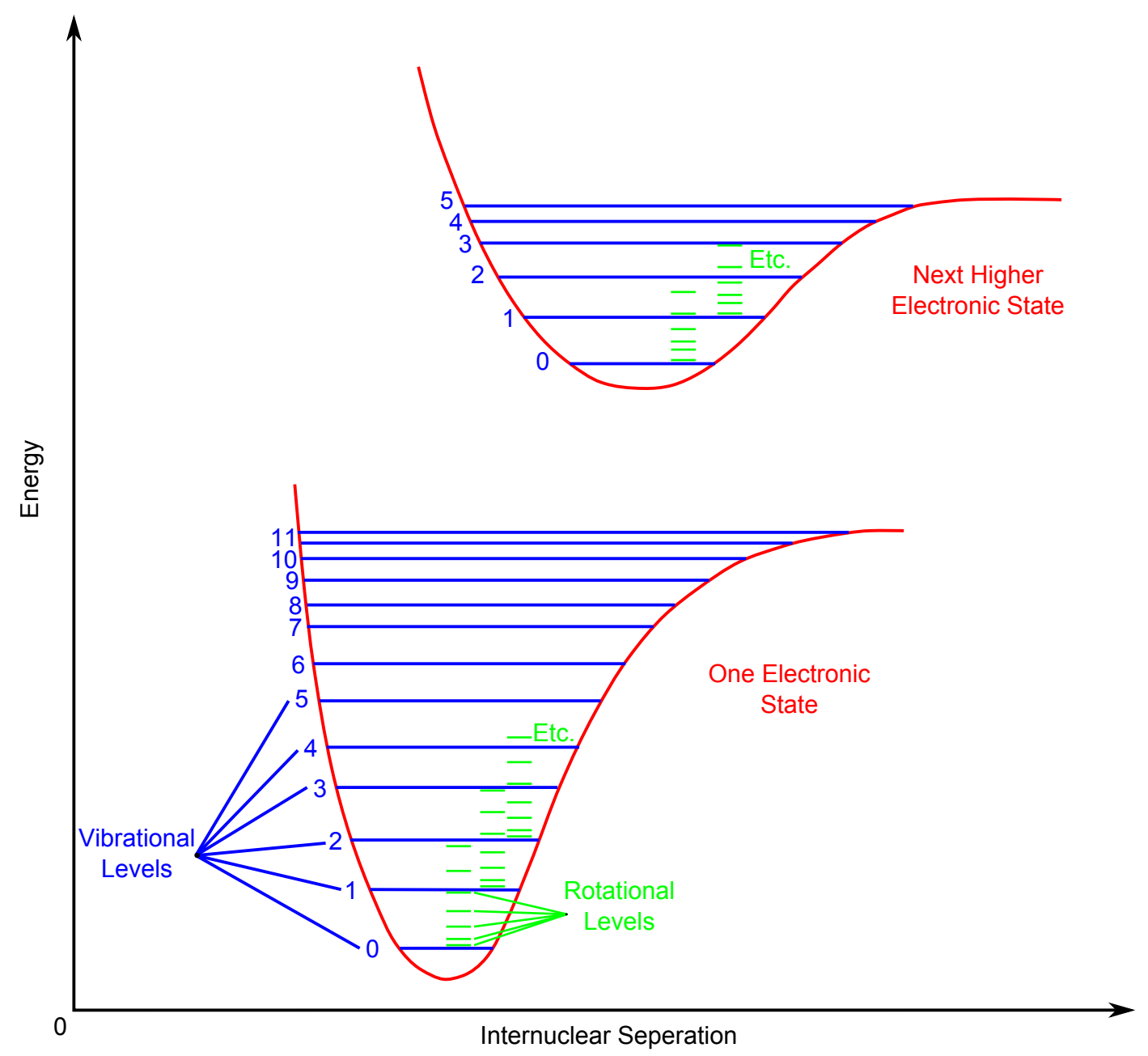

Figure 2.1: Thermochemical energy storage mechanisms; electronic, vibrational and rotational. Adapted from [19]

As a molecule or atom transitions from a higher energy state to a lower one, it can release its stored energy as a photon. The energy of this photon, measured by its wavelength or frequency, is determined by the energy transition undertaken by the particle. It is possible to observe a transition in which a molecule loses energy from more than one energy mode in a single transition, resulting in many possible emission wavelengths. The number of possible energy transitions is determined by the size and complexity of the molecule. A simple particle such as hydrogen has only a limited number of possible energy transitions in the visible wavelength range and thus produces photons at only a few wavelengths, corresponding to those transitions. A more complex element such as iron has many more possible transitions in the same wavelength range and thus produces many more spectral lines in the visible wavelength range as shown in Figure 2.2. 

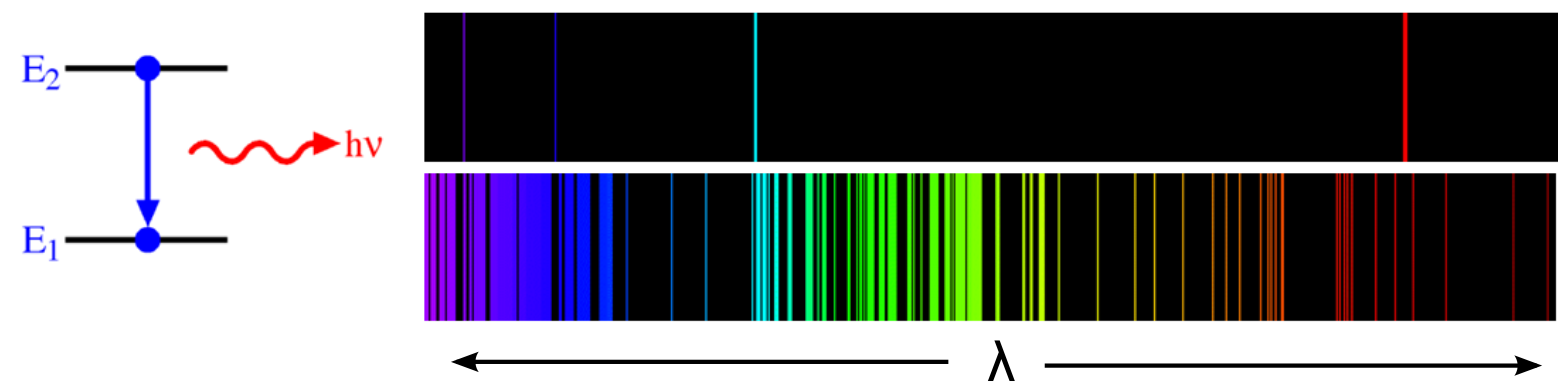

Figure 2.2: (Left) - Photon emission in the visible wavelength range through spontaneous transition to a lower energy state. (Right) - Spectral lines possible as a result of quantum transitions. Hydrogen spectrum (top) with minimal lines due to a small number of possible transitions and iron (bottom) with many different quantum transitions possible resulting in numerous spectral lines. Adapted from [40]

To observe spectral lines and determine their intensities, from which information about temperature and number density can be deduced, a spectroscopy system is required. A spectroscopy system uses a diffraction device to disperse an incoming signal into its various wavelength components. The integrated spectral radiance, $I$, of a given spectral line is a function of the transition probability, as defined by the Einstein coefficient $A_{u l}$, the number density in the upper energy state, $n_{u}$ and the energy difference between the upper and lower states of the transition, $E_{u}$ and $E_{l}$. This relationship is described in Equation 2.2.

$$
I\left(W a t t / \mathrm{cm}^{3} s r\right)=n_{u} \frac{A_{u l}}{4 \pi}\left(E_{u}-E_{l}\right)
$$

To infer the number density of the emitting state from the intensity of the spectral line, the distribution of excited energy levels is required. If a gas is known to be in thermochemical equilibrium, the number of particles $N_{i}$, in energy level $E_{i}$, can be calculated with the Boltzmann distribution using Equation 2.3.

$$
\frac{N_{i}}{N}=\frac{g_{i} \exp \left(\frac{-E_{i}}{k_{B} T}\right)}{Z(T)}
$$

To determine the Boltzmann distribution, the degeneracy $g_{i}$, Boltzmann constant $k_{B}$, equilibrium temperature $T$ and partition function $Z(T)$ are required. However in flight at super orbital velocities, the shock layer is rarely entirely in chemical and thermal equilibrium. At velocities greater than $2.5 \mathrm{~km} / \mathrm{s}$, the energy imparted on the air is enough to cause two body collisions that dissociate molecules of oxygen and nitrogen, as depicted for example by Equations 2.4 and 2.5 . 


$$
\begin{aligned}
& \mathrm{O}_{2}+M \longrightarrow 2 \mathrm{O}+M \\
& N_{2}+M \longrightarrow 2 N+M
\end{aligned}
$$

As shown in Figure 2.3, these effects grow in severity with increasing velocity and eventually ionisation of the shock layer occurs.

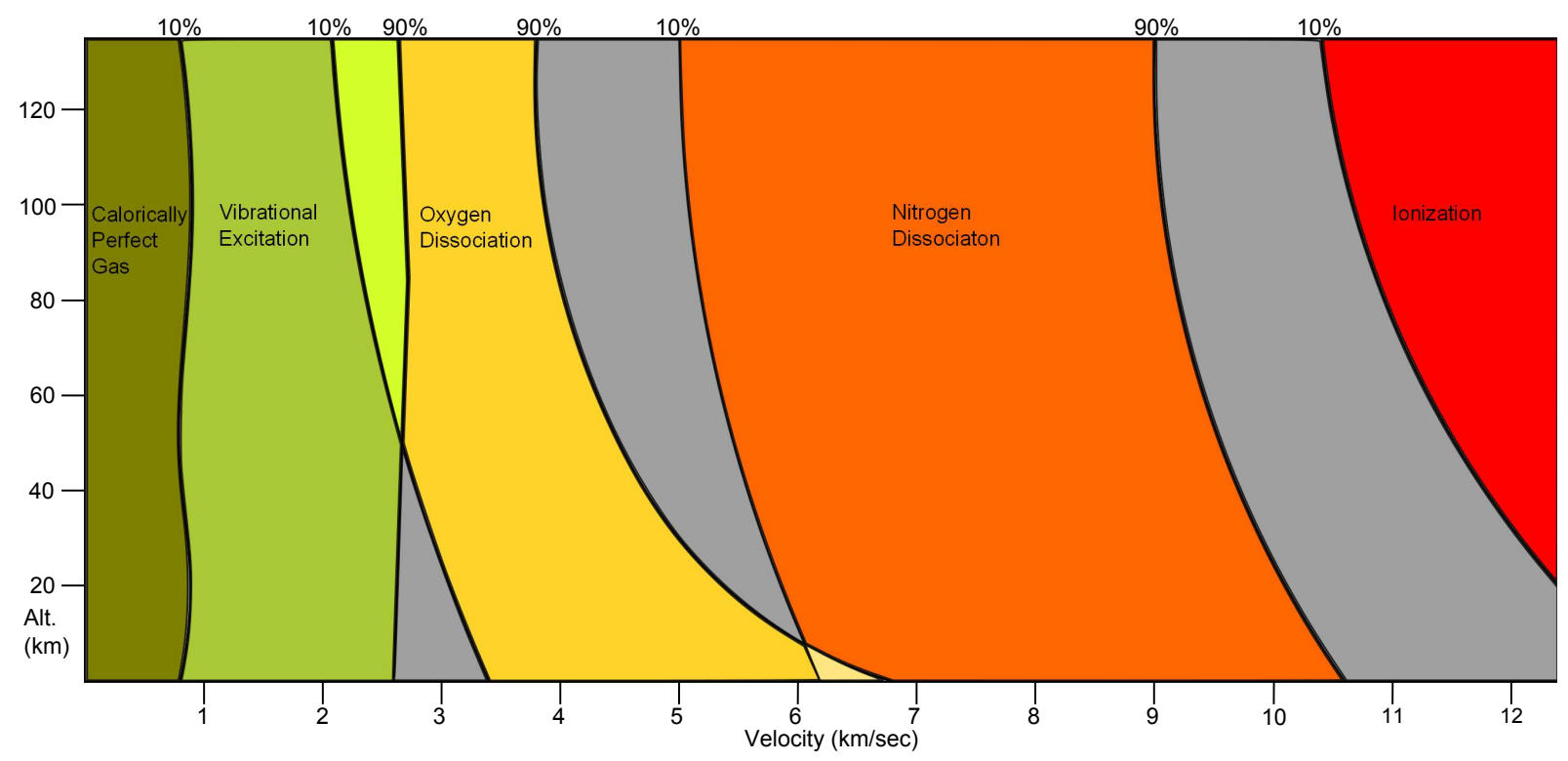

Figure 2.3: Effects of shock heating in air at varying velocities and altitudes. Adapted from Anderson [18]

There is a finite amount of time, or number of particle collisions, required for the gas in the shock layer to reach thermochemical equilibrium. The length scale required to reach equilibrium depends on the free stream velocity, providing heat or energy to the gas, and shock layer pressure, determining the frequency of collisions between particles. If the shock stand off is not larger than the length scale required to reach equilibrium, the shock layer gas composition and temperatures remain in a state of non-equilibrium. For larger shock stand offs there is an initial non-equilibrium region produced, followed by an equilibrium region, and finally boundary layer at the surface of the re-entry body, as shown in Figure 2.4 . 

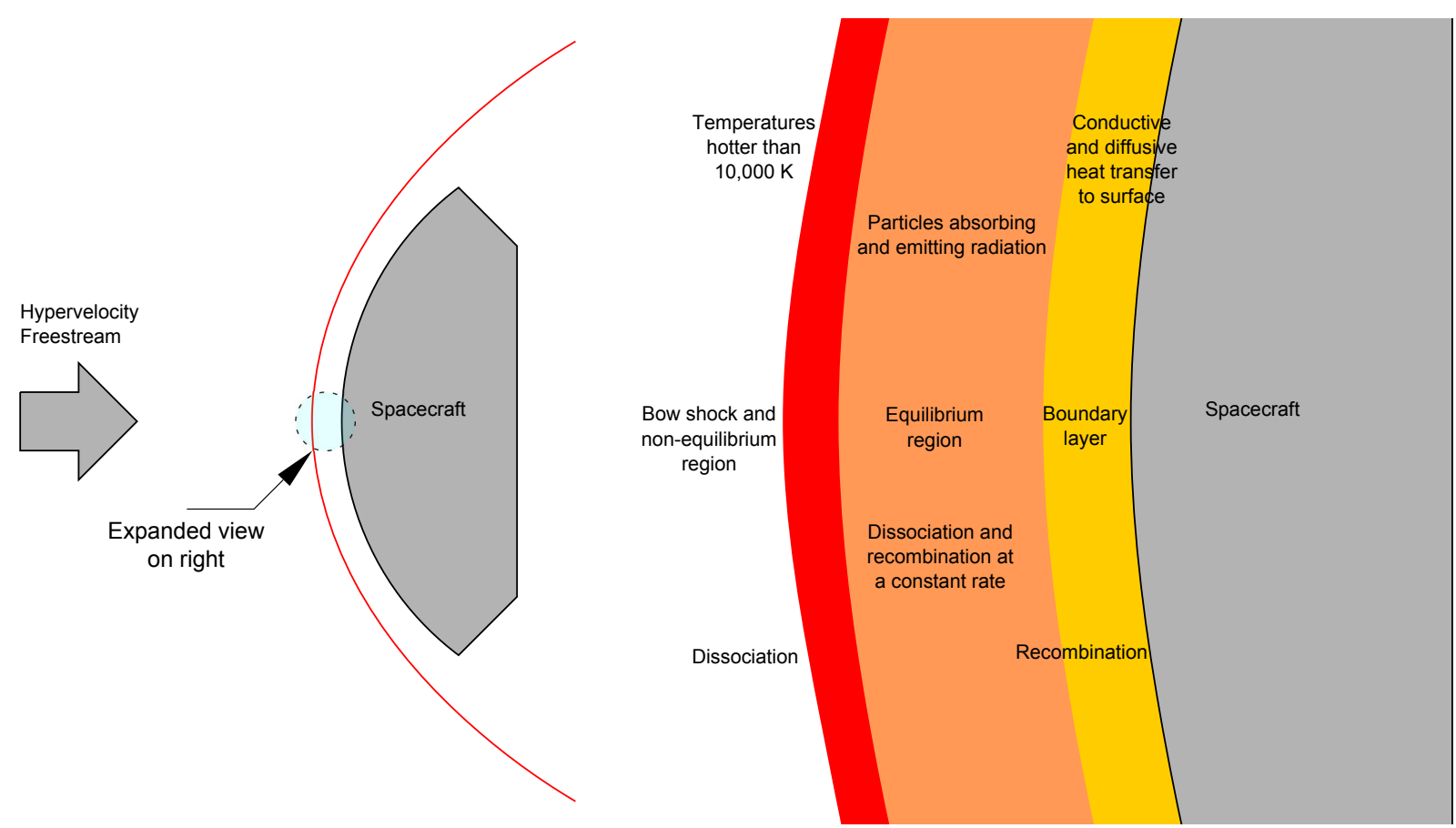

Figure 2.4: Depiction of the varying regions possible within a shock layer. Adapted from [18.

To calculate the radiative heat flux from the non-equilibrium regions of the shock layer, the gas composition along with knowledge of the various temperatures is required. Using these values, mathematical multi-temperature or collision radiative models can be used to model the non-equilibrium state of the shock layer and compute the radiative heat flux [41, 39, 42, 43, 44].

\subsection{Black Body Radiation and Self-Absorption}

A black body radiator is an idealised source of radiation that has defined radiative properties. At any given temperature, a black body radiator will radiate the maximum possible for any source at all wavelengths. A grey body radiator has a constant emissivity coefficient and therefore emits a fixed fraction of the black body emission at all wavelengths. In practice, many physical bodies approximate black or grey body radiators. Radiative transitions producing spectral lines in the VUV can also approach the black body limit. Due to the high energy of VUV emissions in air and the high density of particles in the upper energy state of the transitions involved, much of the radiation is self-absorbed as the spectral line strength would otherwise exceed the Planck curve limit as shown in Figure 2.5. The black body emission $B_{\lambda}$, for a given wavelength $\lambda$, and temperature $T$, follows Planck's law as shown in Equation 2.7. 


$$
B_{\lambda}=\frac{2 h c^{2}}{\lambda^{5}} \frac{1}{\exp \left(\frac{h c}{\lambda k_{B} T}\right)-1}
$$

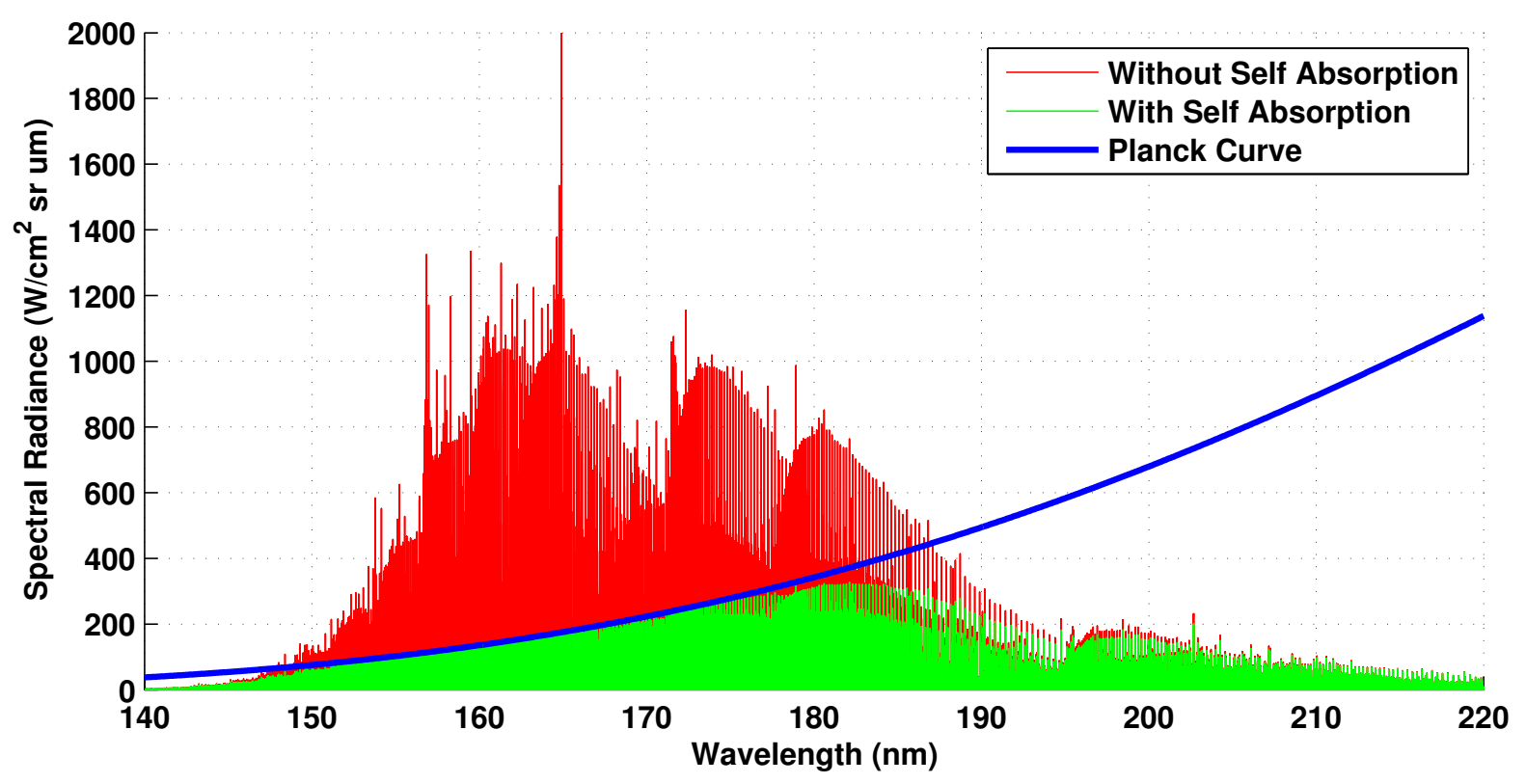

Figure 2.5: Planck's law limiting radiative emission and causing self-absorption to occur. Simulation conducted using Specair for $\mathrm{CO}(4+)$ at a temperature of $6,600 \mathrm{~K}$, pressure of $2,000 \mathrm{~Pa}$ and $1.0 \mathrm{~cm}$ depth of radiating flow field.

\subsection{Flow Field Radiation Coupling Effects}

Throughout the shock layer, photons are constantly being absorbed and re-emitted. Whilst the majority of this radiation either reaches the surface as radiative heat flux or is radiated out of the shock layer, a portion of this energy is reabsorbed within the shock layer. A good example of this is the VUV region. Bose [45] found that more than 99\% of VUV radiation is either self-absorbed due to the Planck curve limit, or absorbed by the boundary layer before reaching the surface. Yet the small fraction of VUV radiation that reaches the surface is still responsible for up to $40 \%$ of the total radiative heat flux [46, 45].

Absorption of VUV radiation by molecular oxygen or ablative products in the boundary layer can increase the temperature of the boundary layer and consequently increase convective heat flux. The location at which this radiation is absorbed determines whether the absorbed radiative heat flux becomes convective heat flux onto the surface or is merely convected downstream. Park found that absorption must occur close to the surface, within 
the boundary layer for it to be converted to convective heat flux [47]. If the radiation is absorbed near the outer edge of the boundary layer or further from the surface, it will be convected downstream. This coupling effect of radiative heat transfer to convective heating is enhanced by the recombination processes that occur near the cooler surface of the spacecraft, causing the recombination of oxygen, which strongly absorbs VUV radiation.

Conversely, the energy that is radiated out of the shock layer can have a significant effect on reducing the heat flux to the thermal protection system. If the radiant energy emitted is small relative to the total energy of the shock heated gas, there will be minimal change to the fluid dynamics within the shock layer and consequently the incident heat flux. However, at higher enthalpy flows where the radiant energy is a significant proportion of the total energy, emission of radiation from the shock layer can significantly reduce the temperature of the shock layer through a phenomenon known as radiative cooling. Radiative cooling has the potential to drastically alter the environment a thermal protection system must endure and therefore requires detailed analysis [48]. The level of coupling between the radiation and the flow field can be estimated with the Goulard number [49]:

$$
\tau=\frac{4 q_{r}}{\rho_{\infty} U_{\infty}^{3}}
$$

As a rule of thumb, when the Goulard number is larger than 0.01, the fluid dynamics and radiation are considered to be strongly coupled [143].

\subsection{Measurement of Emission Spectra}

The bulk of the data presented in this thesis is acquired through a series of optical components coupled to a spectrometer and recording device. A spectrometer is a device that is capable of dispersing a signal into its electromagnetic spectrum. This spread of electromagnetic radiation is detected by a measuring device, most commonly an intensified charge-coupled device or ICCD. Spectroscopic ICCDs with a two-dimensional array were used in this study to enable spatial resolution in one direction and spectral resolution in the second. Factors external to the spectroscopy system that influence the image obtained include the design of the optical system, spectral line broadening processes and absorbing gases in the optical path. 


\subsubsection{Design of Optical Systems}

The design of an optical system determines the magnification, location and intensity of the image formed. Through basic optical relations readily available in textbooks such as Williams [50], the position of optical elements can be calculated. Where physical constraints such as optical chamber sizes do not allow optical elements to be placed in a particular location, different focal lengths can be used to calculate more preferred positions. The sharpness of an image depends on the quality of the optical components and the minimisation of aberrations in the layout design. The depth of field must also be a consideration in the design process as it dictates the apertures required, strongly influencing the intensity of the image produced.

\section{Aberrations}

There are six types of aberrations that can occur, blurring or distorting the recorded image. These aberrations are spherical aberration, comatic aberration, astigmatism, curvature of field, distortion and chromatic aberration and are detailed in Appendix A. The first five aberrations listed above are known as monochromatic aberrations as they are independent of wavelength. Spherical aberrations, astigmatism and curvature of field can all be reduced by the application of an aperture that effectively reduces the surface area of the focussing element used. This results in small angles of curvature being introduced into the system. Distortion is also linked to aperture size and can be minimised by placing the aperture as close to the focussing optical element as possible. Comatic aberration is reduced by keeping the angle of reflecting optics to a minimum, thus reducing the change in optical path across the beam width. Finally, to avoid chromatic aberration, focussing mirrors can be used instead of lenses as their focal length is independent of wavelength.

\section{Depth of Field}

The depth of field $(D o F)$, represents the distance either side of the focal point that will be sharp in the image. Sharpness of an image is based on the circle of confusion created in the image by a point at the position of the object. For example, an infinitely small point in reality may appear as a circle in the image with a diameter of $0.2 \mathrm{~mm}$, thus having a circle of confusion of $0.2 \mathrm{~mm}$. In photography, an image is considered optically sharp when the circle of confusion is $0.2 \mathrm{~mm}$ or smaller when the observer is viewing the image from a distance of $25 \mathrm{~cm}$. For the purposes of this study, where each pixel on the 
recording device can be interrogated independently, it is important to achieve a circle of confusion as small as possible to avoid smearing data across pixels. The depth of field is controlled by varying the F-number $F_{N}$, that limits the solid angle of light received by the focussing optics from a point in space as depicted in Equation 2.8 [51].

$$
D_{o} F=\frac{2 F_{N} C s_{o} f^{2}\left(s_{o}-f\right)}{f^{4}-F_{N}^{2} C^{2} s_{o}^{2}}
$$

where $C$ is the circle of confusion, $s_{o}$ is the distance from the focussing optics to the object and $f$ is the focal length. The $\mathrm{F}$-number is the ratio of the focal length of the focussing element to the limiting aperture diameter, $A_{p}$, as shown in Equation 2.9 .

$$
F_{N}=\frac{f}{A_{p}}
$$

Table 2.1 shows the variation in circle of confusion as a function of aperture size. The large magnification used in this example is detrimental to the sharpness of the image as it effectively moves the focussing optics closer to the light source, thus increasing the solid angle seen. Having an appropriate depth of field is imperative when resolving the shock layer in front of a model. If the circle of confusion is too large it will not be possible to resolve the shock layer.

Table 2.1: Effect of varying aperture on circle of confusion for a $500 \mathrm{~mm}$ focal mirror system with a magnification of 1.5 and a depth of field of $100 \mathrm{~mm}$

\begin{tabular}{l|ll}
\hline Aperture $(\mathrm{mm})$ & F-Number & Circle of Confusion $(\mathrm{mm})$ \\
\hline 100 & 5.0 & 8.8 \\
75 & 6.7 & 6.6 \\
50 & 10 & 4.4 \\
25 & 20 & 2.2 \\
10 & 50 & 0.88 \\
5.0 & 100 & 0.44 \\
\hline
\end{tabular}

Conversely a larger solid angle allows for a higher signal to noise ratio on the recording device. To find the maximum aperture that can be used, without loss in depth of field, it is important to investigate the total effect of all aberrations combined. This was estimated by writing a ray tracing program [52], however due to minor imperfections in the curvatures of surfaces and position of optical elements, an accurate answer can only be achieved experimentally. A method that can be readily used to measure the total circle of confusion created is to place a sharp object in the imaging plane and observe how 
sharp it appears on the recording device. Knowing the size of the pixels on the recording device, the number of pixels over which the edge is spread can be converted to a circle of confusion measurement. This method can also be used to check the sharpness at the edges of the depth of field.

\subsubsection{Spectral Line Broadening Processes}

The recorded shape of a spectral line is dependent on the instrument function and several physical broadening processes. The instrument function alters the shape of a spectral line due to a finite entry slit width, resolution of the spectrometer and the pixel size of the recording device. The physical broadening processes relevant to this study are Doppler broadening, natural broadening and pressure broadening.
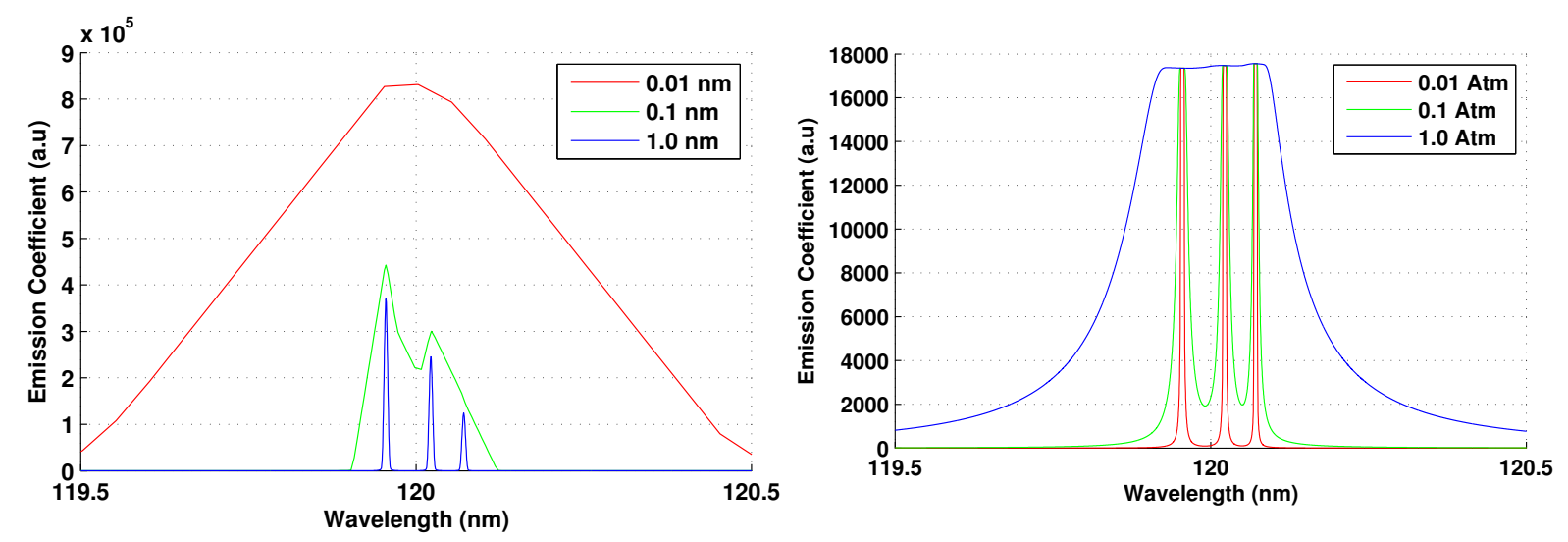

Figure 2.6: Broadening effects on an atomic oxygen triplet calculated using Specair. (Left) - Effect of varying the instrument function on observed spectral lines at 10,000 K using STP air with a $1 \mathrm{~cm}$ depth of radiating flow field. (Right) - Effect of broadening at varying pressures of pure nitrogen. Calculated at $7,000 \mathrm{~K}$ and $1 \mathrm{~cm}$ depth of radiating flow field with no instrument function convolution.

Doppler broadening occurs as molecules and atoms are travelling at varying velocities with respect to the observation point. Different velocities produce slightly altered observed wavelengths leading to the appearance of a broadened spectral line. Natural broadening is the spreading of energy levels over their radiative lifetimes, as described by the Heisenberg uncertainty principle. Pressure broadening occurs when collisions involving a radiating particle disturb its energy levels. This perturbation is also dependent on the type of collision partner. Broadening due to collisions with a charged particle is labelled Stark broadening, collisions with a neutral species is Van der Waals broadening and resonance broadening is due to collisions with a like particle. The influence of varying instrument 
functions and pressures on spectral line shape is depicted in Figure 2.6. Further details on spectral line broadening can be found in textbooks such as Samson and Thorne [53, 54].

\subsubsection{Absorption Cross-Sections}

To overcome the strong absorption of VUV radiation by molecular oxygen and water vapour, accurate data on the level of absorption at varying conditions is a powerful tool in the design of instrumentation systems and analysis of measured data. The absorption cross-sections dictate the vacuum pressures and flush purities required in the instrumentation system as well as the wavelengths to interrogate to test for any absorption. The absorption cross-sections used in this study for molecular oxygen absorption are obtained from $\mathrm{Lu}$ [20] for $108 \mathrm{~nm}$ to $180 \mathrm{~nm}$ and Yoshino [21] for the $\mathrm{O}_{2}$ Runge-Schumann bands between $180 \mathrm{~nm}$ and $192 \mathrm{~nm}$. These two data sets were selected as they presented the highest resolution datasets matching previous lower resolution studies of Ahmed, Hudson, Gibson and Watanabe [55, 56, 57, 58]. The water vapour absorption cross-sections are from Mota [22] and are relevant in this study due to the out-gassing of water vapour produced in high vacuum chambers. To convert absorption cross-sections $(\sigma)$ to signal absorption fractions, the absorption cross-section must first be converted to an absorption coefficient $(\alpha)$ using Equation 2.10.

$$
\alpha=\sigma \times n=C \times \frac{P}{T \cdot k_{B}}
$$

where $n$ is the number density of the absorbing species, $C$ is the mole fraction of the molecule, $P$ is the gas pressure, $T$ is the temperature and $k_{B}$ is the Boltzmann constant.

To calculate the intensity of the transmitted signal $I$, as a function of the initial signal $I_{o}$ and optical path length $l$, Beer-Lambert's law is applied:

$$
I=I_{o} \cdot \exp (-\alpha . l)
$$




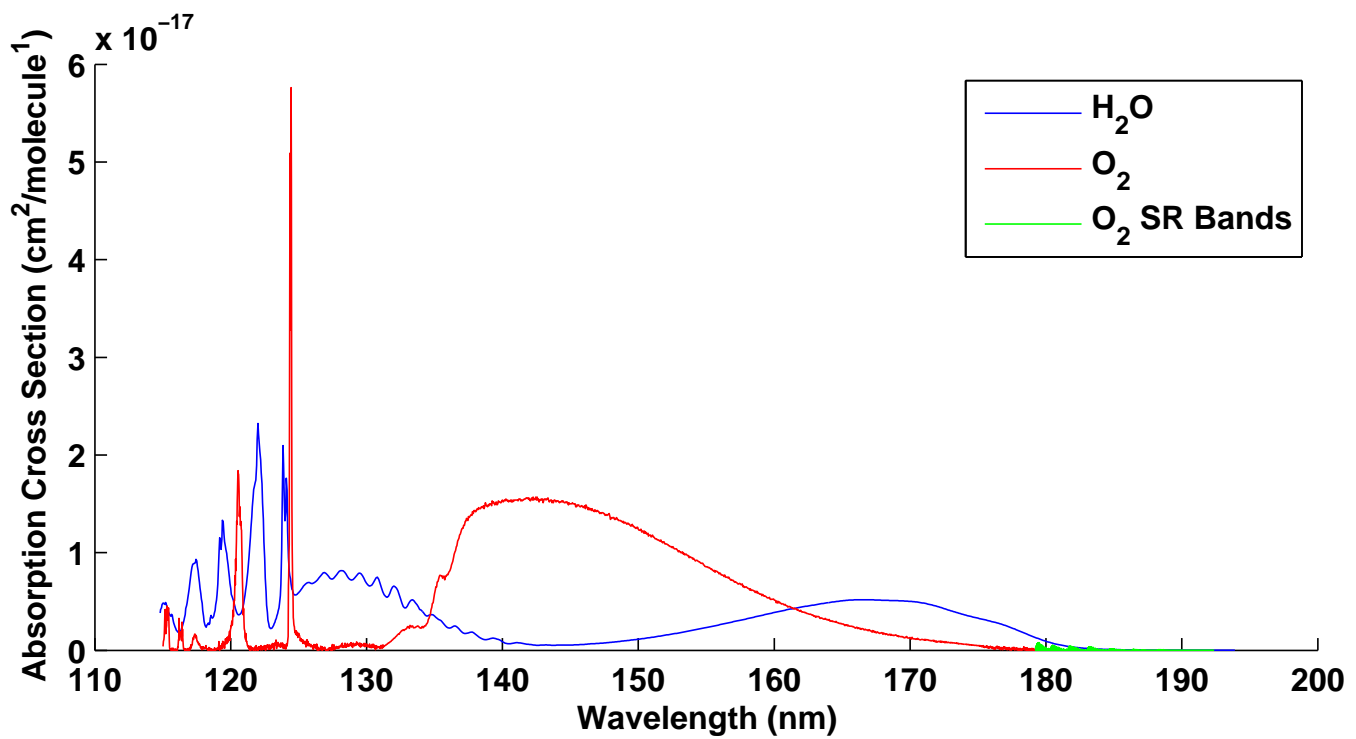

Figure 2.7: Absorption cross-sections for molecular oxygen [20], $\mathrm{O}_{2}$ Schumann-Runge bands [21] and water [22] 


\section{Chapter 3}

\section{Literature Review}

Radiative heating for hypersonic craft has been the subject of significant research for over 60 years. Study into this field began in the late 1950s with the focus of aerothermodynamic heating for lunar return conditions and ballistic missiles. Due to the lack of computational power at the time, empirical correlations were preferred over computational modelling. Whilst correlations such as Lees and Fay and Riddell were available for the prediction of convective heating, none existed for radiative heat flux [59, 60]. To predict radiative heating in the 1950s and 1960s, state of the art inviscid flow field solvers were coupled to chemistry models. During the mid 1960s computational models had advanced to the stage where viscous flow field solvers were coupled to equilibrium chemistry models capable of predicting non-gray spectral data [61]. Extensive reviews on radiative heating research carried out in the late 1950s and 1960s can be found in Belotserkovskii, Boughner and Anderson [62, 63, 64].

The only available ground testing facilities in the 1950s and 1960s for radiative heating studies were shock tunnels. Shock tunnels were used in one of two ways: observing the shock wave as it traverses down the tunnel and placing model at the exit of the tunnel. Observation of the shock wave as it traverses the shock tunnel required specialised equipment to complete all required measurements in the short period of time that the shock wave was in the field of view. Even at measurement intervals as short as a microsecond, the shock wave moves $10 \mathrm{~mm}$ in a laboratory frame of reference at a velocity of $10 \mathrm{~km} / \mathrm{s}$. The main advantage of this configuration was the ability to recreate the real gas effects observed in flight and for this reason this configuration is still employed today.

By placing a model at the exit of the shock tunnel, a standing shock wave is created around the model. This approach allows for the shock wave to be formed in a fixed frame of reference and increases the total enthalpy of the flow in a frame of reference. A disadvantage of this configuration is the altered state of the test gas as it has been 
shock processed causing it to become partially dissociated and ionised. This alteration of the test gas results in an inability to capture real gas effects and produce a shock layer comparable to that of flight. An additional drawback of this shock tunnel configuration is the inability to place models larger than a fraction of the core flow. This limitation is due to the extremely short test times of the shock tunnel not allowing the test flow to establish steady state over the model. To more accurately recreate shock layers around models and quantify the radiative heat flux, the use of expansion tubes was proposed.

The expansion tube concept was first introduced by Resler and Boxsom in 1952 [65]. The idea behind the expansion tube was to use an unsteady longitudinal expansion through a tube to increase the enthalpy of the test gas. This approach allowed for the temperature of the test gas to remain low enough throughout the experiment to avoid high levels of dissociate and ionisation, resulting in a test gas more representative of flight conditions and the ability to reproduce real gas effects and shock layers. A detailed study of this concept was carried out by Trimpi in 1962 and it predicted several advantages over shock tubes including increased test times and the ability to attain velocities twice those possible by conventional shock tubes [66]. The increase in test time allowed for larger models to be placed in the tunnels leading to the possibility of scaling studies. Experiment work began on expansion tubes in the 1966 to increase the performance envelope of ground testing facilities to match the requirements of the Apollo program [67]. To validate the ground testing and computational work being carried out to design the Apollo re-entry capsule, flight experiments were deemed necessary.

\subsection{Flight Experiments}

Two flight campaigns were carried out in the 1960s to complement the growing array of ground testing facilities used to investigate radiative heat flux and obtain a set of benchmark flight data. The Flight Investigation of Re-Entry (FIRE) experiments of 1964 and 1965 were designed to measure the heat flux experienced during re-entry on an Apollo shaped capsule. The planned trajectory of the mission, presented in Figure 3.1, resulted in the capsule achieving a velocity of $11.35 \mathrm{~km} / \mathrm{s}$ at $122 \mathrm{~km}$ altitude, with a $0^{\circ}$ angle of attack and $-14^{\circ}$ flight path [68, 69]. The FIRE I flight experienced telemetry and control problems during the second half of the re-entry resulting in questionable accuracy of the experimental results. The FIRE II flight however, was greatly successful in obtaining data throughout the trajectory and is still regarded as benchmark data for testing of computational models [68, 70, 69, 71]. Through a series of calorimeters and radiometers 
located on the fore and after body, FIRE II was able to measure total heating rates as well as spectrally resolved radiative heat fluxes at a range of wavelengths.

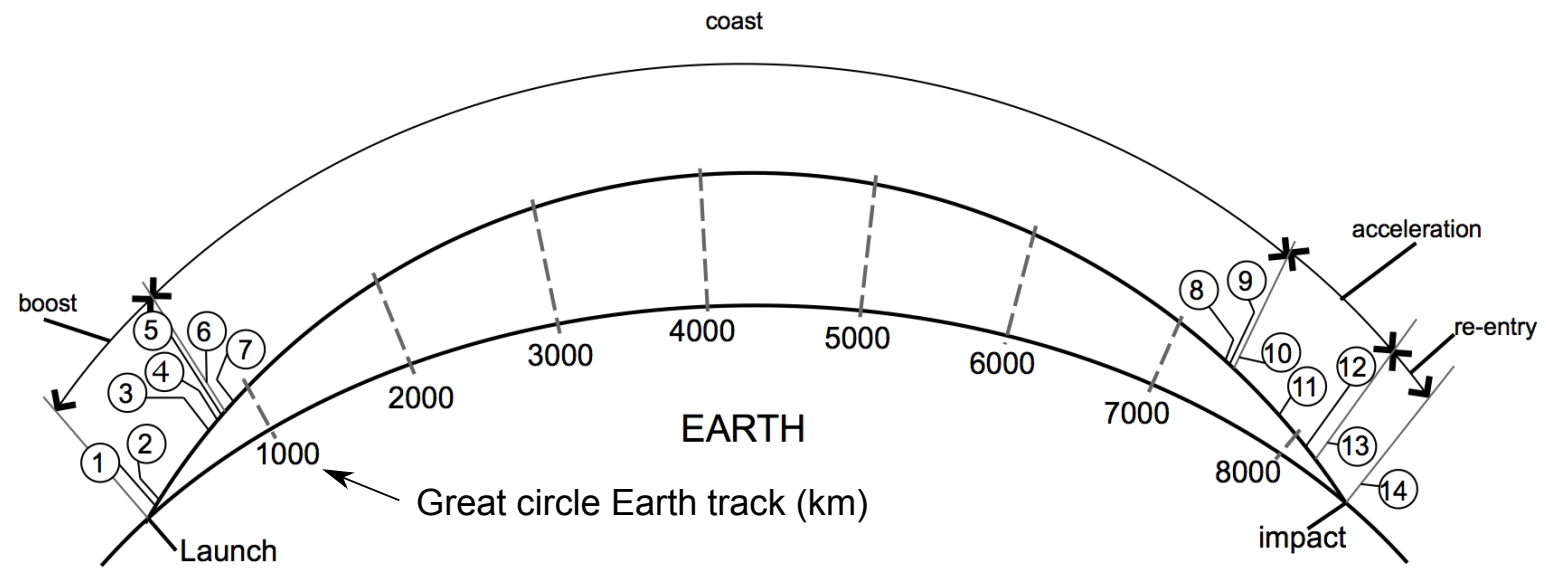

1 Booster engine cut-off

2 Booster jettison

3 Sustainer engine cut-off

4 Shroud separation

5 Vernier engine cut-off

6 Spacecraft separation

7 Velocity package start

8 Spin-up

9 Velocity package shell jettison

10 Antares ignition

11 Antares burnout

12 Re-entry package separation

13 Seperation rocket ignition

14 Impact

Figure 3.1: FIRE II mission trajectory. Adapted from [23]

The Apollo 4 capsule flown in 1967 was a prototype of the final manned Apollo capsule and the first test of the three stage Saturn V rocket. The rocket placed the capsule into orbit at $192 \mathrm{~km}$ altitude and after two rotations around Earth the third stage rocket was reignited, setting the capsule on an Earth-intersecting trajectory with an apogee of $17,346 \mathrm{~km} \mathrm{[72]}$. The capsule re-entered the atmosphere at a velocity of $11.14 \mathrm{~km} / \mathrm{s}$ at $122 \mathrm{~km}$ altitude, with a $22^{\circ}$ angle of attack and $-7.077^{\circ}$ flight path [43, 72. During reentry, a radiometer mounted at the stagnation point was used to measure the radiative heat flux [73]. The difference between the Apollo 4 capsule and the FIRE II capsule was the ablating heat shield installed on the Apollo 4, instead of beryllium as used for FIRE II. The Apollo 4 capsule remains the only re-entry craft with on-board instrumentation mounted behind an ablating shield and no flight experiments at velocities greater than $6.0 \mathrm{~km} / \mathrm{s}$ with any on-board radiation measurements have been flown since. 


\subsection{Planetary Exploration and Non-Equilibrium Radiation}

Having successfully completed a manned lunar return mission in 1969, radiative heating research began to focus on Mars entry and Earth return conditions [74, 75]. Naturally, this also led to research on radiative heating for entry into Venus and the outer planets such as Jupiter, Saturn and Uranus [75, 76]. In 1978, NASA's Pioneer-Venus probes entered Venus' atmosphere where they collected aerothermodynamic heating data using thermocouples embedded in the heat shields [77]. In attempts to reconstruct measurements made by the Pioneer-Venus mission, Grose and Nealy showed that if non-equilibrium effects were considered, the radiative heating nearly doubled relative to the equilibrium prediction and this spurred research into non-equilibrium flows [78].

Detailed studies into non-equilibrium radiation in air did not begin until the early 1980s $[79,80,81,82,83,84$. This was predominantly due to a lack of computing power required to solve thermodynamic and chemical non-equilibrium Navier-Stokes flow field models. The increased interest in non-equilibrium air was a result of the proposed Aeroassist Orbital Transfer Vehicle concept and the Aeroassist Flight Experiment [85, 86]. These vehicles were designed to decelerate at high altitudes and due to the subsequent low densities, the shock layer would predominantly remain in a state of non-equilibrium. The Non-Equilibrium Air radiation code, NEQAIR [87], developed during this time is still used in modelling radiation today.

\subsection{Re-Entry Observation Missions}

At the turn of the $21^{\text {st }}$ century, three sample return craft, Genesis, Stardust and Hayabusa, re-entered the Earth at super-orbital velocities [88, 89, 90]. These missions provided the first opportunity since the 1960s to take spectral and thermal measurements during reentry of a capsules. All three craft had no on-board instrumentation so all measurements had be taken from large distances. Even with modern advancements in measurement techniques, working at distances in the order of 100's of kilometres did not allow for spatial resolution to be obtained through the shock layer. For each mission, spectral data was obtained from on-board NASA aircraft and various ground based observation points 91, 92, 93. The data collected through these observation missions produced another set of benchmark data for the validation of computational models. 
Table 3.1: Re-entry flight observation missions of the 2000s.

\begin{tabular}{l|lll}
\hline & Genesis & Stardust & Hayabusa \\
\hline Re-entry year & 2004 & 2006 & 2010 \\
Velocity $(\mathrm{km} / \mathrm{s})$ & 11.04 & 12.9 & 12.2 \\
Sample & Solar wind & Comet tail particles & Comet surface dust \\
Result & Failure & Success & Success \\
\hline
\end{tabular}

\subsection{Ground Testing Facilities}

Since the 1960s, ground testing facilities have evolved from the use of just shock tunnels to a variety of approaches to create features of planetary entry flows. No one facility is capable of accurately producing entry flows representative of the entire trajectory, whilst maintaining flow for long enough to test materials and produce ablation processes. To create high enthalpy flows experienced at the high velocity end of super orbital trajectories requires large amounts of power and such flows can only be sustained for fractions of a second. Facilities that create the highest enthalpy flows are compressed gas or electric arc driven shock tubes and expansion tubes. For longer duration hypersonic flows, arc jets are commonly used. Arc jets use an electrical arc between two electrodes to heat the test gas and channel it into a nozzle that accelerates the gas to hypersonic velocity. Whilst this type of facility provides a longer duration test time, it has certain major disadvantages. As the test gas has already been heated to very high temperatures, causing the composition of the test gas to be drastically altered, the non-equilibrium gas chemistry effects experienced in flight cannot be produced. The altered gas chemistry also impedes the recreation of hypersonic shock layers and flight aerodynamics [94]. As such, expansion tubes and shock tubes are traditionally used to study gas chemistry effects through the shock layer and arc jets have been used to test material properties and ablation.

Continuous facilities such as subsonic plasma torches can also be used to study high temperature gas effects and test materials. Induction coupled plasma torches, such as the one used in this study, produce a high temperature gas representative of the equilibrium part of the shock layer at temperatures in excess of $8,000 \mathrm{~K}$. At such temperatures it is possible to simulate the heating loads experienced by a super orbital entry and create representative boundary layer interactions with ablative products from the surface. The disadvantage of the plasma torch is that the flow is often subsonic and as such the shock wave and non-equilibrium region cannot be produced at high enthalpies. The advantages of an induction plasma torch over an arc jet is the cleaner flow produced due to the lack of 
the electrodes interacting directly with the plasma and the capability of longer duration test times.

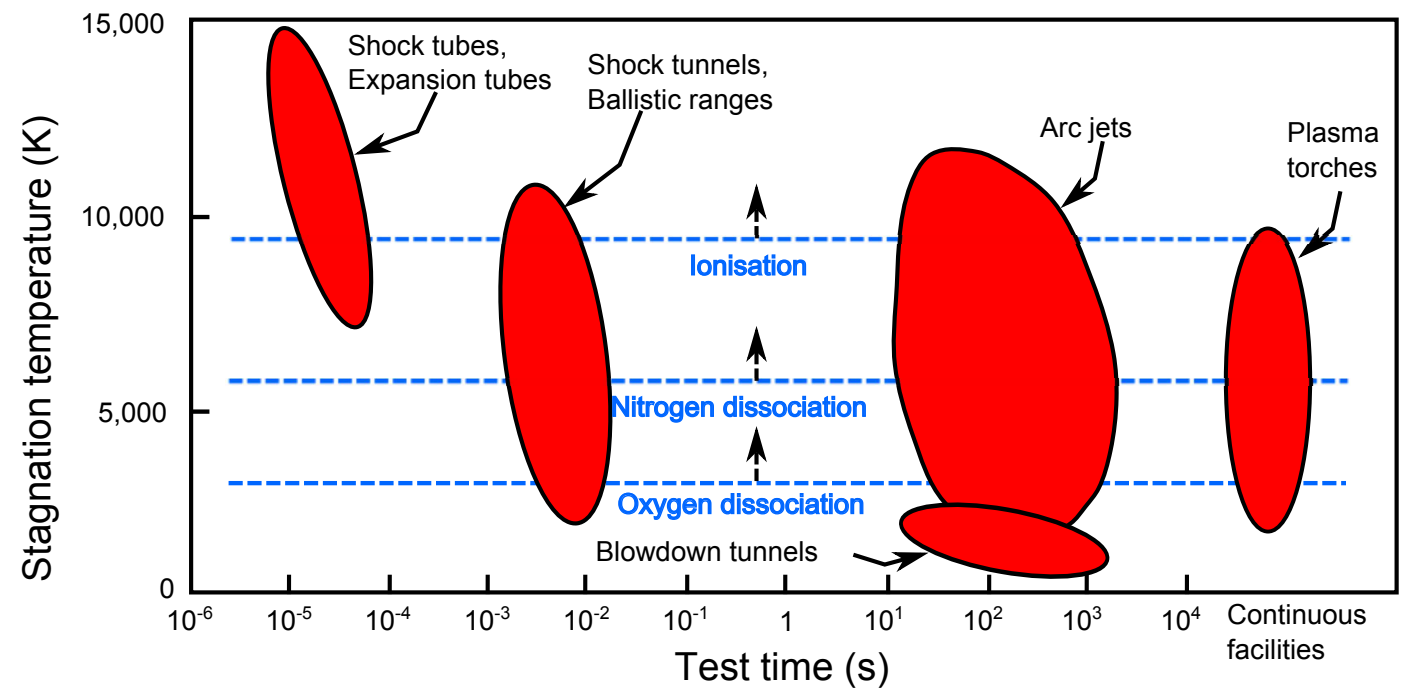

Figure 3.2: Stagnation temperature and test time ranges of various ground testing facilities. Adapted from Bertin [24].

\subsubsection{X2 Expansion Tube}

The X2 expansion tube at The University of Queensland was the main ground testing facility used in this study. X2 is a free piston, compressed gas driven expansion tube capable of producing flows of up to $75 \mathrm{MJ} / \mathrm{kg}$ for test times in excess of $100 \mu \mathrm{s}$. A range of configurations is possible for this facility, such as the use of detachable nozzles, additional driver sections and a range of orifice plates used to vary the internal diameter at the primary diaphragm. For this study a single driver tube with a $208 \mathrm{~mm}$ nozzle configuration as shown in Figure 3.3 was used.

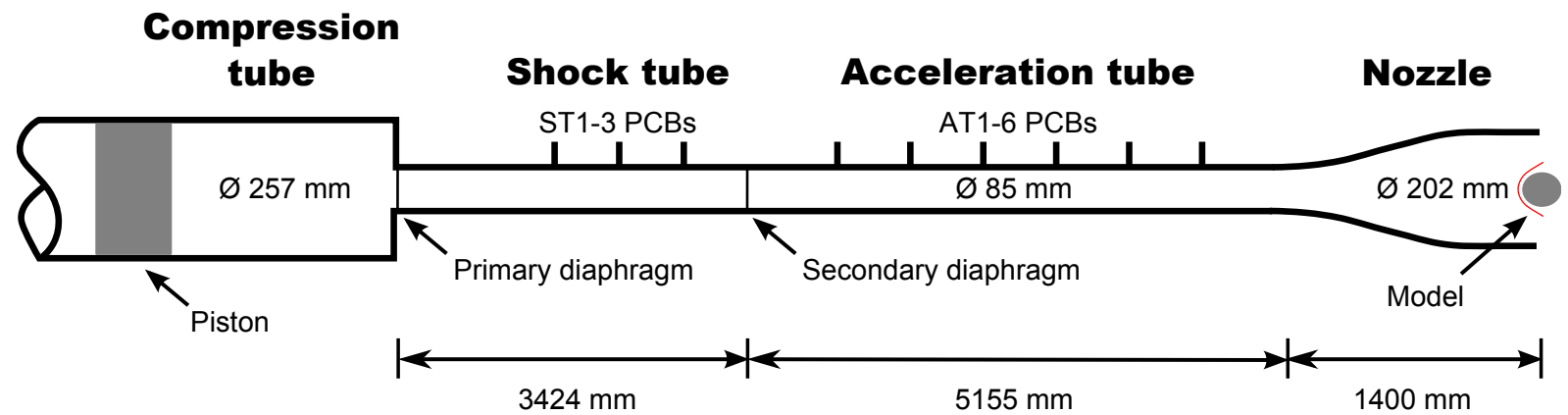

Figure 3.3: Schematic of the X2 expansion tube configuration used for this study. ST1-3 and AT1-6 PCBs are the shock tube and acceleration tube pressure transducers. 
$\mathrm{X} 2$ can be broken down into four main sections; the driver, shock tube, acceleration tube and nozzle. Before an experiment can be carried out, pressures in the various sections need to be set accurately and according to the condition required. To begin the experiment, the piston is released from the launcher, rapidly accelerating due to the high pressure air in the reservoir and compressing the driver gas in the compression tube. Once the pressure in the compression tube is greater than that of the reservoir, the piston begins to decelerate. The pressure in the compression tube continues to rise as the kinetic energy of the piston is imparted upon the driver gas. At a pressure of approximately $30 \mathrm{MPa}$ the primary steel diaphragm ruptures and a normal shock wave is generated in the shock tube.

As the normal shock wave travels through the shock tube, it processes the test gas increasing its temperature and pressure whilst accelerating it to a velocity in the order of a few $\mathrm{km} / \mathrm{s}$. The secondary diaphragm at the downstream end of the shock tube is typically made from Mylar or a sheet of thin aluminium. The immense force from the normal shock wave shatters the diaphragm and the shock wave continues into the acceleration tube. The shock processed test gas behind the acceleration gas interface expands into the acceleration tube and through unsteady expansion processes, the test gas continues to accelerate into the nozzle, where it undergoes a final expansion to reach the desired test conditions. The amount of expansion is regulated by the initial acceleration gas pressure and the profile of the nozzle used. The end of steady test time is marked by he arrival of the $\mathrm{u}$-a wave at the test section.

The determination of gas chemistry in the free stream flow produced at the exit of the nozzle is a complex undertaking because of the range of pressures and temperatures experienced by the test gas during an experiment. Due to the high pressure and relatively low primary shock velocity in the shock tube, the test gas can be shown to be at, or close to, equilibrium conditions at the moment of secondary diaphragm rupture. As the test gas undergoes a series of unsteady expansions in the acceleration tube, the pressure and temperature of the gas is decreasing as it accelerates. Different parts of the test gas will experience different flow path lengths at each pressure and temperature and therefore have varied amounts of time to transition towards equilibrium chemistry. This can lead to fluctuating chemical composition during the test time. In modelling this process, the largest uncertainties arise for the thermal and chemical non-equilibrium that occurs as the gas is processed by unsteady expansions. This can lead to phenomena such as vibrational freezing and requires well resolved multi-temperature models. The modelling process is further complicated by the need for time resolved simulations with fine spatial resolution to resolve the shock wave and the boundary layer growth for the entire length of the 
expansion tube.

Various analytical and numerical approaches have been used to approximate the state of the chemistry [95, 96, 97, 98], however none have been successful in definitively calculating the gas chemistry state [99]. The best approximation to date was conducted by Neely [100], in which it was shown that at high enthalpy conditions, completely expanding the test gas at equilibrium gas chemistry resulted in good agreement with the measured static pressure values. This study validated the use of the equilibrium assumption to estimate the flow chemistry. Recent advances in computational power have allowed for the commencement of full facility simulations to be developed using finite rate chemistry to determine the time resolved free stream conditions produced [101]. The development of these simulations are currently a major research topic and are outside the scope of this study. For the purposes of this study, the free stream conditions produced are assumed to be constant and at chemical equilibrium.

\subsubsection{Plasma Torch at Ecole Centrale Paris}

The plasma torch at Ecole Centrale Paris is a TAFA Model 66 inductively coupled plasma torch. An inductively coupled plasma torch imparts energy into a plasma through an oscillating magnetic field. This concept was introduced by Reed in 1961 and it has not changed since [102]. As a gas travels through a quartz tube inside a coaxial induction coil carrying a radio frequency $(\mathrm{RF})$ current, the free electrons are forced to spin due to the axial magnetic field produced by the RF current. The free electrons continue to be accelerated as the electromagnetic forces act upon them and this increase in velocity is an effective increase in the translational temperature of the electrons. Through collisions kinetic energy is transferred to heavy particles causing them to gain energy and ionise. Once enough free electrons are produced, the plasma becomes self sustained.

Whilst necessary for the creation of a sustainable plasma, the ionisation of a gas also limits the influence of the induction coil. The electromagnetic field produced by the plasma creates an adverse magnetic field that shields the electromagnetic waves emanating from the induction coil and limits their penetration into the plasma. The result of this phenomenon is strong heating at the outer edges of the plasma and minimal heating in the centre. This effect was experimentally shown by Eckert [103] and is presented in Figure 3.5 . 


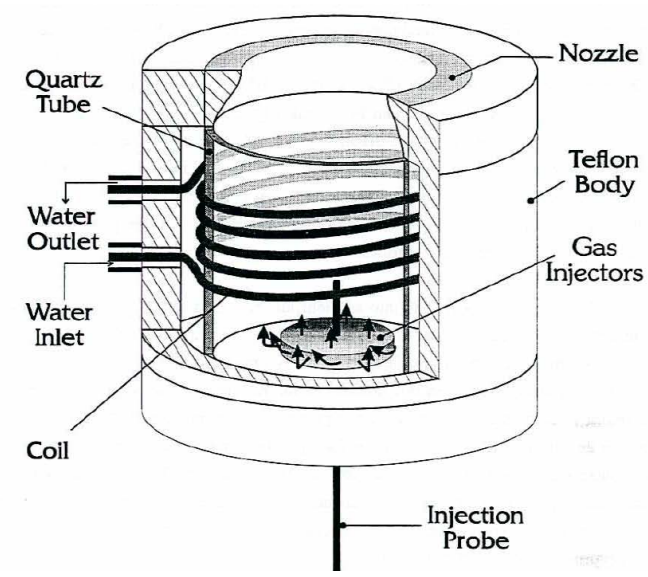

Figure 3.4: Schematic of the plasma torch [25]

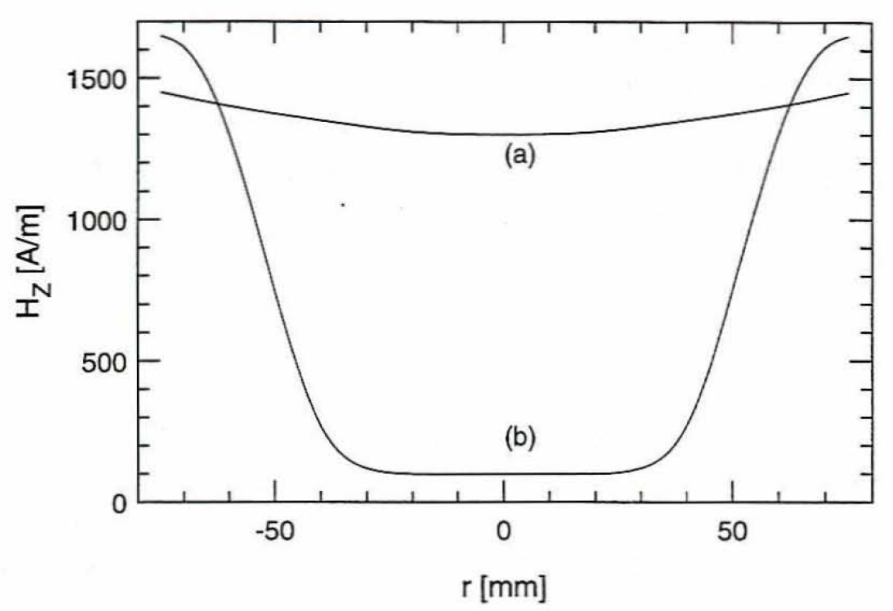

Figure 3.5: Intensity of the magnetic field without a discharge (a) and with a discharge (b). 25]

It is possible to calculate the penetration depth of the electromagnetic waves, also known as the skin depth $d$, through the Channel approximation shown in Equation 3.1 [104.

$$
d=\sqrt{\frac{1}{\pi \mu_{0} \sigma f}}
$$

Laux found that for the plasma torch used in this study, at an average temperature of $8,000 \mathrm{~K}$, with the torch operating in air at atmospheric pressure and at $4 \mathrm{MHz} \mathrm{RF}$, the penetration depth of the electromagnetic waves is approximately $9 \mathrm{~mm}$ [25]. The internal diameter of the torch is $76 \mathrm{~mm}$ and thus the skin depth covers only approximately $25 \%$ of the plasma. The internal $75 \%$ must be heated through convection, radiation and conduction. It is possible to increase the skin depth through a reduction in the frequency 
however this would result in a decrease in the coupling efficiency $(\kappa)$, as shown by Equation $3.2[25]$.

$$
\kappa=\sqrt{2} \frac{r_{n}}{d}
$$

As free electrons are required to sustain the plasma, particular steps must be taken to start the plasma torch. For start up, argon gas flows through the torch while being ionised with a Tesla coil. Argon is used as it has a lower ionisation energy than air and contains a relatively large number of electrons in its outer shell. Argon also has a low specific heat, enabling higher temperatures at lower power, and its low thermal conductivity compare to air ensures a hot region is maintained with limited diffusion. Once the plasma is established with argon, the test gas is slowly introduced whilst increasing the power input to the plasma. Through the gradual addition of test gas and incremental increases in power, a sufficient number of electrons to sustain the plasma are produced.

\section{Physical Characteristics}

The TAFA model 66 plasma torch used in this study is powered by a radio frequency $4 \mathrm{MHz}$ LEPEL Model T-50-3 power supply capable of delivering $120 \mathrm{~kW}$ to the oscillator plates. A fraction of this line power is lost as heat in the oscillator tube and consequently the maximum RF power output of the oscillator plates that can be directly delivered to the plasma is $50 \mathrm{~kW}$. Through a combination of thermocouples at the inlet and outlet of the generator, and turbine flow meters in the flow lines, it is possible to measure the heat lost to the torch and generator cooling systems. Subtracting this from the total power input, the amount of power delivered to the plasma is calculated.

The induction coil is a five turn copper coil with an average diameter of $86 \mathrm{~mm}$ housed between a quartz tube and a Teflon body. The inner diameter of the $3 \mathrm{~mm}$ thick quartz tube is $76 \mathrm{~mm}$. Quartz is used due to its high thermal resistance and transparency to electromagnetic waves. Teflon is used for the outer body as it absorbs electromagnetic radiation produced and acts as an electrical insulator. The coil itself is cooled with deionised water and this technique is preferred over the simpler air cooling method due to the high heat fluxes produced. Deionised water is used to prevent arcing between the coils.

At the base of the quartz tube are gas injectors capable of injecting gas axially, radially and with a swirl component. Axial flow provides large flow velocities that limit the heat 
addition to the gas and is only used during start up with argon. Radial injectors provide flow that travels through the quartz tube in an axisymmetrical manner. Swirl injectors provide a gas layer close to the quartz tube to prevent overheating and reduce heat loss to the cooling water. In addition, swirl injectors create low pressure regions that promote recirculation of the test gas through the induction regions and create an evenly heated and stable plasma. Through the variation of radial and swirl injector flow rates it is possible to establish various radial temperature profiles. High swirl profiles produce a more uniform temperature through the plasma whereas low swirl flows produce a more pronounced temperature peak in the centre. The main drawback of the even temperature profile, high swirl condition, is the reduction in maximum temperature attainable.

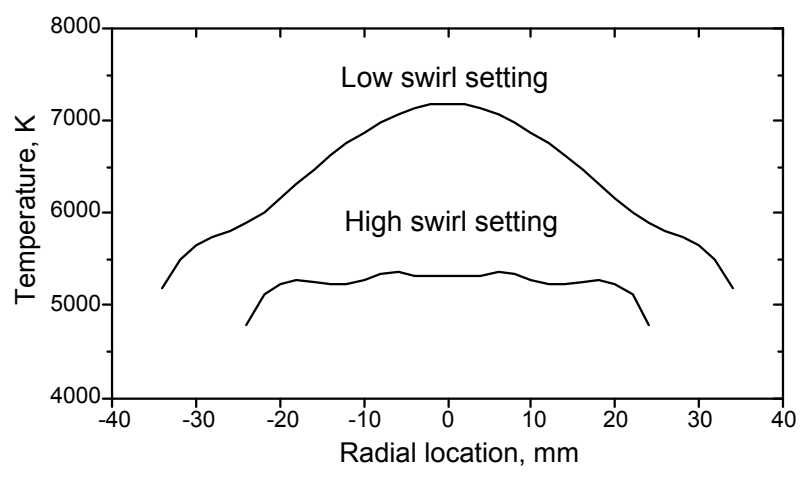

Figure 3.6: Representative temperature profiles for a high swirl and low swirl condition. Adapted from Laux [105].

At the exit of the quartz heating tube, a converging nozzle is attached. Nozzles varying from $70 \mathrm{~mm}$ in diameter to $10 \mathrm{~mm}$ in diameter are currently available for this system. The nozzle is selected based on the velocity, density and size of the flow required. The velocities that can be produced range from $10 \mathrm{~m} / \mathrm{s}$ with the $70 \mathrm{~mm}$ nozzle to $1 \mathrm{~km} / \mathrm{s}$ with the $10 \mathrm{~mm}$ nozzle. The nozzle diameter chosen for this study was $50 \mathrm{~mm}$ as it produced the largest, even depth of radiating plasma.

\subsubsection{Ground Testing Radiation Data}

In the regions of a re-entry trajectory where radiative heat flux is a significant contributor, five main types of facilities are used to recreate the radiating shock layer; arc jet tunnels, shock tunnels, expansion tubes, ballistic ranges and plasma torches. A review of facilities around the world found that more than 15 are capable of providing emission spectroscopy measurements in flows at the required enthalpies [106, 107, 108, 109, 110, 111, 112, 113, 
114, 115, 116, 117, 118, 119, 120, 121. All of these facilities are listed in Table 3.2 and are capable of emission spectroscopy measurements from the ultraviolet (UV) to the near infra-red (NIR). Combined, these facilities have produced a large body of spectral data that is available for testing of computational models.

Table 3.2: Emission spectroscopy capabilities of various hypersonic facilities around.

\begin{tabular}{llllll}
\hline Wavelength & VUV & UV & VIS & NIR & IR \\
Range (nm) & $<200$ & $200-390$ & $390-700$ & $700-1100$ & $>1100$ \\
\hline Shock Tunnels and Expansion Tubes & & & \\
\hline EAST [107, 108] & $\mathrm{x}$ & $\mathrm{x}$ & $\mathrm{x}$ & $\mathrm{x}$ & $\mathrm{x}$ \\
HIEST [109] & $\mathrm{x}$ & $\mathrm{x}$ & $\mathrm{x}$ & $\mathrm{x}$ & \\
X2 [110] & $\mathrm{x}$ & $\mathrm{x}$ & $\mathrm{x}$ & $\mathrm{x}$ & \\
LENS XX [11] & & $\mathrm{x}$ & $\mathrm{x}$ & $\mathrm{x}$ & \\
X3 [110] & & $\mathrm{x}$ & $\mathrm{x}$ & $\mathrm{x}$ & \\
\hline
\end{tabular}

Plasma Wind Tunnels and ARC Jets

\begin{tabular}{|c|c|c|c|c|c|}
\hline NASA ARC 112 & $\mathrm{x}$ & $\mathrm{x}$ & $\mathrm{x}$ & $\mathrm{x}$ & \\
\hline Scirocco [113, 114] & & $\mathrm{x}$ & $\mathrm{x}$ & $\mathrm{x}$ & \\
\hline Plasmatron [115, 114] & & $\mathrm{x}$ & $\mathrm{x}$ & $\mathrm{x}$ & \\
\hline DLR L3K [116, 114] & & $\mathrm{x}$ & $\mathrm{x}$ & $\mathrm{x}$ & \\
\hline IRS PWK [117] & & $\mathrm{x}$ & $\mathrm{x}$ & $\mathrm{x}$ & \\
\hline Onera F4 [118, 114] & & $\mathrm{x}$ & $\mathrm{x}$ & $\mathrm{x}$ & \\
\hline \multicolumn{6}{|l|}{ Plasma Torches } \\
\hline EM2C ICP & $\mathrm{x}$ & $\mathrm{x}$ & $\mathrm{x}$ & $\mathrm{x}$ & $\mathrm{x}$ \\
\hline PHEDRA [119, 120, 114] & & $\mathrm{x}$ & $\mathrm{x}$ & $\mathrm{x}$ & \\
\hline \multicolumn{6}{|l|}{ Ballistic Ranges } \\
\hline HFFGAF [121, 114] & & $\mathrm{x}$ & $\mathrm{x}$ & $\mathrm{x}$ & \\
\hline
\end{tabular}

Outside of the facilities used in this study, only three were found to have published emission spectroscopy measurements in the VUV; the Arc-Jet at NASA, the Electric Arc Shock Tunnel (EAST) at NASA and the High Enthalpy Shock Tube (HIEST) at Japanese Aerospace Exploration Agency (JAXA). As recently as 2009, due to the lack of calibrated data published it was stated that "no good experimental data is available for radiation in the VUV' [108].

The first VUV experimental measurements relevant to re-entry studies were published by 
Palumbo in 1997 [26]. This experiment was conducted in an arc jet facility at a velocity of $7.8 \mathrm{~km} / \mathrm{s}$ and a pressure of $51 \mathrm{kPa}$. At this velocity, the VUV radiative heat flux is estimated to be less than $10 \%$ of the total radiative heat flux [26]. Palumbo attempted to measure the radiative heat flux through a helium cooled window on the surface of a blunt model, as shown in Figure 3.7. To avoid absorption by atmospheric air, a high vacuum was achieved in the optical diagnostics section behind the window, allowing the VUV radiation to travel unattenuated to the spectroscopy system. The hot dissociated gas of the arc jet meant that free stream radiation was also recorded as incident VUV heat flux on the surface of the model and due to a lack of calibration tools, the recorded data was stated to be accurate to within a factor of two. Even with the lack of accurate quantitative data, this system showed it was possible to measure VUV radiation through the surface of a model in hypersonic facilities.

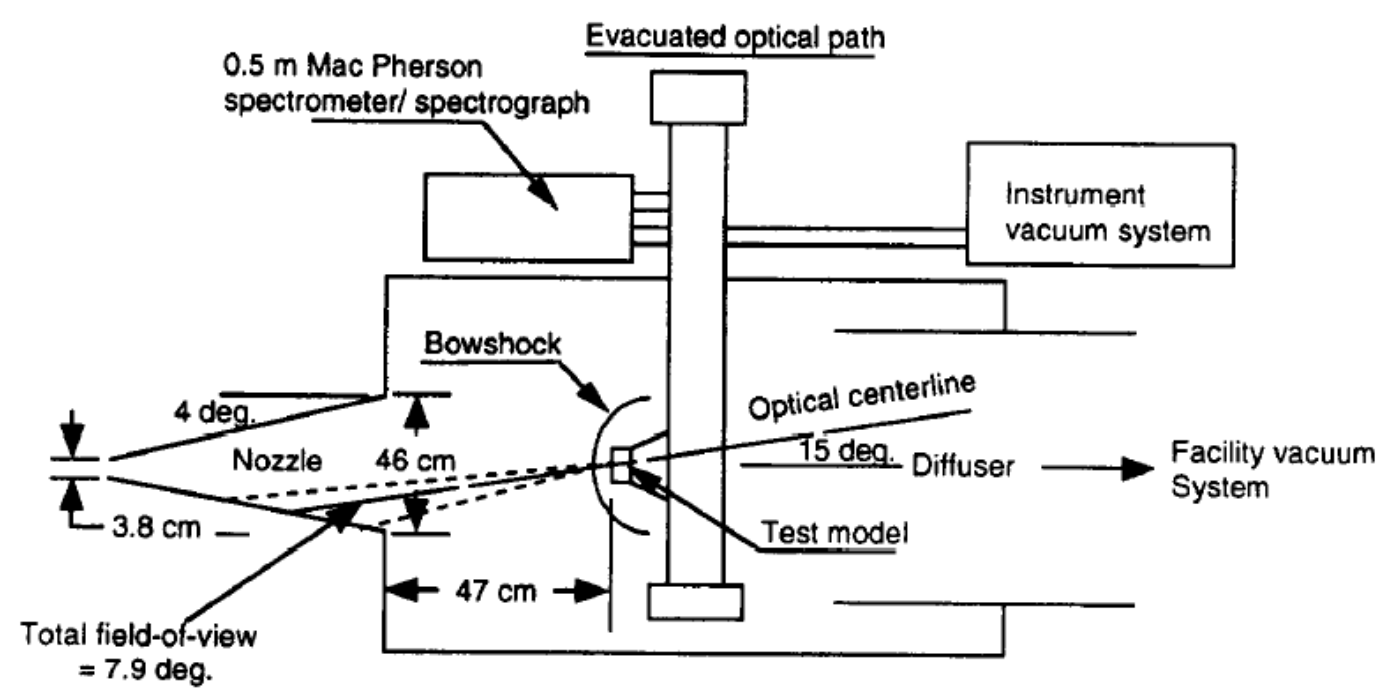

Figure 3.7: Schematic arrangement of the Palumbo arc jet experiment [26]

In 2009, publications describing the VUV spectroscopy systems installed on the HIEST shock tube at Chofu Aerospace Centre at JAXA and the EAST facility at NASA were published. Both systems are capable of measuring radiation down to $120 \mathrm{~nm}$ [108, 122]. The JAXA facility has published data covering conditions from $7.8 \mathrm{~km} / \mathrm{s}$ to $10.4 \mathrm{~km} / \mathrm{s}$ at a range of pressures [122, 123] and the EAST facility datasets cover a range of velocities from $9.5 \mathrm{~km} / \mathrm{s}$ to $15.5 \mathrm{~km} / \mathrm{s}$ at various pressures [124, 125]. Both of these facilities were operated in shock tube mode observing the shock wave traverse down the length of the tube and no models were used. 


\subsection{Radiation Modelling Programs}

To analyse experimental data and predict heat fluxes, computational radiation modelling programs have been created. The most commonly used radiation models in literature are the non-equilibrium air radiation code NEQAIR [87, hypersonic air radiation algorithm HARA [126, 42] and Specair [25]. NEQAIR computes atomic emission spectra based on the atomic spectral database of the National Institute of Standards and Technology (NIST) [127]. Molecular band structure is also computed the main rovibrational and rovibronic transitions of air components. Continuum radiation is calculated with the absorption cross-sections of Peach [128].

The HARA radiation model also uses atomic transitions obtained from NIST along with the Opacity Project database [129]. For continuum radiation, HARA uses a curve fit of Opacity Projects TOPbase instead of the Peach cross-sections as used by NEQAIR [126, 130. The molecular band structures are computed using the smeared-rotational band model [131]. Whilst these models are commonly seen in the literature, they will not be used in any computations in this study due to their unavailability as they are proprietary models of NASA.

The third computational model commonly used, Specair, will be the computational model used in this study. Specair is a code that was built on a previous version of the NEQAIR code. Specair uses the atomic line transitions available from NIST along with a series of external databases such as the Harvard-Smithsonian Center for Astrophysics [132. Unlike NEQAIR, Specair does not have a collisional-radiative model for excited electronic states. Instead, the populations of energy levels are calculated using an external solver or assumed to follow Boltzmann distributions at a specified rotational, vibrational and electronic temperatures. 


\section{Chapter 4}

\section{Expansion Tube Experiments}

The following chapter describes the process undertaken to reach the final model design and the variations used. The emission spectroscopy system built for this study is detailed and the reasoning behind the selections of various components utilised is outlined. The optical configurations selected to image the shock layers and the techniques applied to overcome the complexities of spectral measurements in the VUV are explained. An outline of the calibration process carried out along with validation of the calibration images acquired is provided. Following the discussions of the emission spectroscopy system, the operating conditions designed and experimentally validated are defined as well as the design of a shot validity analysis tool. An analysis of the uncertainties involved in the experimental measurements is conducted before the results of a repeatability study are presented. Finally, the datasets measured during this experimental campaign are presented and analysed.

\subsection{Model Design}

\subsubsection{Measuring VUV Radiation Through the Surface}

There has been one previous attempt at UQ to measure spectrally resolved radiative heat flux on the surface of a model by Kurtz in 1997 [133. The technique used by Kurtz was to mount an optical fibre onto the surface of a model. Kurtz found that it was possible to obtain qualitative data using optical fibres but there were numerous problems associated with exposing optical fibres to the shock layer. The high temperature behind the shock wave caused cladding to vaporise leading to a range of problems including ablation and changes in the angle of refraction resulting in unquantifiable capture angles. 
These problems resulted in an inability to quantify the data. These factors, coupled with the lack of readily available optical fibres capable of transmitting into the deep VUV, meant a new approach was required.

To avoid the use of optical fibres, a concept similar to that of the Palumbo experiment discussed in Section 3.4.3 was designed [26]. The Palumbo experiments were conducted in a long duration arc-jet tunnel and required the model to be actively cooled. Test times in the X2 expansion tube are in the order of hundreds of microseconds and thus models do not require active cooling. This enabled a simpler design of the model and removed all cooling gases that would interfere with the shock layer. The prototype design shown in Figure 4.1, was trialled with a turning mirror housed behind a cylindrical tube with a blunt perspex window as the model. A high speed camera was set up to observe the shock wave through the window as it moved along the expansion tube before impacting the model. This initial design proved to be highly successful as it was able to track the shock wave, as well as observe the shock wave rupture the secondary diaphragm and impact on the model.

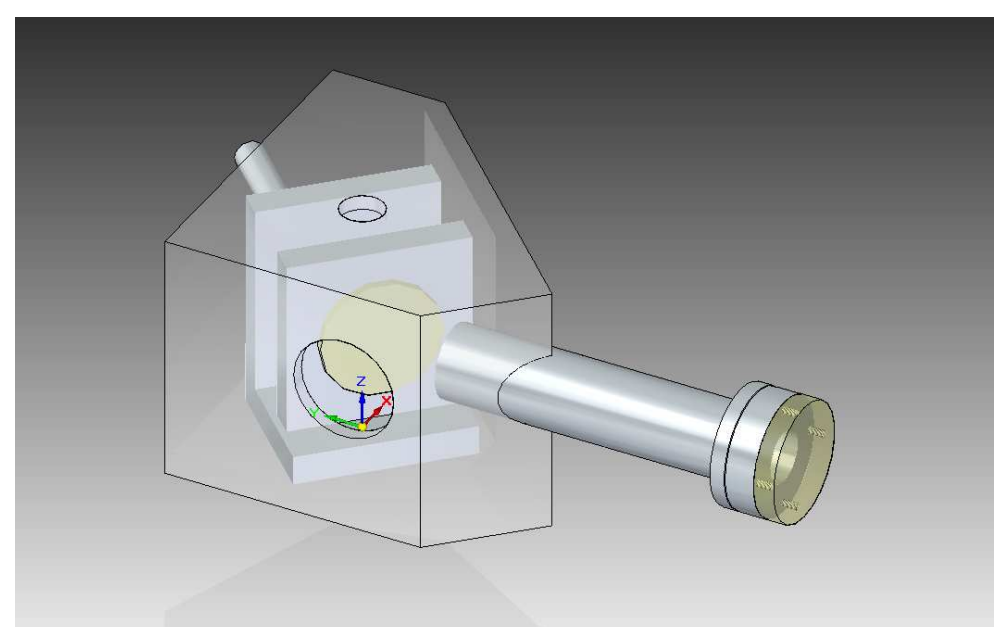

Figure 4.1: Proof of concept model with perspex front end and open mirror housing.

Following the successful proof of concept testing, the front end of the prototype was modified to house optical windows that would be used in the final VUV experiments. The optical windows selected for the final experiments were $2.0 \mathrm{~mm}$ thick magnesium fluoride windows to meet transmission requirements in the VUV. Based on the thermal expansion coefficients and material strength properties, it was calculated that the windows should be able to withstand the high temperatures during the test time. It was expected that the unsteady flow and driver gas arrival after the test time would heat the windows enough to damage the surface quality and influence the transmission properties. As the 
test time is at the beginning of the experiment, this degradation in surface finish did not effect measurements taken during the steady test flow as a new window was used for each experiment.

Test shots were conducted at experimental conditions and it was found that all windows maintained structural integrity during the test time. However, during the unexpanded gas and driver gas regions of the flow, the windows suffered significant structural damage. In some cases fragments of the secondary diaphragm impacted the window and were able to penetrate through it, damaging optical components. Figure 4.2 illustrates the result of Mylar fragments impacting the window after the experiment test time.
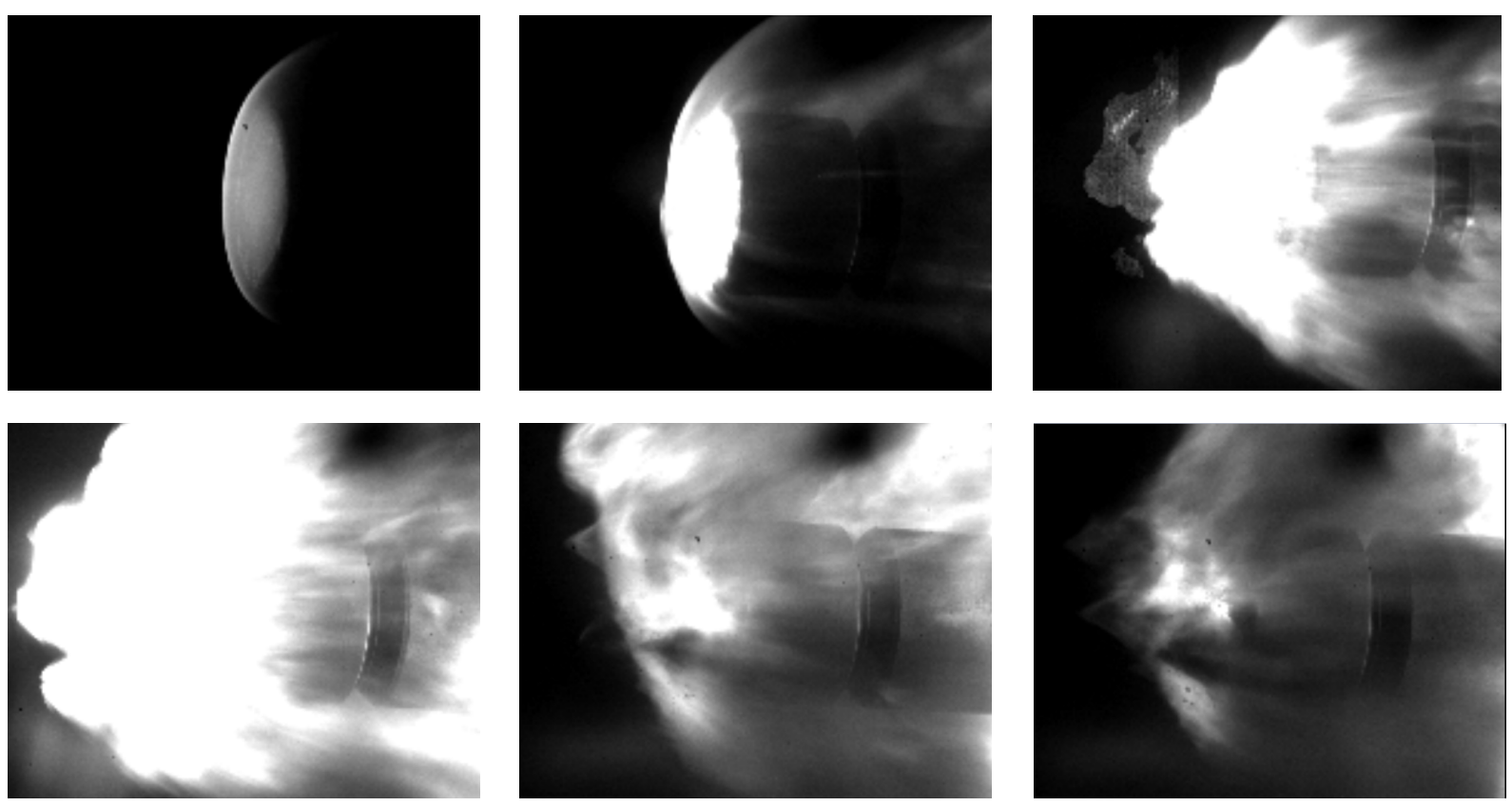

Figure 4.2: Mylar diaphragm fragments impacting on windows. The window during the steady test time can be seen in the top left image. Top right image shows the arrival of a large piece of Mylar diaphragm and its impact on the window.

To reduce the damage from diaphragm fragments, the Mylar material was substituted with thin aluminium sheet for the secondary diaphragm. Unlike the plastic behaviour of Mylar, the aluminium sheet disintegrates upon contact with the shock wave and does not remain in large pieces as it is accelerated towards the model. This was evident through visual inspection of the model after a shot and the high speed video observing the shock wave. In the final design of the model and mirror housing, an additional window was placed behind the primary window. In case of failure or any small fragments being released from the rear of the optical window, the secondary window would prevent any damage occurring to the optical mirror. 
The high vacuum housing for the mirror was hollowed out from a single round piece of stainless steel to reduce potential leak paths. The walls of the housing were designed to be $30.0 \mathrm{~mm}$ thick to allow for an o-ring to be machined into the top and to withstand numerous experiments in the harsh flows of the expansion tube. At the front end of the mirror housing an evacuated light tube was installed to serve as a model mount. The length of the tube was selected to be $150 \mathrm{~mm}$ to ensure there would be no interference from shock waves reflecting from the mirror housing. A sliding seal was created perpendicular to the flow to allow for an evacuated light path to transport the signal out of the test section and into the external collection optics. Pitot and static pressure probes were installed on the top of the mirror housing for additional diagnostic capabilities. A schematic of the final model configuration is displayed in Figure 4.3.

\section{Edge of test section}

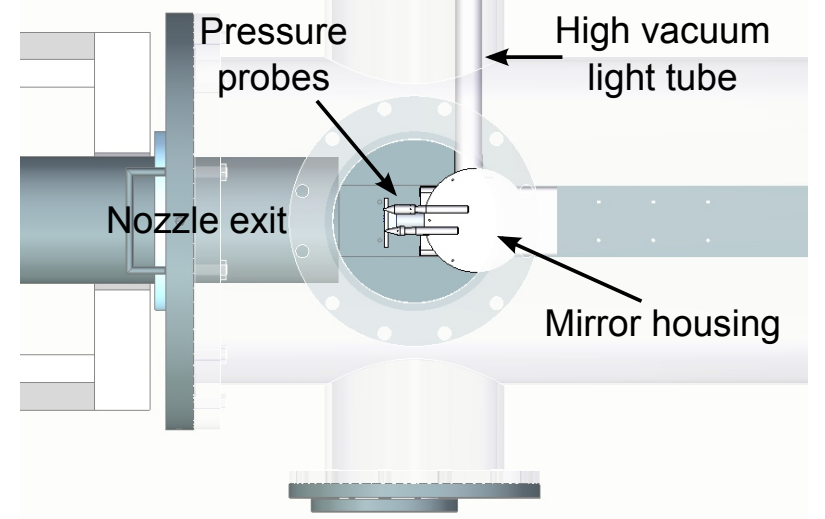

Figure 4.3: Top down view of high vacuum path to model with mirror housing assembly and pressure probes.

Due to the large size of the model, the forces placed on the support railing within the test section are significant. By estimating the mirror housing as a flat plate, the calculated force on the support structure was in the order of two tonnes. Additional braces, as shown in Figure 4.4, were required to ensure no drift in the location of the model between experiments occurred. 


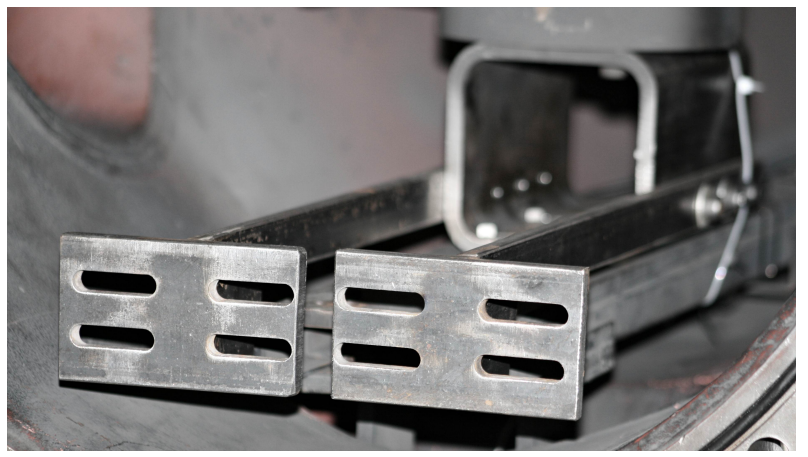

Figure 4.4: Additional bracing installed to ensure model position is maintained.

\subsubsection{Measuring VUV Radiation Across the Surface}

An optical path of $1.0 \mathrm{~m}$ at a pressure of 0.1 Torr, a typical operating pressure in the test section of X2, results in a loss of $85 \%$ of the radiation signal between $135 \mathrm{~nm}$ and $150 \mathrm{~nm}$, and, at certain absorption lines between $115 \mathrm{~nm}$ and $130 \mathrm{~nm}$, the entire signal would be absorbed. To eliminate this absorption by molecular oxygen in the free stream flow and the test section, an evacuated light tube was installed. At the front end of the light tube, a fence housing a window was mounted. The fence allows for the window to be placed close to the model without causing any interference to the shock layer being observed.

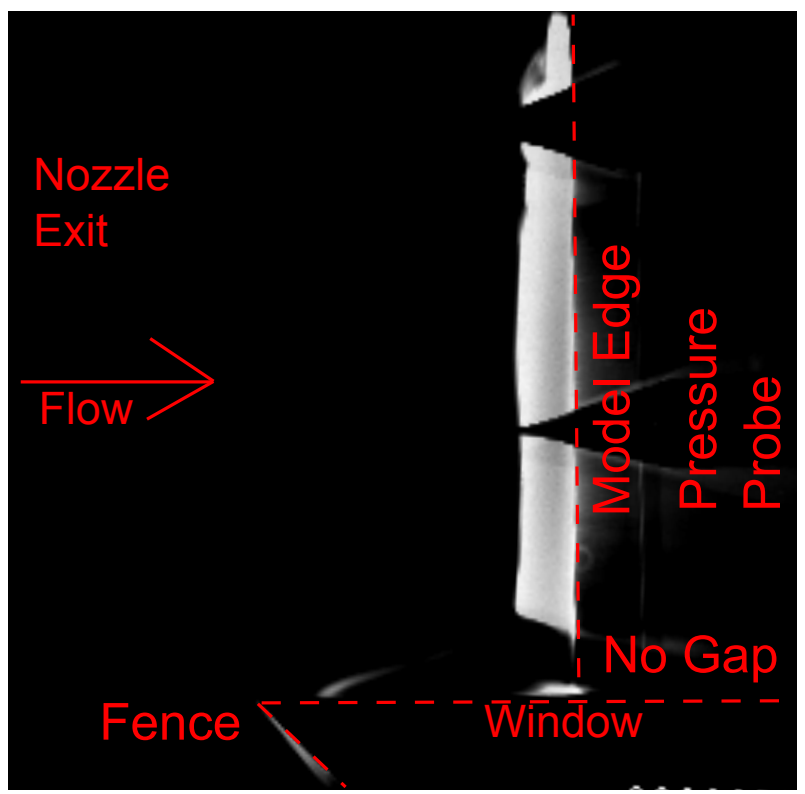

Figure 4.5: Top down view of initial fence and window position with no gap to the blunt model. 
This concept was trialled with the fence sitting flush against the side of the model, ensuring there was no possible molecular oxygen between the radiating shock layer and the evacuated light tube. A small disturbance from the fence was expected but it was assumed that this would only influence a small fraction of the total radiating shock layer. By repeating the experiment with a shorter model, the influence of the shock layer interaction could be negated and radiation from a steady region calculated. Testing revealed the shock wave produced by the fence was too unsteady and greatly perturbed the shock layer created by the model, as shown in Figure 4.6. There was also a stagnation region produced between the fence and the model, further increasing the detrimental effect of the fence.
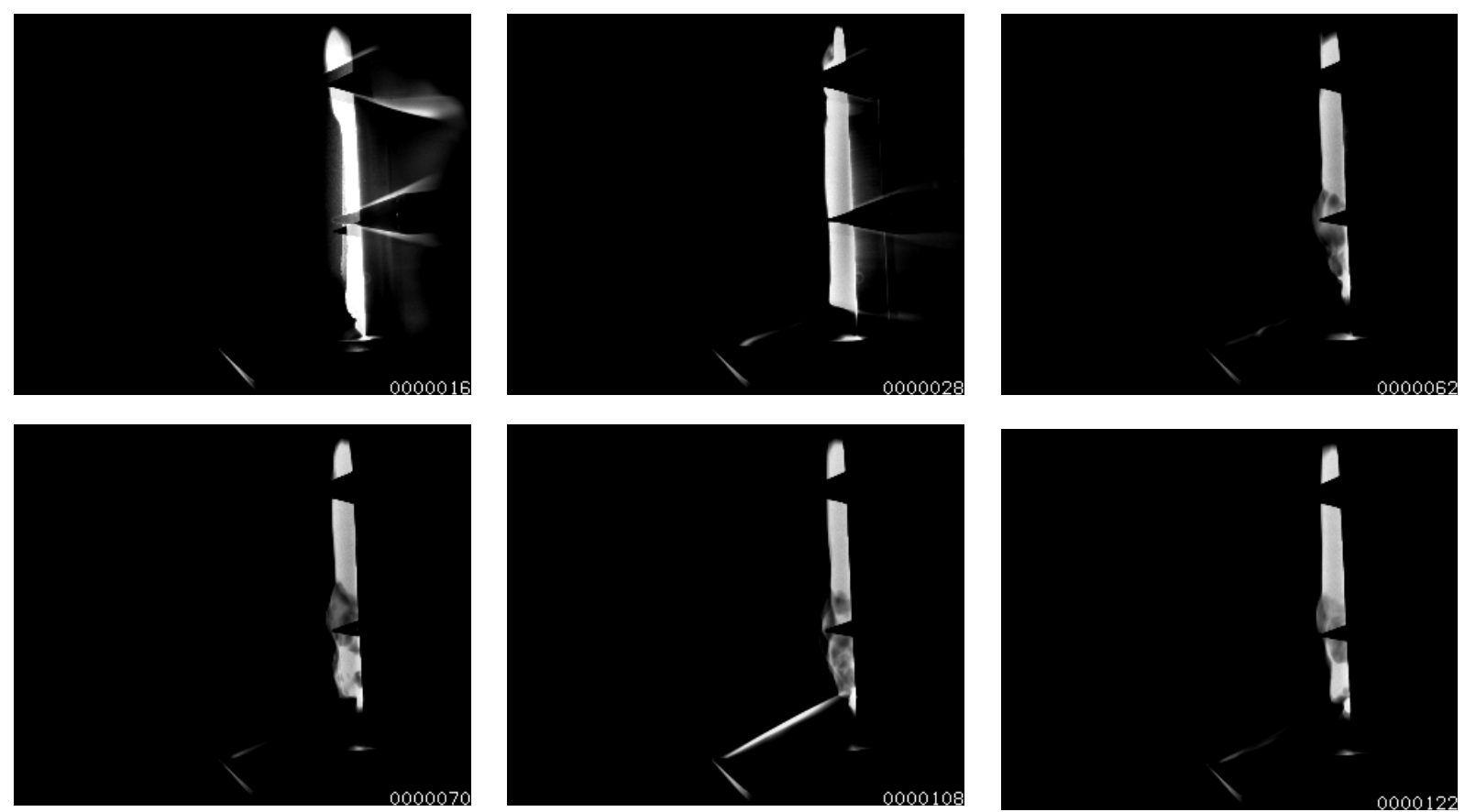

Figure 4.6: Perturbations created by the fence and stagnation region between the fence and the model.

To avoid shock wave interactions, different size gaps between the fence and the model were experimented with. A gap of $10 \mathrm{~mm}$, as shown in Figure 4.8, was selected as it caused no interference with the shock layer whilst maintaining a small enough core flow region where the absorption was calculated to be negligible. The absorption in such a small gap was found to be negligible due to the low pressure of the test gas coupled with the lower mass fraction of molecular oxygen within the core flow. The apparent gap between the window and the model is effectively reduced further by the shock wave from the fence that dissociates the molecular oxygen in the flow, also ensuring a cold boundary layer containing molecular oxygen does not form on the viewing window. 


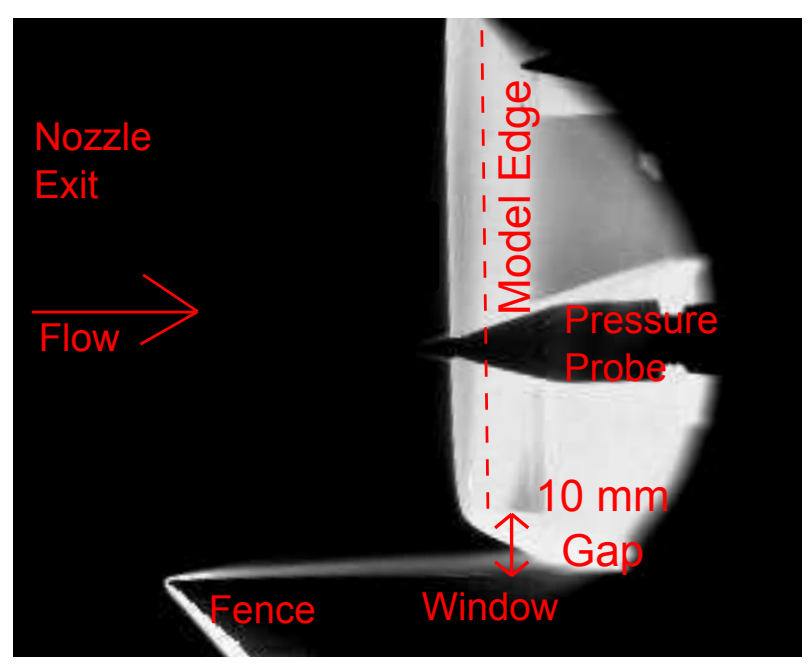

Figure 4.7: Top down view of second fence and window position with a $10 \mathrm{~mm}$ gap to the blunt model.
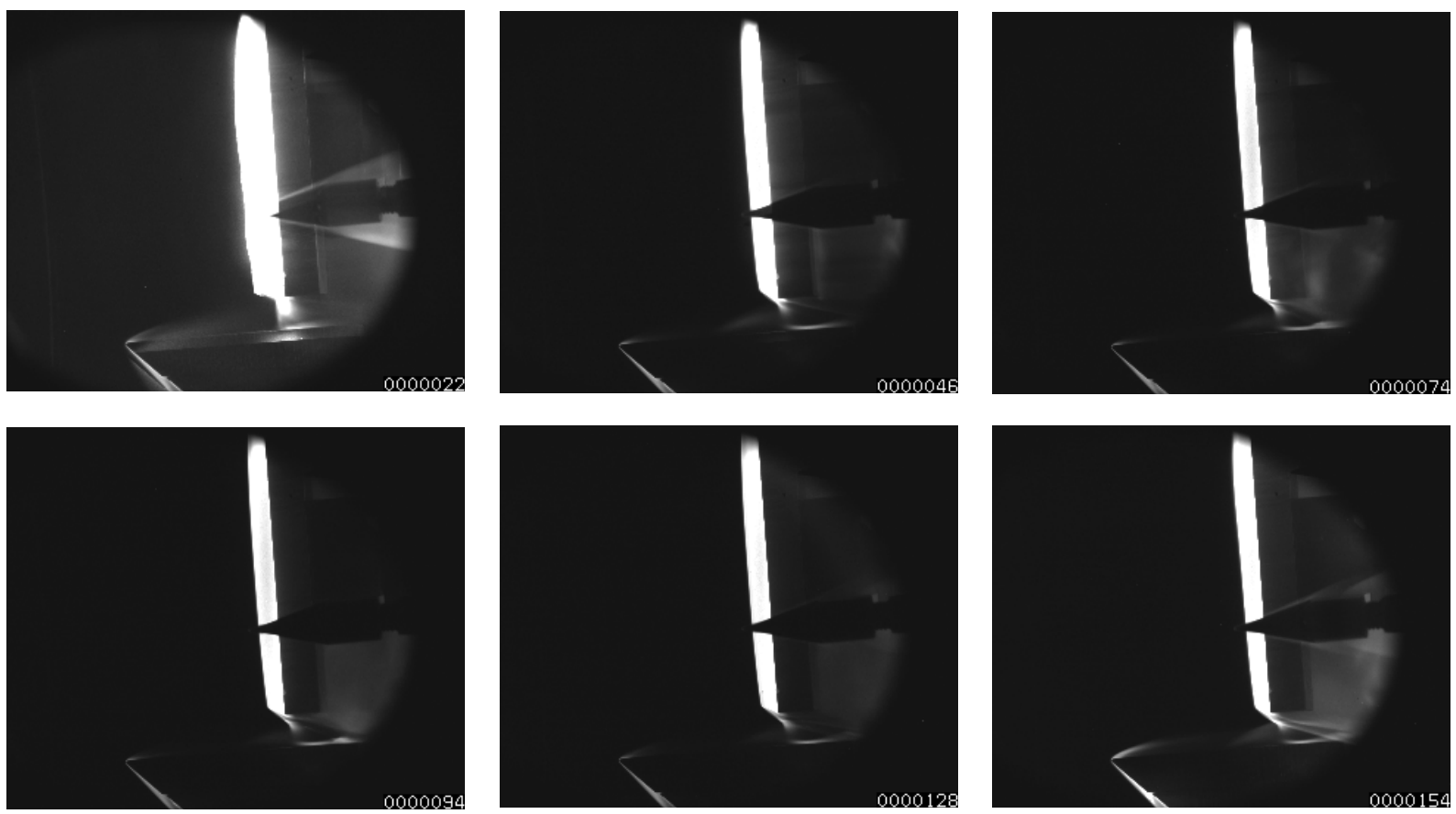

Figure 4.8: Steady flow for over $130 \mu$ s achieved with a $10 \mathrm{~mm}$ gap. Counter in bottom right of each image is video time in $\mu \mathrm{s}$ 


\subsubsection{Model Shape}

To place a window in the surface of a model without disturbing the flow field around the model, a flat area was required. A simple two dimensional shape was desired and thus a flat bar, $25 \mathrm{~mm}$ tall and $90 \mathrm{~mm}$ wide was selected. Such a blunt shape is able to provide a large shock stand off allowing for a larger shock layer to be studied and driving flows closer to equilibrium.

A two-dimensional shape was selected for ease of computational modelling. No literature was found describing the influence of the aspect ratio on three-dimensional shock stand off distance for a flat faced body. A first order approximation was carried out using a simplified three-dimensional shock layer region and the conservation of mass principle. The approximately planar nature of the shock wave over the model allowed for the shock region to be modelled as a rectangular shock layer, as shown in Figure 4.9.

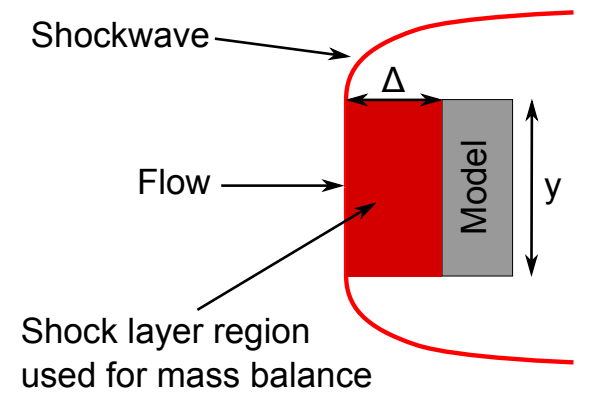

$\Delta=$ Shock stand off

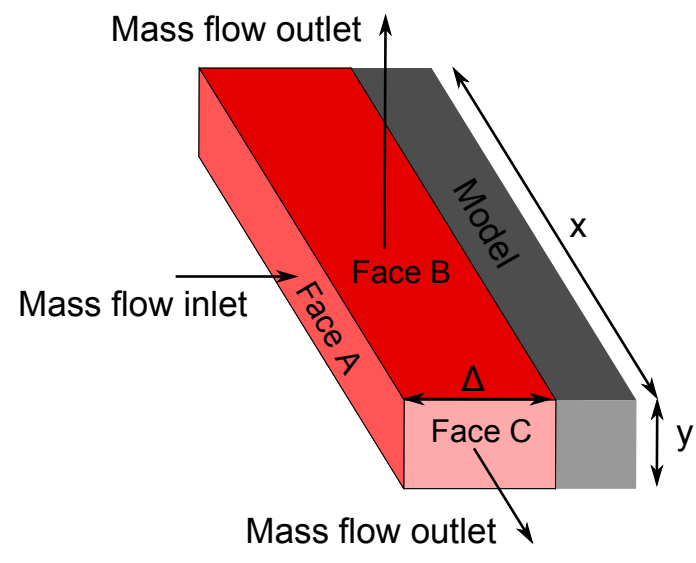

Faces $D$ and $E$ mirror faces $B$ and $C$

Figure 4.9: Left - Side on view of idealised two-dimensional shock stand off. Right - Three dimensional view of idealised region used for mass flow balance.

The shock stand off, $\Delta$, can be estimated by equating the mass flow into the shock layer through face A, and out of the shock layer through faces B to E, noting that the model acts as a boundary to the flow.

$$
\dot{m}_{I N}=\rho U A_{\text {Face } A}=\dot{m}_{O U T}=\rho^{\prime} U^{\prime} A_{\text {Faces B to E }}
$$

Where $\rho^{\prime}$ and $U^{\prime}$ are the post shock density and velocity. Substituting $A_{\text {Face } A}=x y$ and 
$A_{\text {Faces B to } E}=2(x \Delta+y \Delta)$, the shock stand off relationship equates to

$$
\Delta_{3 D}=\frac{\rho U x y}{\rho^{\prime} U^{\prime} 2(x+y)}
$$

In the $2 \mathrm{D}$ case, $\mathrm{x}>>\mathrm{y}$ and therefore $\mathrm{x}+\mathrm{y}$ can be approximated to $\mathrm{x}$, giving

$$
\Delta_{2 D}=\frac{\rho U x y}{\rho^{\prime} U^{\prime} 2 x}
$$

Taking the ratio of the two shock stand off estimates gives

$$
\begin{aligned}
\frac{\Delta_{3 D}}{\Delta_{2 D}} & =\frac{\rho U x y}{\rho^{\prime} U^{\prime} 2(x+y)} \frac{\rho^{\prime} U^{\prime} 2 x}{\rho U x y} \\
\frac{\Delta_{3 D}}{\Delta_{2 D}} & =\frac{x}{x+y}
\end{aligned}
$$

By substituting the aspect ratio, AR, the relationship can be further simplified

$$
\text { substituting } \begin{aligned}
A R & =\frac{x}{y} \\
\frac{\Delta_{3 D}}{\Delta_{2 D}} & =\frac{A R}{A R+1}
\end{aligned}
$$

A plot of this relationship is shown in Figure 4.10 for aspect ratios between 0.1 and 20 . The aspect ratio produced by the model used in this study is 3.6 and was predicted to create a shock stand off of $76 \%$ of the idealised two-dimensional value. Higher aspect ratios were not possible due to window size requirements and free stream size limits.

A drawback to such a blunt shape was the lack of velocity gradients within the shock layer causing an increase in steady flow establishment time around the model and increased sensitivity to flow perturbations. Preliminary experiments conducted with the high speed camera indicated an unsteady shock layer due to small fragments being entrained in the test gas, impacting the model and vaporising, as shown in Figure 4.11. A new series of pre-experiment procedures, such as compressed gas cleaning of the tubes and chemical cleaning of the aluminium diaphragm, were established to further remove any particles from the expansion tube. It was concluded that there was a reduction in the number of particles in the flow, but not all particles could be removed from the expansion tube and experiments in which particles entered the shock layer would have to be repeated. 


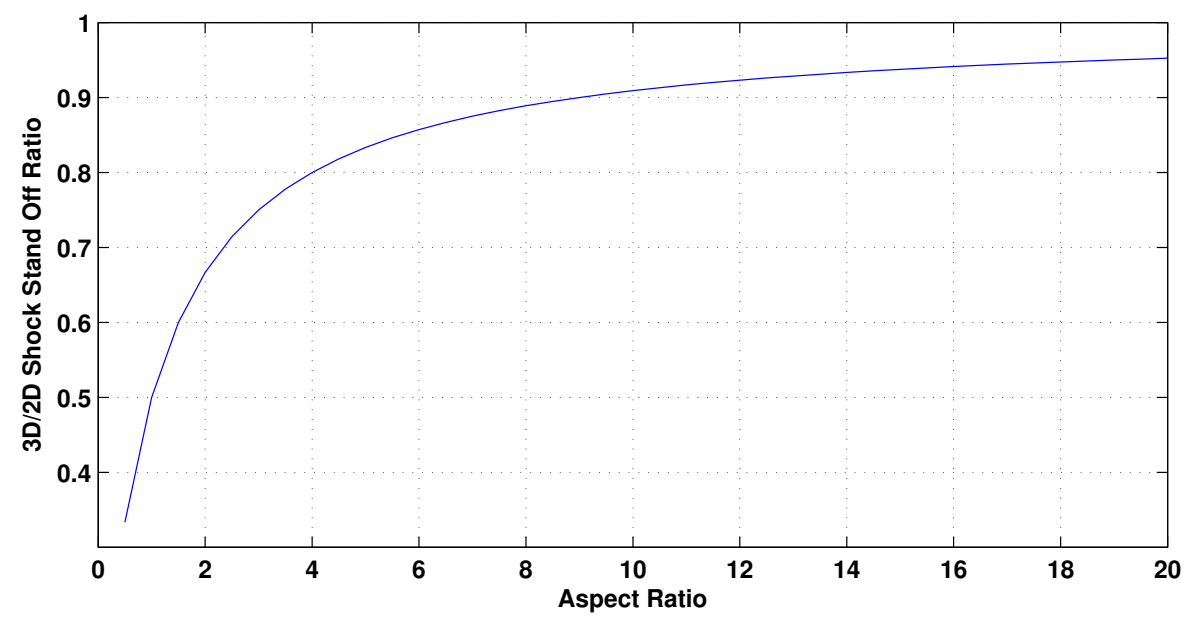

Figure 4.10: Shock stand off correction for varying aspect ratio square bodies in supersonic and hypersonic flow. An aspect ratio of 3.6 produces a 0.76 correction factor between twodimensional and three-dimensional shock stand offs.
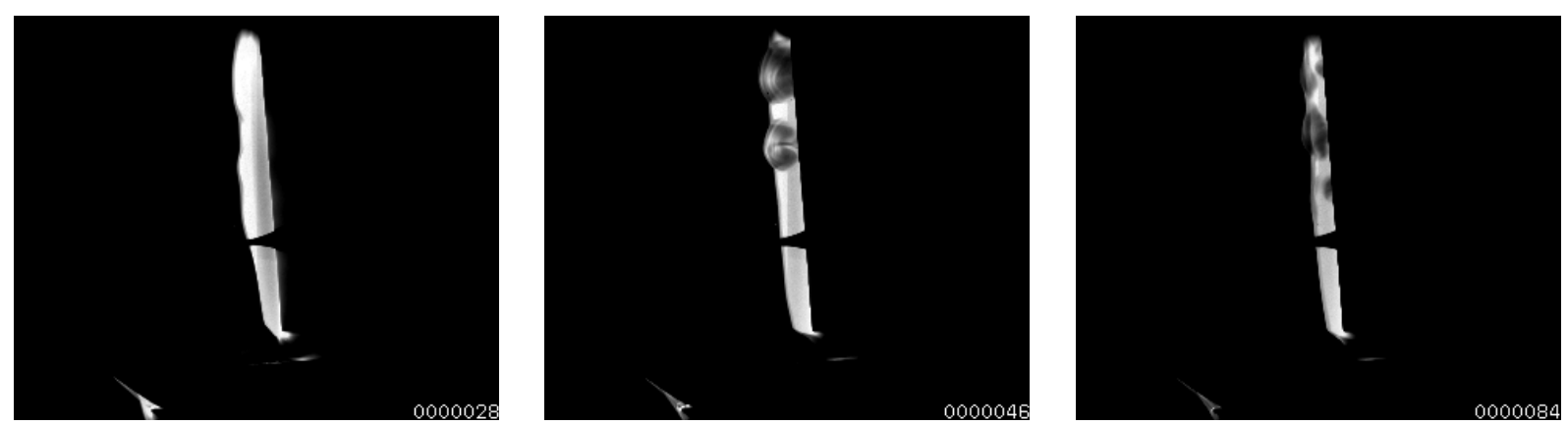

Figure 4.11: Top down view of vaporised aluminium impacting the surface. Flow from left to right. 


\subsubsection{Self-Absorption Experiments}

Varying depths of radiating flow fields are required to study the effects of self-absorption on the measured emission spectrum. To create the required variations in depth of radiating flow fields, two-dimensional cylindrical models with lengths of $20 \mathrm{~mm}, 45 \mathrm{~mm}$ and $90 \mathrm{~mm}$ were used. The shock layer formed around each two-dimensional model is comprised of a uniformly radiating mid-section and three dimensional non-uniform flow at either edge. This approach results in each model having nominally the same three dimensional edge effects but a varying length of steady two-dimensional radiating gas in the mid-section, as shown in Figure 4.12. By changing the length of this mid-section, it was possible to study the variation in observed radiation as a function of depth of the radiating flow field.

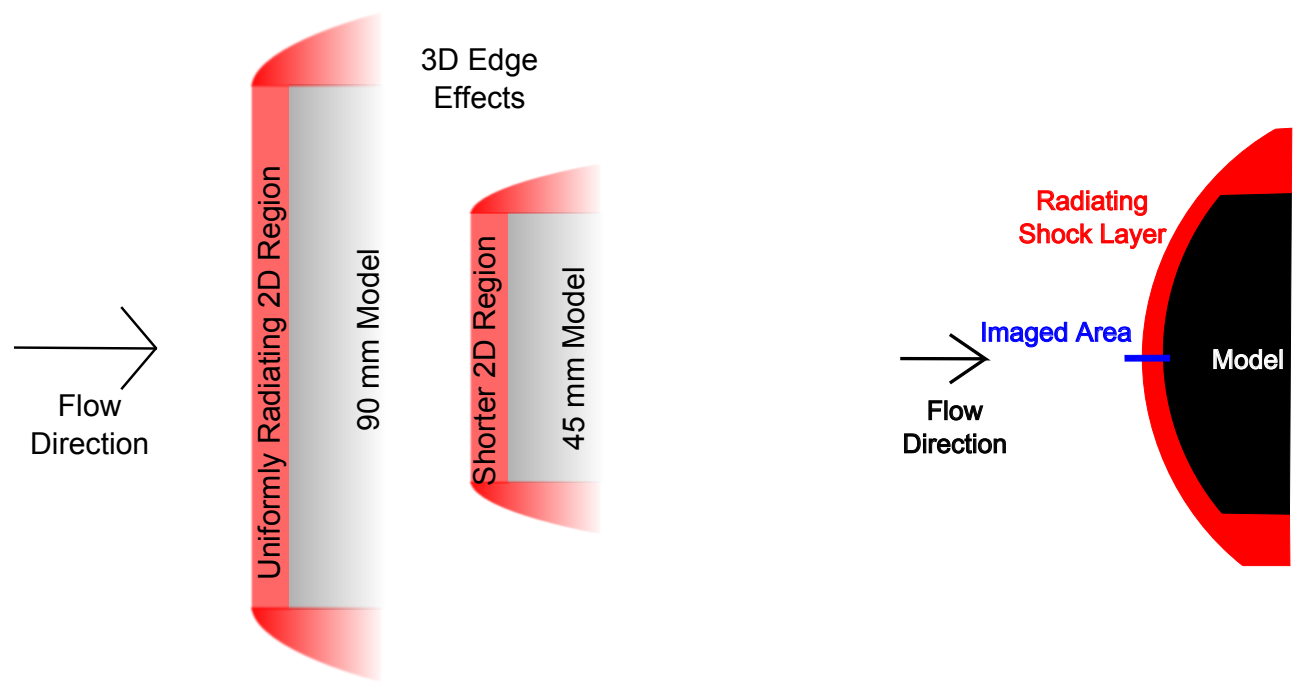

Figure 4.12: Self-absorption experiments concept, top down view (left). Area imaged spatially onto spectrometer when looking across the cylinder (right).

A cylindrical model with a radius of curvature of $19 \mathrm{~mm}$ was selected for these experiments as a flat bar was no longer required to house a viewing window. The curvature of a cylinder was preferred as it greatly reduced the model's sensitivity to perturbations in the free stream flow, consequently reducing the number of unusable experimental measurements. The aspect ratio required to match two-dimensional computational analysis was also reduced based on the experimental study carried out by Eichmann [27]. Eichmann showed that an aspect ratio of 2.5 with a radius of curvature of $7.5 \mathrm{~mm}$ produced a negligible shock stand off correction factor. A direct three-dimensional shock stand off correction factor cannot be extrapolated from these results for the $19 \mathrm{~mm}$ radius of curvature used in these experiments, but it can be ascertained that a curved cylindrical model greatly reduces the three-dimensional shock stand off correction factor. 


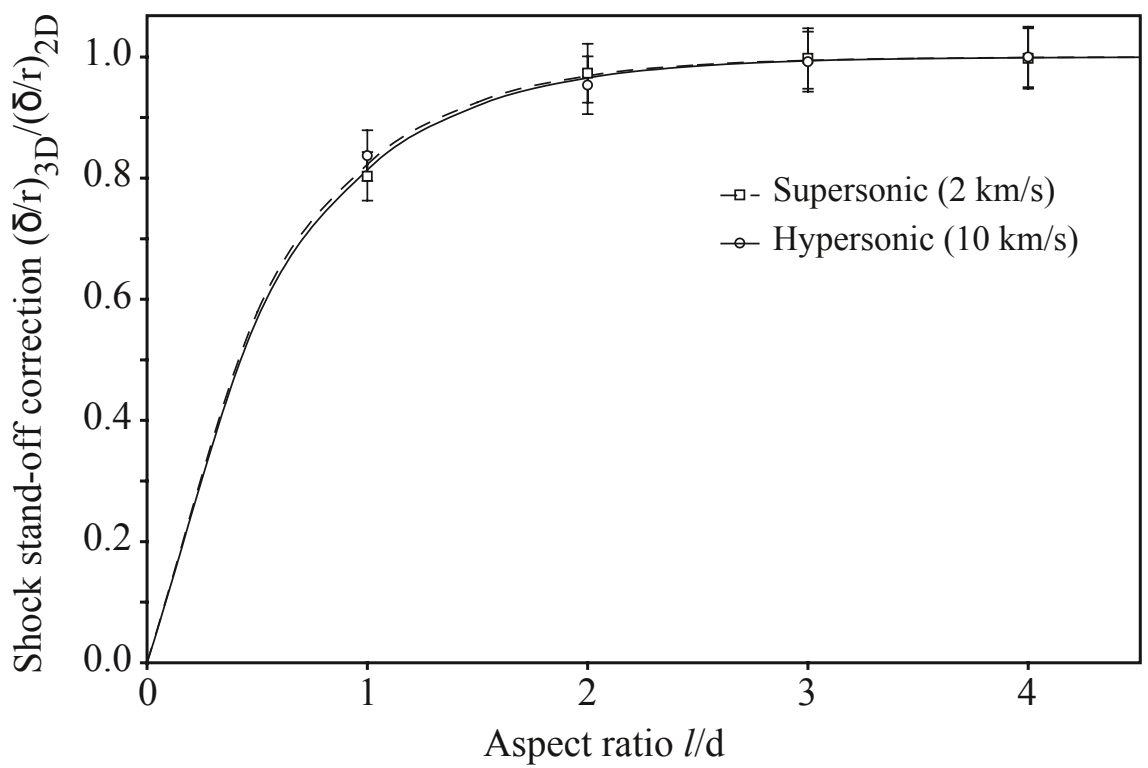

Figure 4.13: Shock stand off correction for varying aspect ratio cylinders in supersonic and hypersonic flow [27].

\subsection{VUV Emission Spectroscopy System}

To achieve high quality spectral data, the spectroscopy system must be matched to the experiment and the optical configuration used to focus the incoming signal. Experiments on the X2 expansion tube are in the order of 10s of microseconds in duration and the depth of radiating gas being observed can be up to $100 \mathrm{~mm}$. Test times of this duration require highly sensitive systems to achieve a good signal to noise ratio and the relatively large optical depth results in small apertures, increasing the F-number of the system. To cover the VUV radiative transitions of interest in one image, a spectral range of $60-80 \mathrm{~nm}$ was required.

Based on the requirements of the experiment, there were three monochromator designs considered in the selection of the VUV spectroscopy system: Czerny-Turner, turning concave grating and normal incidence concave grating, also known as the Seya-Namioka design. A Czerny-Turner uses a focussing mirror to collimate the signal entering at the slit and direct it towards the diffraction grating. The diffraction grating diffracts the signal and directs it towards a focussing mirror, which focusses the signal onto the recording device. A depiction of the Czerny-Turner configuration is presented in Figure 4.14.

The turning concave grating and normal incidence concave grating designs use a single optical component within the monochromator, a concave grating, to diffract and focus the light. The turning concave grating design turns the incoming signal by 90 degrees to the 


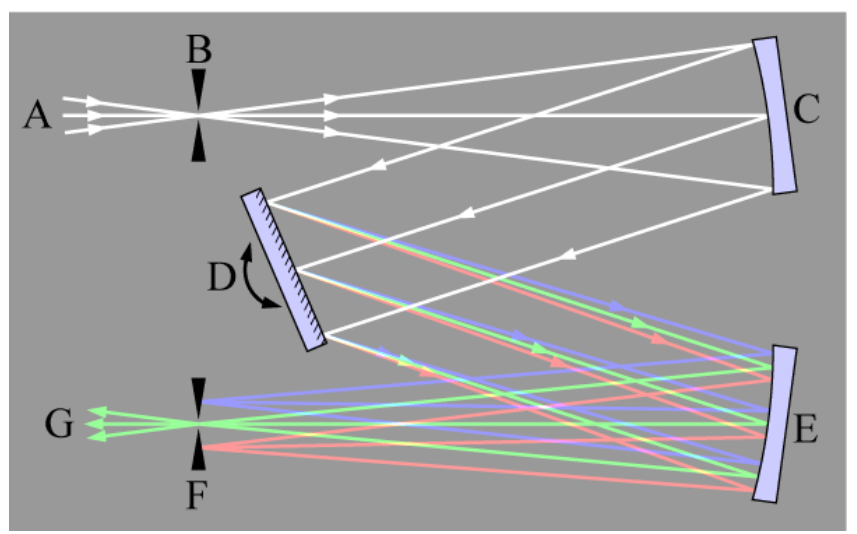

Figure 4.14: Depiction of a Czerny-Turner configuration. Light (A) is focused onto an entrance slit (B) and is collimated by a curved mirror $(\mathrm{C})$. The collimated beam is diffracted from a rotatable grating (D) and the dispersed beam re-focussed by a second mirror (E) at the exit slit $(\mathrm{F})$. Each wavelength of light is focussed to a different position at the slit, and the wavelength which is transmitted through the slit $(\mathrm{G})$ depends on the rotation angle of the grating. [134]

exit slit and is generally used in smaller monochromators. These monochromators have the advantage of a smaller volume that is round and therefore easily manufactured for high vacuum. Normal incidence concave grating or Seya-Namioka designs are reserved for longer focal length monochromators and result in a more rectangular shape. Whilst this design results in a larger volume that is harder to evacuate, there are substantial gains in image quality due to the smaller turning angle of the focussing optics and the cam mechanism that enables the grating position to be in focus at a large range of turning angles. Idealised representations of concave grating designs are presented in Figure 4.46 .

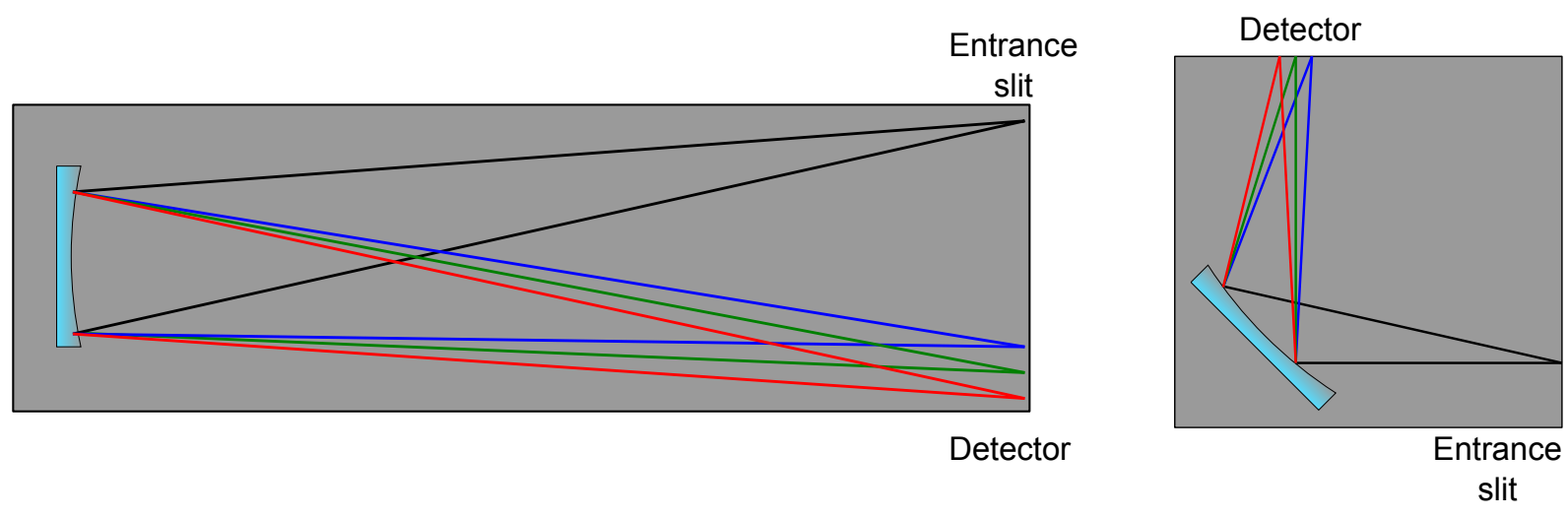

Figure 4.15: Left - Normal incidence concave grating Seya-Namioka monochromator. Right - Turning concave grating design.

The only recording device considered in the selection of the spectroscopy system was an ICCD and a full description of its design is given in Appendix B. All ICCDs considered for this study were spectroscopic ICCDs with a spectral to spatial pixel number ratio of 4:1. 
Three spectroscopy systems that were within the constraints of the project were found and the properties are summarised in Table 4.1. The idealised spectral resolution of the system was determined by the spectral range imaged upon the ICCD by the monochromator divided by the number of pixels.

Table 4.1: Comparison of the three spectroscopy systems considered.

\begin{tabular}{l|l|l|l}
\hline Spectrometer & Acton VM504 & McPherson 234 & McPherson 225 \\
\hline Monochromator design & Czerny-Turner & Turning concave & Seya-Namioka \\
Focal length (mm) & 390 & 200 & 1000 \\
F-number & 5.4 & 4.5 & 10.4 \\
Grating line density (g/mm) & 600 & 1200 & 300 \\
Spectral range (nm) & 75.6 & 72 & 60 \\
ICCD resolution (pixels) & $1024 \times 256$ & $2048 \times 512$ & $2048 \times 512$ \\
Spectral resolution (nm/pixel) & 0.12 & 0.1 & 0.06 \\
\hline
\end{tabular}

The McPherson 234 monochromator was considered due to its small size and affordability. Through its small size, this monochromator is capable of obtaining a large spectral range in a single image. However, this severely limits any possible high resolution spectroscopy in future studies. Whilst it can provide good spectral resolution with the $1200 \mathrm{~g} / \mathrm{mm}$ grating, it's maximum attainable resolution is limited by it's short focal length. The main drawback of this design is the large turning angle of the focussing grating leading to reduced image quality and this factor led to this monochromator not being selected.

The Acton VM504 monochromator has the perfect spectral range for VUV studies in the expansion tube using a $600 \mathrm{~g} / \mathrm{mm}$ grating. Its relatively small $\mathrm{F}$-number means that a large solid angle can be directed into the entry slit. The ICCD attached to this system has the larger $26 \mu \mathrm{m}$ pixel size resulting in enhanced sensitivity. The VM504 also has the added bonus of a grating turret, that would enable the user to change the grating while the monochromator was still under vacuum.

Whilst the grating turret, small F-number and large pixel size are valuable in a range of spectroscopy applications, they are not especially useful in expansion tube experiments. Due to the extremely short test time, it is not possible to change the grating during an experiment and thus the turret feature cannot be utilised. The small F-number gains are negated by the small aperture required to achieve a depth of field that would render the entire model in focus. The greater sensitivity gained by the large pixel size results in a decrease in spectral and spatial resolution. And any gains in sensitivity are offset by the 
additional mirrors required in the Czerny-Turner design. These reasons led to the VM504 model not being selected for these experiments.

The McPherson NOVA 225 monochromator was selected for this series of experiments due to its simplistic design and superior spectral resolution. Due to a minimum number of optical elements and a long focal length, this monochromator is capable of maintaining a strong signal whilst returning a well resolved, broad spectral range. The monochromator F-number is well matched to the external focussing optics and thus the relatively larger Fnumber is not detrimental. The NOVA 225 also has the ability to carry out extremely high resolution spectroscopy with the use of readily available finer gratings and an integrated calibration lamp ensures a relatively straight-forward calibration procedure.

\subsubsection{Optical Configuration}

Appropriate configuration of the optics external to the spectroscopy system are fundamental in achieving a sharp image of the experiment and maximising the signal to noise ratio attainable. For this study, two optical systems were produced to image the shock layer across the surface and through the surface of a model. Both optical systems created utilised a focussing mirror with a focal length of $500 \mathrm{~mm}$. The across surface optical path consisted of one flat mirror, additional to the focussing mirror, to deflect the signal towards the spectrometer slit. The through surface optical path required two flat mirrors to deflect the signal out of the test section and through to the spectrometer. Both optical configurations are shown in Figure 4.16.

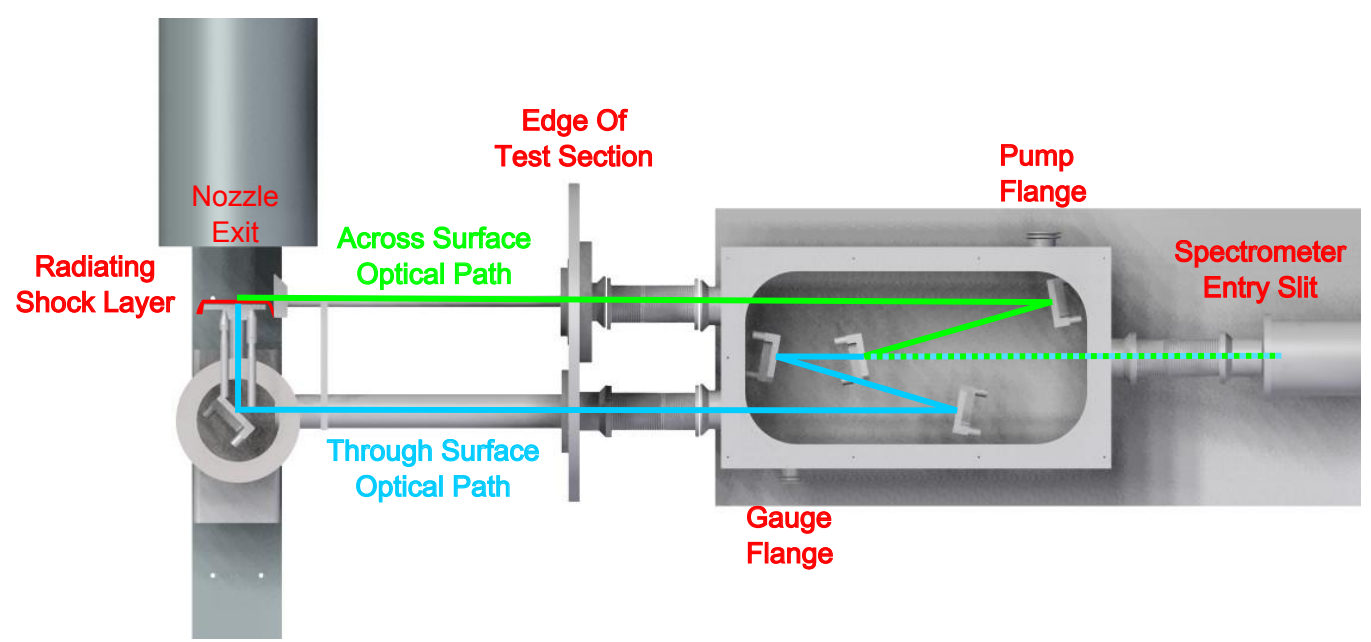

Figure 4.16: Imaging optics to observe radiation across and through the surface of the models. Image drawn to scale. 
A magnification of 0.85 was selected to ensure the entire shock layer was imaged on the $6.66 \mathrm{~mm}$ available in the spatial direction of the ICCD array. This magnification resulted in $7.8 \mathrm{~mm}$ of the test section being imaged, accounting for the expected shock stand off of approximately $6.5 \mathrm{~mm}$ and any small movements of the model between experiments. An aperture of $5.0 \mathrm{~mm}$ was initially selected as it provided a circle of confusion of $0.18 \mathrm{~mm}$ with a depth of field of $90 \mathrm{~mm}$. Such a small aperture greatly reduced the signal strength leading to a poor signal to noise ratio and infeasibly long exposure time requirements. The aperture was consequently increased to $10 \mathrm{~mm}$, providing a factor of four increase in signal strength with only a factor of two increase in circle of confusion.

The depth of field required for through surface measurements was much smaller and therefore a larger aperture of $30.0 \mathrm{~mm}$ could be utilised. Maintaining a magnification of 0.85 to allow for any misalignments and movement of the test section during pumping, the circle of confusion calculated for this system was $0.10 \mathrm{~mm}$ for a depth of field of $8.5 \mathrm{~mm}$. This calculated value for the circle of confusion is an underestimate of the actual value as the total circle of confusion would be increased by aberrations resulting from other optical components and alignment. The final details of the optical systems designed are presented in Table 4.2 .

Table 4.2: Details of optical systems produced.

\begin{tabular}{l|ll}
\hline Optical path & Across surface & Through Surface \\
\hline Focussing mirror to model centreline (mm) & 1088 & 1088 \\
Focussing mirror to slit (mm) & 925 & 925 \\
Total optical path (mm) & 2013 & 2013 \\
Aperture (mm) & 10.0 & 30.0 \\
F-Number & 50.0 & 16.7 \\
Depth of field (mm) & 90 & 8.5 \\
Calculated circle of confusion (mm) & 0.35 & 0.10 \\
\hline
\end{tabular}

\subsubsection{High Vacuum Optical Chamber Design}

A high vacuum chamber was constructed to house the optical components in an environment void of molecular oxygen that would absorb VUV radiation. To aid with the evacuation process, the optical chamber built for this study was designed to have a minimal internal volume and made from stainless steel to reduce out gassing [135]. To align the spatial imaging direction of the spectrometer with the stagnation stream line, the 
spectrometer itself was rotated by 90 degrees instead of using additional optical components to rotate the image. This rotation resulted in a decrease in the height requirements of the optical chamber and a decrease in the number optical components required leading to fewer reflectance losses.

The optical chamber was designed so that the user could switch between observing an emission spectrum across the surface of a model, to observing through the surface of a model, without having to physically move the spectrometer and optical chamber assembly. This is highly advantageous as the spectrometer and optical chamber assembly weigh in the order of $400 \mathrm{~kg}$ due to their construction of thick stainless steel. To further aid the alignment of the system, the spectrometer table was extended to create a platform for the optical chamber, as shown in Figure 4.18. This allowed for the optical chamber and spectrometer to be rigidly aligned and consequently reduce the degrees of freedom within the system.

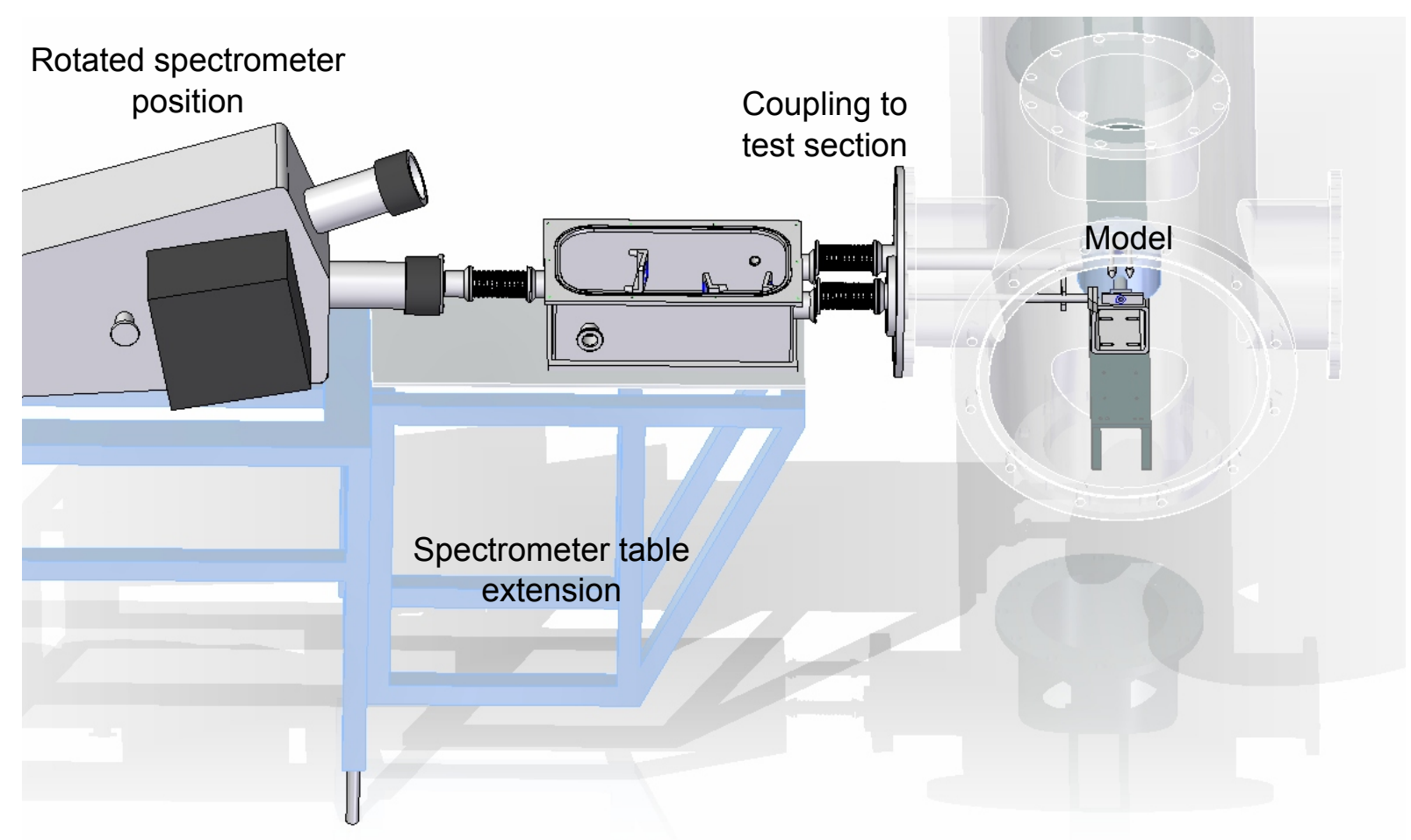

Figure 4.17: Extension of spectrometer table and rotation of spectrometer for enhanced compatibility with test section.

The optical chamber was constructed using stainless steel to prevent distortion under vacuum forces that can result in small changes in the optical angles leading to large distortions of the image. The base plate was constructed using $16 \mathrm{~mm}$ thick, 304 grade stainless steel. To reduce the weight of the optical chamber, thinner $6.0 \mathrm{~mm}$ thick plates were used for the side walls. This was possible as deformation of the side walls would not 
effect the optical alignment. Conforming to high vacuum manufacturing techniques, the chamber was welded on the insides of the joining surfaces with one continuous bead. A finite element analysis was conducted to ensure deformation of the bottom surface was below $0.05 \mathrm{~mm}$ and the thinner side walls would not deform more than $0.5 \mathrm{~mm}$.

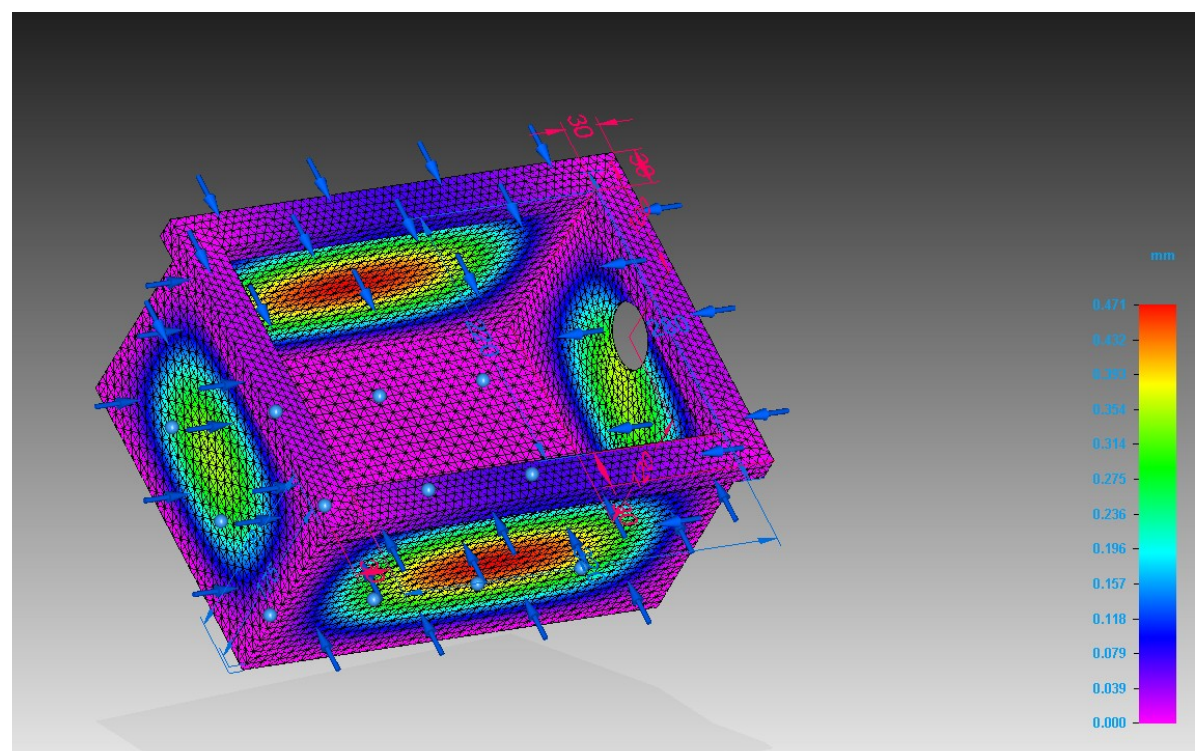

Figure 4.18: Deformation of the optical chamber with an atmospheric pressure force.

\subsubsection{Alignment of Optical Components}

The across surface optical components external to the spectrometer were aligned by passing a laser through two holes of $2.0 \mathrm{~mm}$ diameter, mounted onto the edges of the model as shown in Figure 4.19 left. Sliding rails were installed in the optical chamber to ensure optical components were in line with the optical entrances and exit of the chamber. Two optical poles mounted onto the sliding rails were used to ensure the optical chamber and spectrometer assembly was square with the model surface as shown in Figure 4.19 right. Using this approach it was possible to confirm the optical line of sight was level and across the centreline of the model.

Alignment for the through surface optical path was a much greater challenge as the change in position under vacuum had a substantial impact on the optical alignment. For the across surface configuration, small changes in model axial location during or between shots were not important, as with the magnification factor of 0.85 used, the observed strip was significantly longer than the shock stand off region, which would always lie in the recorded region of the spectrometer slit. However for the through surface observations, the use of the 45 degree mirror in the test section itself meant that any axial displacement 

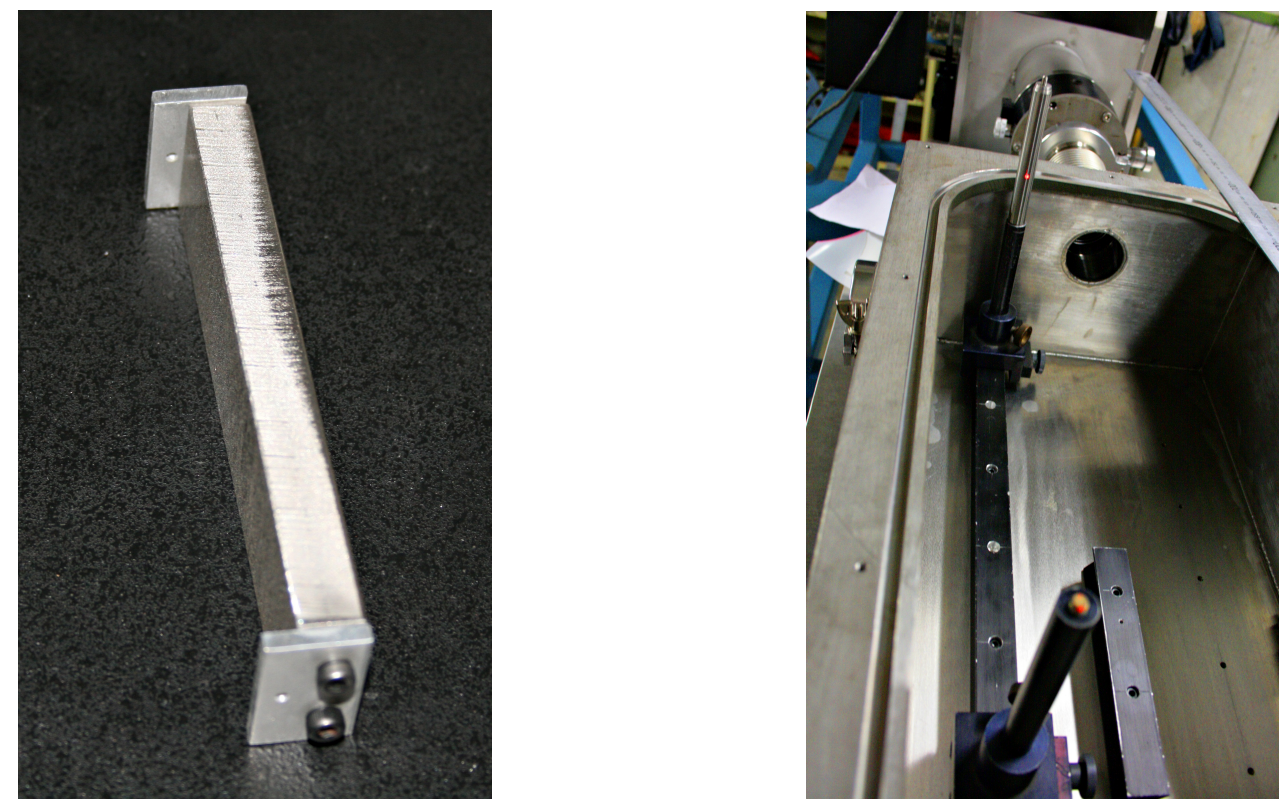

Figure 4.19: (Left) - Alignment tool mounted onto the edges of the flat model.(Right) Installation of railings in the optical chamber and the mounting poles used in the alignment process.

error would steer the beam in a transverse direction off the centreline. To eliminate this error, a chain block was employed in the manner shown in Figure 4.20 to move the test section into the same position as under vacuum. This allowed access to the model so a light source could be placed for alignment, something that would be impossible under vacuum conditions.

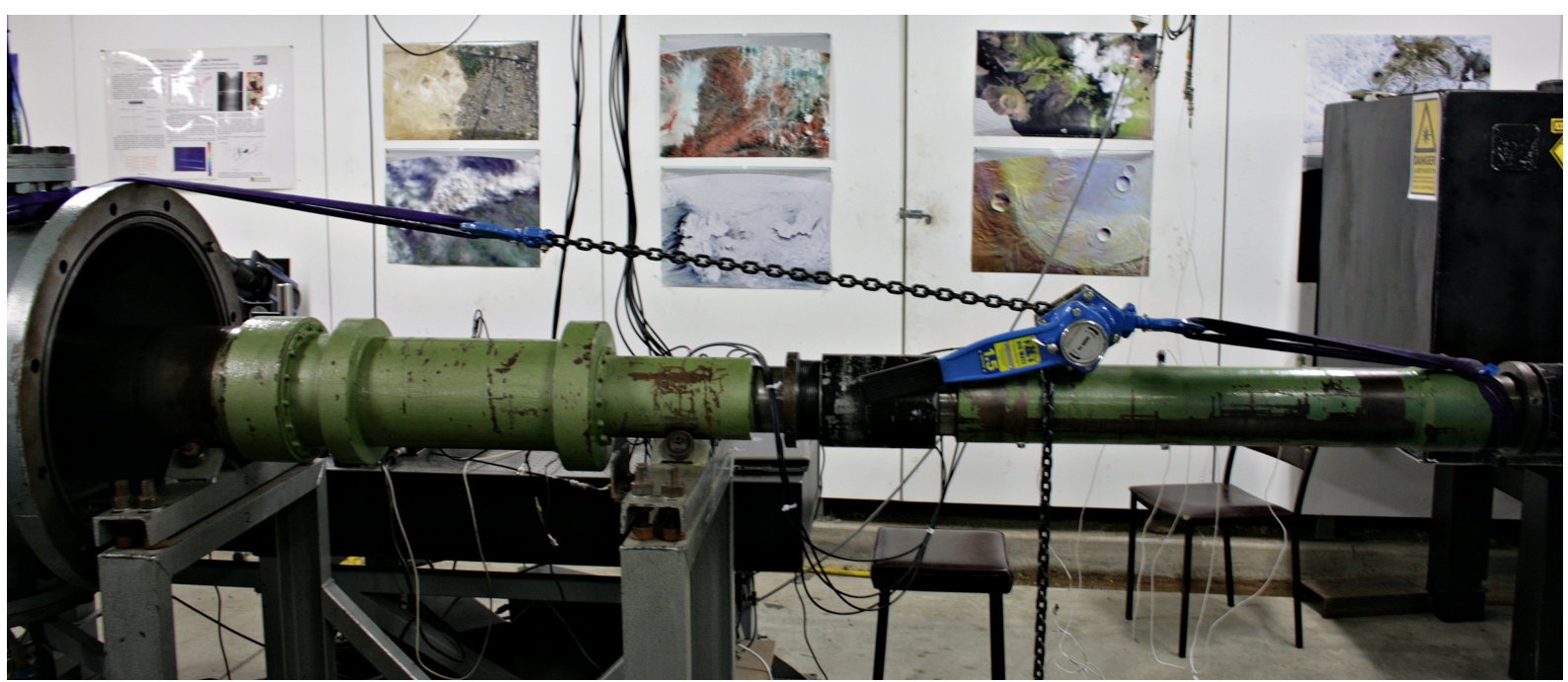

Figure 4.20: A chain block was used to move the test section into firing position. This was required to account for movement of the test section due to vacuum forces. 
To test the positioning of the optical components, sharp edged alignment tools were placed in the position of the model and imaged onto the ICCD. Using this approach it was discovered that the spectral focal plane and the spatial focal plane were independent due to the inherent astigmatism of the spectrometer design. The focal point of the spatial plane was measured to be $35 \mathrm{~mm}$ in front of the slit and the focal point of the spectral plane was the at slit. This measurement was carried out by setting the slit width at $2.0 \mathrm{~mm}$ and placing a sharp 90 degree edge to cover the upper left quarter of the slit. The results of this test, displayed in Figure 4.21, show the shadow produced on the ICCD is sharp in the spatial direction but blurred in the spectral location. Below the edge of the shadow, the slit location is sharp in the spectral plane. To maintain spectral focus, the position of the ICCD was not altered. Instead the image was focussed onto the spatial focal point of the grating and not the slit.

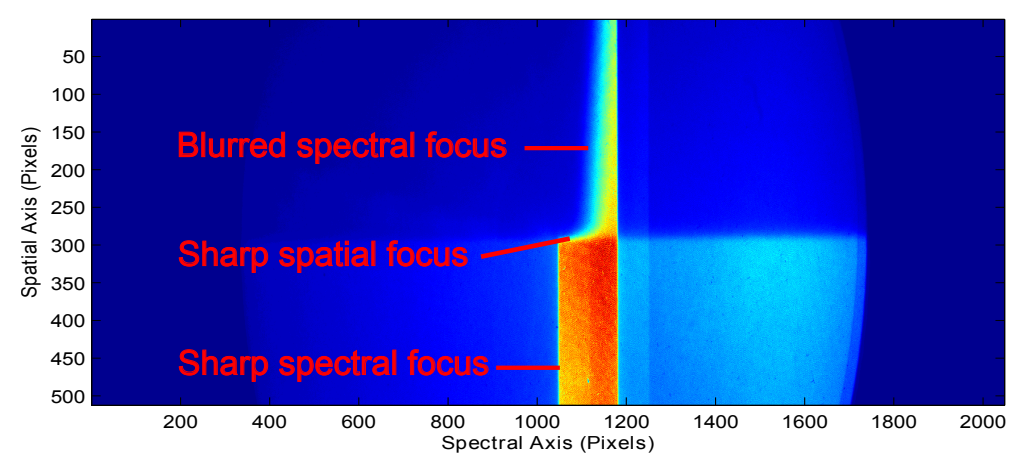

Figure 4.21: Blurred spectral focus at spatial focal point and sharp spectral focus at slit location.

\subsubsection{Vacuum Pressure Requirements}

Molecular oxygen and water vapour are strong absorbers of VUV radiation. Generally high vacuum is employed to remove it from optical paths and VUV spectroscopy systems. The level of absorption at a given pressure and path length can be calculated using absorption coefficients, which are calculated using the number density of a particular molecule in a gas and the absorption cross-section. For this study, the absorption crosssections from $\mathrm{Lu}$ were used in conjunction with the equations outlined in Section 2.3.3 [20. Figures 4.22 and 4.23 show the level of absorption per metre of optical path through air and pure water vapour. 


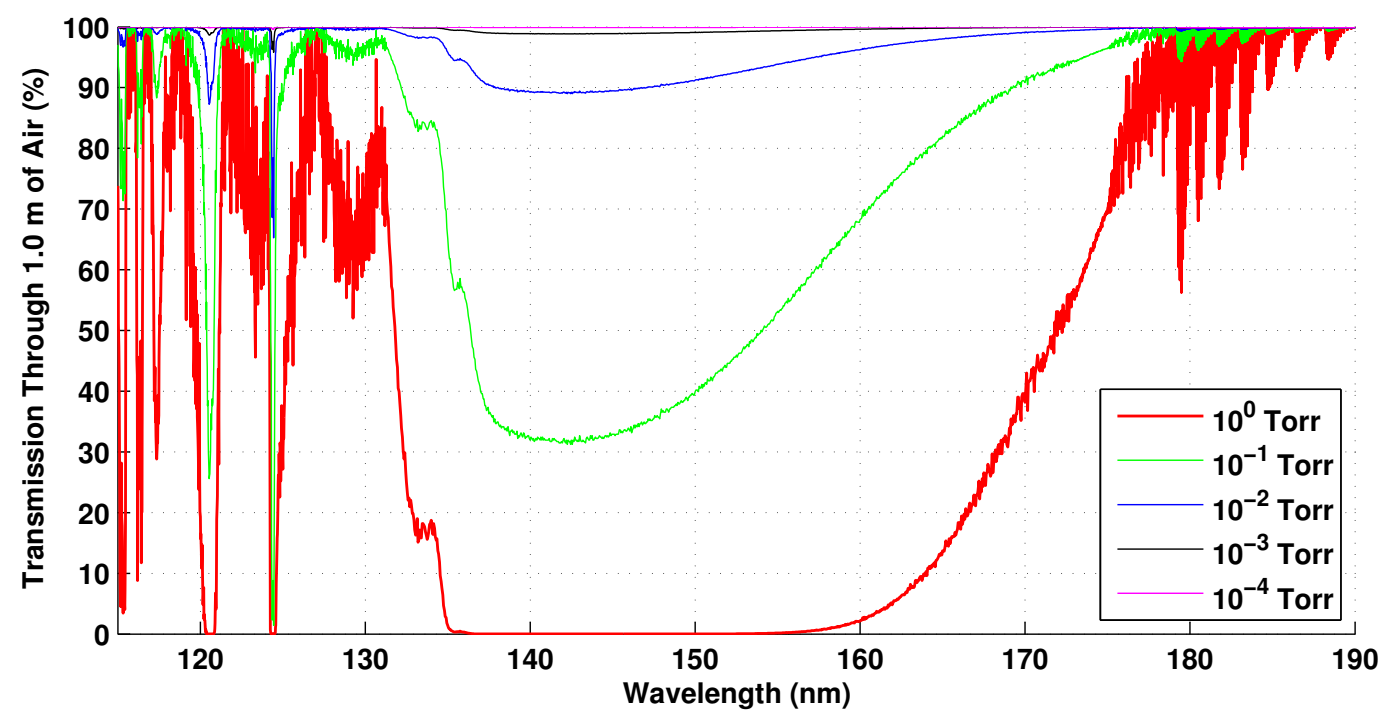

Figure 4.22: Transmission percentage of VUV radiation through $1.0 \mathrm{~m}$ of air at varying pressures.

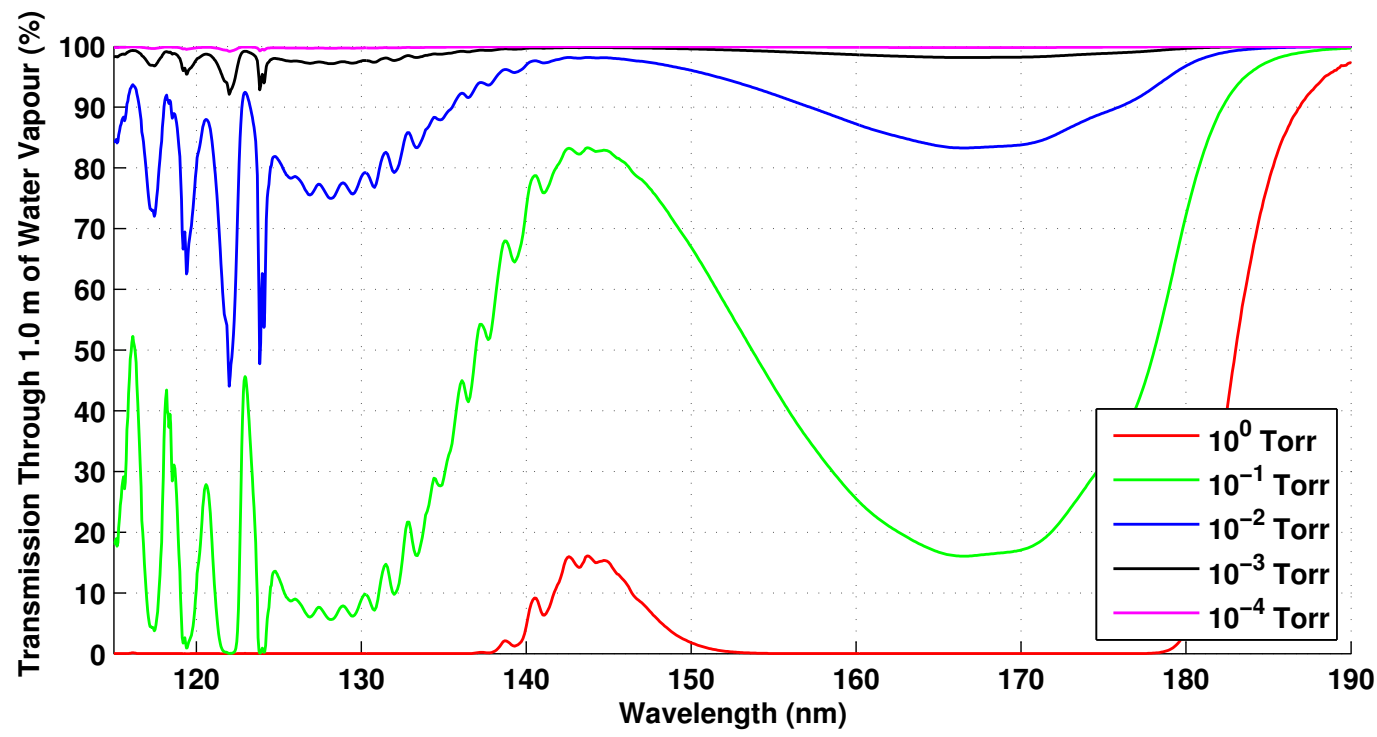

Figure 4.23: Transmission percentage of VUV radiation through $1.0 \mathrm{~m}$ of water vapour at varying pressures.

From Figures 4.22 and 4.23 , it is evident that pressures in the order of $10^{-4}$ Torr are required to achieve close to $100 \%$ transmission. To quantify the percentage of signal transmitted through the optical system constructed for this study, calculations were conducted using a $401.3 \mathrm{~cm}$ optical path accounting for the external optical path length of $201.3 \mathrm{~cm}$ and $200.0 \mathrm{~cm}$ for internal optical path length of the spectrometer. The number density of oxygen was calculated at room temperature with a concentration of 0.21 . This value of molecular oxygen concentration is an overestimate as the percentage of air in the optical path will drop as a result of water vapour from out-gassing being introduced 
into the mixture. To set an upper limit for water vapour absorption, the concentration of water vapour was estimated to be 0.50 in the optical path. Based on these parameters, it was calculated that a pressure of $10^{-4}$ Torr will result in approximately $1.5 \%$ absorption at the strongest absorption lines of water vapour and molecular oxygen, as shown in Figure 4.24. It was concluded that this pressure was sufficient to negate all absorption by molecular oxygen and water vapour in the optical light path.

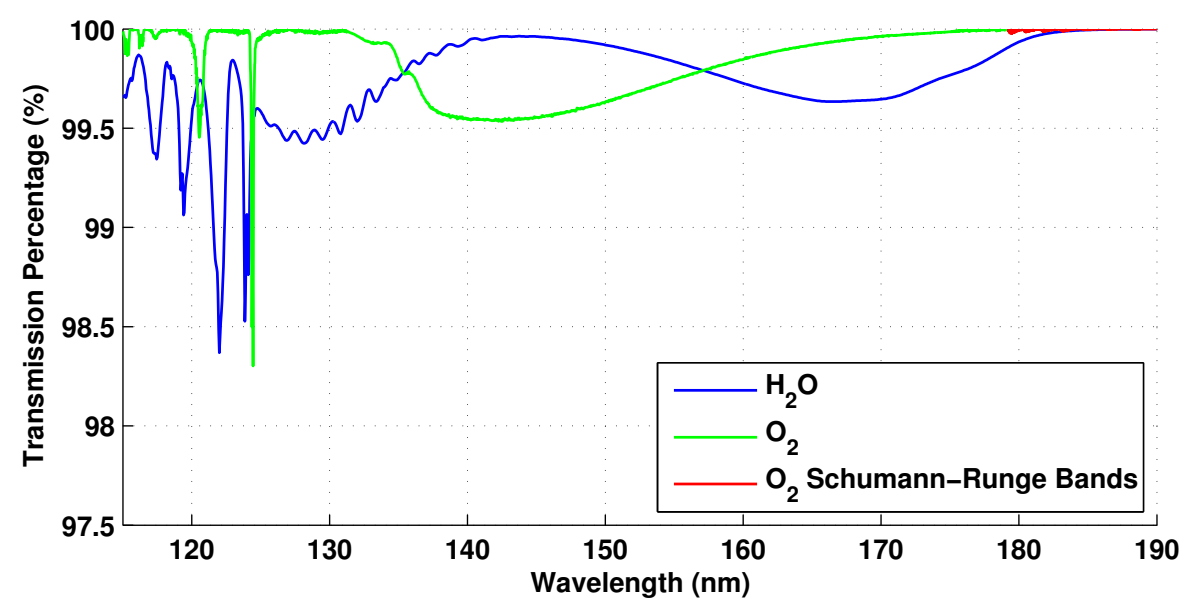

Figure 4.24: Results of a $10^{-4}$ Torr vacuum pressure on the transmission of the VUV radiation through an optical path of $401.3 \mathrm{~cm}$ with a molecular oxygen mole fraction of 0.21 and a water vapour mole fraction of 0.5 .

No single pump was available to reduce the pressure from atmospheric to the required level of vacuum as shown in Figure 4.25. To cover the pressure range required, a two stage pumping system consisting of a $150 \mathrm{~L} / \mathrm{s}$ Leybold Heraus PT150 turbo molecular pump and a $4.2 \mathrm{~m}^{3} / \mathrm{h}$ Leybold Trivac D4B/WS rotary vane pump was selected [136]. A turbo molecular pump was selected for the second stage of the pumping system as it is an oil free pump. This is important as oil mist in pumping systems can make its way out through the inlet of the pump and when coupled to an optical system, the oil mist can coat optical components, greatly reducing their performance and in some cases, destroying the optical coating.

Where possible, internal apertures of the vacuum path were kept to a maximum to avoid conductance losses experienced in the rarefied regime. The conductance loss through a tube of constant cross-section, D, at a length of L, can be calculated using Equation 4.8 [135].

$$
C=\frac{12.1 D^{3}}{L+1.33 D}
$$




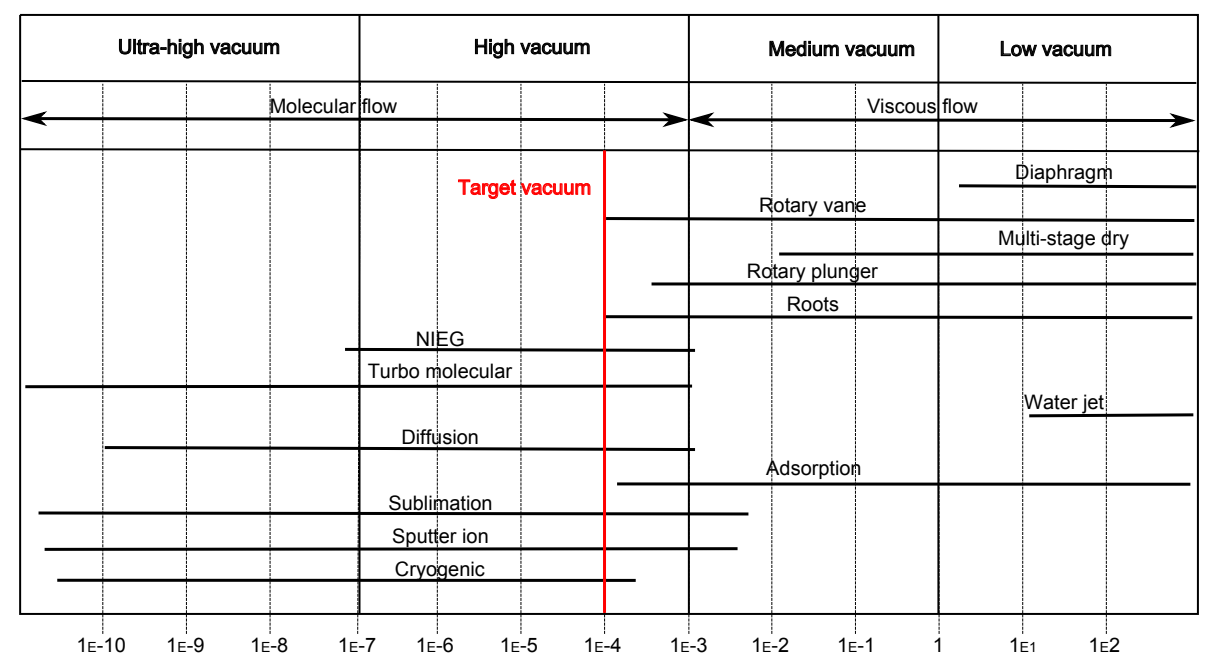

Figure 4.25: Array of vacuum pumping options available and their ranges. Source [28]

A pumping speed of $150 \mathrm{~L} / \mathrm{s}$ at the exit of a pump will drop to $22.7 \mathrm{~L} / \mathrm{s}$ at the exit of 500 $\mathrm{mm}$ long bellows with an internal diameter of $50 \mathrm{~mm}$ [135]. Turns and bends through the high vacuum path were also minimised as they also severely decrease conductance. A 90 degree elbow, with a $40 \mathrm{~mm}$ internal diameter and $7.5 \mathrm{~cm}$ effective lengths in each direction, will result in a conductance loss of 77\%, as calculated by Equation 4.9 [135].

$$
C=\frac{9.86 D^{3}}{L_{1}+L_{2}+0.665 D}
$$

\subsection{Calibration}

The only quantitative optical data requiring calibration on the expansion tube experiments is the VUV spectroscopy system. To calibrate this system, a deuterium lamp calibrated using the electron storage ring, BESSY II, at the Physikalisch-Technische Bundesanstalt was used [36]. The deuterium lamp has a known spectral radiance and can therefore be used to conduct an in situ calibration. To position the lamp at the location of the radiating shock layer, an adapter was constructed. The design of the adapted allows for the lamp to be observed using the optical path for through surface, as shown in Figure 4.26, and across surface measurements. A calibration factor between the measured spectrum and the known spectral radiance of the lamp can be calculated and applied to experimental data to convert intensity counts on the ICCD to spectral radiance. This approach is commonly referred to as in situ calibration and is preferred over other methods as it accounts for all reflectances and transmissions of optical elements experimentally and in one single calibration. The relationship between the calibration factor, counts on 
the ICCD and spectral radiance is a function of wavelength and expressed by Equation 4.10.

$$
\text { calibration factor }(\lambda)=\text { system } \operatorname{sensitivity}(\lambda)=\frac{\text { ICCD counts }}{\text { known spectral radiance }}
$$

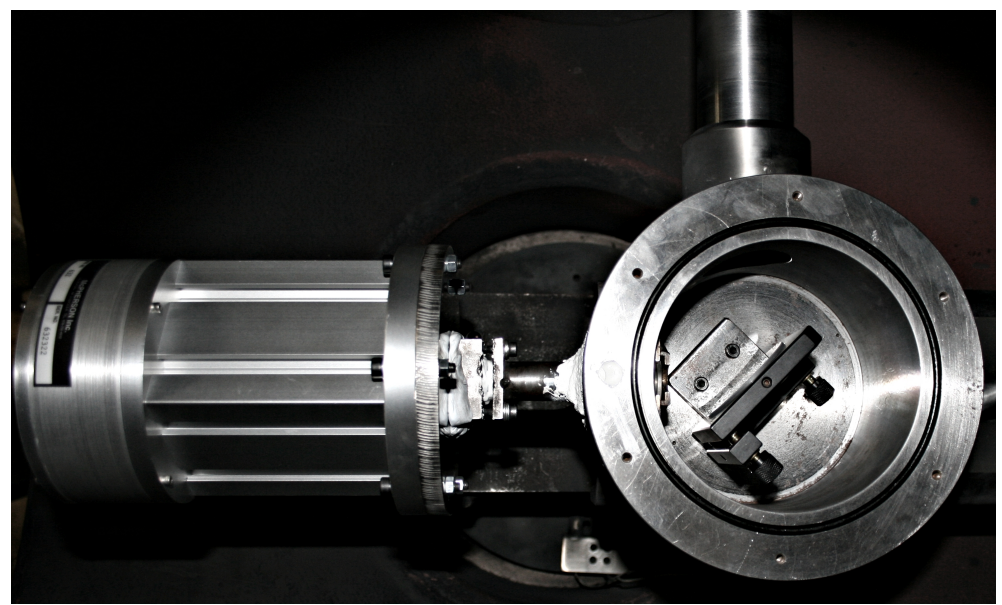

Figure 4.26: Calibration lamp mounted in place of the model.

Due to the position of the radiating element within the lamp and the spectral output of the lamp, a full in situ calibration was not possible. The radiating element within the calibration lamp is situated approximately $80 \mathrm{~mm}$ from the front edge as shown in Figure 4.27. This was acceptable for calibration of the across surface optical path as the radiating element is within the depth of field. For the through surface optical system, the radiating element is outside of the depth of field and thus a true in situ calibration is not possible. To account for this, the depth of field was increased by reducing the aperture on the focussing mirror to $10 \mathrm{~mm}$ instead of $30 \mathrm{~mm}$ as used in experiments. This approached required the assumption that the focussing mirror reflects evenly within the inner $30 \mathrm{~mm}$, which is within the central clear aperture of $80 \%$, as specified by the manufacturer [137.

The spectral radiance of the deuterium lamp is much lower than that of the radiating shock layers observed in experiments resulting in longer exposure times for the ICCD during calibration. To relate the longer exposure time used in calibration to experimental data, a relationship between exposure time and intensity on the camera was established. This was done using two ranges of exposure times with varying slit widths. Slit widths of $25 \mu \mathrm{m}$ and $100 \mu \mathrm{m}$ were required to prevent saturation of the camera at longer exposure times and maintain a good signal to noise ratio at shorter exposure times. The total counts across the entire ICCD were integrated after the background was removed and the results indicate that the camera has a linear response to variation in exposure time. 


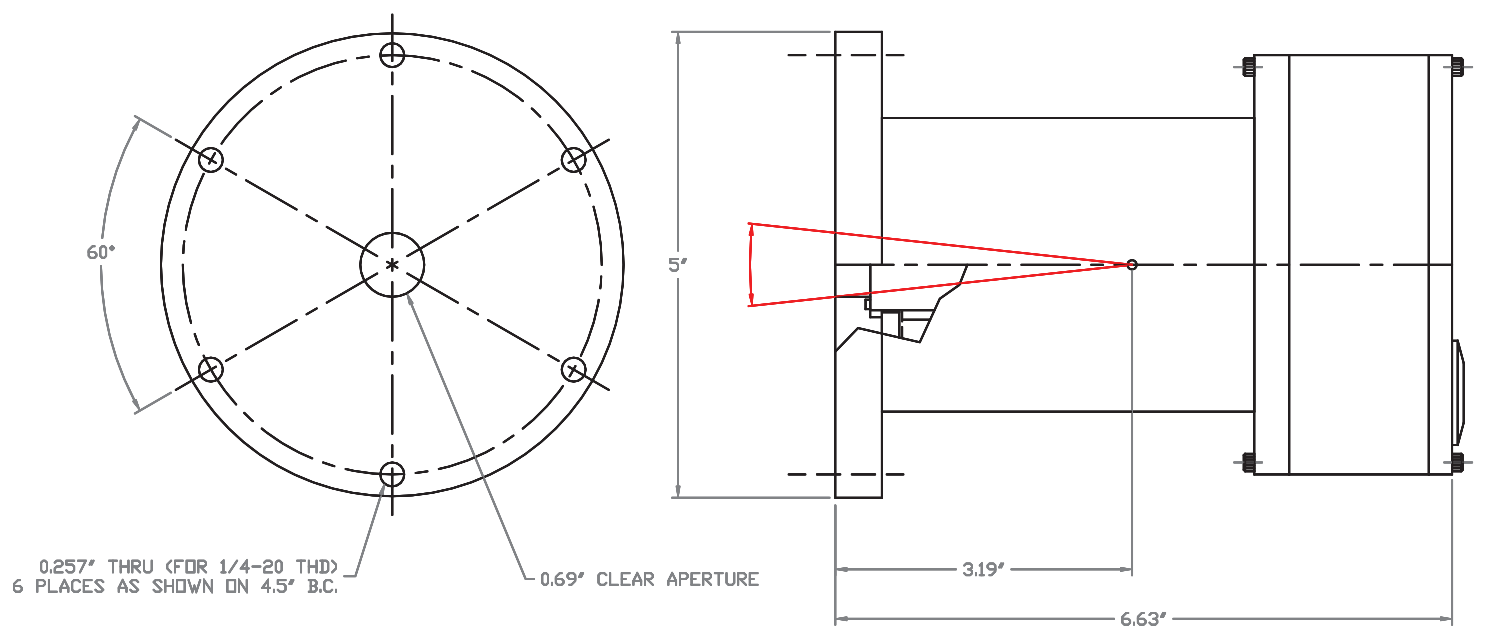

Figure 4.27: Calibration lamp internal dimensions

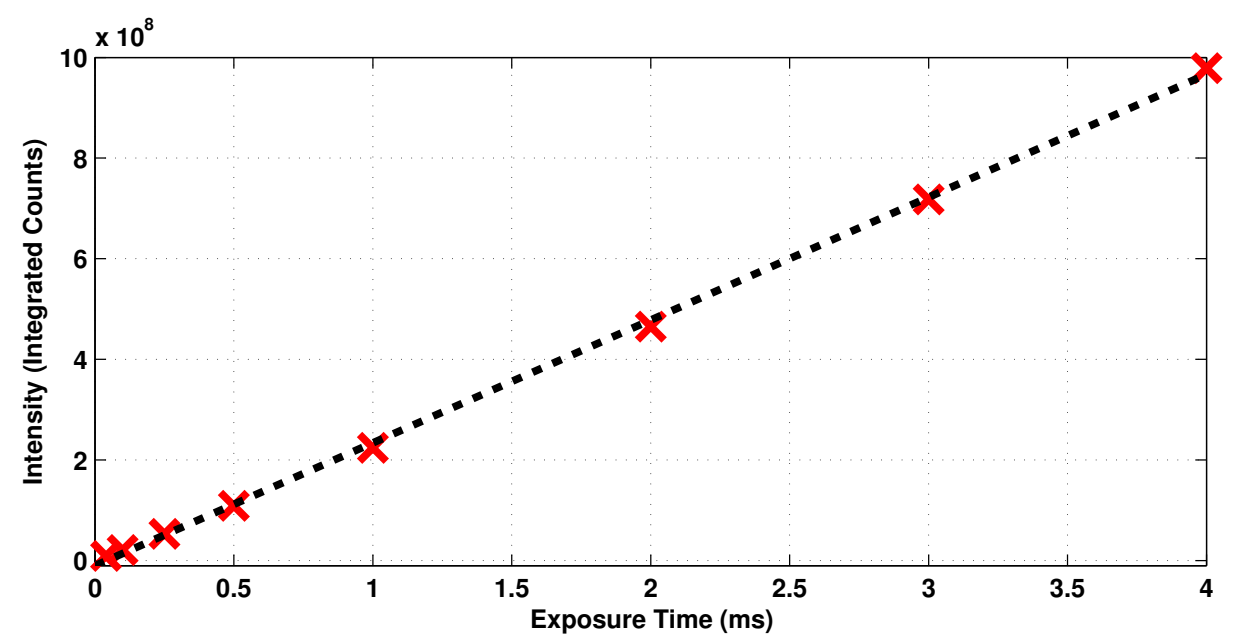

Figure 4.28: Linear response of the camera with variation in exposure time of the ICCD 
As in situ calibration was conducted using a limited range of pixels at the centre of the ICCD array, it was necessary to ensure there is no variation in sensitivity across the ICCD. Images were taken near the top, centre and bottom of the ICCD and the results were integrated and compared in Figure 4.29. Longer wavelengths were used for this study as it was not possible to adjust the optics under the vacuum conditions required for VUV measurements. It was found that the variation in ICCD sensitivity was negligible across the array of pixels and therefore calibration with the central pixels could be used as a representative calibration for the entire ICCD. A similar calibration for wavelength sensitivity of pixels was not required as the grating position is maintained between calibration and experiments.
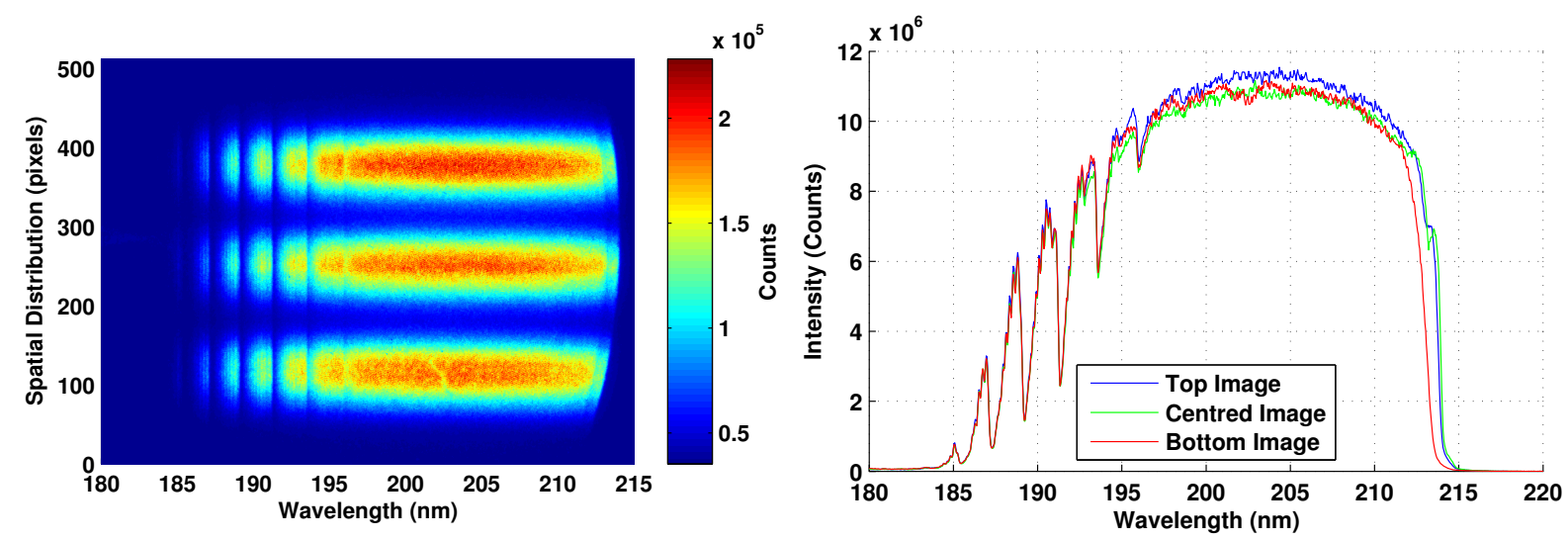

Figure 4.29: Left - Superimposed calibration images depicting ICCD array ranges tested. Right - Results of integrating each image indicate there negligible variation in pixel sensitivity across the ICCD. The slight discrepancy at the longer wavelength cut off is due to the circular shape of the intensifier clipping the signal.

\subsubsection{Validity of Calibration Data}

Before applying the calibration dataset, it was important to test the validity of the acquired calibration lamp spectra. There were four factors considered that were deemed capable of corrupting the recorded data:

- Absorption of the signal by molecular oxygen or water vapour, caused by outgassing

- The calibration source not reaching steady state emission

- A low signal to noise ratio

- Alignment of the ICCD with the spectrometer 
Signal absorption in calibration measurements can lead to an overestimation of experimental values. As the absorption cross-sections of molecular oxygen and water vapour have strong absorption lines in the deep VUV, it is possible to deduce if there is any absorption occurring by checking for sharp unexpected features in the measured spectrum. Figure 4.30 shows the scaled values of the absorption cross-section superimposed on the measured calibration spectrum. At the strong molecular oxygen absorption line at 124.4 $\mathrm{nm}$, there is no relative drop in measured signal strength, compared to the known lamp radiance data, therefore it can be concluded that there is no absorption occurring. Similarly, there is no drop in relative signal strength at the $122.0 \mathrm{~nm}$ water vapour absorption line, again indicating there is no absorption occurring.

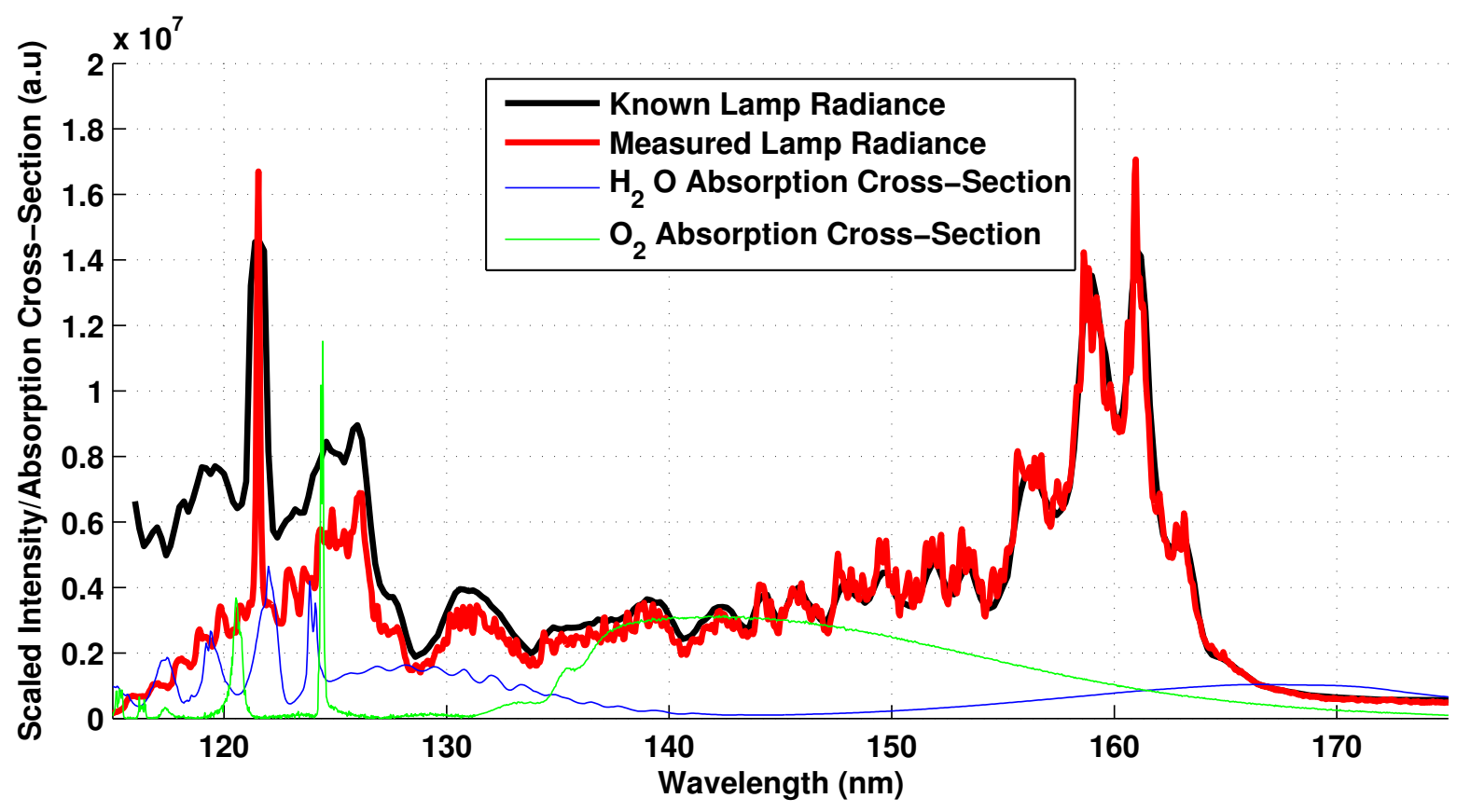

Figure 4.30: Scaled absorption cross-sections of water vapour and molecular oxygen superimposed on the measured and known spectral datasets.

Calibration sources take a finite amount of time to reach the steady state emission conditions at which they are originally calibrated. To ensure the deuterium lamp reached steady state before calibration images were recorded, a study was conducted to observe the change in emission during the warm up of the lamp. Figure 4.31 shows a slight variation in signal output between 2 minutes of operation and all other values. These results indicate that the lamp has reached steady state after a period of 5 minutes. To completely remove any error due to lamp start up, the lamp was allowed to warm up for 20 minutes before any calibration data was recorded. 


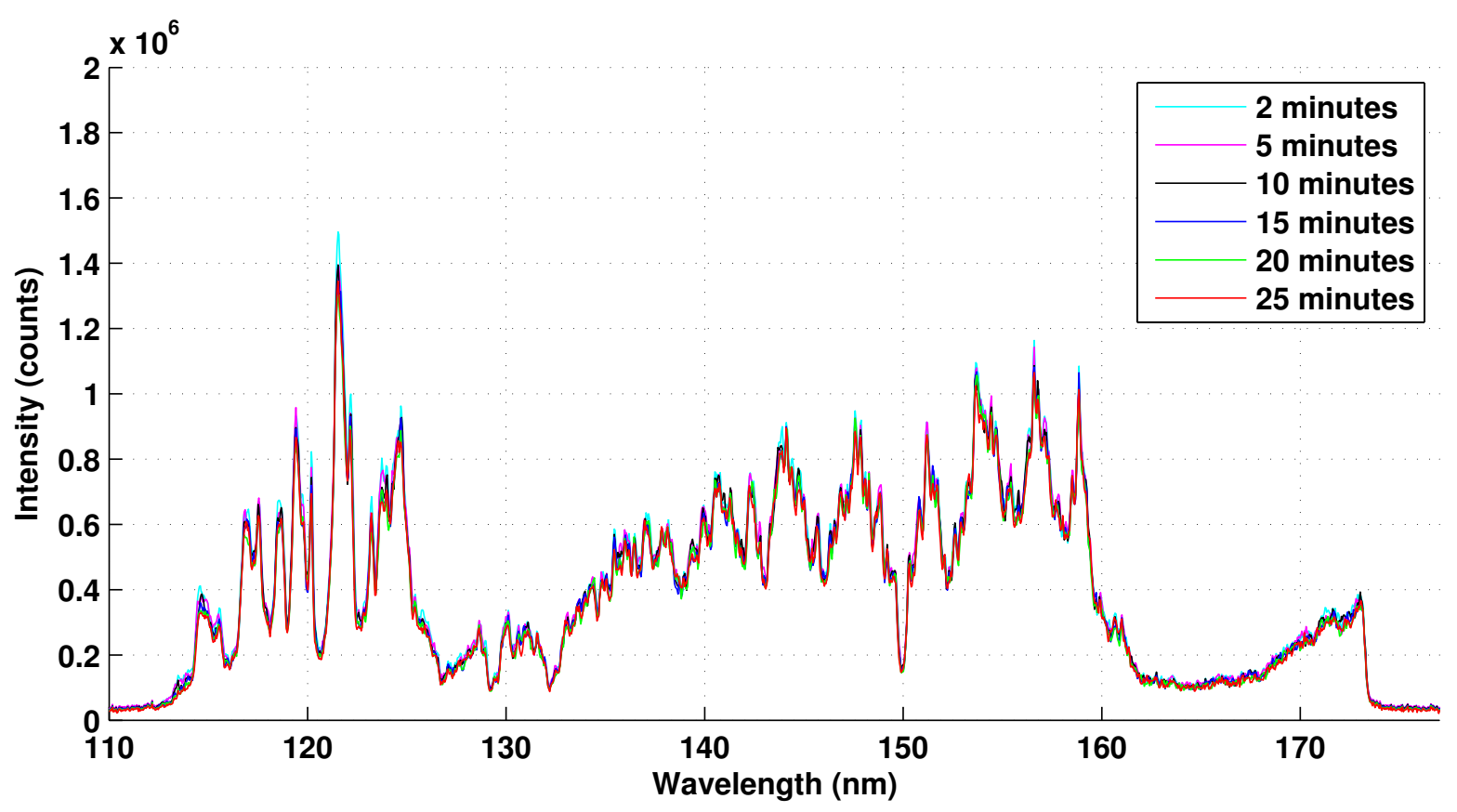

Figure 4.31: Spectra of the deuterium lamp during start up

To ensure a high signal to noise ratio, 25 accumulations of the lamp spectrum were acquired. The peak spatial intensity values of the spectrum are used for calibration and therefore a plateau region in the spatial direction is required. As the lamp is not a uniformly radiating source, this region is limited to a fraction of a millimetre. To achieve a better signal to noise ratio, more pixels can be averaged across. However in doing so, the peak spectral radiance value is reduced. As a compromise, pixels within $2 \%$ of the peak value were averaged across to obtain the spectral radiance for calibration. For the through surface optical system calibration, 7 pixels were used and the difference between the averaged value and the peak value was $0.81 \%$. For the across surface system calibration, 11 points were used with a $0.75 \%$ variance between the average and peak values.

Alignment of the ICCD array with the grating grooves is carried out manually and this can result in slight imperfections in the recorded image. To test this, spectral regions were integrated, normalised and plotted against spatial pixels as shown in Figure 4.32. If the ICCD is oriented perfectly, all spectral regions should peak at the same spatial pixel and any variation represents a degree of rotation between the CCD and the grating. This is an important factor to consider as it can result in the selection of pixels at certain wavelengths that are not at the peak spectral radiance values. 


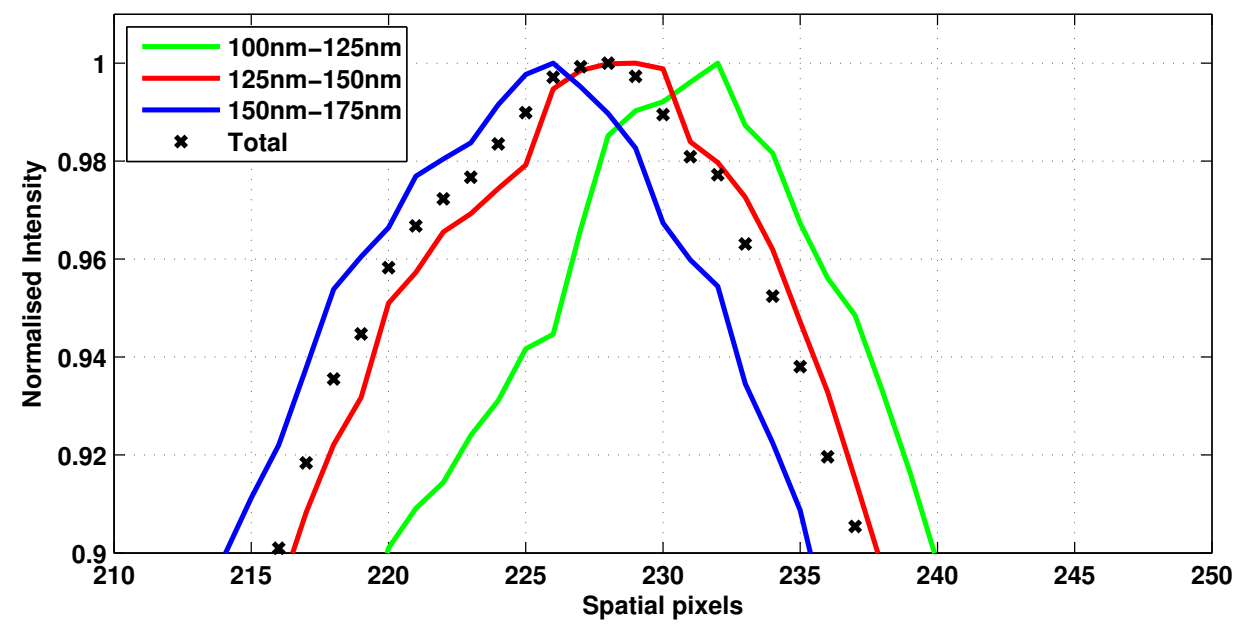

Figure 4.32: Spatial variation in the location of peak radiation output due to a twisting angle between the grating and the camera

As evidenced in Figure 4.32, the spectral peaks do not align spatially and therefore it is concluded that there is a slight twisting angle between the ICCD and grating grooves. By plotting the peak spatial pixel at each wavelength, a twist angle of 0.38 degrees was discovered. It was calculated that a 0.38 degree twist angle would result in a calibration error as high as $3.2 \%$ when averaging 11 pixels at the assumed peak spectral radiance position. A small angle such as 0.38 degrees is difficult to correct physically with the available alignment mechanisms on the spectrometer but could be easily corrected through matrix manipulation in data processing. Through matrix rotation the peak spectral radiance position was corrected and this was confirmed by integrating spectral regions once again as shown in Figure 4.33.

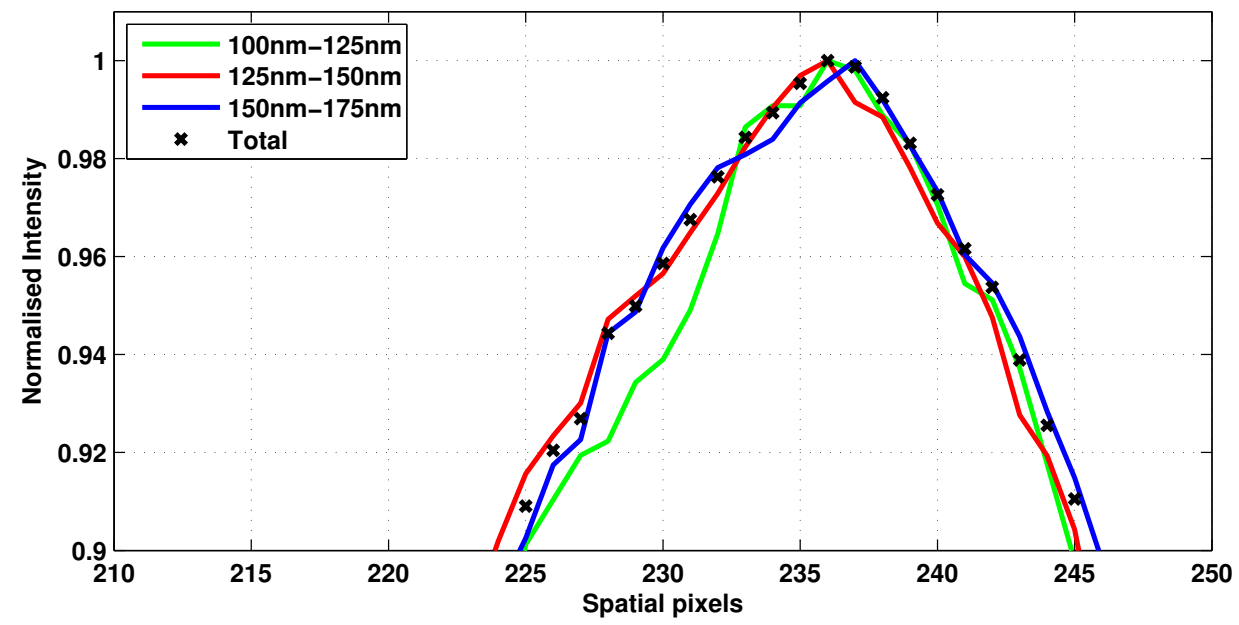

Figure 4.33: Results of a 0.375 degree rotation of the recorded image on the position of the peak spectral radiation output. 


\subsubsection{Calculation of the Calibration Factor}

Having ensured the applicability and validity of the calibration dataset as described above, two steps remained in the calculation of the calibration factor; matching the spectral bandwidth of the given calibration data and accounting for the viewing window at the end of the sealed optical path that needs to be removed during calibration. The deuterium lamp is calibrated using a spectral bandwidth $0.8 \mathrm{~nm}$ (FWHM) between $116 \mathrm{~nm}$ and $170 \mathrm{~nm}$ and $1.6 \mathrm{~nm}$ for wavelengths higher than $170 \mathrm{~nm}$. The spectral resolution of the system is much better than the spectral bandwidths used in the calibration and therefore a technique is required to reduce the height of the peaks and broaden them to match the given dataset. The VUV spectroscopy system has a resolution of $0.05 \mathrm{~nm}$ per pixel and therefore 18 pixels were averaged across using a moving average function. The results of the spatial averaging with the given and measure lamp spectrum is presented in Figure 4.34.

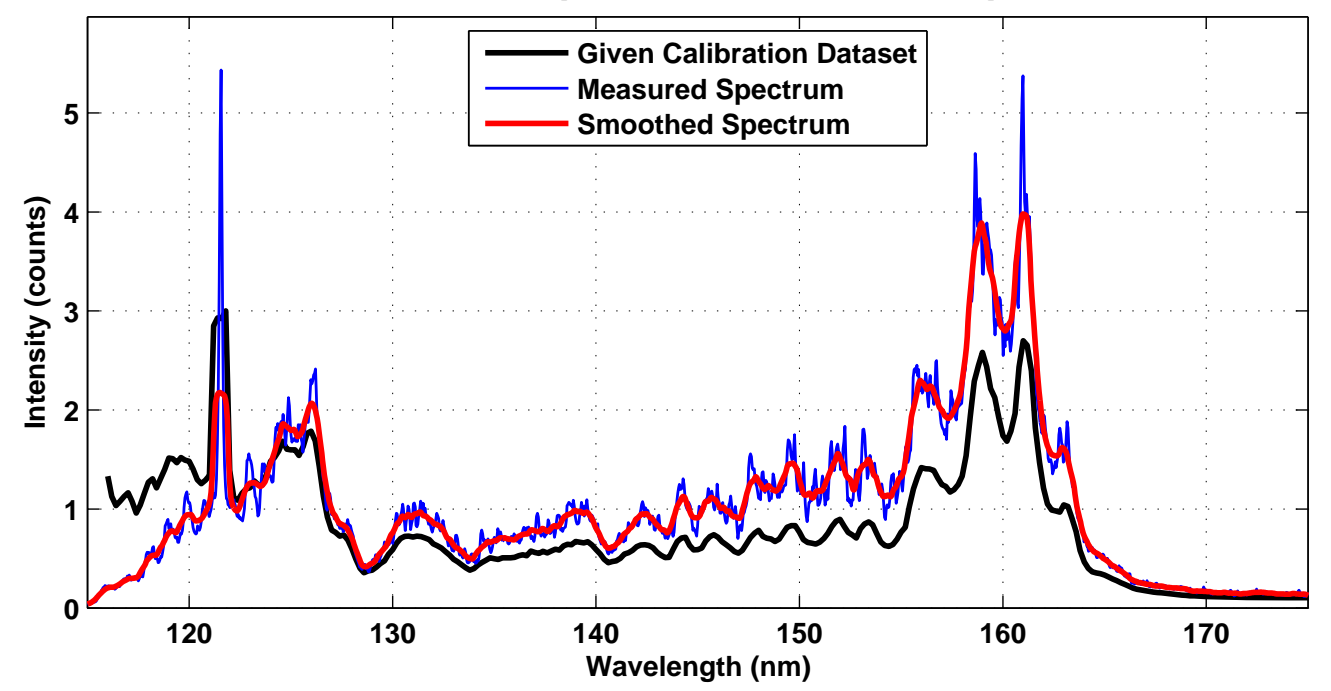

Figure 4.34: Spectral averaging or smoothing of measured data to achieve a spectral bandwidth matching that of the given calibration dataset.

Transmission of the windows was found experimentally by placing an additional window at the aperture of the focussing mirror and comparing the spectrum with the original calibration spectrum. As the window was placed in front of a mirror, the signal passed through it twice, simulating two windows in the system. This was preferable to a single pass through the window as a larger difference would be observed in the transmission, allowing for a more accurate final measurement. The transmission calculated was spectrally averaged to remove any sharp features that were most likely due to physical characteristics of the measurement system. The final calculated transmission of the magnesium fluoride windows applied in the calibration of experimental data is shown in Figure 4.35 . 


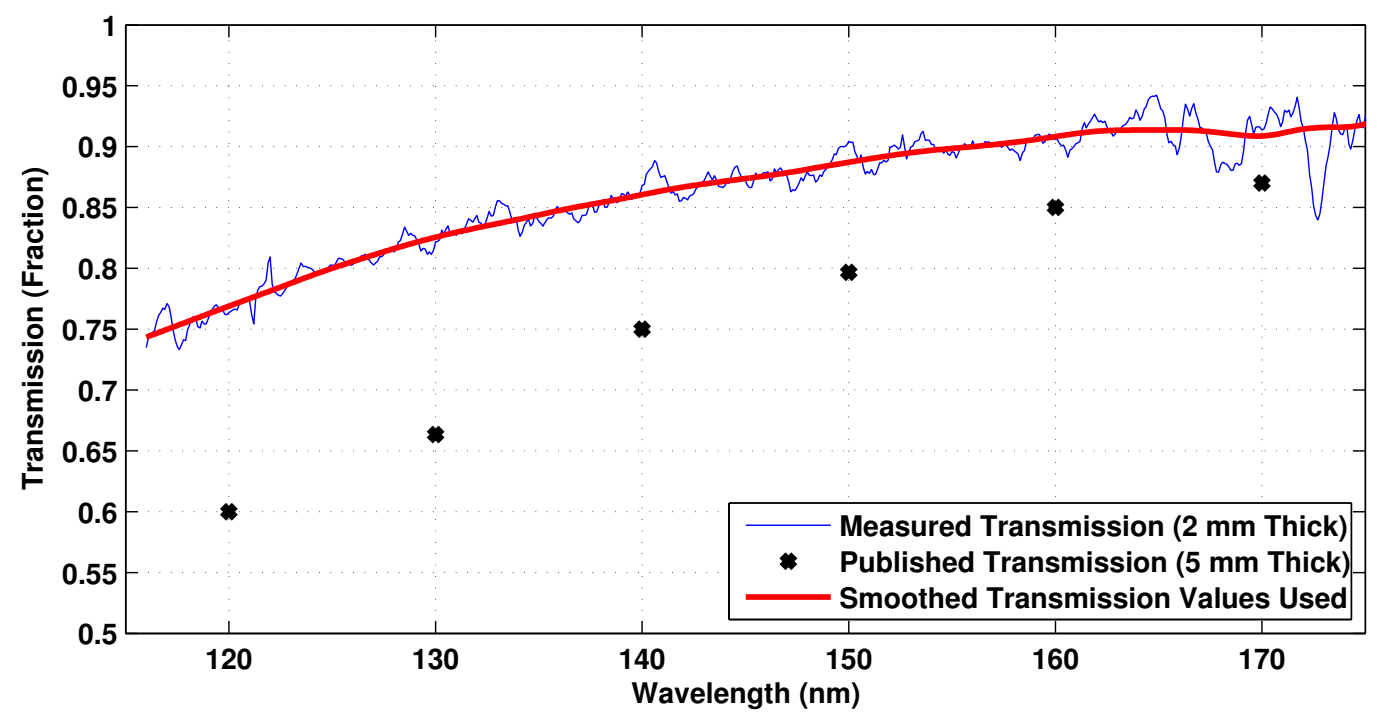

Figure 4.35: Measured and published transmissions of magnesium fluoride windows. Publish transmission from Crystran [29].

The final measured system sensitivity for both optical systems is displayed in Figure 4.36. The through surface optical system has a much higher sensitivity throughout the wavelength range, even with an additional mirror in the optical system. This is due to the use of standard UV focussing mirror, instead of a VUV enhanced mirror, in the across surface measurements. A VUV enhanced focussing mirror was not available at the time of the across surface measurements.

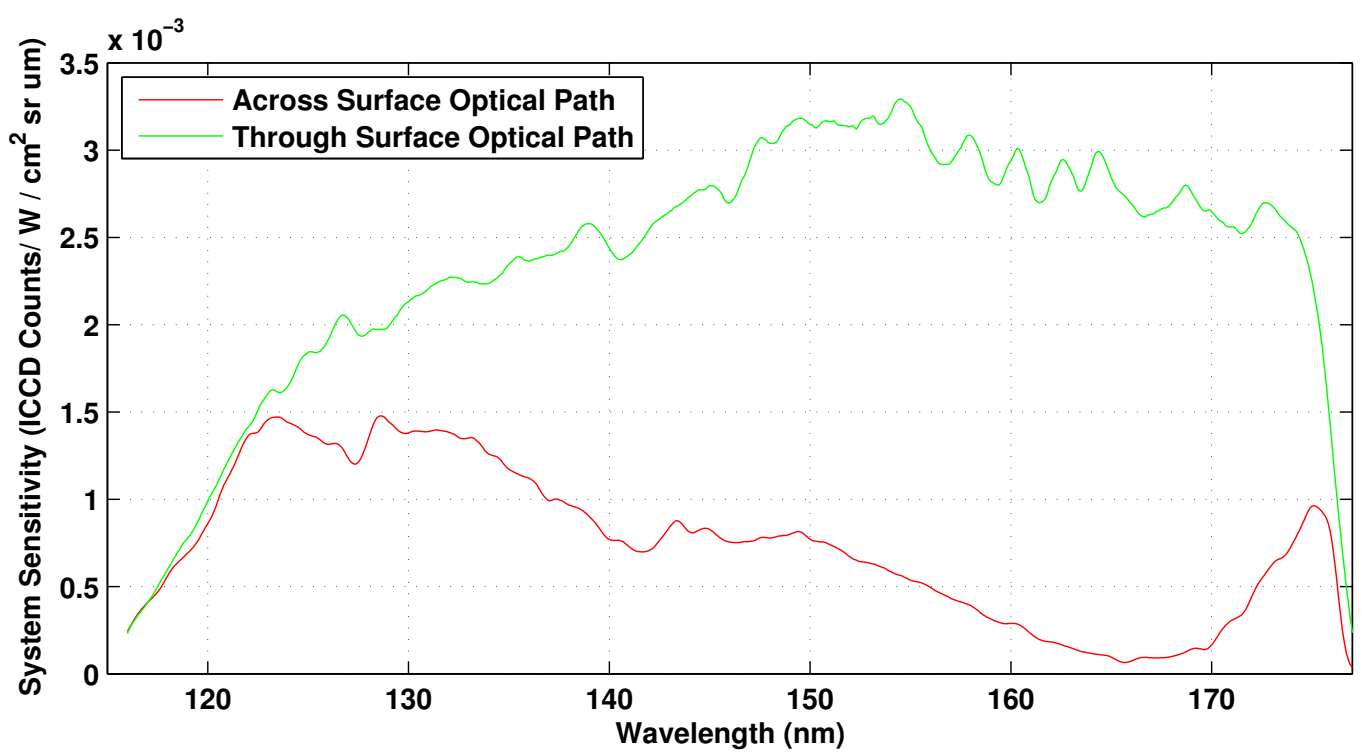

Figure 4.36: Final system sensitivity measured for both optical systems. The across system sensitivity here is calculated for a $10.0 \mathrm{~mm}$ aperture. 


\subsection{Operating Conditions}

For this study two conditions were targeted; $10.0 \mathrm{~km} / \mathrm{s}$ flight equivalent velocity and $12.0 \mathrm{~km} / \mathrm{s}$ flight equivalent. Higher velocities were preferred as radiative heating contributes a greater fraction of the total heat flux. Increased radiative emission also produces stronger signals allowing higher quality measurements to be taken in the expansion tube.

Previous shot data and basic shock wave relations, as outlined in Gildfind [30], were utilised to compute the fill pressures required. These estimates were tested by the numerical simulation code Pitot to more accurately calculate flow conditions [138]. Numerical simulation was necessary as the basic shock relations found in Gildfind assume calorically perfect gases whereas Pitot uses equilibrium gas chemistry from the CEA look up tables [139]. Fill pressures computed using this approach are outlined in Table 4.3 .

Table 4.3: Fill pressures for condition 1. Uncertainties taken from Gildfind [30]

\begin{tabular}{l|lll}
\hline Section & $\begin{array}{l}\text { Gas Mixture } \\
\text { Condition 1 }\end{array}$ & $\begin{array}{l}\text { Gas Mixture } \\
\text { Condition } 2\end{array}$ & Pressure \\
\hline Reservoir & Lab Air & Lab Air & $6,850 \pm 50 \mathrm{kPa}$ \\
Driver & $90 \%$ He 10\% Ar & $100 \%$ He & $92.8 \pm 0.2 \mathrm{kPa}$ \\
Shock Tube & Dry Air & Dry Air & $3,000 \pm 200 \mathrm{~Pa}$ \\
Acceleration Tube & Lab Air & Lab Air & $10.0 \pm 0.15 \mathrm{~Pa}$ \\
and Test Section & & & \\
\hline
\end{tabular}

To validate the calculated free stream values from Pitot, the primary shock wave velocity, secondary shock wave velocity, static pressure at the inlet of the nozzle and pitot pressure measured at the exit of the nozzle were compared with experimental values. Pitot pressures were obtained in the test section using an array of 15 degree conical glancing impact pressure probes in a rake formation, as shown in Figure 4.37. Using the rake formation it is possible to measure a radial distribution of the pitot pressures and estimate the core flow produced. The temporal resolution of the pitot probes allow for an estimate of the steady test time achieved by the condition. Detailed design and testing of the conical pressure probes is outlined in Gildfind [30]. The results of the Pitot calculations and comparisons with experimental testing are shown in Table 4.4. 
Table 4.4: Free stream test conditions calculated using Pitot and available measured values.

\begin{tabular}{l|ll}
\hline Condition $\mathbf{1}$ & & \\
\hline Parameter & Calculated & Measured \\
\hline Primary shock velocity $(\mathrm{km} / \mathrm{s})$ & 4.76 & $4.7 \pm 0.12$ \\
Secondary shock velocity $(\mathrm{km} / \mathrm{s})$ & 9.57 & $9.6 \pm 0.30$ \\
Static pressure at nozzle entry $(\mathrm{Pa})$ & 4,710 & $5,000 \pm 750$ \\
Pitot pressure $(\mathrm{kPa})$ & 115 & $115 \pm 11.5$ \\
Test gas velocity $(\mathrm{km} / \mathrm{s})$ & 9.71 & $\mathrm{~N} / \mathrm{A}$ \\
Test gas temperature $(\mathrm{K})$ & 2,495 & $\mathrm{~N} / \mathrm{A}$ \\
Test gas Mach number & 10.6 & $\mathrm{~N} / \mathrm{A}$ \\
Test gas density $\left(\mathrm{kg} / \mathrm{m}^{3}\right)$ & $1.26 \times 10^{-3}$ & $\mathrm{~N} / \mathrm{A}$ \\
Test gas enthalpy $(\mathrm{MJ} / \mathrm{kg})$ & 50.3 & $\mathrm{~N} / \mathrm{A}$ \\
Test gas static pressure $(\mathrm{Pa})$ & 930 & $\mathrm{~N} / \mathrm{A}$ \\
\hline & & \\
Condition 2 & & \\
\hline Parameter & Calculated & Measured \\
\hline Primary shock velocity $(\mathrm{km} / \mathrm{s})$ & 5.64 & $5.7 \pm 0.15$ \\
Secondary shock velocity $(\mathrm{km} / \mathrm{s})$ & 11.7 & $11.5 \pm 0.40$ \\
Static pressure at nozzle entry $(\mathrm{Pa})$ & 4,460 & $7,000 \pm 1750$ \\
Pitot pressure $(\mathrm{kPa})$ & 135 & $140 \pm 28$ \\
Test gas velocity $(\mathrm{km} / \mathrm{s})$ & 11.8 & $\mathrm{~N} / \mathrm{A}$ \\
Test gas temperature $(\mathrm{K})$ & 2,794 & $\mathrm{~N} / \mathrm{A}$ \\
Test gas Mach number & 11.9 & $\mathrm{~N} / \mathrm{A}$ \\
Test gas density $\left(\mathrm{kg} / \mathrm{m}^{3}\right)$ & $1.00 \times 10^{-3}$ & \\
Test gas enthalpy $(\mathrm{MJ} / \mathrm{kg})$ & 74.5 & $\mathrm{~A}$ \\
Test gas static pressure $(\mathrm{Pa})$ & 870 & \\
\hline & & \\
\hline & & \\
\hline
\end{tabular}




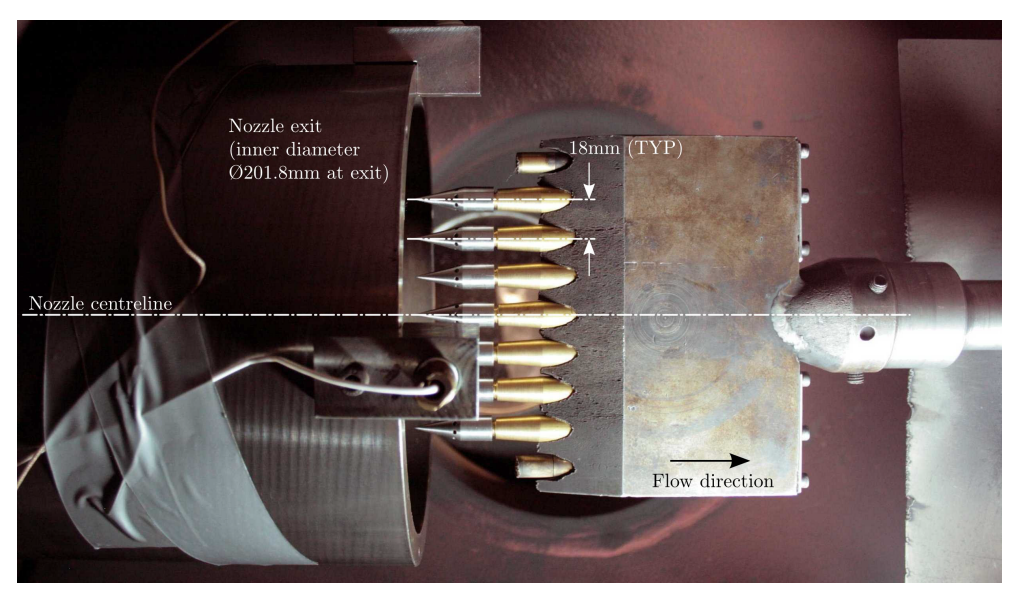

Figure 4.37: Pitot rake arrangement with conical glancing pressure probes for conditions testing. [30]

All experimentally measured values for condition 1 were found to be in good agreement with the computed values of Pitot and consequently the test gas properties calculated were deemed to be accurate. The computed shock velocities and pitot pressure were again in good agreement with measured values for condition 2 , however the static pressure computed at the entry of the nozzle was approximately 35\% lower than the measured value. Without any additional experimental data available at this stage, it was not possible to further constrain this condition and investigate the pressure discrepancy.

The total enthalpy at a point in a chemically reacting flow consists of kinetic, thermal and chemical components. For free stream flight conditions in hypervelocity flows, the thermal contribution is generally negligible upstream of any bow shock or viscous heating mechanisms. However, in laboratory simulated flows, there is commonly a significant amount of enthalpy tied up in chemical and thermal modes, due to the shock based processes involved in energising the flows. To give a useful comparison with flight conditions in ambient air, an equivalent flight velocity is defined which has the same total enthalpy as the laboratory condition. Using the enthalpy balance shown in Equation 4.11, where $U$ is the velocity in the expansion tube and $U_{e}$ is the flight equivalent velocity, flight equivalent velocities of $10.0 \mathrm{~km} / \mathrm{s}$ and $12.2 \mathrm{~km} / \mathrm{s}$ were calculated.

$$
H=C_{P} T+\frac{U^{2}}{2}=\frac{U_{e}^{2}}{2}
$$

An estimate of steady test time, free stream flow diameter or core flow size and variation of pitot pressure with distance from the exit of the nozzle can be experimentally measured using the pitot rake. These parameters are critical in the design of experiments that make the most of the core flow and test time. A steady test time is estimated to be from 
the arrival of the test gas until the arrival of higher pressure, unexpanded gas signalling the arrival of the $\mathrm{u}$-a wave. Using this approach and the pitot pressure traces shown in Figure 4.38, test times of $150 \mu$ s and $100 \mu$ s were estimated for condition 1 and condition 2 respectively.

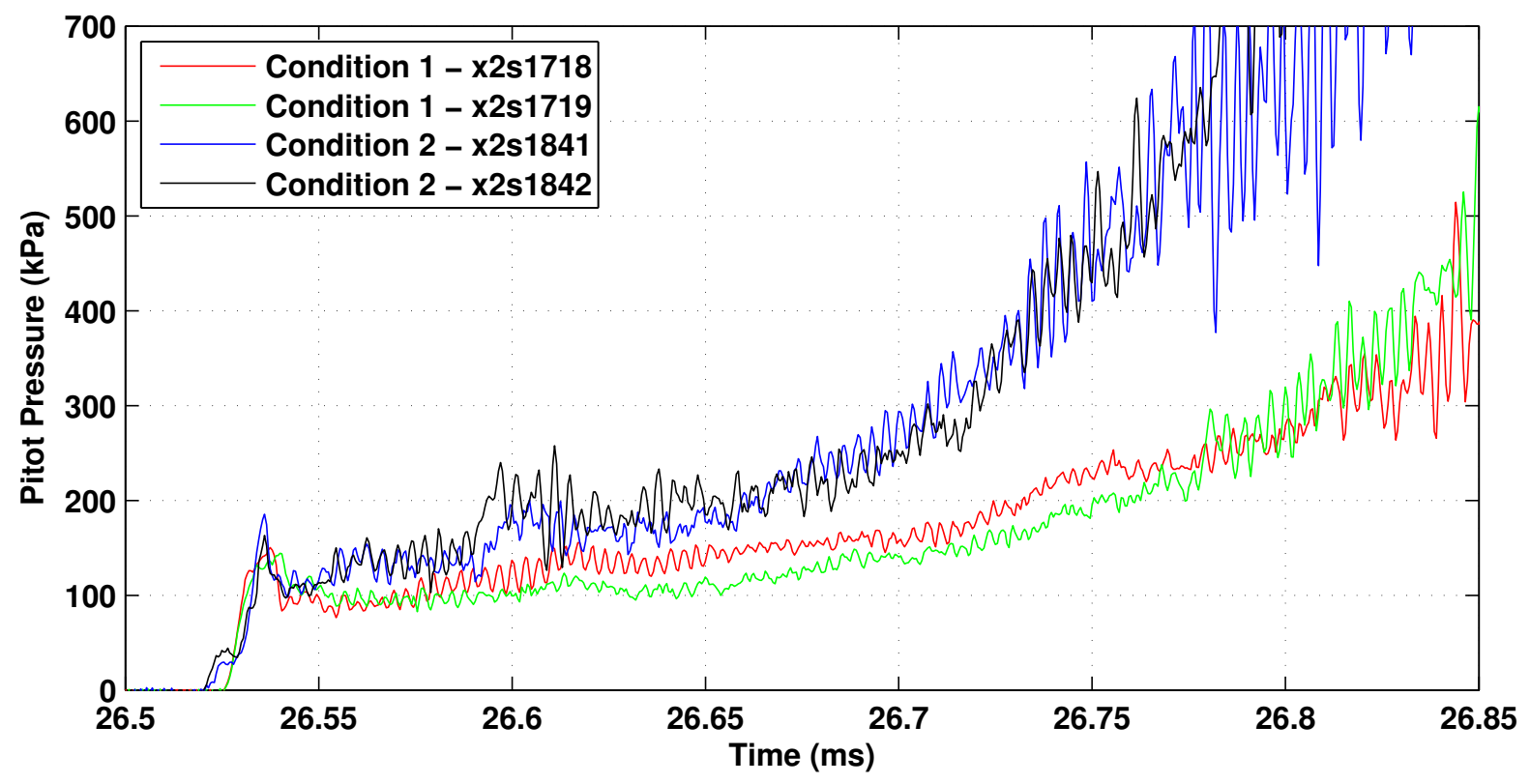

Figure 4.38: Experimental pitot traces for condition 1 and 2. Estimated test time for condition 1 is $26.55 \mathrm{~ms}$ to $26.70 \mathrm{~ms}$ and estimated test time for condition 2 is $26.55 \mathrm{~ms}$ to $26.65 \mathrm{~ms}$.

A study of core flow size and variation of pitot pressure with distance from the exit of the nozzle was conducted for condition 1 and the results are presented in Figure 4.39. A core flow size of $175 \mathrm{~mm}$ was estimated at the nozzle exit and this value dropped to approximately $100 \mathrm{~mm}$ at a distance of $200 \mathrm{~mm}$ from the nozzle exit. The core flow estimates were made using the pitot rake and therefore the spatial resolution is limited to the $18 \mathrm{~mm}$ between the pitot probes. The pitot pressure appears to remain consistent for up to $200 \mathrm{~mm}$ from the nozzle exit and begins to drop thereafter. 


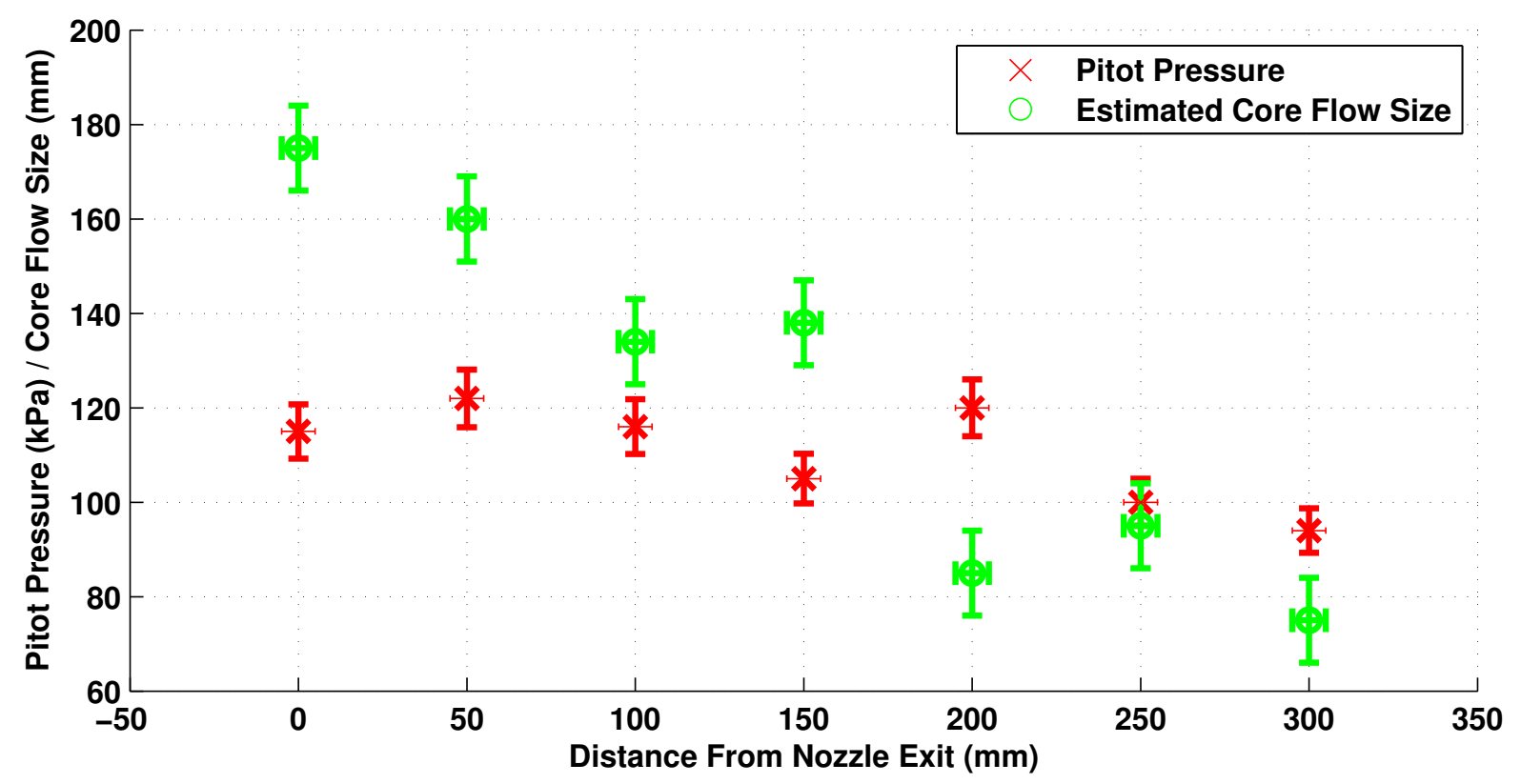

Figure 4.39: Measured core flow size and variation in pitot pressure at distances from the nozzle exit for condition 1

\subsubsection{Experimental and Computational Uncertainties}

There are numerous aspects to consider in the uncertainty analysis that directly affect the measured and computed values of the shock speeds, static pressure and pitot pressure. These include variation in fill pressures, uncertainties in pressure transducer measurements and location, record rate of the data acquisition system and effective area ratio of the nozzle. The shock speed is calculated based on shock arrival time, as determined by a large pressure rise, at the given locations of the pressure transducers. Knowing the uncertainty in pressure transducer location, $\delta \mathrm{x}$ is $\pm 2 \mathrm{~mm}$ and the record rate is $2.5 \mathrm{MHz}$, leading to an uncertainty, $\delta \mathrm{t}$ of $\pm 0.2 \mu \mathrm{s}$, a maximum and minimum possible shock speed can be determined using Equations 4.12 and 4.13 .

$$
\begin{aligned}
& U_{\text {maximum }}=\frac{\text { maximum dx }}{\text { minimum dt }}=\frac{(x 2+\delta x)-(x 1-\delta x)}{(t 2-\delta t)-(t 1+\delta t)}=\frac{\Delta x+2 \delta x}{\Delta t-2 \delta t} \\
& U_{\text {minimum }}=\frac{\text { minimum dx }}{\text { maximum dt }}=\frac{(x 2-\delta x)-(x 1+\delta x)}{(t 2+\delta t)-(t 1-\delta t)}=\frac{\Delta x-2 \delta x}{\Delta t+2 \delta t}
\end{aligned}
$$

The uncertainty in static pressure is a combination of the fluctuations in the measured values and the uncertainty of $2 \%$ in the pressure transducer reading. As evident in Figure 4.40 , the fluctuations in the measured values are greater than the $2 \%$ uncertainty of the transducer and therefore a $15 \%$ total uncertainty is estimated for the static pressure. 
These factors also apply to the measured pitot pressures but as the signal to noise ratio is higher, the uncertainty is estimated to be $10 \%$.
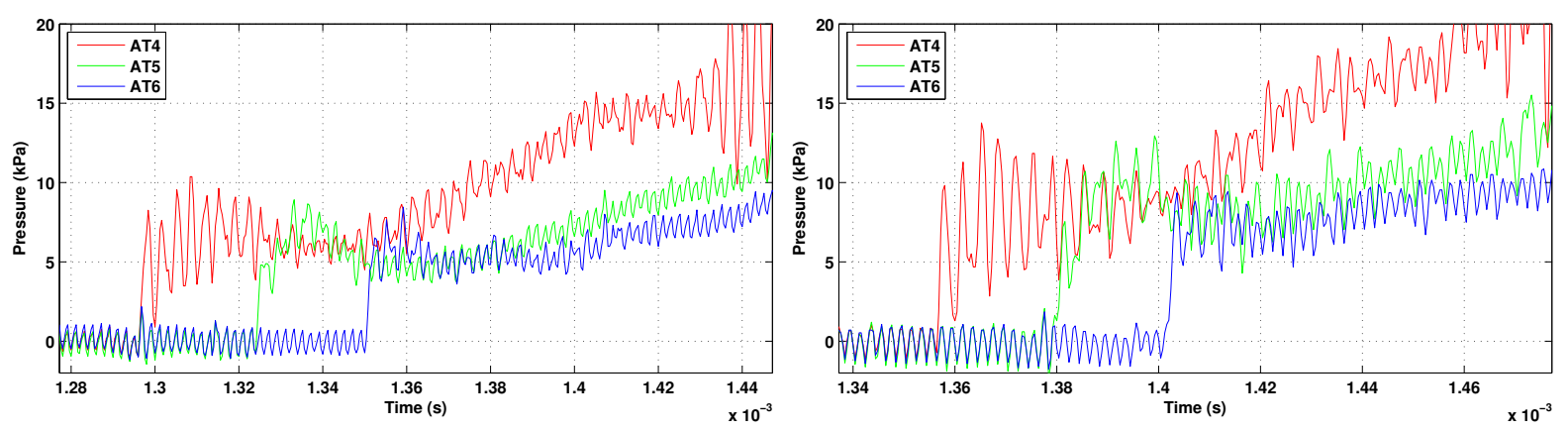

Figure 4.40: Measured static pressure values at the downstream end of the acceleration tube for condition 1 (Left) and condition 2 (Right).

The expansion process in the nozzle is condition sensitive, and depends on the Mach number and chemistry of the incoming flow, and on the displacement thickness of the boundary layer at the end of the expansion tube. The nozzle has a fixed geometrical area ratio of 5.03, but the effective expansion ratio of the core flow may be very different to that due to the wave processes involved, the displacement thickness which restricts the mass flow, and the growth of the boundary layer during the expansion [140]. Numerical simulation of the flows may take several paths, depending on time and resources available. Full axisymmetric numerical simulation in time accurate reacting flow can be very effective, but is generally too time consuming to run in parallel with an intensive experimental program. However, a quasi one-dimensional approach, using a nozzle area ratio chosen to match the pitot measurements from the test section can be used to give good estimates of the flow conditions. The nozzle area ratio selected for the computation was 4.1 as it provided the best match with experimental measurements.

\subsection{Shot Validity Analysis Tool}

A shot validity analysis tool was created to ensure the comparability of one experiment to another. This tool utilised a time resolved radiative emission measurement system, a high speed imaging camera, high temporal resolution shock speed measurement system, static pressure measurements at various locations on the expansion tube and a pitot pressure probe mounted on the model. The data acquisition system used in this study was National Instruments PXI-6133 and PXI-5105 data acquisition cards mounted in a PXI-1042Q chassis. All static and pitot pressure measurements as well as the time resolved radiative emission measurements were recorded on the PXI-6133 data acquisition card at 
a $2.5 \mathrm{MHz}$ record rate. The high speed imaging camera was capable of record rates up to $1 \mathrm{Mhz}$ and the high temporal resolution shock speed measurement system was monitored using the PXI-5105 card with a record rate of $60 \mathrm{MHz}$. Details of the time resolved radiative emission measurement system, high speed imaging camera and high temporal resolution shock speed measurement system are described in the following sections.

\subsubsection{Time Resolved Radiative Emission Measurement}

Time resolved radiative emission measurements are a powerful tool used to monitor the state of the shock layer formed over a model. This diagnostic tool is sensitive to changes in shock layer chemistry, temperature and consequently free stream conditions. To achieve temporally resolved emission measurements at a given wavelength, a monochromator is coupled with a photomultiplier tube (PMT). A PMT is a highly sensitive device capable of converting incident light to an electrical signal output with a response time in the order of several nano seconds. A schematic of its operation is shown in Figure 4.41. When coupled with a monochromator and a high speed recording device, it is possible to obtain the time evolution of a small spectral region during an experiment. The sensitive area is not divided into pixels and as such there is no spatial resolution possible with a PMT. The wavelength region to be studied is set through the grating position and the size of the exit slit on the monochromator.

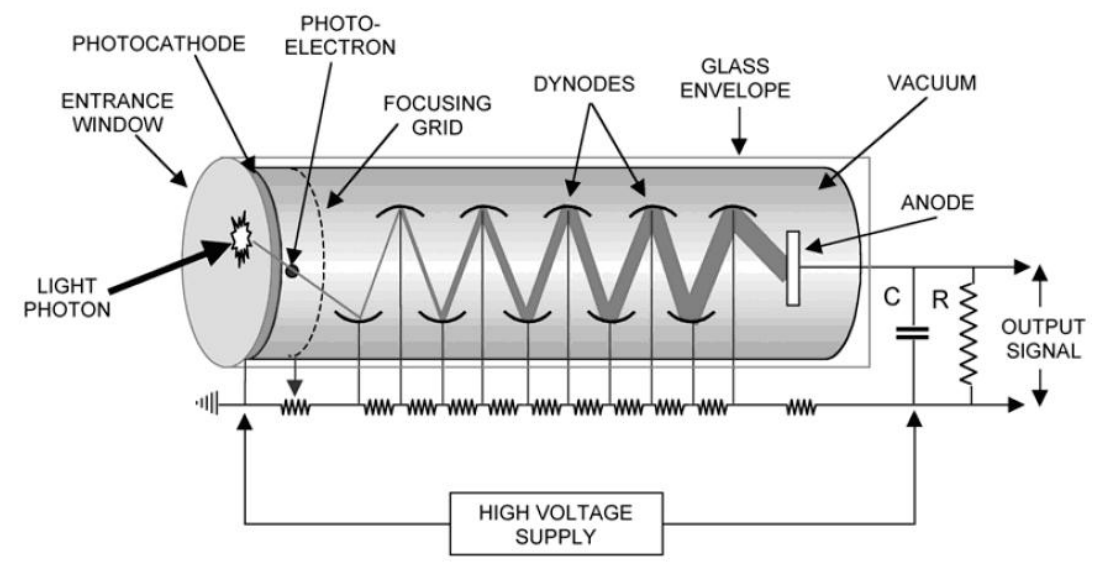

Figure 4.41: Schematic of a photomultiplier tube. 31]

The optical system depicted in Figure 4.42 was used to focus radiation into the visible and near infra-red (VNIR) spectrometer and instead of the diffracted radiation reaching the ICCD, a turning mirror within the monochromator was used to direct it to a PMT. The signal from the PMT was fed into a custom built amplifying terminator module to drive 
the signal through the coaxial cable to the data acquisition system. This was required to overcome the capacitance of the coaxial cable integrating the signal and saturating the recording channel. The impedance was also matched to remove any ringing through the cable that would introduce noise in the signal. The PMT circuit was channelled into the PXI-6133 card capable of recording at $2.5 \mathrm{MHz}$.

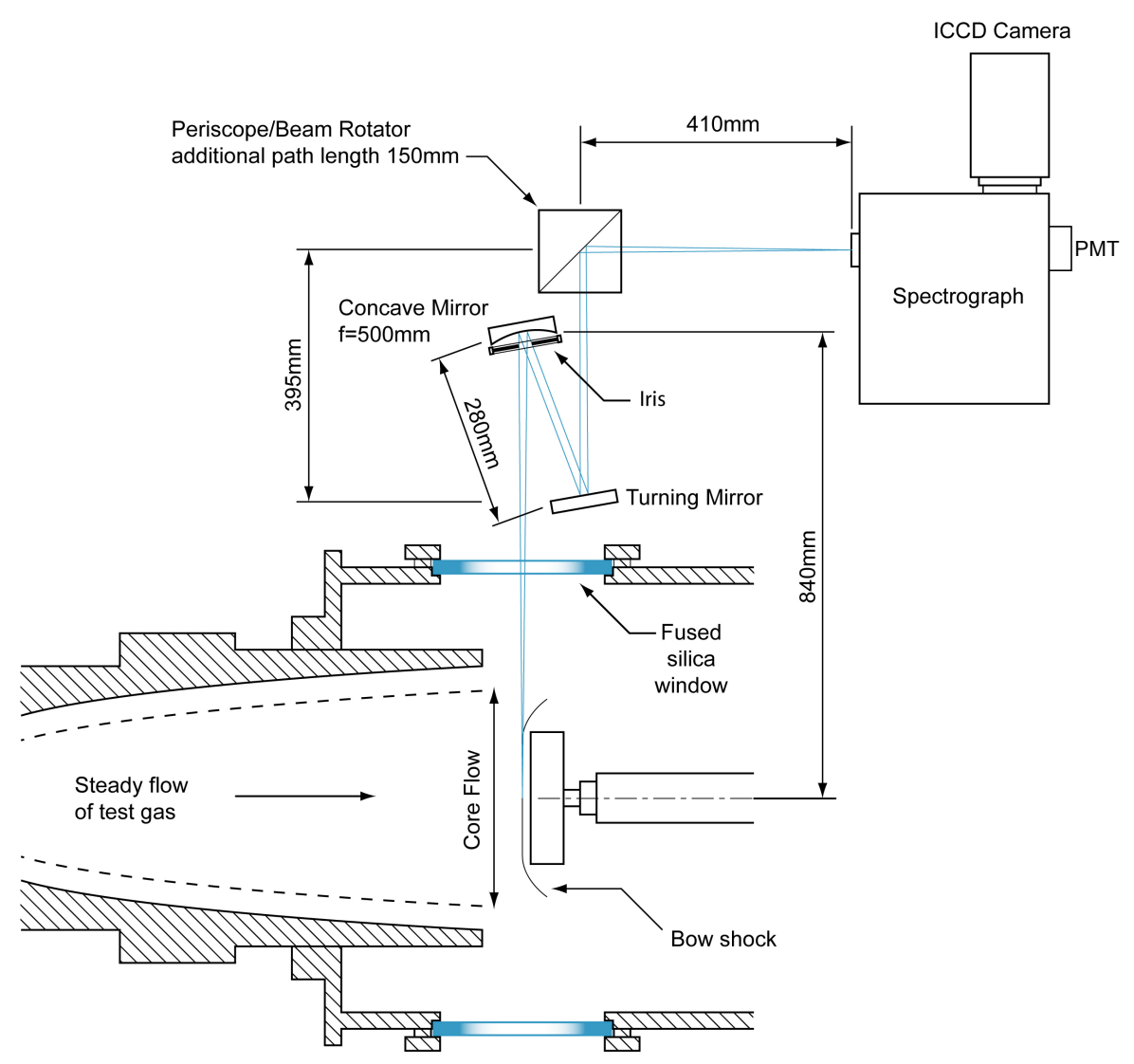

Figure 4.42: Optical system used with VNIR and UV spectroscopy system now used with a PMT. Adapted from 32 ]

\subsubsection{High Speed Imaging System}

To visualise shock wave interactions and monitor the steadiness of the radiating shock layer, a Shimadzu HPV-1 high speed camera with a Nikon Zoom-Nikkor 100-300mm $\mathrm{f} / 5.6 \mathrm{~s}$ lens was used. The HPV-1 is capable of recording at $1 \mathrm{MHz}$ for a duration of 100 frames at a resolution of $312 \times 260$ pixels. The camera is predominantly sensitive in the visible wavelength range with reduced sensitivity in the UV and NIR bands, as shown in Figure 4.43. A mirror was mounted above the test section so the camera could record a top down view to better observe any interactions between the fence and the model. 
Setting the aperture, frame rate and exposure time correctly allowed for the visualisation of the shock arrival, model start up, steady test time and arrival of the unsteady test gas marking the end of the test time.

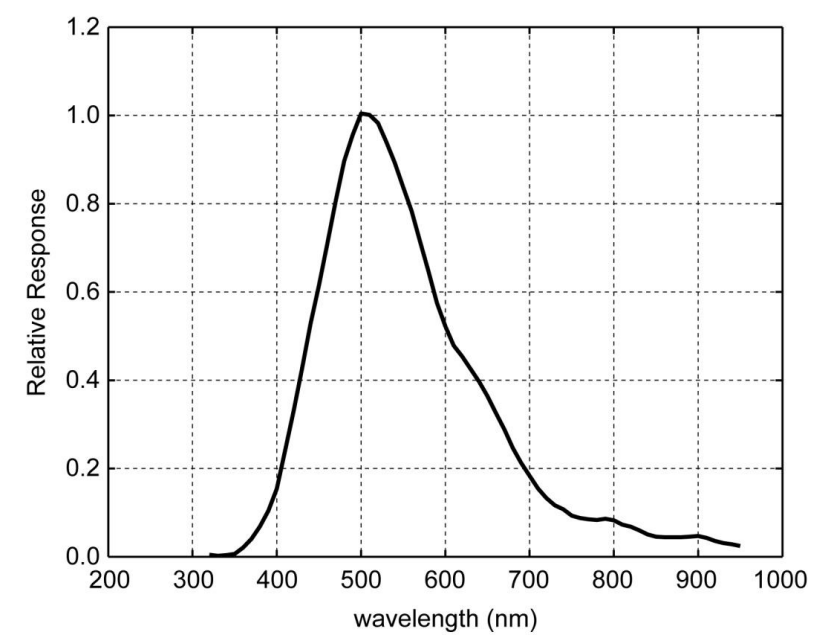

Figure 4.43: Spectral response of the Shimadzu HPV-1 high speed camera [33]

\subsubsection{High Temporal Resolution Shock Speed Measurement System}

As the radiation emitted by a gas is strongly linked to its temperature, which is almost entirely dependent on the velocity of the free stream flow in the hypersonic regime, it is imperative to measure the shock speed with as much accuracy as possible. To complement the current pressure transducer array used to measure shock speeds, an additional four instrumentation ports were designed and installed at the downstream end of the acceleration tube. The instrumentation ports are capable of housing photodiodes or pressure transducers or a combination of the two. For this study, only photodiodes were experimented with as a faster response time was expected due to the lower switching time when compared with pressure transducers. In addition to this, a new data acquisition system was installed allowing for electrical signals to be recorded at $60 \mathrm{MHz}$.

An amplifier and terminator system was designed to couple the photodiodes with the data recorder ensuring high temporal resolution. A schematic of the electronics designed for each photodiode is shown in Figure 4.45. The photodiodes selected were Silicon PIN Photodiode SFH 229FA from OSRAM [34].

Using this system, the uncertainty in measured shock wave velocity between two photodiodes, calculated using the equations outlines in Section 4.2 .1 , was found to be $\pm 150 \mathrm{~m} / \mathrm{s}$ 

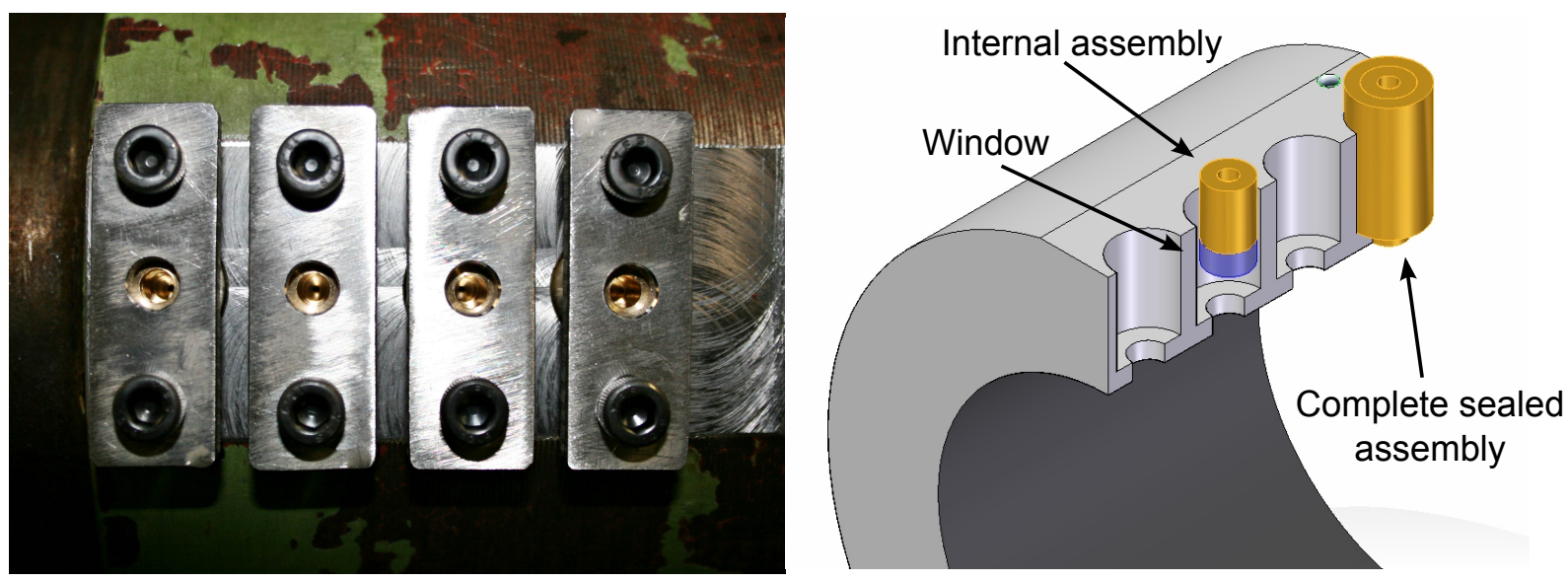

Figure 4.44: Top down view of installed photodiode mount assembly (left). Schematic of photodiode mounts assembly (right).

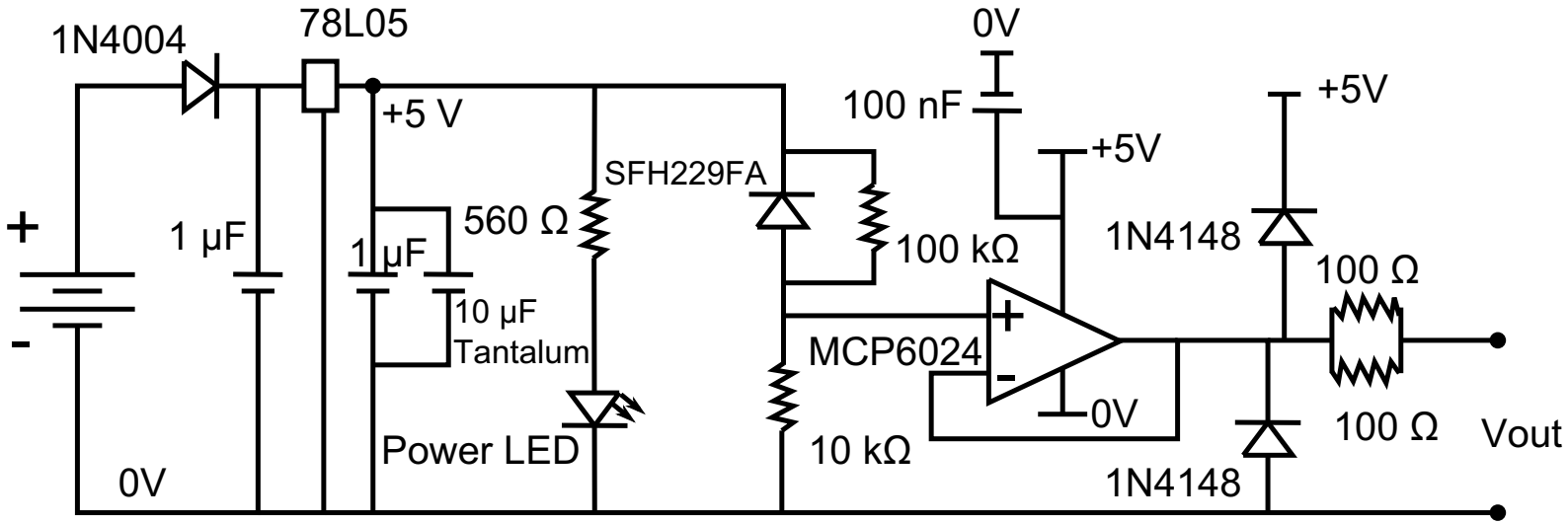

Figure 4.45: schematic of the amplifier and terminator circuit designed for each photodiode. Adapted from Zander [34] 
for condition 1 and $\pm 220 \mathrm{~m} / \mathrm{s}$ for condition 2 . Using only two adjacent points in the array results in a higher uncertainty than the pressure transducer system already in place. However if the shock speed is measured between the first and last photodiodes in the array, the uncertainty drops to $\pm 51 \mathrm{~m} / \mathrm{s}$ for condition 1 and $\pm 74 \mathrm{~m} / \mathrm{s}$ for condition 2 . This uncertainty can be further reduced by plotting the shock arrival times at each location and fitting the values with a linear curve, resulting in a substantial reduction in uncertainties of measured shock velocities over the previous approach.

\section{High Temporal Resolution Shock Speed Measurement System Results}

The shock velocities measured using the photodiode array produced inconsistent results between the photodiodes and the velocities measured using the pressure transducers. The output signal from the photodiodes did not produce a sharp rise as the shock wave travelled past its location. The slow rise produced did not allow for a consistent trigger level to be set. A curve fitting approach that assumed a constant rise time for each photodiode was attempted but also failed to produce consistent values. It was concluded that the slow rise time was a result of the capture angle being too large, non-planar shape of the shock front and the fact the photodiodes measure radiation across the entire diameter of the tunnel, unlike the pressure transducers that measure pressure only at the edge of the flow. Based on these factors, the data obtained using the photodiode array was not used in the shot validity analysis.

\subsubsection{Sample Results}

The set of shock layer and free stream monitoring systems described were used to test the comparability of each shot. At the bottom of each spectral dataset presented in Appendices C and D, a shot validity analysis plot is displayed showing the scaled intensity of the time resolved radiative emission measurement system, pitot pressure and trigger time for each image recorded on the high speed camera. The exposure time of the VUV emission spectroscopy system is also displayed in the plot to indicate when the spectral data was acquired. A sample shot validity analysis is shown in Figure 4.47. 


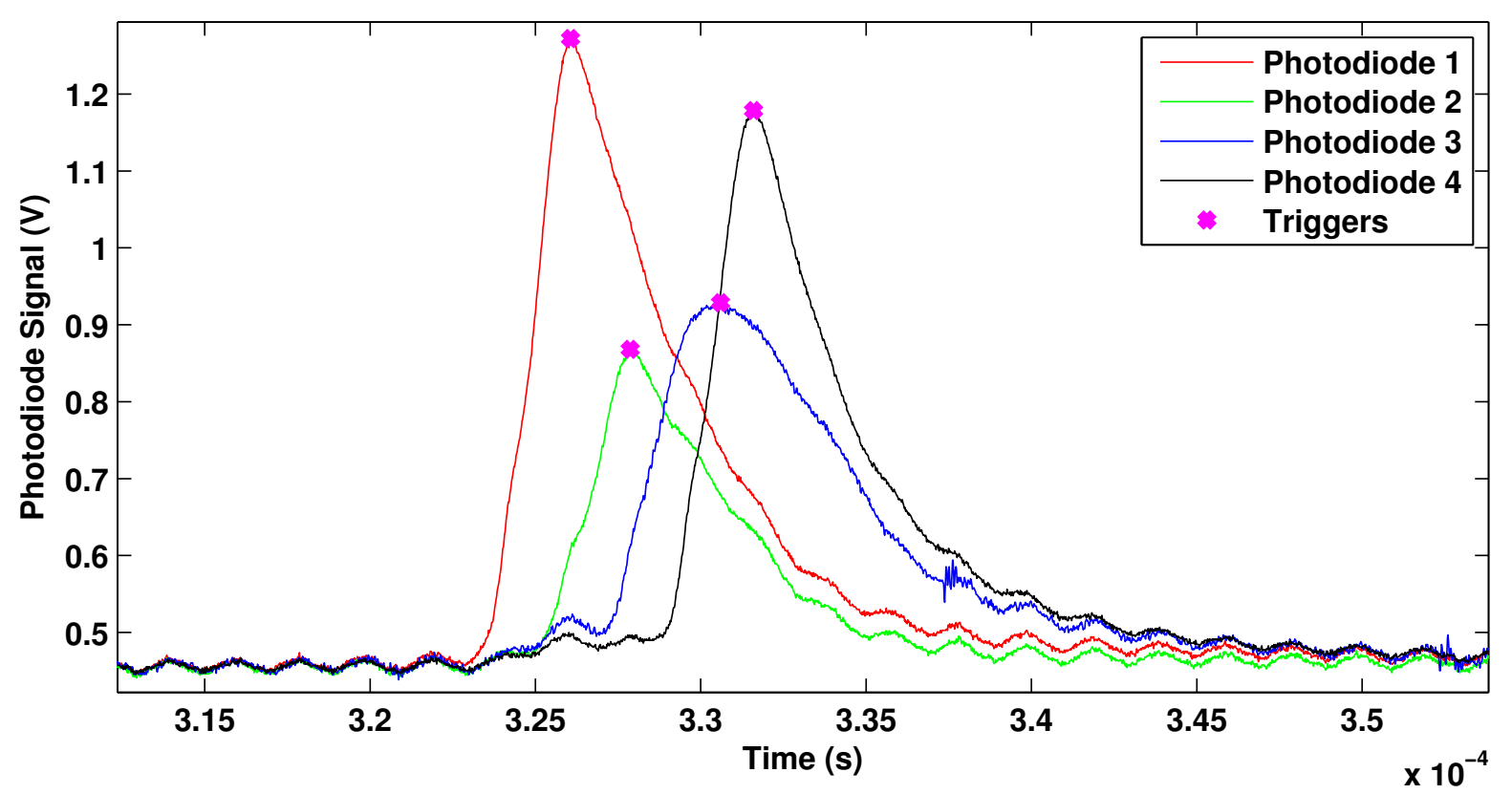

Figure 4.46: Signal traces from the photodiode array. Shock speed calculations between the three pairs of photodiodes produces $11.1 \mathrm{~km} / \mathrm{s}, 7.3 \mathrm{~km} / \mathrm{s}$ and $20.0 \mathrm{~km} / \mathrm{s}$. A curve of best fit predicts $10.1 \mathrm{~km} / \mathrm{s}$.

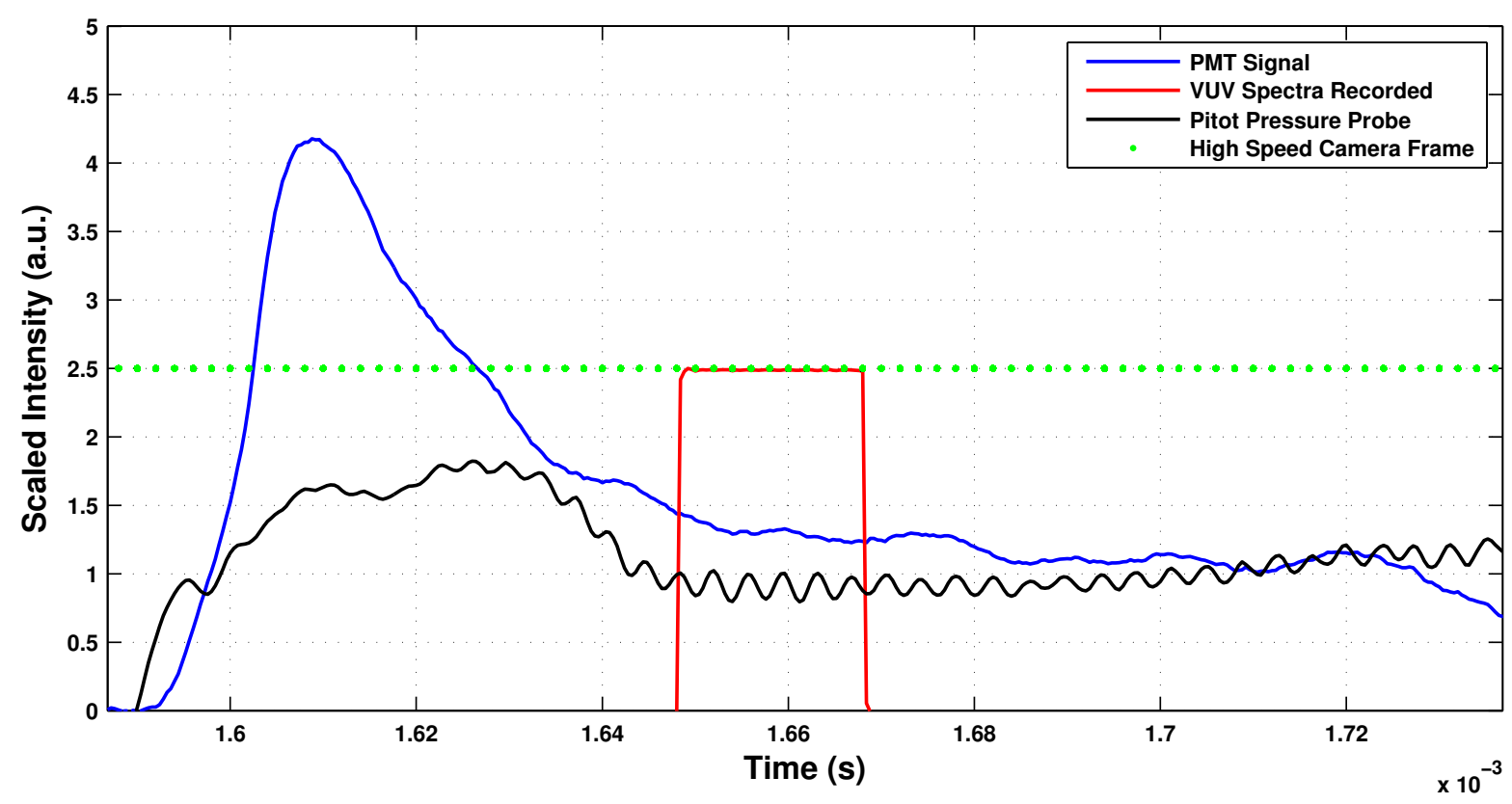

Figure 4.47: Shot validity graph showing ICCD exposure time in reference to the scaled intensity of the PMT data, the pitot pressure measured at the model and the points in time an image is recorded on the high speed camera. 


\subsection{Repeatability}

Shot-to-shot variation was tested by measuring spectral radiation across a $90 \mathrm{~mm}$ long cylindrical model. Radiative emission in the VUV spectral range is strongly dependent on the temperature of the radiating flow field due to its strongly self-absorbing nature. Consequently, the radiative emission is strongly coupled with the velocity of the free stream flow making a VUV emission spectrum a highly sensitive method to test experiment repeatability. For these experiments the evacuated light tube and fence system was removed to prevent damage to windows. With the evacuated light tube removed, there was absorption of the emitted radiation in the cold regions of the free stream flow and outer regions of the test section. Provided the free stream conditions produced and test section pressure remained consistent, the level of absorption would remain constant each test and could therefore be disregarded for repeatability tests.

To ensure an accurate measure of repeatability, all spectral lines from contaminants were identified and removed, as shown in Figure 4.48. Common contaminants found in X2 flow are carbon and iron from the walls of the tunnel and aluminium from the secondary diaphragm. Fragments from the secondary diaphragm are able to enter the test gas flow as the diaphragm is positioned in front of the test gas at the time of firing. Whilst only vaporised aluminium is able to accelerate fast enough to be in the radiating shock layer during test time, the intensity with which it radiates in the VUV makes these lines apparent on the spectrum. 


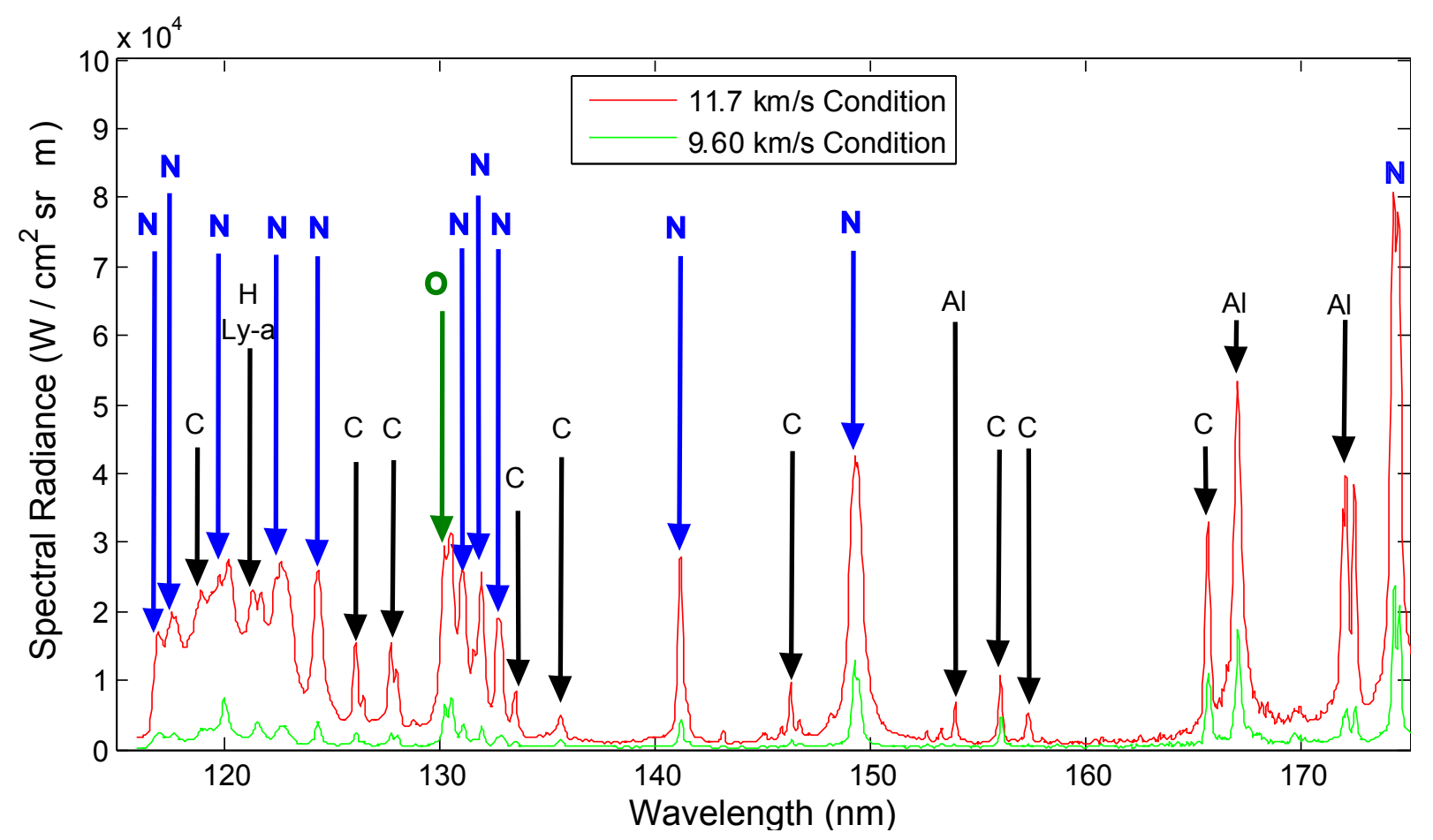

Figure 4.48: Chemical species responsible for each spectral line observed.

\subsubsection{Condition 1}

Spectral measurements taken during the repeatability study for condition 1 are shown in Figure 4.49 .

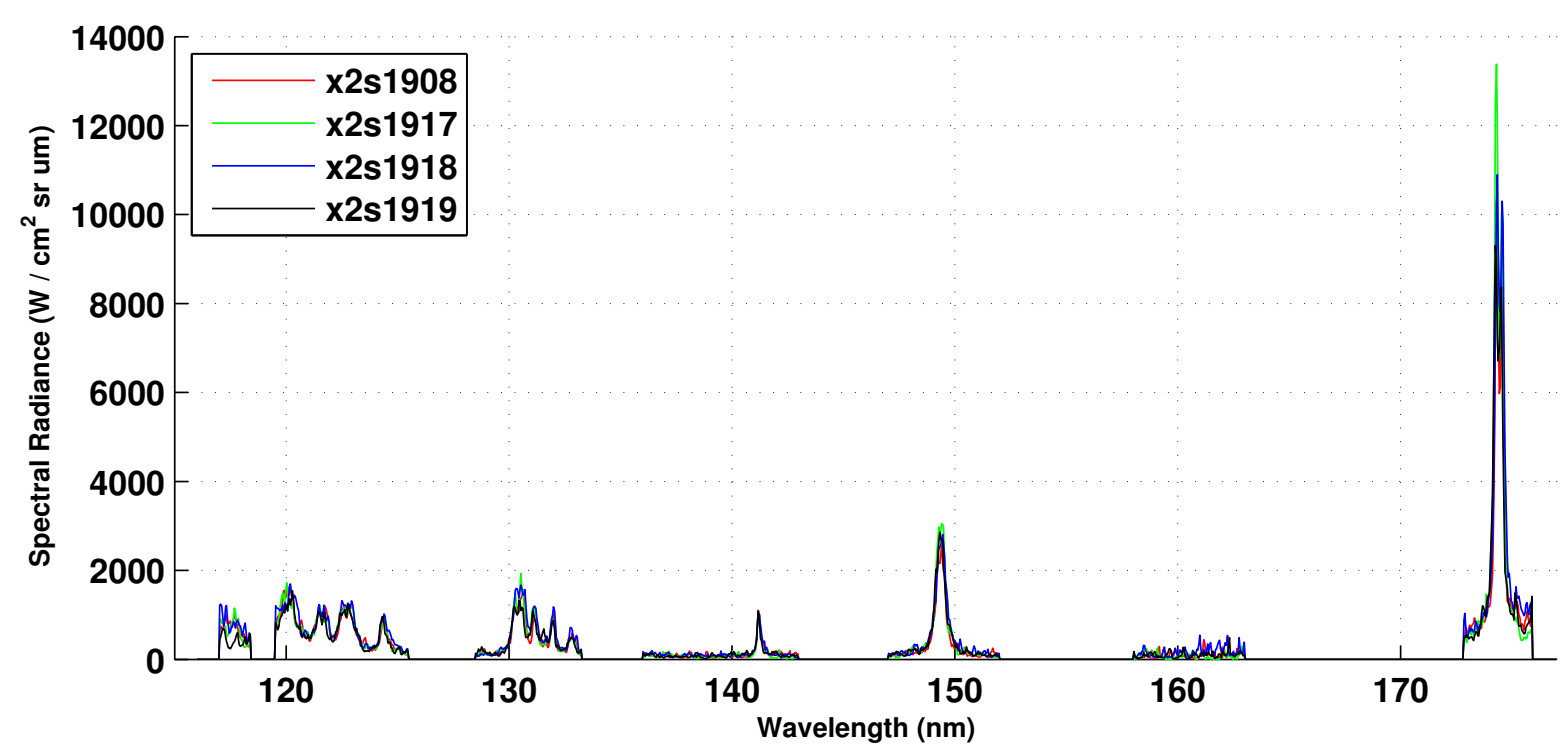

Figure 4.49: Results from repeatability study of condition 1 across the VUV range.

Figure 4.49 indicates the repeatability for condition 1 across the VUV range is better than 
15\%. This high level of repeatability is highlighted when observing the more sensitive shorter wavelength half of the VUV spectrum shown in Figure 4.50. Complete datasets for all shots used in the repeatability study are presented in Appendix C.

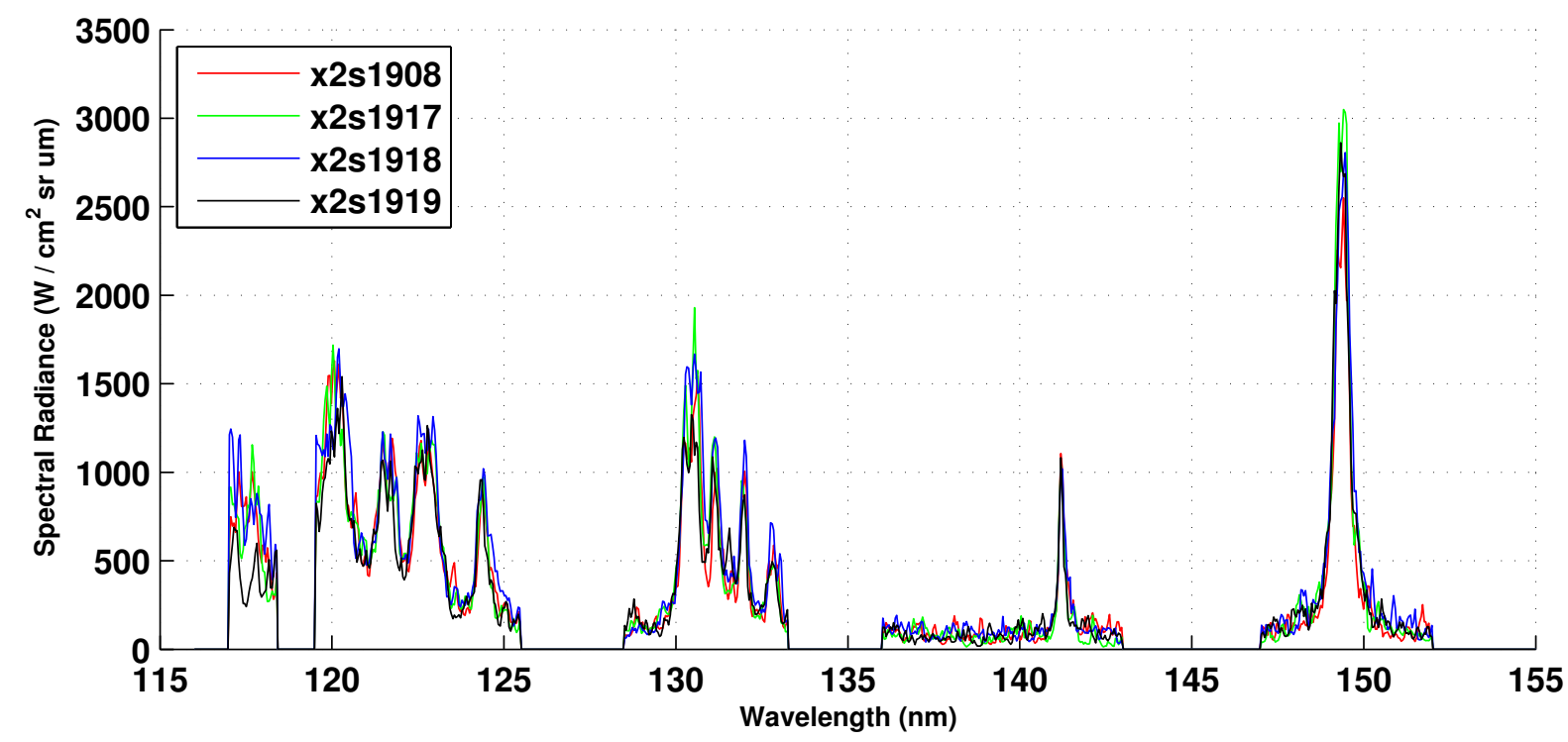

Figure 4.50: Results from repeatability study of condition 1 for the lower half of the VUV range.

The total integrated spectral radiance is another highly sensitive indicator of repeatability. As shown in Table 4.5, the maximum variance in total integrated spectral radiance for this condition is $20 \%$.

Table 4.5: Total integrated counts and normalised integrals for condition 1 when observing across the surface of the model.

\begin{tabular}{c|cc}
\hline Shot & Total Integrated Counts & Normalised By x2s1918 \\
\hline x2s1908 & $3.81 \mathrm{e}+05$ & 0.85 \\
$\mathrm{x} 2 \mathrm{~s} 1917$ & $4.08 \mathrm{e}+05$ & 0.91 \\
$\mathrm{x} 2 \mathrm{~s} 1918$ & $4.49 \mathrm{e}+05$ & 1.00 \\
$\mathrm{x} 2 \mathrm{~s} 1919$ & $3.68 \mathrm{e}+05$ & 0.82
\end{tabular}

Integration across wavelength bands can also be used to investigate the repeatability of regions across the spectrum and investigate if a particular region is more sensitive than others. For this analysis, six wavelength ranges were selected as outlined in Table 4.6 and the results of this are analysis are shown in Figure 4.51. From Figure 4.51 it can be deduced that each region has a maximum variance of $20 \%$ between shots with the exception of shot x2s1919 in the wavelength range of $115 \mathrm{~nm}$ to $119 \mathrm{~nm}$. 
Table 4.6: Wavelength regions selected for integration analysis.

\begin{tabular}{l|llllll}
\hline Wavelength Range & 1 & 2 & 3 & 4 & 5 & 6 \\
\hline Wavelength $(\mathrm{nm})$ & $<119$ & $119-127$ & $127-135$ & $135-145$ & $145-155$ & $>155$ \\
\hline
\end{tabular}

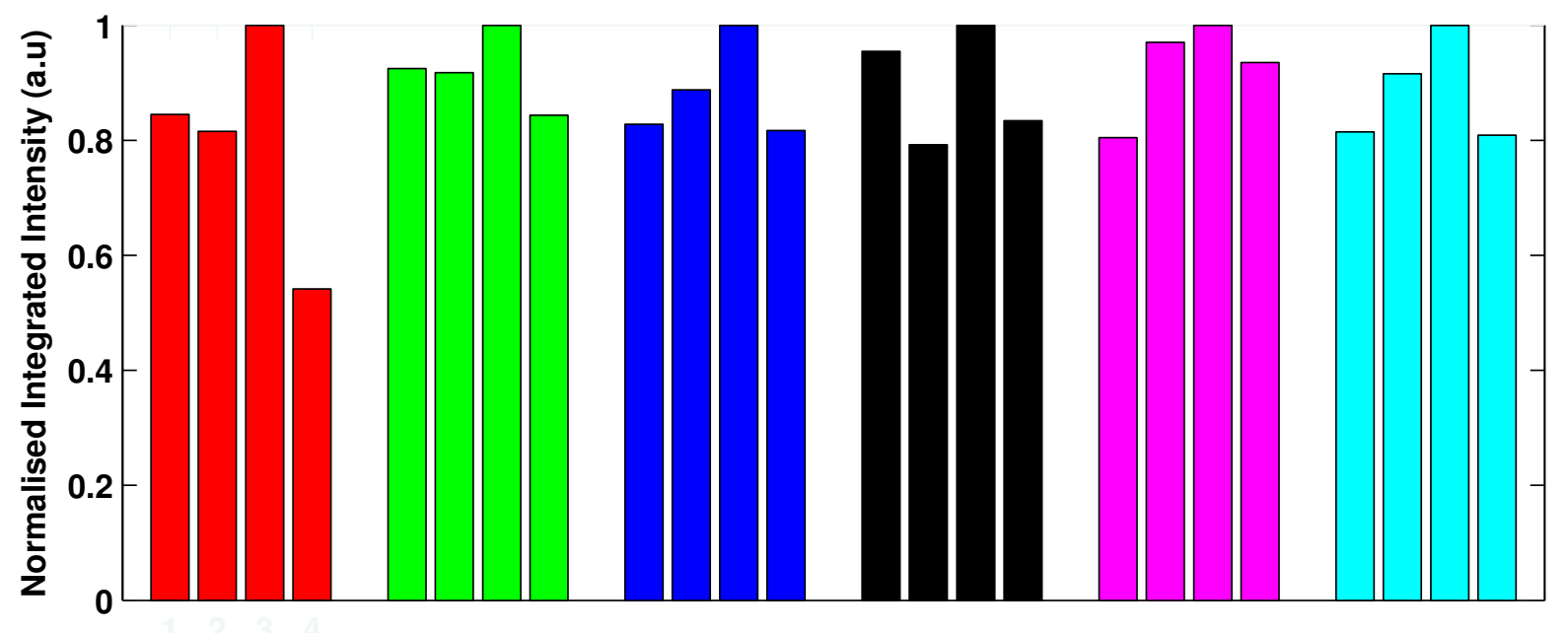

Figure 4.51: Results from the integration analysis of condition 1 across the VUV wavelength bands. Wavelength range 1 - red, wavelength range 2 - green, wavelength range 3 - blue, wavelength range 4 - black, wavelength range 5 - magenta, wavelength range 6 cyan. Shot order from left to right is x2s1908, x2s1917, x2s1918 and x2s1919. 
An estimate of temperature variation required to create a $20 \%$ variation in integrated spectral intensity was carried using Planck's law between $120 \mathrm{~nm}$ and $180 \mathrm{~nm}$. This was possible as spectral lines in the VUV are expected to be strongly self-absorbing due to the Planck curve limit at the pressures and temperatures achieved in the expansion tube. The results of this calculation, shown in Figure 4.52 , indicate a variation of less than $200 \mathrm{~K}$ is required to achieve $20 \%$ variation in integrated spectral intensity at $10,000 \mathrm{~K}$. Consequently, it can be stated that the integrated spectral intensity is temperature sensitive to the power of 9.2 at $10,000 \mathrm{~K}$ when integrated between $120 \mathrm{~nm}$ and $180 \mathrm{~nm}$.

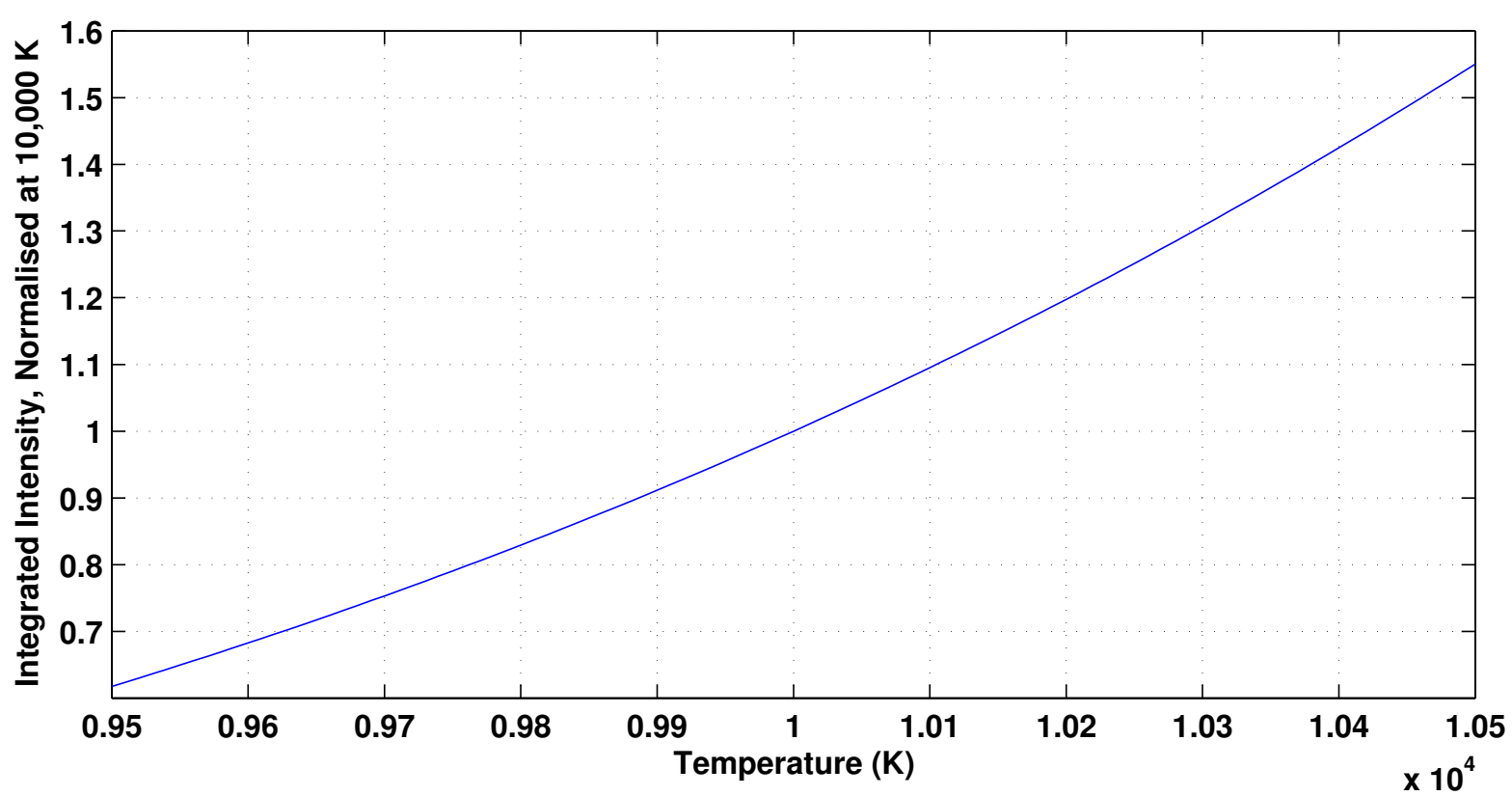

Figure 4.52: Integrated values of the Planck curve between $120 \mathrm{~nm}$ and $180 \mathrm{~nm}$ at various temperatures, normalised at 10,000 K.

\subsubsection{Condition 2}

As shown in Figure 4.53, the results from the repeatability study of condition 2 show a decrease in measured intensity each shot. This consistent reduction in intensity is attributed to surface degradation of the viewing window occurring. Potential reasons for window damage occurring at condition 2 and not at condition 1 may include the higher energy flow at this condition causing more diaphragm and contaminant particles in the test section to come into contact with the viewing window and the expansion fan angle at the nozzle exit directing more flow towards the viewing port. Due to the variation in transmission of the viewing window between shots, a measure of repeatability for condition 2 cannot be quantified. 


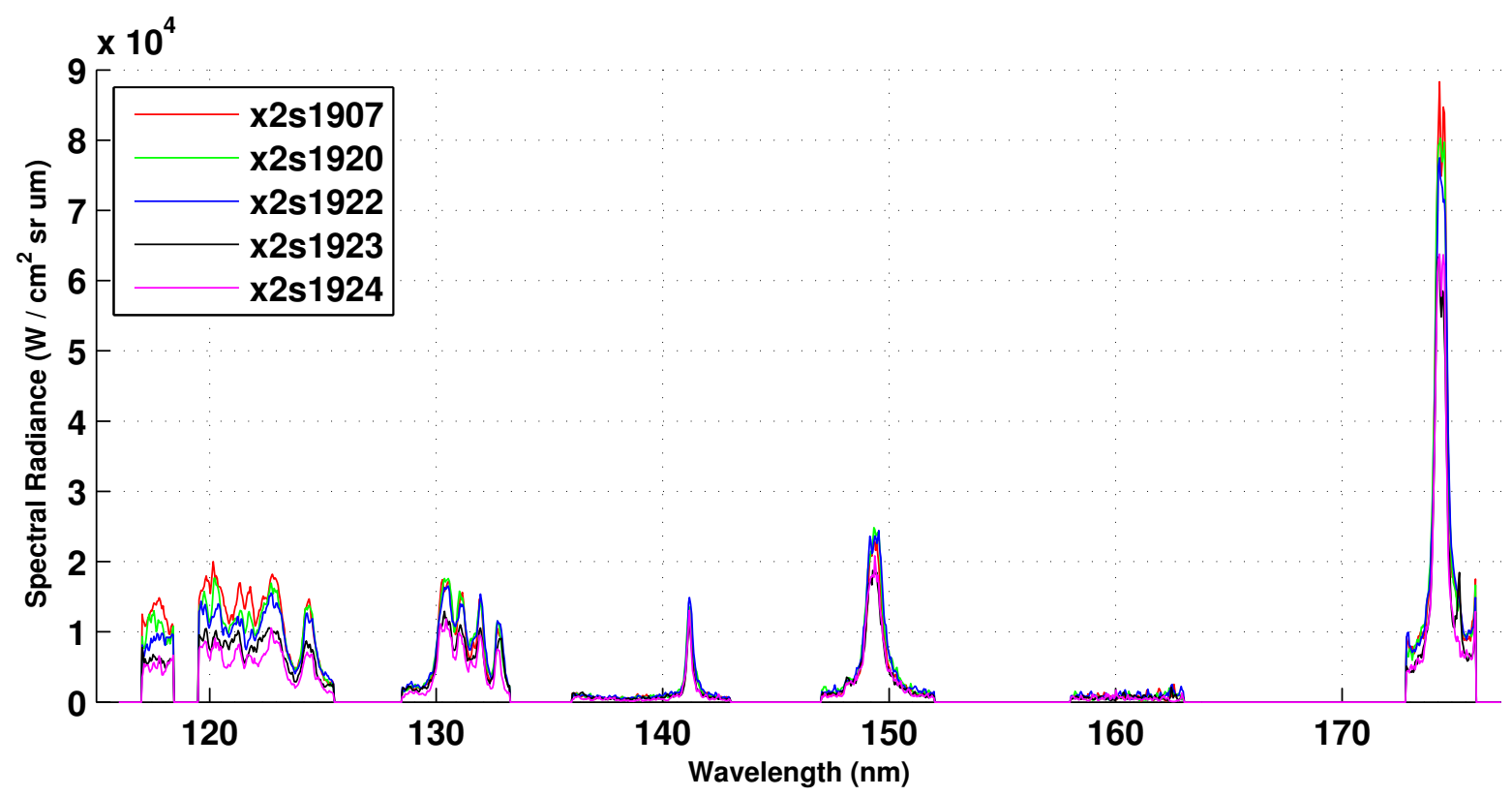

Figure 4.53: Results from repeatability study of condition 2.

\subsection{Uncertainty Analysis}

Two driving uncertainty factors are the repeatability of the expansion tube flow conditions and calibration of the emission spectroscopy system. As the VUV spectrum is highly sensitive to temperature and consequently flow conditions, the repeatability study shows that the expansion tube is capable of producing VUV emission spectra to within $15 \%$. As outlined by Brandis [141], five terms are the most influential in calculating the level of uncertainty in emission spectroscopy; radius of the aperture, distance to the aperture, the width of the radiating slug of gas, optical magnification and optical losses. As the shock layer is formed over models with precise dimensions, the width of the radiating shock layer is known accurately. The other four uncertainties are features of the optical focussing system. Through the use of an in situ calibration, all of these uncertainties are accounted for as all of the components are calibrated with any imperfections that they may contain. Consequently the emission spectroscopy system uncertainty is limited to the uncertainty of the calibration lamp itself and these values are outlined in Table below. 
Table 4.7: Relative uncertainty of the calibration lamp [36].

\begin{tabular}{lll}
\hline Spectral Range $(\mathrm{nm})$ & Spectral Bandwidth $(\mathrm{nm})$ & $\begin{array}{l}\text { Relative Expanded } \\
\text { Uncertainty }(\mathrm{k}=2)\end{array}$ \\
\hline $116.0-120.4$ & 0.8 & $14 \%$ \\
$120.6-122.6$ & 0.8 & $36 \%$ \\
$122.8-170.0$ & 0.8 & $14 \%$ \\
$172.0-410.0$ & 1.6 & $7 \%$ \\
\hline
\end{tabular}

\subsection{Expansion Tube Experimental Datasets}

The following section presents data obtained on the X2 expansion tube along with the process undertaken to convert a raw image to the final spectrum analysed. The test conditions produced and trigger times for each experiment were monitored using the time resolved radiation measurement system, high speed camera and pitot pressure probe mounted on the model as described in Section 4.1. Combined these tools were used in a shot validity analysis to ensure experimental data obtained was comparable. The data obtained was calibrated using the method outlined in Section 4.3 and full data sets for all shots selected for analysis are presented in Appendix D.

\subsubsection{Sample Image Processing}

Figure 4.54 shows the raw image acquired on the ICCD when viewing across a shock wave. The horizontal axis of the image represents wavelength and the vertical axis represents the spatial location. Through the identification of known spectral lines and the measured dispersion of the grating, it is possible to convert the horizontal pixels to wavelength. Conversion of the vertical pixels to spatial locations is carried out using the known size of each pixel and the magnification of the external optical system. The orientation of each image results in the shock front appearing towards the top of the image and the model edge towards the bottom.

The raw intensity measured is recorded as counts on the ICCD. There is a level of background counts produced on each image due to electronic and thermal noise. To remove this background, a dark frame is taken after each experiment and subtracted from the measured spectrum. This approach does not account for any spurious light that may reach the ICCD during the experiment. Due to the short test times of the expansion tube it is not possible to change the position of the grating during the test gas flow to account 


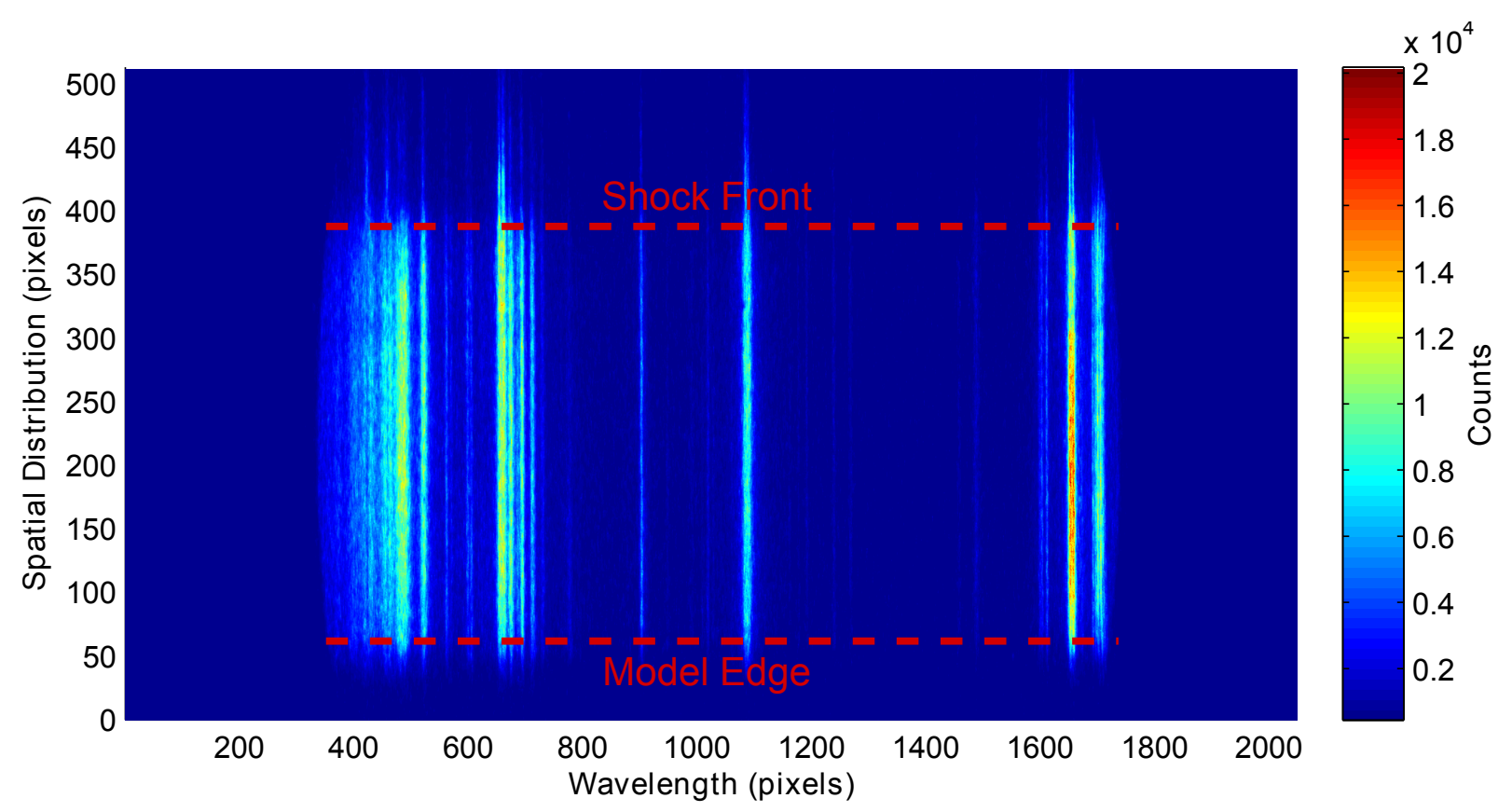

Figure 4.54: Raw image acquired on the ICCD across at flat $90 \mathrm{~mm}$ model at condition 2 .

for this. By dividing the counts recorded on each pixel by the calculated calibration factor at the corresponding wavelength, it is possible to obtain the spectral radiance measured. This conversion is illustrated by Equation 4.3, shown again below.

$$
\text { calibration factor }(\lambda)=\operatorname{system} \operatorname{sensitivity}(\lambda)=\frac{\text { ICCD counts }}{\text { known spectral radiance }}
$$

A measure of spectral emission as a function of spatial position is achieved through the integration of each row. This allows for the selection of a spatially uniform emission region over which the spatially resolved spectrum can be averaged to provide a high signal to noise ratio spectrum. Each image used in this study is presented in Appendices $\mathrm{C}$ and $\mathrm{D}$ and contains a spatial emission profile of the shock layer and red lines indicating the region spatially averaged across. The spatially averaged spectrum obtained through this process is presented as the resulting spectrum for each experiment. 

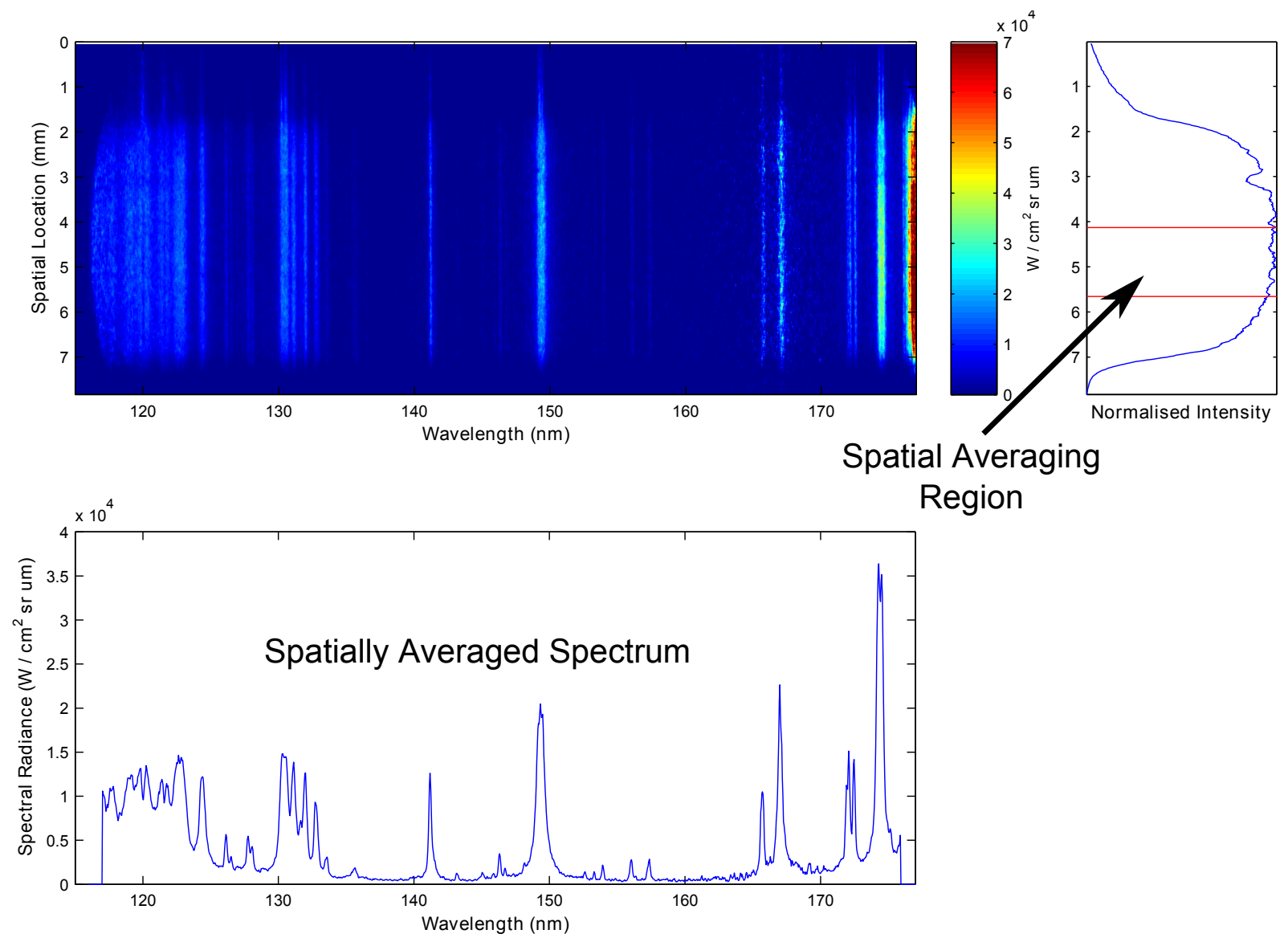

Region

Figure 4.55: (Top Left) - Calibrated image across a $90 \mathrm{~mm}$ model at condition 2. (Top Right) - Spatial distribution of recorded emission and spatial region averaged across. (Bottom) - Spatially averaged spectrum produced. 


\subsubsection{Through Surface and Across Surface Spectral Comparison}

The following section presents spectral data measured across the surface of a model superimposed on spectral data measured through the surface. By comparing spectra in this manner, it was possible to investigate absorption by molecular oxygen within the boundary layer. The integrated values of the spectral data obtained were also analysed to calculate a ratio for the integrated heat fluxes of both optical paths measurements. All contaminants in the spectra, as outlined in Section 4.5.1, have been removed from the data presented and the calculation of integrated values.

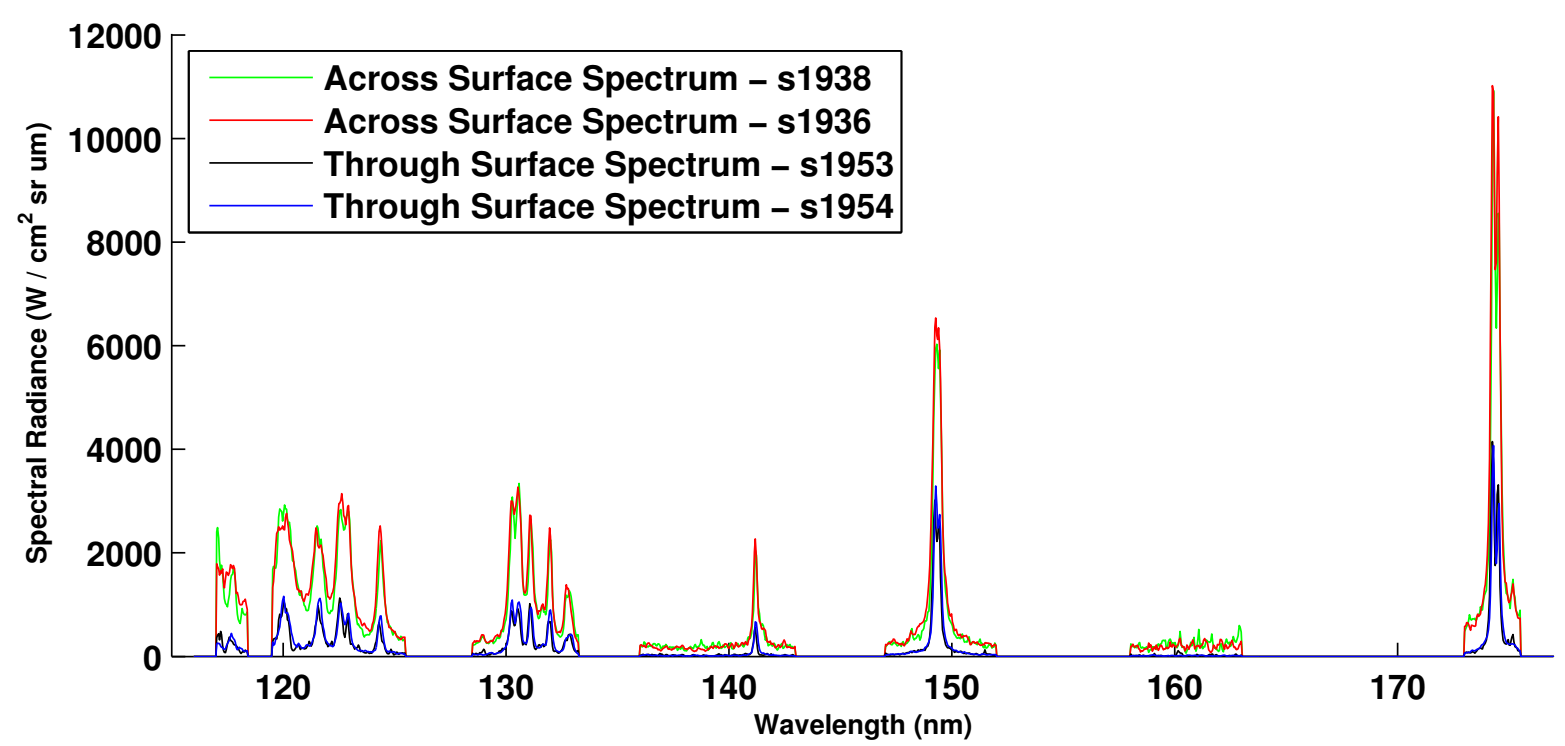

Figure 4.56: Comparison of across surface and through surface spectral measurements at condition 1.

The depth of radiating flow field when measuring across the surface is $90 \mathrm{~mm}$ and approximately $5.5 \mathrm{~mm}$ when measuring through the surface of the model, resulting in a ratio of approximately 16. The measured spectral intensity ratios of approximately three and four for the two conditions, are much lower than the ratio of the depths of radiating flow field. The reduced spectral intensity ratio is attributed to the strongly self-absorbing nature of VUV radiation and the line of sight through the non-equilibrium region observed through the surface of the model. Simulations and experiments investigating the self-absorption length scales and the size of the non-equilibrium and equilibrium regions of the shock will be presented in Section 4.8.3 and Chapter 5 . 


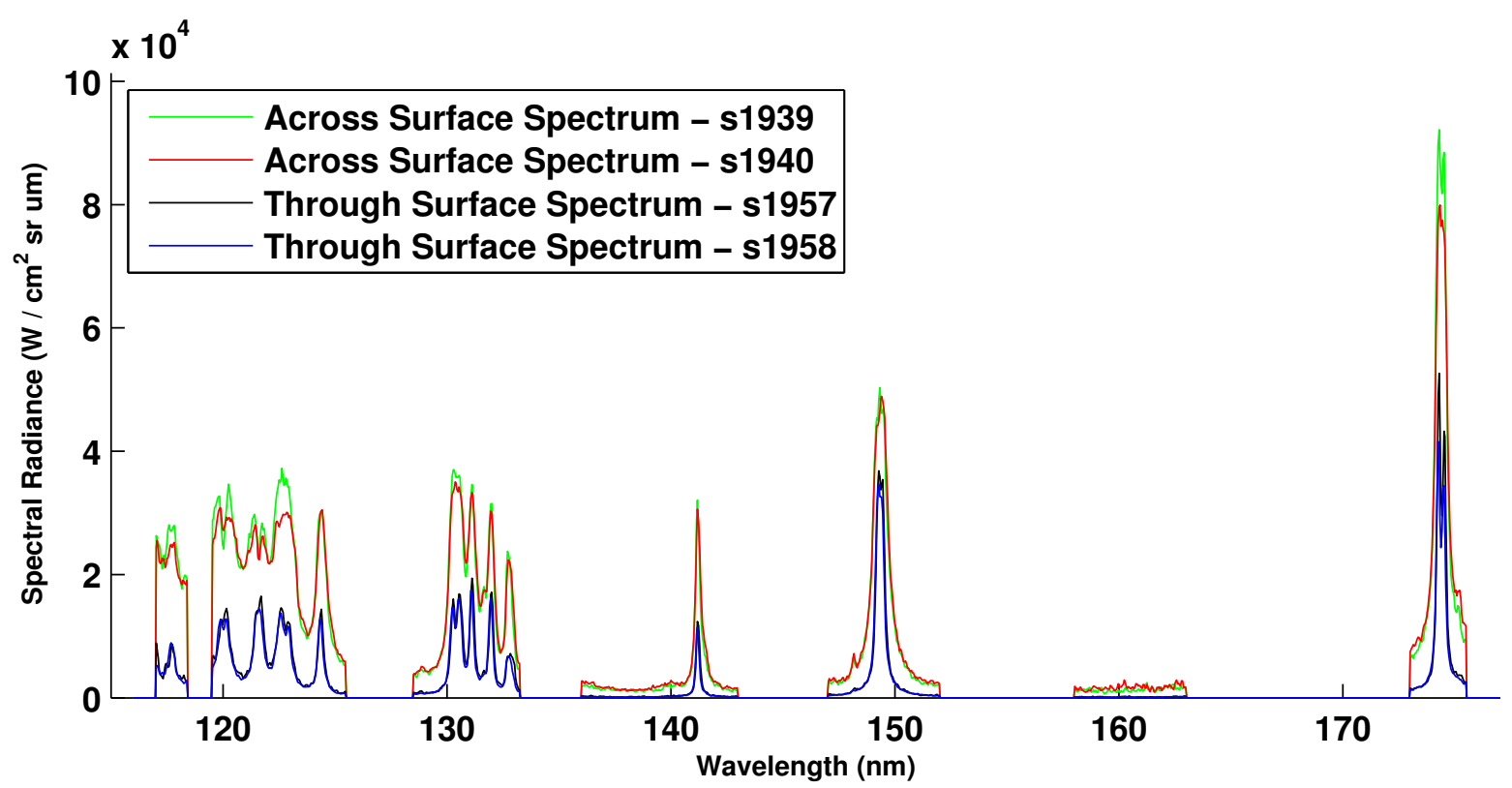

Figure 4.57: Comparison of across surface and through surface spectral measurements at condition 2 .

\section{Absorption by Molecular Oxygen in Boundary Layer}

To investigate potential absorption by molecular oxygen in the boundary layer, nine atomic lines presented in Table 4.8 were compared. Intensity ratios measured through and across the surface were calculated and plotted in Figure 4.58. Scaled oxygen absorption cross-section values were superimposed upon the ratios to determine if the ratios vary with respect to wavelength in the same manner as the molecular oxygen absorption cross-sections.

Table 4.8: Nine atomic lines selected for analysis of boundary layer absorption

\begin{tabular}{ll}
\hline Wavelength $(\mathrm{nm})$ & Radiating Atom \\
\hline 120.0 & Nitrogen \\
124.3 & Nitrogen \\
130.5 & Oxygen \\
131.1 & Nitrogen \\
132.0 & Nitrogen \\
141.2 & Nitrogen \\
149.3 & Nitrogen \\
174.3 & Nitrogen \\
174.5 & Nitrogen \\
\hline
\end{tabular}




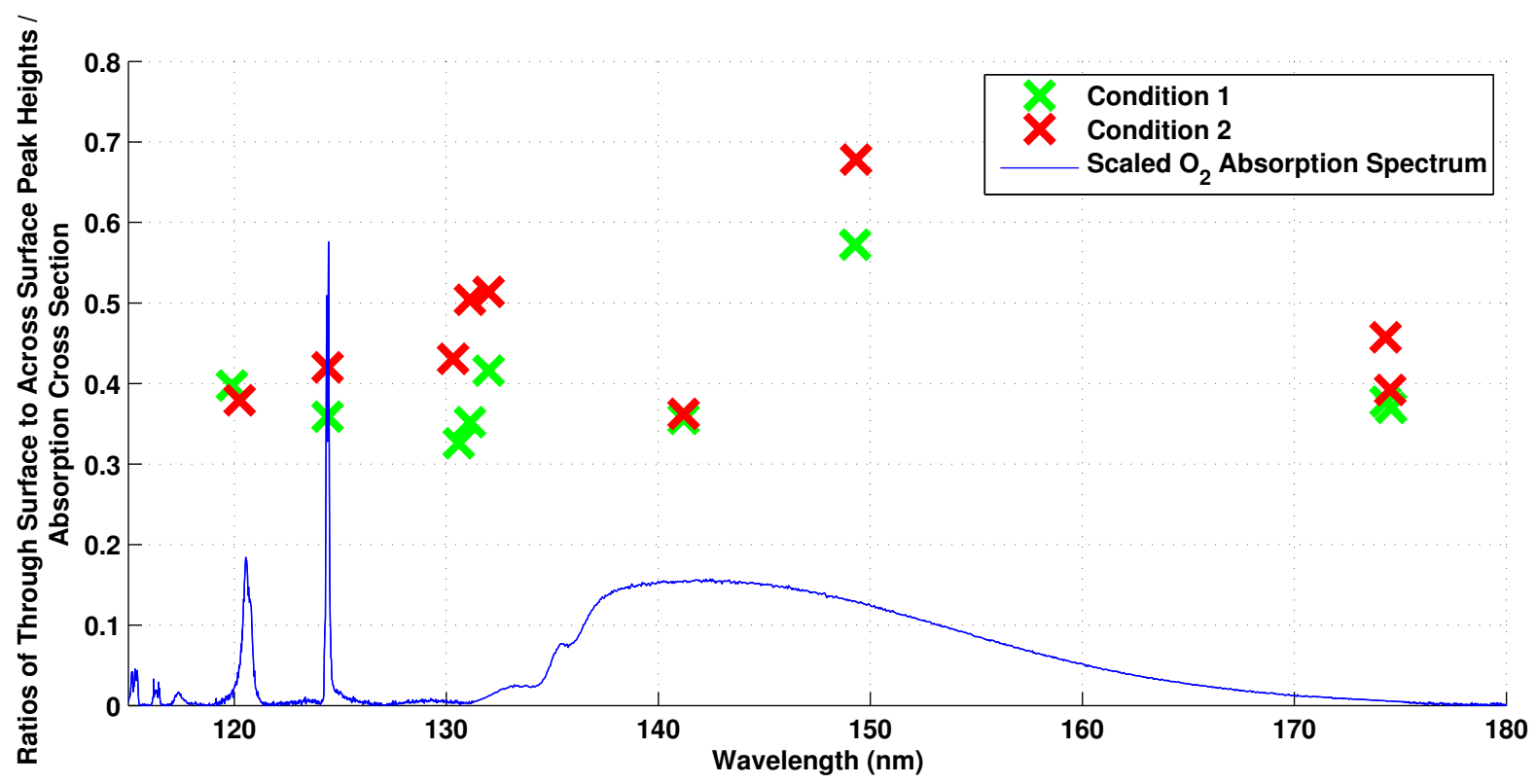

Figure 4.58: Ratios of the selected peak heights when observing through the surface compared to across the surface. Molecular oxygen absorption spectrum superimposed to investigate boundary layer oxygen absorption.

The intensity ratios do not follow the same trend as the molecular oxygen absorption spectrum, indicating a lack of absorption in the boundary layer. This lack of absorption suggests that oxygen is not recombining in the boundary layer.

\section{Integrated Heat Flux}

Through the integration of the spectral radiance measured through both optical paths, it is possible to calculate a correlation between radiation observed across a shock wave and the radiation incident on a model. Such a correlation provides a test bed for computational analysis and validation for measurements made across a shock wave in shock tunnels being applied in the design of thermal protection systems. The integrated values also provide an indicator of the repeatability achieved in these experiments and are presented in Tables 4.9 and 4.10 .

For condition 1, a quarter of the radiation measured in the equilibrium region is found to be incident on the surface. For the second faster condition, this fraction increases to almost a third. To investigate the processes increasing this fraction, further computational modelling is required. The shot to shot variation in these integrated results is substantially lower than the variation observed in the repeatability study. This improvement is attributed to the inclusion of the evacuated light tube removing the potentially 
Table 4.9: Total VUV radiative heat flux measurements across the surface in the equilibrium region and incident on the surface having passed through the boundary layer for condition 1.

\begin{tabular}{l|l|l|l|l}
\hline & Shot 1 & Shot 2 & Average & Discrepancy \\
\hline Across Surface $\left(W / \mathrm{cm}^{2} s r\right)$ & 28.8 & 30.8 & 29.8 & $6.39 \%$ \\
Through Surface $\left(W / \mathrm{cm}^{2} s r\right)$ & 7.65 & 7.23 & 7.44 & $5.76 \%$ \\
Ratio & 0.27 & 0.23 & 0.25 & \\
\hline
\end{tabular}

Table 4.10: Total VUV radiation heat flux measurements across the surface in the equilibrium region and incident on the surface having passed through the boundary layer for condition 2 .

\begin{tabular}{l|l|l|l|l}
\hline & Shot 1 & Shot 2 & Average & Discrepancy \\
\hline Across Surface $\left(W / \mathrm{cm}^{2} s r\right)$ & 400 & 398 & 399 & $0.46 \%$ \\
Through Surface $\left(W / \mathrm{cm}^{2} s r\right)$ & 116 & 129 & 123 & $9.35 \%$ \\
Ratio & 0.29 & 0.32 & 0.31 & \\
\hline
\end{tabular}

variable amount of absorption in the core flow and the test section, as well as any damage sustained by the window during the repeatability tests.

\subsubsection{Self-Absorption Experiments}

Experiments were carried out measuring radiation across the surface of a two-dimensional model with three different lengths; $90 \mathrm{~mm}, 45 \mathrm{~mm}$ and $20 \mathrm{~mm}$. Using the spectral line intensity peaks outlined in Table 4.8, it is possible investigate which lines are selfabsorbing. If a line is not self-absorbing, the ratio of spectral line intensity peaks should be the same as the ratio between the lengths of the model. If the ratio of spectral line intensity peaks is higher than the physical ratio, it is an indication of self-absorption occurring. The closer to unity the ratio, the stronger the level of self-absorption.

From Figure 4.59 it is evident that all of the lines for condition 2 are self-absorbing to some degree as they are well above the ratios of model lengths. In the case of condition 1 , the ratio between the $90 \mathrm{~mm}$ model and $20 \mathrm{~mm}$ model shows a large variation across the wavelength range. This could be due to the three dimensional edge effects becoming more influential for the smaller $20 \mathrm{~mm}$ model or the smaller model not reaching equilibrium emission due to the reduced shock stand off. To investigate if the shock layer has reached equilibrium, a comparison with the equilibrium region measured across the surface of the 

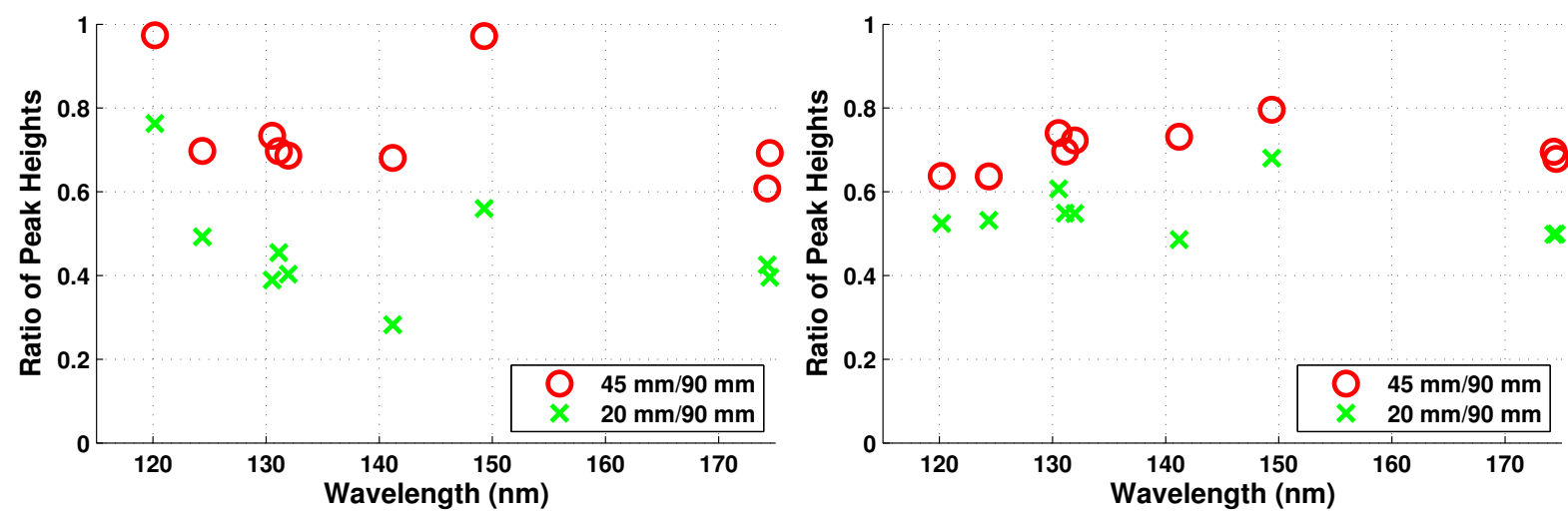

Figure 4.59: (Left) - Ratios of spectral line peaks for condition 1. (Right) - Ratios of spectral line peaks for condition 2. Nitrogen lines selected are $120.0 \mathrm{~nm}, 124.3 \mathrm{~nm}$, $131.1 \mathrm{~nm}, 132.0 \mathrm{~nm}, 141.2 \mathrm{~nm}, 149.3 \mathrm{~nm}, 174.3 \mathrm{~nm}$ and $174.5 \mathrm{~nm}$. Oxygen line at $130.5 \mathrm{~nm}$.

square model can be made. This is possible as the square model produced a larger shock stand-off which in turn produced a larger apparent equilibrium region. Comparisons of the spectra obtained across the equilibrium region of the square and cylindrical models are presented in Figures 4.60 and 4.60

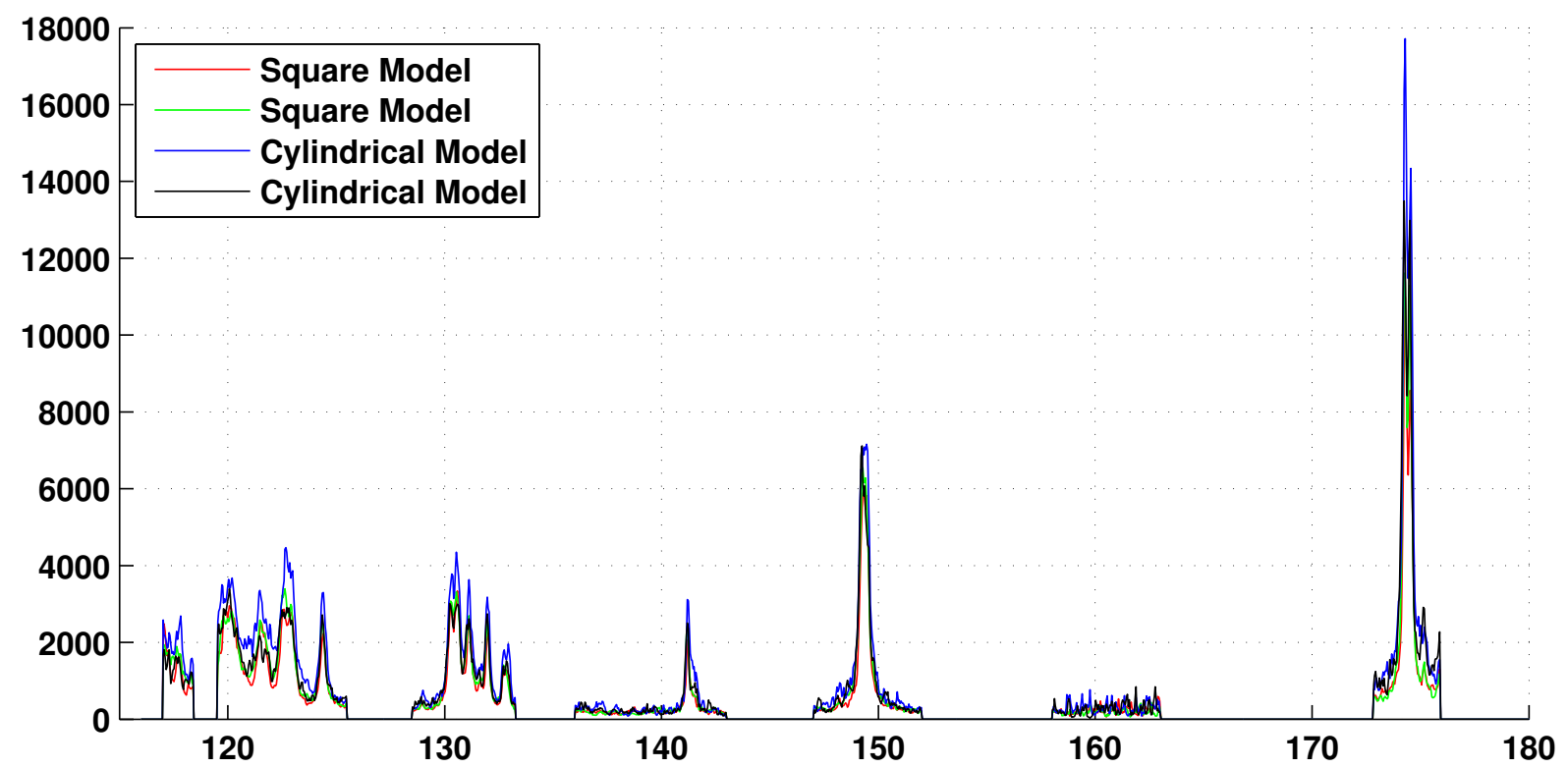

Figure 4.60: Comparison of VUV emission measured across a $90 \mathrm{~mm}$ square and cylindrical model for condition 1.

Figure 4.60 shows a good match between the spectral measurements at condition 1, indicating the cylindrical model has reached steady state emission in the shock layer. The results for condition 2, displayed in Figure 4.61, show a higher spectral emission measurement for the cylindrical model and this indicates the temperature and gas chemistry may 


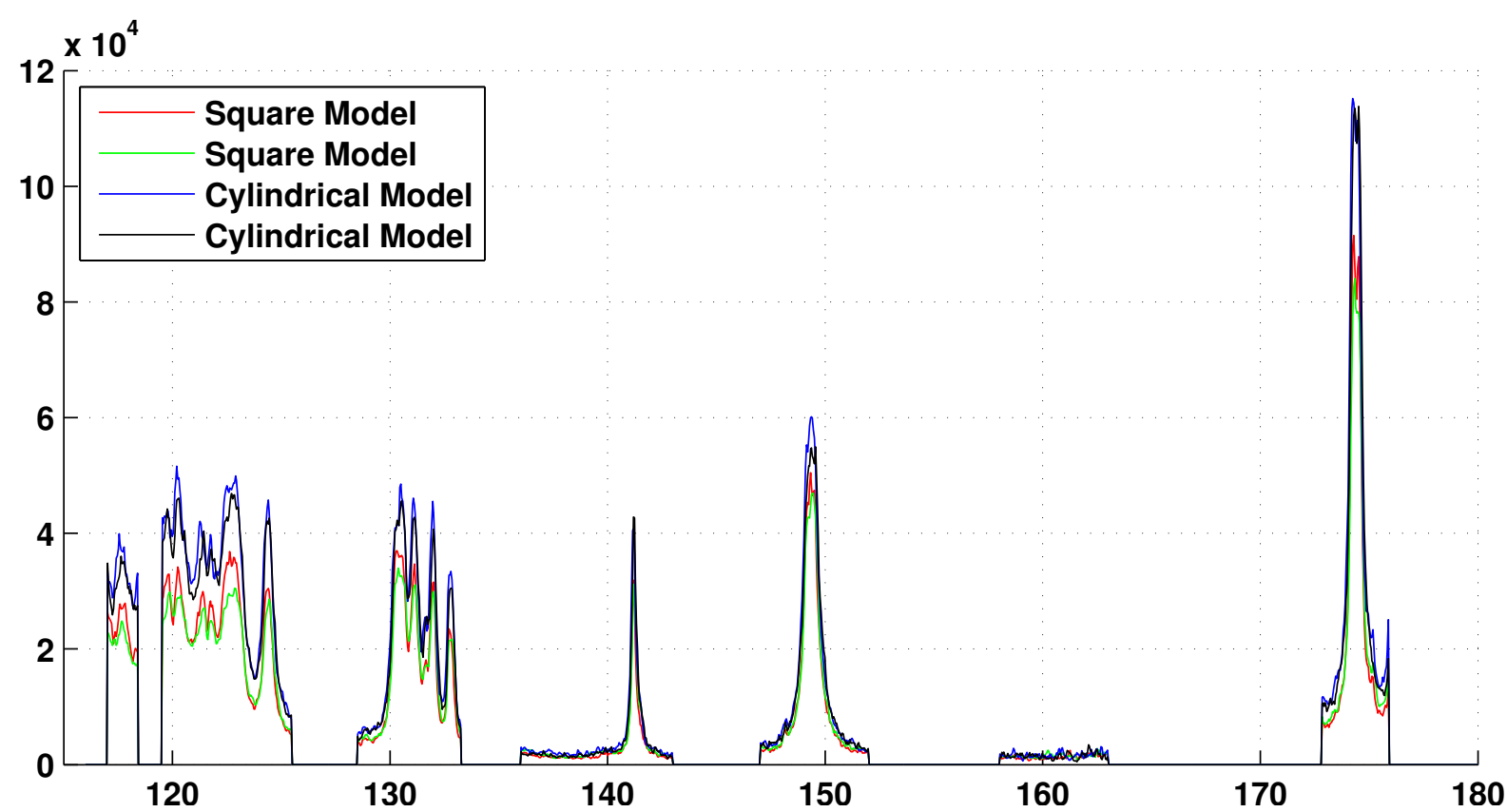

Figure 4.61: Comparison of VUV emission measured across a $90 \mathrm{~mm}$ square and cylindrical model for condition 1.

not have reached a steady state value. A further investigation of the gas chemistry state and temperature variation will be carried out computationally in Section 5.2.

Broadening was also observed in lines that were strongly self-absorbing. Whilst the Planck limit restricts the peak height, there is a significant amount of radiation recorded either side of the peak. This is an important factor to consider as it can significantly increase the amount of total radiative heat flux.
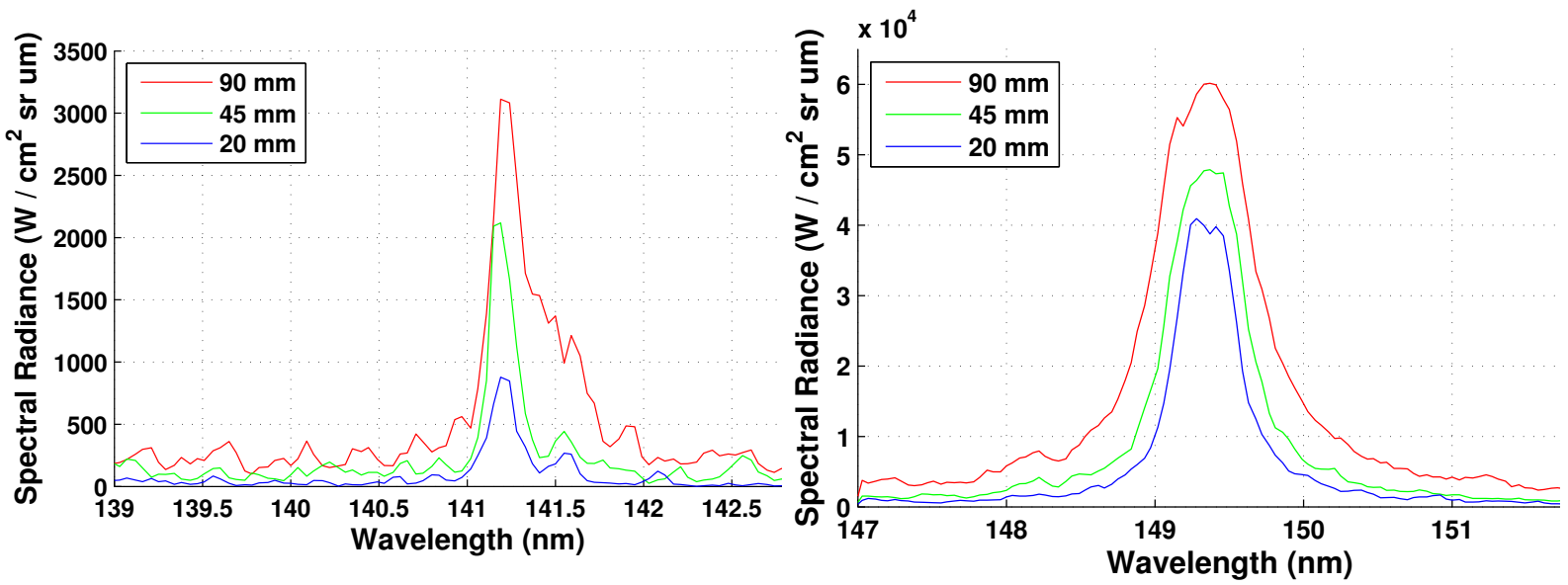

Figure 4.62: (Left) - Minimal peak broadening observed for condition 1 at the $141 \mathrm{~nm}$ line. (Right) - Strong peak broadening observed for the faster test condition on a self-absorbing line. 


\section{Chapter 5}

\section{Computational Reconstruction of Expansion Tube Experiments}

Computational reconstruction is used to investigate the chemical state of the shock layer, steady flow establishment time and compare the computed and measured spectra. Eilmer 3 was the flow field solver selected to compute the flow field around a two-dimensional representation of the models [142]. The upstream boundary conditions were calculated using Pitot and outlined in Section 4.4. The inflow gas chemistry was assumed to be in thermochemical equilibrium and calculated using CEA [139]. In reality, the free stream gas chemistry is much more complicated due to the varying times of flight experienced by the test gas through the unsteady expansions in the acceleration tube. As discussed in Section 3.4.1, in the absence of full finite rate chemistry simulations of the expansion tube, the best estimate of free stream chemistry is equilibrium.

The finite-rate reaction scheme used to compute the gas chemistry state in the shock layer was the two temperature Park model[84]. A structured grid with no clustering, as shown in Figure 5.1, was selected for both models. The grid produced a stagnation streamline resolution of $0.208 \mathrm{~mm}$ per cell for the square model and $0.167 \mathrm{~mm}$ per cell for the cylindrical model. This grid was selected as it produced the highest resolution possible for completion of the required simulations within the time frame of this study. A grid resolution study was not carried out but is prescribed as future work. A sample of the code used to generate the grids, gas models and boundary conditions is presented in Appendix E. 

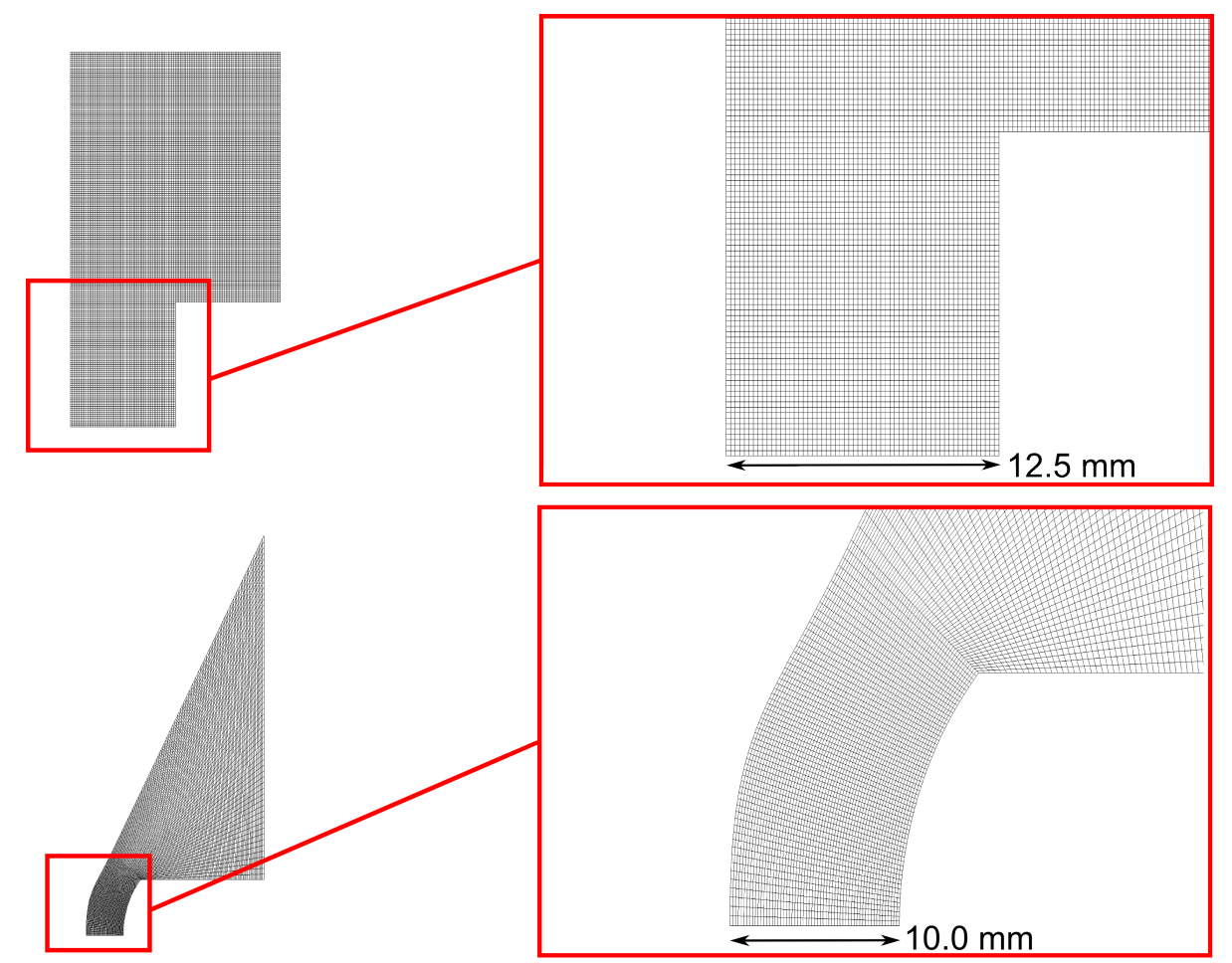

Figure 5.1: Structured grids used in the simulation of both models.

\subsection{Across and Through Surface Model Results}

Eilmer 3 is able to provide temporal resolution and could therefore be used to estimate flow establishment time over the model. This was of importance for the blunt square model which creates limited velocity gradients within the shock layer, resulting in additional time being required to achieve steady state. The initial flow over the model in experiments consists of accelerator gas, and this slug of gas can form a short steady flow. Whilst the duration of this flow is too short to form steady flow over the model, it is still capable of forming a boundary layer and creates an easier boundary condition for the test gas to start in when it arrives. For this simulation, there was no accelerator gas region and therefore an upper limit on start up time could be calculated. Results of this study, shown in Figure 5.2 , indicate steady state flow is established in $35 \mu \mathrm{s}$, validating the $40 \mu$ s delay between shock arrival and ICCD exposure used in experiments. 


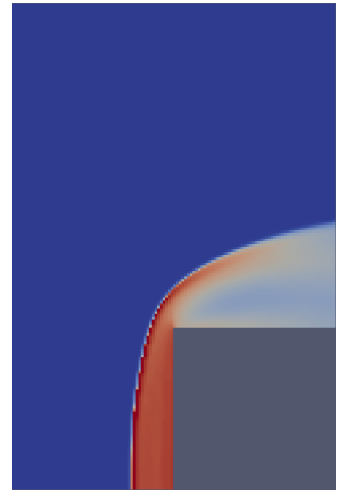

Shock Arrival

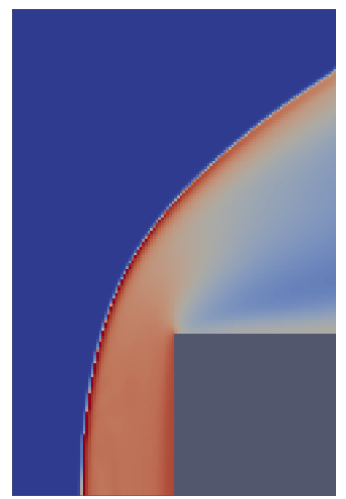

$25 \mu \mathrm{s}$

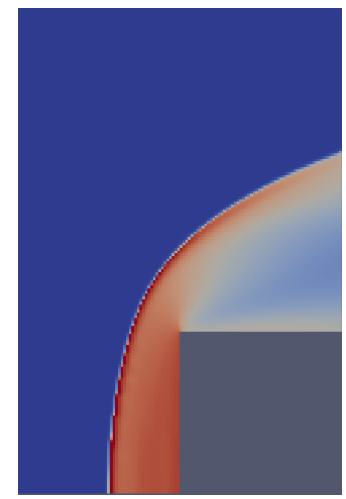

$5 \mu \mathrm{s}$

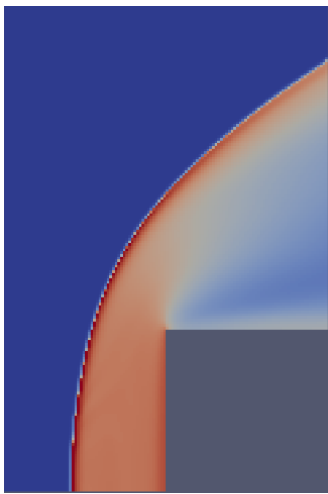

$35 \mu \mathrm{s}$

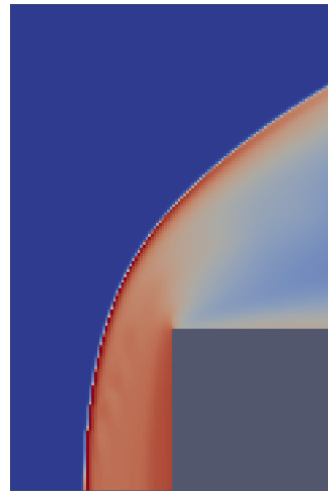

$15 \mu \mathrm{s}$

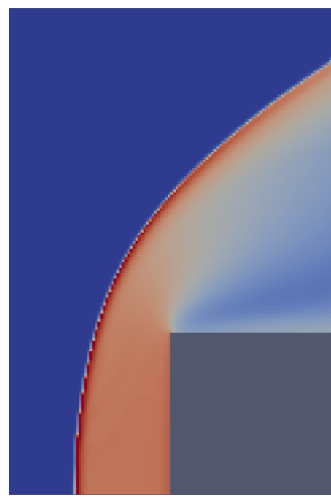

$55 \mu \mathrm{s}$

Figure 5.2: Time resolved images of shock arrival and flow establishment over the model.

From the computed gas properties along the stagnation streamline, it was possible to estimate the shock stand-off and variation of the gas properties through the shock layer. It was also possible to determine if the shock layer reached chemical and thermal steady state. The temperatures and mass fractions computed along the stagnation streamline for both conditions are displayed in Figures 5.3 and 5.4 . 


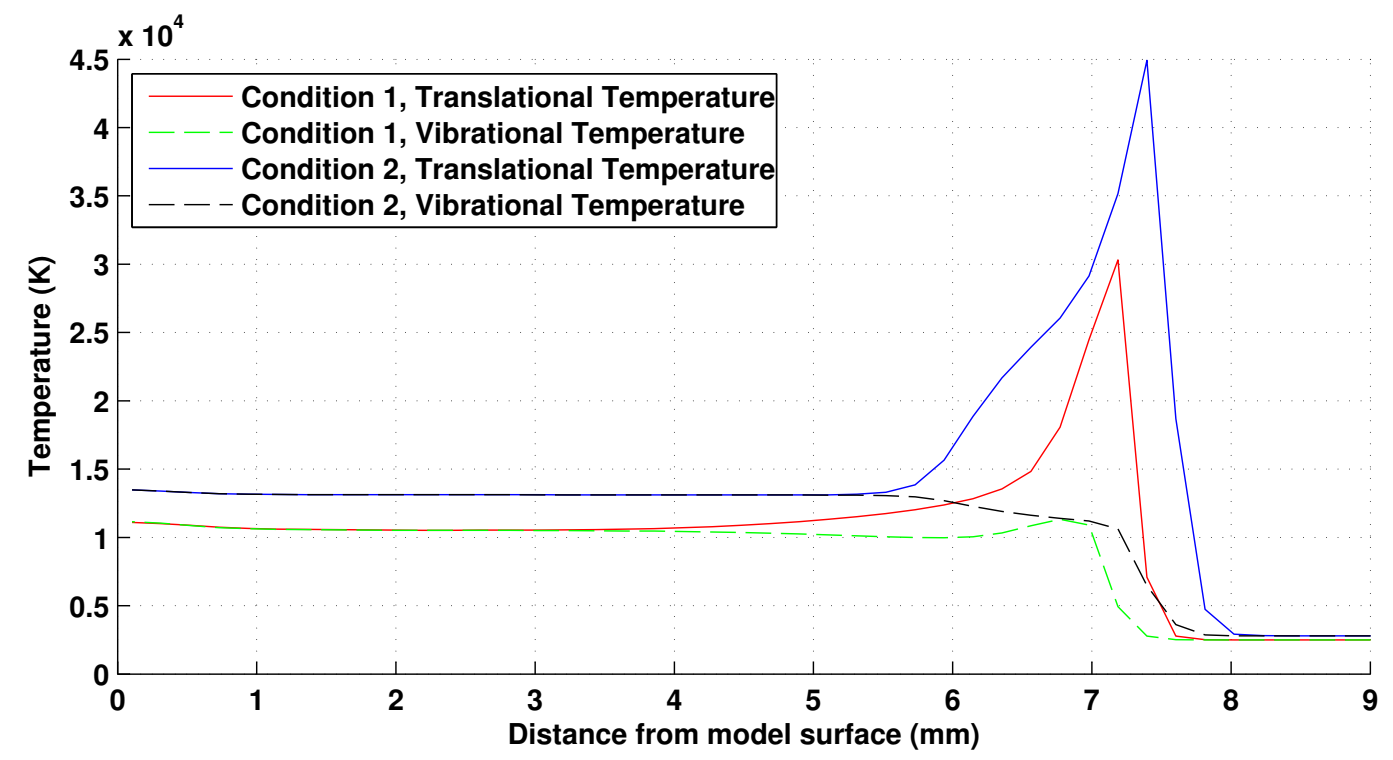

Figure 5.3: Computed stagnation streamline temperatures.

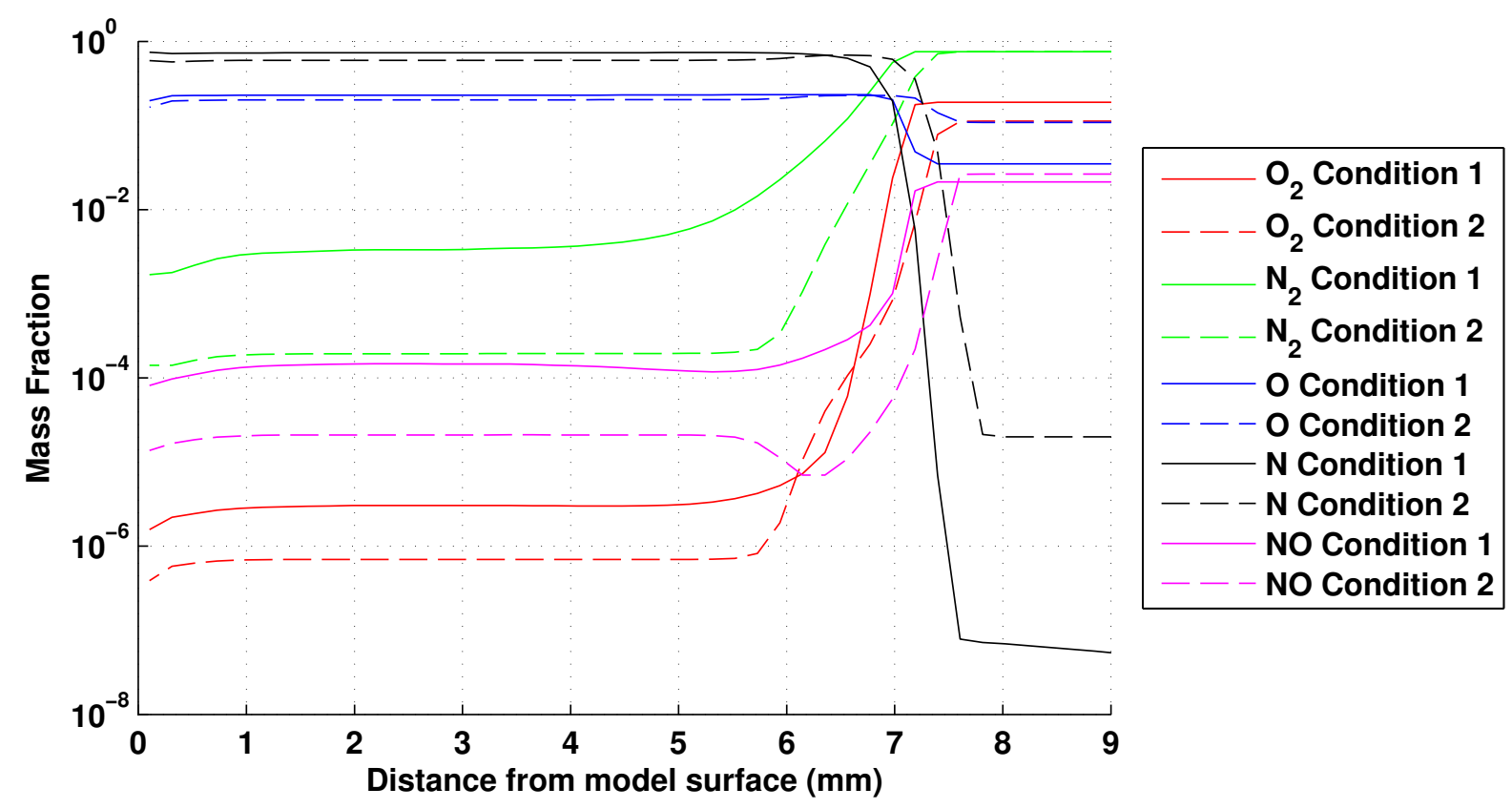

Figure 5.4: Computed mass fractions at the stagnation streamline. 
The stagnation temperature and mass fraction plots indicate the shock layer reaches steady state after approximately $1.6 \mathrm{~mm}$ and $2.1 \mathrm{~mm}$ for conditions 1 and 2 respectively. The experimental spectral emission measurements indicated that condition 2 achieved steady emission in a shorter length scale than condition 1 and this is in good agreement with the computed stagnation streamline values. The pressures, temperatures and mass fractions computed are displayed in Table 5.1 .

The computed two-dimensional results predicted shock stand off distances of $7.2 \mathrm{~mm}$ and $7.6 \mathrm{~mm}$ for conditions 1 and 2 respectively. The experimentally measured values of $5.1 \mathrm{~mm}$ and $5.5 \mathrm{~mm}$ are approximately $72 \%$ and $73 \%$ of the idealised two-dimensional shock stand off distance computed and this is in good agreement with the predicted value of $76 \%$ calculated in Section 4.1.3.

Table 5.1: Steady region properties calculated using Eilmer 3 for both conditions with the flat model.

\begin{tabular}{|l|c|c|}
\hline & Condition 1 & Condition 2 \\
\hline Equilibrium Temperature $(\mathrm{K})$ & 10,500 & 13,140 \\
\hline Pressure $(\mathrm{kPa})$ & 113 & 132 \\
\hline \multicolumn{2}{|c|}{ Mass Fractions Taken 1 mm From Model Surface } \\
\hline $\mathrm{O}_{2}$ & $3.04 \mathrm{e}-6$ & $6.91 \mathrm{e}-7$ \\
\hline $\mathrm{N}_{2}$ & $3.31 \mathrm{e}-3$ & $7.91 \mathrm{e}-5$ \\
\hline $\mathrm{N}$ & $7.31 \mathrm{e}-1$ & $5.94 \mathrm{e}-1$ \\
\hline $\mathrm{O}$ & $2.29 \mathrm{e}-1$ & $2.02 \mathrm{e}-1$ \\
\hline $\mathrm{NO}$ & $1.47 \mathrm{e}-4$ & $2.07 \mathrm{e}-5$ \\
\hline $\mathrm{N}^{+}$ & $3.00 \mathrm{e}-2$ & $1.70 \mathrm{e}-1$ \\
\hline $\mathrm{O}^{+}$ & $5.91 \mathrm{e}-3$ & $3.36 \mathrm{e}-2$ \\
\hline $\mathrm{N}_{2}^{+}$ & $1.05 \mathrm{e}-4$ & $7.19 \mathrm{e}-5$ \\
\hline $\mathrm{O}_{2}^{+}$ & $7.75 \mathrm{e}-7$ & $8.45 \mathrm{e}-7$ \\
\hline $\mathrm{NO}^{+}$ & $1.73 \mathrm{e}-4$ & $5.66 \mathrm{e}-5$ \\
\hline $\mathrm{e}^{-}$ & $1.38 \mathrm{e}-6$ & $7.82 \mathrm{e}-6$ \\
\hline
\end{tabular}

The computed temperatures, pressures and mass fractions were input into Specair to calculate the emission spectra they would produce and are presented in Table 5.1. To compute a comparable spectrum, the instrument broadening function was required and this was estimated to be $0.4 \mathrm{~nm}$ based on the $121.5 \mathrm{~nm}$ deuterium lamp spectral line. The depth of radiating flow field was set to $9 \mathrm{~cm}$ for the across surface spectral calculations and $0.75 \mathrm{~cm}$ for the through surface calculations, matching the length of the model and the computed shock stand off distance. The computed spectra for condition 1 is displayed 
in Figures 5.5 and 5.6 and the spectra for condition 2 is displayed in Figures 5.7 and 5.8 . For comparison, experimentally measured spectral data is superimposed on each plot.

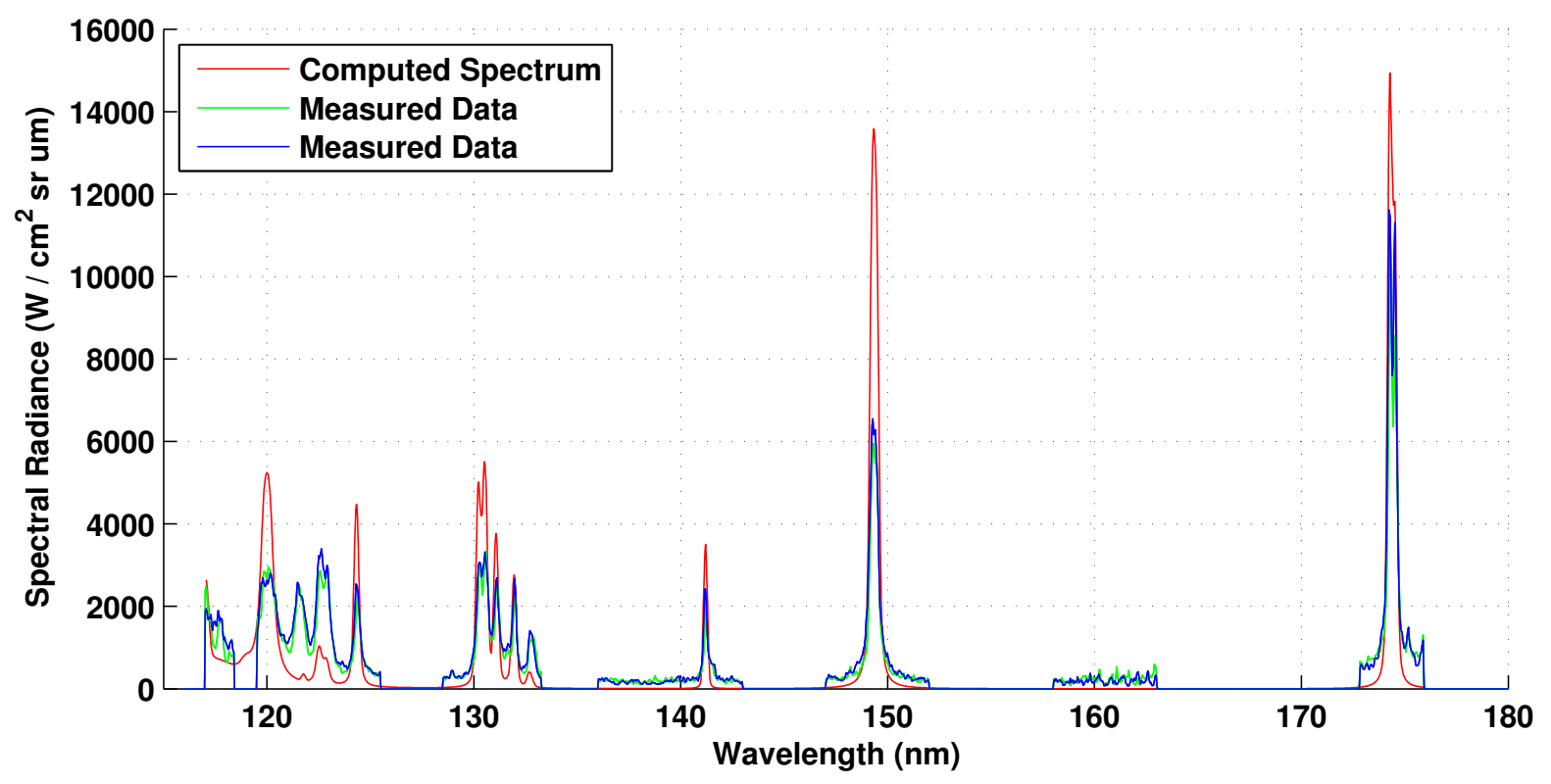

Figure 5.5: Computed and experimentally measured across surface spectra for condition 1.

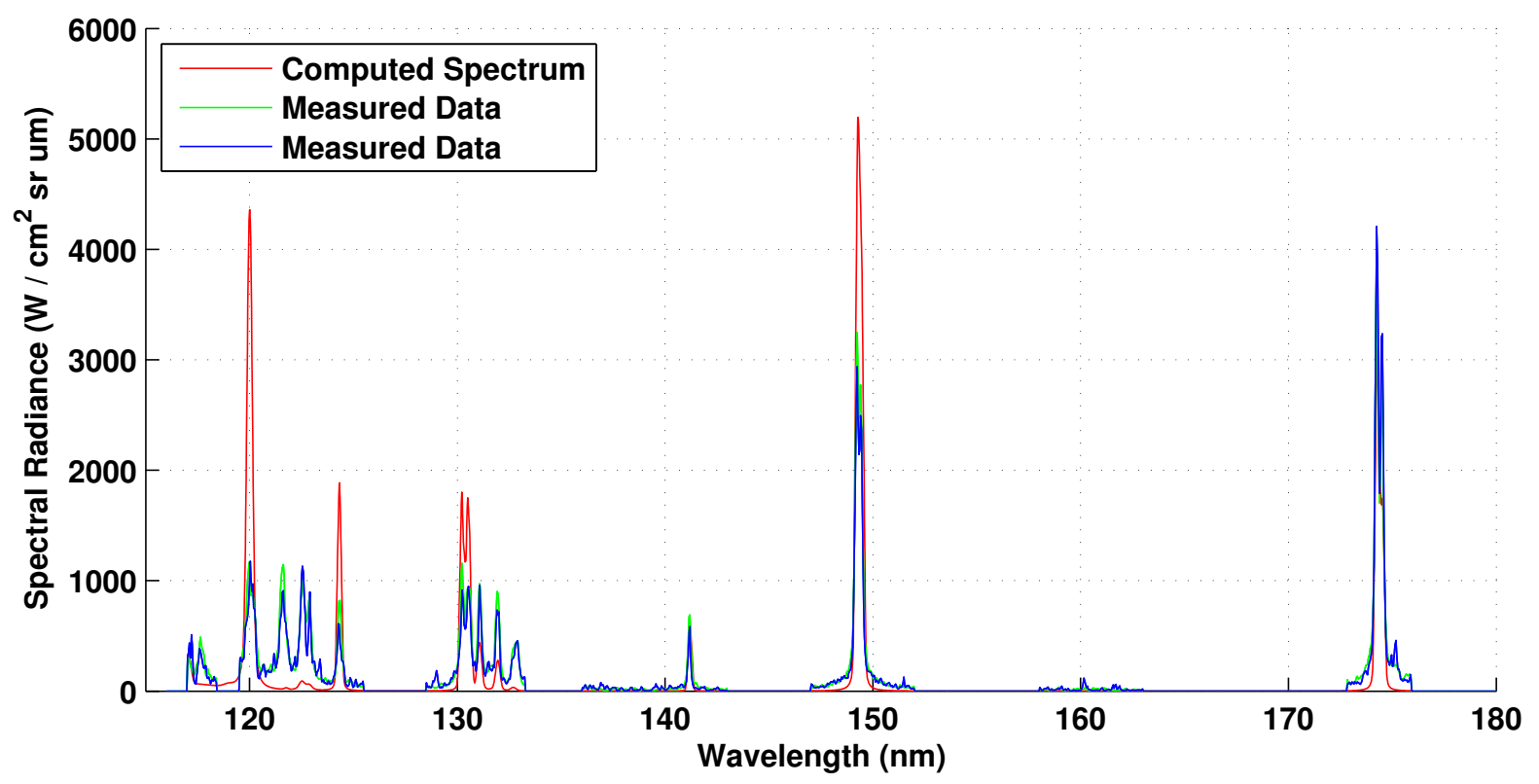

Figure 5.6: Computed and experimentally measured through surface spectra for condition 1. 


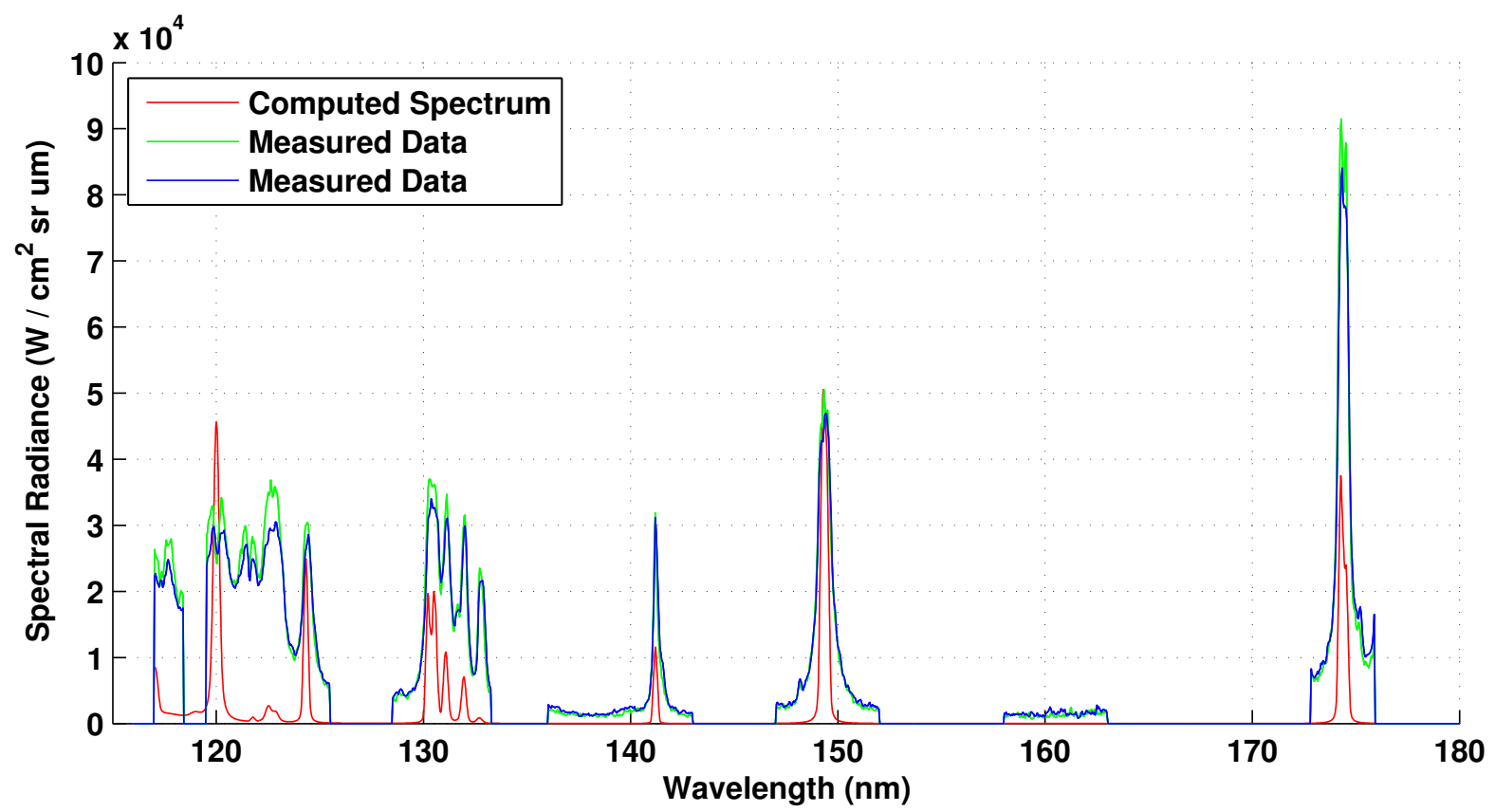

Figure 5.7: Computed and experimentally measured across surface spectra for condition 2.

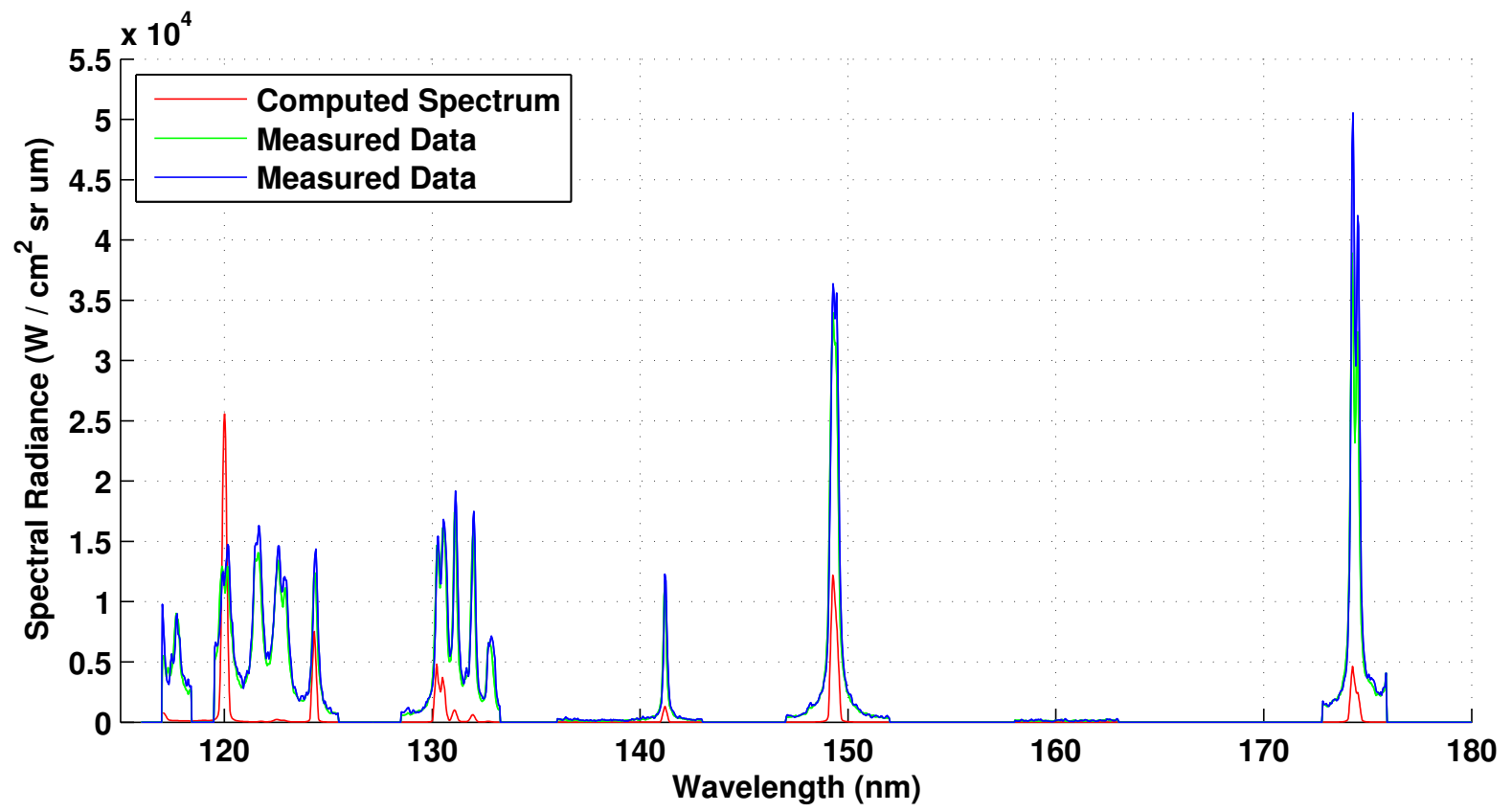

Figure 5.8: Computed and experimentally measured through surface spectra for condition 2 .

In all four comparisons, the computed spectra over predicted the $120.0 \mathrm{~nm}$ spectral line. Additionally at condition 1 , the $124.3 \mathrm{~nm}, 130.5 \mathrm{~nm}, 131.1 \mathrm{~nm}$ and $149.3 \mathrm{~nm}$ lines are all over predicted by the computed spectrum. Conversely all of the spectral lines at 
condition 2, with the exception of the $120.0 \mathrm{~nm}$ line, are under predicted. The spectral lines measured at $121 \mathrm{~nm}$ and $122 \mathrm{~nm}$ are significantly weaker in the computed spectra and warrant further investigation.

Integrated values of the measured and computed spectra between $115 \mathrm{~nm}$ and $180 \mathrm{~nm}$ are presented in Table 5.2. At condition 1 the variance between the computed and measure spectra exceeds $100 \%$ however the ratio of across surface to through surface spectral radiance is in good agreement. The computed values at condition 2 under predict the measured values and also under estimate the ratio of across surface to through surface spectral radiance.

Table 5.2: Integrated values for the VUV spectrum and ratios of observed across surface measurements to the through surface measurements.

\begin{tabular}{|l|l|c|c|c|}
\hline & & \multicolumn{2}{|c|}{ Integrated Total $\left(\mathrm{W} / \mathrm{cm}^{2} \mathrm{sr}\right)$} & Variance (\%) \\
\hline & & Computed & Measured & \\
\hline Condition 1 & Across Surface & 67.9 & 29.8 & 130 \\
\hline & Through Surface & 15.9 & 7.44 & 110 \\
\hline & Ratio & 0.23 & 0.25 & -6.3 \\
\hline Condition 2 & Across Surface & 205 & 399 & -49 \\
\hline & Through Surface & 43.3 & 123 & -65 \\
\hline & Ratio & 0.21 & 0.31 & -31 \\
\hline
\end{tabular}

The simulations presented in this section have shown significant disagreement between the computed and measured spectral radiances. It is important to note that these are currently not state of the art simulations and require further grid refinement to resolve the non-equilibrium region and boundary layer so that a true line of sight spectrum can be calculated.

These simulations also do not accounted for any radiation coupling effects and this can drastically change the temperature, and consequently the chemistry, of the radiating shock layer. An estimate of the effect of radiation coupling was made using the Goulard number. The measured incident radiative heat flux was used to estimate the total radiative heat flux assuming the contribution from the VUV region was one third and a half for conditions 1 and 2 respectively. These assumptions gave radiative heat fluxes of $1.41 \mathrm{MW} / \mathrm{m}^{2}$ and $15.5 \mathrm{MW} / \mathrm{m}^{2}$ for the two conditions, and coupled with the free stream properties presented in Table 4.4, the Goulard numbers calculated were 0.0012 and 0.0097 for conditions 1 and 2 respectively. As a rule of thumb, when the Goulard number is larger than 0.01, the fluid dynamics and radiation are considered to be strongly coupled [143]. Based on 
this approximation, the calculated Goulard numbers indicate that condition 1 is loosely coupled whereas condition 2 is strongly coupled. It is currently not possible to further investigate the effects of coupling in the simulations presented, however, radiation coupling is currently being implemented in Eilmer 3 and these simulations will be used as a platform for future computational reconstructions of the dataset presented in this study.

\subsection{Self-Absorption Experiments}

Eilmer 3 was used to compute the flow field around a two-dimensional representation of the cylindrical model used to carry out the self-absorption experiments described in Section 4.8.3. Using the computed flow field, estimates of shock stand-off and variation of shock layer temperature and gas composition along the stagnation stream line were obtained. The temperature profiles obtained are displayed in Figure 5.9 for both conditions and the mass fractions computed are displayed in Figure 5.10.

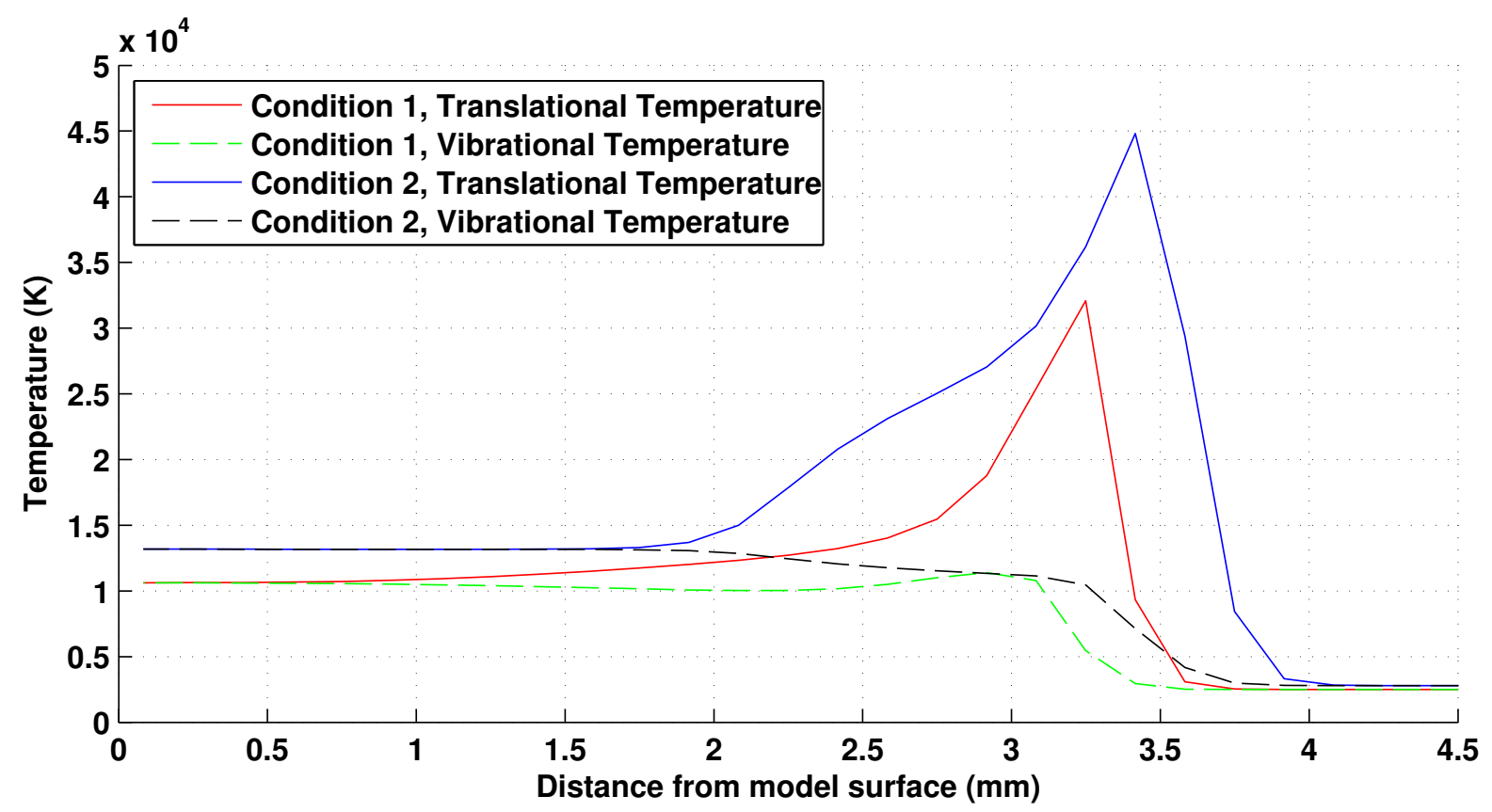

Figure 5.9: Computed stagnation streamline temperatures at both conditions.

The computed shock stand-off distance is measured as the distance from the model to the peak translational temperature and is found to be approximately $3.2 \mathrm{~mm}$ for condition 1 and $3.4 \mathrm{~mm}$ for condition 2. These values are in good agreement with the experimentally measured values of $3.4 \mathrm{~mm}$ and $3.3 \mathrm{~mm}$ for conditions 1 and 2 respectively using the $90 \mathrm{~mm}$ model. The $45 \mathrm{~mm}$ and $20 \mathrm{~mm}$ models produced shock stand-offs smaller than the $90 \mathrm{~mm}$ 
model for both conditions and this was attributed to three dimensional effects becoming more prominent at the lower aspect ratios of the models, as shown in Figure 4.13 [27].

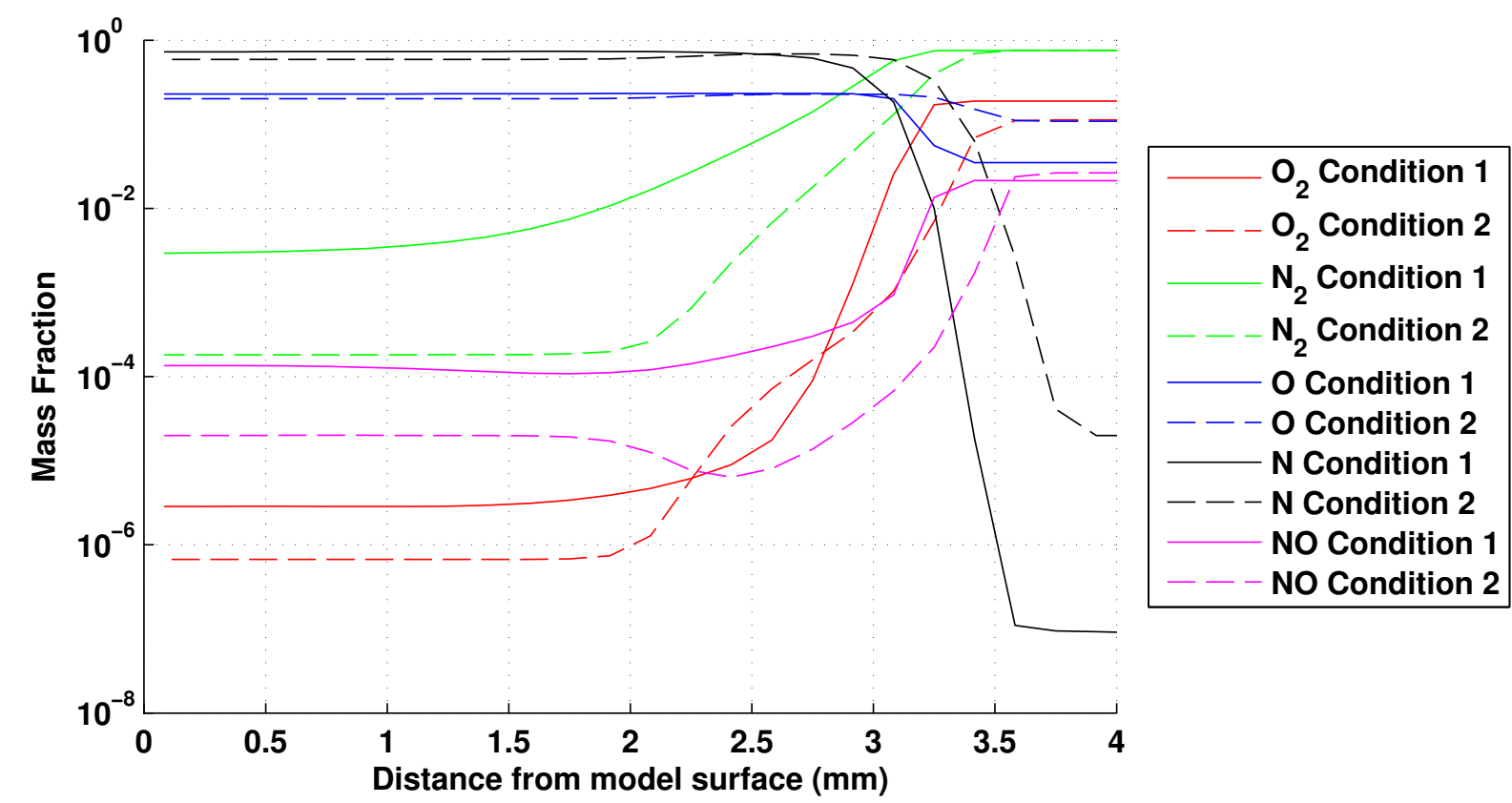

Figure 5.10: Computed stagnation streamline mass fractions using the Park reaction scheme at both conditions.

It is apparent from Figure 5.10 that the mass fractions asymptote within the shock layer after approximately $1.5 \mathrm{~mm}$ and $1.7 \mathrm{~mm}$ for conditions 1 and 2 respectively. As the shock stand-off distances are larger than these length scales, and the regions spatially averaged across to obtain the experimental spectra did not include the initial non-equilibrium regions, the mass fractions computed for the asymptotic regions were used to calculate comparable spectra. The temperatures, pressures and mass fractions computed in the steady region of the shock layer are detailed in Table 5.3. The spectra computed with these values at depths of radiating fields of $90 \mathrm{~mm}, 45 \mathrm{~mm}$ and $20 \mathrm{~mm}$ are presented in Figures 5.11 to 5.16 and are superimposed with experimentally measured spectra. 
Table 5.3: Steady region properties calculated using Eilmer 3 for both conditions with the cylindrical model.

\begin{tabular}{|l|c|c|}
\hline & Condition 1 & Condition 2 \\
\hline Equilibrium Temperature $(\mathrm{K})$ & 10,600 & 13,200 \\
\hline Pressure $(\mathrm{kPa})$ & 113 & 131 \\
\hline \multicolumn{2}{|c|}{ Mass Fractions Taken 1 mm From Model Surface } \\
\hline $\mathrm{O}_{2}$ & $2.86 \mathrm{e}-6$ & $6.70 \mathrm{e}-7$ \\
\hline $\mathrm{N}_{2}$ & $2.97 \mathrm{e}-3$ & $1.82 \mathrm{e}-4$ \\
\hline $\mathrm{N}$ & $7.29 \mathrm{e}-1$ & $5.90 \mathrm{e}-1$ \\
\hline $\mathrm{O}$ & $2.29 \mathrm{e}-1$ & $2.01 \mathrm{e}-1$ \\
\hline $\mathrm{NO}$ & $1.35 \mathrm{e}-4$ & $2.00 \mathrm{e}-5$ \\
\hline $\mathrm{N}^{+}$ & $3.25 \mathrm{e}-2$ & $1.74 \mathrm{e}-1$ \\
\hline $\mathrm{O}^{+}$ & $6.38 \mathrm{e}-3$ & $3.44 \mathrm{e}-2$ \\
\hline $\mathrm{N}_{2}^{+}$ & $1.04 \mathrm{e}-4$ & $7.08 \mathrm{e}-5$ \\
\hline $\mathrm{O}_{2}^{+}$ & $7.83 \mathrm{e}-7$ & $8.39 \mathrm{e}-7$ \\
\hline $\mathrm{NO}^{+}$ & $1.66 \mathrm{e}-4$ & $5.53 \mathrm{e}-5$ \\
\hline $\mathrm{e}^{-}$ & $1.49 \mathrm{e}-6$ & $8.00 \mathrm{e}-6$ \\
\hline
\end{tabular}

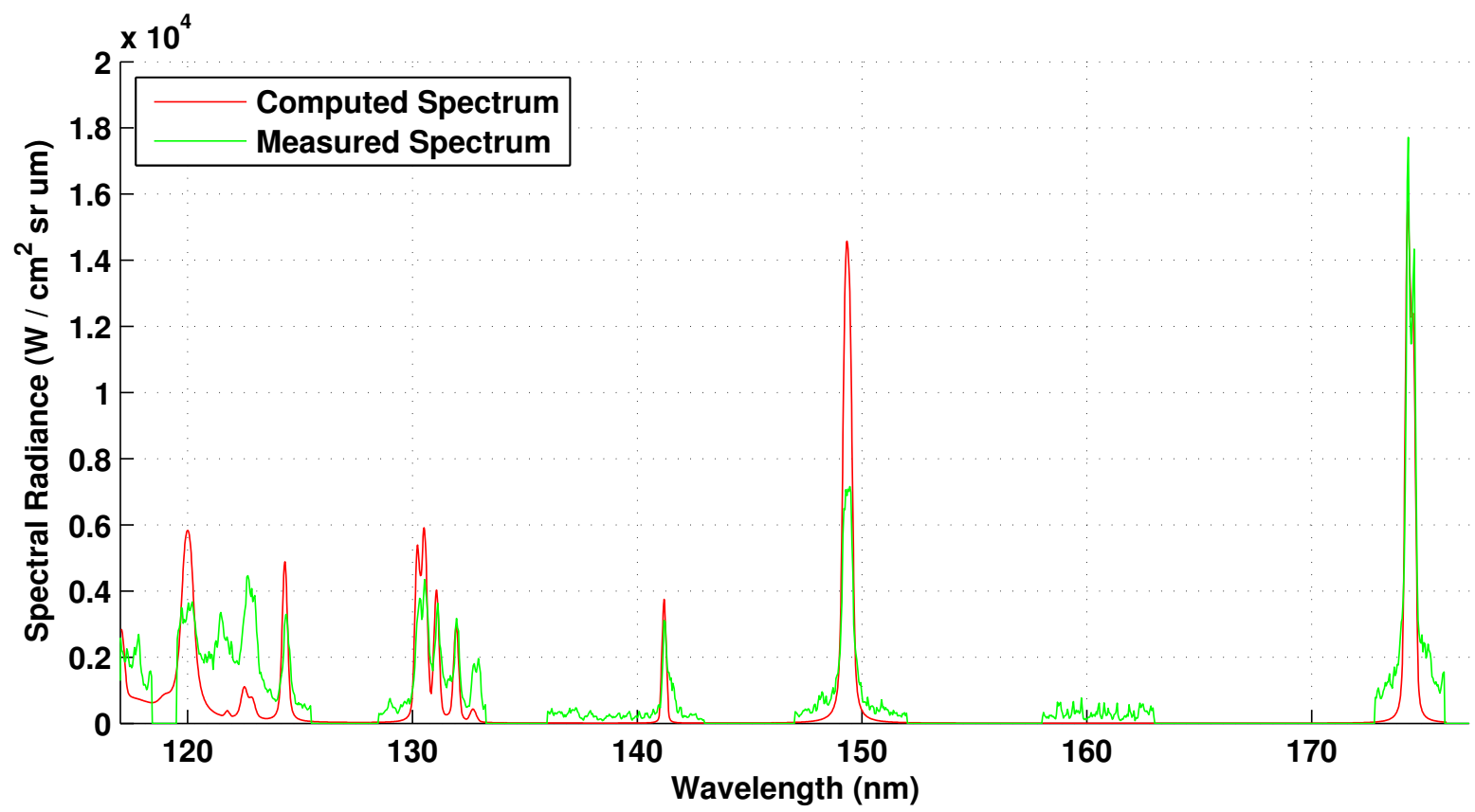

Figure 5.11: Computed and spectra across a $90 \mathrm{~mm}$ cylindrical model at condition 1. 


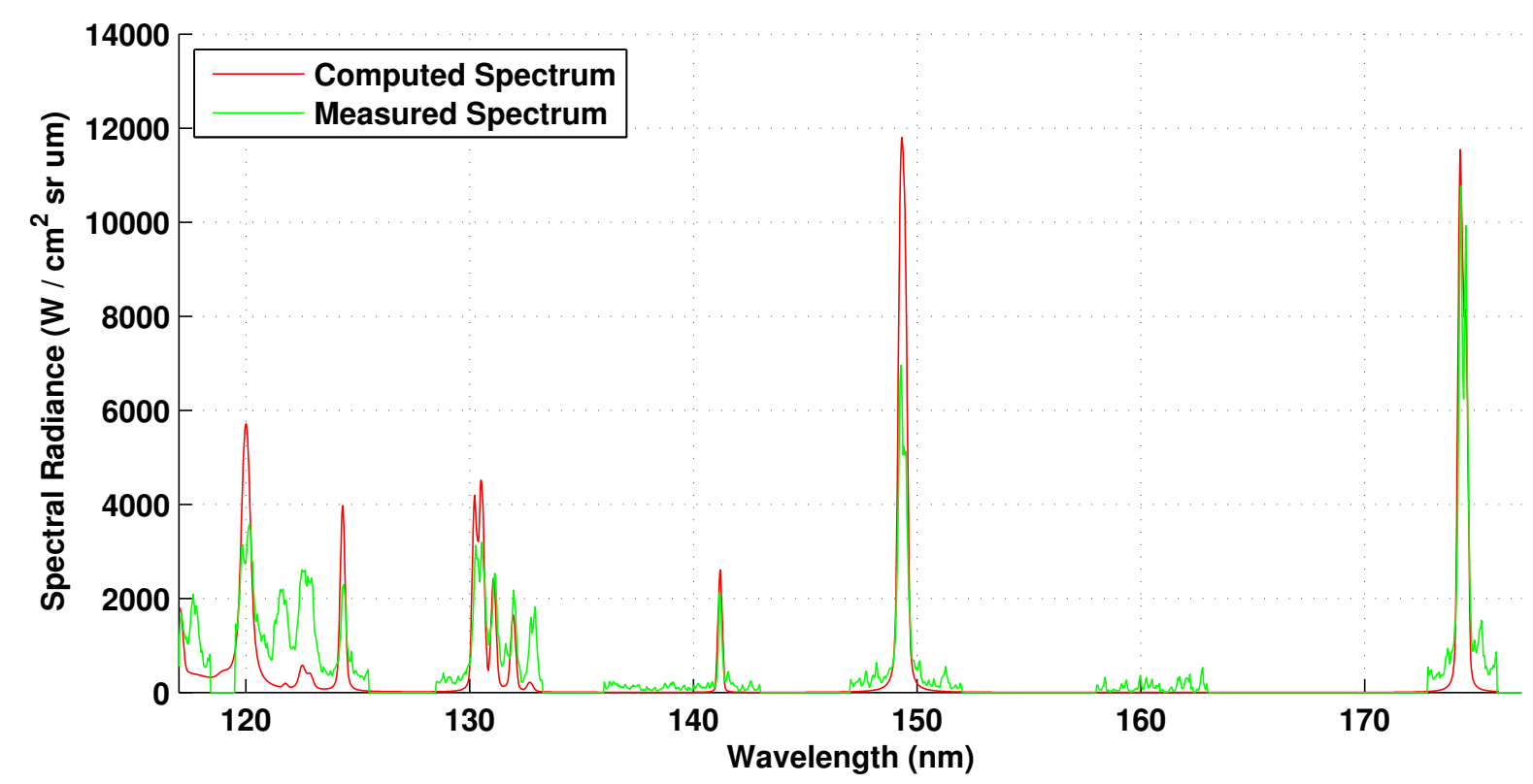

Figure 5.12: Computed and measured spectra across a $45 \mathrm{~mm}$ cylindrical model at condition 1.

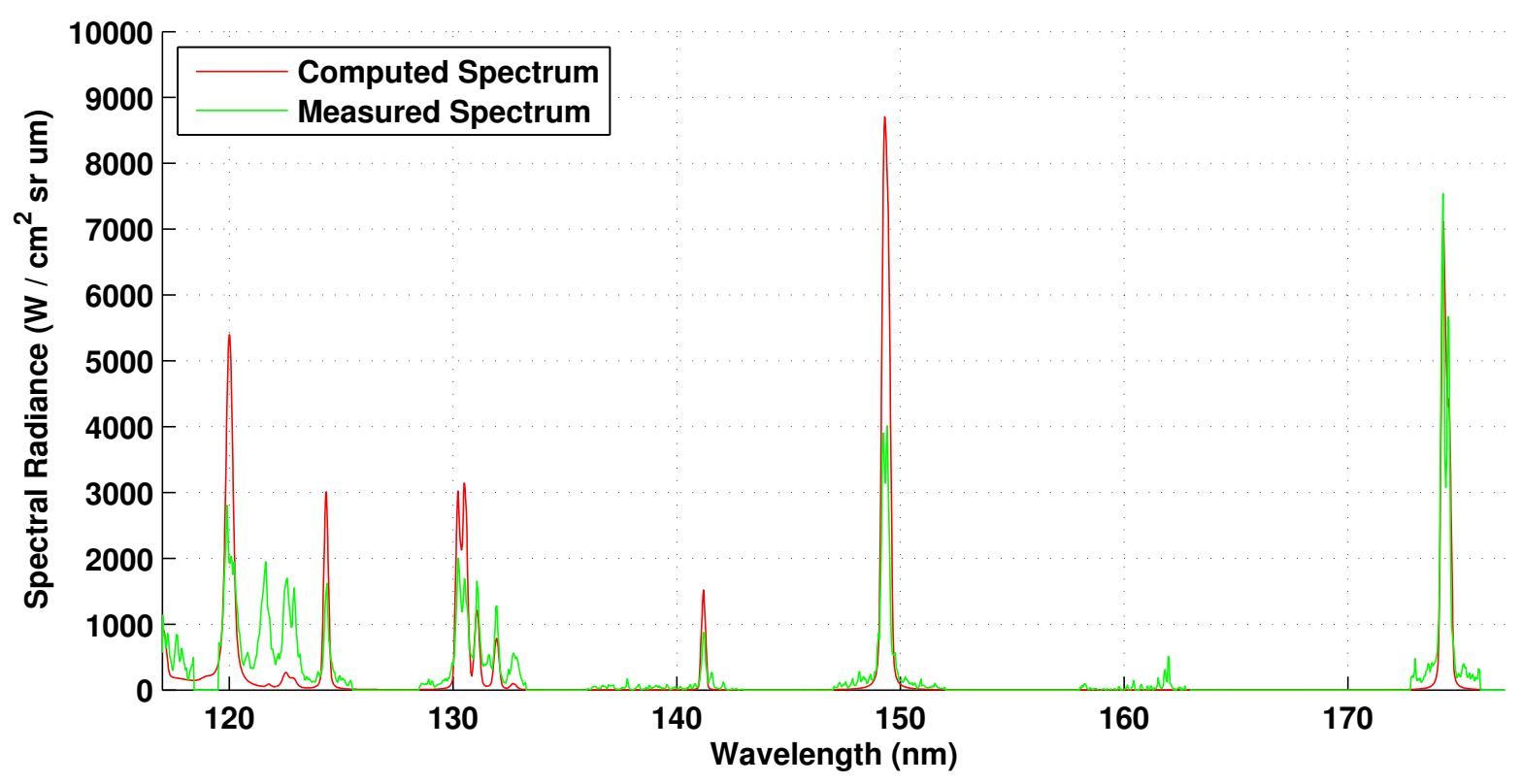

Figure 5.13: Computed and measured spectra across a $20 \mathrm{~mm}$ cylindrical model at condition 1. 


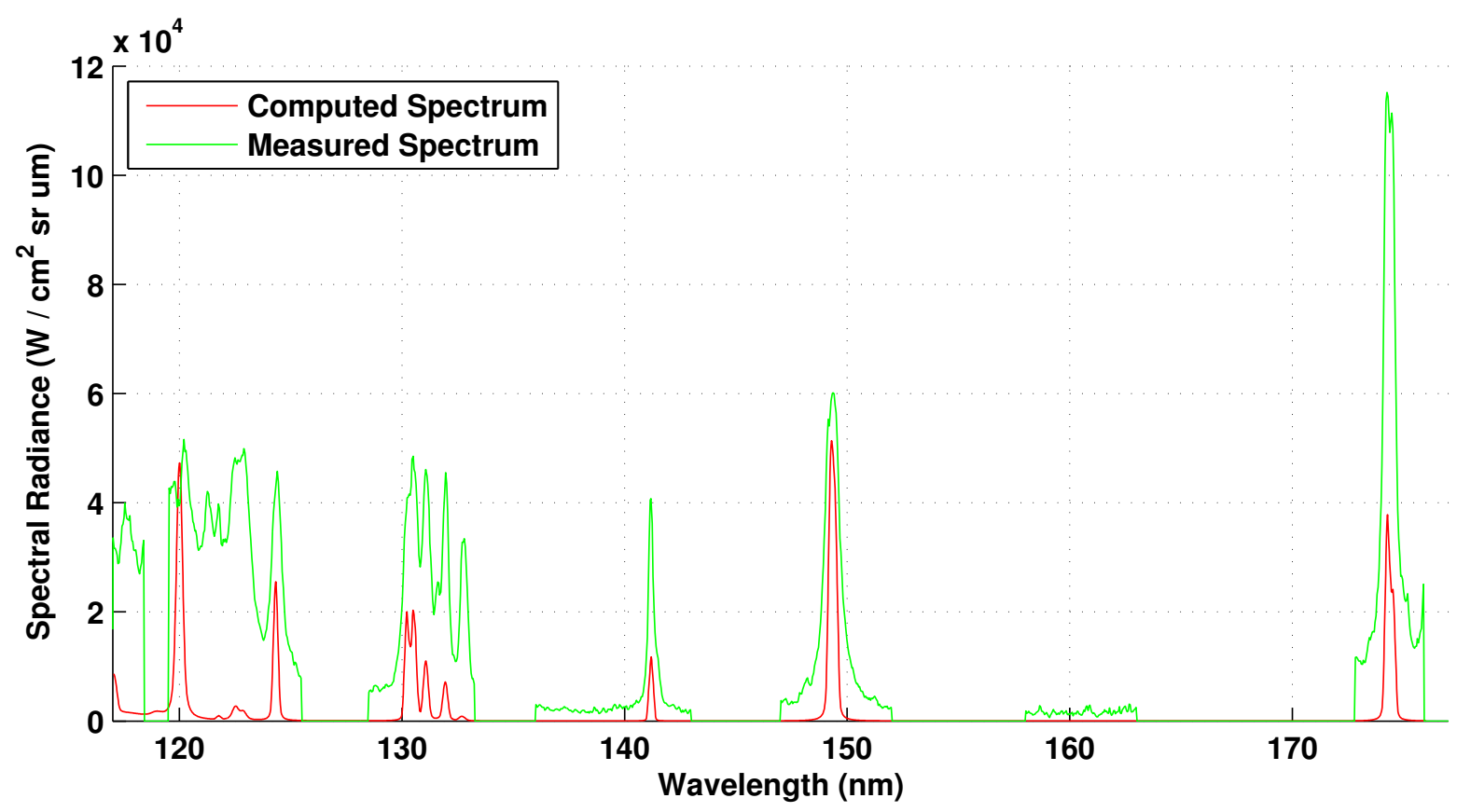

Figure 5.14: Computed and measured spectra across a $90 \mathrm{~mm}$ cylindrical model at condition 2.

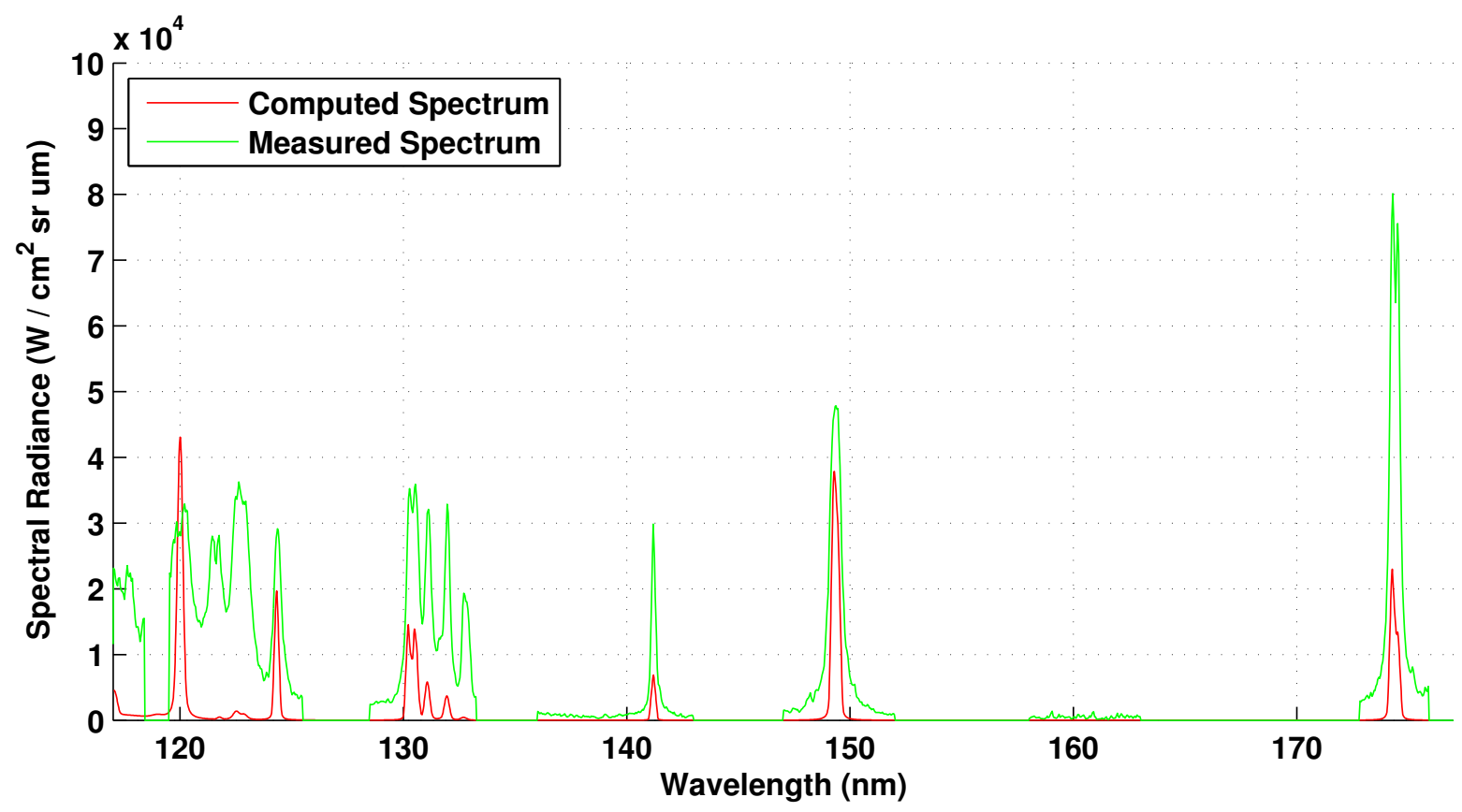

Figure 5.15: Computed and measured spectra across a $45 \mathrm{~mm}$ cylindrical model at condition 2 . 


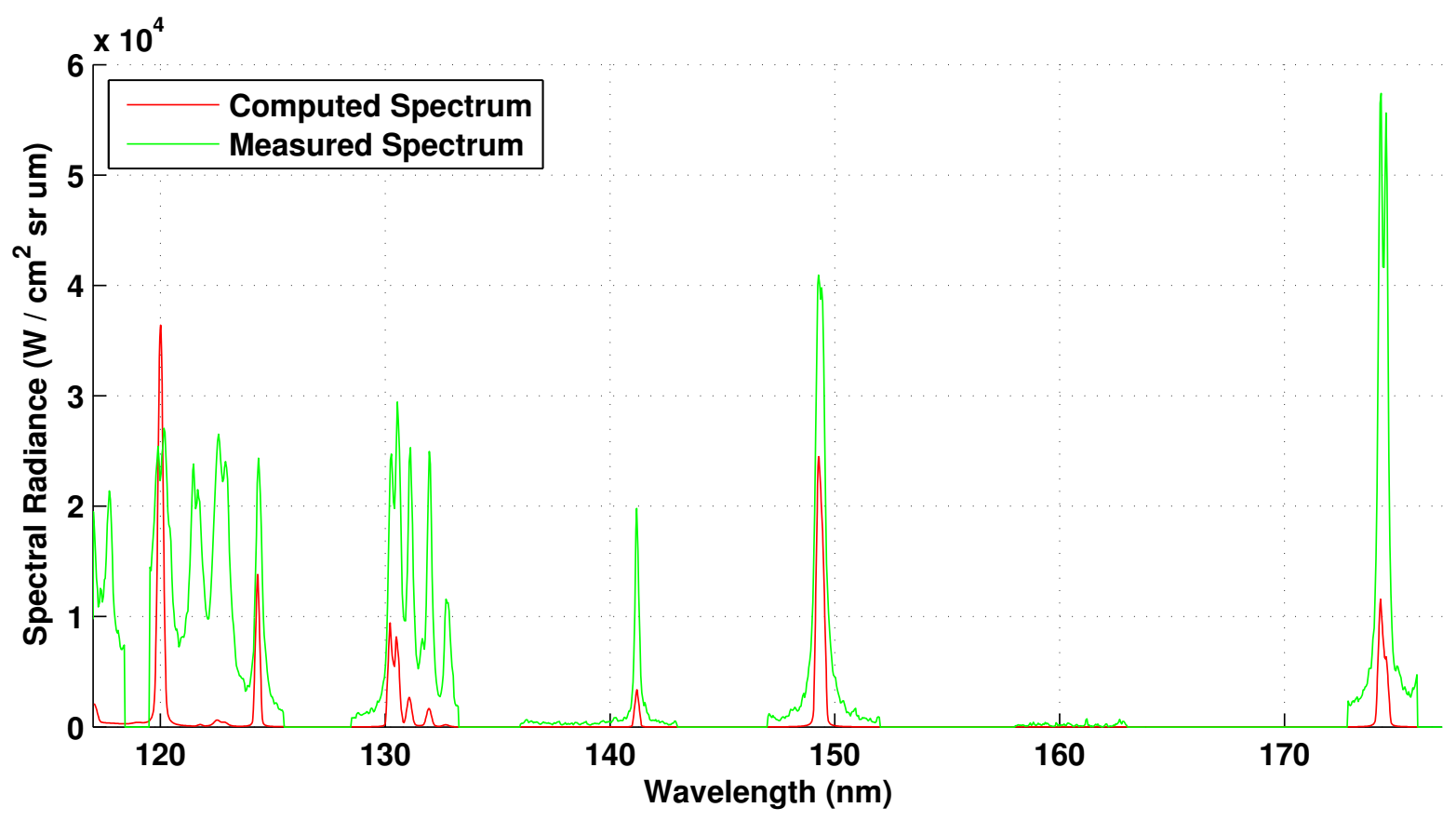

Figure 5.16: Computed and measured spectra across a $20 \mathrm{~mm}$ cylindrical model at condition 2 .

It was found that the computed spectra over predicted all spectral lines at condition 1 and under predicted all spectral lines at condition 2 except the $120.0 \mathrm{~nm}$ atomic nitrogen line. Similar to the analysis of self-absorption experimental data in Section 5.2, using the peak intensities from the $90 \mathrm{~mm}$ depth of radiating flow field as a normalising reference, some indication of the significance of self-absorption can be found from the ratios of the peak magnitudes for different depths of radiating flow fields. A ratio closer to unity indicates a strongly self-absorbing line whereas a ratio equal to the ratio of the radiating flow fields indicates an optically thin line. The results of this analysis are displayed in Figures 5.17 and 5.18 . 


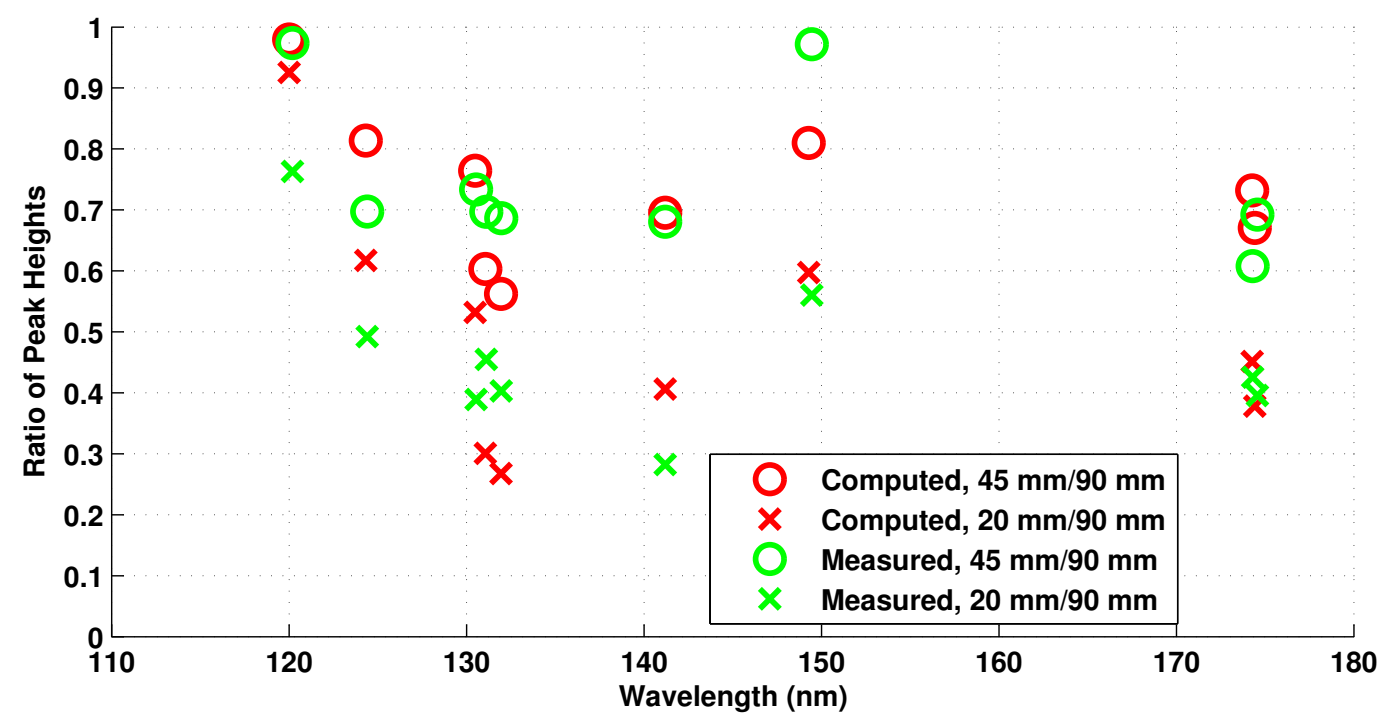

Figure 5.17: Experimentally measured and computed spectral line peak height ratios for condition 1.

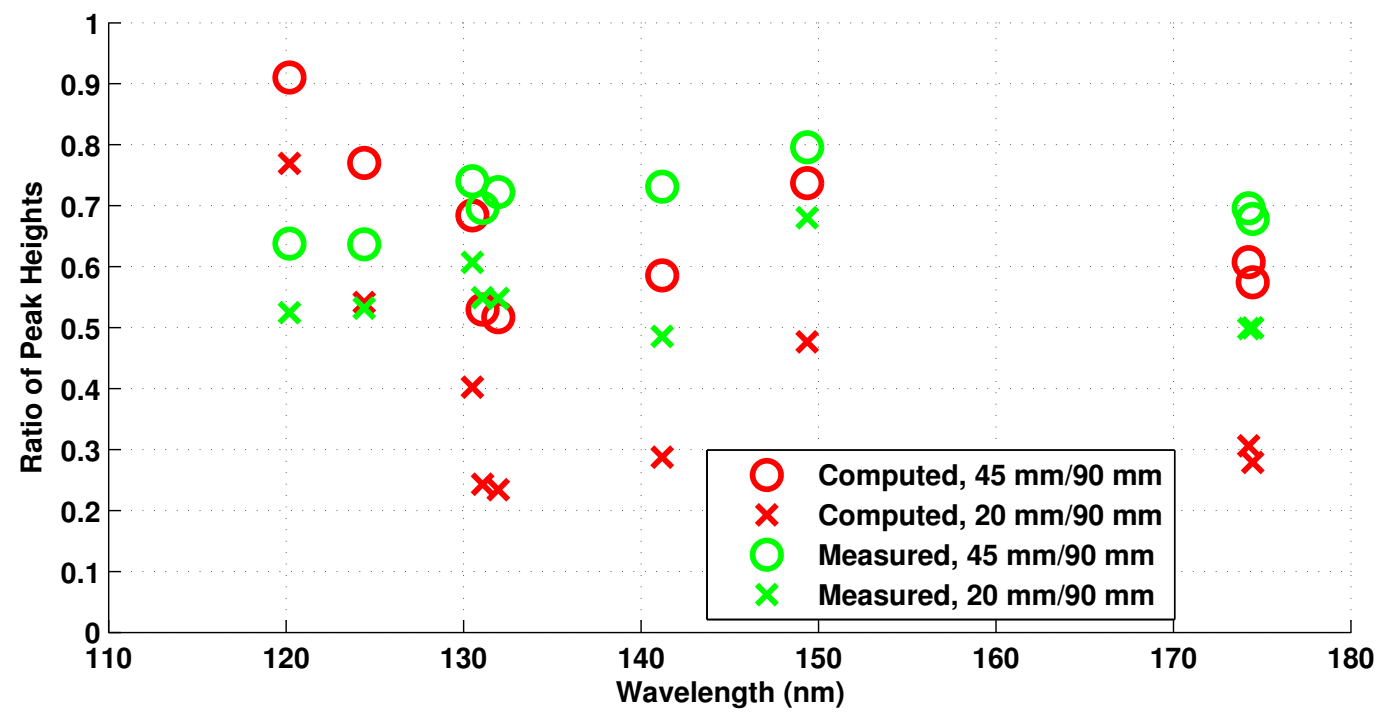

Figure 5.18: Experimentally measured and computed spectral line peak height ratios for condition 2.

The computed ratios of spectral line intensities showed varying levels of agreement across the spectral range with the measured ratios. In general, the computed ratios at condition 1 matched more closely with the measured values, especially for the $20 \mathrm{~mm}$ model as shown by the large scatter of all the ' $\mathrm{x}$ ' markers for condition 2 .

An estimate of the length scale over which each line is self-absorbed was made by plotting normalised spectral line intensities as a function of depth of radiating flow field, as shown in Figures 5.19 and 5.20 . If a line is strongly self-absorbing, the spectral intensity will rise 
and asymptote more sharply and if a line is optically thin, the spectral line intensity will scale with the depth of radiating flow field.
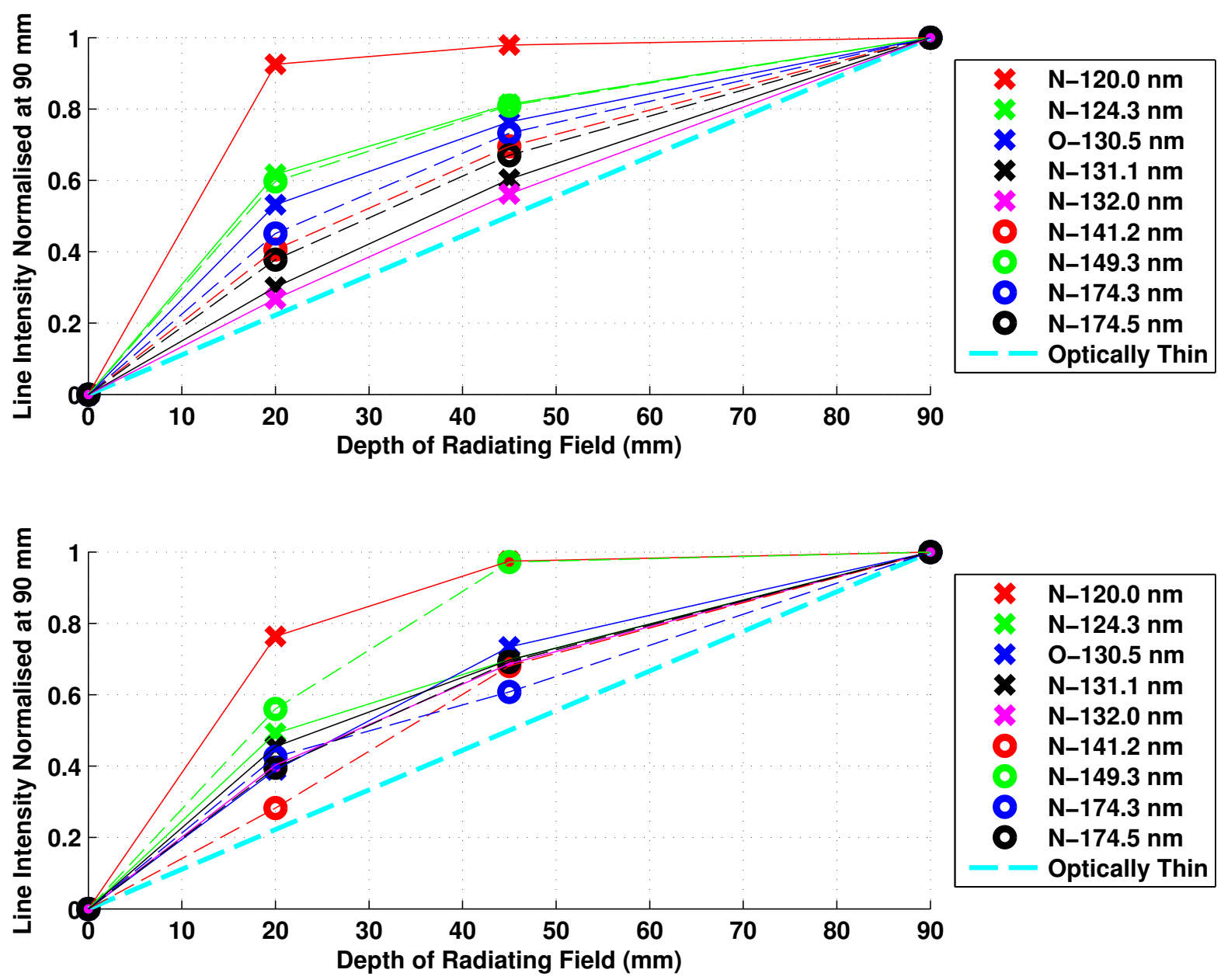

Figure 5.19: Spectral line intensities for condition 1 as a function of depth of radiating flow field, normalised at $90 \mathrm{~mm}$ depth of radiating flow field. (Top) - Computed results. (Bottom) - Experimental measurements. 

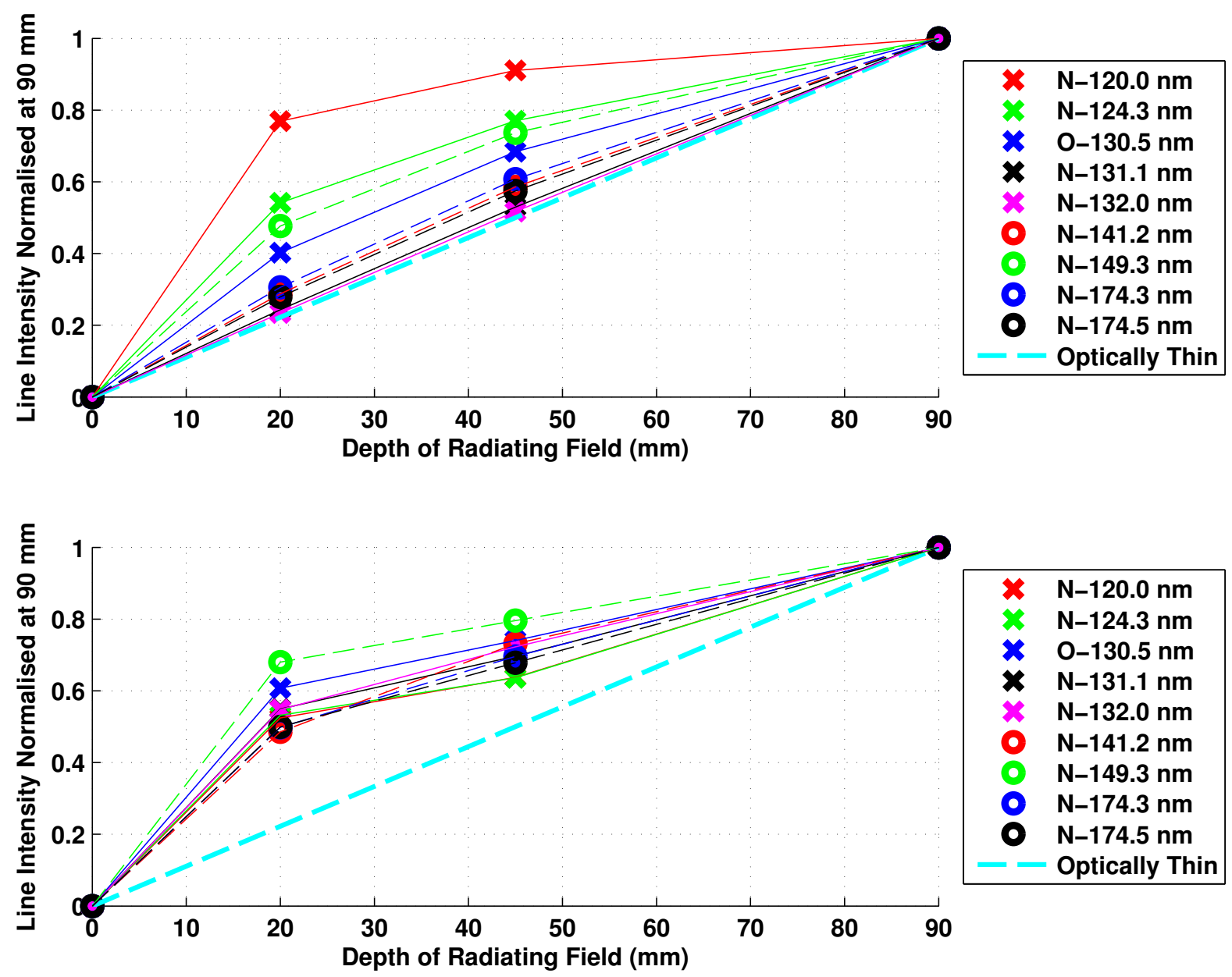

Figure 5.20: Spectral line intensities for condition 2 as a function of depth of radiating flow field, normalised at $90 \mathrm{~mm}$ depth of radiating flow field. (Top) - Computed results. (Bottom) - Experimental measurements.

It is evident from Figures 5.19 and 5.20 that all spectral lines observed are self-absorbing as they are above the optically thin ratio. In all cases except the measured values at condition 2, the $121.0 \mathrm{~nm}$ atomic nitrogen line is shown to be strongly self-absorbing achieving over $90 \%$ intensity in $20 \mathrm{~mm}$ of radiating gas for condition 1 and $80 \%$ intensity in $20 \mathrm{~mm}$ of radiating gas for condition 2. Once again, the computed values agree more closely with condition 1 and this can be attributed to the stronger radiation coupling that is expected at condition 2, which will ultimately effect the size and shape of the spectral line.

A comparison of spectral line broadening was conducted between the computed and measured spectra for the $141.2 \mathrm{~nm}$ line at condition 1 and the $149.3 \mathrm{~nm}$ line at condition 2 . The measured and computed spectral lines are presented in Figures 5.21 and 5.22. The computed spectral line width at $141.2 \mathrm{~nm}$ was in good agreement with the measured value, however there appears to be a peak $141.5 \mathrm{~nm}$ in the measured spectrum across the $90 \mathrm{~mm}$ 
model that was not apparent in the computed spectra. The cause of this anomaly has not yet been identified. The calculated spectral line broadening at $149.3 \mathrm{~nm}$ was found to be an under prediction of the measured value. A possible explanation for this discrepancy may be an underestimation of the electron number density, reducing the Stark broadening width.

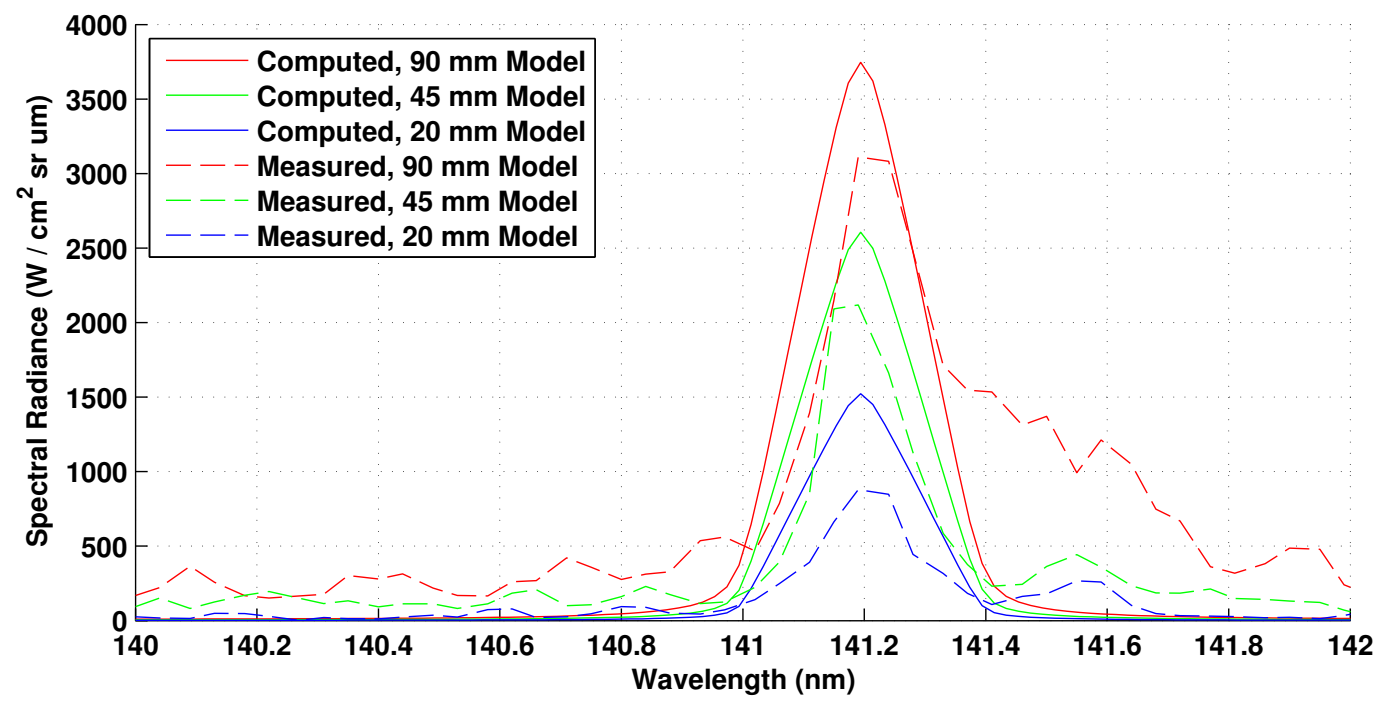

Figure 5.21: Experimentally measured and computed spectral line widths for the $141.2 \mathrm{~nm}$ peak at condition 1 .

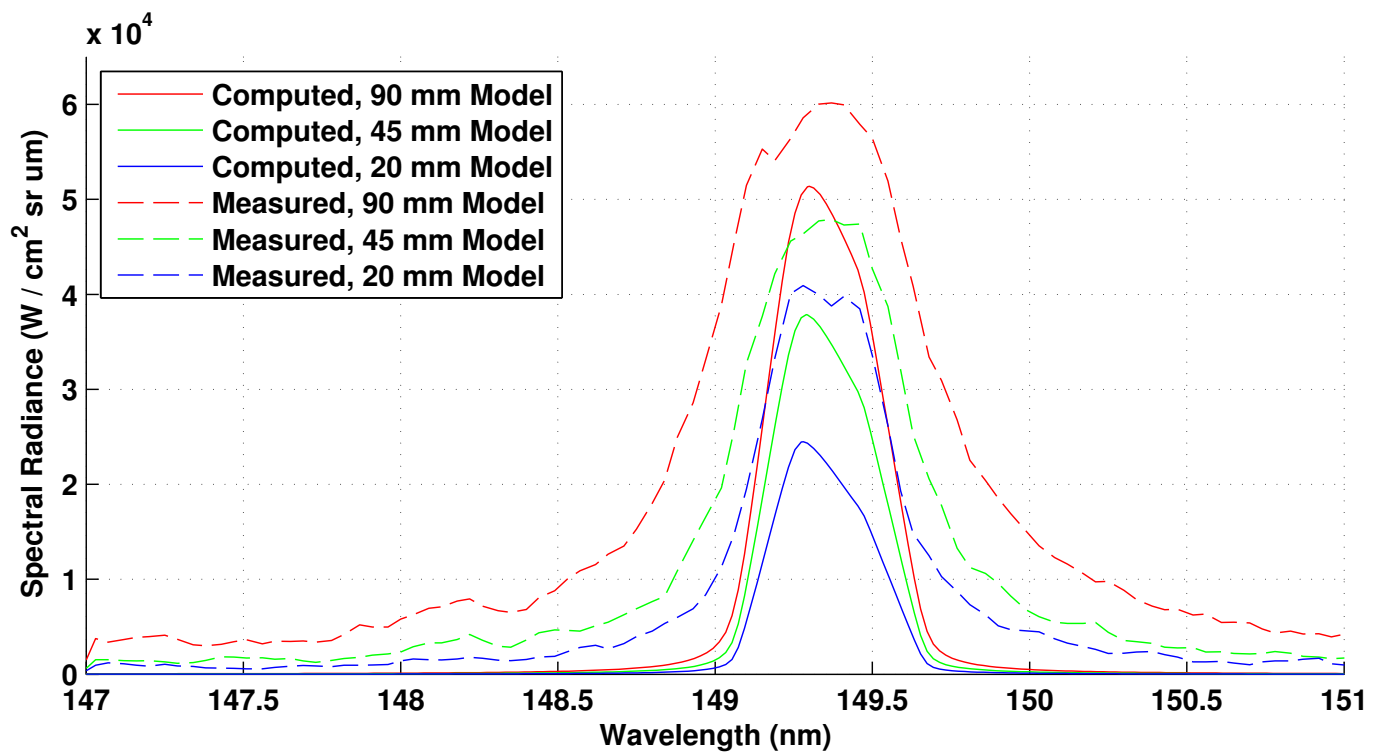

Figure 5.22: Experimentally measured and computed spectral line widths for the $149.3 \mathrm{~nm}$ peak at condition 2 . 


\subsection{Conclusions}

In this chapter simulations have been presented for both conditions and both models using Eilmer 3 as the flow field solver and the Park two-temperature reaction scheme to compute the finite rate chemistry. The properties computed for the steady region of the shock layer were used as inputs into Specair to compute spectra and the calculated spectra was compared with measured values. The comparisons revealed large discrepancies between the computed and measured values. The discrepancies are attributed to a lack of grid resolution, radiation coupling and assumed equilibrium free stream chemistry. Through the further development of the simulations presented, these discrepancies can be reduced and a more accurate comparison made with the modelling techniques available. 


\section{Chapter 6}

\section{Plasma Torch Experiments}

The goal of the plasma torch experiments was to obtain absolute measurements of radiative transitions in the VUV at well known thermochemical equilibrium conditions. Measurements of a radiating field known to be at thermochemical equilibrium allow for the validation of Einstein coefficients used to calculate spectral line intensities, without the complexities of non-equilibrium effects observed in shock layers produced in the X2 expansion tube.

The following chapter presents the design methodology followed to obtain and calibrate VUV emission spectral measurements on the plasma torch. The operating conditions that influence the final flow produced are discussed and the approach used to determine the radial temperature profile outlined. This is followed by an explanation of the optical configuration designed as well as of the actively cooled assembly used to place the viewing window at the edge of the plasma. An approach using a fence traversed through the plasma to study the effects of various depths of radiating flow field is explained. Finally the results from this set of experiments are presented and compared with computations and previously presented data.

\subsection{Operating Conditions}

There are numerous variables that control the flow produced by the plasma torch and these are controlled through the power supply, gas flow settings and the nozzle attached. The power supply has three variables that control the energy supplied to the induction coil: number of coil links, grid number and power input setting. These settings directly control the plate voltage, plate current, grid voltage and grid current. It is important to tune these variables in order to achieve maximum efficiency and ensure voltage and 
current values for the oscillator tube and plate are within the allowable ranges. The gas inflow rates, varied through the radial and swirl settings, also influence the maximum power that can be delivered to the plasma. A study was conducted to achieve a condition that allowed for high power and the final settings selected are outlined below. It should be noted that the final inflow rate selected was based not only on maximum power, but also the required radial temperature profile for the study.

Table 6.1: Operational parameters for tuned plasma torch condition.

\begin{tabular}{ll}
\hline Operational parameter & Setting \\
\hline Coil link & 1 \\
Grid number & 13.10 \\
Plate voltage & $11.2 \mathrm{kV}$ \\
Plate current & $5.5 \mathrm{~A}$ \\
Power setting & $62 \mathrm{~kW}$ \\
Air mass flow rate & $1.4 \mathrm{~g} / \mathrm{s}$ \\
Nozzle diameter & $50 \mathrm{~mm}$ \\
Plasma velocity at nozzle exit & $20 \mathrm{~m} / \mathrm{s}$ \\
Air injection ratio & $40 \mathrm{SLPM}$ radial and 20 SLPM swirl
\end{tabular}

\section{Temperature Profile}

The plasma temperature profile was calculated using the absolute spectral radiance of the atomic oxygen triplet at $777 \mathrm{~nm}$ as prescribed in MacDonald [144] and Laux [25]. The optical system shown in Figure 6.1. consisting of two 15 degree off-axis parabolic mirrors was used to image the plasma $27.5 \mathrm{~mm}$ above the nozzle exit onto the $25 \mu \mathrm{m}$ wide, $6.66 \mathrm{~mm}$ high slit of a spectroscopy system. The optical system was designed with a translational mount allowing for measurements to be made at intervals of $1 \mathrm{~mm}$ across the plasma. The spectroscopy system used for this study was an Acton Research Spectra Pro SP2758-P spectrometer coupled to a Princeton Instruments PI-MAX:1024-UV-18 mm ICCD. The PI-MAX ICCD provides 1024 pixels in the spectral direction and 256 pixels in the spatial direction. Combined with a pixel size of $26 \mu \mathrm{m} \times 26 \mu \mathrm{m}$, the slit is imaged over $6.66 \mathrm{~mm}$ in the spatial direction. Two gratings were used with this system; a $1200 \mathrm{~g} / \mathrm{mm}$ grating blazed at $300 \mathrm{~nm}$ for measurements for the oxygen triplet at $777 \mathrm{~nm}$ and a $2400 \mathrm{~g} / \mathrm{mm}$ grating blazed at $150 \mathrm{~nm}$ for measurements in the VUV. With an entrance slit width of $25 \mu \mathrm{m}$, the instrument broadening full-width at half-maximum (FWHM) was measured 
using HeNe laser at $632.8 \mathrm{~nm}$ to be $0.055 \mathrm{~nm}$ with the $1200 \mathrm{~g} / \mathrm{mm}$ grating and $0.0156 \mathrm{~nm}$ with the $2400 \mathrm{~g} / \mathrm{mm}$ grating [8]. A correction factor was applied to adjust the measured instrument function with the $2400 \mathrm{~g} / \mathrm{mm}$ for use in the VUV according to the reciprocal linear dispersion equation for a Czerny-Turner spectrometer, shown in Equation 6.1:

$$
\frac{f \lambda}{d x}=\frac{\lambda}{2 f}\left(\tan (\phi)+\sqrt{\left(\frac{2 d \cos (\phi)}{m \lambda}\right)^{2}-1}\right.
$$

where $f$ is the focal length of the spectrometer, $d$ is the spacing between the grooves of the grating, $m$ is the order of the dispersion, and $\phi$ is the half angle between the centre of the grating and the centre of the two mirrors. The Spectra Pro SP2758-P is a $750 \mathrm{~mm}$ focal length spectrometer with a half angle of $11^{\circ}$ resulting in a FWHM of $0.0303 \mathrm{~nm}$ for spectral range of $170 \mathrm{~nm}$ to $200 \mathrm{~nm}$ when observing first order [8].

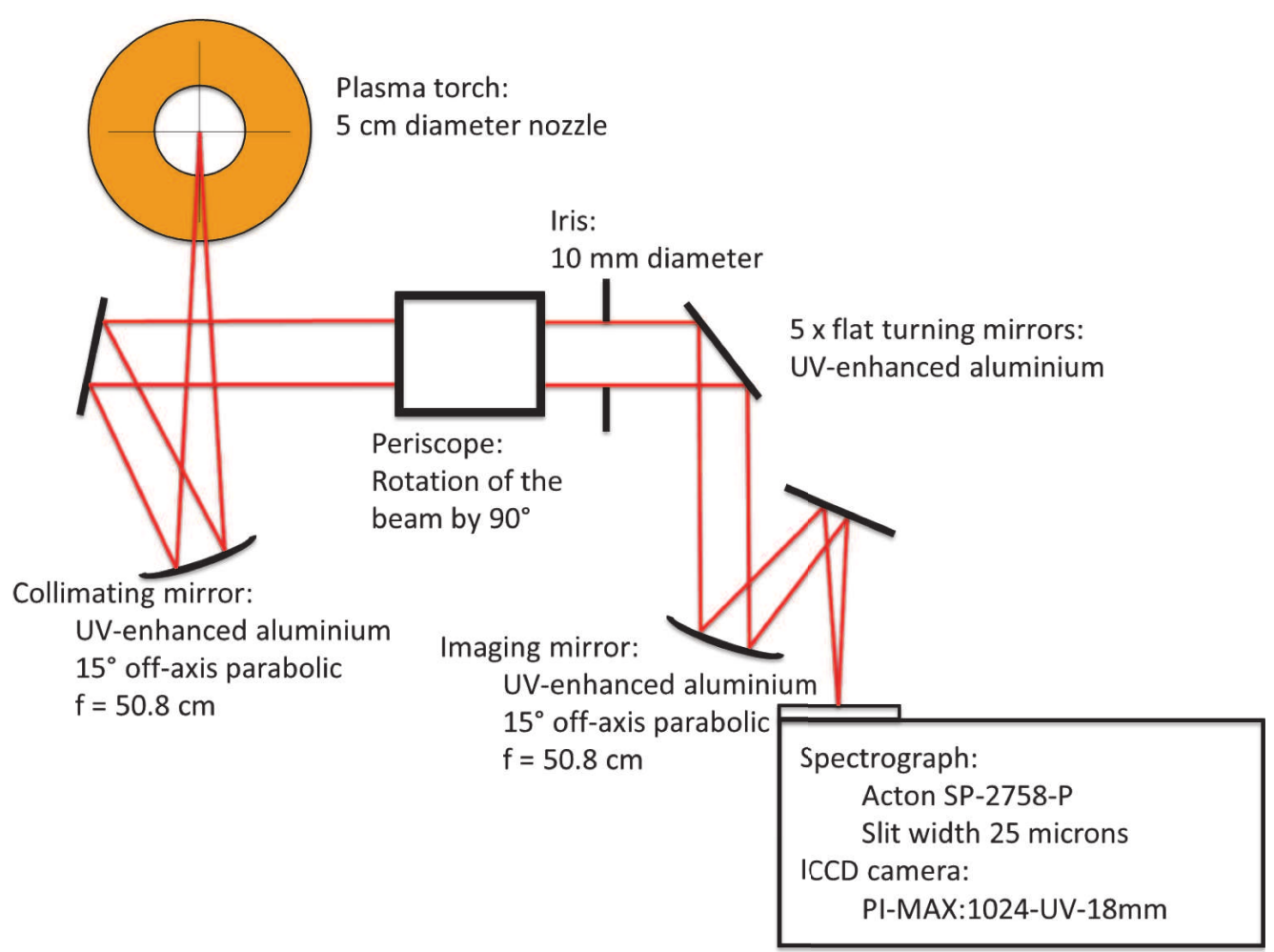

Figure 6.1: Optical system used to measure the atomic oxygen triplet at $777 \mathrm{~nm}[8]$.

Absolute calibration of the system was conducted using a tungsten ribbon lamp and an infra-red pyrometer to measure the temperature of the ribbon. As the emissivity of the tungsten ribbon, and transmission of the glass housing of the ribbon is known, it is possible to calculate the absolute grey body emission of the lamp. Using the calculated spectral radiance of the lamp, a conversion factor from intensity counts to spectral radiance on 
the ICCD is readily found, as described in Section 4.3. The temperature profile for this condition, shown in Figure 6.2, was determined by Jacobs et al. and showed a peak temperature of $6,700 \mathrm{~K}$ dropping to $5,100 \mathrm{~K}$ at a radial distance of $20 \mathrm{~mm}$ from the centreline of the plasma with an uncertainty of $3 \%$ [8]. The uncertainty of $3 \%$ in static temperature was calculated assuming perfect axisymmetry of the plasma torch flow and results in a $12 \%$ uncertainty in enthalpy. The centreline enthalpy for this condition was calculated to be $21.8 \mathrm{MJ} / \mathrm{kg}$ using the CEA program [139].

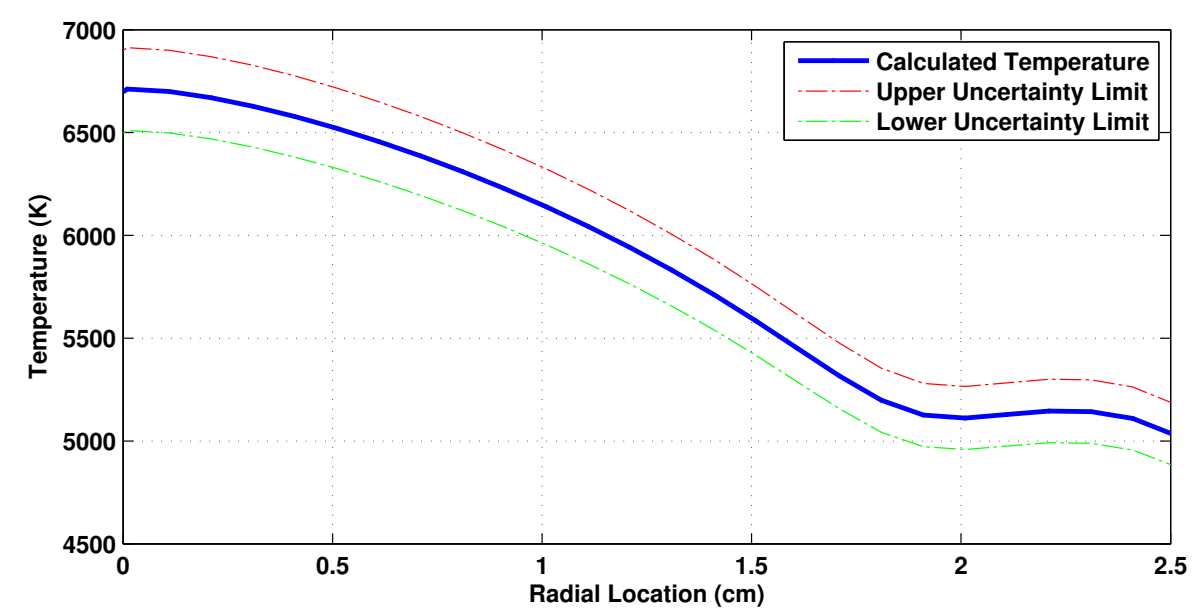

Figure 6.2: Measured temperature profile with upper and lower uncertainty limits.

\section{Gas Composition Profile}

The plasma torch operates at atmospheric pressure and the plasma has previously been shown to be in thermochemical equilibrium [25]. Using the known pressure of one atmosphere and the measured temperature profile, it is possible to use the CEA code to calculate the gas composition at radial locations within the plasma [139]. Gas compositions for three representative radial locations are shown in Table 6.2. At each location the mole fraction of the strongly VUV absorbing oxygen molecule is less than $1.7 \times 10^{-3}$. 
Table 6.2: Mole fractions for three representative plasma torch locations

\begin{tabular}{l|lll}
\hline Radial Location $(\mathrm{cm})$ & 0.0 & 1.3 & 2.0 \\
Temperature $(\mathrm{K})$ & 6,700 & 5,900 & 5,100 \\
\hline $\mathrm{N}$ & 0.384 & 0.145 & 0.0327 \\
$\mathrm{O}$ & 0.277 & 0.314 & 0.325 \\
$\mathrm{~N}_{2}$ & 0.327 & 0.524 & 0.616 \\
$\mathrm{O}_{2}$ & $7 \times 10^{-5}$ & $3.0 \times 10^{-4}$ & $1.7 \times 10^{-3}$ \\
$\mathrm{NO}$ & $3.9 \times 10^{-3}$ & $8.6 \times 10^{-3}$ & 0.0167 \\
$\mathrm{e}-$ & $4.8 \times 10^{-4}$ & $1.9 \times 10^{-4}$ & $5 \times 10^{-5}$ \\
\hline
\end{tabular}

\subsection{Optical System Design}

The optical diagnostic system used in these experiments was an emission spectroscopy system consisting of an Acton Research Spectra Pro SP2758-P coupled to a Princeton Instruments PI-MAX ICCD, as outlined in Section 6.1. This spectroscopy system was not vacuum capable and therefore a nitrogen flush was employed to remove molecular oxygen from the optical path. A nitrogen flushing approach had never been applied to experiments on the plasma torch and no guidelines were available regarding the flushing requirements for the spectroscopy system being used. Therefore a proof of concept study was carried out to examine the purity of nitrogen required in the optical path to allow for transmission of VUV radiation, and if this level was physically attainable. A sealed system external to the spectrometer was built around a vacuum chamber and used to house the focussing optical components. A magnesium fluoride window housed in an actively cooled assembly sealed the nitrogen flushing system at the plasma torch end. Active cooling of the viewing window allowed it to be placed close to the plasma to remove molecular oxygen absorption in ambient air and reduce the temperature of the window to decrease thermal stresses and avoid cracking.

\subsubsection{Proof of Concept Testing - Removal of Molecular Oxygen Absorption Through a Nitrogen Flush}

To achieve transmission in the VUV with the Spectra Pro SP2758-P spectrometer, molecular oxygen must be removed from the optical line of sight using a purge gas. Nitrogen was selected as it has no absorption bands in the wavelength range of interest and has higher resistance to arcing compared to other potential flush gases such as argon. Helium was not selected due to its comparatively high cost. 
The effect of molecular oxygen contaminants on the level of radiation transmitted at room temperature was calculated using the Lu absorption coefficients at wavelengths between $130 \mathrm{~nm}$ and $182 \mathrm{~nm}$ and Yoshino for the Schumann-Runge bands [20, 21]. Absorption by water vapour was ignored as there would be minimal out-gassing occurring due to the lack of vacuum pressures in this system [145]. The absorption coefficients for various impurities of molecular oxygen in the nitrogen flush were calculated using a pressure of $108 \mathrm{kPa}$ at room temperature. The optical path used for this calculation was $3.0 \mathrm{~m}$, representative of the optical path within the spectrometer. The results of these calculations are presented in Figure 6.3.

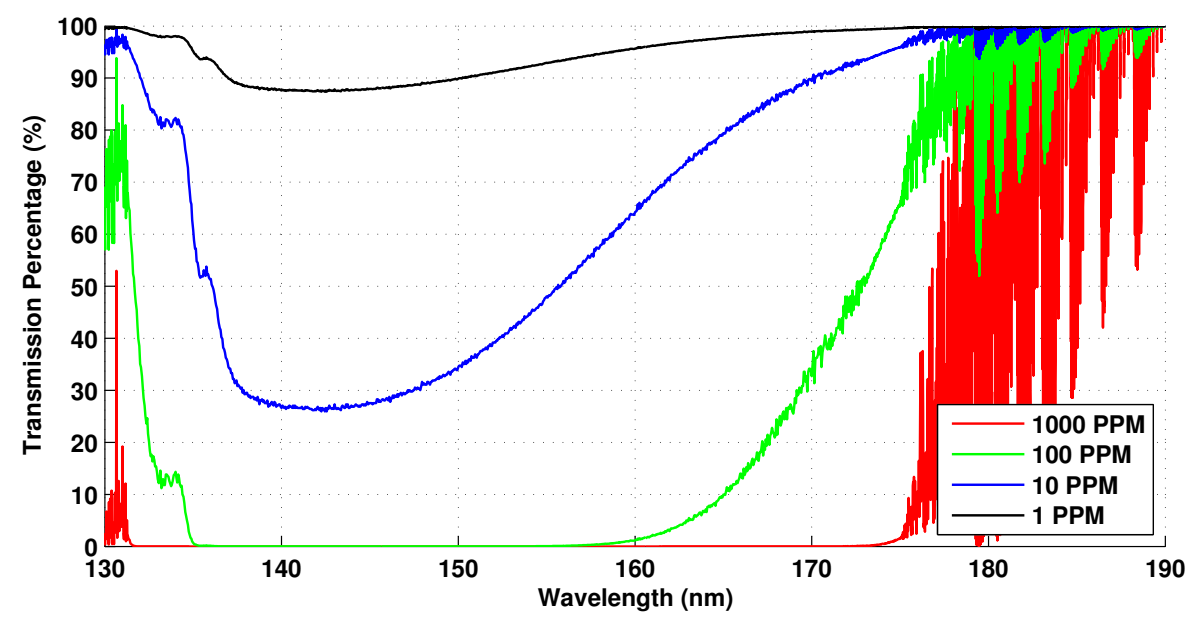

Figure 6.3: Influence of molecular oxygen contaminants on transmitted signal through an optical path of $3 \mathrm{~m}$.

To achieve better than $90 \%$ transmission down to $130 \mathrm{~nm}$, a molecular oxygen contamination level of less than 1 part per million (PPM) was required. This is better than the purity of a standard gas bottle and consequently unattainable for the entire optical path. A more realistic goal of $10 \mathrm{PPM} \mathrm{O}_{2}$ enabling $95 \%$ transmission of the nitrogen doublet at $174 \mathrm{~nm}$ was settled upon. At this level of contamination with the flush purity remaining constant, it would be theoretically possible to take measurements down to $130 \mathrm{~nm}$ and calibrate for any absorption occurring in the optical line of sight.

Initial testing of the nitrogen flushing concept was conducted using an argon mini-arc as a VUV radiation source and a nitrogen flow purity of 5 PPM. The mini-arc was placed at the entrance slit of the spectrometer and nitrogen was flushed through the spectrometer, as shown in Figure 6.4. There was a single inlet and two effective outlets for the nitrogen; the optical entry slit and a port at the front end of the ICCD. A line pressure of $8 \mathrm{kPa}$ above atmosphere was set to maintain a positive pressure differential to prevent atmospheric molecular oxygen diffusing into the chamber, and allowing all leak paths to 
be additional nitrogen outlets and ensuring there would be no build-up of pressure within the spectrometer that could damage it.

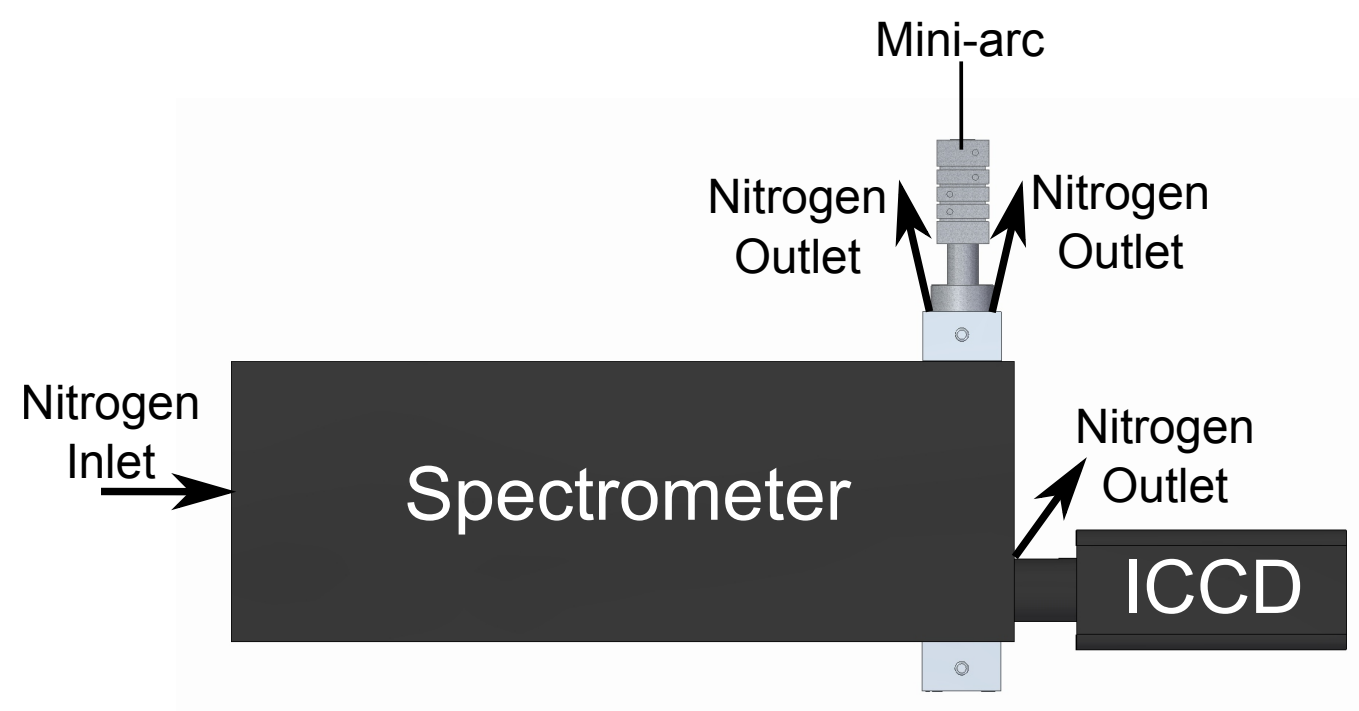

Figure 6.4: Initial nitrogen flush testing configuration with the mini-arc placed at the optical entrance of the spectrometer whilst nitrogen is flushed through it.

To investigate the time scales required to remove molecular oxygen from the optical path and achieve steady state purity, images were taken every minute observing the $\mathrm{O}_{2}$ Schumann-Runge bands between $182 \mathrm{~nm}$ and $192 \mathrm{~nm}$. The results of this investigation are presented in Figure 6.5.

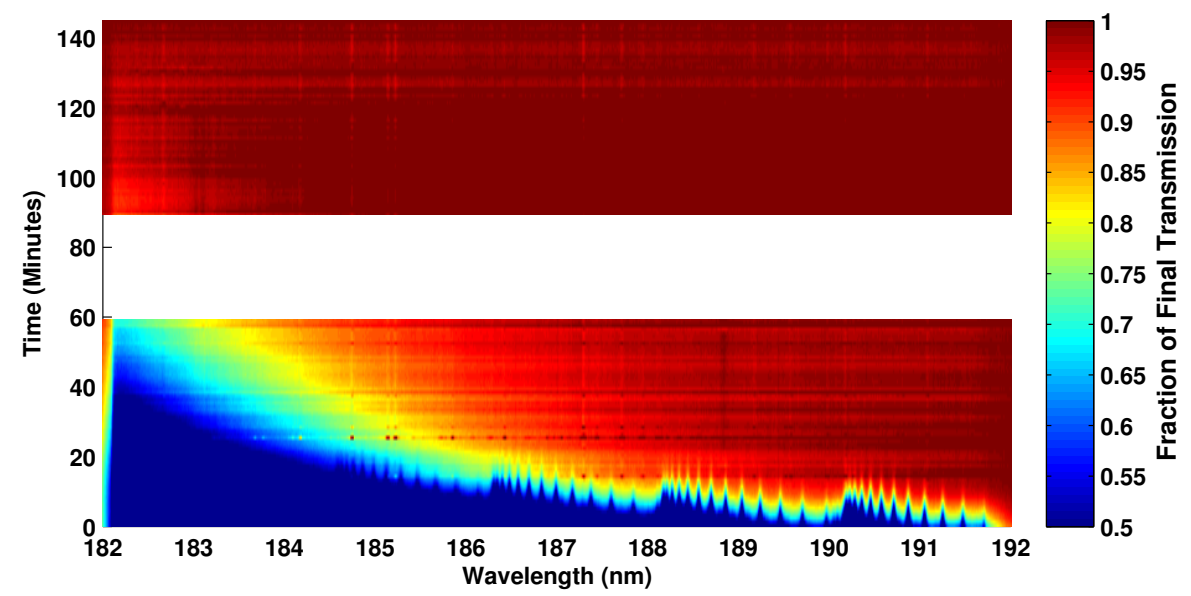

Figure 6.5: Increase in transmission through the optical path during 145 minutes of flushing the spectrometer with nitrogen. Spectra were not recorded in this wavelength range between 60 and 90 minutes.

The results of this experiment showed that the signal strength ceased to increase after 90 minutes for wavelengths longer than approximately $184 \mathrm{~nm}$ and there was minimal change 
in intensity for wavelengths shorter than $184 \mathrm{~nm}$ after 120 minutes. To observe the effects of the flush over the $170 \mathrm{~nm}$ to $180 \mathrm{~nm}$ spectral range, the experiment was repeated and the results are presented in Figure 6.6.

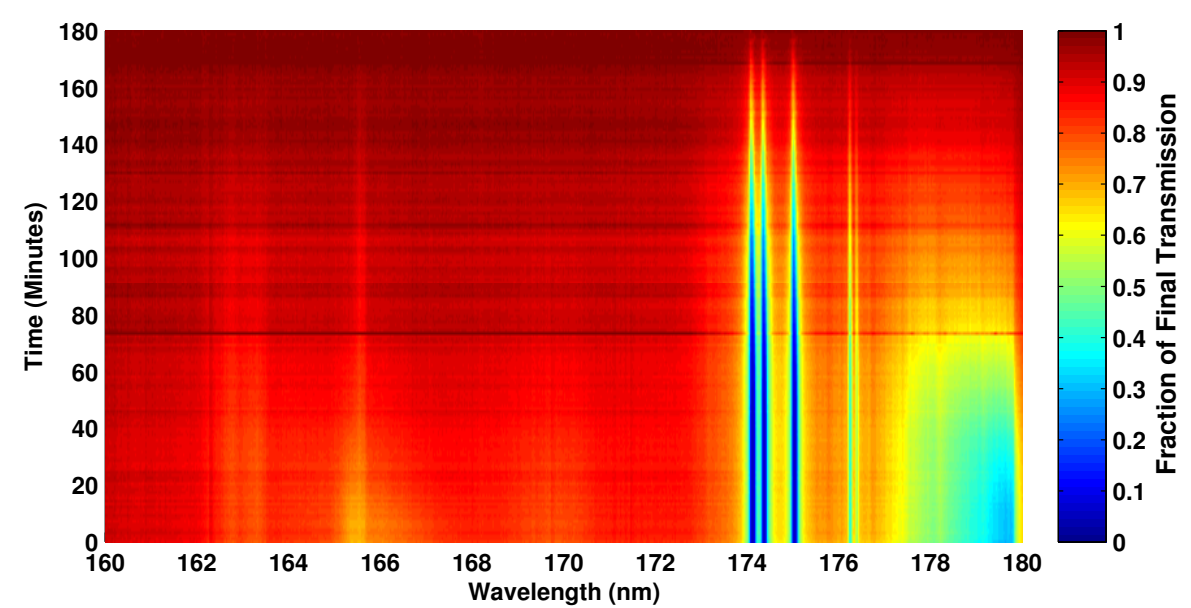

Figure 6.6: Increase in radiation transmission between $170 \mathrm{~nm}$ and $180 \mathrm{~nm}$ during 180 minutes of flushing the spectrometer with nitrogen.

In the wavelength range of $170 \mathrm{~nm}$ to $180 \mathrm{~nm}$, several atomic lines from contaminants were apparent in the spectral data. Two of the lines observed correspond to the nitrogen doublet at $174 \mathrm{~nm}$, due to contaminants within the argon gas flowing into the mini-arc and the rest of the spectral lines are due to carbon from the electrodes. Taking spectral images every minute for 180 minutes showed that the signal strength, and consequently flush purity, did not reach steady state in that time and the $174 \mathrm{~nm}$ nitrogen doublet did not appear on the spectrum at counts above background for over an hour into the flush. To determine if the flush purity was close to steady state, the nitrogen flush was continued for 20 hours. The spectra obtained are presented in Figure 6.7.

After 20 hours of flushing the optical path with molecular nitrogen, the transmitted spectral emission of the argon mini-arc increased by almost an order of magnitude. Due to the long flushing time required, a single high purity bottle could not be used for the entire flush. Consequently, a bottle bank of nitrogen with $5 \mathrm{PPM} \mathrm{O}_{2}$ was used for 18 hours and switched to a $0.1 \mathrm{PPM} \mathrm{O}_{2}$ bottle for 4 hours before the experiment. It was assumed that if the flush purity had not completely reached steady state, flushing for this amount of time would result in minimum change in $\mathrm{O}_{2}$ concentration during experiments and the subsequent calibration, allowing for any absorption to be accounted for. 


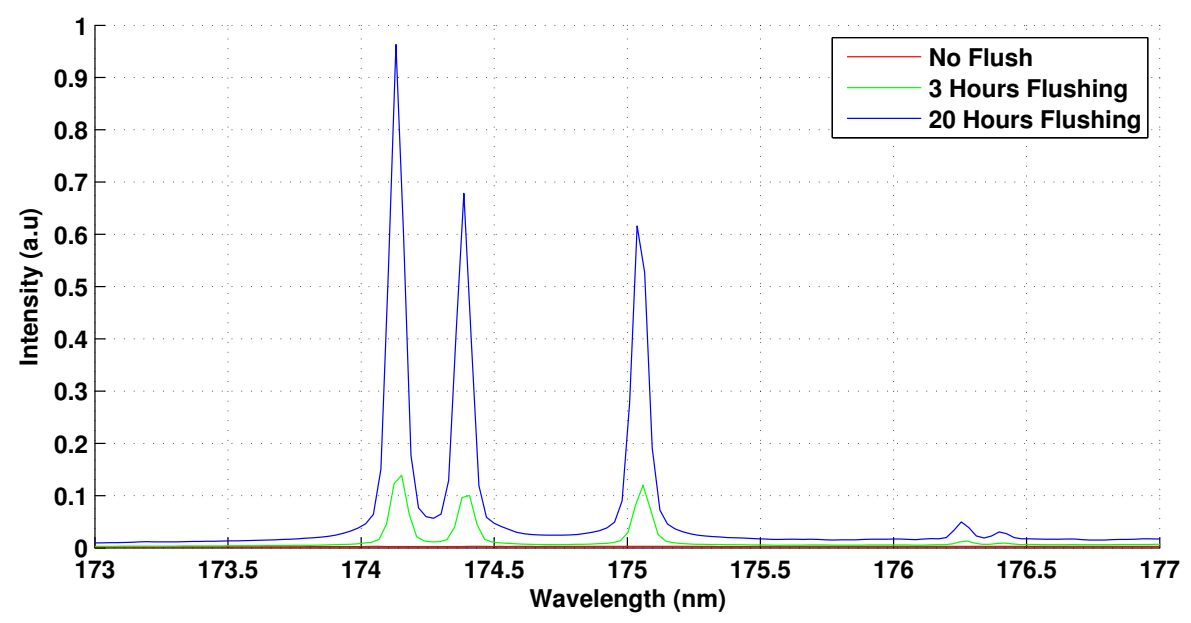

Figure 6.7: Increase in transmission through the optical path of the nitrogen and carbon lines between $173 \mathrm{~nm}$ and $177 \mathrm{~nm}$ after 0,3 and 20 hours of flushing the spectrometer with nitrogen.

\subsubsection{General Set-up for VUV Diagnostics of Plasma Emission}

The optical system designed for imaging the radiation emitted by the plasma onto the spectroscopy system consisted of a collimating $\mathrm{f}=500 \mathrm{~mm}$ mirror and a focussing $\mathrm{f}=500 \mathrm{~mm}$ mirror, resulting in a magnification of one. Both mirrors were coated with the Princeton Instruments \#1600 coating providing a peak reflectance at $160 \mathrm{~nm}$. To minimise aberrations, the turning angle between the two mirrors was kept to a minimum. An aperture of $10 \mathrm{~mm}$ was placed on the focussing mirror to ensure the entire plasma was within the depth of field and a slit width of $25 \mu \mathrm{m}$ was selected to provide high spectral resolution.

To remove molecular oxygen from the optical path of the external focussing optics, a sealed optical chamber was designed. This chamber was designed to also be suitable for high vacuum if the spectroscopy system is upgraded in the future. Consequently, the optical chamber was constructed using stainless steel plates to counteract deflections under atmospheric force and reduce out gassing. Vacuum flanges were installed on the chamber and modified to act as an inlet and outlet for the nitrogen flush. A schematic of the optical configuration and the nitrogen flow path is presented in Figure 6.8.

To connect the optical chamber to the spectrometer, an adapter that sealed onto the slit interface was designed. A vacuum fitting was installed onto the adapter allowing it to be connected to the optical chamber using a standard vacuum bellows. A plastic tube with a sliding seal was used at the plasma torch end of the optical chamber to isolate the system from the high voltage at the plasma torch head. To seal the system at the plasma torch end, a magnesium fluoride window was glued into a water cooled assembly. Water 


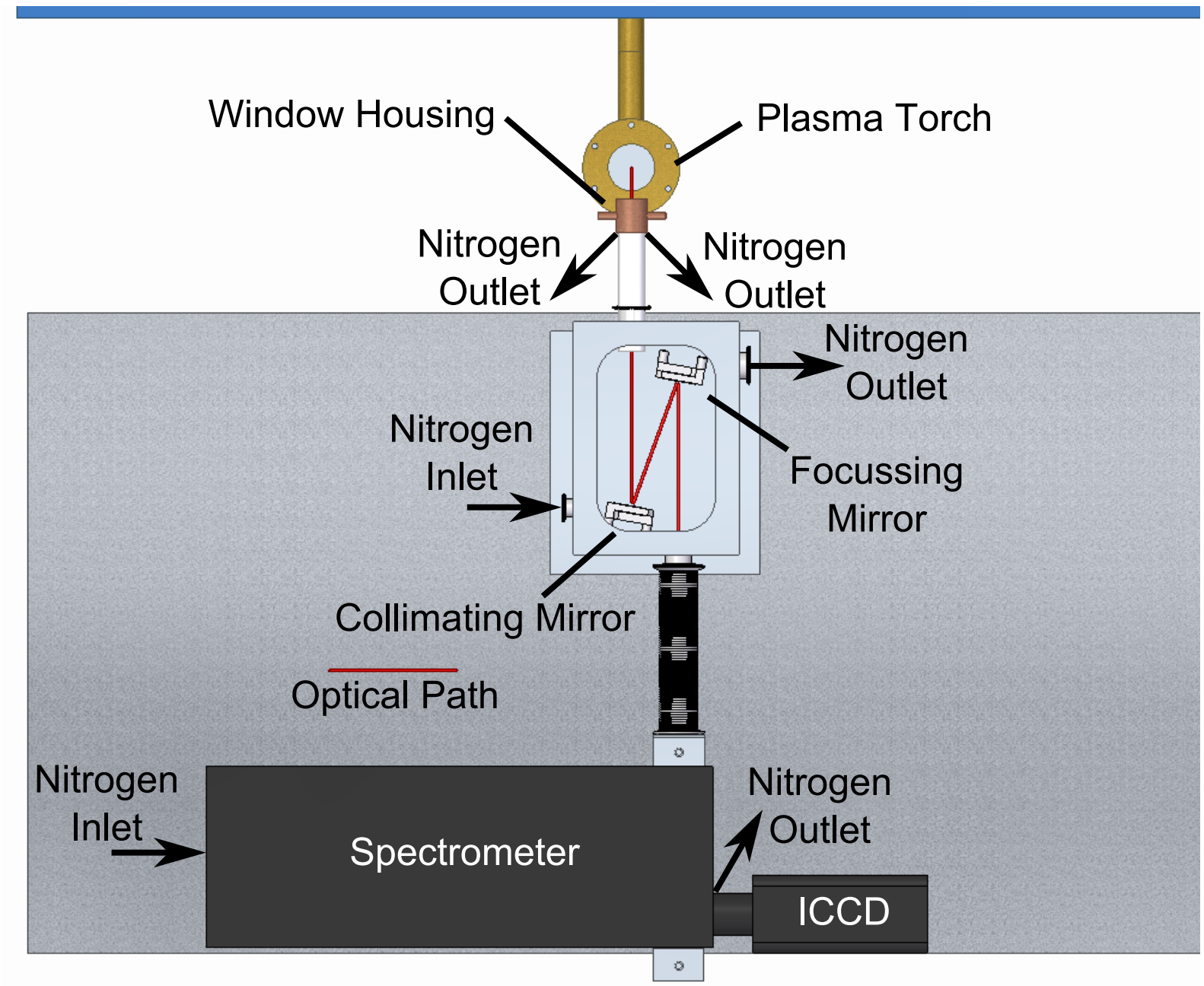

Figure 6.8: Optical configuration and nitrogen flow path for plasma torch experiments. 
cooling was required to prevent the window from cracking or being damaged as a result of the high heat flux from the plasma torch. An image of the final system set up is shown in Figure 6.9.

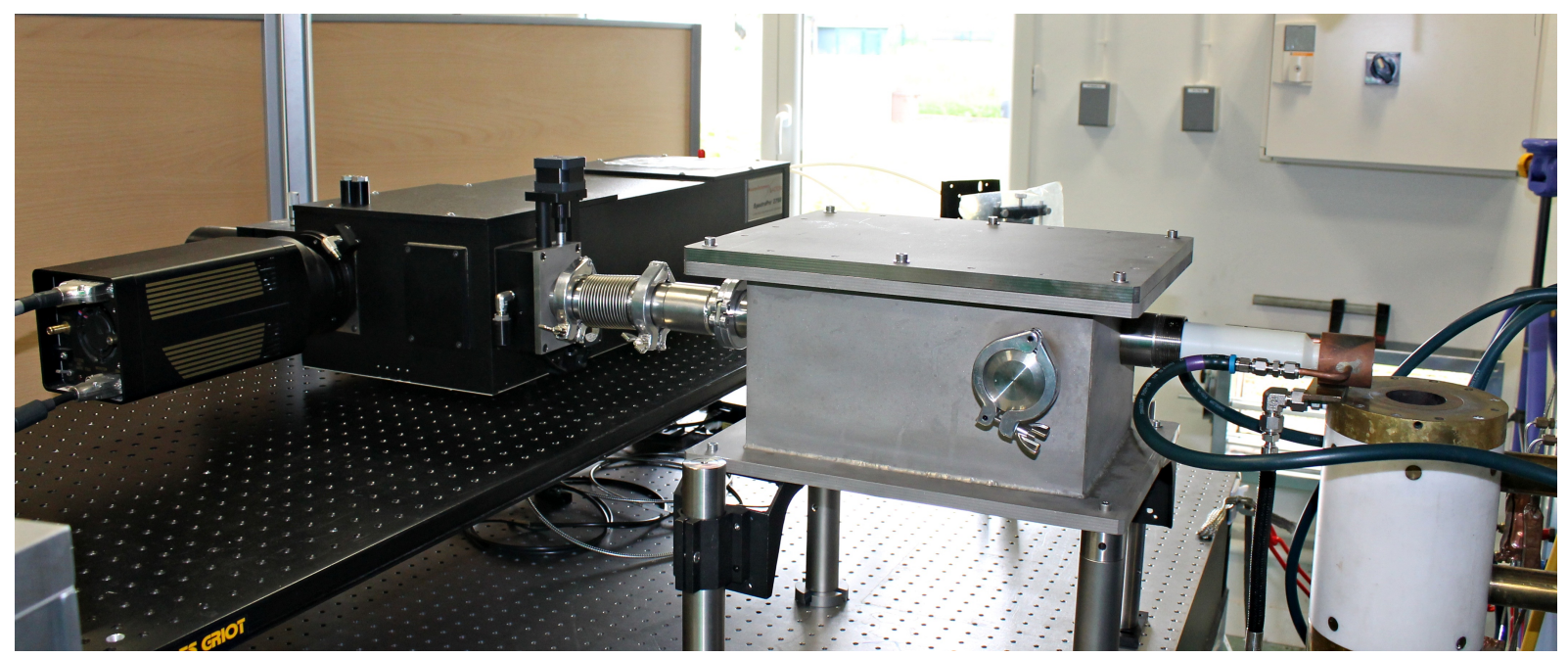

Figure 6.9: Final set up of the spectrometer, optical chamber, water cooled window housing and the plasma torch.

\subsubsection{Window Housing Design}

To avoid absorption from molecular oxygen between the sealed optical path and the plasma, the viewing window was required to be positioned at the edge of the plasma. This meant the window needed to withstand air flowing over it at approximately 4,000 K at $20 \mathrm{~m} / \mathrm{s}$. The window was not recessed to prevent a cooled pocket of air that may contain molecular oxygen from forming between the window and the plasma. A copper housing with water flowing through it was designed to cool the window, as shown in Figure 6.10. Copper was selected as it provides high thermal conductivity, ensuring the heat loads on the window were efficiently transferred to the cooling water. A high thermal conductivity epoxy, Omegabond 101 [146], was used to ensure there was no thermally insulating barrier between the window and the copper surface. A schematic of the window housing assembly is presented in Figure 6.10.

To test the design of the copper window housing, a heat transfer analysis was conducted using the heat transfer module of COMSOL Multiphysics [147]. The thermal and structural values for the magnesium fluoride windows and Omegabond epoxy were taken from Crystran and Omega [29, 146]. An axisymmetric simulation was conducted on the copper housing with a heat flux calculated using a flow temperature of $4,000 \mathrm{~K}$ and velocity of 


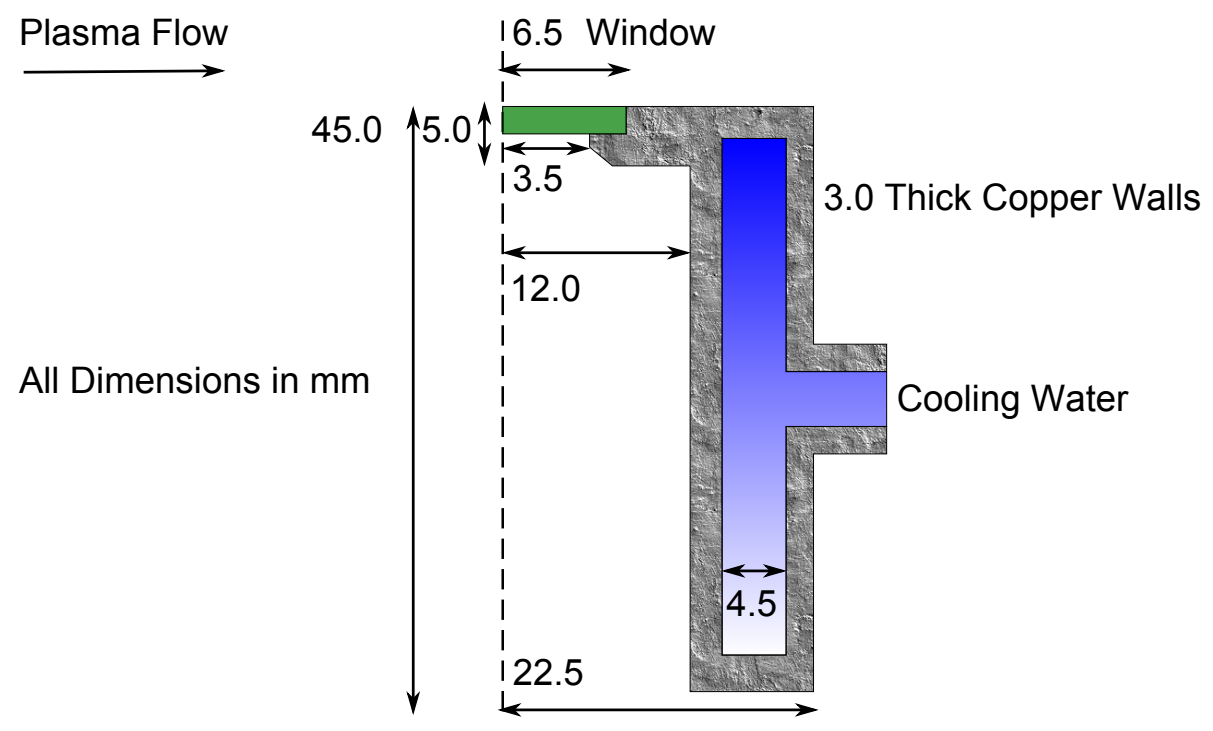

Figure 6.10: Final design of the water cooled window housing assembly.

$20 \mathrm{~m} / \mathrm{s}$ resulting in a convective heat transfer coefficient of $35 \mathrm{~W} / \mathrm{m}^{2} \mathrm{~K}$. The radiative heat flux was not considered at the window as it is transparent. The thickness of the epoxy between the window and the copper housing was estimated to be $0.1 \mathrm{~mm}$.

The thermal conductivity of magnesium fluoride is higher in the perpendicular direction of the central axis than the parallel direction [29]. The average of the two values, $27.3 \mathrm{~W} / \mathrm{mK}$, was used as this would produce an overestimate of the maximum temperature because the higher perpendicular thermal conductivity is more influential, as the thermal load must travel radially to the cooled edges. Whilst it is possible to obtain temporal resolution using COMSOL, a steady state solution was required due to the long test times the window needed to endure. The results of this analysis are presented in Figure 6.11.

The heat transfer analysis showed the window reaches a maximum temperature of $329 \mathrm{~K}$ at the centre and a minimum temperature of $304 \mathrm{~K}$ at the coldest edge. This variance in temperature produces a thermal stress of $30.7 \mathrm{MPa}$ in the radial direction and $47.3 \mathrm{MPa}$ in the parallel direction. Both of these values are below the apparent elastic limit of 49.6 $\mathrm{MPa}$ for magnesium fluoride and therefore the temperature difference of $25 \mathrm{~K}$ across the window was deemed acceptable.

To experimentally test the active cooling and the importance of molecular oxygen absorption at the edge of the plasma, the viewing window was placed at a distance of $10 \mathrm{~mm}$ from the edge of the nozzle and gradually moved closer whilst obtaining spectra of the plasma. It was not possible to move the window during operation of the plasma torch and consequently the window had to endure the start-up procedure and plasma stabilisation time for each test. Five spectra were acquired at distances of $10 \mathrm{~mm}, 7.5 \mathrm{~mm}, 6 \mathrm{~mm}, 5 \mathrm{~mm}$ 
and $4 \mathrm{~mm}$ from the edge of the nozzle. The results of this experiment are presented in Figure 6.12.

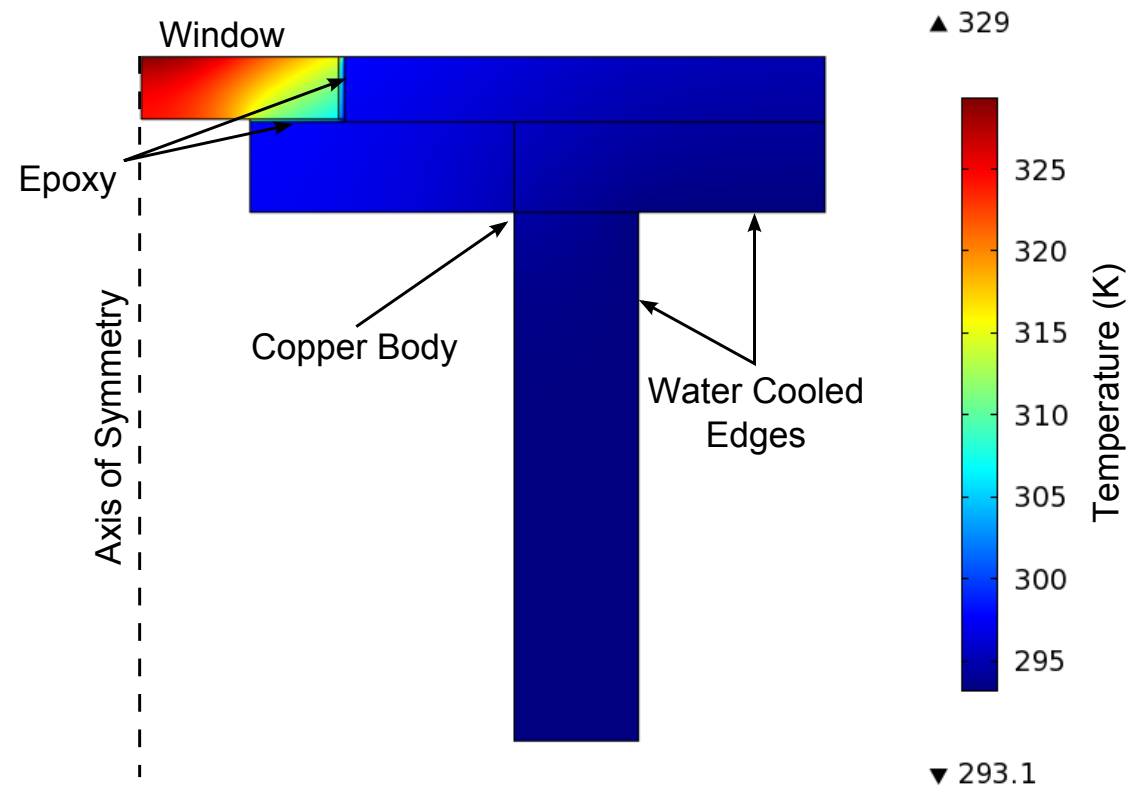

Figure 6.11: Results of the COMSOL Multiphysics simulation showing the steady state temperature of the window within the water cooled copper housing.

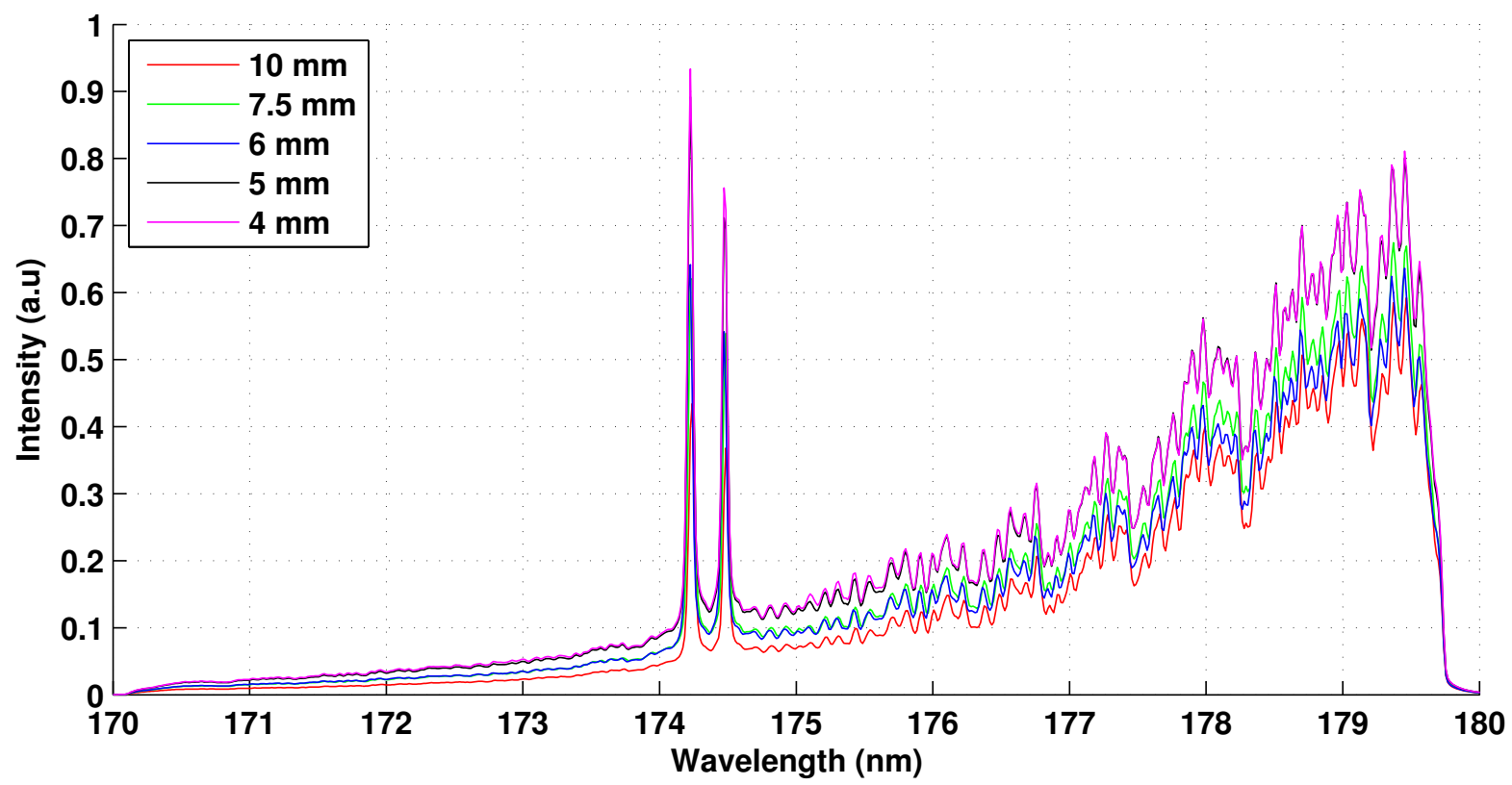

Figure 6.12: Plasma emission spectra obtained in the VUV with the water cooled window housing at $10 \mathrm{~mm}, 7.5 \mathrm{~mm}, 6 \mathrm{~mm}, 5 \mathrm{~mm}$ and $4 \mathrm{~mm}$ from the edge of the nozzle. 
The results of this study indicated that there was absorption occurring at distances greater than $5 \mathrm{~mm}$ from the nozzle and there was no change in measured intensity between $4 \mathrm{~mm}$ and $5 \mathrm{~mm}$. To confirm if there was negligible absorption within $6 \mathrm{~mm}$ of the nozzle edge, another study was conducted at distances of $5 \mathrm{~mm}, 4 \mathrm{~mm}, 3 \mathrm{~mm}, 2 \mathrm{~mm}$ and $1 \mathrm{~mm}$ from the edge of the nozzle. The results of this investigation are presented in Figure 6.13 .

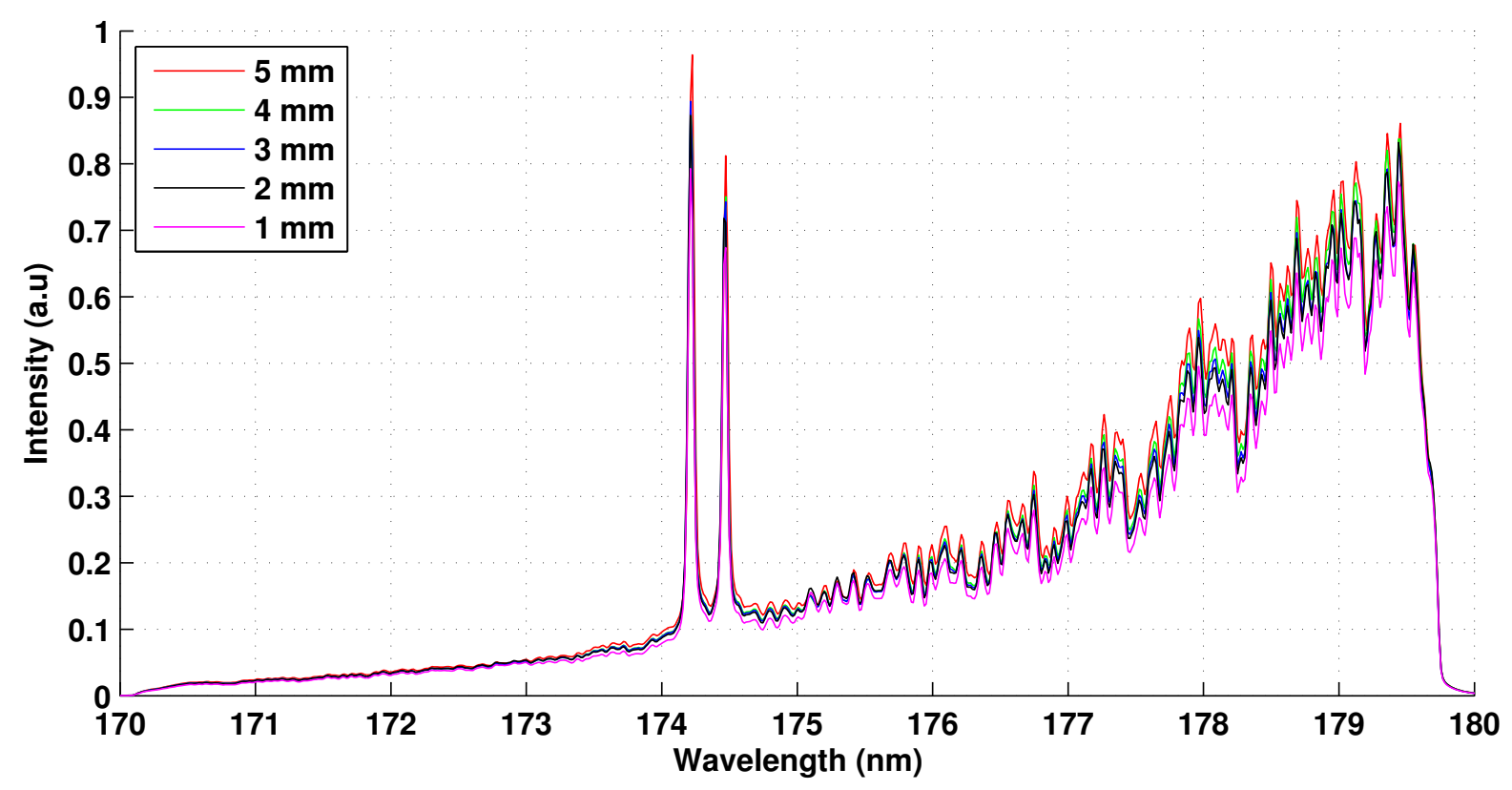

Figure 6.13: Plasma emission spectra obtained in the VUV with the window housing at $5 \mathrm{~mm}, 4 \mathrm{~mm}, 3 \mathrm{~mm}, 2 \mathrm{~mm}$ and $1 \mathrm{~mm}$ from the edge of the nozzle.

The measured spectra showed that there was no increase in signal strength between $5 \mathrm{~mm}$ from the plasma edge and $2 \mathrm{~mm}$ from the plasma edge. At a distance of $1 \mathrm{~mm}$ from the plasma edge, the signal strength dropped and this was attributed to surface degradation of the window. Throughout these experiments, spectral measurements were also taken of the $777 \mathrm{~nm}$ atomic oxygen triplet. As the $777 \mathrm{~nm}$ emission is not absorbed by molecular oxygen, it was possible to test if the increases observed in VUV signal strength were due to the reduction in molecular oxygen in the line of sight or due to other factors such as window transmission degradation. Integrated and normalised values of the spectral images obtained are shown in Figure 6.14.

From Figure 6.14, it is apparent that the VUV signal strength is increasing between $10 \mathrm{~mm}$ and $4 \mathrm{~mm}$ from the nozzle edge whilst the NIR signal remains fairly constant. This indicates molecular oxygen is absorbing the VUV signal at distances greater than $4 \mathrm{~mm}$ from the plasma edge. The results from the experiments at distances closer than $5 \mathrm{~mm}$ from the nozzle edge indicate surface degradation is occurring as both the VUV and NIR signals are decreasing. The fact that the signal strength in the VUV spectral 


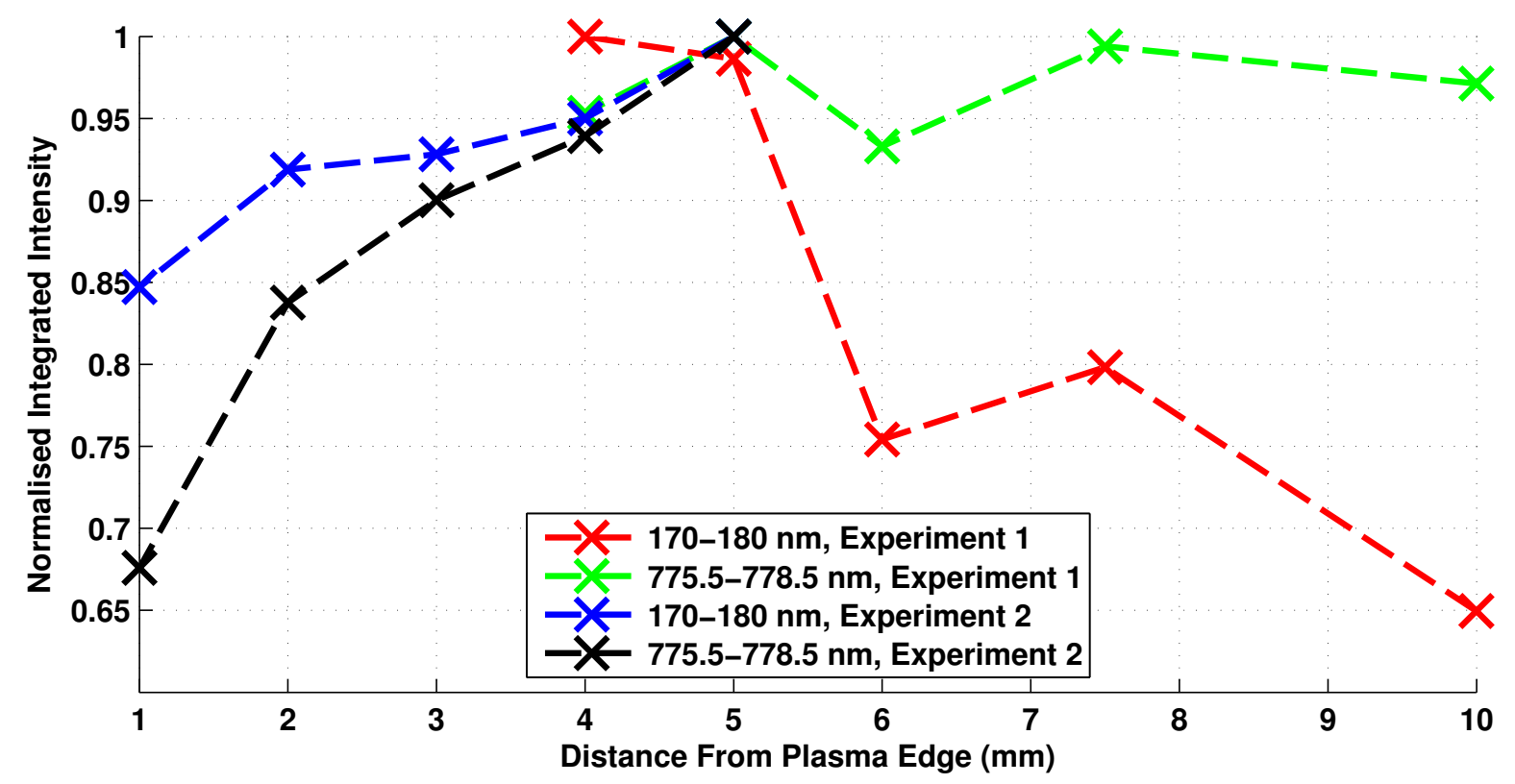

Figure 6.14: Integrated and normalised values of spectral measurements in the VUV between $170 \mathrm{~nm}$ and $180 \mathrm{~nm}$ and in the NIR between $775.5 \mathrm{~nm}$ and $778.5 \mathrm{~nm}$ for distances between $10 \mathrm{~mm}$ and $1 \mathrm{~mm}$. Experiment 2 - Measurements between $1 \mathrm{~mm}$ and $4 \mathrm{~mm}$. Experiment 1 - Measurements between $4 \mathrm{~mm}$ and $10 \mathrm{~mm}$.

range does not decrease as rapidly as the NIR with decreasing distance from the plasma, indicates increases in signal strength obtained through the removal of molecular oxygen from the optical path. The final position of the window selected was $5 \mathrm{~mm}$ from the edge of the nozzle to reduce window degradation and limit absorption by molecular oxygen.

\subsubsection{Calibration}

An in-situ calibration of the spectroscopy system was conducted using an argon miniarc with a known spectral radiance from $125 \mathrm{~nm}$ to $400 \mathrm{~nm}$. To carry out the in-situ calibration, the mini-arc was placed in the position of the plasma. A shroud with a flow of nitrogen was installed between the mini-arc and the viewing window to remove absorption from molecular oxygen, as shown in Figure 6.15. The calibration was conducted after the experiment to account for any surface degradation of the viewing window that may have occurred during the experiment. The conversion from intensity counts on the ICCD to spectral radiance was carried out following the technique described in Section 4.3.

To obtain the peak radiance of the mini-arc spectrum required for calibration, the spatially resolved spectrum was averaged across 10 pixels either side of the peak spatial value. An example image between $171 \mathrm{~nm}$ and $179 \mathrm{~nm}$ is shown in Figure 6.16. 


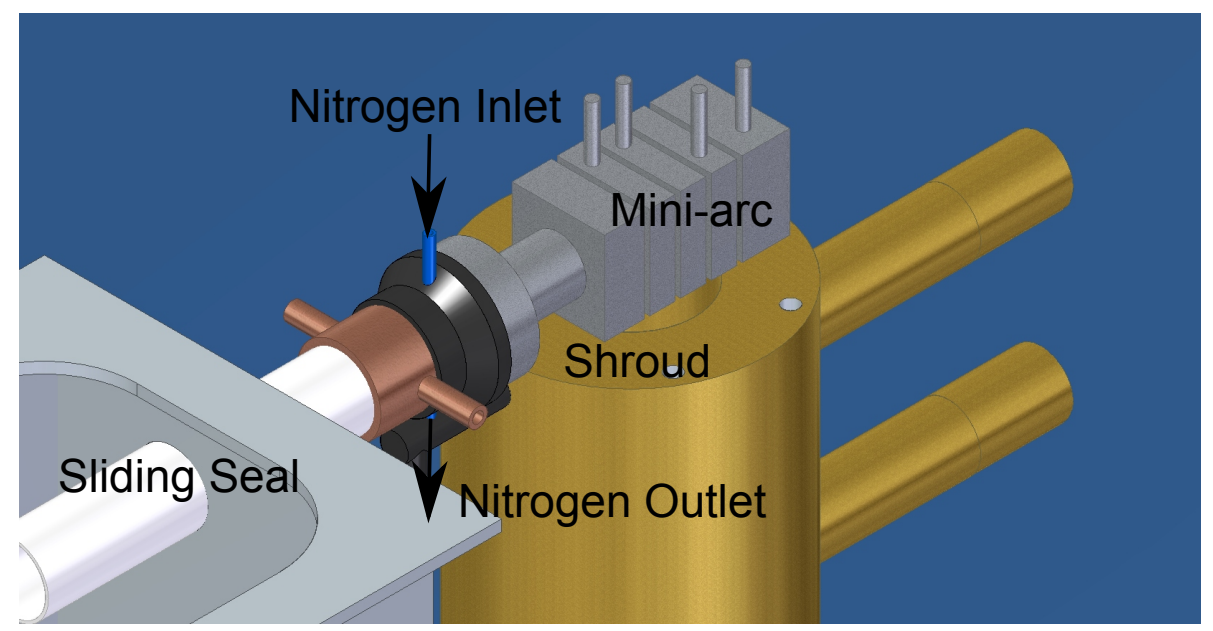

Figure 6.15: Schematic of the in-situ calibration approach with a shroud to contain the nitrogen flush used to remove molecular oxygen between the viewing window and the mini-arc.
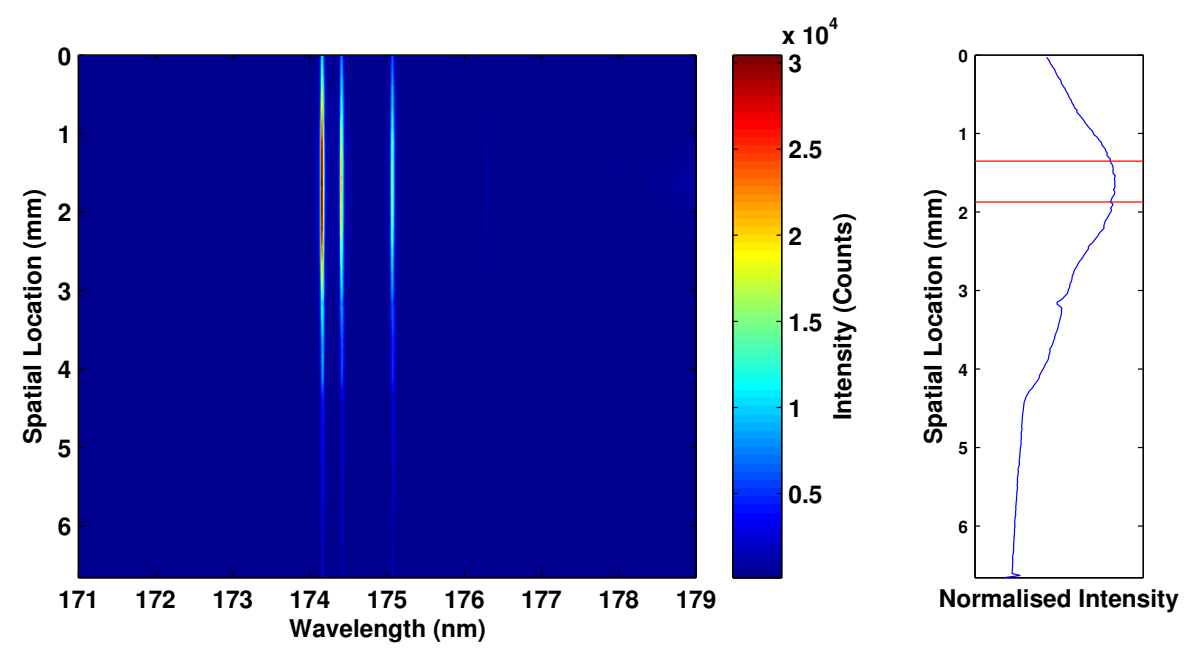

Figure 6.16: Spectral response of the system using in-situ calibration between $171 \mathrm{~nm}$ and $179 \mathrm{~nm}$ with interpolation across contaminant spectral lines. 
The spectral images obtained using the mini-arc contain strongly radiating atomic nitrogen and carbon lines in the wavelength range being investigated, as shown in Figure 6.17. The appearance of the nitrogen is believed to be due to contaminants in the argon supply and potential entrainment of atmospheric air. Carbon is also believed to have entered the system through the flush gas flow and its presence is enhanced by carbon build-up on the electrodes. These spectral lines are identified in the spectrum presented in Figure 6.17.

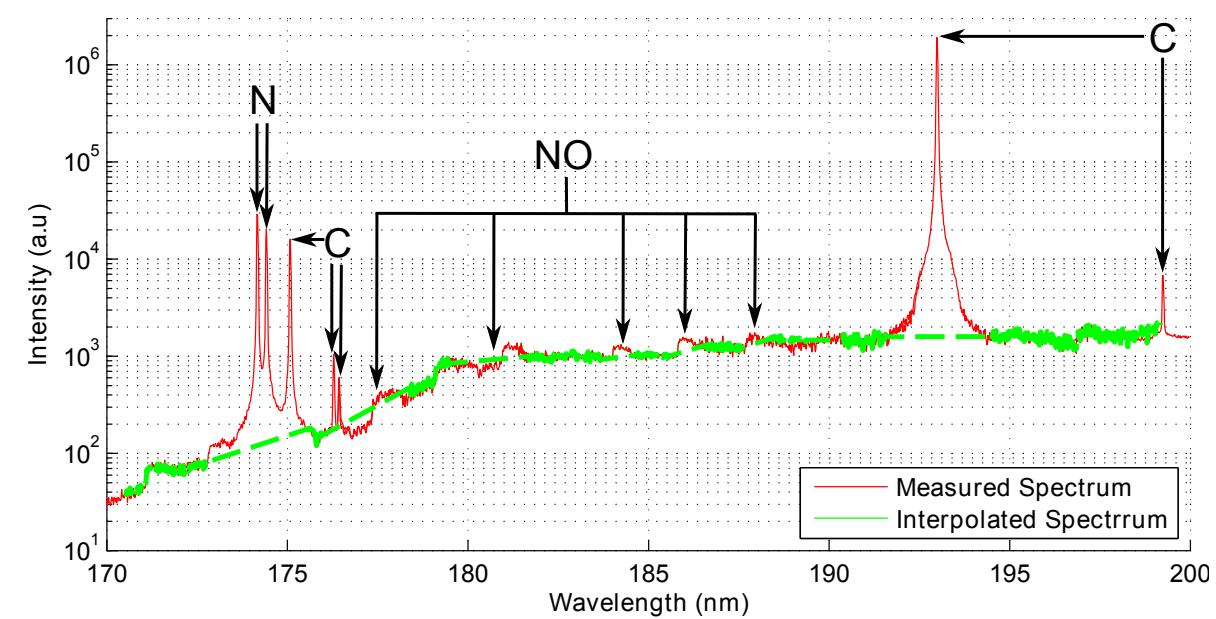

Figure 6.17: Spectral data obtained using the mini-arc with nitrogen and carbon contamination lines.

To determine the system response, the measured spectrum was divided by the known spectral radiance of the mini-arc, as described in Equation 4.10, The regions containing spectral lines from contaminants were removed and system response values were interpolated across them, as shown in Figure 6.18.

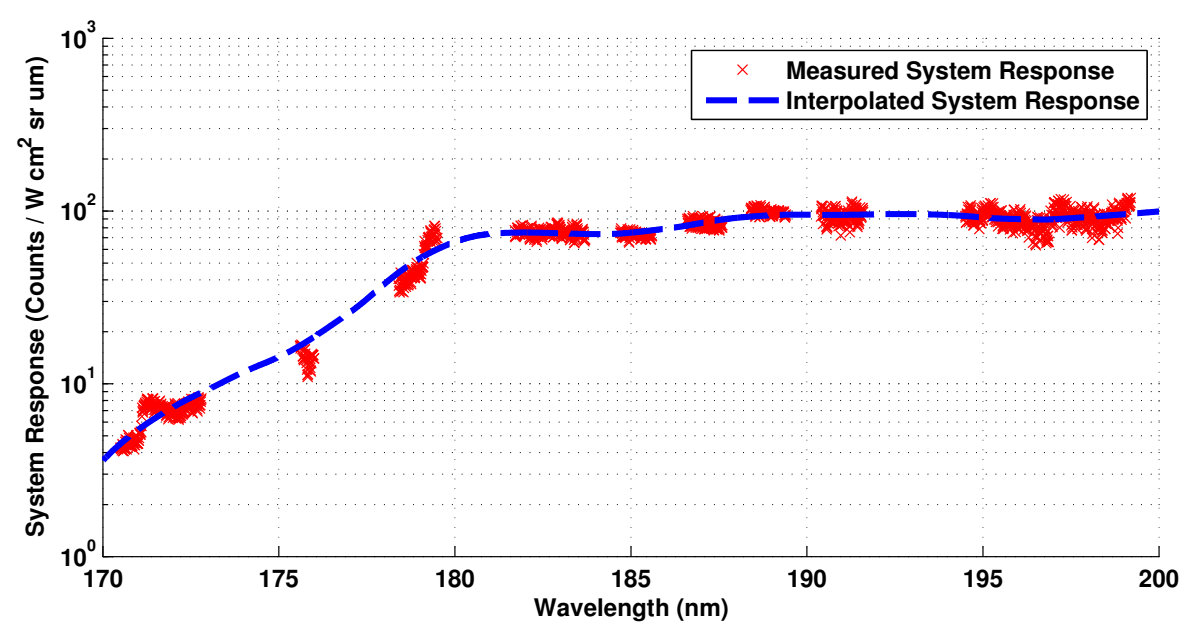

Figure 6.18: Spectral response of the system using in-situ calibration between $170 \mathrm{~nm}$ and $200 \mathrm{~nm}$ with interpolation across contaminant spectral lines. 


\section{Spectroscopy System Uncertainty Analysis}

Calibration of the spectroscopy system was conducted using an in-situ technique immediately after the experimental measurements were conducted. This approach ensured the purity of the nitrogen flush remained constant between the experiment and the calibration measurements, and all optical component transmissions, reflectances and apertures are accounted for in the spectrum acquired. Consequently, the total uncertainty of the calibrated measurements is a result of the uncertainty in the mini-arc output and positioning.

The argon mini-arc was constructed and operated in accordance with the radiometric standards in the vacuum ultraviolet as defined by the Center for Radiation Research at the National Measurement Laboratory [148]. The variables that control the output of the mini-arc include the power supplied to the arc, the gas flow rate and purity, the transmission of the window and the stability of the arc. The alignment of the miniarc with the optical capture system can also introduce additional variables and must be considered in the uncertainty analysis. Finally, there is also an uncertainty in the primary standard itself. All of these factors are discussed in detail in the calibration report [148] and are summarised in Table 6.3 as they apply to the calibration of these experiments. The total uncertainty for the spectroscopy system was found to be $21.4 \%$ for wavelengths shorter than $140 \mathrm{~nm}$ and $16.4 \%$ for wavelengths longer than $140 \mathrm{~nm}$.

Table 6.3: Contributing factors in the total uncertainty of the in-situ mini-arc calibration.

\begin{tabular}{l|ll}
\hline Variable & Uncertainty $\lessgtr 140 \mathrm{~nm}(\%)$ & Uncertainty $\geq 140 \mathrm{~nm}(\%)$ \\
\hline Current & 0.4 & 0.4 \\
Gas flow rate & $\sim 0$ & $\sim 0$ \\
Mini-arc pressure & 1 & 1 \\
Alignment & 4 & 4 \\
Purity & $\sim 0$ & $\sim 0$ \\
Stability & 1 & 1 \\
Window transmission & 5 & 5 \\
Primary standard & 10 & 5 \\
\hline Total & 21.4 & 16.4 \\
\hline
\end{tabular}




\subsection{Spectral Measurements Between $170 \mathrm{~nm}$ and $200 \mathrm{~nm}$}

Spectral images were acquired between $170 \mathrm{~nm}$ and $200 \mathrm{~nm}$ with the torch operating at the conditions prescribed in Section 6.1. A sample uncalibrated, spatially resolved spectral image of the plasma is presented in Figure 6.19.
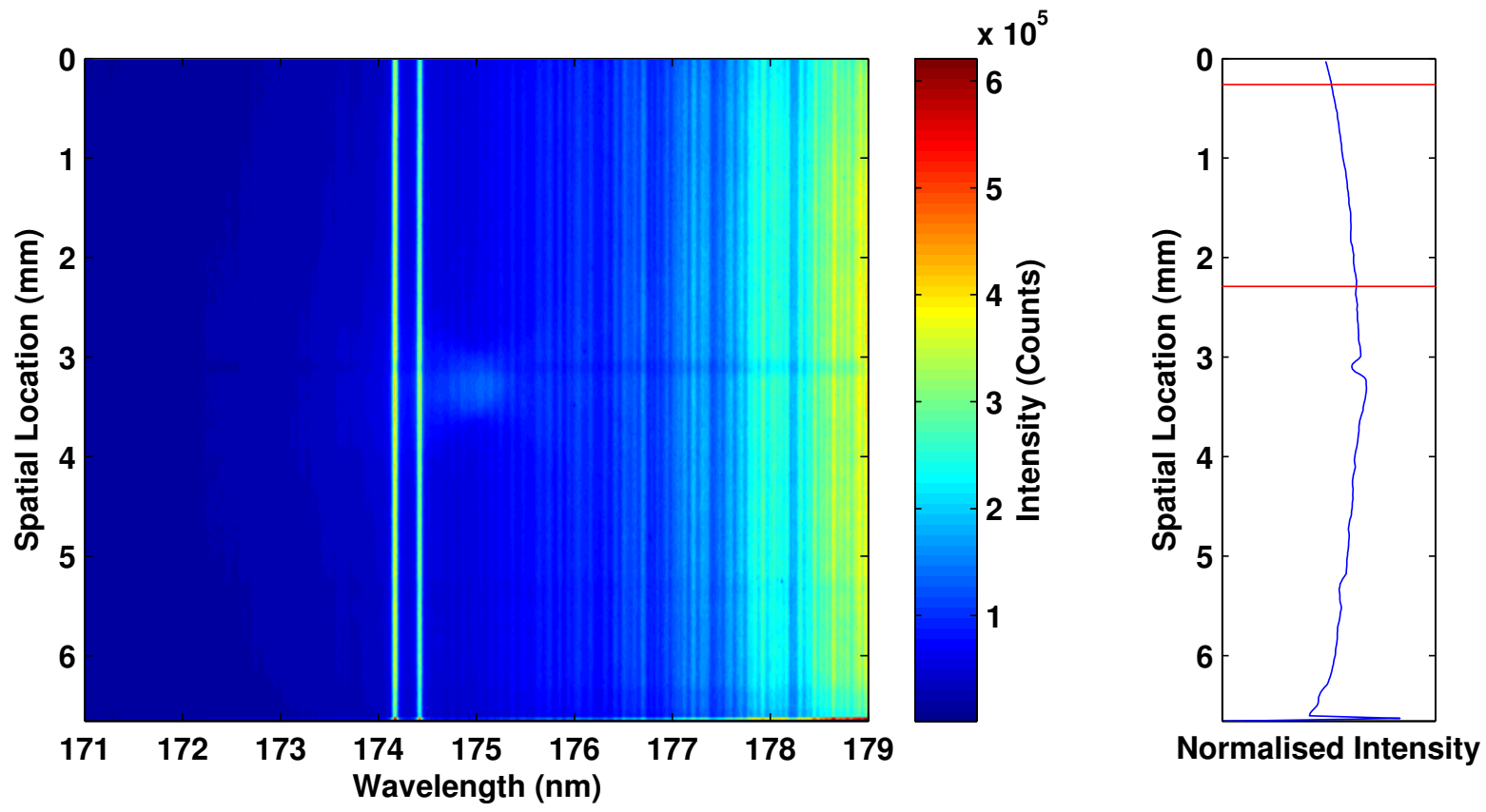

Figure 6.19: Uncalibrated, spatially resolved spectral image of plasma torch emission between $171 \mathrm{~nm}$ and $179 \mathrm{~nm}$.

To discard the spurious light and row of lower sensitivity pixels at the centre of the ICCD array due to damage from previous experiments, the spatially resolved image was averaged across the region bracketed by the red lines in Figure 6.19. Spatial averaging was employed to produce spectra with better overall signal to noise ratio. The uncalibrated results obtained between $170 \mathrm{~nm}$ and $200 \mathrm{~nm}$ are presented in Figure6.20, Even at long exposure times, no usable spectral data could be acquired below $170 \mathrm{~nm}$. This is attributed to the expected drop in system response coupled with an increasing molecular oxygen absorption coefficient at shorter wavelengths. 


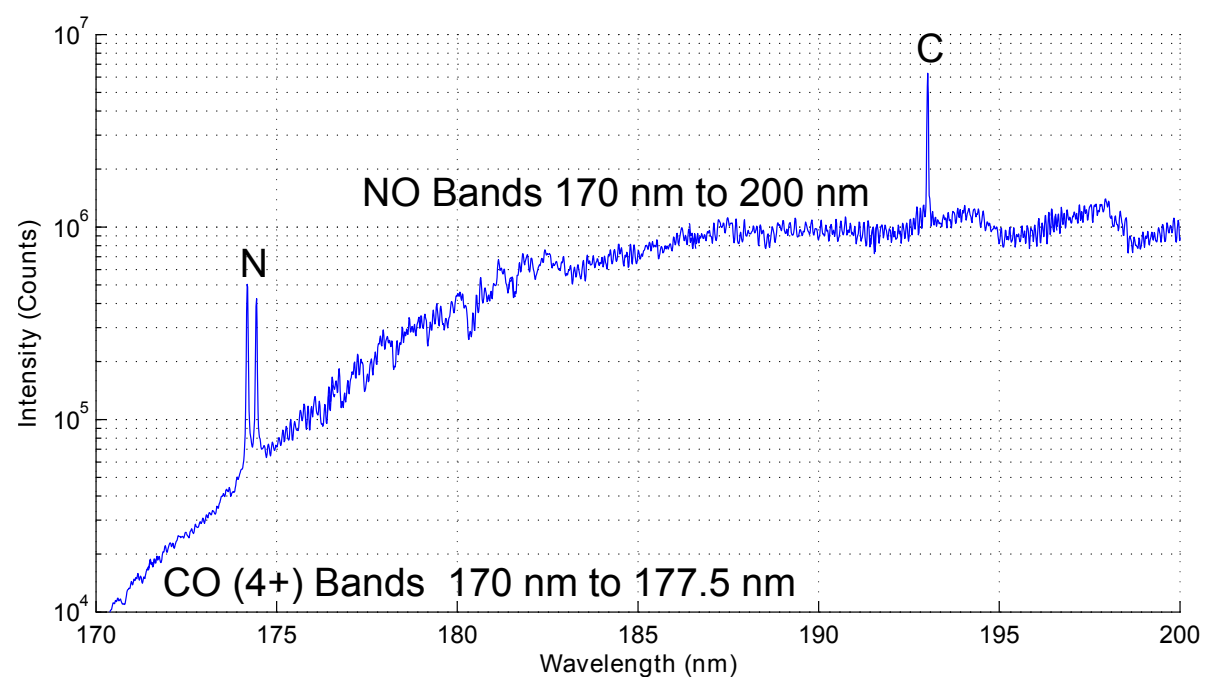

Figure 6.20: Uncalibrated spectrum of plasma emission from $170 \mathrm{~nm}$ to $200 \mathrm{~nm}$.

The spectral lines in the uncalibrated image were identified as the atomic nitrogen doublet at $174 \mathrm{~nm}$ and atomic carbon at $193 \mathrm{~nm}$. There was also spectral band structure observed indicating the presence of nitric oxide and carbon monoxide. The measured spectrum was divided by the previously calculated calibration factor to produce the calibrated spectrum shown in Figure 6.21.

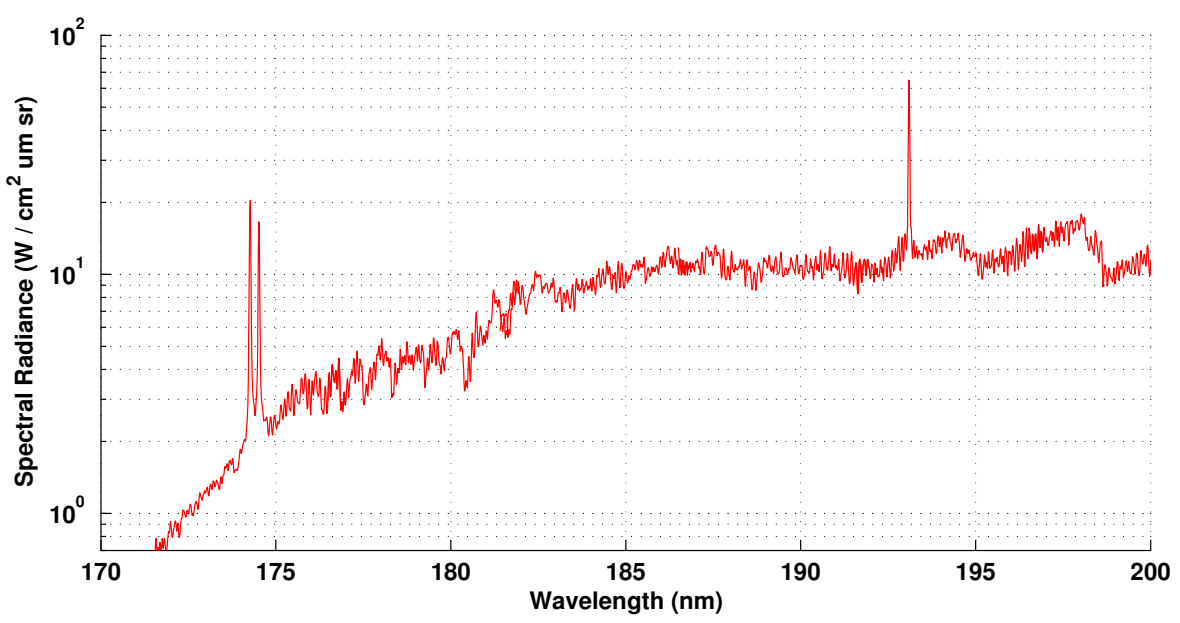

Figure 6.21: Calibrated and spatially averaged spectrum of plasma emission from $170 \mathrm{~nm}$ to $200 \mathrm{~nm}$.

The calibration images used to calibrate spectral data were acquired at the conclusion of the experiment. To ensure experimental measurements taken at the start of the experiment were comparable to the measurements taken at the end of the experiment, the first spectral measurements taken during the experiment were repeated after 52 minutes of sustained plasma torch flow over the viewing window. Through this method the effects of surface degradation sustained by the window during the experiment could also be 
quantified. A comparison of the images is presented in Figure 6.22.

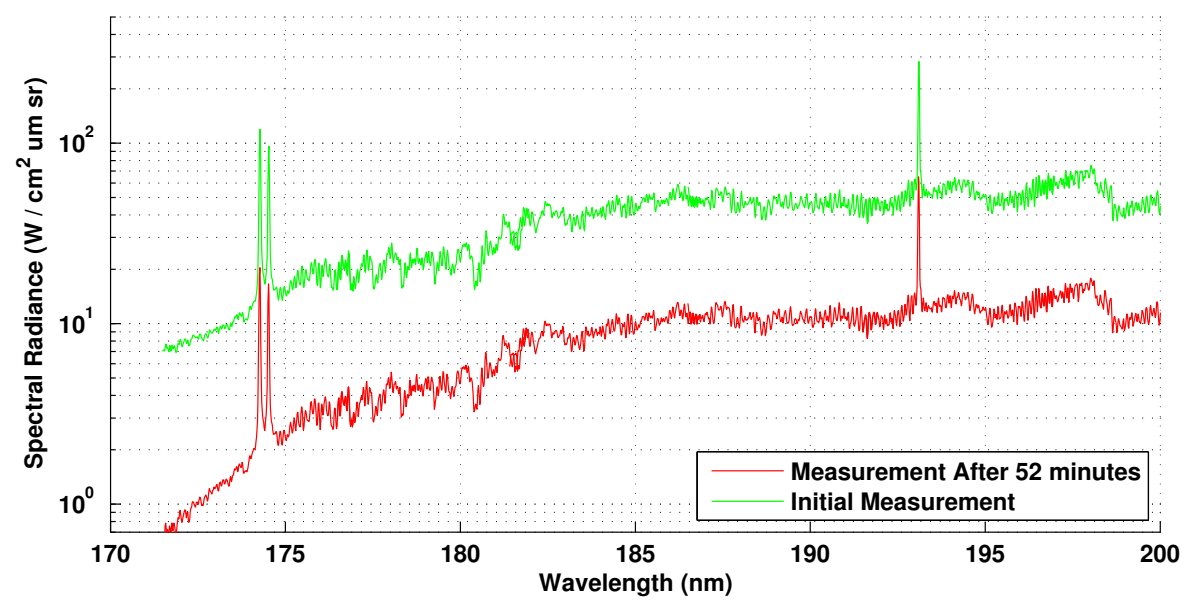

Figure 6.22: Calibrated and spatially averaged spectra at torch start up and after 52 minutes of operation.

During the 52 minutes of plasma torch operation, the measured spectral intensity dropped by approximately 85\%. This significant drop in performance is attributed to surface degradation due to thermal stresses and the interaction of particles in the flow field with the window surface.

\subsubsection{Comparisons With Simulations and Previous Studies}

The flow field of the plasma torch is known to be in thermochemical equilibrium and the radial temperature profile has been experimentally measured to within $3 \%$ [ 8 . Using the measure temperature profile, the mole fractions were calculated using the CEA program at spatial increments of $1 \mathrm{~mm}$. From these values a spectrum was computed by Specair using the radiative transport equation along 50 slabs in order to compute the total emitted spectrum [8]. The computed spectrum convolved with the experimental instrument function is compared with the measured values in Figure 6.23 .

The computed spectrum showed good agreement with the measured spectrum at longer wavelength with the discrepancy between the two datasets increasing with decreasing wavelength. This trend follows the molecular oxygen absorption cross-section and therefore the discrepancy at shorter wavelengths is attributed to absorption by residual molecular oxygen between the viewing window and the plasma. 


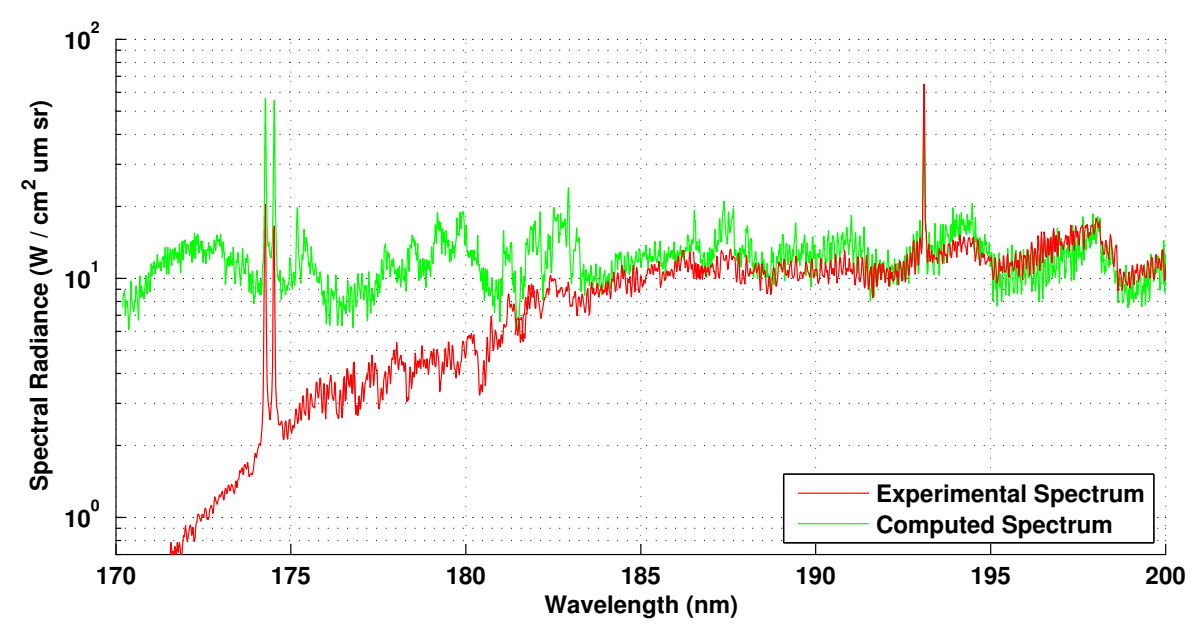

Figure 6.23: Comparison of the measured spectrum and the spectrum computed by Specair.

A non-in-situ calibration of the system was conducted by Jacobs [8] using the mini-arc positioned at the entrance slit of the spectrometer, as shown in Figure 6.24. Calibration of the external optical components was conducted using the transmission and reflectance values provided by the supplier. This calibration was applied to the measured spectrum and is presented in Figure 6.24.

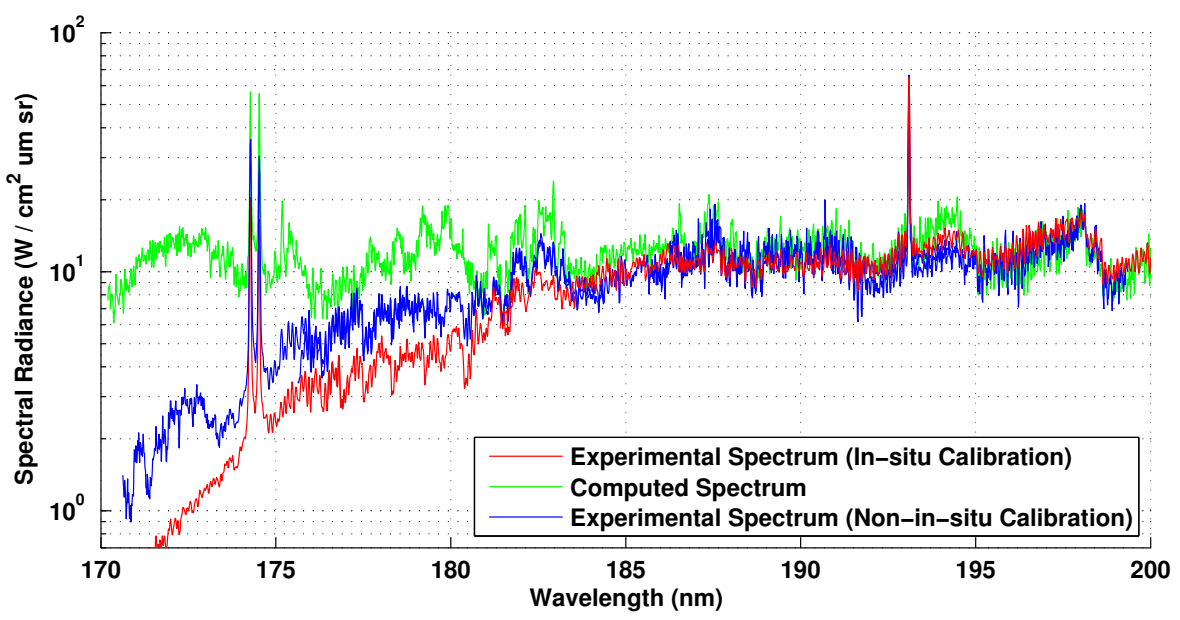

Figure 6.24: Comparison of measured spectra calibrated using an in-situ approach and a non-in-situ calibration and the spectrum computed by Specair.

The non-in-situ calibration matched well with the in-situ calibration and simulations for wavelengths higher than $183 \mathrm{~nm}$. Below $183 \mathrm{~nm}$, the non-in-situ calibration predicts a higher spectral radiance than the in-situ calibration but is still below the computed values of Specair. This is once again attributed to absorption by molecular oxygen between the viewing window and plasma. A potential explanation for the higher values produced 
by the non-in-situ calibration is absorption by molecular oxygen occurring between the mini-arc and the entrance slit of the spectrometer whilst the calibration images were being acquired. The nitrogen exiting the spectrometer slit had been assumed to be sufficient to remove molecular oxygen in the light path but no measurements were made to validate this. Any absorption between the spectrometer and the mini-arc would reduce the calculated sensitivity of the system resulting in a higher value computed for the measured spectrum.

Molecular oxygen absorption cross-sections from $\mathrm{Lu}$ and Yoshino were used to estimate the path lengths of ambient air required to achieve the $35 \%$ and $70 \%$ absorption observed in the non-in-situ and in-situ calibrations respectively [20, 21]. These calculations were made assuming a molecular oxygen concentration of 0.21 at atmospheric pressure and ambient temperature. The results of these calculations are presented in Figure 6.25 .

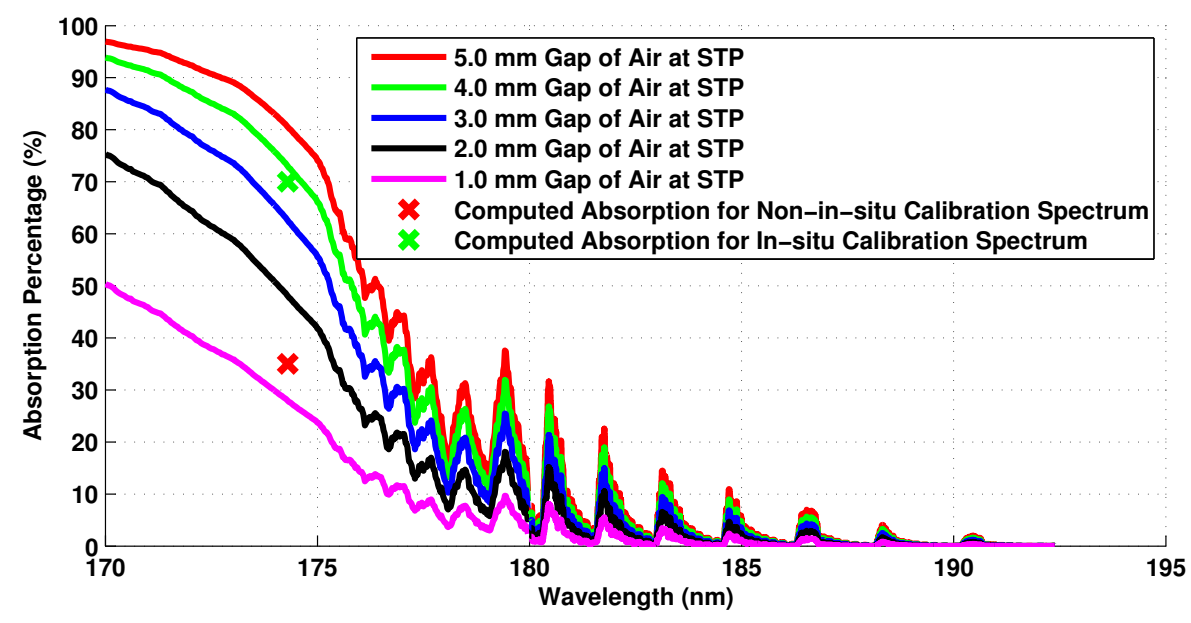

Figure 6.25: Radiation absorption of a slab of air at ambient temperature and atmospheric pressure with a molecular oxygen mole fraction of 0.21 at over various path lengths. The level of absorption predicted by the comparison of Specair simulation and the measured values using the two calibration approaches at a distance of $5 \mathrm{~mm}$ from the plasma edge are also presented.

At a path length of $5 \mathrm{~mm}$ the radiation absorption level is predicted to be $80 \%$. This value can only be used as an estimate as the concentration of molecular oxygen at the edge of the plasma is not known and the absorption cross-sections used were measured at room temperature. There is also the potential of atmospheric air becoming entrained in the flow at the edge of the plasma and further altering the gas composition. Elevated temperatures and reduced concentrations of molecular oxygen would significantly reduce the calculated level of absorption. 
This preliminary experiment has shown the importance of eliminating or quantifying the absorption in the air gap between the window and the plasma. This can be achieved through the optimisation of the window housing assembly or a mating device capable of removing molecular oxygen in the optical path. Optimisation of the window housing assembly would be aide not only in the reduction of the air gap between the window and the plasma, but also improve window cooling leading to an increase in durability of the window and a consequent reduction in the variability of window transmission properties.

\subsection{Self-Absorption Experiment}

To investigate the influence of the depth of the radiating flow field on the measured spectral intensity and broadening of spectral lines, a traversable water cooled fence was designed. The fence acts as a mechanism to vary the line of sight as it is traversed through the radiating flow field. It is made from copper with a flow of water through it to prevent melting. A round pipe with an external diameter of $7.5 \mathrm{~mm}$ was used to minimise flow disturbances and reflections, which are assumed to be negligible due to the rough surface finish of the pipe and the surface oxidation that occurs during the initial weathering experiments. A schematic of the approach is presented in Figure 6.26 and images of the fence in the plasma during operation are presented in Figure 6.27. The cooled copper

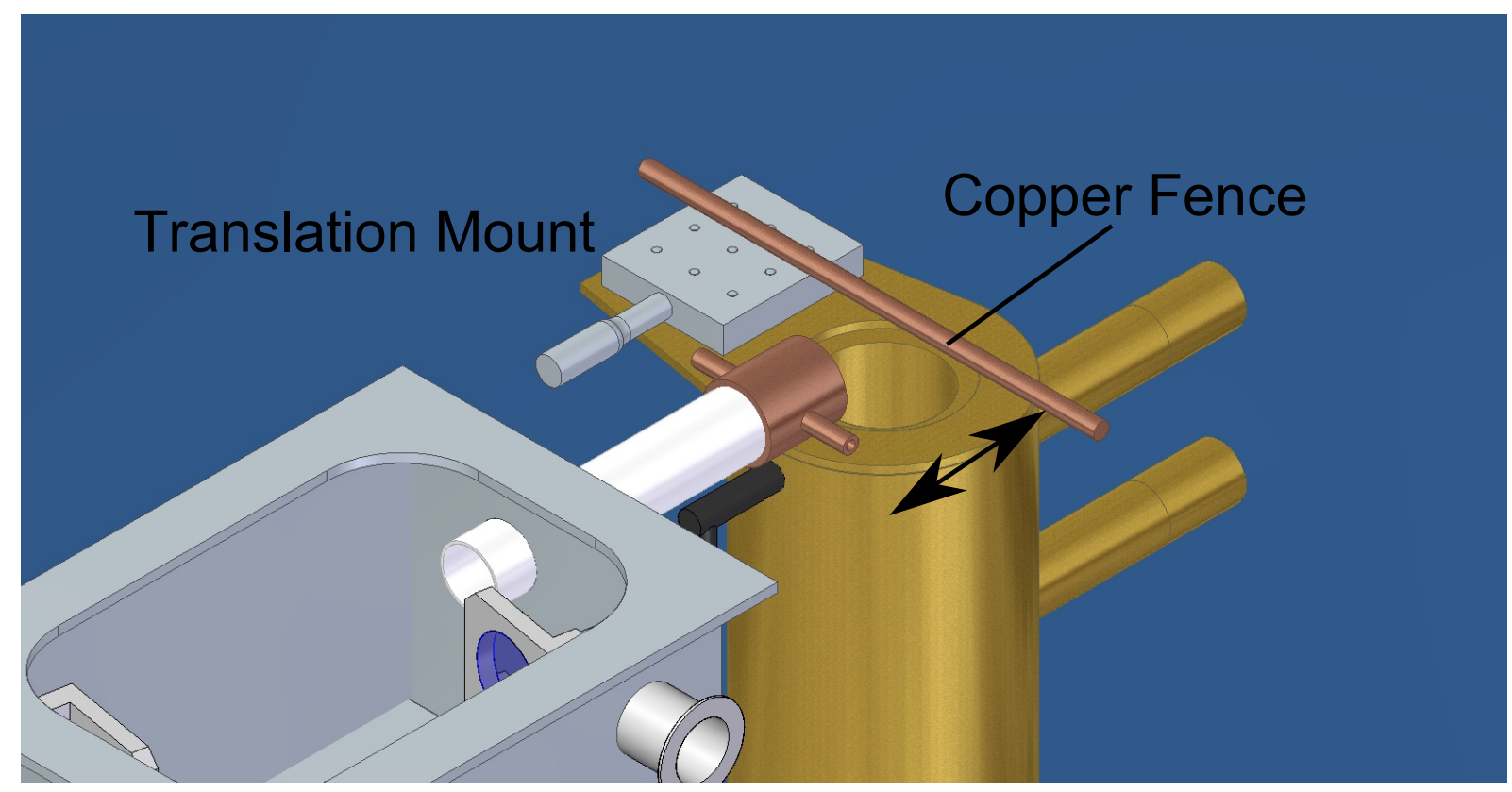

Figure 6.26: Concept of the traversable water cooled fence across the plasma to limit the depth of radiating flow field observed. 

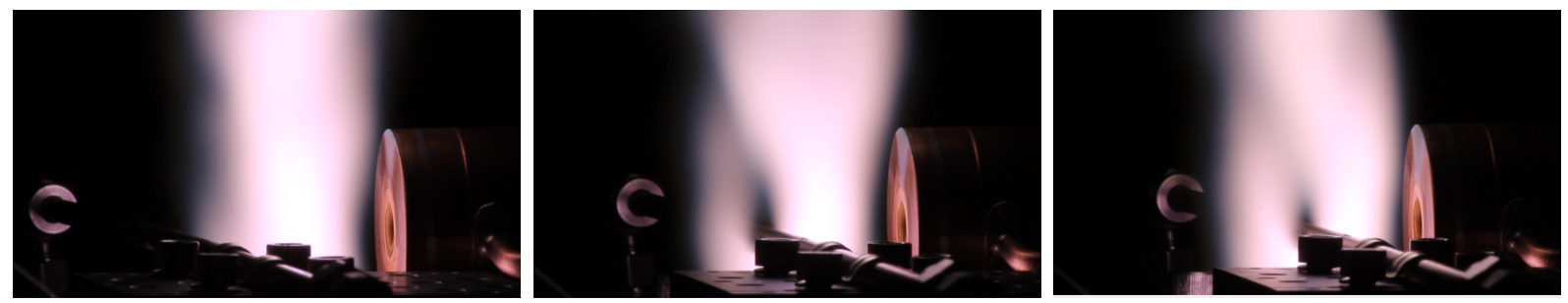

Figure 6.27: Water cooled fence during operation.

pipe was attached to an optical translation mount so that it could be traversed in a controlled manner during plasma torch operation. A rotating universal joint and a $2 \mathrm{~m}$ plastic rod were attached to the translation mount enabling the translation of the pipe to be conducted from a safe distance through an electrically isolated medium.

\subsubsection{Measurements}

To investigate self-absorption of the nitrogen doublet at $174 \mathrm{~nm}$, the fence was traversed through the plasma with spectral images taken at $1 \mathrm{~mm}$ spacings. The complete experiments lasted longer than 45 minutes to complete and consequently the viewing window transmission could not be assumed to be constant between measurements. For relative comparison, the acquired spectral images were normalised at the $174.3 \mathrm{~nm}$ spectral line peak. Spectral images of the oxygen triplet at $777 \mathrm{~nm}$ were also obtained for a comparison with a known optically thin spectral transition at the operating temperatures. The entire depth of the plasma could not be traversed without shutting down the plasma torch and readjusting the copper fence position on the mount and consequently two separate datasets were created. To relate both data sets, the central $4 \mathrm{~mm}$ of the plasma were measured twice and used to scale the datasets appropriately. The results of this experiment are displayed in Figure 6.28. It can be seen from Figure 6.28 that the intensities of $174.3 \mathrm{~nm}$ and $174.5 \mathrm{~nm}$ lines do not reduce proportionally with the optically thin $777.2 \mathrm{~nm}$ spectral line. This indicates that a significant portion of the VUV radiation from the rear half of the plasma is self-absorbed.

Measured spectra of the $174 \mathrm{~nm}$ nitrogen doublet were normalised and compared at varying depths of radiating flow to investigate spectral line broadening. Images were taken at $1 \mathrm{~mm}$ spacing through the plasma and representative images at $45 \mathrm{~mm}, 35 \mathrm{~mm}, 25 \mathrm{~mm}$ and $15 \mathrm{~mm}$ depths of radiating flow field are presented in Figure 6.29. Figure 6.29 indicates that no apparent broadening is measured in the atomic nitrogen lines at $174.3 \mathrm{~nm}$ and $174.5 \mathrm{~nm}$ with a change in depth of radiating flow field. The $174.5 \mathrm{~nm}$ spectral line increases in relative intensity at a depth of radiating flow field of $15 \mathrm{~mm}$ due to a significant 
reduction in the peak temperature of the observed plasma flow field, as will be shown in the following section.

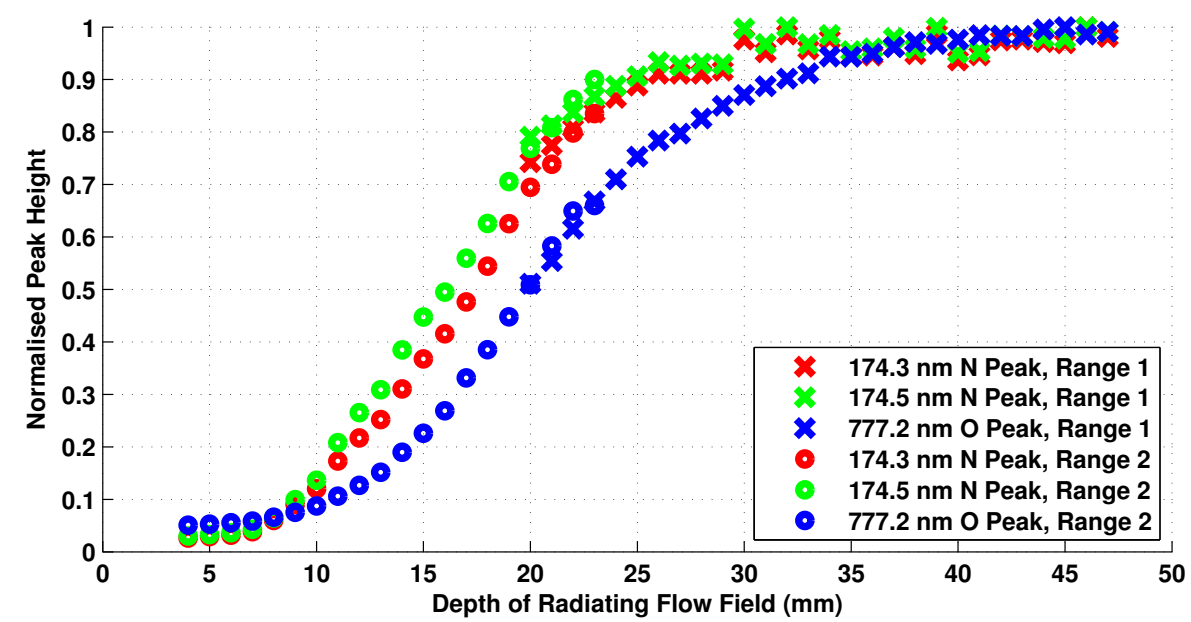

Figure 6.28: Spectral line peak intensities at various depths of radiating flow fields normalised using the measured intensities at a $50 \mathrm{~mm}$ depth of radiating flow field. Spectral lines compared are the $174.3 \mathrm{~nm}$ and $174.5 \mathrm{~nm}$ atomic nitrogen lines and the $777.2 \mathrm{~nm}$ atomic oxygen line.

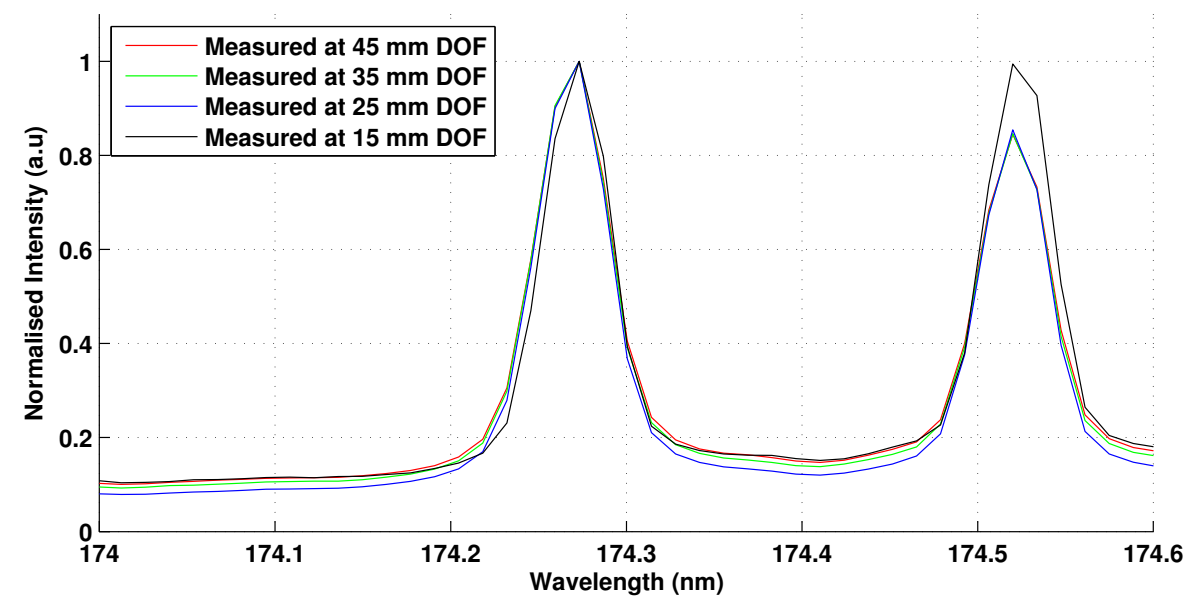

Figure 6.29: Normalised spectral lines of the $174.3 \mathrm{~nm}$ and $174.5 \mathrm{~nm}$ nitrogen doublet at varying depths of radiating flow field. 


\subsubsection{Comparison With Simulations}

Specair was used to computed spectra at varying depths of radiating flow fields for comparison with measured values. For depths of radiating flow fields greater than half of the plasma, the temperature used for the computation was 6,700 K. For the $15 \mathrm{~mm}$ depth of radiating flow field calculation, the temperature was set to the measured maximum value of $6,150 \mathrm{~K}$ to more accurately model the change in relative heights of the nitrogen doublet over the outer $15 \mathrm{~mm}$ of the plsama. To produce a more accurate match with measured values, the FWHM of the instrument broadening function used for this calculation was $0.040 \mathrm{~nm}$. The results of this simulation are presented in Figure 6.30.

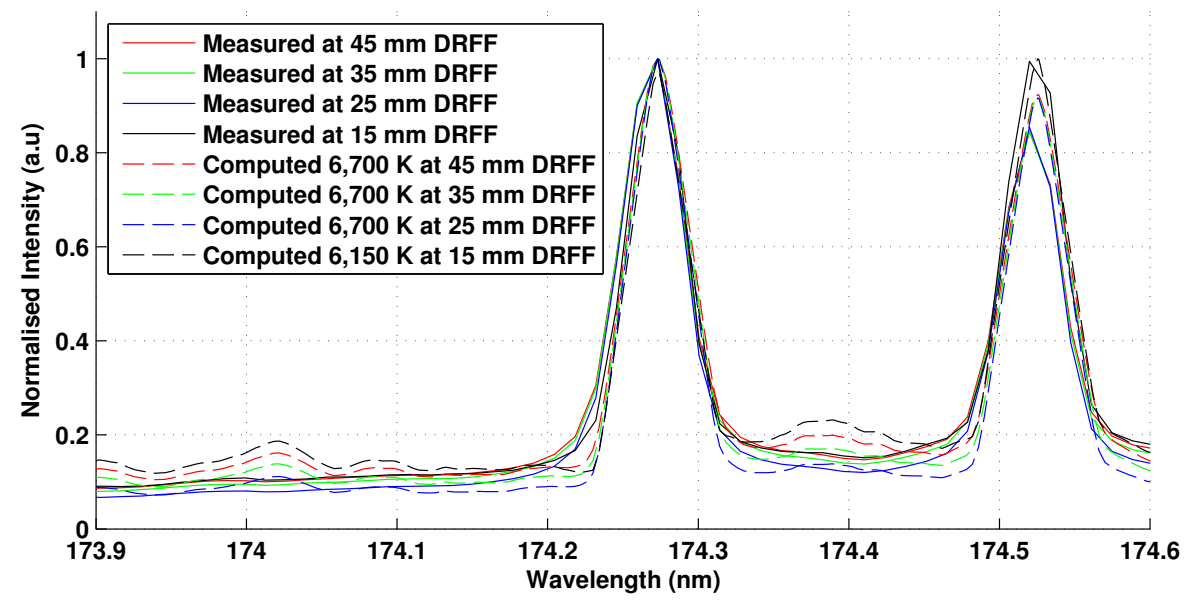

Figure 6.30: Measured and computed normalised spectral lines of the $174.3 \mathrm{~nm}$ and $174.5 \mathrm{~nm}$ nitrogen doublet at varying depths of radiating flow field.

It is apparent in Figure 6.30 that the relative intensity of the two lines is a function of the temperature and depth of plasma. The computations also show that the line width is likely dominated by instrument broadening. Idealising the plasma as a uniform $4.5 \mathrm{~cm}$ slug of gas radiating at the measured peak temperature of $6,700 \mathrm{~K}$, an upper limit for the instrument broadening function required to observe broadening of the plasma was estimated. Figure 6.31 depicts the simulated spectral line profile for various instrument broadening functions for the $174.27 \mathrm{~nm}$ and $174.53 \mathrm{~nm}$ atomic nitrogen spectral lines. It is apparent from Figure 6.31 that the calculated instrument broadening function of $0.030 \mathrm{~nm}$ FWHM (green,) and the experimentally matched value of $0.040 \mathrm{~nm}$ FWHM (red), are significantly broader than the line widths of the plasma emission. Using Figure 6.31, it was estimated that an instrument broadening function smaller than $0.005 \mathrm{~nm}$ FWHM is required in order to measure the $174 \mathrm{~nm}$ nitrogen spectral line widths of a $4.5 \mathrm{~cm}$ diameter plasma at $6,700 \mathrm{~K}$. Consequently, the current experimental system developed will be capable of quantifying 
spectral line broadening of the plasma emission when coupled with a higher resolution spectroscopy system in the future.
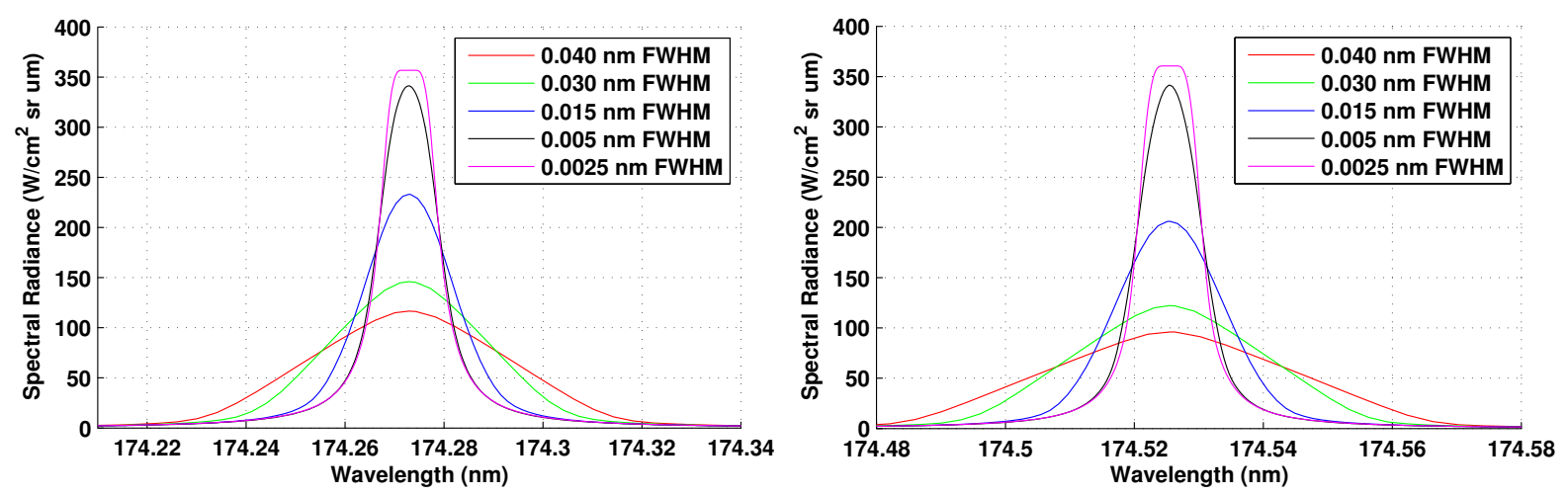

Figure 6.31: (Left) - Spectral line profile for various instrument broadening functions for the $174.27 \mathrm{~nm}$ atomic nitrogen spectral line. (Right) - spectral line profile for various instrument functions for the $174.53 \mathrm{~nm}$ atomic nitrogen spectral line. Both simulations conducted at atmospheric pressure at $6,700 \mathrm{~K}$ with a $4.5 \mathrm{~cm}$ depth of radiating flow field. 


\section{Chapter 7}

\section{Conclusions}

The goal of this study was to obtain calibrated spectral measurements in the VUV range to enable investigations of physical processes occurring in a shock layer that influence the amount of incident radiative heat flux. This goal was achieved through a three stage process of system development and categorisation, measurements across and through the surface of a model and measurements made observing varying depths of radiating flow fields. The X2 expansion tube at The Centre for Hypersonics was the primary facility used in this study to create the high enthalpy flows representative of super orbital re-entry trajectory points. An extension of this study was the development and categorisation of a VUV emission spectroscopy system for use on the plasma torch at Ecole Centrale Paris, which was used to obtain a complementary set of experimental results at lower temperatures.

Two conditions were designed and categorised on the X2 expansion tube representative of $10.0 \mathrm{~km} / \mathrm{s}$ and $12.2 \mathrm{~km} / \mathrm{s}$ re-entry trajectory points. An optical system was designed that utilised a viewing window in the surface of a blunt model allowing for spectral measurements of incident VUV radiation through the surface to be made. A second optical system measured radiation across the surface of the model in a spatially resolved manner. Spatial resolution allowed for the observation of the equilibrium and non-equilibrium regions of the shock layer. To observe the effects of varying depths of radiating flow field, experiments were carried out using two-dimensional models with lengths of $90 \mathrm{~mm}, 45 \mathrm{~mm}$ and $20 \mathrm{~mm}$.

The VUV emission spectroscopy system designed for the expansion tube experiments utilised a McPherson NOVA 225 spectrometer coupled to an Andor iStar VUV enhanced intensified charge coupled device. To transport the emitted radiation from the model to the detection system without absorption by molecular oxygen, an evacuated light tube sealed with a magnesium fluoride window was used. An in-situ calibration of the VUV 
spectroscopy system was conducted using a deuterium lamp located in the position of the radiating shock layer. A repeatability study of this system returned a value of $15 \%$ for measurements of spectral line intensity and $20 \%$ for integrated intensity between $115 \mathrm{~nm}$ and $180 \mathrm{~nm}$.

Spectrally resolved emission measurements were carried out between $115 \mathrm{~nm}$ and $180 \mathrm{~nm}$ on the expansion tube. The integrated spectral radiance measured through the surface in this spectral range was found to be $7.44 \mathrm{~W} / \mathrm{cm}^{2} \mathrm{sr}$ for the $10.0 \mathrm{~km} / \mathrm{s}$ condition and $123 \mathrm{~W} / \mathrm{cm}^{2} \mathrm{sr}$ for the $12.2 \mathrm{~km} / \mathrm{s}$ condition. The integrated spectral radiance measured across the $90 \mathrm{~mm}$ long model was four times higher for the $10.0 \mathrm{~km} / \mathrm{s}$ condition and three times higher for the $12.2 \mathrm{~km} / \mathrm{s}$ condition. Measurements made with varying depths of radiating flow field revealed all spectral lines in the VUV to be self-absorbing to varying degrees for the $10.0 \mathrm{~km} / \mathrm{s}$ condition and strongly self-absorbing for the $12.2 \mathrm{~km} / \mathrm{s}$ condition. Significant spectral line broadening of the strongly self-absorbing lines was also observed.

Eilmer 3 was used to compute the flow field around the two dimensional models using the Park reaction rate scheme. Specair was used to generate emission spectra using the temperatures and pressures computed and compared with experimental measurements. Large discrepancies between the computed and measured values were found. These discrepancies were attributed to a lack of grid resolution, no radiation coupling implemented within the code and assumed equilibrium chemistry of the free stream conditions produced by X2. Specair was also used to compute spectra at varying depths of radiating flow fields as observed in experiments. The results of these simulations indicated self-absorption of all spectral lines investigated and showed good agreement with the measured values for most spectral lines. Comparisons of spectral line width revealed an under prediction of measured values.

To complement the VUV emission spectroscopy measurements made on the X2 expansion tube, a VUV emission spectroscopy system was designed for use on the plasma torch at Ecole Centrale Paris. This study showed promising potential as it allowed for the observation of spectral lines at known thermochemical equilibrium conditions, avoiding the complexities of the non-equilibrium regions observed in the expansion tube. The test condition used for this study produced a peak temperature of $6,700 \mathrm{~K}$ and with an uncertainty of $3 \%$.

Molecular oxygen from the optical path was removed through the use of a nitrogen flush through the spectrometer and a light tube positioned at the edge of the plasma. The light tube was sealed with a magnesium fluoride window in an actively cooled copper assembly. Thermal stress analysis of the window was conducted using the COMSOL Multiphysics 
program and it determined the designed assembly would cool the window sufficiently such that it did not exceed the apparent elastic limit. Experiments were conducted to measure surface degradation of the window and absorption by molecular oxygen at varying distances from the edge of the plasma. It was concluded that surface degradation of the viewing window was occurring at distances closer than $5 \mathrm{~mm}$ from the edge of the plasma and molecular oxygen was still absorbing a fraction of the radiation at this distance.

In-situ calibration of the plasma torch VUV emission spectroscopy system was carried out using an argon mini-arc positioned at the location of the plasma. A shroud with a flow of nitrogen was created to remove molecular oxygen between the mini-arc and the viewing window. The uncertainty of the measured spectra was calculated to be $21.4 \%$ for wavelengths shorter than $140 \mathrm{~nm}$ and $16.4 \%$ for wavelengths longer than $140 \mathrm{~nm}$. Due to the observed degradation of the window surface during the test time and unquantified level of absorption between the viewing window and the plasma, a large uncertainty was estimated for the measured data. The final measured spectrum was compared with values previously presented using a non-in-situ calibration and a spectrum computed using Specair and suggestions were made to improve the system.

This study has addressed one of the major design challenges of super orbital re-entry spacecraft: the modelling and validation of radiative heat transfer to the surface of a vehicle. In doing so, two VUV emission spectroscopy systems have been established on two unique facilities. These systems have allowed for investigations in the variation of spectral radiance observed across a shock layer compared to the spectral radiance measured through the surface of a model. The effects of self-absorption on spectral line intensity and the broadening of spectral lines in the VUV as a function of depth of radiating flow field has also been investigated. Measurements made across and through the surface of a model have provided the first set of calibrated results for the validation of computational codes used to predict incident radiative heat flux. Measurements made with a varying depth of radiating flow field provide a unique set of experimental data for the validation of radiation transport models and broadening coefficients. Computational simulations of the flow field conducted with Eilmer 3, in conjunction with the Park reaction rate scheme, were used to generate spectra with Specair and the results were compared against experimentally measured values. 


\subsection{Future Work}

In regards to measurements of radiating flows and validation of modelling programs, recommendations for future work include the following:

- Comparisons of the experimentally measured and computed spectra with external radiation modelling codes. This would provide information on the accuracy of the databases used and the theories applied in radiation transport computations.

- Measurements using the established VUV spectroscopy systems for planetary entry studies in Martian atmosphere gas. In particular the study of the $\mathrm{CO}(4+)$ system which is predicted to be a substantial contributor to the total radiative flux.

- High resolution spectroscopic measurements of the $120 \mathrm{~nm}$ region to investigate the discrepancies with simulations observed in this region and improved resolution of the spectral lines observed.

- Additional diagnostic tools in the categorisation of the operation of expansion tube. This includes upgrades to the photodiode array established during this study. One potential approach to achieve better results from the photodiode array is the application of lenses to focus on one particular point in the shock front instead of integrating over the entire non-planar region. An array of photodiodes or pressure transducers at the exit of the nozzle would also aid in constraining flow conditions. Finally, spectroscopic measurements of the free stream flow to investigate the chemical composition and temperature of the flow would be a valuable tool in modelling the operation of the X2 facility.

- Grid resolution studies to ensure the simulation of the non-equilibrium region is accurately resolved. Higher resolution simulations capable of better resolving the boundary layer and oxygen recombination would also provide valuable comparisons with the measured data.

- Further optimisation of the plasma torch operating parameters to achieve higher temperature flows that are more directly applicable to re-entry shock layers allowing for self-absorption and broadening to be further investigated. This could also be achieved through the use a smaller cooling assembly allowing for the higher temperature flow closer to the nozzle exit to be studied.

- Quantification of the absorption occurring between the edge of the plasma and the viewing window. This could be achieved through computational modelling and experimental measurements of a calibration source across the gap. 
- Upgrades to the VUV emission spectroscopy system used on the plasma torch including the procurement of a vacuum capable spectrometer and the use of a translating optical table. A vacuum capable spectrometer combined with the already built high vacuum optical chamber would allow measurements to be made a wavelengths shorter than $170 \mathrm{~nm}$. A translating table would enable the viewing window assembly to be inserted into the plasma for short periods of time, reducing surface degradation.

- A sapphire window could be installed instead of the currently used magnesium fluoride window whilst a nitrogen flush is being utilised on the plasma torch spectroscopy system. As the current system is not capable of measuring below $170 \mathrm{~nm}$, a sapphire window would be sufficient in this wavelength range whilst providing superior thermal properties to aid in the prevention of surface degradation.

- Simulations of spectral line broadening using the measured temperature profile of the plasma to calculate the instrument function required to quantify spectral line broadening, and consequent installation of a higher resolution spectroscopy system to carry out spectral line broadening measurements. 


\section{Bibliography}

[1] U. A. Sheikh. Vacuum ultraviolet measurements on the surface of a blunt body for re-entry flows. In 11th International Workshop on Shock Tube Technology, University of Queensland, Brisbane, Australia., 2011.

[2] U. A. Sheikh, R. G. Morgan, and T. J. McIntyre. Self-absorption of vacuum ultraviolet radiation in superorbital flows. In 5th International Workshop on Radiation of High Temperature Gases in Atmospheric Entry, Barcelona, Spain, 2012.

[3] U. A Sheikh, R. G. Morgan, F. Zander, T. N. Eichmann, and T. J. McIntrye. Vacuum ultraviolet emission spectroscopy system for superorbital reentries. In 18th AIAA International Space Planes and Hypersonic Systems and Technologies Conference, Tours, France., 2012.

[4] U. A Sheikh, C. Jacobs, C. O. Laux, R. G. Morgan, and T. J McIntyre. Measurements of high enthalpy radiating flows in the vacuum ultraviolet. In 29th International Symposium on Shock Waves, Madison, WI, USA., 2013.

[5] U. A. Sheikh, C. Jacobs, R. G. Morgan, C. O. Laux, and T. J. McIntyre. Reentry radiative heating studies in the vacuum ultraviolet. In Aerospace Thematic Workshop., Aussois, France, 2013.

[6] U. A Sheikh, C. O. Laux, R. G. Morgan, and T. J McIntyre. Through surface and across surface vacuum ultraviolet spectral measurements in an expansion tube. In 44th AIAA Thermophysics Conference, San Diego, California, USA., 2013.

[7] U. A Sheikh, R. G Morgan, and T.J McIntyre. Vacuum ultraviolet spectral measurements for superorbital earth entry in the x2 expansion tube. AIAA (Submitted), 2014.

[8] C Jacobs, U.A Sheikh, M MacDonald, and C.O Laux. Vacuum ultraviolet radiation studies in a plasma torch facility from 170 - $200 \mathrm{~nm}$. In 44th AIAA Thermophysics Conference, San Diego, California, USA., 2013. AIAA. 
[9] P. Leyland, T. McIntyre, U. Sheikh, T. Eichmann, F. Zander, R. Morgan, S. Loehle, T. Hermann, F. deFillippis, E. Trifoni, and G. Cillo. Vuv radiation measurements for radiation-abalation coupling. In 5th International Workshop on Radiation of High Temperature Gases in Atmospheric Entry, Barcelona, Spain.

[10] R. G. Morgan, T. J. McIntyre, and U. A. Sheikh. Simulation of radiating flows in impulsive facilities. In 49th AIAA Aerospace Sciences Meeting including the New Horizons Forum and Aerospace Exposition, Orlando, Florida, 2011.

[11] R. G. Morgan, U. A Sheikh, and D. E. Gildfind. Standing shock formation in a non-reflected shock tube. In The 28th International Symposium on Shock Waves, Manchester, UK., 2011.

[12] H Porat, U. A Sheikh, R. G Morgan, and T. J McIntyre. Vacuum ultraviolet emission spectroscopy at titan and mars atmospheric entry conditions. In 44th AIAA Thermophysics Conference, San Diego, California, USA., 2013.

[13] F. Zander, Morgan R. G., Sheikh U. A., Buttsworth D. G., and Teakle P. R. Hot wall reentry testing in hypersonic impulse facilities. AIAA, 51:476-484, 2012.

[14] F. Zander, Morgan R. G., Sheikh U. A., Buttsworth D. G., and Teakle P. R. Hot wall testing methodology for impulse facilities. In 18th AIAA International Space Planes and Hypersonic Systems and Technologies Conference, Tours, France, 2012.

[15] F Zander, P. A Jacobs, U. A Sheikh, and R. G Morgan. High speed imaging of spherical shock standoff in hypervelocity flows. In 18th Australasian Fluid Mechanics Conference. Australasian Fluid Mechanics Society, 2012.

[16] F Zander, P. A Jacobs, U. A Sheikh, D. Buttsworth, and R. G Morgan. High speed imaging of $\mathrm{x} 2$ expansion tube flows. In 30th International Congress on High-Speed Imaging and Photonics, 2012.

[17] P Leyland, T McIntyre, R Morgan, P Jacobs, F Zander, U Sheikh, T Eichmann, E Fahy, O Joshi, G Duffa, et al. Radiation-ablation coupling for capsule reentry heating via simulation and expansion tube investigations. In 5th European Conference for Aeronautics and Space Sciences (EUCASS), 2013.

[18] J. D. Anderson. Hypersonic and high temperature gas dynamics. McGraw-Hill, New York, 1989. 88-312811 John D. Anderson, Jr. ill ; 25cm. McGraw-Hill series in aeronautical and aerospace engineering. Includes index. Includes bibliography: p.

[19] R. Eisberg and R. Resnick. Quantum physics. John Wiley New York, 1974. 
[20] H. C. Lu, K. K. Chen, H. F. Chen, B. M. Cheng, and J. F. Ogilvie. Absorption cross section of molecular oxygen in the transition $\mathrm{e} 3 \mathrm{u}-\mathrm{v}=0-\mathrm{x} 3 \mathrm{~g}-\mathrm{v}=0$ at $38 \mathrm{k}$. Astronomy and Astrophysics, 520:A19, 2010.

[21] K Yoshino, D. E. Freeman, J. R. Esmond, and W. H. Parkinson. High resolution absorption cross section measurements and band oscillator strengths of the $(1,0)$ $(12,0)$ schumann-runge bands of $o_{2}$. Planetary and Space Science, 31(3):339-353, 1983.

[22] R Mota, R Parafita, A Giuliani, M-J Hubin-Franskin, JMC Lourenco, G Garcia, SV Hoffmann, NJ Mason, PA Ribeiro, M Raposo, et al. Water vuv electronic state spectroscopy by synchrotron radiation. Chemical physics letters, 416(1):152-159, 2005.

[23] B. R Capra. Aerothermodynamic Simulation of Subscale Models of the FIRE II and Titan Explorer Vehicles in Expansion Tubes. PhD thesis, 2006.

[24] J. J Bertin. Hypersonic aerothermodynamics. AIAA, 1994.

[25] C. O. Laux. Optical Diagnostics and Radiative Emission of Air Plasmas. PhD thesis, 1993.

[26] G. Palumbo, R. A. Craig, E. W. Whiting, and C Park. Measured specific intensity from 130 to $900 \mathrm{~nm}$ at the stagnation point of a model in an arcjet flow of $7.8 \mathrm{~km} / \mathrm{sec}$. Journal of Quantitative Spectroscopy and Radiative Transfer, 57(2):207-236, 1997.

[27] T. N Eichmann, T. J McIntyre, A. I Bishop, S. Vakata, and H. Rubinsztein-Dunlop. Three-dimensional effects on line-of-sight visualization measurements of supersonic and hypersonic flow over cylinders. Shock Waves, 16(4-5):299-307, 2007.

[28] J.A. Eichmeier and M.K. Thumm. Vacuum Electronics: Components and Devices. Springer-Verlag Berlin Heidelberg, 2008.

[29] Crystran. Magnesium fluoride datasheet. Website, November 2013.

[30] D. E. Gildfind. Development of High Total Pressure Scramjet Flow Conditions using the X2 Expansion Tube. PhD thesis, 2012.

[31] Website University of Illonois https://wiki.engr.illinois.edu/display/BIOE414/Home. Schematic of a photomultiplier tube.

[32] T.N Personal Communication: Eichmann. 
[33] Shimadzu. Private communication. 2010.

[34] OSRAM Opto Semiconductors. Silicon pin photodiode with very short switching time, version 1.0, 2007.

[35] Princeton Instruments. Pi-max user manual, 4411-0069 version 5.e, 2006.

[36] Physikalisch-Technische Bundesanstalt. Calibration certificate, 2011.

[37] J. H. Grinstead, M. C. Wilder, J. Olejniczak, D. W. Bogdanoff, G. A. Allen, K. Dang, and M. J. Forrest. Shock-heated air radiation measurements at lunar return conditions. In 46th AIAA Aerospace Sciences Meeting and Exhibit., Reno, NV, 2008. AIAA.

[38] B. Kleb and C.O. Johnston. Uncertainty analysis of air radiation for lunar return shock layers. In AIAA Atmospheric Flight Mechanics Conference and Exhibit, Honolulu Hawaii, 2008. AIAA.

[39] C. Park. Review of chemical kinetic problems of future nasa missions. ii earth entries. Journal of Thermophysics and Heat Transfer, 7(3):385 to 398, 1993.

[40] Website http://en.wikipedia.org/wiki/Emission spectrum. Emission spectrum of hydrogen, iron and energy transition.

[41] D. F Potter. Modelling of radiating shock layers for atmospheric entry at Earth and Mars. PhD thesis, 2011.

[42] C. O. Johnston, B. R. Hollis, and K. Sutton. Non-boltzmann modeling for air shock-layer radiation at lunar-return conditions. Journal of Spacecraft and Rockets, 45(5):879-890, 2008.

[43] C.O Johnston. Nonequilibrium Shock-Layer Radiative Heating for Earth and Titan Entry. Phd dissertation, 2006.

[44] C. O Laux. Radiation and nonequilibrium collisional-radiative models. von Karman Institute lecture series, 7, 2002.

[45] D. Bose, E. McCorkle, C. Thompson, and J. Grinstead. Analysis and model validation of shock layer radiation in air. In 46th AIAA Aerospace Sciences Meeting and Exhibit, Reno, Nevada, 2008. AIAA.

[46] C. O Laux, R. J Gessman, and C. H Kruger. Modeling the uv and vuv radiative emission of high-temperature air. In AIAA, 28th Thermophysics Conference, volume 1, 1993. 
[47] C. Park, S. Y. Hyun, and K. S Chang. Contemporary problems of radiation. In Proc. of The 3rd Int. Workshop on Radiation of High Temperature Gases in Atmospheric Entry, Heraklion, Greece.

[48] AM Feldick, Michael Modest, and DA Levin. Closely coupled flowfield-radiation interactions for flowfields created during hypersonic reentry. In 40th Thermophysics Conference, Seattle, WA, Jun, pages 23-26, 2008.

[49] R Goulard. The coupling of radiation and convection in detached shock layers. Journal of Quantitative Spectroscopy and Radiative Transfer, 1(3):249-257, 1961.

[50] C.S Williams and O.A Becklund. OPTICS: A Short Course for Engineers and Scientists. Robert E. Krieger Publishing Company, 1986.

[51] F. L Pedrotti, L. M Pedrotti, and L. S Pedrotti. Introduction to Optics. AddisonWesley, 3rd edition, 2006.

[52] U.A Sheikh. 3d ray tracing program. Technical report, Mechanical Engineering, University of Queensland, 2010.

[53] J. A. R. Samson and D. L. Ederer. Vacuum ultraviolet spectroscopy. Academic Press, San Diego, 1998. 98202438 edited by J.A.R Samson and D.L. Ederer. ill. ; $24 \mathrm{~cm}$. Experimental methods in the physical sciences, v. 31 v.32. Includes bibliographical references and index.

[54] Anne P. Thorne, U. Litzn, and S. Johansson. Spectrophysics : principles and applications. Springer, Berlin ; New York, 1999. 99010573 A. Thorne, U. Litzn, S. Johansson.

[55] S. M Ahmed, I Kanik, and R Link. Temperature-dependent photoabsorption cross section measurements of o2 at the oi-1304 a triplet emission lines. Chemical physics letters, 259(5):545-553, 1996.

[56] R. D Hudson, V. L Carter, and J. A Stein. An investigation of the effect of temperature on the schumann-runge absorption continuum of oxygen, 1580-1950 a. Journal of Geophysical Research, 71(9):2295-2298, 1966.

[57] S. T Gibson, H. P. F Gies, A. J Blake, D. G McCoy, and P. J Rogers. Temperature dependence in the schumann-runge photoabsorption continuum of oxygen. Journal of Quantitative Spectroscopy and Radiative Transfer, 30(5):385-393, 1983. 
[58] K Watanabe, Edward CY Inn, and M. Zelikoff. Absorption coefficients of oxygen in the vacuum ultraviolet. The Journal of Chemical Physics, 21:1026, 1953.

[59] L. Lees. Laminar heat transfer over blunt-nosed bodies at hypersonic flight speeds. Journal of Jet Propulsion, 26(4):259-269, 1956.

[60] J.A. Fay and F.R Riddell. Theory of stagnation point heat transfer in dissociated air. Journal of Aeronautical Sciences, 25(2):73-85, 1958.

[61] H Hoshizaki and L. E Lasher. Convective and radiative heat transfer to an ablating body. AIAA Journal, 6(8):1441-1449, 1968.

[62] O. M Belotserkovskii, L.M Biberman, S. Bronin, V. N Formin, and A. N Lagarkov. Hypersonic gas flow around a blunt object and heating of the object, with an account of radiation(stagnation point heating in hypersonic gas flow past blunt bodies, considering radiative transfer effects on shock wave temperature and density distribution, wave separation, etc). High Temperature, 7:476-488, 1969.

[63] R. E Boughner, R. C Burns, R Goulard, and H. F Nelson. Flow of radiating gas around a probe entering a planetary atmosphere(radiating gas flows during hypersonic planetary reentry, discussing atmospheric composition, shock layer characteristics, nonequilibrium flows, etc). High Temperature, 7:489-510, 1969.

[64] J. D Anderson. An engineering survey of radiating shock layers. AIAA Journal, $7(9): 1665-1675,1969$.

[65] Resler E.L. and Boxsom D.E. Very high mach number principles by unsteady flow principle. Cornell University Graduate School of Aerodynamic Engineering, 1952.

[66] R. L Trimpi. A preliminary theoretical study of the expansion tube: a new device for producing high-enthalpy short-duration hypersonic gas flows. National Aeronautics and Space Administration, 1962.

[67] J. J Jones and J. A Moore. Exploratory study of performance of langley pitot model expansion tube with a hydrogen driver. tn d-3421. Technical report, NASA, 1966.

[68] E. S. Cornette. Forebody temperatures and calorimeter heating rates measured during project fire ii reentry at 11.35 kilometers per second. Technical Memorandum, NASA Langley Research Center, (TM X-1305), 1966.

[69] T. H Slocumb. Project fire flight ii afterbody temperatures and pressures at 11.35 kilometers per second. NASA TM X-1319, 1966. 
[70] D. L. Cauchon. Radiative heating results from the fire ii flight experiment at a reentry velocity of 11.4 kilometers per second. NASA TM X-1402, 1967.

[71] C.O. Johnston, B.R. Hollis, and K. Sutton. Nonequilibrium stagnation-line radiative heating for fire ii. Journal of Spacecraft and Rockets, 45(6), 2008.

[72] National Aeronautics and Space Administration. National space science data center. Technical report, National Aeronautics and Space Administration, 2013.

[73] RC Reid Jr, WC Rochelle, and JD Milhoan. Radiative heating to the apollo command module: Engineering predictions and flight measurements. NASA TMX58091, 1972.

[74] WS Rigdon, RB Dirling, and M Thomas. Stagnation point heat transfer during hypervelocity atmospheric entry, volume 1462. National Aeronautics and Space Administration, 1970.

[75] J. N Moss. Radiative viscous-shock-layer solutions with coupled ablation injection. AIAA Journal, 14(9):1311-1317, 1976.

[76] K. Sutton. Radiative heating about outer planet entry probes. Journal of Spacecraft and Rockets, 13(5):294-300, 1976.

[77] M Wakefield and WC Pitts. Analysis of the heat-shield experiment on the pioneervenus entry probes. AIAA 1980, 1494, 1980.

[78] W. L Grose and J. E Neal. Estimates of nonequilibrium radiation for venus entry. AIAA Journal, 13(4):421-424, 1975.

[79] C Park. Calculation of nonequilibrium radiation in the flight regimes of aeroassisted orbital transfer vehicles. 1984.

[80] C. Park. Problems of rate chemistry in the flight regimes of aeroassisted orbital transfer vehicles. 1985.

[81] C. Park. Radiation enhancement by nonequilibrium in earth's atmosphere. Journal of Spacecraft and Rockets, 22(1):27-36, 1985.

[82] C Park. Assessment of two-temperature kinetic model for dissociating and weaklyionizing nitrogen. 1986.

[83] C Park. Assessment of two-temperature kinetic model for ionizing air. Journal of Thermophysics and Heat Transfer, 3(3):233-244, 1989. 
[84] C. Park. Nonequilibrium Hypersonic Aerothermodynamics. Wiley-Interscience, 1990.

[85] GD Walberg. A review of aeroassisted orbital transfer. AIAA Paper, (82-137), 1982.

[86] J. J Jones. The rationale for an aeroassist flight experiment. In AIAA, 22nd Thermophysics Conference, volume 1, 1987.

[87] E. E. Whiting, C Park, Y Liu, J. O. Arnold, and J. A. Paterson. Neqair 96 user manual. NASA Ames Research Center, Moffett Field, CA, 1996.

[88] M. W Lo, B. G Williams, W. E Bollman, D. Han, Y. Hahn, J. L Bell, E. A Hirst, R. A Corwin, P. Hong, K. C Howell, et al. Genesis mission design. Journal of the Astronautical Sciences, 49(1):169-184, 2001.

[89] DE Brownlee, P Tsou, D Burnett, B Clark, MS Hanner, F Horz, J Kissel, JAM McDonnell, RL Newburn, S Sandford, et al. The stardust mission: returning comet samples to earth. Meteoritics \& Planetary Science, 32(4):A22-A22, 1997.

[90] M. Yoshikawa, A. Fujiwara, and J. Kawaguchi. Hayabusa and its adventure around the tiny asteroid itokawa. Highlights of Astronomy, 14:323-324, 2007.

[91] P. Jenniskens, P. Wercinski, J. Olejniczak, G. Allen, P. N Desai, G. Raiche, D. Kontinos, D. Revelle, J. Hatton, R. L Baker, et al. Preparing for hyperseed mac: an observing campaign to monitor the entry of the genesis sample return capsule. Earth, Moon, and Planets, 95(1-4):339-360, 2004.

[92] P. Jenniskens. Observations of the stardust sample return capsule entry with a slitless echelle spectrograph. Journal of Spacecraft and Rockets, 47(5):718-735, 2010.

[93] J. H Grinstead, P. Jenniskens, A. M Cassell, J. Albers, and M. W Winter. Airborne observation of the hayabusa sample return capsule re-entry. AIA A Paper, 3329:2011, 2011.

[94] O Chazot. Experimental studies on hypersonic stagnation point chemical environment. Technical report, DTIC Document, 2006.

[95] David A Bittker and Vincent J Scullin. General chemical kinetics computer program for static and flow reactions, with application to combustion and shock-tube kinetics, volume 6586. National Aeronautics and Space Administration, 1972.

[96] Philippe Sagnier and Lionel Marraffa. Parametric study of thermal and chemical nonequilibrium nozzle flow. AIAA journal, 29(3):334-343, 1991. 
[97] J Gorden Hall. Blunt-nose inviscid airflows with coupled nonequilibrium processes. 1962.

[98] JA Lordi and Robert Edward Mates. Nonequilibrium expansions of high-enthalpy airflows. Technical report, DTIC Document, 1964.

[99] Robert J Bakos and Richard G Morgan. Chemical recombination in an expansion tube. AIAA journal, 32(6):1316-1319, 1994.

[100] AJ Neely. Planetary entry aerothermodynamic studies in a superorbital expansion tube. PhD thesis, 1995.

[101] Peter Jacobs, Richard Morgan, Aaron Brandis, David Buttsworth, Andrew Dann, Mary D'Souza, Troy Eichmann, David Gildfind, Rowan Gollan, Carolyn Jacobs, et al. Design, operation and testing in expansion tube facilities for super-orbital re-entry. In STO-AVT-VKI Lecture Series Radiation and Gas-Surface Interaction Phenomena in High Speed Re-Entry (2013-AVT-218), pages 5-1, 2013.

[102] T. B. Reed. Induction-coupled plasma torch. Journal of Applied Physics, 32, 1961.

[103] H. U. Eckert. Induction plasmas at low frequencies. AIAA Journal, 9(8):1452-1456, 1971.

[104] M. P. Freeman and J.D. Chase. Energy transfer mechanism and typical operating characteristics for the thennal rfplasma generator. Journal of Applied Physics, 39, 1968.

[105] C. O. Laux, M. Mac Donald, C. Jacobs, F. Zander, U. A. Sheikh, D. Lacoste, and R. G. Morgan. Radiation-shapes-thermal protection investigations for high speed earth re-entry. d4.2: Ablation-radiation studies. Technical report, European Union FP-7 Project, 2013.

[106] J. Fournier and O. Chazot. Radiation-shapes-thermal protection investigations for high speed earth re-entry - d2.1 - review of ground facilities and instrumentation for re-entry studies. Technical report, July 2011.

[107] S. P Sharma and C. Park. Operating characteristics of a 60-and 10-cm electric arc-driven shock tube. i-the driver. ii-the driven section. Journal of thermophysics and heat transfer, 4(3):259-265, 1990.

[108] B. A. Cruden, R. Martinez, J. H. Grinstead, and J. Olejniczak. Simultaneous vacuum ultraviolet through near ir absolute radiation measurement with spatiotemporal resolution in an electric arc shock tube. AIAA paper, 4240:2009, 2009. 
[109] K Itoh, S Ueda, H Tanno, T Komuro, and K Sato. Hypersonic aerothermodynamic and scramjet research using high enthalpy shock tunnel. Shock Waves, 12(2):93-98, 2002 .

[110] R. G. Morgan, T. McIntyre, D. Buttsworth, P. A. Jacobs, D. Potter, A. Brandis, R. Gollan, C. Jacobs, B. Capra, M. McGilvary, and T Eichmann. Shock and expansion tube facilities for the study of radiating flows. Annexe to European Space Agency Contract Report AMOD-VKI-TN-011, 2008.

[111] R. Parker, A. Dufrene, M. MacLean, M. Holden, P. DesJardin, J. Weisberger, and D. Levin. Emission measurements from high enthalpy flow on a cylinder in the lens-xx hypervelocity expansion tunnel. 2013.

[112] A Balter-Peterson, F. Nichols, B. Mifsud, and W Love. Arc jet testing in nasa ames research center thermophysics facilities. In AIAA Materials Specialist ConferenceCoating Technology for Aerospace Systems, volume 1, 1992.

[113] S. Caristia, F. De Filippis, A. Del Vecchio, and E. Graps. Scirocco pwt facility for high temperature material assembly testing. In 54 th International Astronautical Congress, 2003.

[114] A Louzet and A Lemal. Radiation-shapes-thermal protection investigations.

[115] A Bottin, M Carbonaro, V Vander Haegen, and S Paris. Predicted \& measured capability of the vki $1.2 \mathrm{mw}$ plasmatron regarding re-entry simulation. In Aerothermodynamics for space vehicles, volume 426, page 553, 1999.

[116] D. G Fletcher and M Playez. Characterization of supersonic and subsonic plasma flows. AIAA Paper, 3294:2006, 2006.

[117] M. Auweter-Kurtz and T. Wegmann. Overview of irs plasma wind tunnel facilities. Technical report, DTIC Document, 2000.

[118] P. Sagnier and V. Jean-Luc. Flow characterization in the onera $f 4$ high-enthalpy wind tunnel. AIAA journal, 36(4):522-531, 1998.

[119] A. A Ndiaye and V Lago. Optical spectroscopy investigation of n2-ch4 plasma jets simulating titan atmospheric entry conditions. Plasma Sources Science and Technology, 20(1):015015, 2011.

[120] V Lago, A Lebé, M Dudeck, S Pellerin, T Renault, and P Echegut. Entry conditions in planetary atmospheres: emission spectroscopy of molecular plasma arcjets. Journal of thermophysics and heat transfer, 15(2):168-175, 2001. 
[121] T. N Canning, A Seiff, and C. S James. Ballistic-range technology. Technical report, DTIC Document, 1970.

[122] Takayanagi H. Suzuki T. Fujita K. Yamada, G. Analysis of shock layer radiation in vacuum-ultraviolet region for hayabusa return conditions. In 48th AIAA Aerospace Sciences Meeting Including the New Horizons Forum and Aerospace Exposition., Orlando, Florida., 2010.

[123] G. Yamada, H. Takayanagi, T. Suzuki, and K. Fujita. Vacuum ultraviolet spectroscopy for shock layer radiation measurement. AIAA paper, 1454:2009, 2009.

[124] A. M. Brandis, C. O. Johnston, B. A. Cruden, D. K. Prabhu, and D. Bose. Validation of high speed earth atmospheric entry radiative heating from 9.5 to $15.5 \mathrm{~km} / \mathrm{s}$. In 43rd AIAA Thermophysics Conference, New Orleans, Louisiana, 2012. AIAA.

[125] C. O Johnston, A. M Brandis, and D. Bose. Radiative heating uncertainty for hyperbolic earth entry, part 3: Comparisons with electric arc shock-tube measurements. Journal of Spacecraft and Rockets, 50(1):48-55, 2013.

[126] C. O. Johnston, B. R. Hollis, and K. Sutton. Spectrum modeling for air shock-layer radiation at lunar-return conditions. Journal of Spacecraft and Rockets, 45(5):865$878,2008$.

[127] Y. Ralchenko, F. C. Jou, D. E. Kelleher, A. E. Kramida, A Musgrove, J Reader, W. L. Wiese, and K Olsen. Nist atomic spectra database (version 4.0). National Institute of Standards and Technology, Gaithersburg, MD, 2010.

[128] G Peach. Continuous absorption coefficients for non-hydrogenic atoms. Memoirs of the Royal Astronomical Society, 73:1, 1970.

[129] The Opacity Project Team. Bristol and philadelphia: Institute of physics publishing, 1995.

[130] W. Cunto, C. Mendoza, F. Ochsenbein, and C. J. Zeippen. Topbase at the cds. The Opacity Project. Selected Research Papers, Atomic Data Tables for S to Fe, page 53, 1997.

[131] L. H. Chambers. Predicting radiative heat transfer in thermochemical nonequilibrium flow fields. NASA TM-4564, 1994.

[132] R. L. Kurucz and B. Bell. Harvard-smithsonian center for astrophysics. Preprint, (4080), 1995. 
[133] J. Kurtz, R. Palmer, A. Thomas, T. McIntyre, and T. Steinberg. Bow-shock radiation on model surfaces in super-orbital flows. In 21st International Symposium on Shock Waves, Keppel Island, Great Barrier Reef, Queensland., 1997.

[134] Website http://en.wikipedia.org/wiki/File:Czerny turner.png. Czerny-turner configuration.

[135] A. Roth. Vacuum Technology. Elsevier, 1990.

[136] Leybold Turbo Molecular Pump System Manual.

[137] Princeton Instruments Personal Communication: Acton.

[138] C. James, D. E Gildfind, R. G Morgan, P. A Jacobs, and F. Zander. Designing and simulating high enthalpy expansion tube conditions. In Submitted to 2013 AsiaPacific International Symposium on Aerospace Technology, Sunport Hall, Takamatsu, Japan, 2013.

[139] B. J. McBride and S. Gordon. Computer program for calculating and fitting thermodynamic functions. Technical report, NASA Technical Report, RP-1271, 1992.

[140] O. Sudnitsin. Design and testing of expansion tube with area change. Master's thesis, 2000.

[141] A Brandis. Experimental study and modelling of non-equilibrium radiation during titan and martian entry. 2009.

[142] P. A. Jacobs and R. J Gollan. The eilmer3 code: User guide and example book. Technical report, Mechanical Engineering, The University of Queensland, Brisbane, Australia, 2008.

[143] Grant Palmer, Gary Allen, Chun Tang, and Jim Brown. Coupled fluids-radiation analysis of a high-mass mars entry vehicle. AIAA Paper, 3495, 2011.

[144] M. E MacDonald, C. M Jacobs, C. O Laux, and R. G Morgan. Measurements of air plasma/ablator interactions in a $50 \mathrm{kw}$ inductively coupled plasma torch. 2013.

[145] C. W Chang. Examination of material outgassing in the air and purging flow environments. In Optical Science and Technology, the SPIE 49th Annual Meeting, pages 107-115. International Society for Optics and Photonics, 2004.

[146] Omega. User Guide - Omegabond OB-101. 
[147] COMSOL Multiphysics - Heat Transfer Module, User Guide, version 4.0a edition.

[148] Jules Z Klose, John Mervin Bridges, and William R Ott. Radiometric Standards in the Vacuum Ultraviolet. US Department of Commerce, National Bureau of Standards, 1987. 


\section{Appendix A}

\section{Optical Aberrations}

The following section outlines aberrations that can occur in optical systems. The aberrations outlined are spherical aberration, comatic, astigmatism, curvature of field, distortion and chromatic aberration.

Spherical Aberration A theoretically perfect lens is able to converge all parallel rays to a single, infinitesimally small point. For a real lens the angle at which light is diffracted is dependent on the distance from the centre of the lens the ray intersects. The further away from the optical axis the ray enters, the stronger the effects of diffraction will be. The strengthening diffraction causes the beam to be turned at a sharper than required angle resulting in a spatially blurred focal point. This can be observed in Figure A.1.A. The top image in Figure A.1.A shows a perfect lens diffracting a collimated beam to a precise focal point. The bottom image shows the effects of spherical aberration; the rays that enter the lens further from the optical axis are diffracted at a sharper angle and thus do not pass through the focal point.

Comatic A coma or comatic aberration occurs due to a change in magnification across the entrance of a lens. This change in magnification occurs as the optical path length is different depending on where the ray makes contact with the focussing element. Comatic aberration is an extension of spherical aberration in that the light entering the lens at varying distances to the optical axis are bent more sharply coupled with the fact that there will be light entering off-axis. When this occurs the light will not converge and this can cause asymmetry in an image. The further off axis the rays enter, the stronger the effects of comatic aberration will be.

Astigmatism Astigmatism occurs when the rays in perpendicular planes have two different focal lengths. This form of aberration is the cause of the circle of confusion. The circle of least confusion, commonly used as an indicator for the sharpness of an image, is 
known as the point half way between the two foci[50 Confirmation]. Figure A.1.C shows the effects of astigmatism on the light paths of the rays.

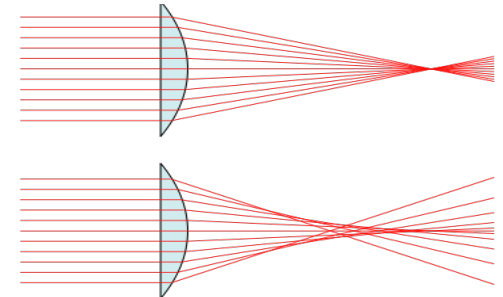

A

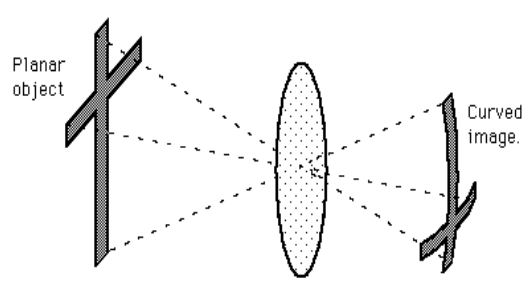

D

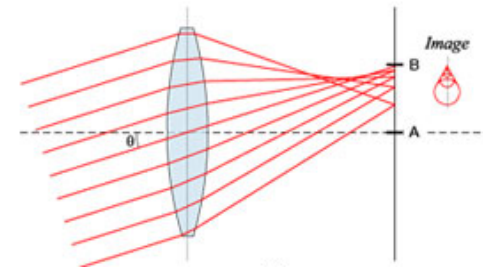

B
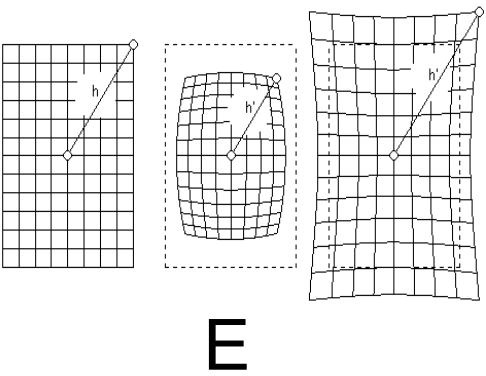

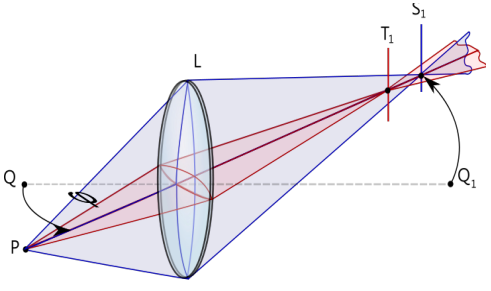

C

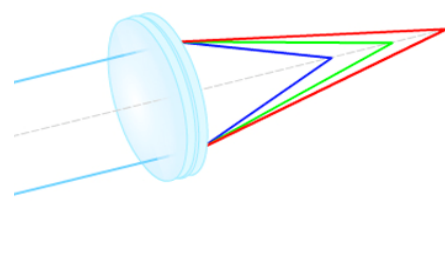

$\mathrm{F}$

Figure A.1: Effects of A. spherical aberration, B. comatic, C. astigmatism, D. curvature of field, E. distortion and F. chromatic aberration

Curvature of Field Curvature of field causes a planar object to be projected as a curved non-planar image. Near the centre of the image there is no curvature but as the light enters at sharper angles, the optical axis is bent more tightly due to comatic aberration. This causes the outer radii of the image to be curved more sharply as shown in Figure A.1.D. The effect of this is curvature of the outer radii of the image and only a small central section of the image remaining in the focal plane.

Distortion Distortion is a type of aberration that warps an image as a whole and is influenced by the position of the aperture. If the position of the aperture is between the lens and the object, a barrel effect will occur as shown in the centre image of Figure A.1.E. If the position of the aperture is between the lens and the image, a pincushion effect will occur as shown in the right image of Figure A.1.E.

Chromatic Aberration The focal length of a lens is dependent on the refractive index of the material. Any variation in refractive index as a function of wavelength, will result in varying focal lengths for radiation of varying wavelengths. Consequently different wavelengths will focus at varying positions creating chromatic aberration. Longitudinal chromatic aberration is known as the change in distance to the focal point for varying wavelengths. Lateral chromatic aberration is known as the change in image size due to the variation of the focal point at various wavelengths. 


\section{Appendix B}

\section{ICCD}

The recording device at the exit of the monochromator is the final device that contributes to the performance of a spectroscopy system. The measurement device for all of the monochromators used in this study was an ICCD and as such it is the only recording device that will be discussed here. An ICCD is capable of converting a fraction of the photons that reach the photocathode surface into electrons. The ratio of electrons released for the given number of photons defines the quantum efficiency. The quantum efficiency is a function of wavelength and as such ICCDs are designed for particular applications and are different for the UV and VNIR cameras. The released electron at the back of the photocathode travels through a microchannel plate, which is composed of millions of microchannels coated with materials of high secondary emission yield. Due to the angle of the microchannels, the electron has a high probability of contacting the high secondary emission yield walls that release more electrons, thus amplifying the number of electrons at the exit of the microchannel plate exponentially. Through the variation of the potential voltage difference across the microchannel plate, the user can control the number of electrons that will exit the microchannel plate and effectively control the intensity of the signal the will finally reach the CCD.

The electrons that exit the microchannel plate enter a strong electronic field that accelerates them towards a phosphor screen. The electrons striking the phosphor screen cause the release of photons, typically in the green wavelength range at which the CCD is most sensitive. The material selection of the phosphor screen is critical in the design process as the phosphor screen continue to emit photons for short periods of time after an electron has struck them. This is especially critical for experiments conducted on short duration facilities such as expansion tubes where the experiment duration can be shorter than the decay time. This factor can also lead to non-linearity in the exposure time of the camera and intensity recorded on the CCD. 


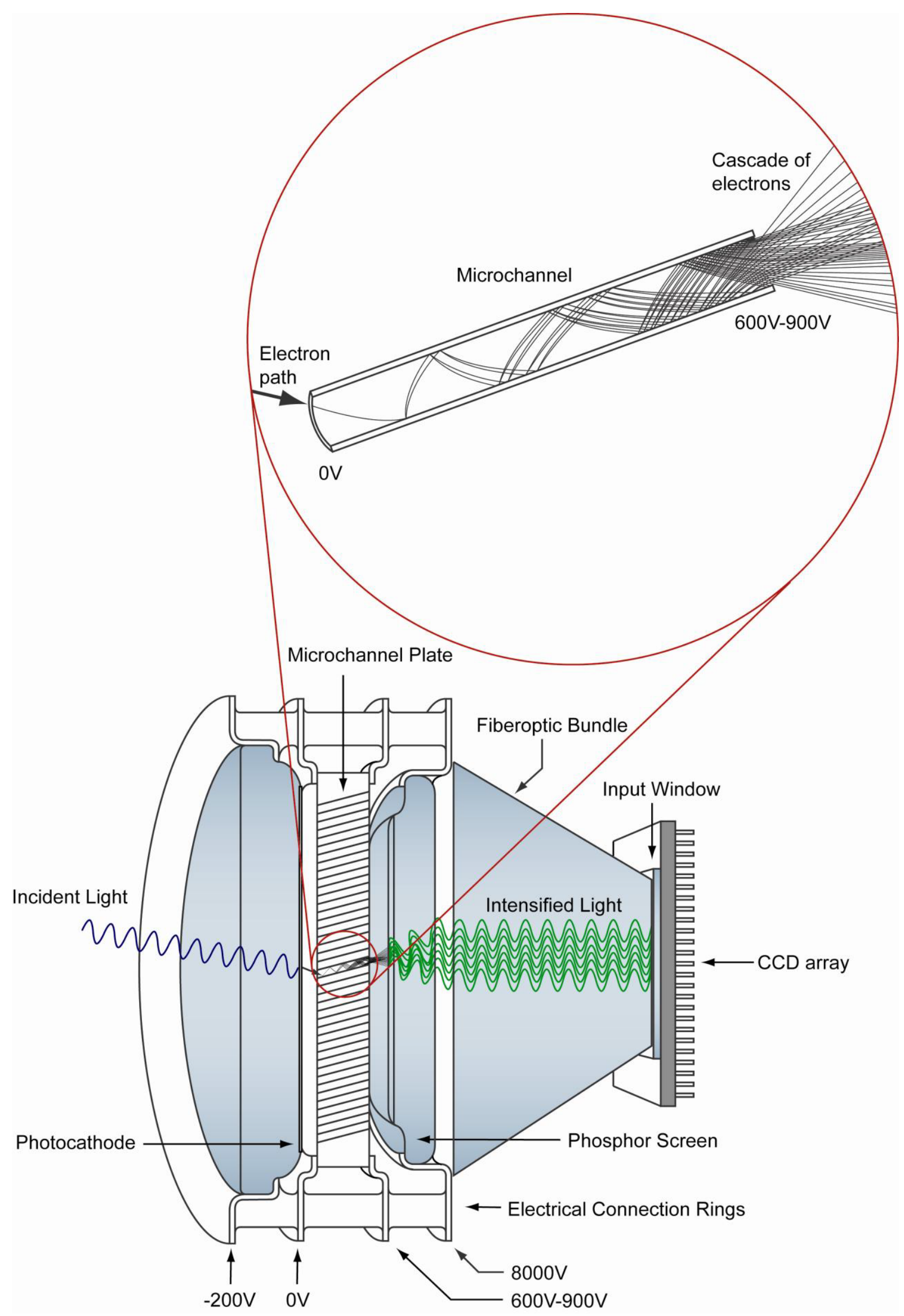

Figure B.1: Cross section of a Princeton Instruments Generation II Image Intensifier and CCD array. 35] 
Finally, the photons released by the phosphor screen are conducted to the CCD array through a fibre optic bundle. The CCD is a device with an array of pixels that are capable of converting the intensity of light impacting them into charge through the photoelectric effect. These pixels, consisting of capacitors with a photoactive coating of silicon, are arranged in a two-dimensional array to provide the spatial and spectral resolution required in imaging spectroscopy. The size of these pixels is important in achieving a balance between signal to noise ratio and resolution. A better resolution can be achieved with smaller pixels however this would reduce the photoactive area of a pixel, in turn reducing the signal to noise ratio. The CCDs used in the VNIR and UV spectrometers have 1024 pixels in the spectral direction and 256 pixels in the spatial direction. The size of each pixel is $26 \mu \mathrm{m}$ and this provides ample signal for the short test times experienced at high speeds in the expansion tube. 


\section{Appendix $\mathrm{C}$}

\section{Repeatability Testing Shots}

The following chapter presents uncalibrated shot data taken from the side of the dump tank to investigate repeatability of the VUV spectroscopy system.

Table C.1: Shots conducted for repeatability study.

\begin{tabular}{ll}
\hline Condition 1 & Condition 2 \\
\hline x2s1908 & x2s1907 \\
x2s1917 & x2s1920 \\
x2s1918 & x2s1922 \\
x2s1919 & x2s1923 \\
& x2s1924 \\
\hline
\end{tabular}



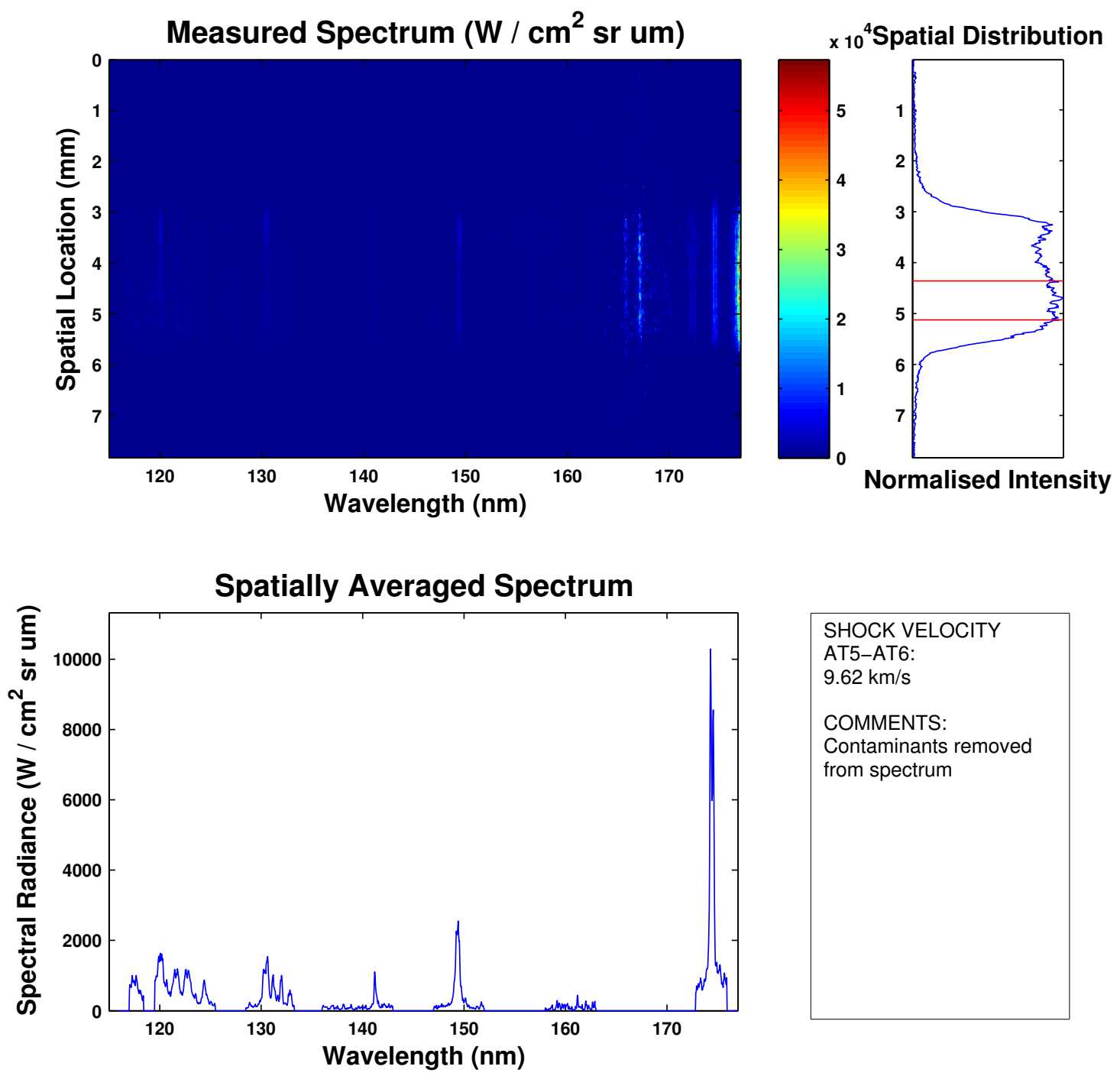

\section{Shot Validity Analysis}

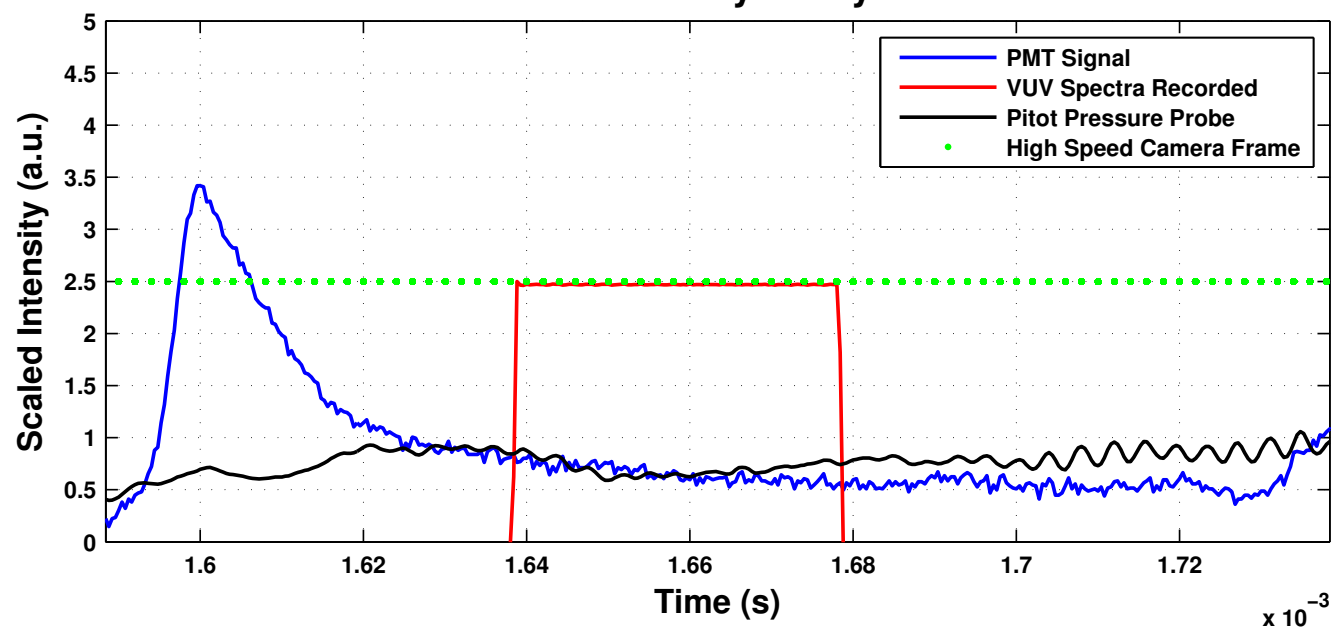

Figure C.1: x2s1908 - Repeatability test shot at condition 1. 

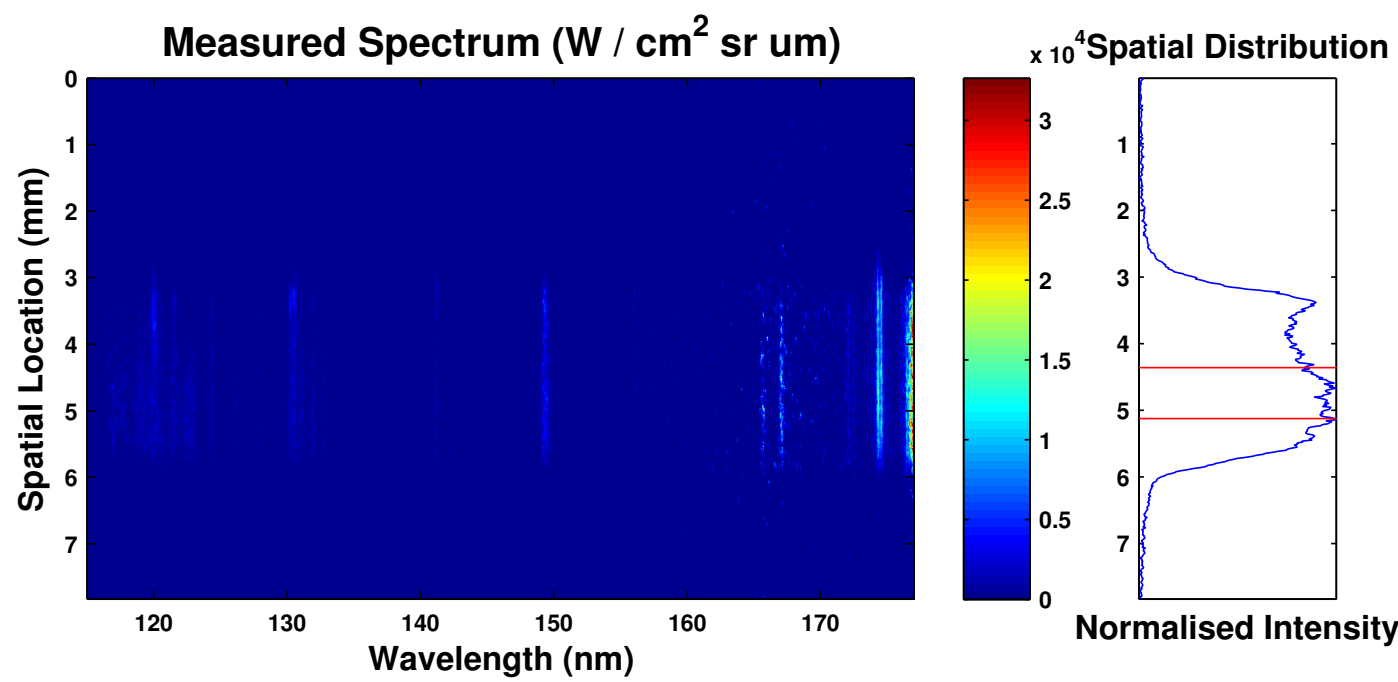

Normalised Intensity

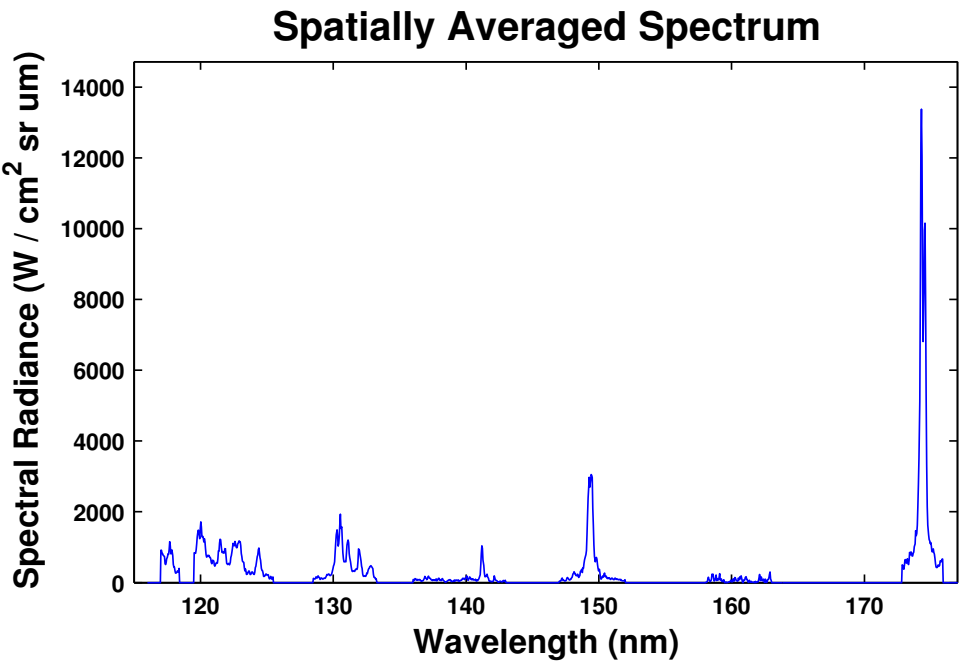

SHOCK VELOCITY

AT5-AT6:

$9.62 \mathrm{~km} / \mathrm{s}$

COMMENTS:

Contaminants removed

from spectrum

Shot Validity Analysis

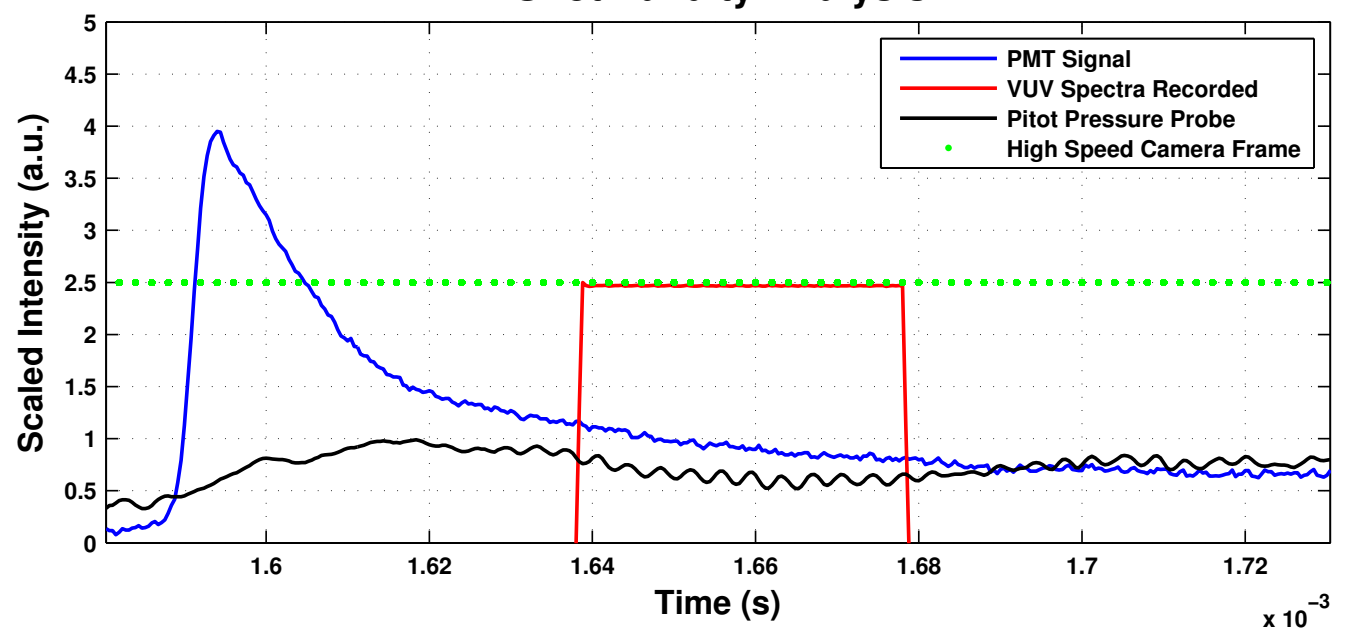

Figure C.2: x2s1917 - Repeatability test shot at condition 1. 

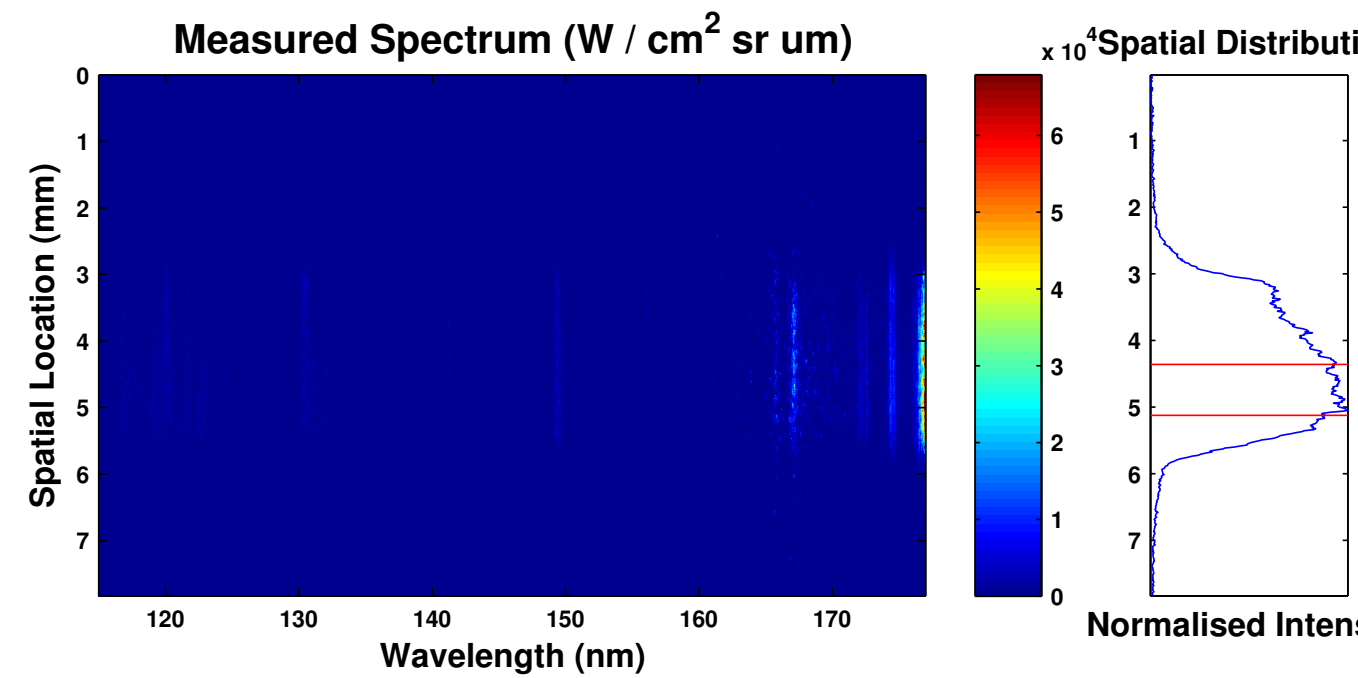

Normalised Intensity
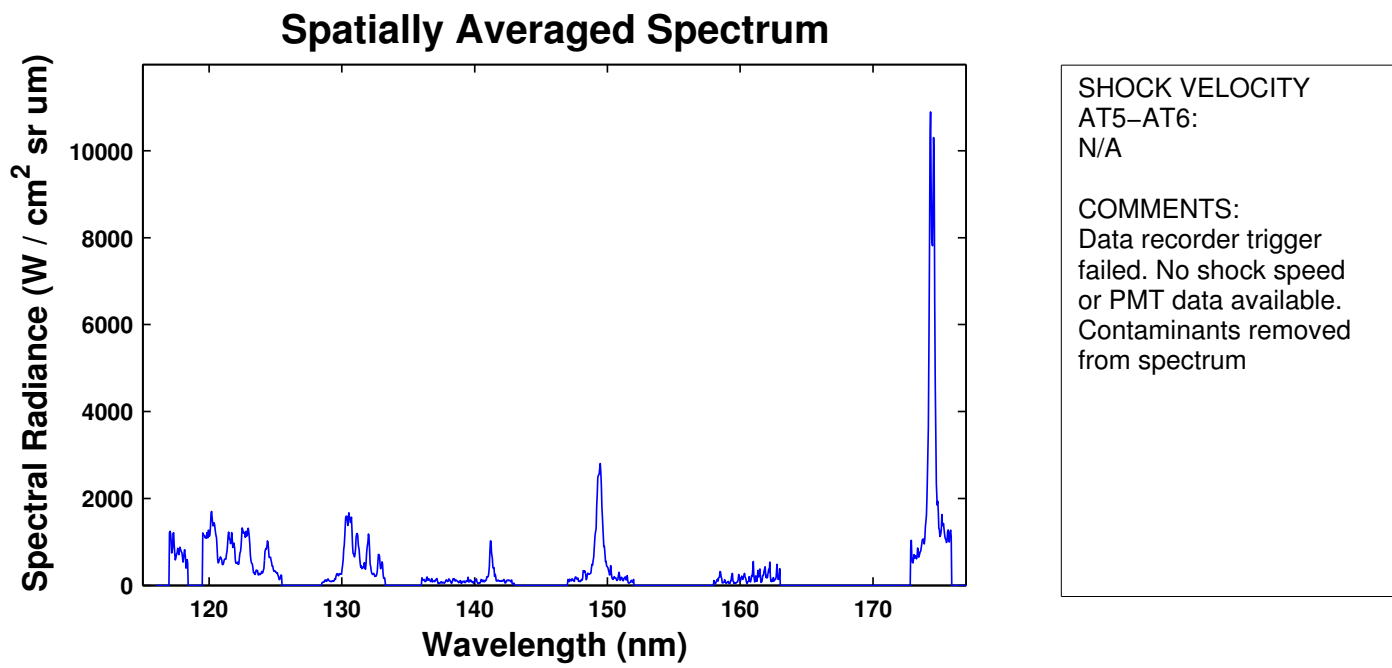

Figure C.3: x2s1918 - Repeatability test shot at condition 1. 

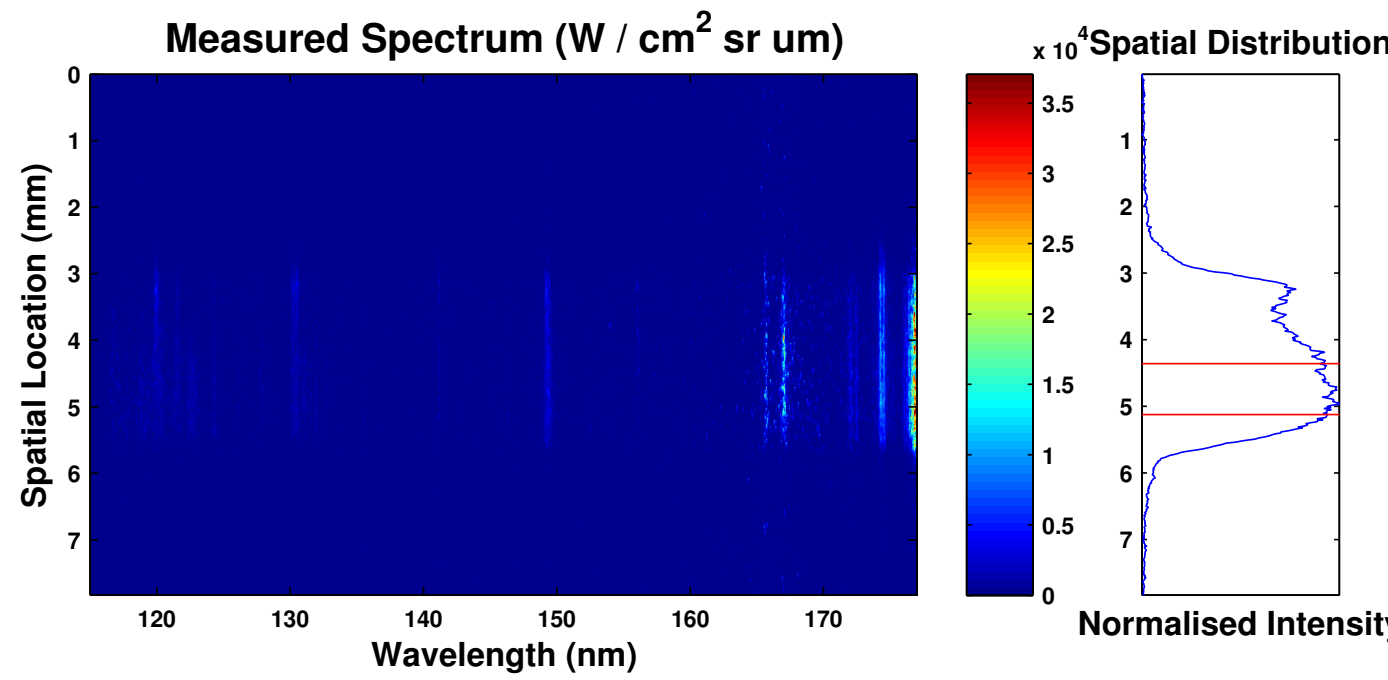

Normalised Intensity

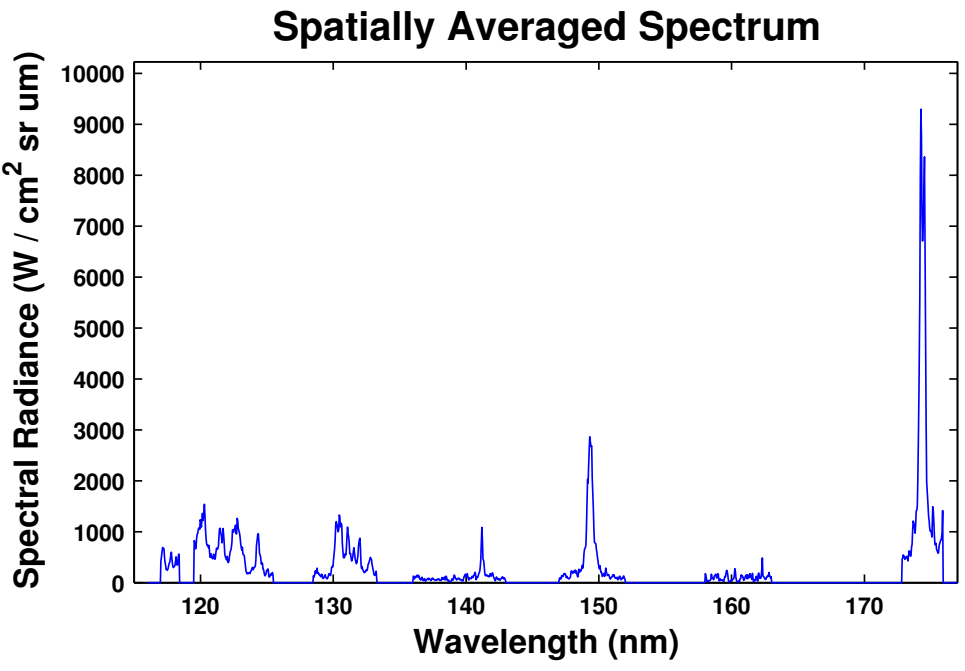

SHOCK VELOCITY

AT5-AT6:

$9.62 \mathrm{~km} / \mathrm{s}$

COMMENTS:

Contaminants removed

from spectrum

Shot Validity Analysis

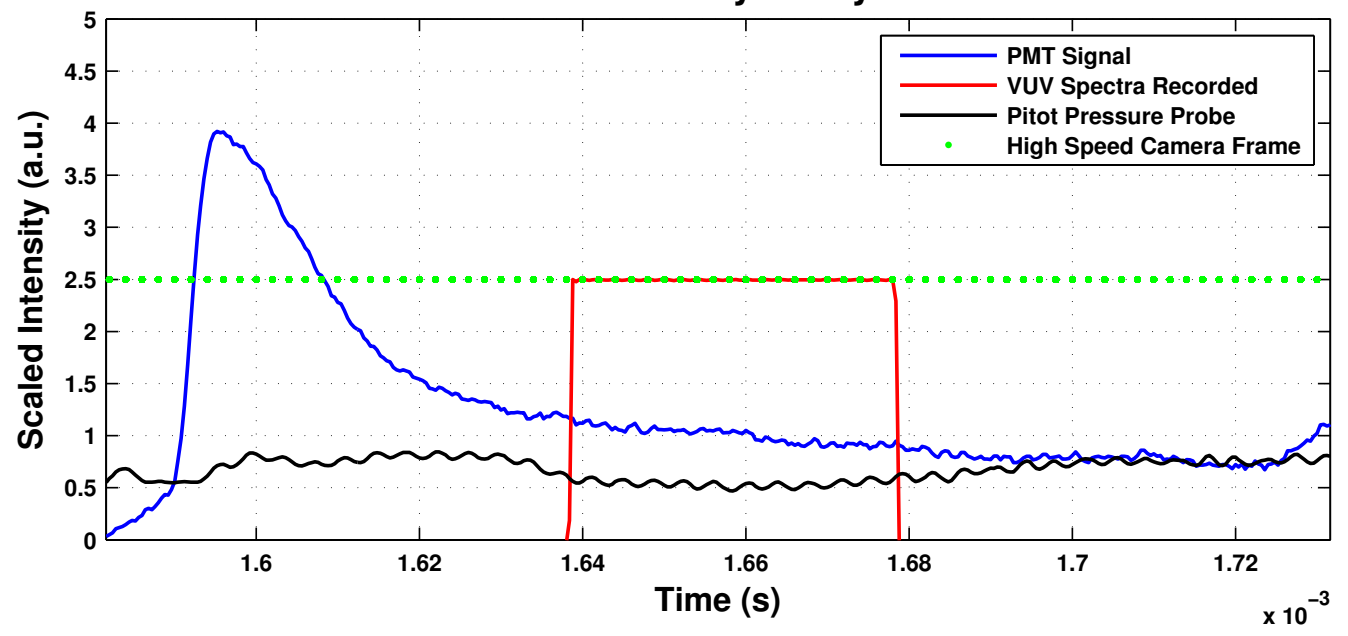

Figure C.4: x2s1919 - Repeatability test shot at condition 1. 

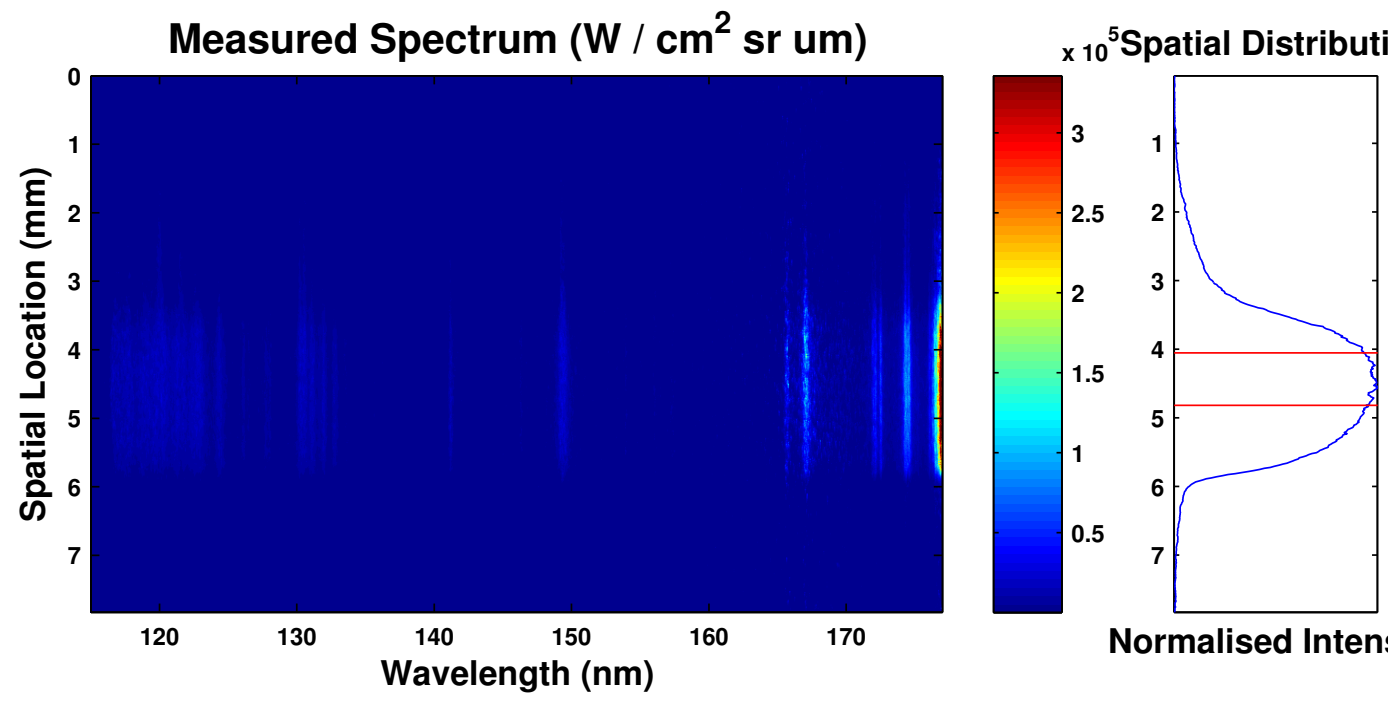

Normalised Intensity

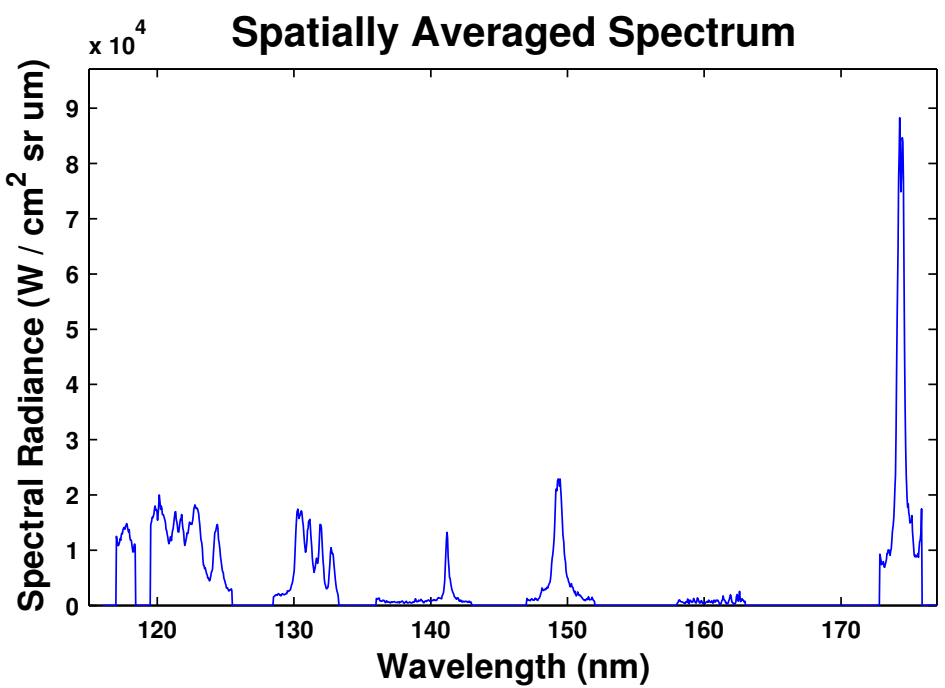

SHOCK VELOCITY

AT5-AT6:

$11.4 \mathrm{~km} / \mathrm{s}$

COMMENTS:

Contaminants removed

from spectrum

Shot Validity Analysis

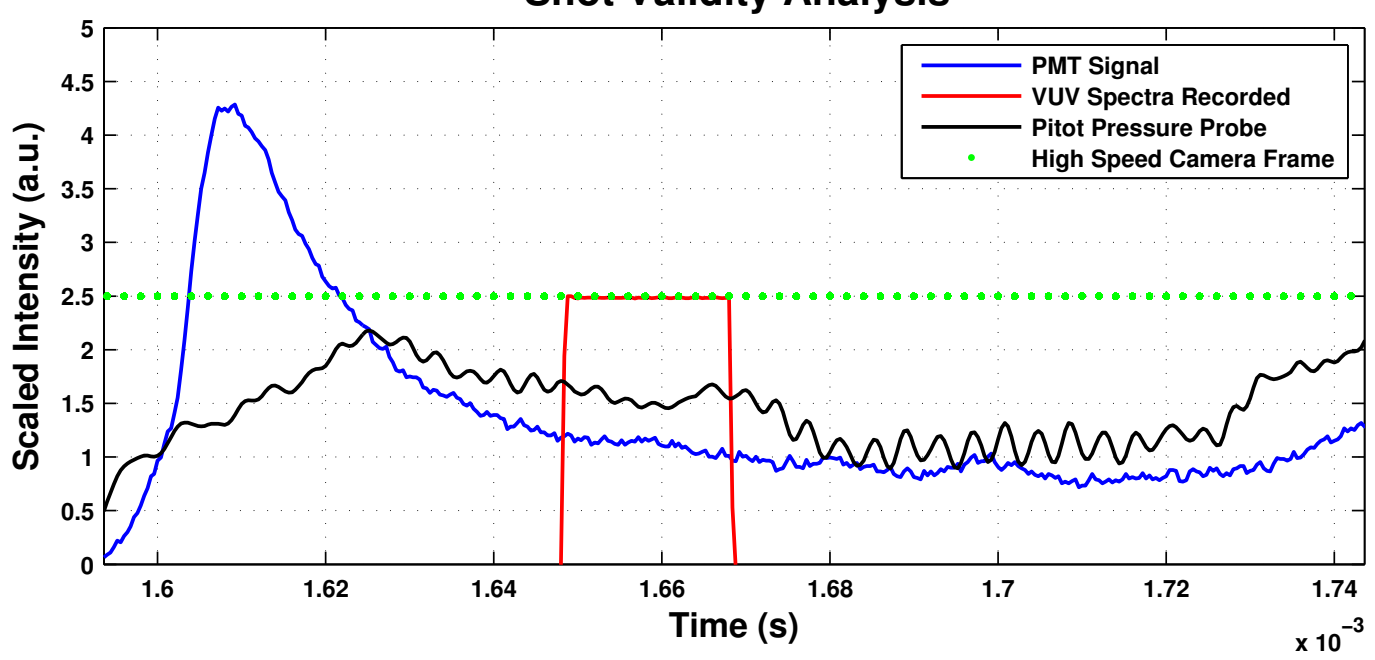

Figure C.5: x2s1907 - Repeatability test shot at condition 2 . 

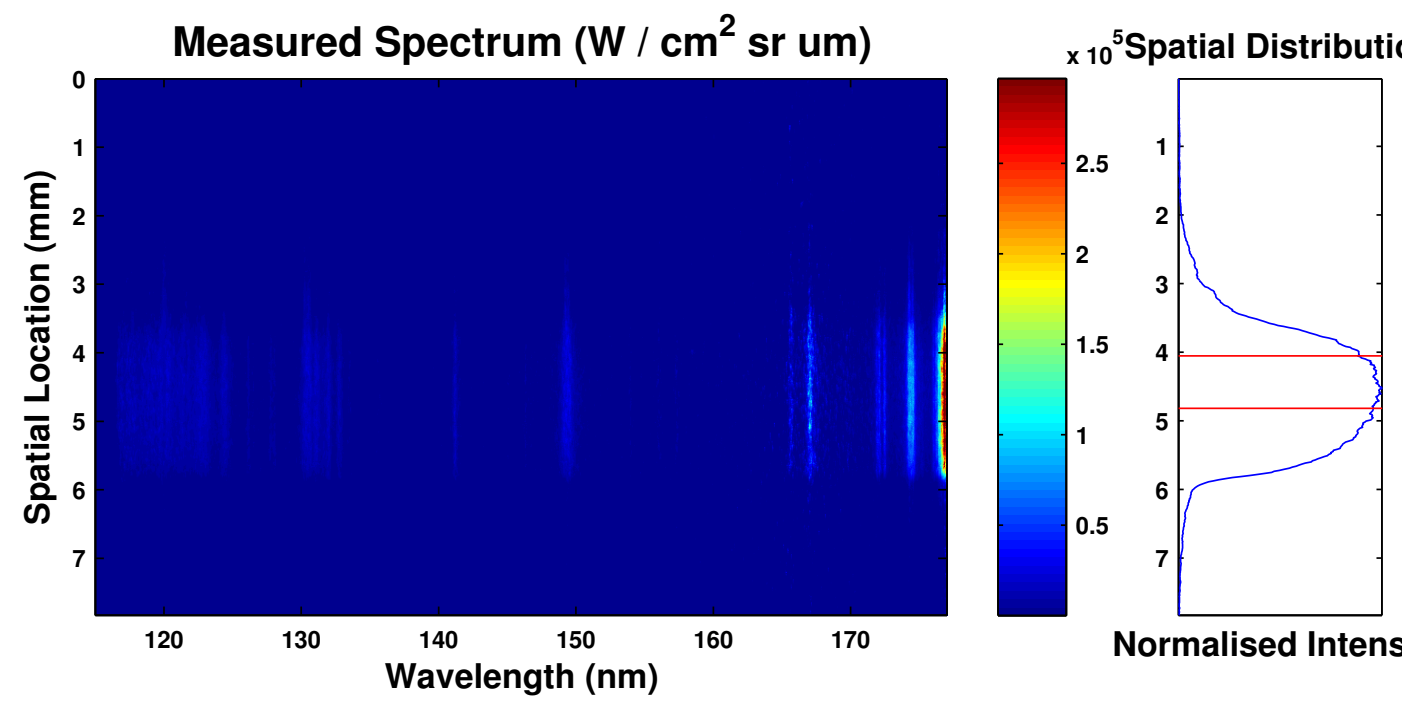

Normalised Intensity
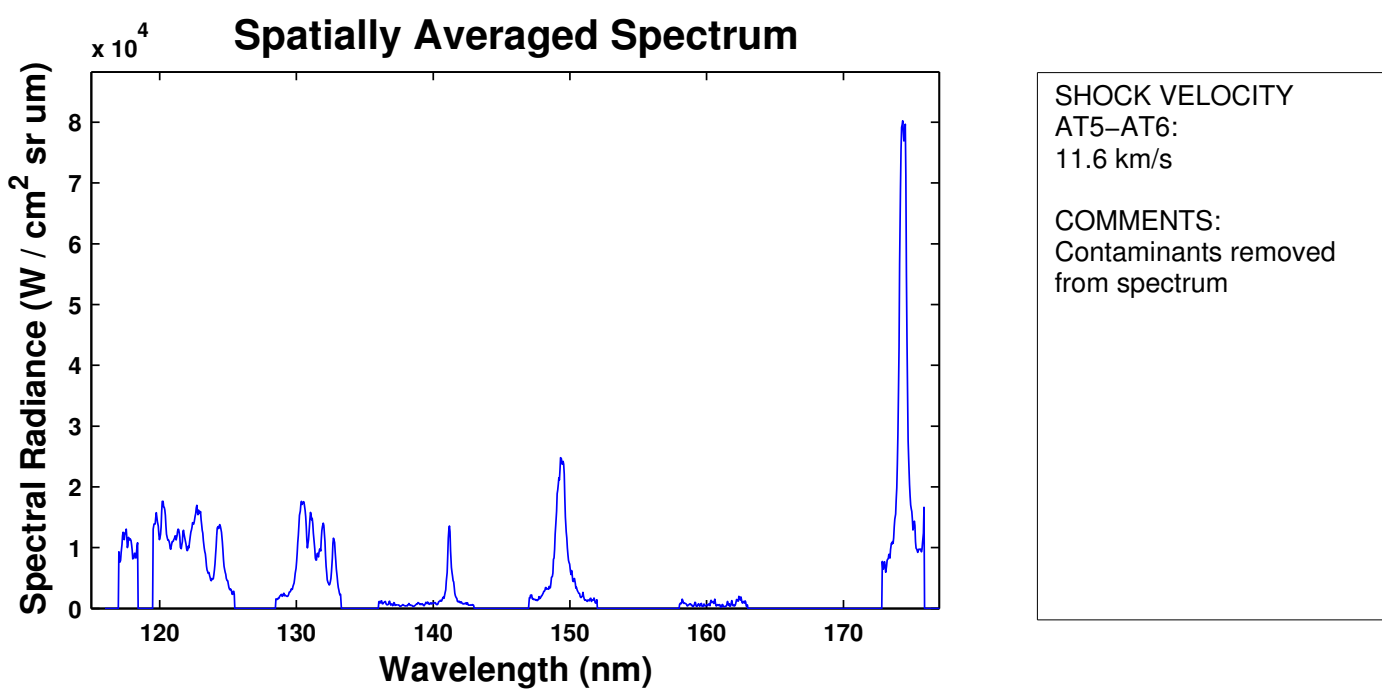

\section{Shot Validity Analysis}

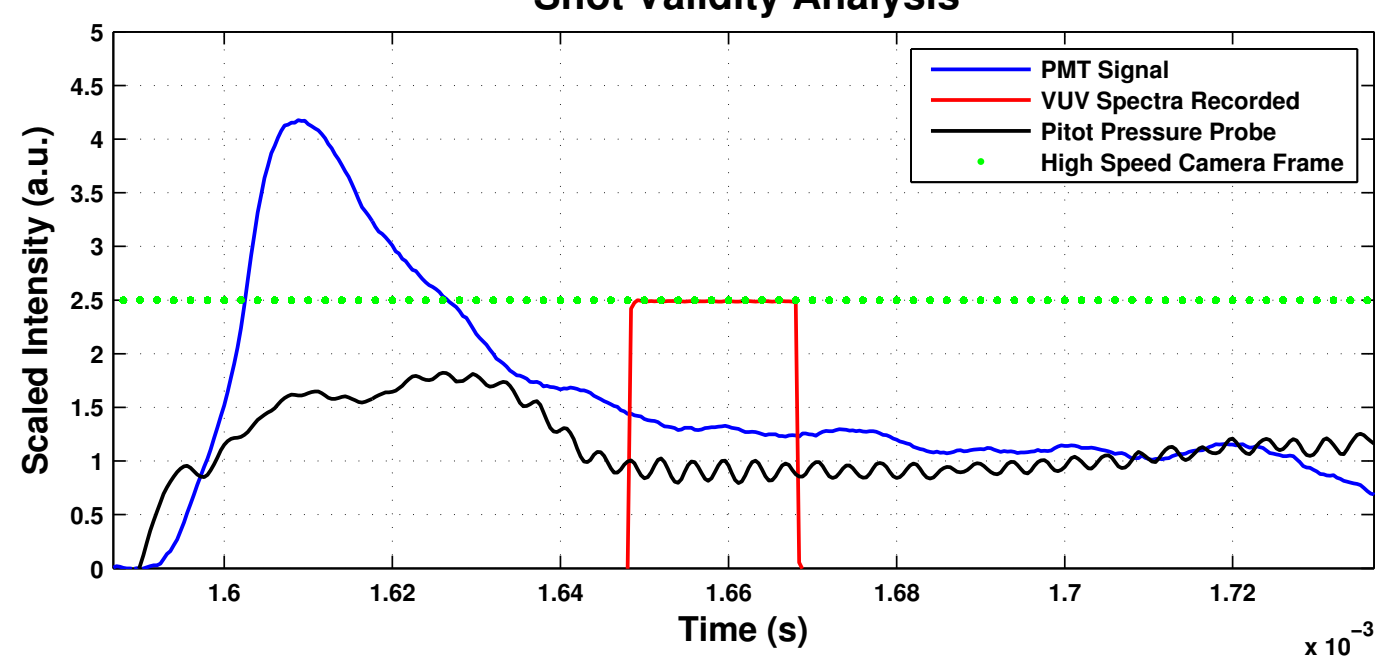

Figure C.6: x2s1920 - Repeatability test shot at condition 2. 

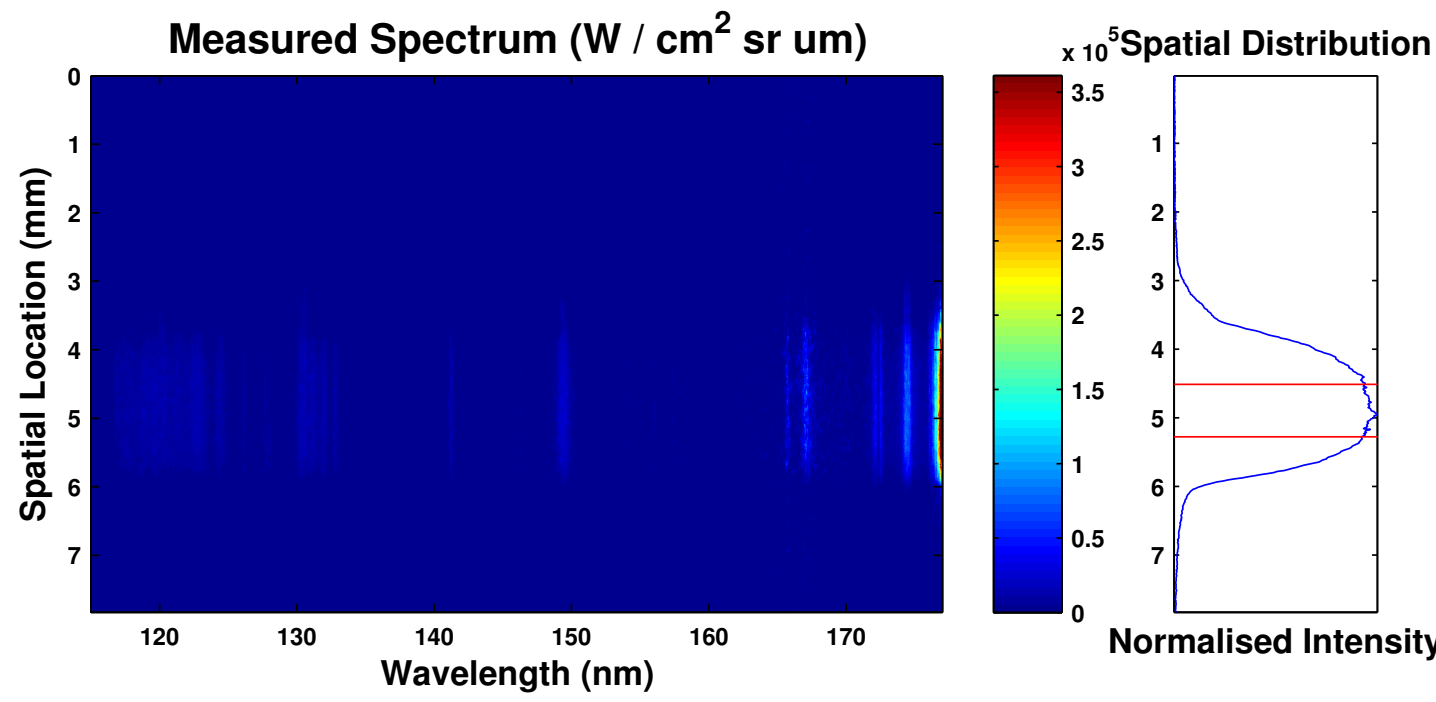

$\times 10^{4} \quad$ Spatially Averaged Spectrum

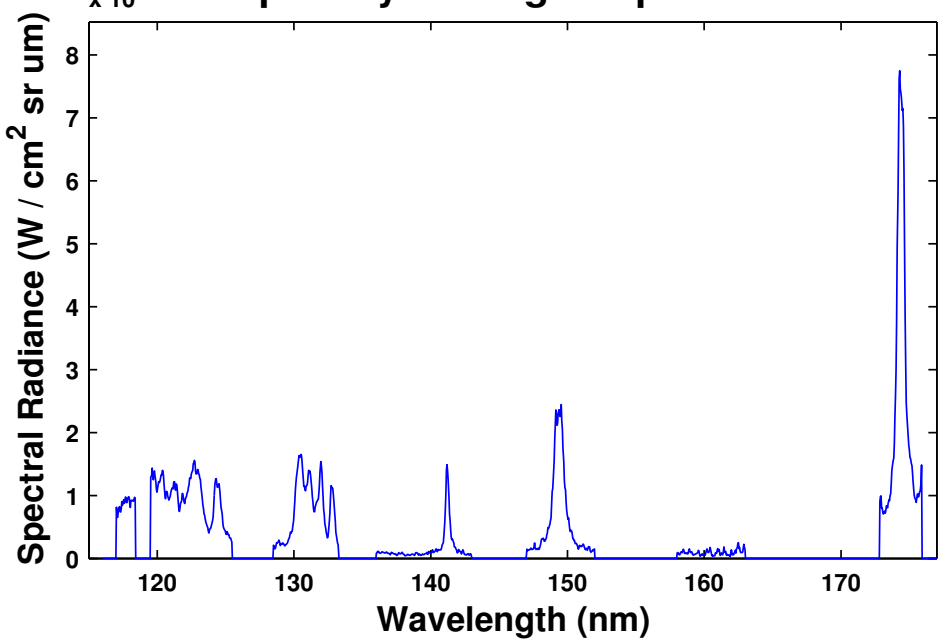

SHOCK VELOCITY

AT5-AT6:

$11.6 \mathrm{~km} / \mathrm{s}$

COMMENTS:

Contaminants removed

from spectrum

Shot Validity Analysis

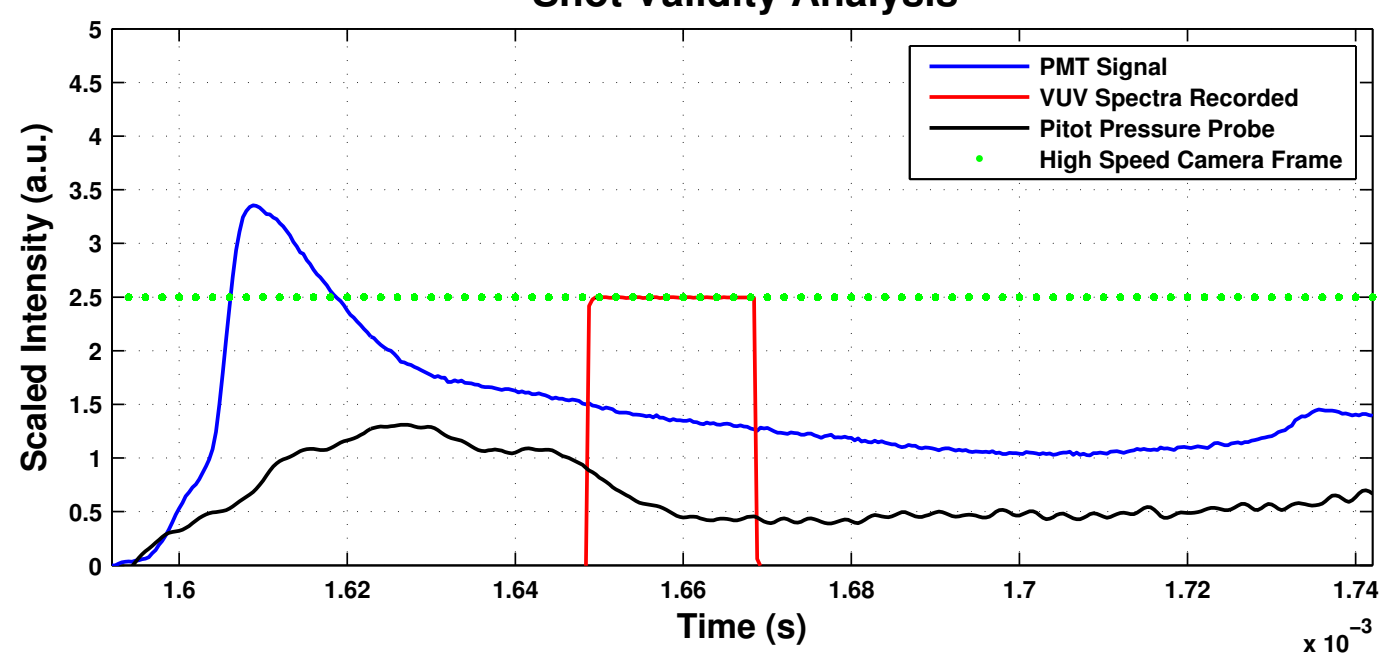

Figure C.7: x2s1922 - Repeatability test shot at condition 2. 

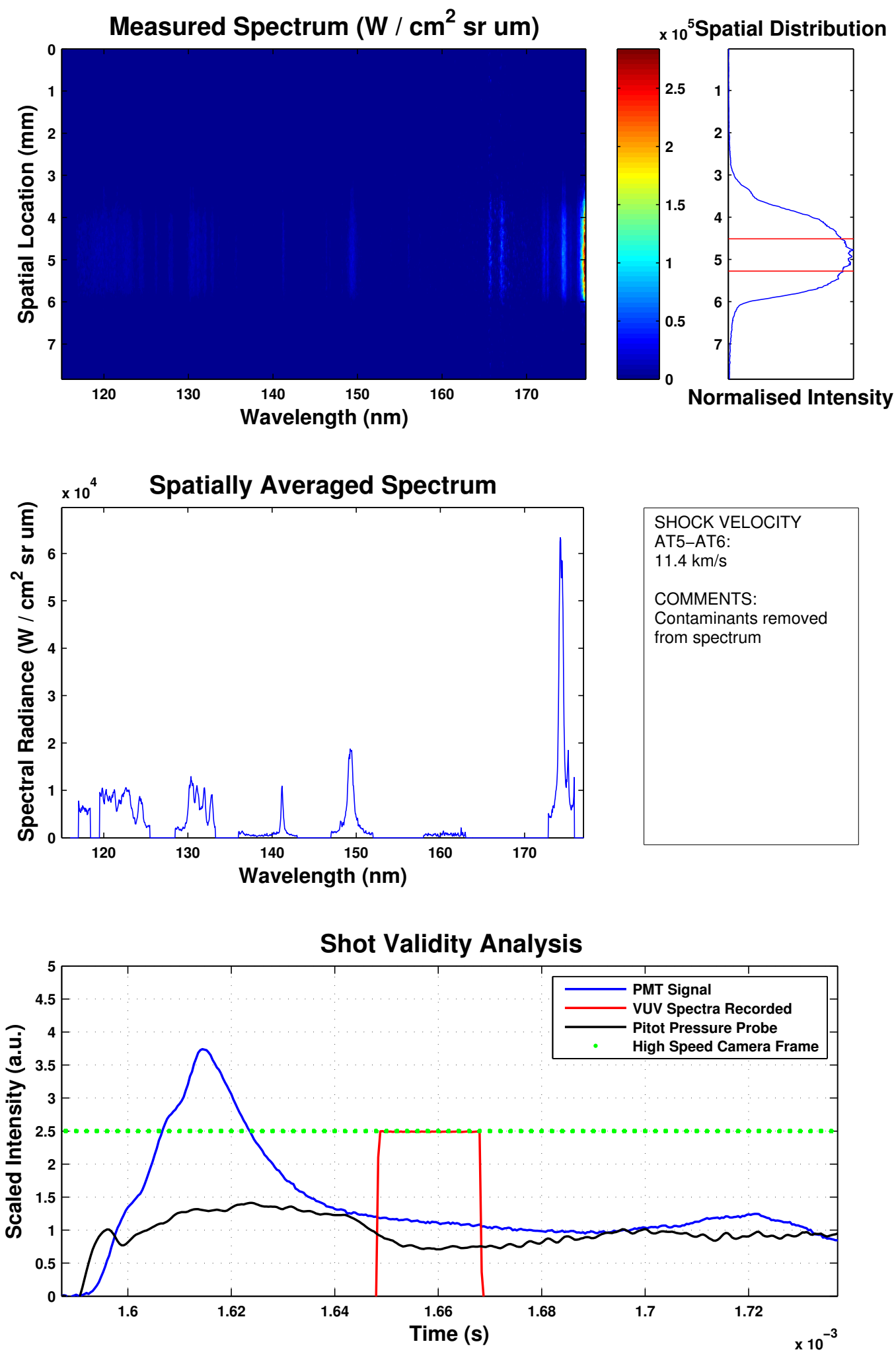

Figure C.8: x2s1923 - Repeatability test shot at condition 2. 

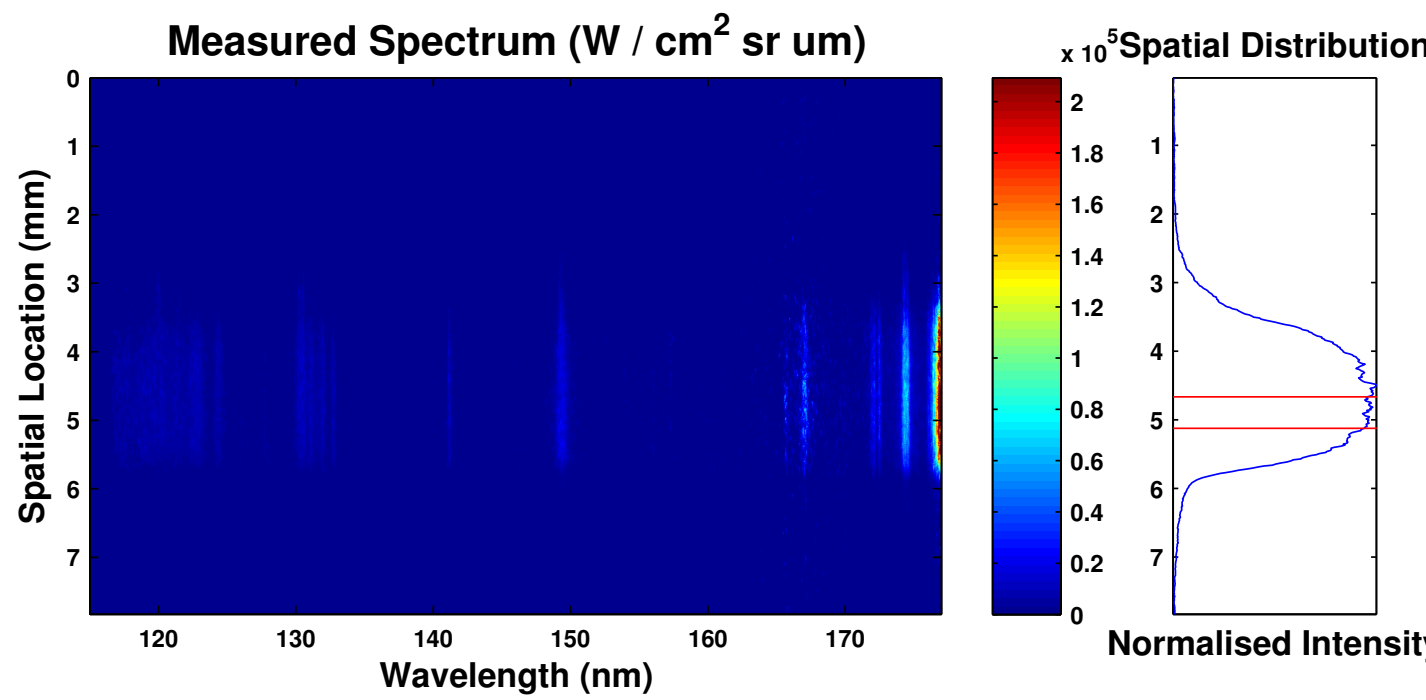

Normalised Intensity

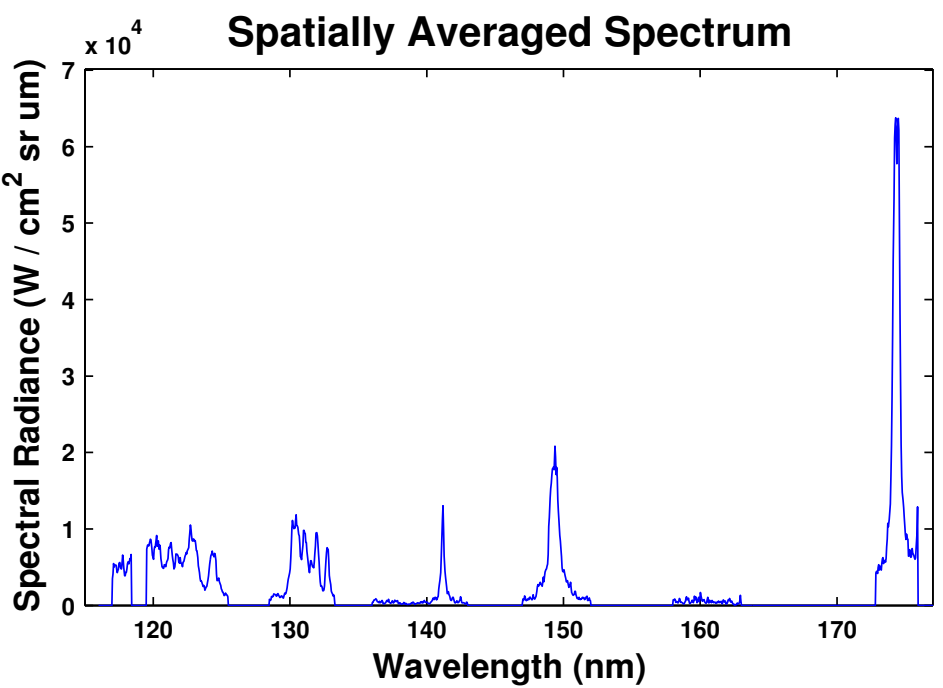

SHOCK VELOCITY

AT5-AT6:

$11.6 \mathrm{~km} / \mathrm{s}$

COMMENTS:

Contaminants removed

from spectrum

Shot Validity Analysis

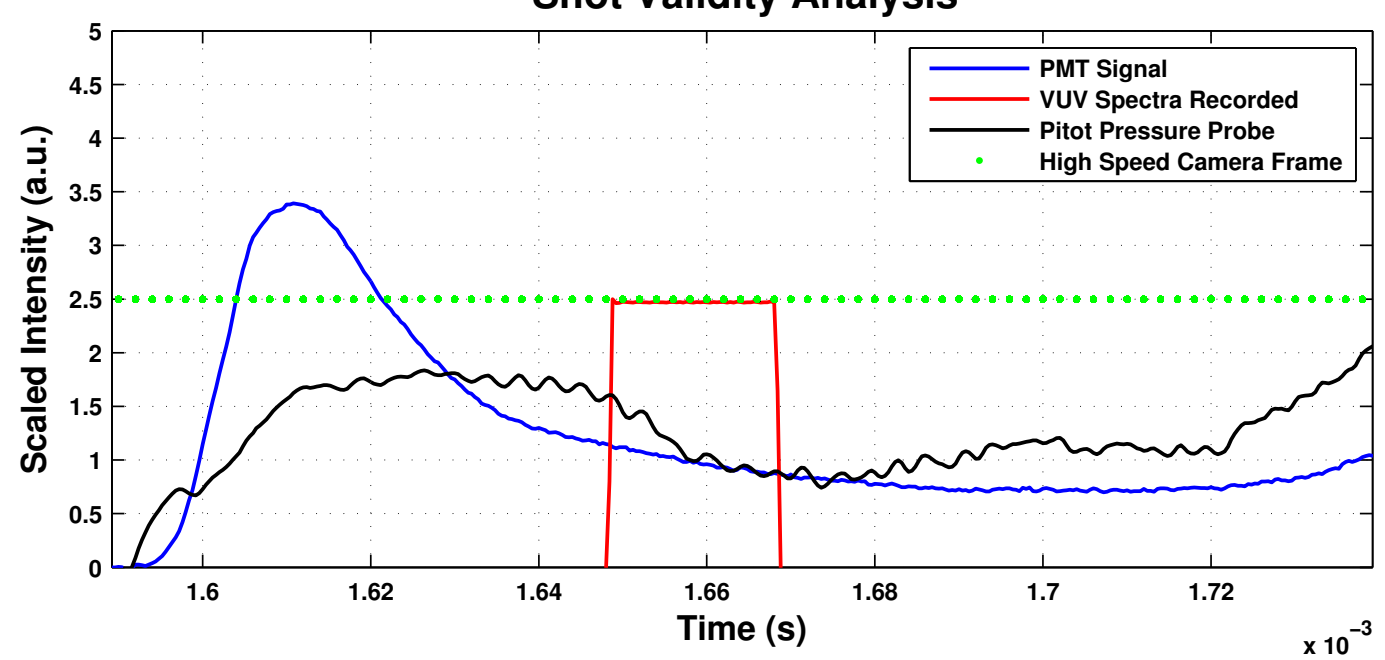

Figure C.9: x2s1924 - Repeatability test shot at condition 2. 


\section{Appendix D}

\section{Calibrated Expansion Tube Datasets}

The following chapter presents all of the calibrated recorded data taken on the X2 expansion tube. The first set of data presented is from the through surface and across surface experiments. Following these spectra, the spectral data from the self-absorption experiments is presents. Repeat shots are also presented.

\section{D.1 Through Surface and Across Surface Experiments}

Table D.1: Shots conducted for across and through surface comparison experiments.

\begin{tabular}{lll}
\hline Condition $\mathbf{1}$ & & \\
\hline Viewing direction & Primary shot & Repeat shot \\
\hline Across surface & $\mathrm{x} 2 \mathrm{~s} 1938$ & $\mathrm{x} 2 \mathrm{~s} 1936$ \\
Through surface & $\mathrm{x} 2 \mathrm{~s} 1954$ & \\
\hline Condition $\mathbf{2}$ & & Repeat shot \\
\hline Viewing direction & Primary shot & x2s1939 \\
\hline Across surface & x2s1940 & x2s1958 \\
Through surface & $\mathrm{x} 2 \mathrm{~s} 1957$ & \\
\hline
\end{tabular}



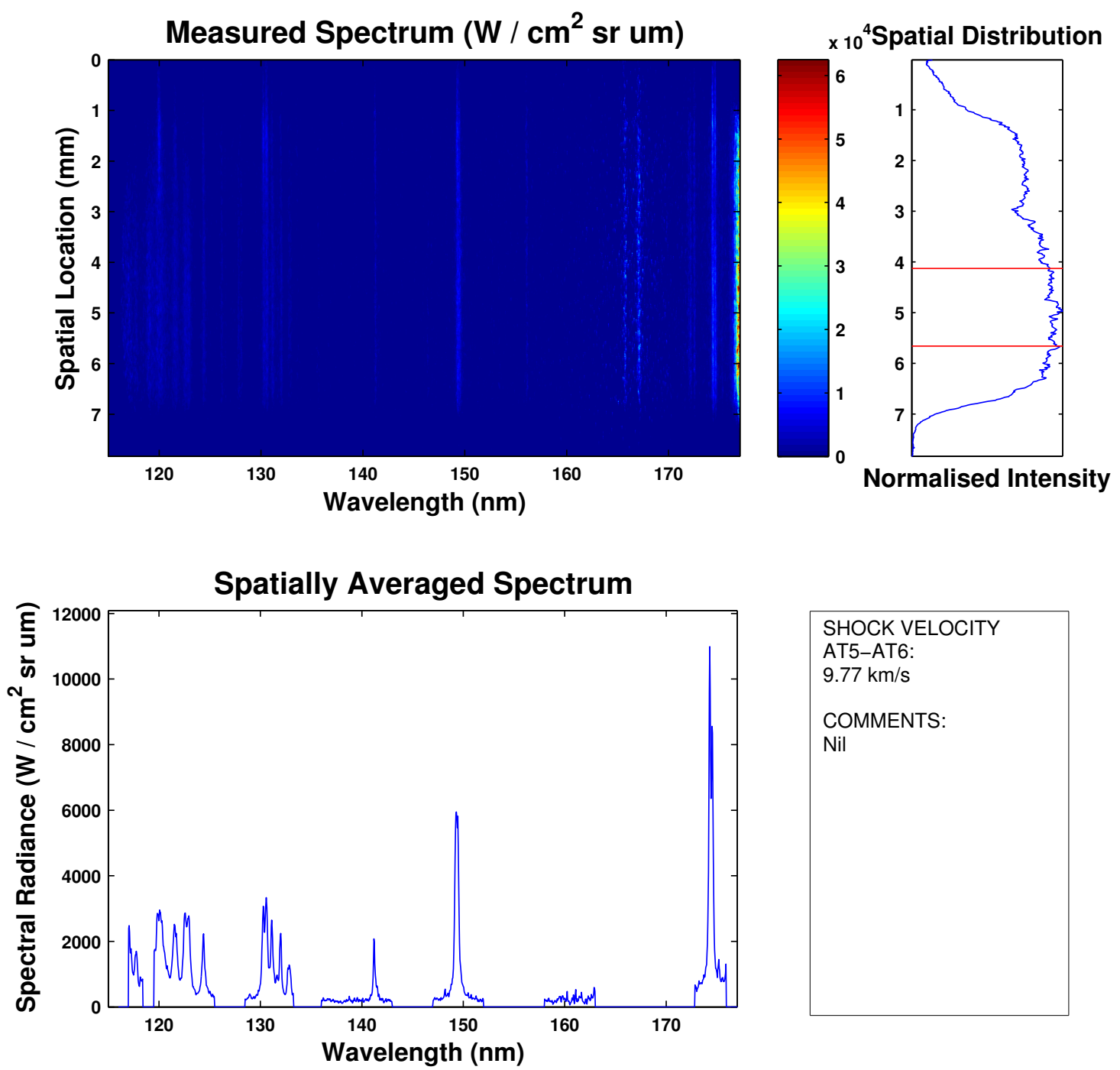

\section{Shot Validity Analysis}

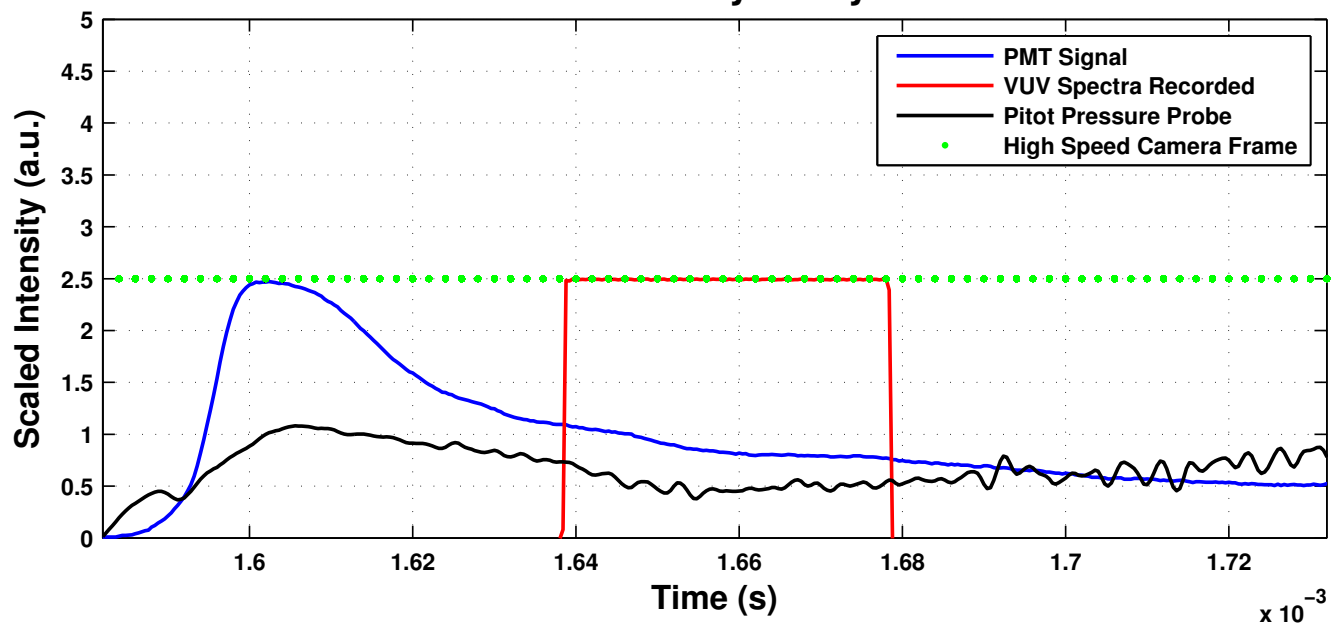

Figure D.1: x2s1938 - Primary shot data for across the surface at condition 1. 

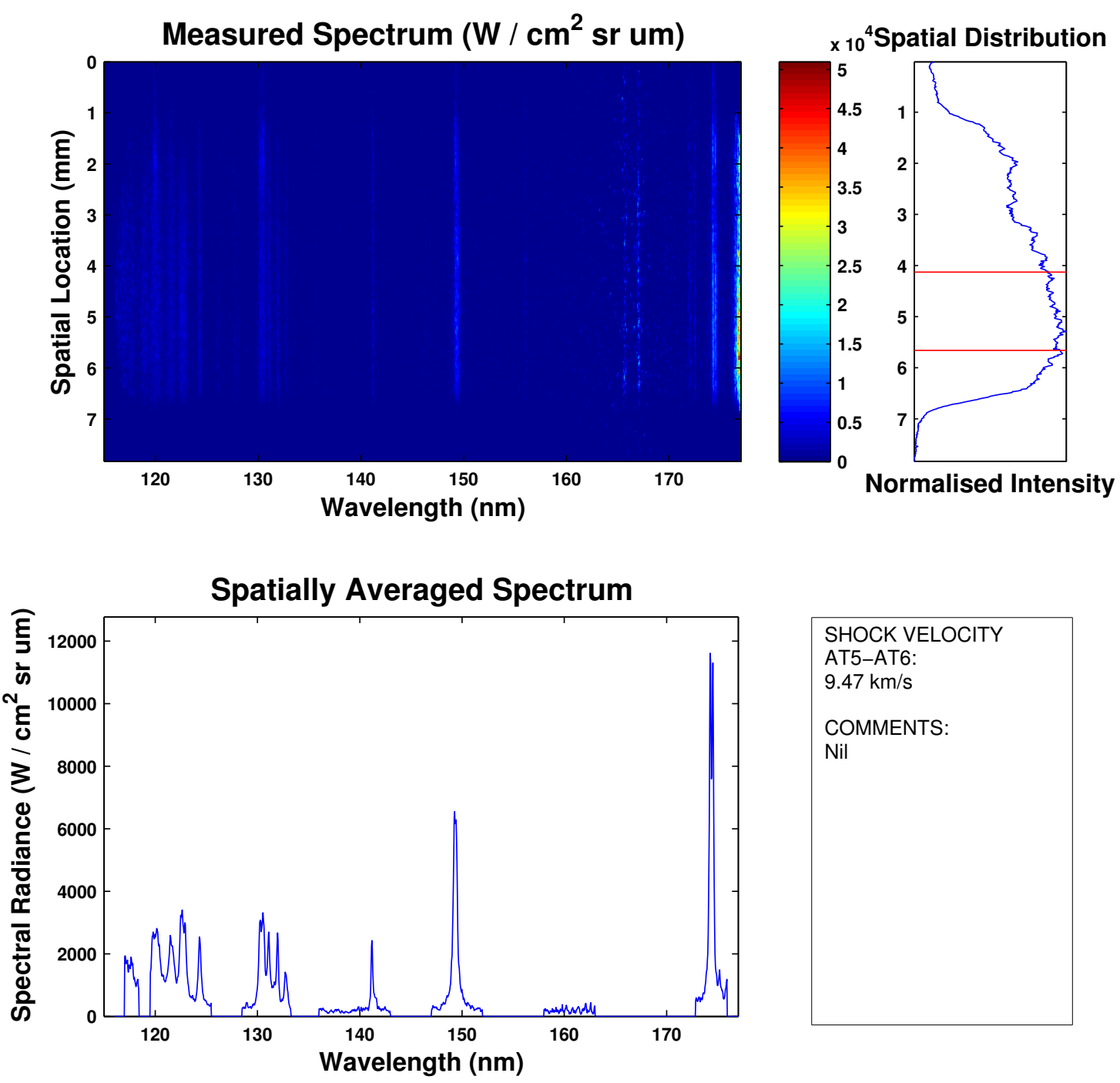

Shot Validity Analysis

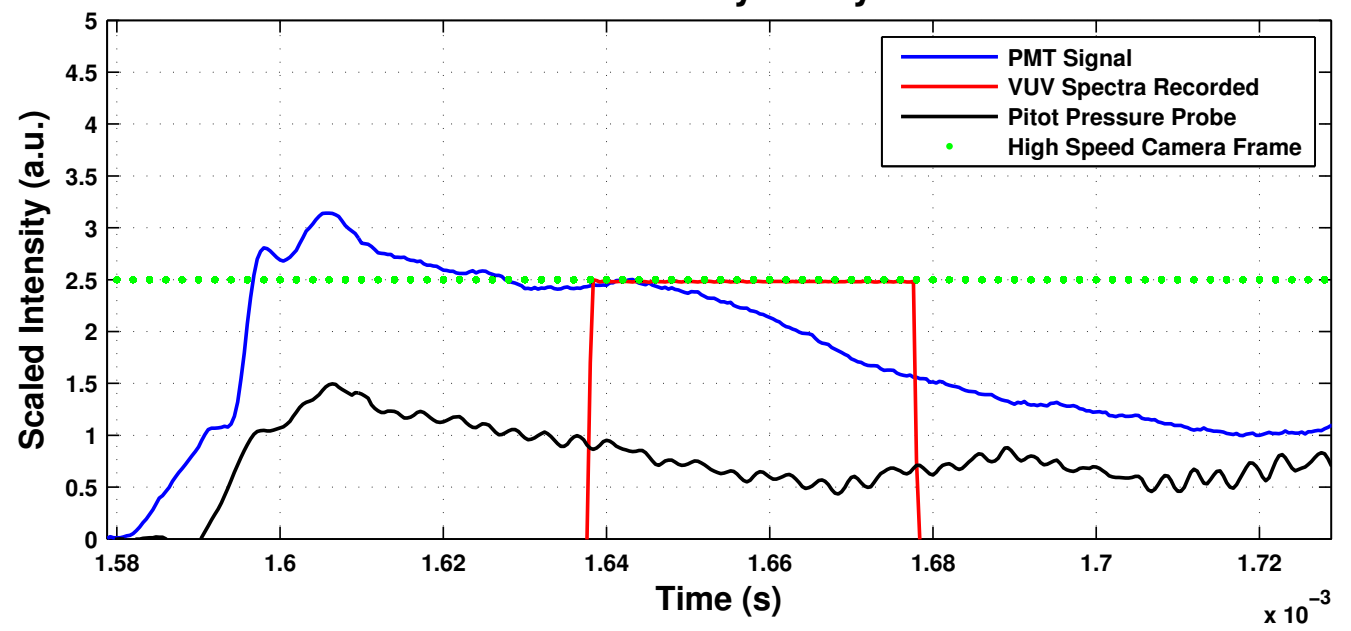

Figure D.2: x2s1936 - Repeat shot data for across the surface at condition 1. 

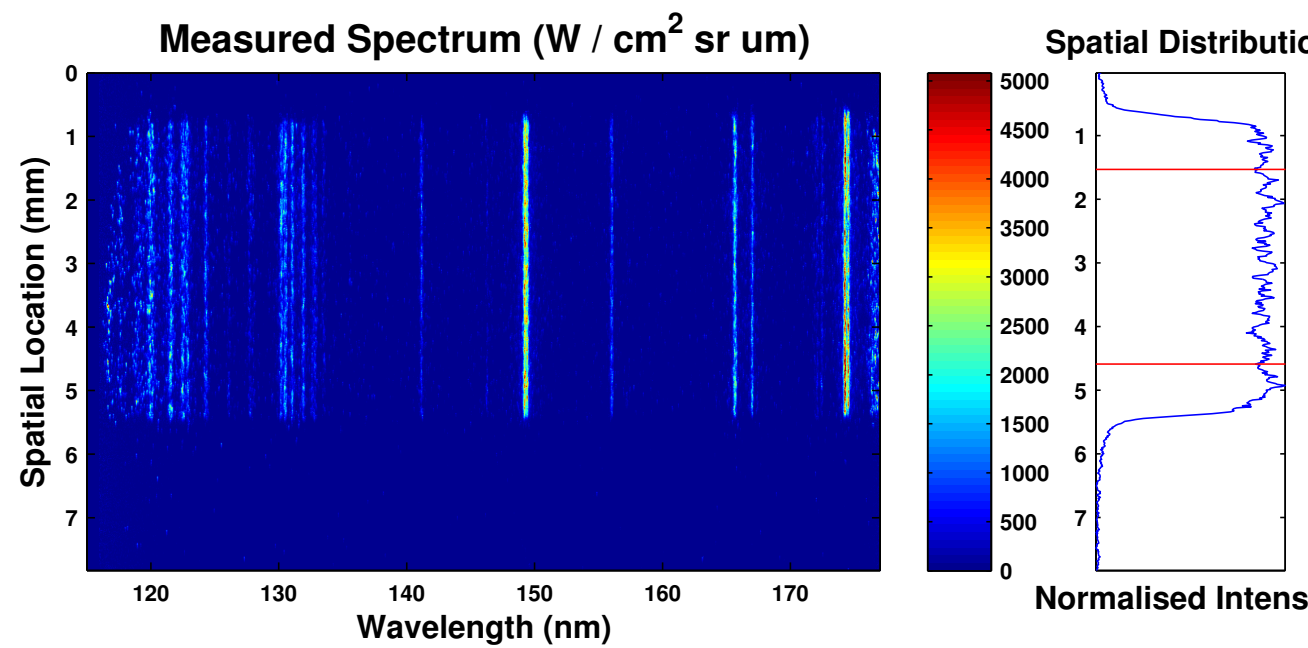

Normalised Intensity
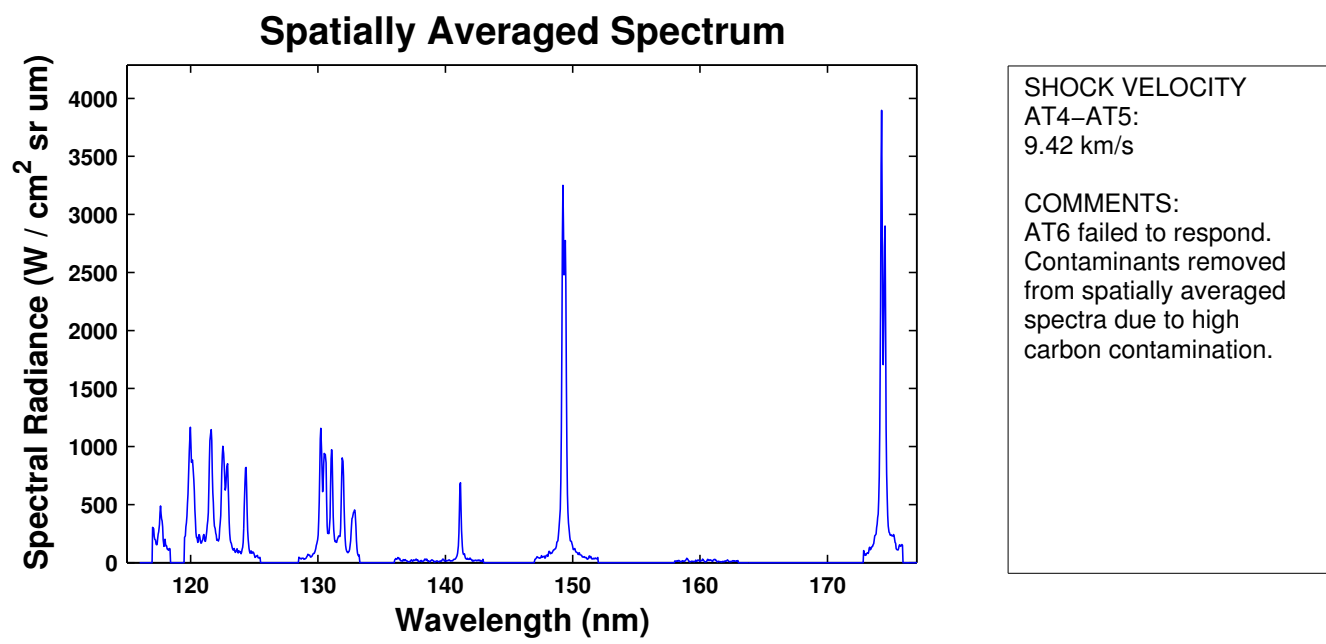

Shot Validity Analysis

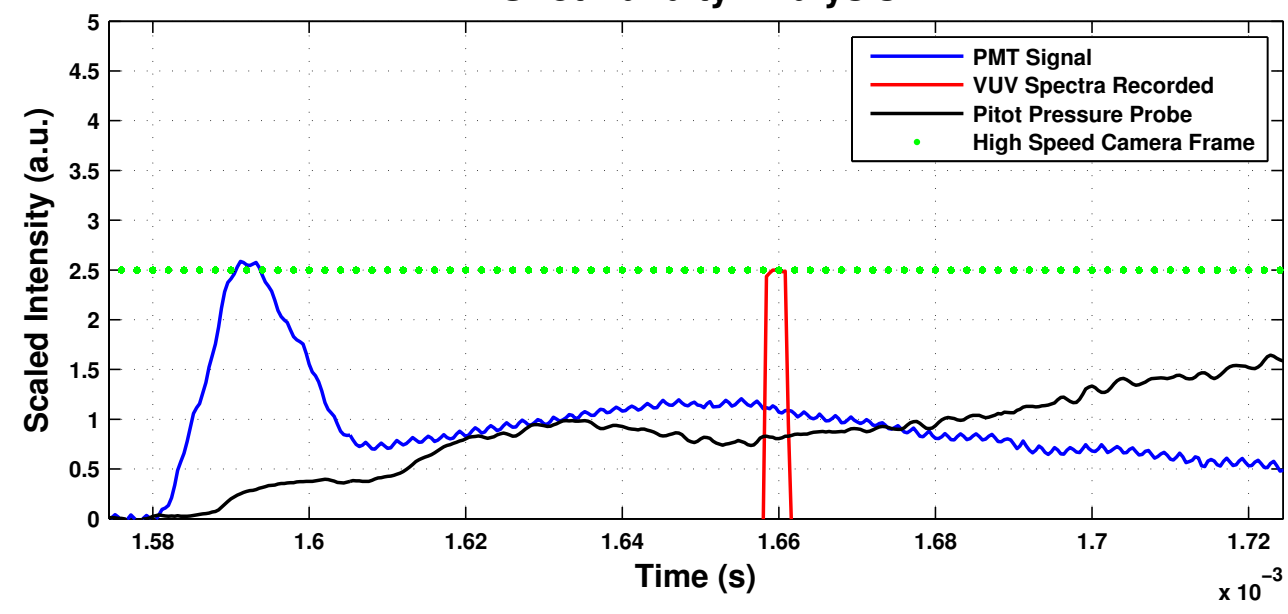

Figure D.3: x2s1954 - Primary shot data for through surface at condition 1. 

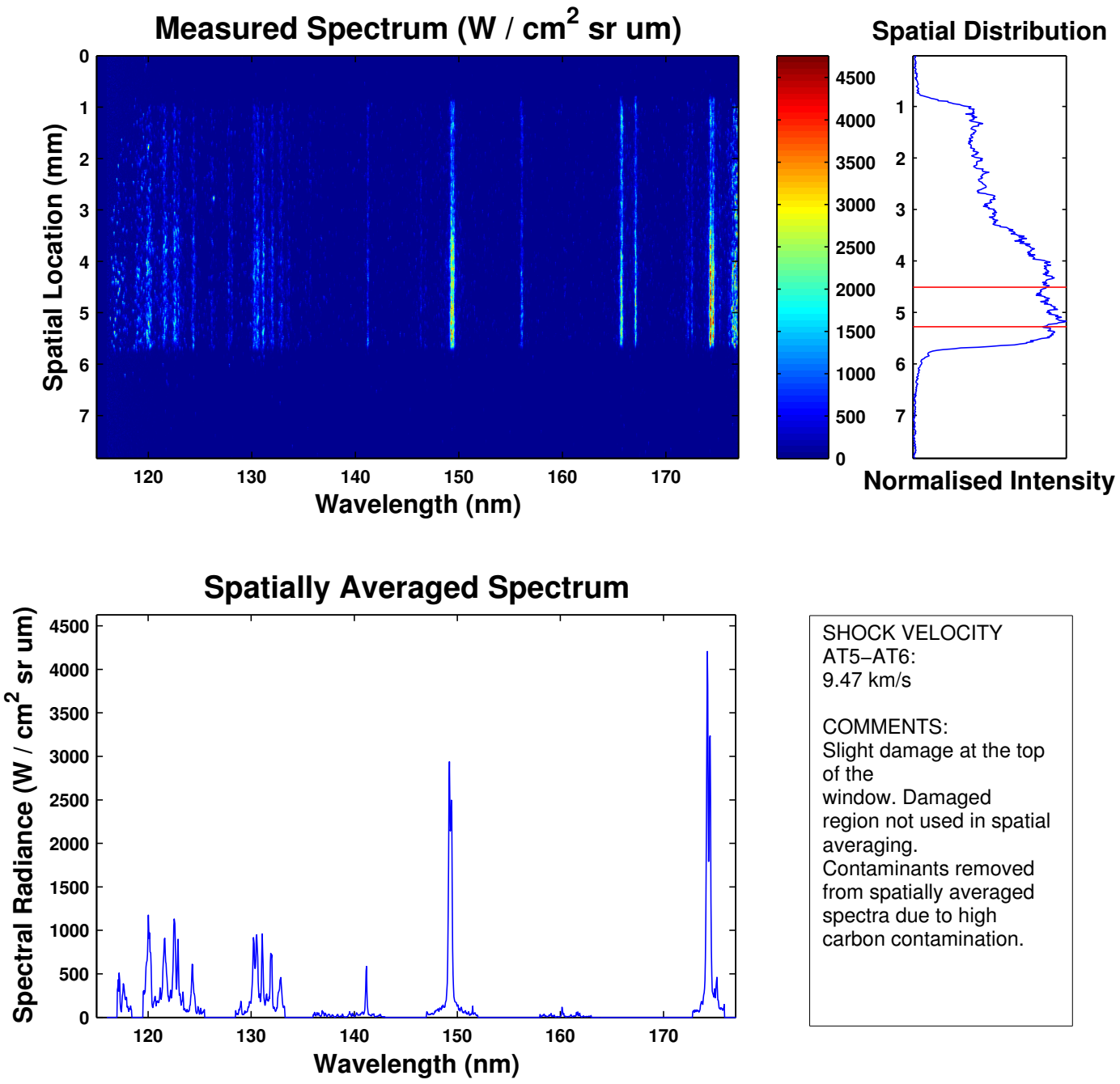

SHOCK VELOCITY AT5-AT6:

$9.47 \mathrm{~km} / \mathrm{s}$

COMMENTS:

Slight damage at the top of the

window. Damaged

region not used in spatial

averaging.

Contaminants removed

from spatially averaged

spectra due to high

carbon contamination.

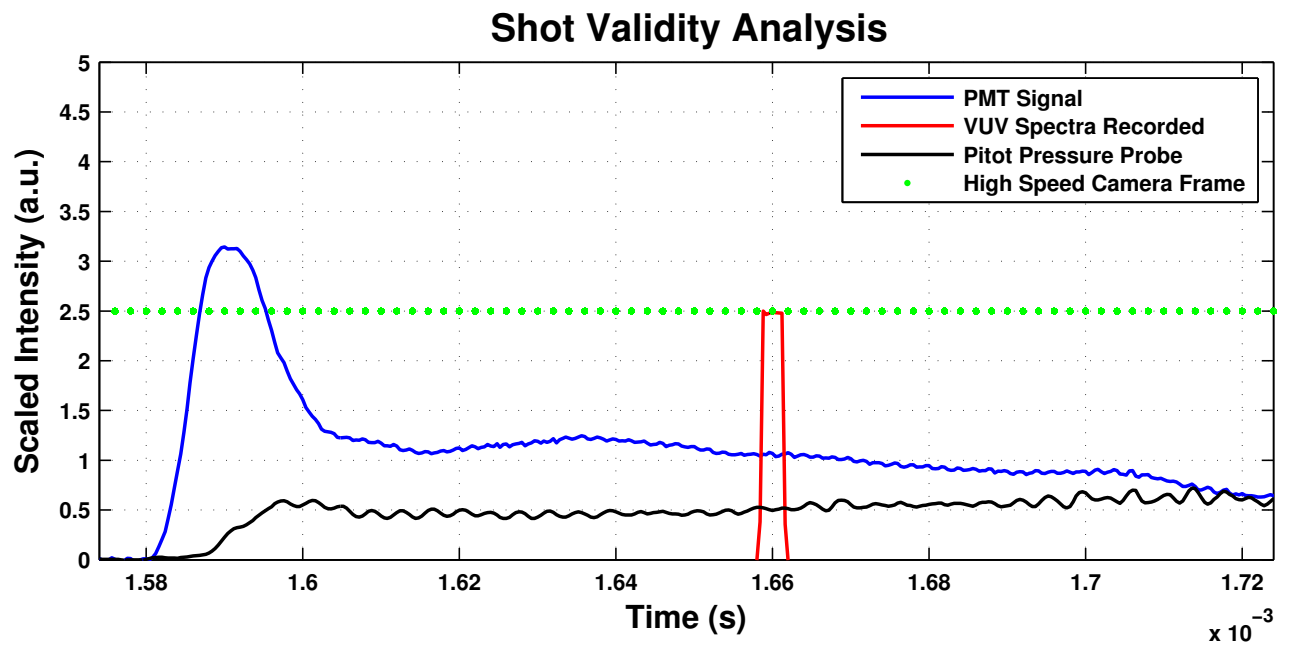

Figure D.4: x2s1953 - Repeat shot data for through surface at condition 1. 

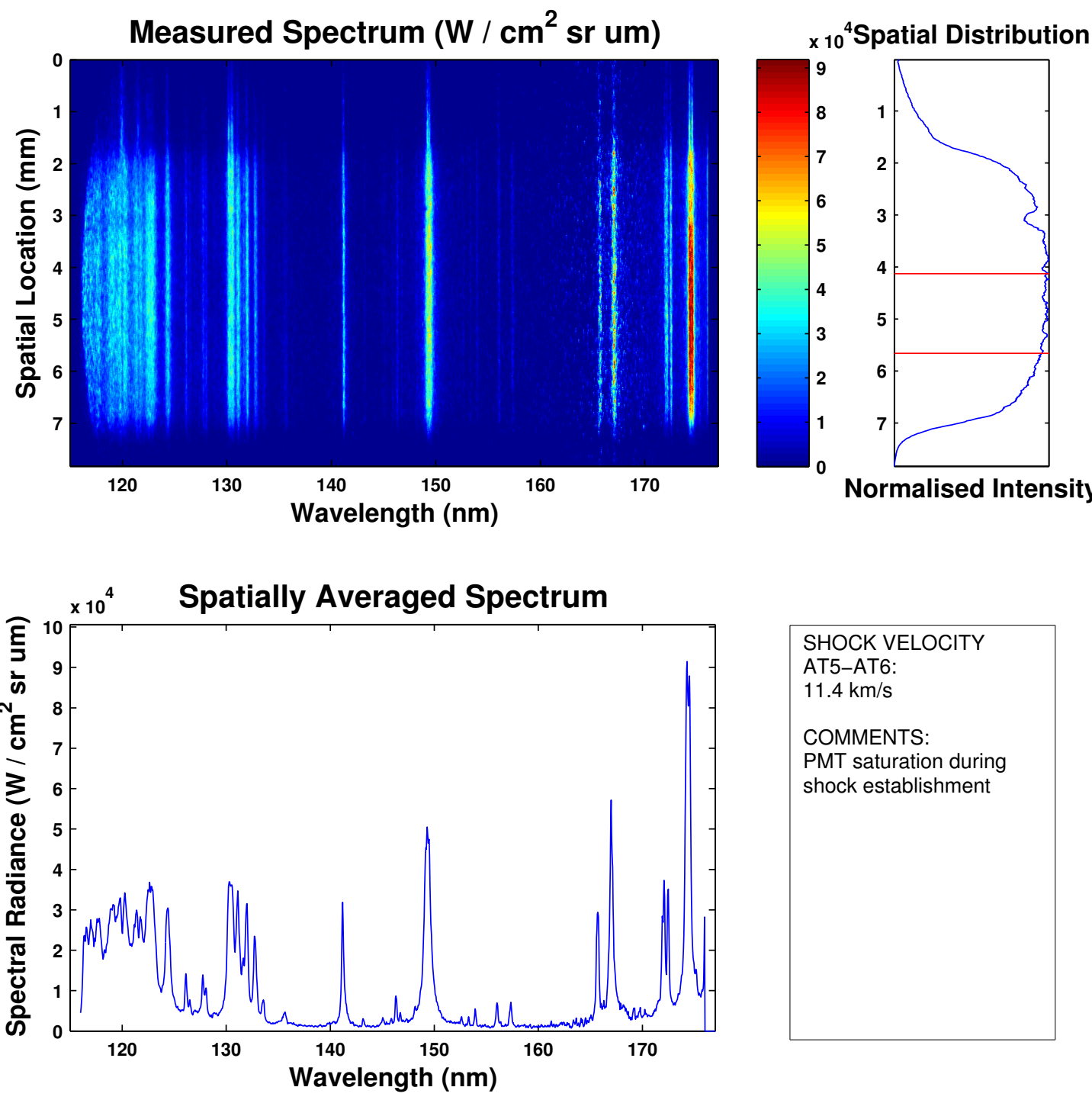

Shot Validity Analysis

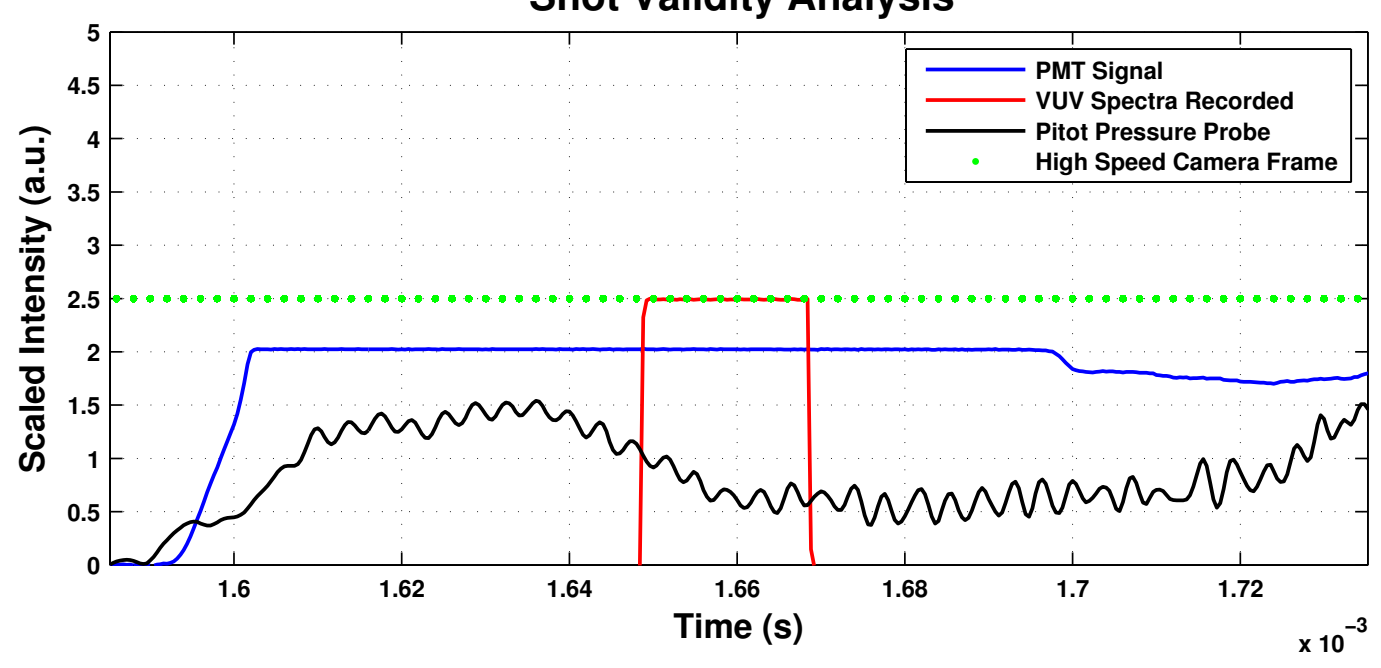

Figure D.5: x2s1940 - Primary shot data for across the surface at condition 2. 

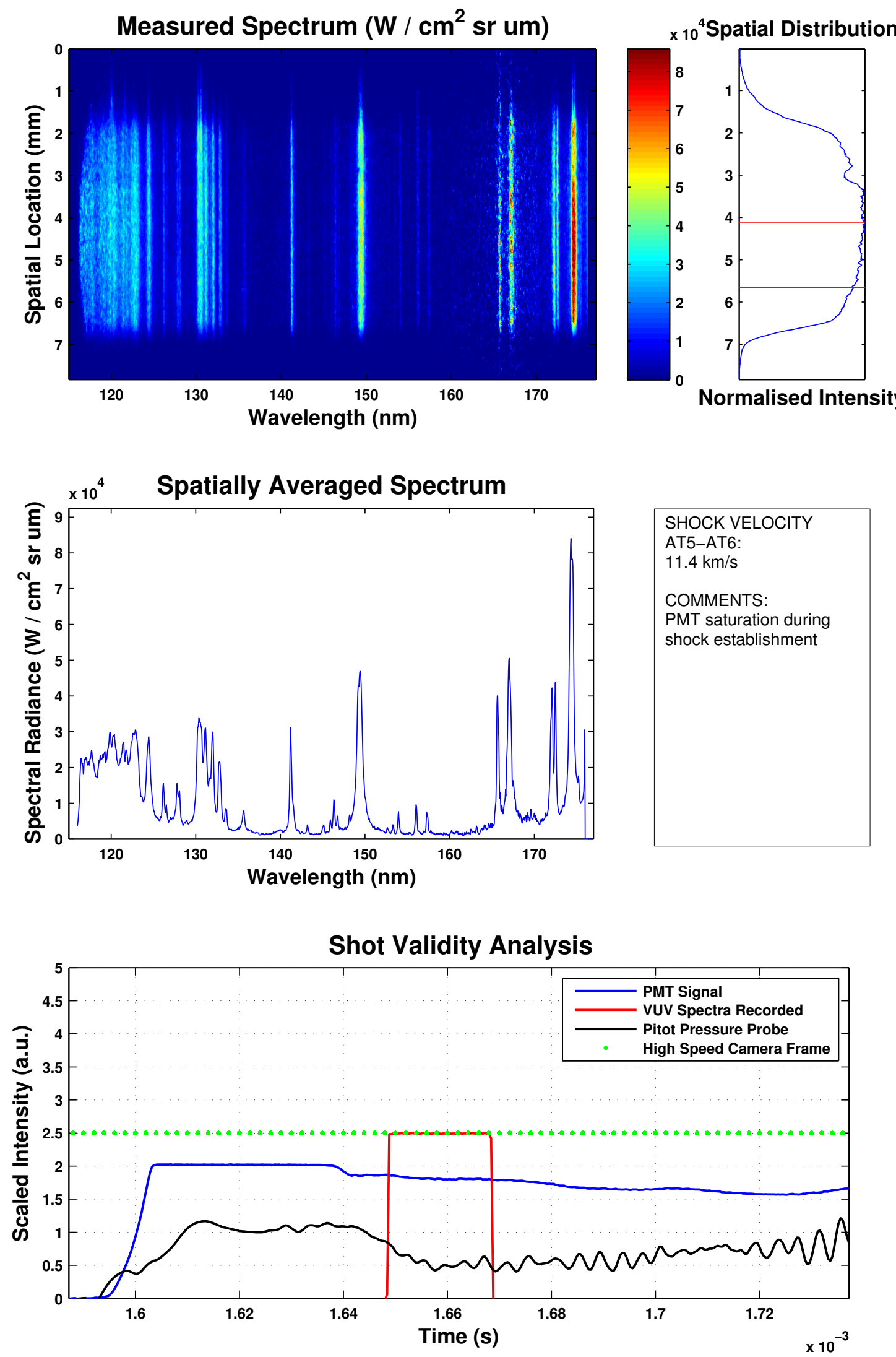

Figure D.6: x2s1939 - Repeat shot data for across the surface at condition 2. 

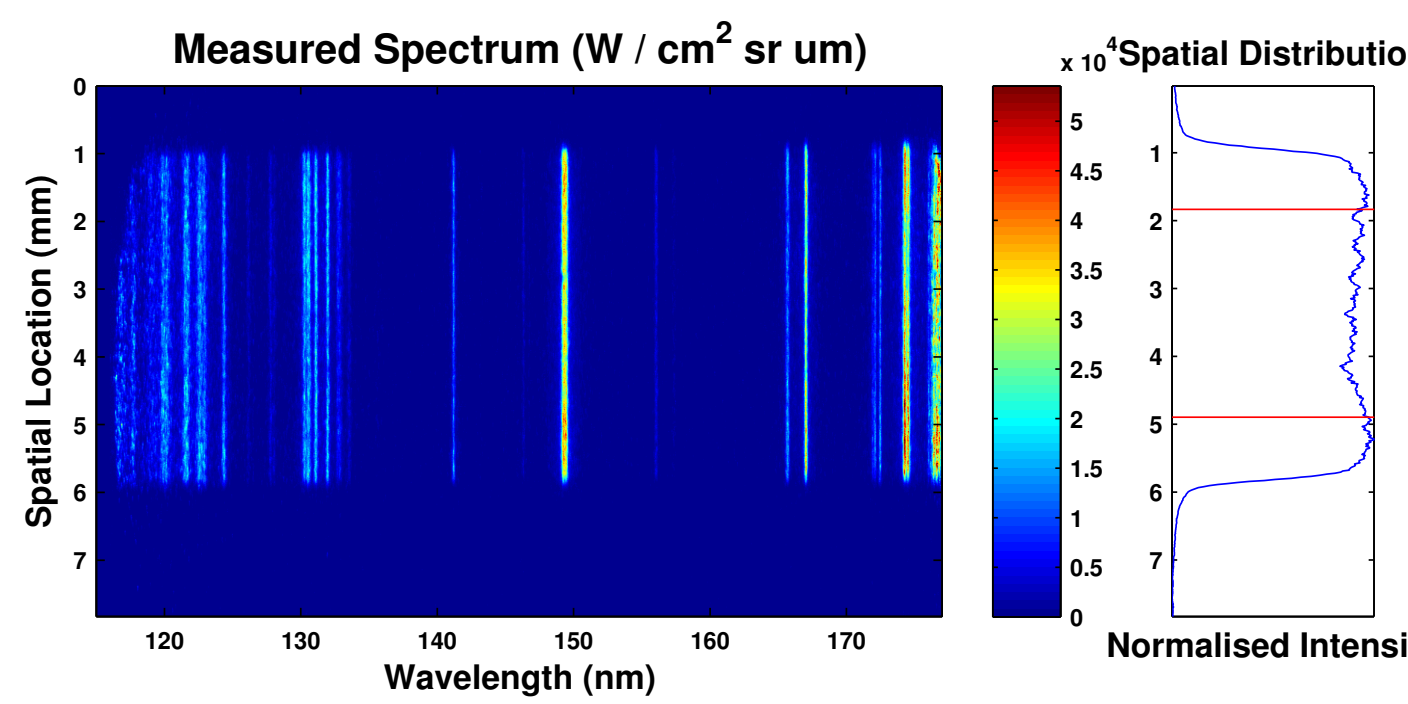

Normalised Intensity

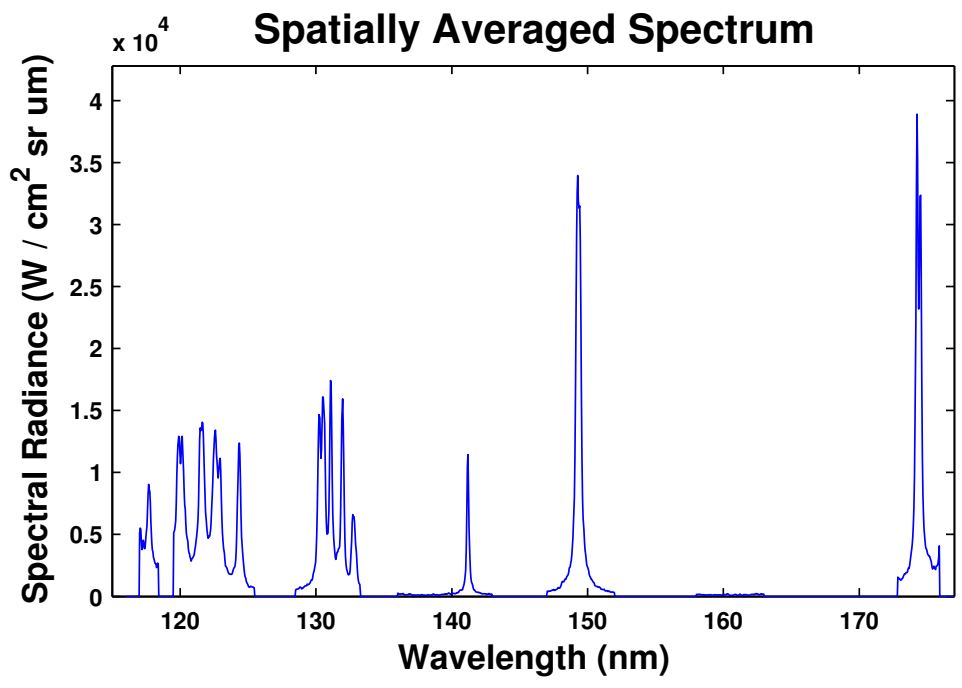

SHOCK VELOCITY

AT5-AT6:

$11.2 \mathrm{~km} / \mathrm{s}$

\section{COMMENTS:}

Contaminants removed from spatially averaged

spectra due to high

carbon contamination.

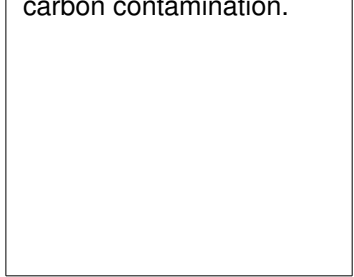

\section{Shot Validity Analysis}

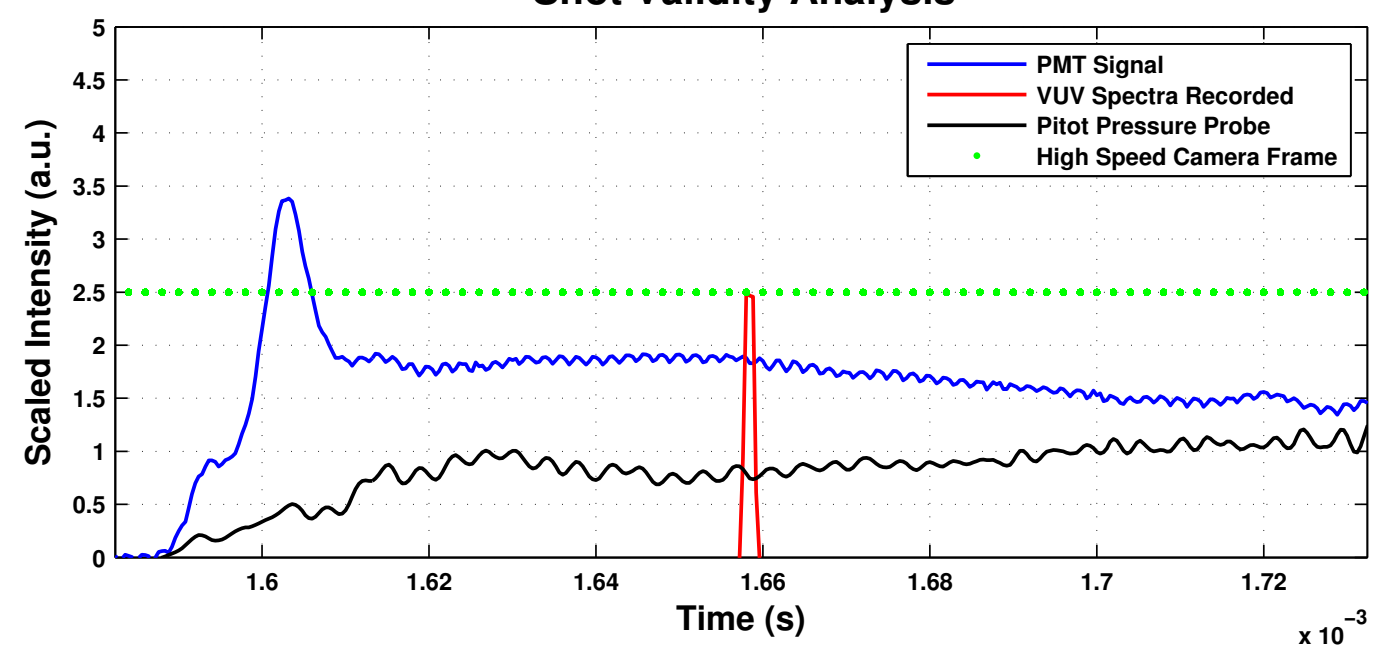

Figure D.7: x2s1957 - Primary shot data for through surface at condition 2. 

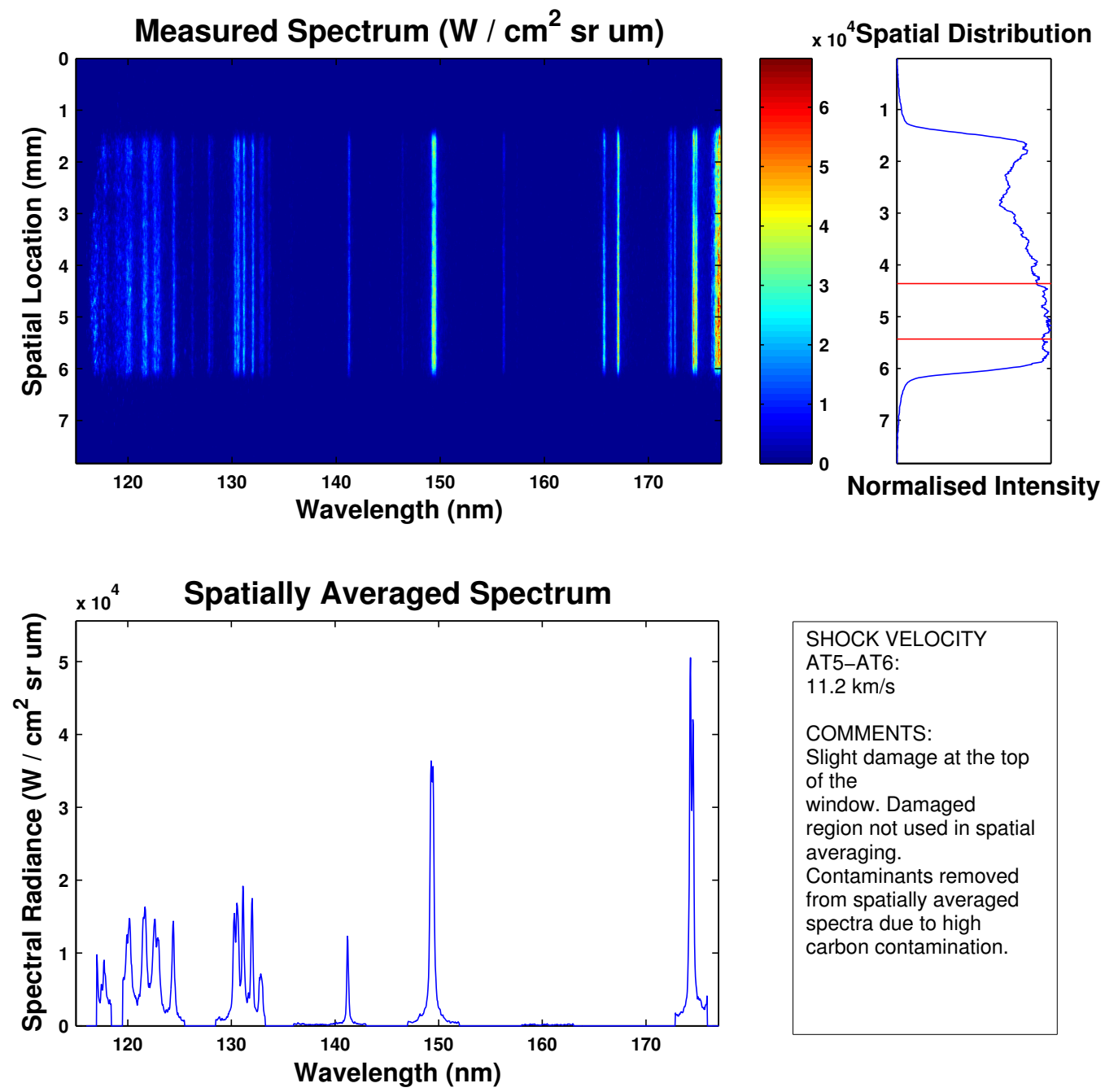

Shot Validity Analysis

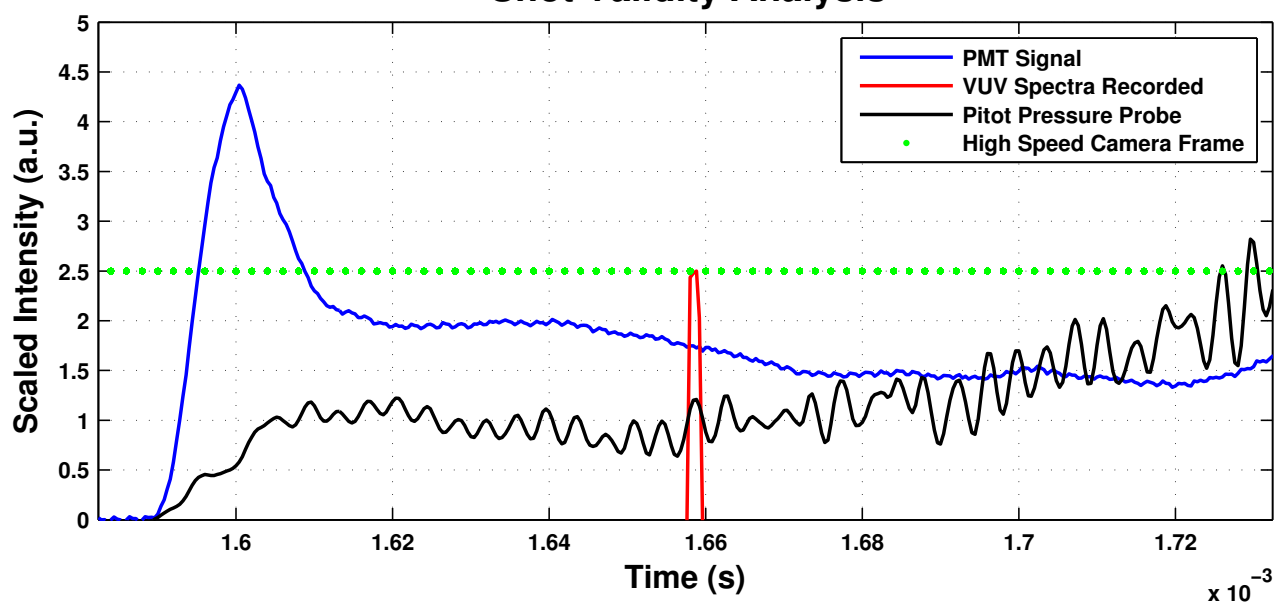

Figure D.8: x2s1958 - Repeat shot data for through surface at condition 2. 


\section{D.2 Self-Absorption Experiments}

Table D.2: Shots conducted for self-absorption experiments with a $19 \mathrm{~mm}$ radius of curvature cylinder.

\begin{tabular}{lll}
\hline Condition $\mathbf{1}$ & & \\
\hline Model length & Primary shot & Repeat shot \\
\hline $90 \mathrm{~mm}$ & $\mathrm{x} 2 \mathrm{~s} 1926$ & $\mathrm{x} 2 \mathrm{~s} 1927$ \\
$45 \mathrm{~mm}$ & $\mathrm{x} 2 \mathrm{~s} 1932$ & $\mathrm{x} 2 \mathrm{~s} 1933$ \\
$20 \mathrm{~mm}$ & $\mathrm{x} 2 \mathrm{~s} 1946$ & \\
\hline Condition $\mathbf{2}$ & & \\
\hline Model length & Primary shot & Repeat shot \\
\hline $90 \mathrm{~mm}$ & x2s1931 & x2s1930 \\
$45 \mathrm{~mm}$ & $\mathrm{x} 2 \mathrm{~s} 1942$ & Nil \\
$20 \mathrm{~mm}$ & $\mathrm{x} 2 \mathrm{~s} 1943$ & x2s1944 \\
\hline
\end{tabular}



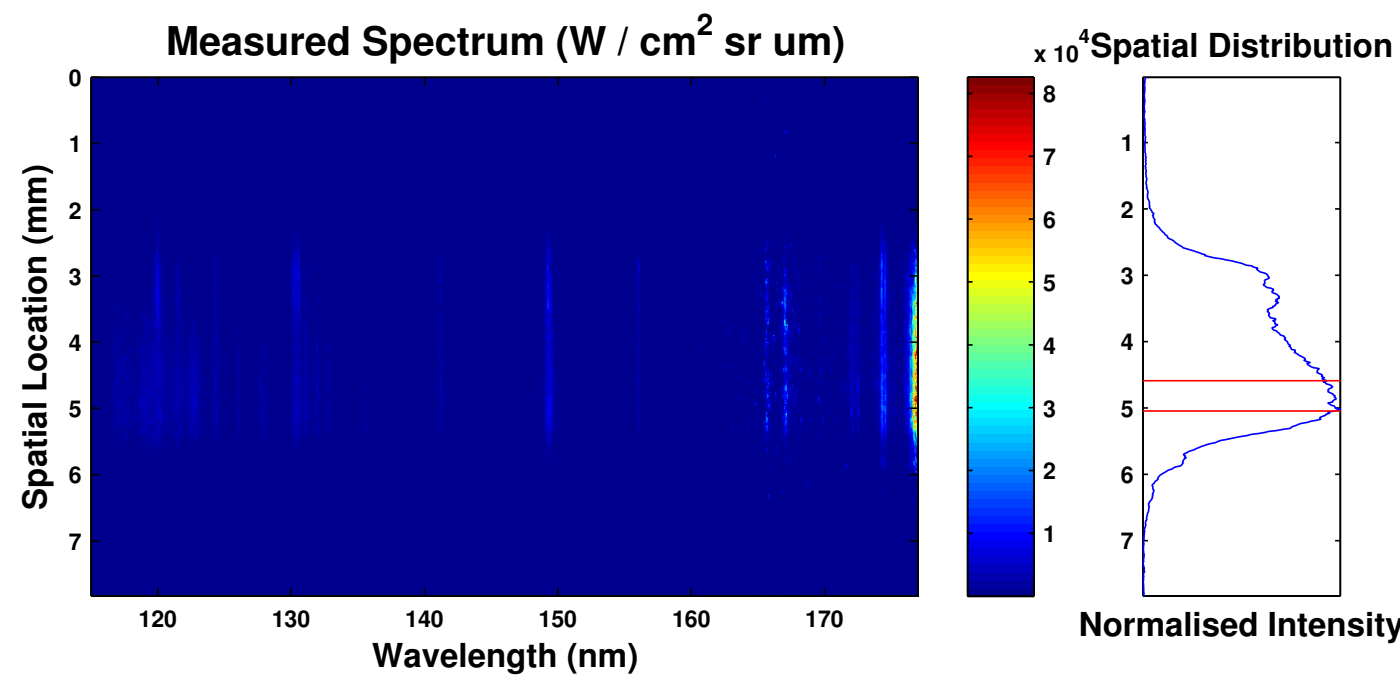

Normalised Intensity
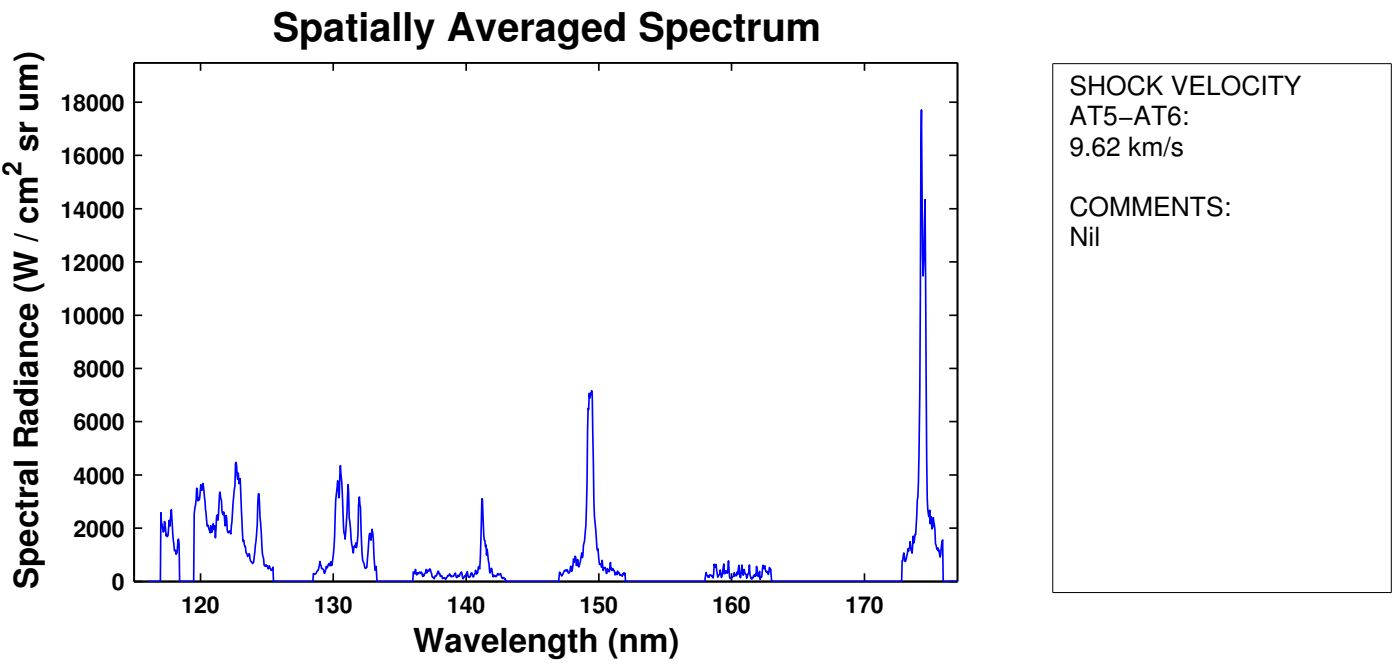

Shot Validity Analysis

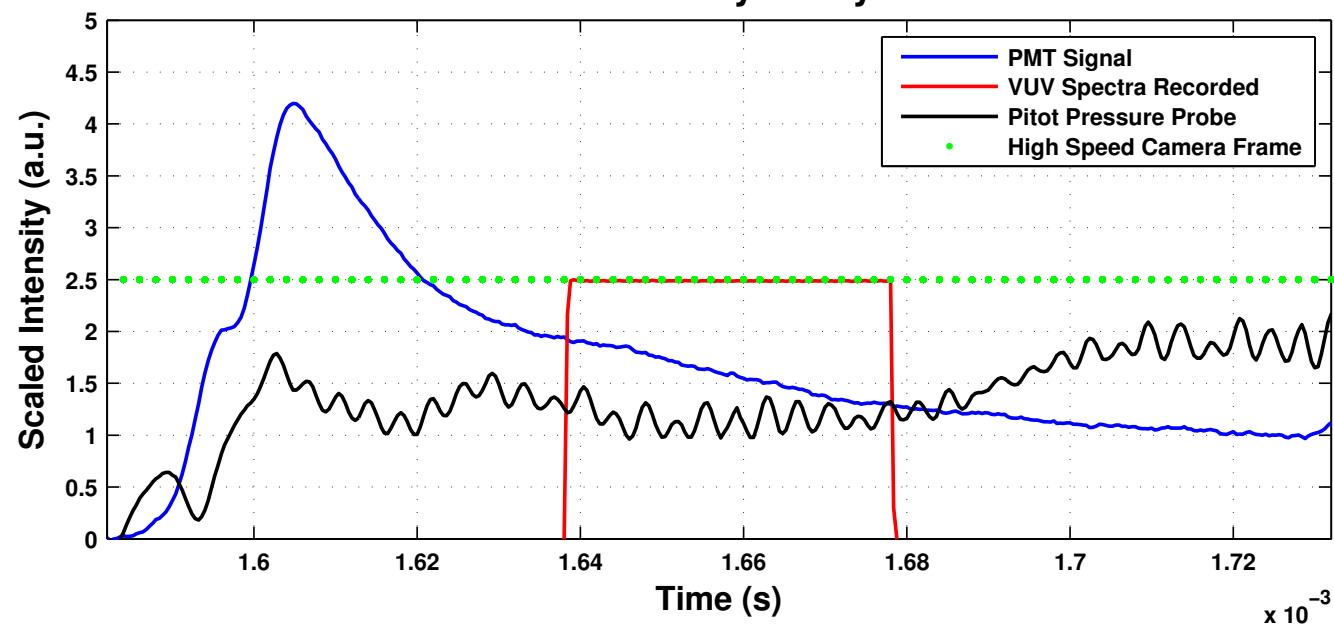

Figure D.9: x2s1926 - Primary shot data for across the surface of a $90 \mathrm{~mm}$ model at condition 1. 

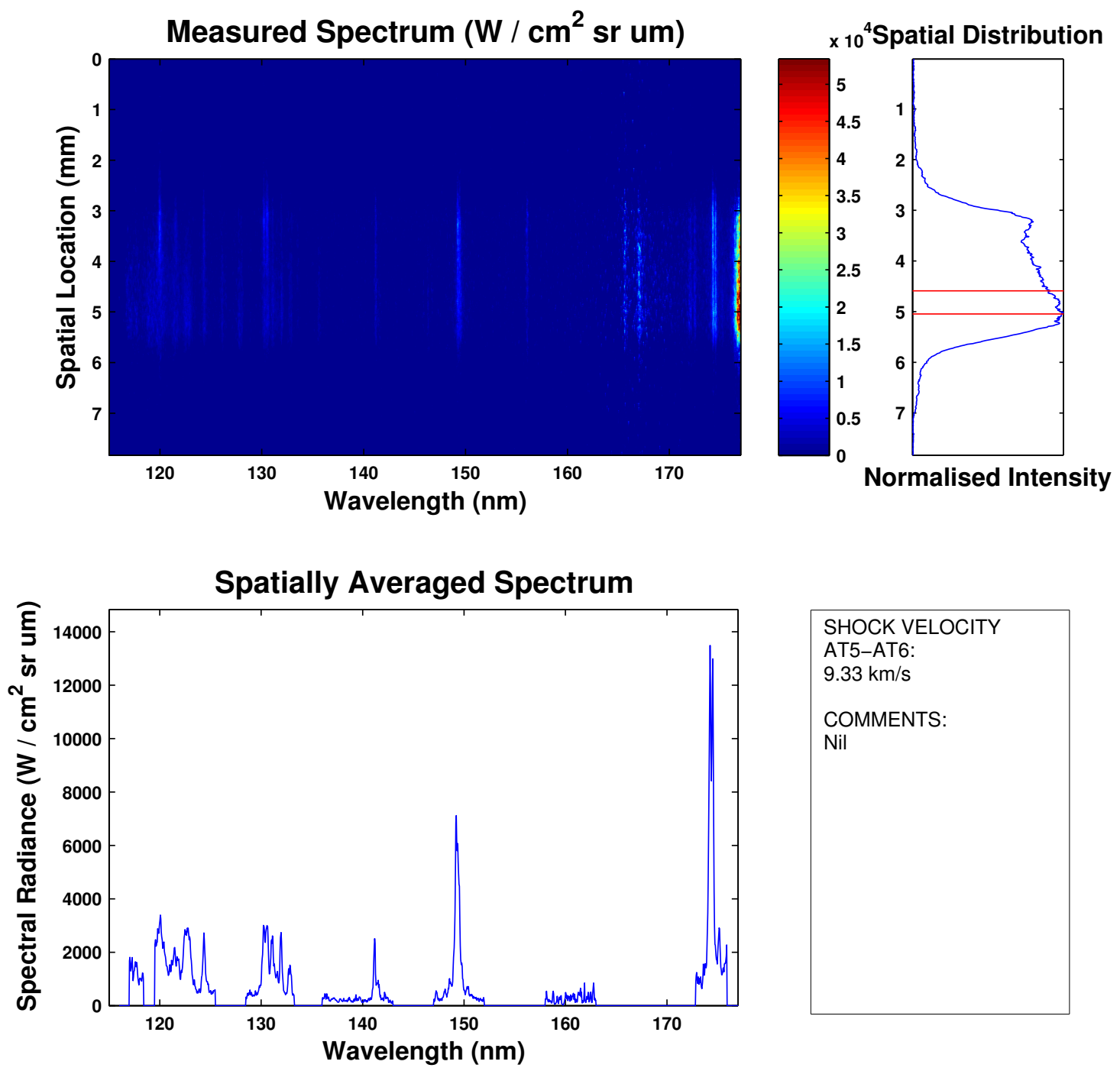

\section{Shot Validity Analysis}

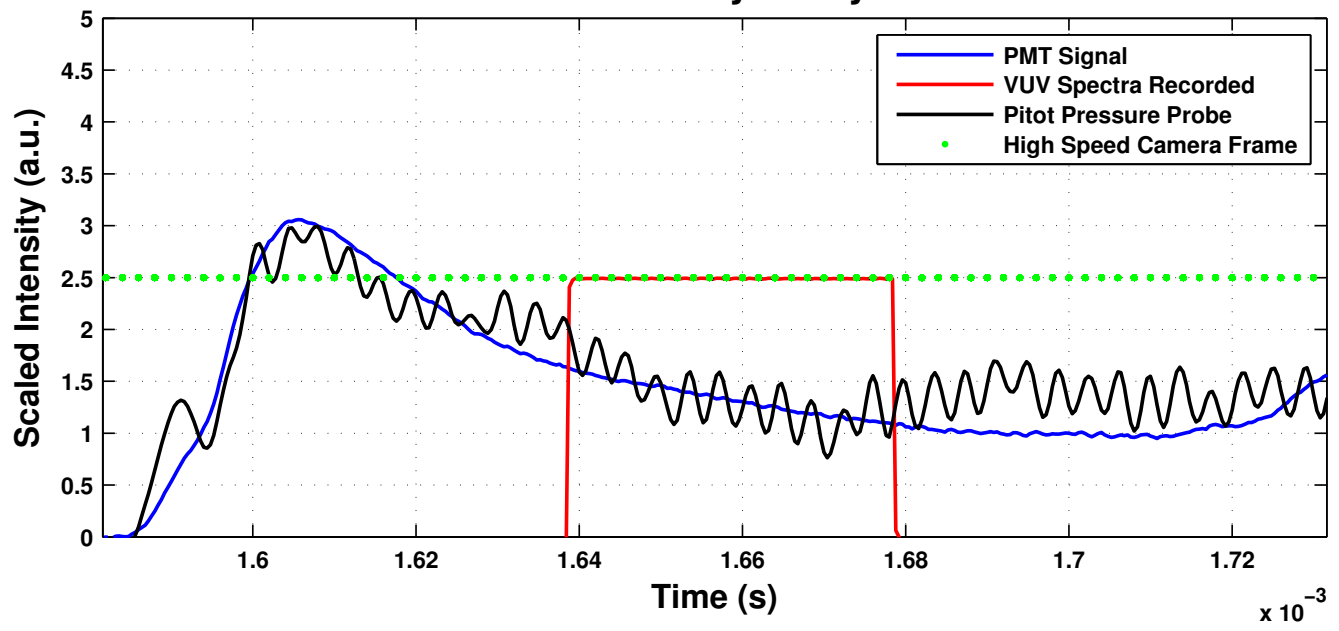

Figure D.10: x2s1927 - Repeat shot data for across the surface of a $90 \mathrm{~mm}$ model at condition 1. 

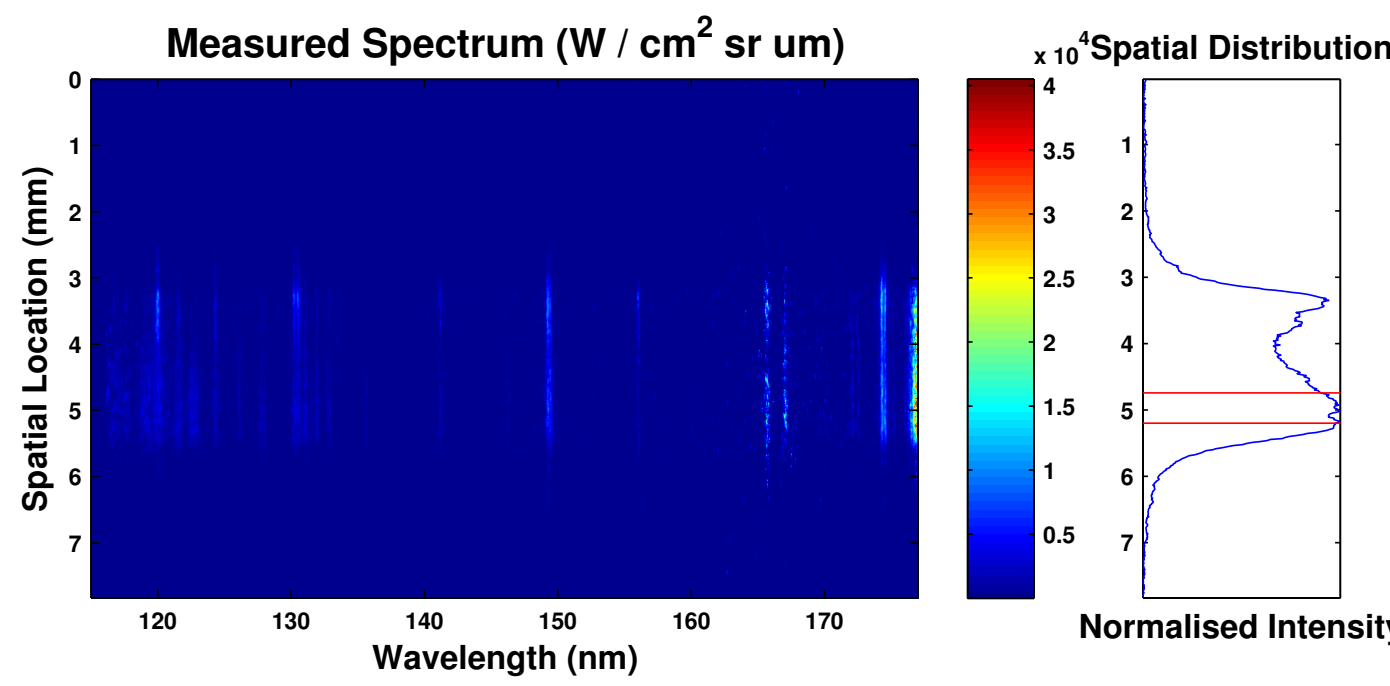

Normalised Intensity
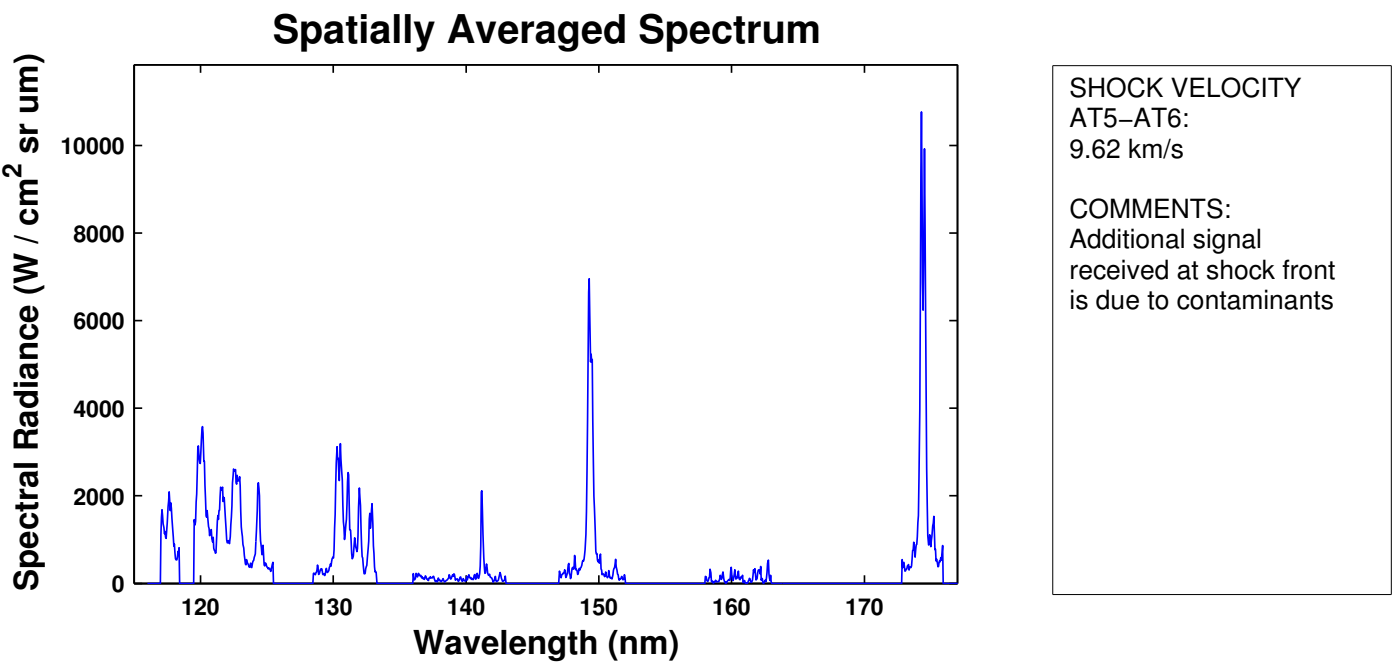

Shot Validity Analysis

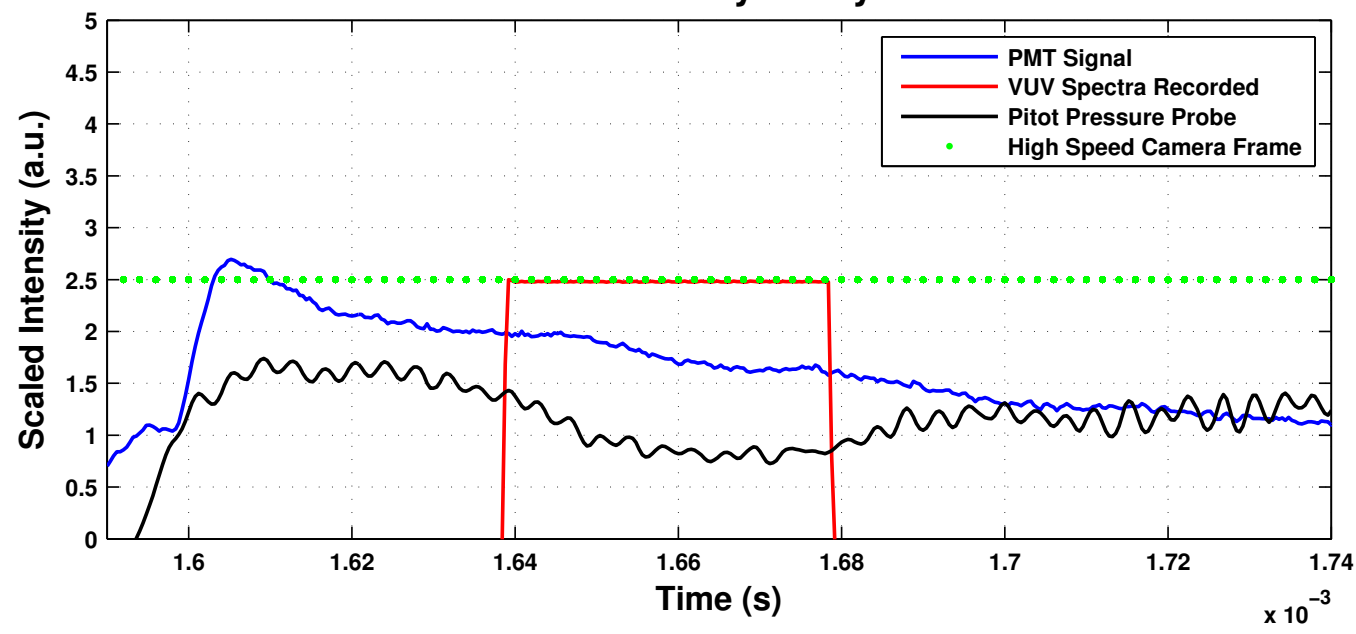

Figure D.11: x2s1932 - Primary shot data for across the surface of a $45 \mathrm{~mm}$ model at condition 1. 

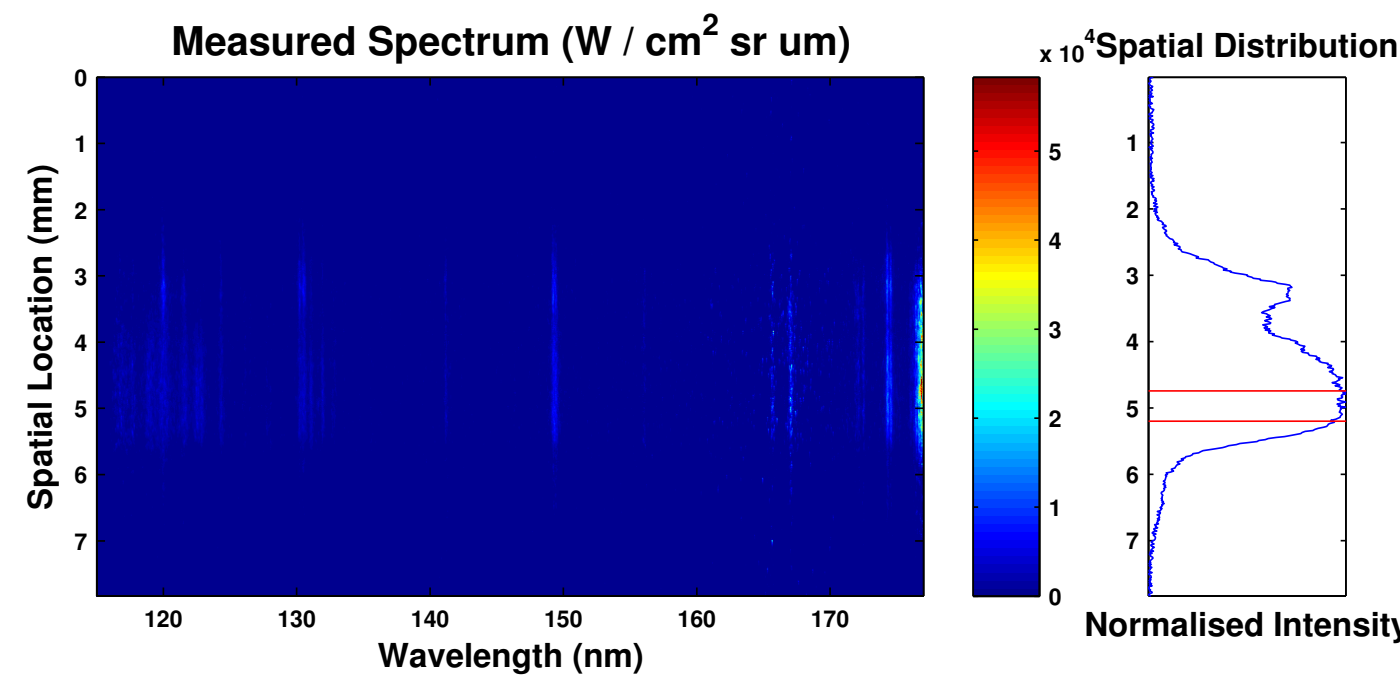

Normalised Intensity
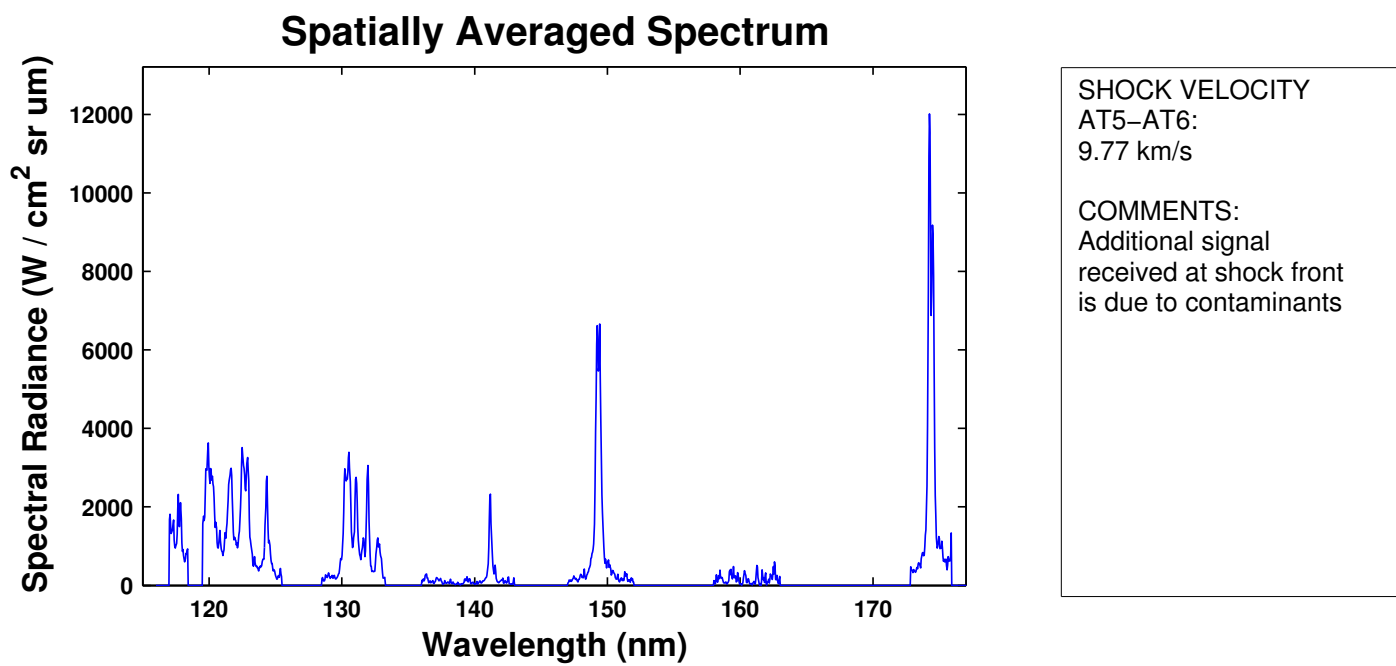

\section{Shot Validity Analysis}

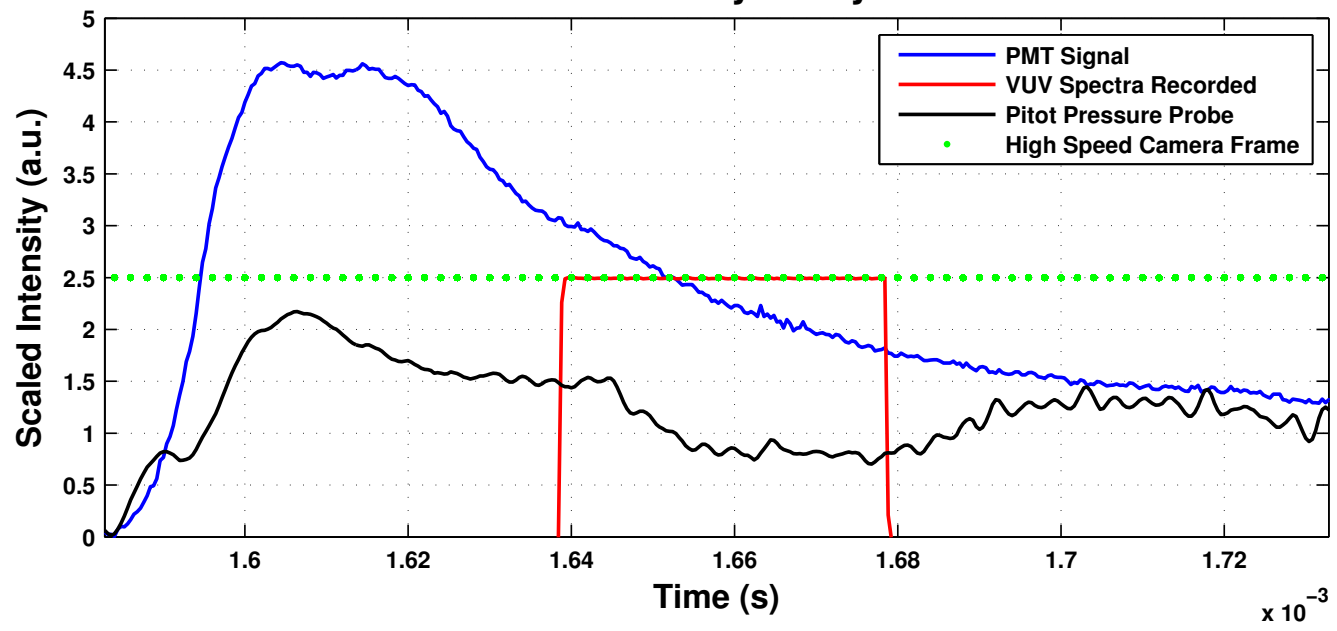

Figure D.12: x2s1933 - Repeat shot data for across the surface of a $45 \mathrm{~mm}$ model at condition 1. 

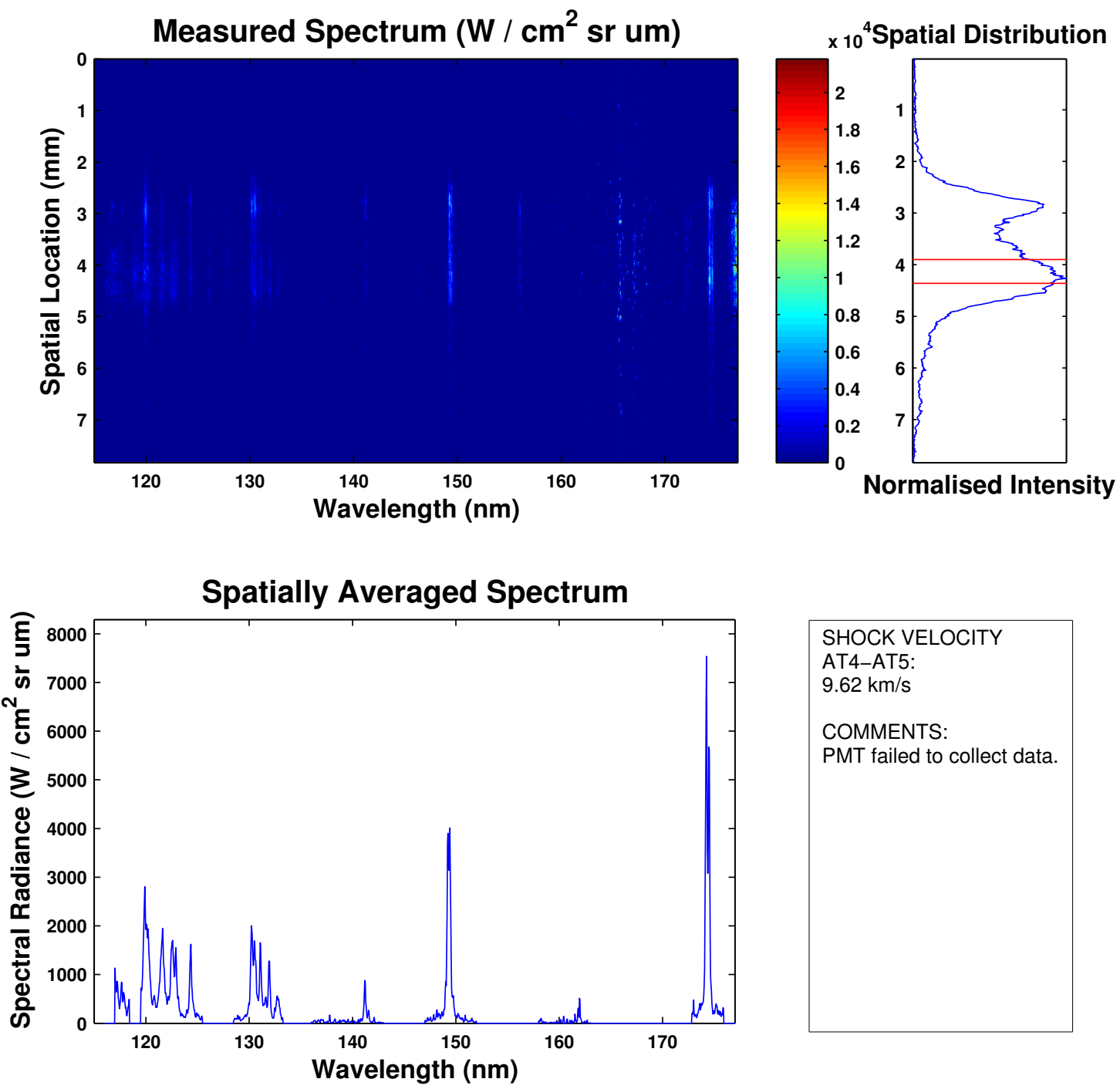

SHOCK VELOCITY

AT4-AT5:

$9.62 \mathrm{~km} / \mathrm{s}$

COMMENTS:

PMT failed to collect data.

Shot Validity Analysis

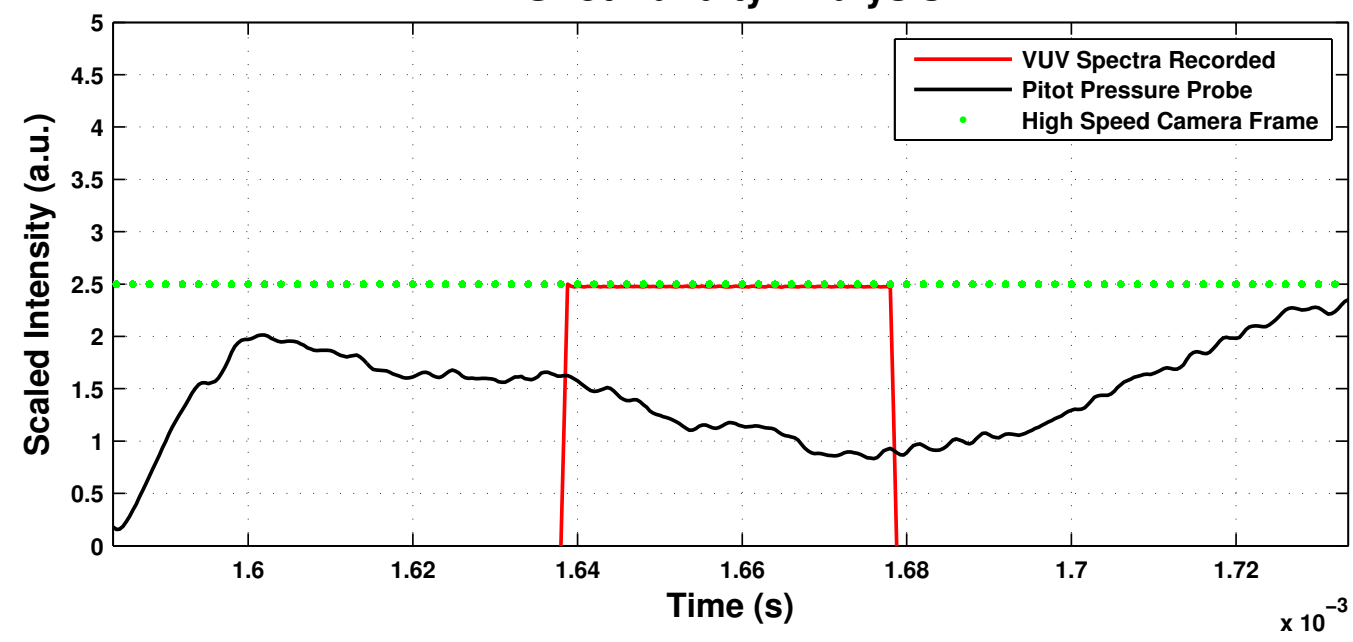

Figure D.13: x2s1946 - Primary shot data for across the surface of a $20 \mathrm{~mm}$ model at condition 1. 

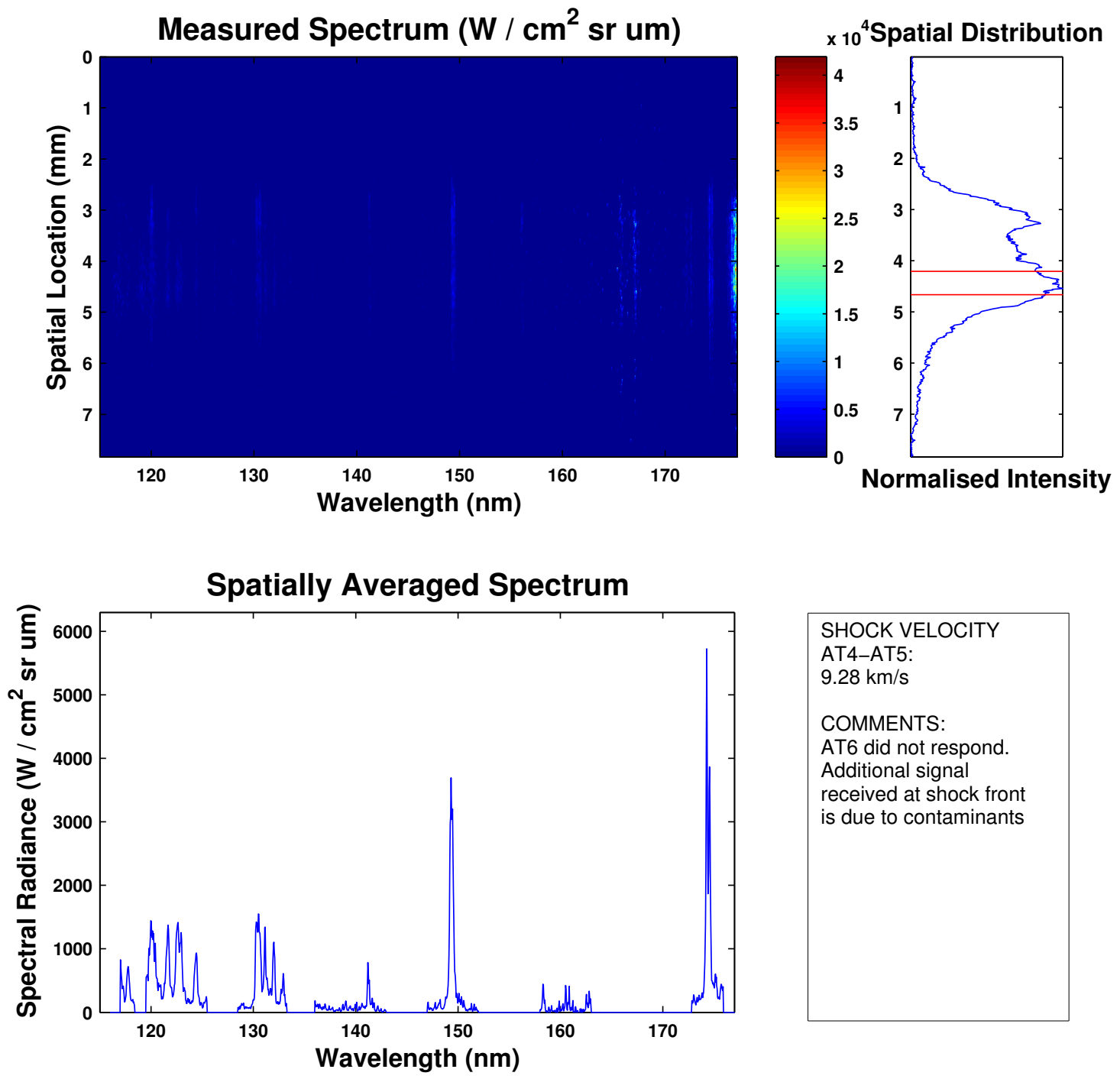

Shot Validity Analysis

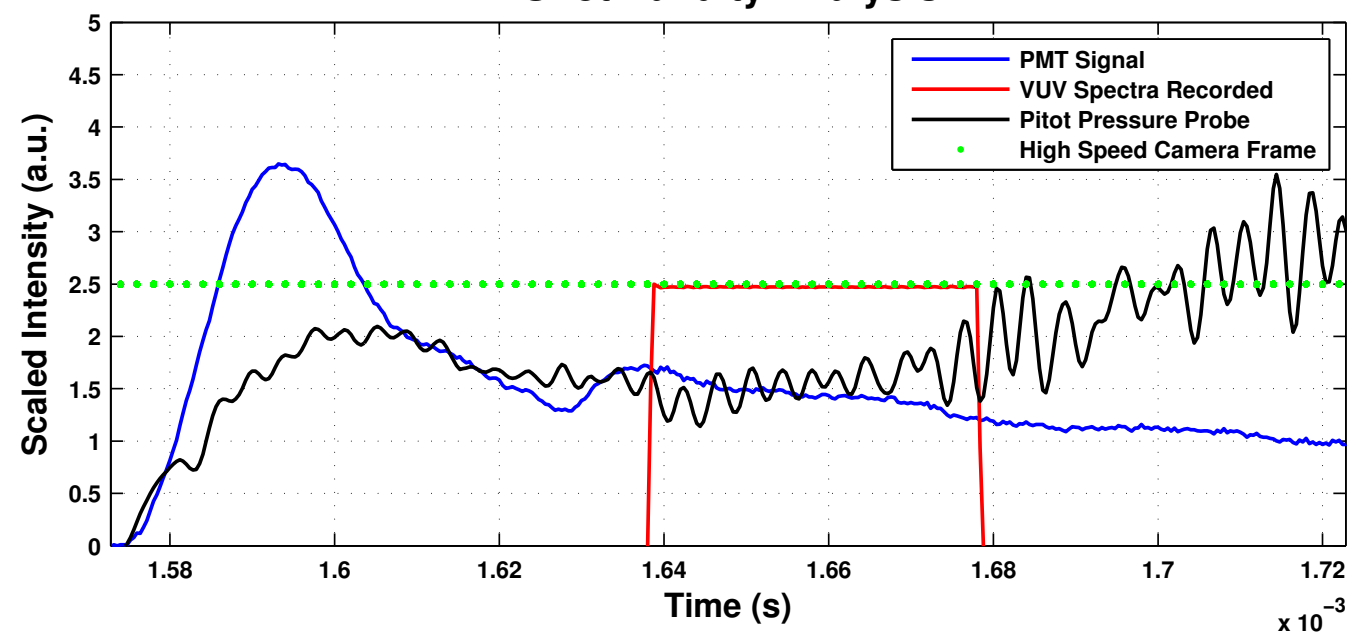

Figure D.14: x2s1945 - Repeat shot data for across the surface of a $20 \mathrm{~mm}$ model at condition 1. 

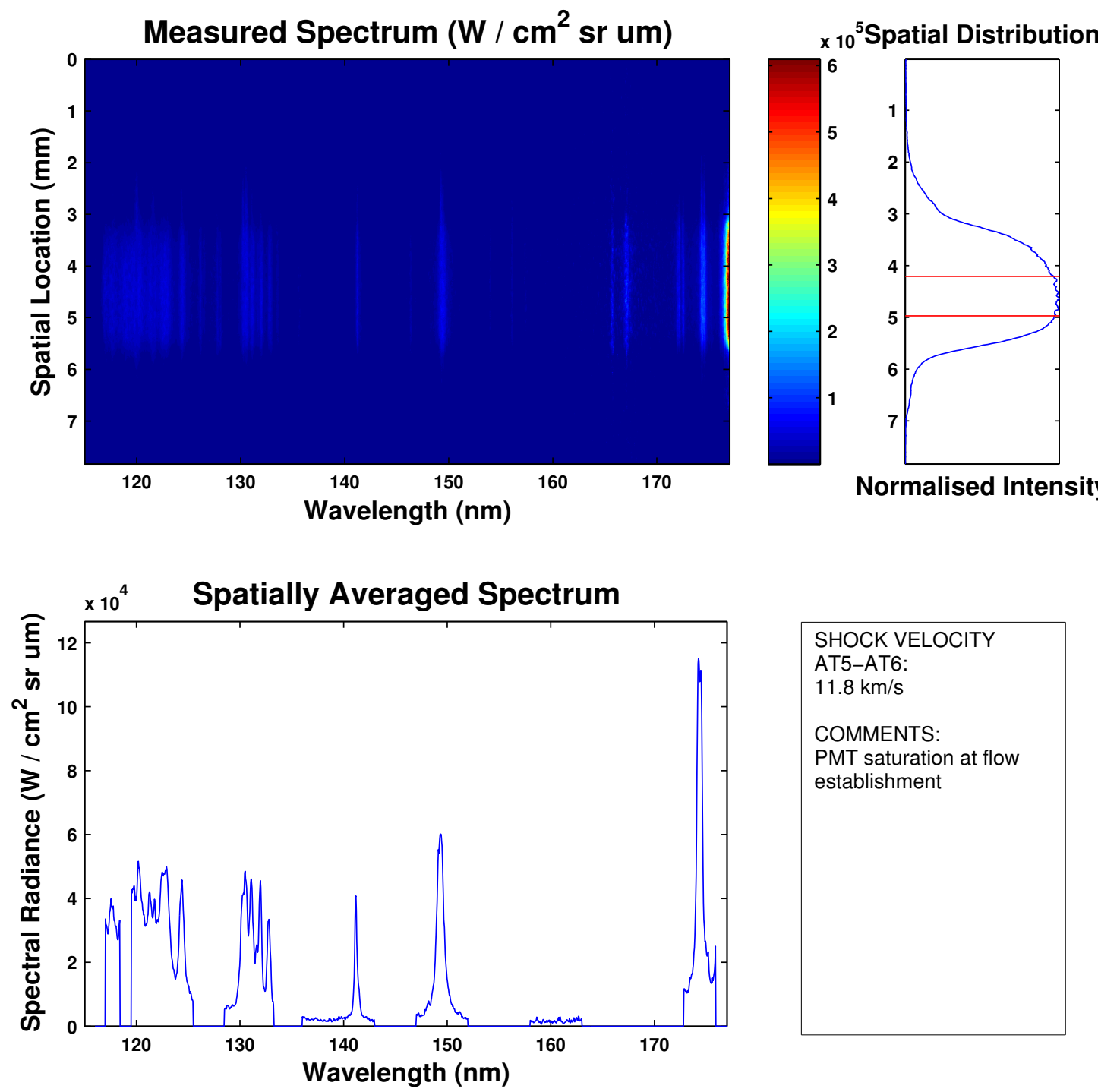

Shot Validity Analysis

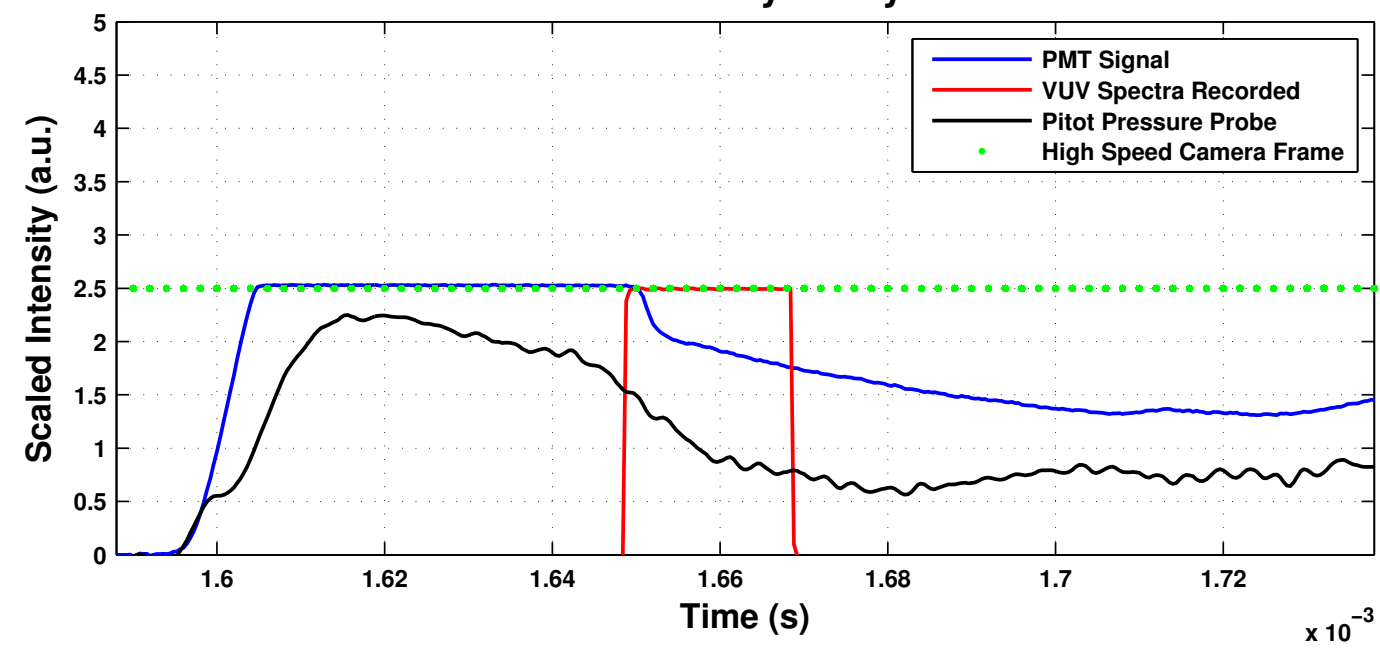

Figure D.15: x2s1931 - Primary shot data for across the surface of a $90 \mathrm{~mm}$ model at condition 2 . 

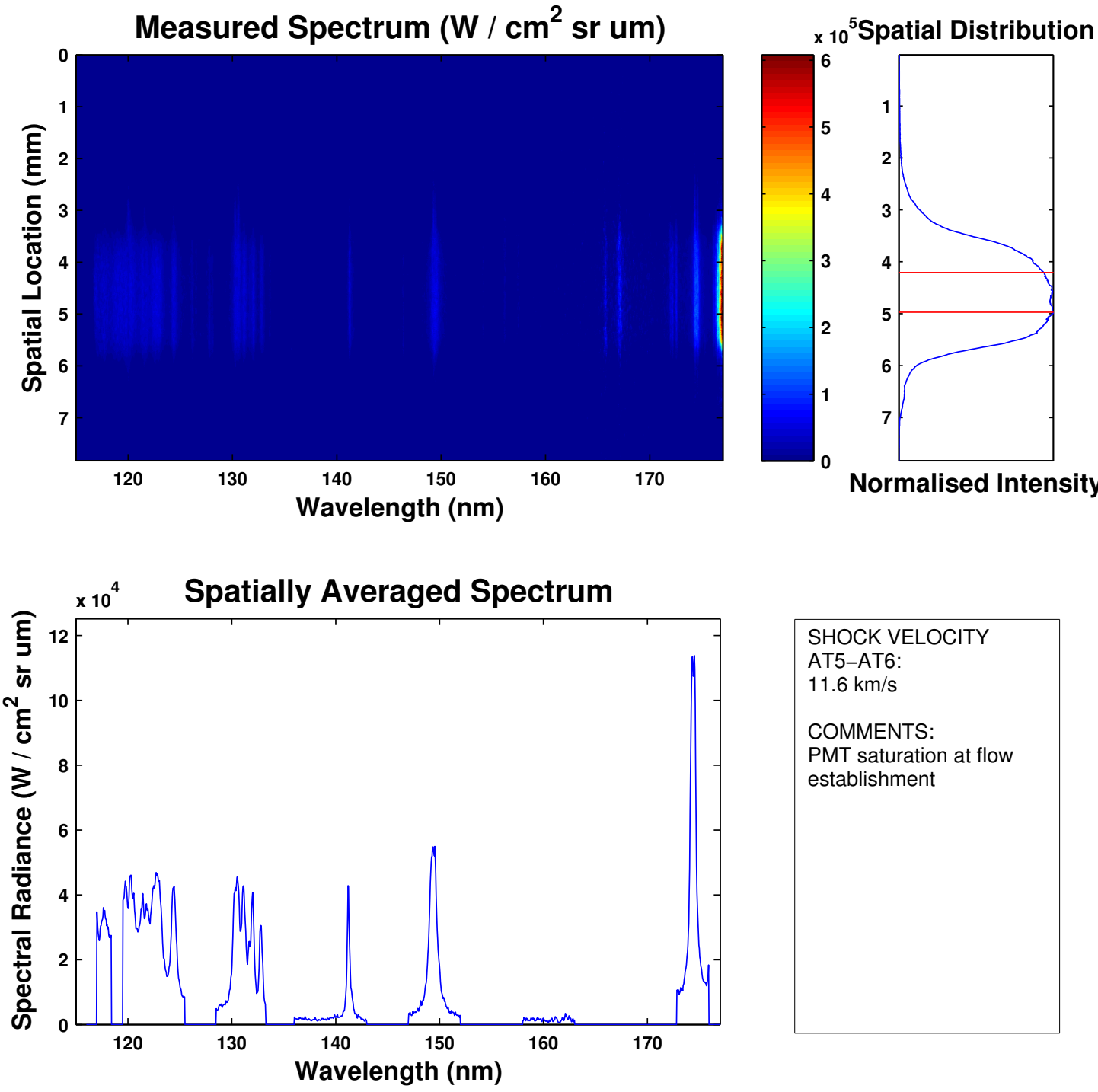

SHOCK VELOCITY

AT5-AT6:

$11.6 \mathrm{~km} / \mathrm{s}$

COMMENTS:

PMT saturation at flow

establishment

Shot Validity Analysis

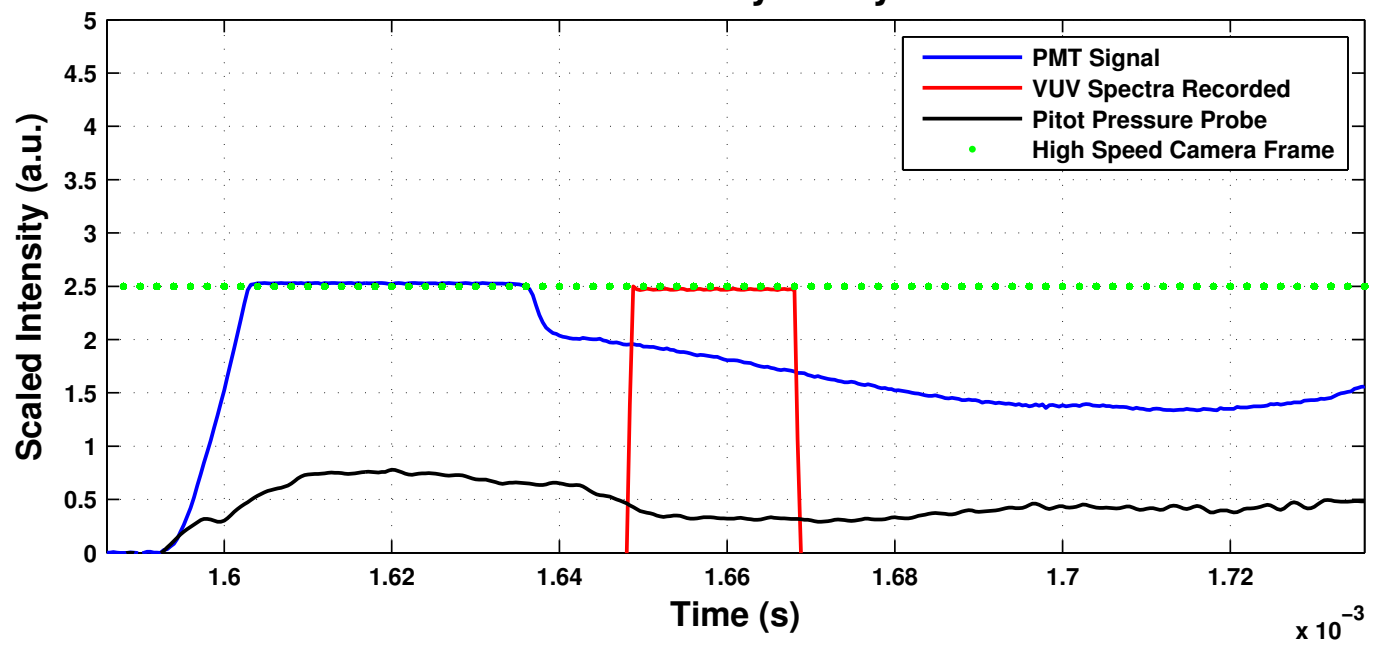

Figure D.16: x2s1930 - Repeat shot data for across the surface of a $90 \mathrm{~mm}$ model at condition 2. 

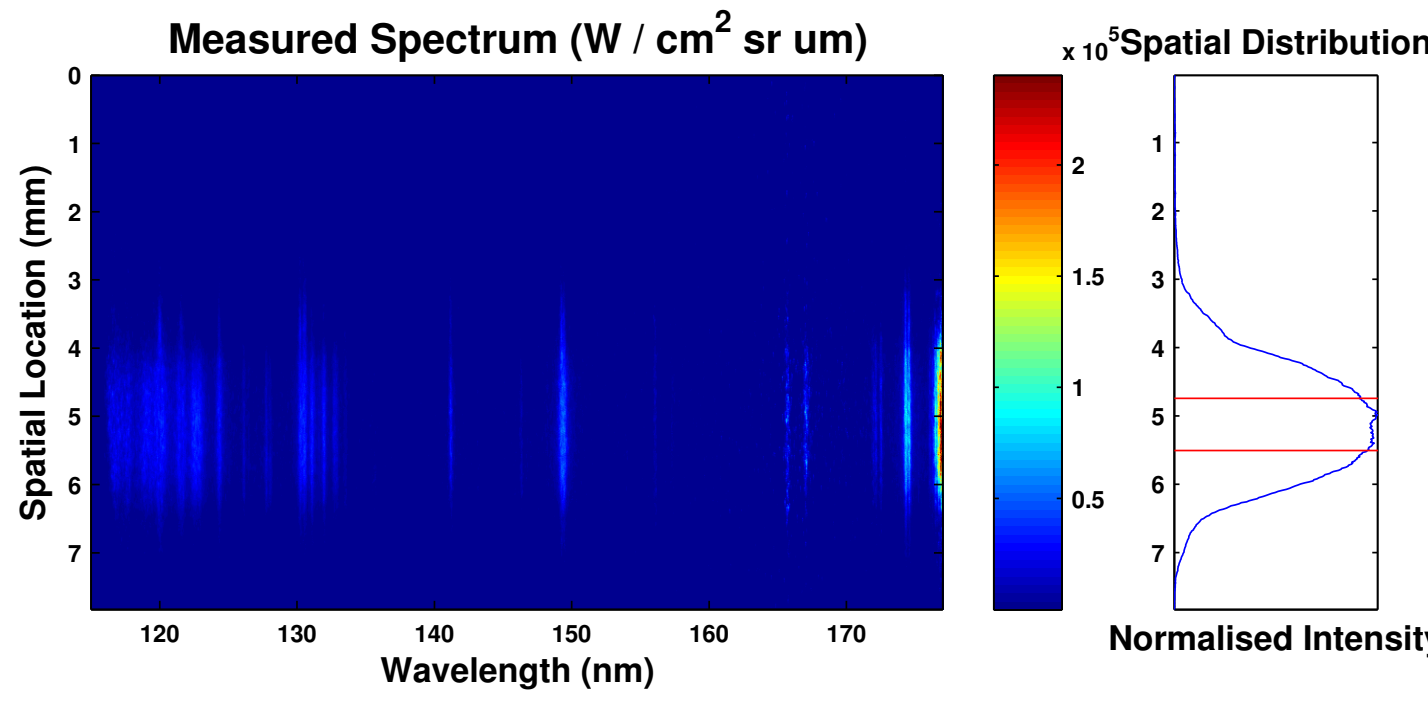

Normalised Intensity
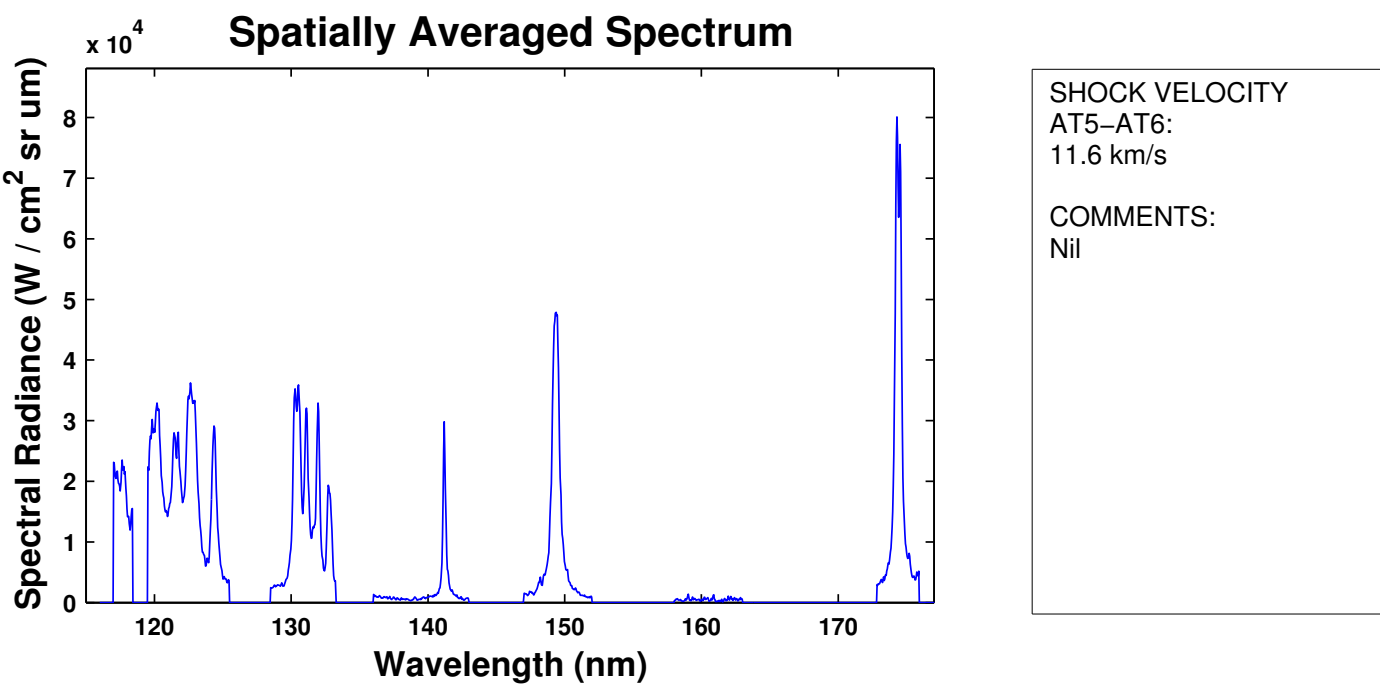

\section{Shot Validity Analysis}

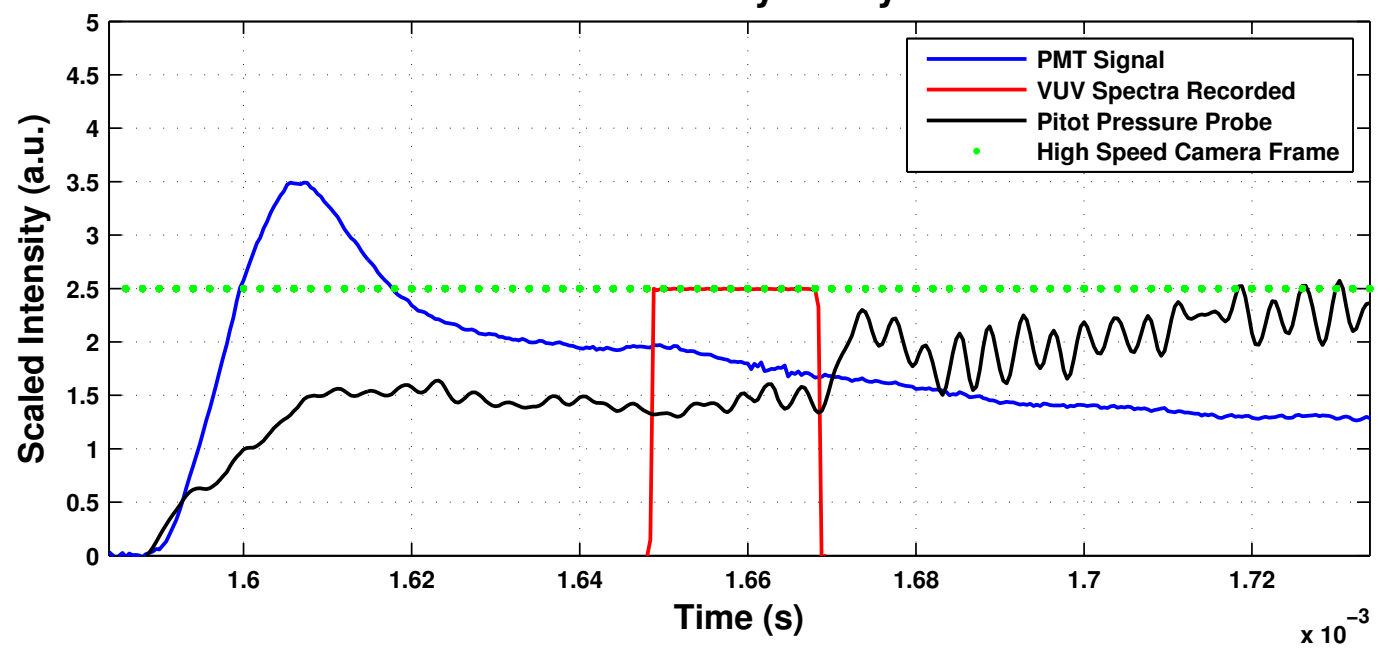

Figure D.17: x2s1942 - Primary shot data for across the surface of a $45 \mathrm{~mm}$ model at condition 2 . 

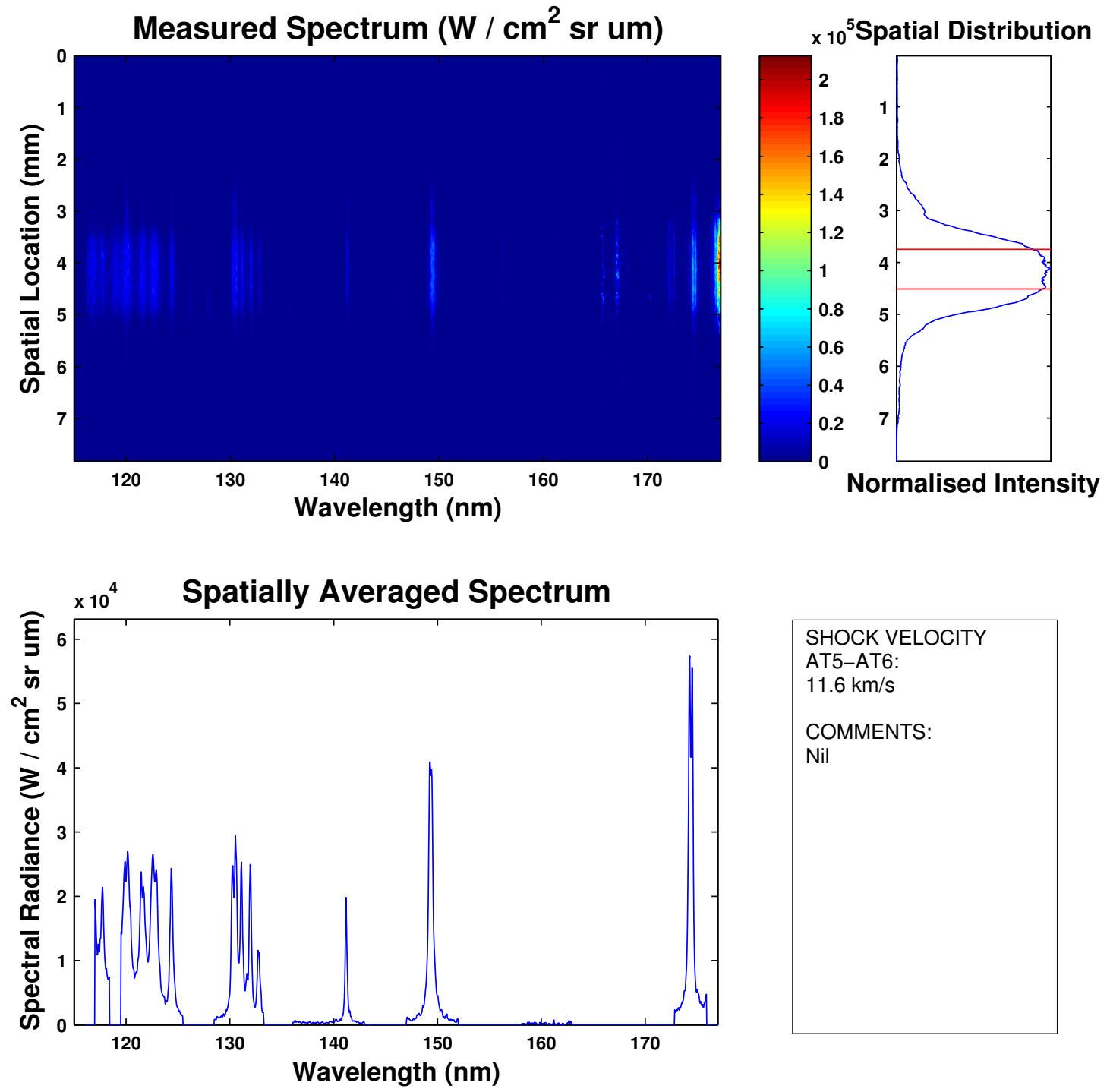

Shot Validity Analysis

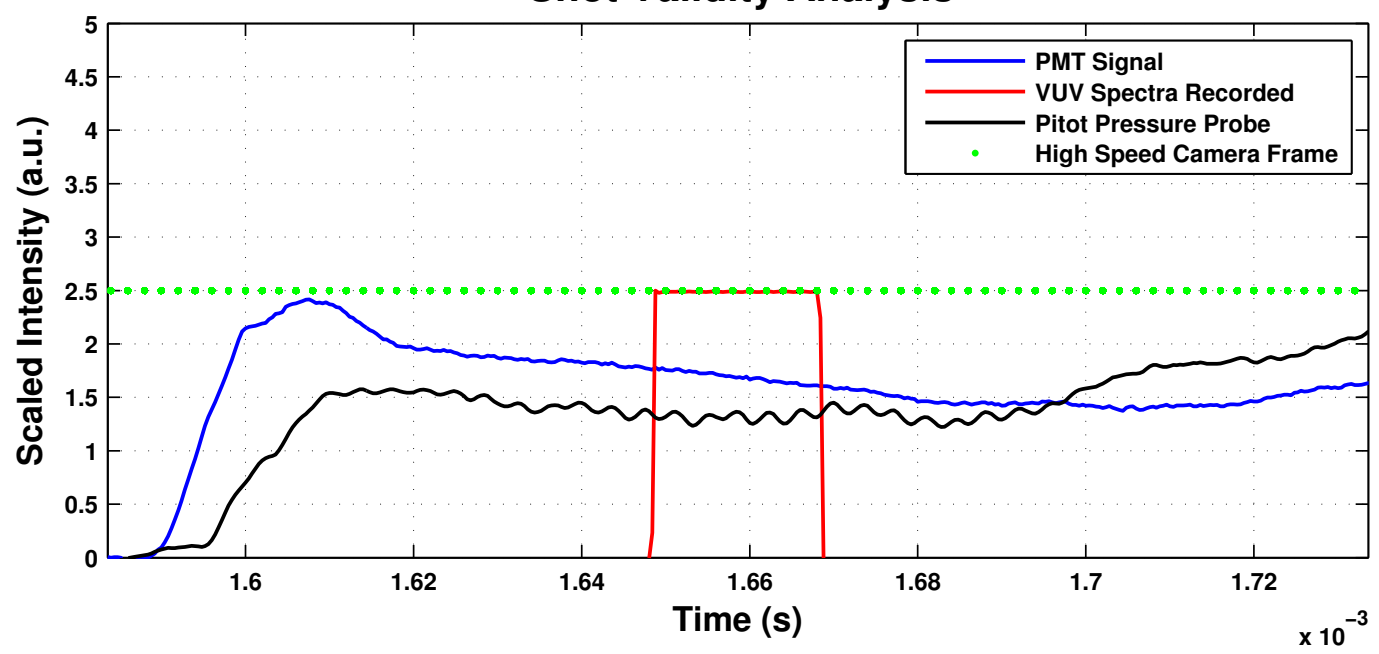

Figure D.18: x2s1943 - Primary shot data for across the surface of a $20 \mathrm{~mm}$ model at condition 2 . 

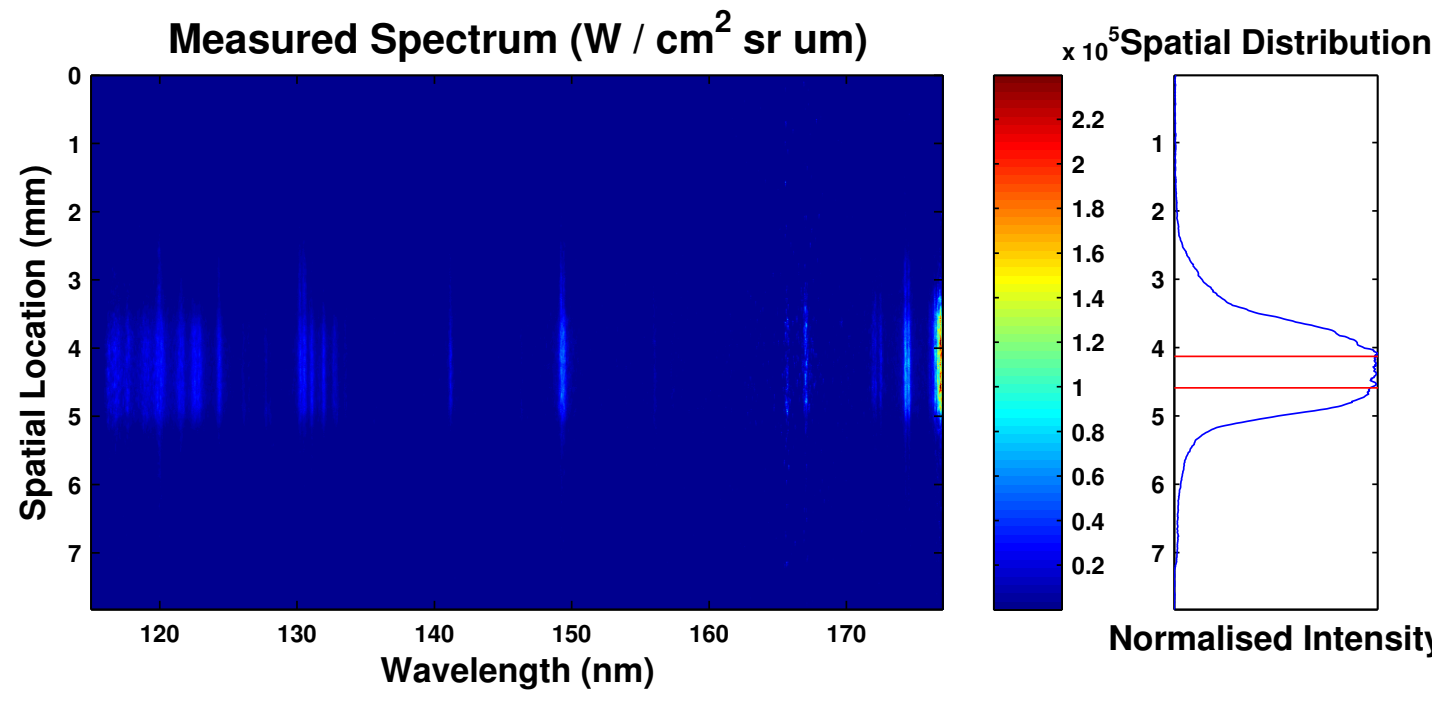

Normalised Intensity
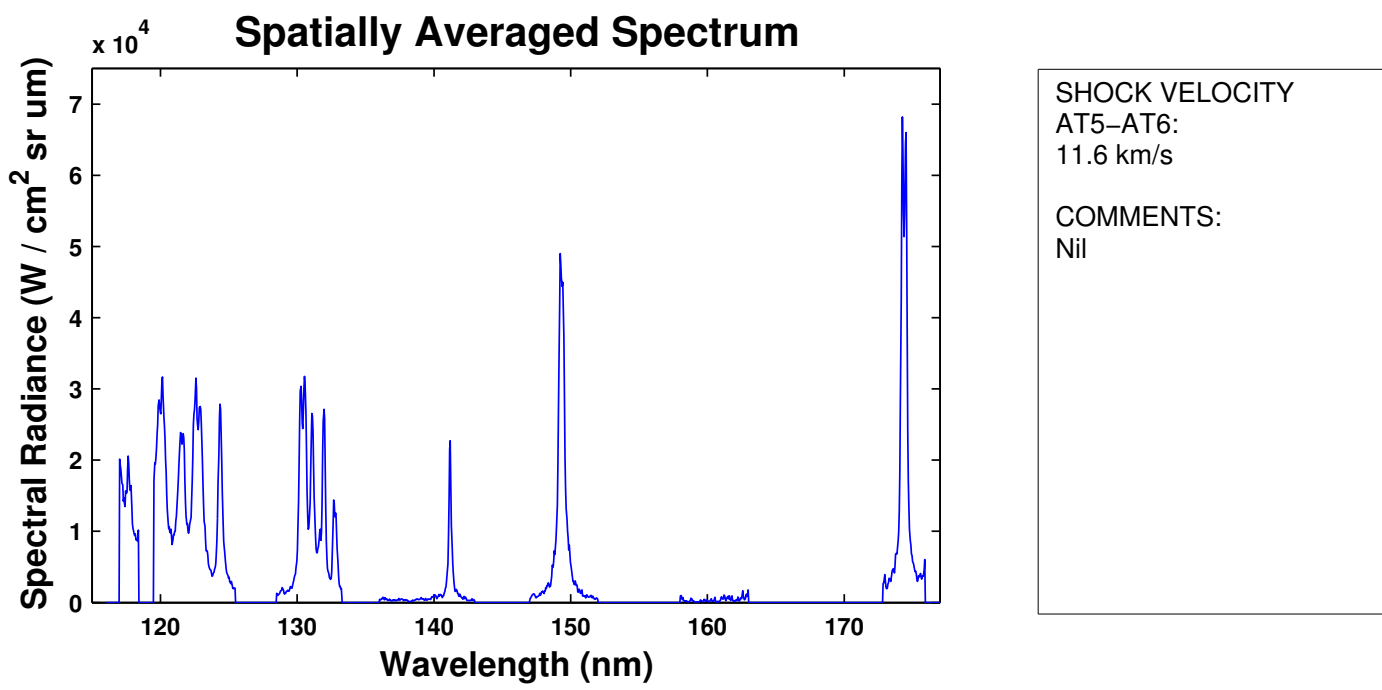

\section{Shot Validity Analysis}

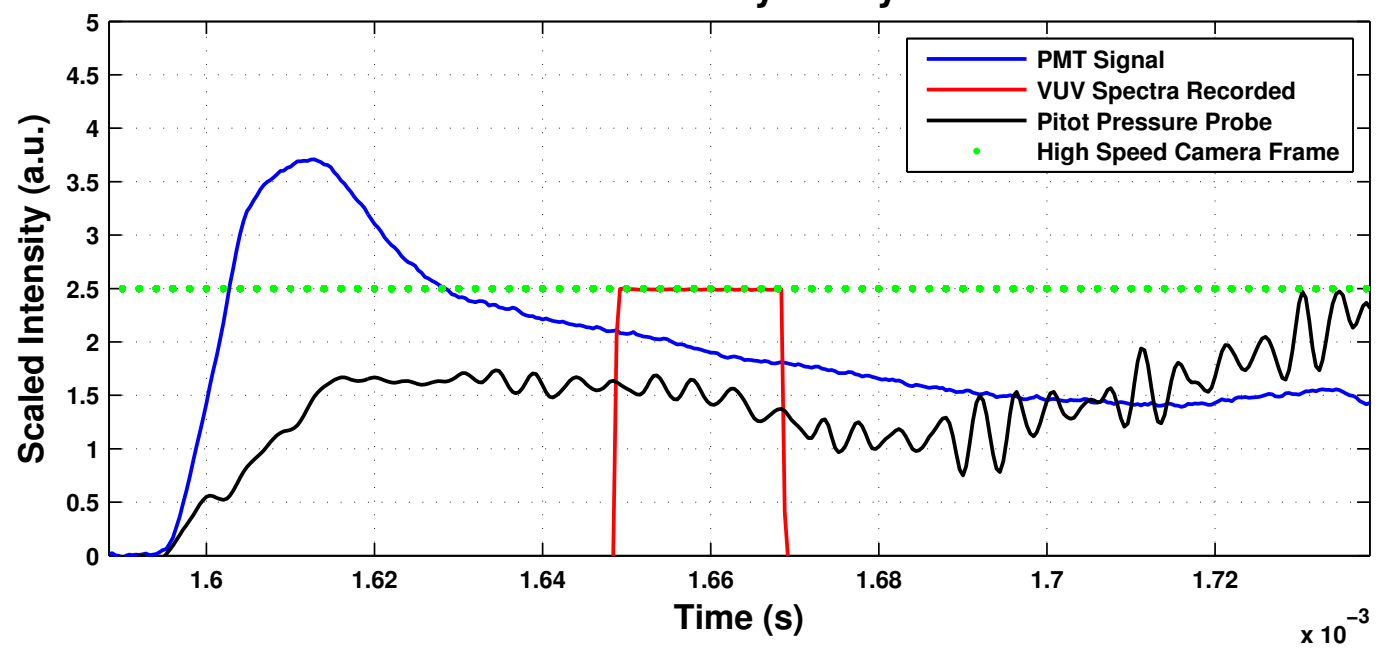

Figure D.19: x2s1944 - Repeat shot data for across the surface of a $20 \mathrm{~mm}$ model at condition 2. 


\section{Appendix E}

\section{Computational Simulation Code}

The following appendix presents the Eilmer script used to create the model shape, grid, gas chemistry and boundary conditions. Two examples are presented:

- Square model at condition 1

- Cylindrical model at condition 2

\section{E.1 Square Model}

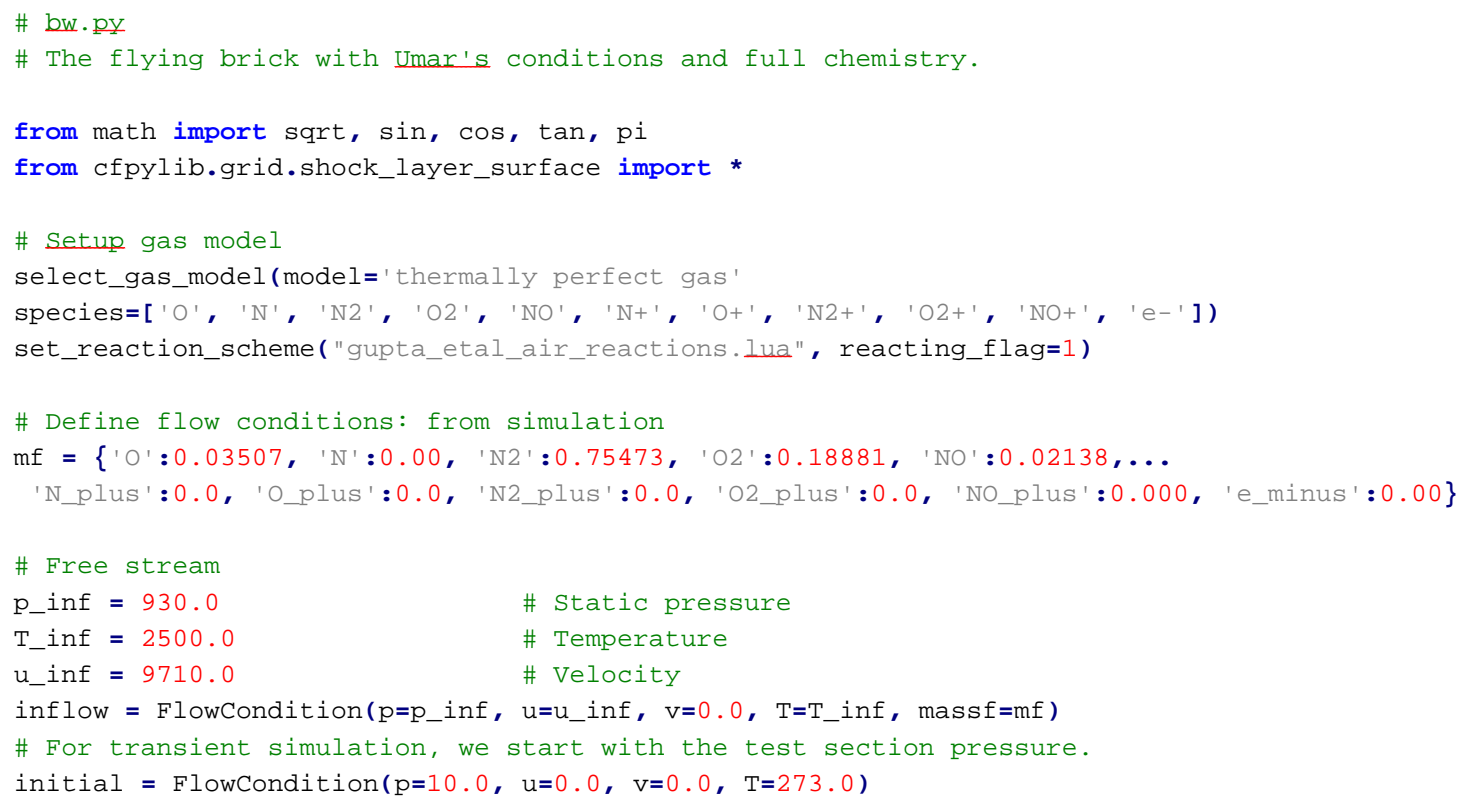


\# The shock angle for a 10 degree ramp with sharp leading edge \# is 20 degrees (read from NACA 1135, chart 2),

\# however, the blunt nose displaces the shock a long way out

\# so we allow some more space.

\# We need to set the boundary high enough to avoid the shock

$\mathrm{R} 3=\mathrm{Rn}+1 *$ delta

$f=\operatorname{Node}\left(-R 3 \star^{\star} \sin (a l p h a), R 3 *^{*} \cos (a l p h a)\right.$, label=' f')

\# Now, put in intermediate control points so that we can use \# cubic Bezier curve for the inflow boundary around the nose \# and a straight line downstream of point $f$.

el = Node (e.x, delta, label='el')

alpha2 $=60.0 / 180.0 * \mathrm{pi}$

$f 1=$ Node(f.x-delta*cos(alpha2), f.y-delta*sin(alpha2), label='f1')

ef $=\operatorname{Bezier}([e, e 1, f 1, f])$

$g=$ Node $\left(x E n d, f \cdot y+(x E n d-f . x) * \tan (a l p h a 2), l a b e l=g^{\prime}\right)$

\# Define straight-line segments between surface and outer boundary.

$e b=\operatorname{Line}(e, b) ; f_{c}=\operatorname{Line}(f, c) ; d g=\operatorname{Line}(d, g) ; f g=\operatorname{Line}(f, g)$

\# Define the blocks using the path segments.

\# Note that the EAST face of regiono wraps around the nose and

\# that the NORTH face of regiono is adjacent to the WEST face

\# of region 1 .

region0 = make_patch (fc, bc, eb, ef)

$\mathrm{cf}=\mathrm{fc} \cdot \mathrm{copy}() ; \mathrm{cf} \cdot$ reverse() \# common boundary but opposite sense

region1 = make_patch $(\mathrm{fg}, \mathrm{dg}, \mathrm{cd}, \mathrm{cf})$

cluster0 = RobertsClusterFunction(0, 1, 1.0)

cluster $1=$ RobertsClusterFunction $(1,0,1.0)$

$\mathrm{nnj0}=60$

nnio $=60$

$\mathrm{nnil}=100$

blk_0 = Block2D(region0, nni=nni0, nnj=nnj0,

cf_list $=[$ cluster0, None, cluster0, None] ,

fill_condition=initial,

xforce_list $=[0,1,0,0])$

blk_1 = Block2D(region1, nni=nnil, nnj=nnj0,

cf_list=[None, cluster 1 , None, cluster 1$]$,

fill_condition=initial,

xforce_list $=[0,0,1,0])$

identify_block_connections()

blk_0.bc_list[WEST] = SupInBC (free_stream)

blk_1.bc_list[NORTH] = SupInBC(free_stream)

blk_1.bc_list [EAST] = ExtrapolateOutBC()

\# We can set individual attributes of the global data object.

job_title = "Blunt Wedge $\mathrm{Rn}="+\operatorname{str}(\mathrm{Rn})$

print job_title

gdata.title = job_title

gdata.axisymmetric_flag $=0$

gdata.viscous_flag $=1$

gdata.flux_calc $=$ ADAPTIVE

gdata.max_time $=50.0 e-6$

print "Final time=", gdata.max_time

gdata.max_step $=500000$

gdata. $d t=5 \cdot 0 e-11$

gdata. $\operatorname{cfl}=0.4$

gdata.stringent_cfl $=1$

gdata.dt_plot $=5 \cdot 0 e-6$

gdata.dt_history = gdata.max_time / 100.0 


\section{E.2 Cylindrical Model}

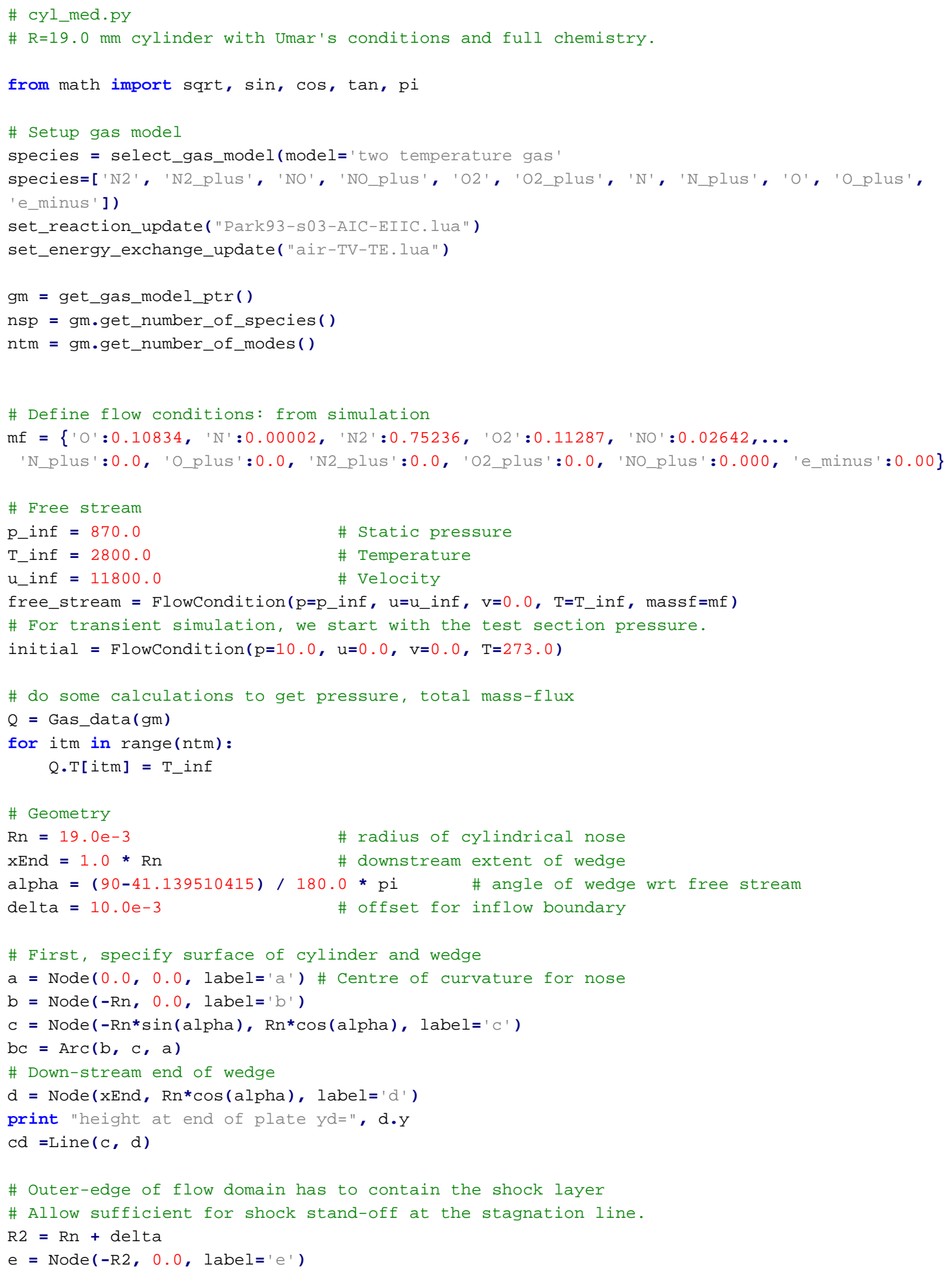


\# The shock angle for a 10 degree ramp with sharp leading edge \# is 20 degrees (read from NACA 1135, chart 2),

\# however, the blunt nose displaces the shock a long way out

\# so we allow some more space.

\# We need to set the boundary high enough to avoid the shock

$\mathrm{R} 3=\mathrm{Rn}+1$ * delta

$f=\operatorname{Node}\left(-R 3 * \sin (a l p h a), R 3 * \cos (a l p h a)\right.$, label=' $\left.f^{\prime}\right)$

\# Now, put in intermediate control points so that we can use

\# cubic Bezier curve for the inflow boundary around the nose

\# and a straight line downstream of point $f$.

el = Node (e.x, delta, label='el')

alpha2 $=60.0 / 180.0 * \mathrm{pi}$

$f 1=$ Node(f.x-delta*cos(alpha2), f.y-delta*sin(alpha2), label='f1')

ef $=\operatorname{Bezier}([e, e 1, f 1, f])$

$g=\operatorname{Node}\left(x E n d, f . y+(x E n d-f . x) * \tan (a l p h a 2), \quad l a b e l=g^{\prime}\right)$

\# Define straight-line segments between surface and outer boundary.

$e b=\operatorname{Line}(e, b) ; f_{c}=\operatorname{Line}(f, c) ; d g=\operatorname{Line}(d, g) ; f g=\operatorname{Line}(f, g)$

\# Define the blocks using the path segments.

\# Note that the EAST face of regiono wraps around the nose and

\# that the NORTH face of regiono is adjacent to the WEST face

\# of region 1 .

regiono = make_patch (fc, bc, eb, ef)

cf $=f(c \cdot c o p y() ; c f \cdot r e v e r s e()$ \# common boundary but opposite sense

region1 = make_patch $(f g, d g, c d, c f)$

cluster0 = RobertsClusterFunction $(0,1,1.0)$

cluster1 = RobertsClusterFunction(1, 0, 1.0)

$\mathrm{nnj0}=60 ; \mathrm{nni0}=60 \mathrm{nnil}=100$

blk_0 = Block2D(region0, nni=nni0, nnj=nnj0,

cf_list $=[$ cluster0, None, cluster0, None],

fill_condition=initial,

xforce_list $=[0,1,0,0])$

blk_1 = Block2D(region1, nni=nnil, nnj=nnj0,

cf_list=[None, cluster1, None, cluster1],

fill_condition=initial,

xforce_list $=[0,0,1,0])$

identify_block_connections()

$\mathrm{blk}$ 0.bc_list [WEST] = SupInBC (free_stream)

$\mathrm{blk} \_1 . \mathrm{bc} \_l i s t[\mathrm{NORTH}]=\operatorname{SupInBC}$ (free_stream)

$\mathrm{blk} \_1 . \mathrm{bc} \_l i s t[\mathrm{EAST}]=$ ExtrapolateoutBC()

\# We can set individual attributes of the global data object.

job_title = "Blunt Wedge $\mathrm{Rn}="+\operatorname{str}(\mathrm{Rn})$

print job_title

gdata.title = job_title

gdata.axisymmetric_flag $=0$

gdata.viscous_flag $=1$

gdata.flux_calc $=$ ADAPTIVE

gdata.max_time $=50.0 e-6$

print "Final time=", gdata.max_time

gdata.max_step $=500000$

gdata. $\cdot d t=5 \cdot 0 e-11$

gdata. $\operatorname{cfl}=0.4$

gdata.stringent_cfl $=1$

gdata. $d t \_p l o t=5.0 e-6$

gdata.dt_history = gdata.max_time / 100.0

HistoryLocation(b.x-0.001, b.y) \# just in front of the stagnation point 